\title{
Hydrographic Data from R/V Endeavor Cruise \#143
}

\author{
by \\ M.C. Stalcup, T.M. Joyce, J.L. Bullister, \\ R.L. Barbour and J.A. Dunworth \\ Woods Hole Oceanographic Institution \\ Woods Hole, Massachusetts 02543
}

March 1988

\section{Technical Report}

Funding was provided by the National Science Foundation under grant Nos. OCE 85-15642 and OCE 85-18372.

Reproduction in whole or in part is permitted for any purpose of the United States Government. This report should be cited as:

Woods Hole Oceanog. Inst. Tech. Rept., WHOI-88-7.

Approved for publication; distribution unlimited.

Approved for Distribution:

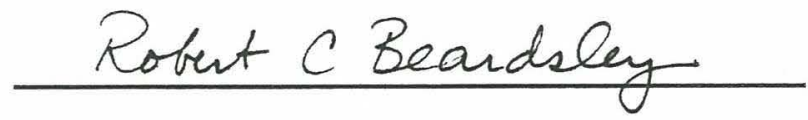

Robert C. Beardsley, Director Department of Physical Oceanography 

TABLE OF CONTENTS

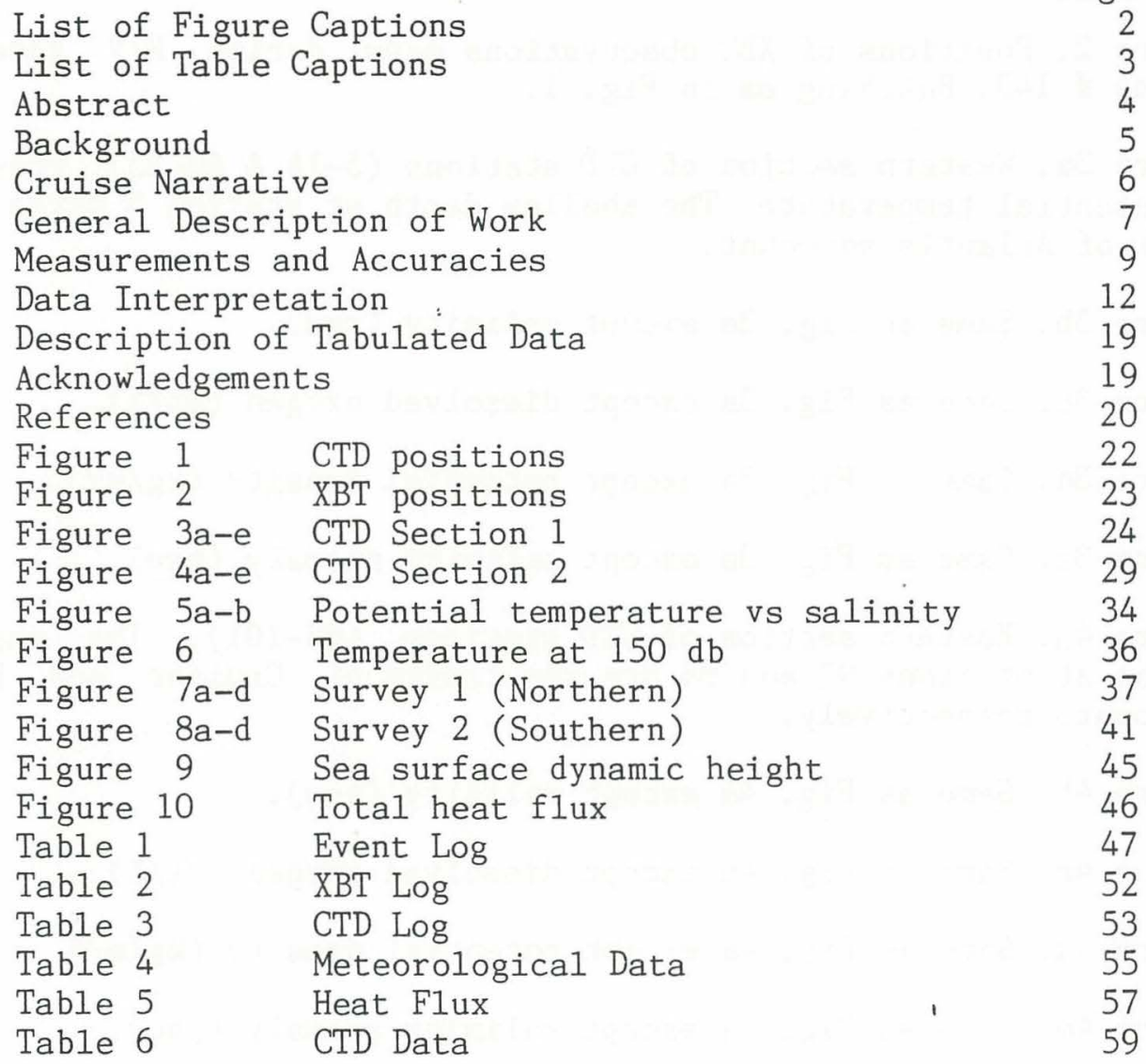




\section{LIST OF FIGURE CAPTIONS}

Figure 1. Positions of CTD-02 stations occupied from May 1-19, 1987 during R/V Endeavor cruise \# 143. The hatching define areas with less than 1000 and less than $3000 \mathrm{~m}$. The positions of several seamounts are noted.

Figure 2. Positions of XBT observations made during R/V Endeavor cruise \# 143. Hatching as in Fig. 1.

Figure 3a. Western section of CTD stations (5-14 \& 48-53). Pressure vs potential temperature. The shallow depth at station 5 marks the flank of Atlantis seamount.

Figure 3b. Same as Fig. 3a except salinity (psu).

Figure 3c. Same as Fig. 3a except dissolved oxygen (ml/1).

Figure 3d. Same as Fig. 3a except potential density $\left(\mathrm{kg} / \mathrm{m}^{3}\right)$

Figure 3e. Same as Fig. 3a except salinity anomaly (psu).

Figure 4a. Eastern section of CTD stations (85-101). The shallow depths at stations 93 and 98 are the flanks of Cruiser and Plato seamounts respectively.

Figure 4b. Same as Fig. $4 a$ except salinity (psu).

Figure 4c. Same as Fig. $4 a$ except dissolved oxygen $(\mathrm{ml} / 1)$.

Figure 4d. Same as Fig. 4a except potential density $\left(\mathrm{kg} / \mathrm{m}^{3}\right)$

Figure 4e. Same as Fig. 4a except salinity anomaly (psu).

Figure 5a. Potential temperature vs salinity diagram for Section 1.

Figure 5b. Potential temperature vs salinity diagram for Section 2.

Figure 6. Potential temperature at a depth of $150 \mathrm{~m}$ during En-143. Data are from CTD and XBT measurements. Hatching as in Fig. 1.

Figure 7a. Pressure, potential temperature, salinity and dissolved oxygen at a potential density of 26.2 during northern survey.

Figure 7b. Same as Fig. 7a except the potential density is 26.3.

Figure 7c. Same as Fig. 7a except the potential density is 26.4 .

Figure 7d. Same as Fig. 7a except the potential density is 26.5. 
Figure 8a. Pressure, potential temperature, salinity and dissolved oxygen at a potential density of 26.2 during southern survey.

Figure 8b. Same as Fig. 8a except the potential density is 26.3.

Figure 8c. Same as Fig. 8a except the potential density is 26.4 .

Figure 8d. Same as Fig. 8a except the potential density is 26.5 .

Figure 9. Dynamic height of the sea surface relative to $300 \mathrm{db}$ for the northern (a) and southern (b) small-scale surveys.

Figure 10. Total heat flux (watts $/ \mathrm{m}^{2}$ ) during En-143 using data from Table 5 .

\section{LIST OF TABLE CAPTIONS}

Table 1. Log of events occurring during En-143. Events are Conductivity-Temperature-Depth stations (C), Expendable Bathythermographs (X), 'bobber' floats and buoys (B) and other (O).

Table 2. Date, time, position and surface temperature and salinity values at XBT launch sites.

Table 3. Date, time, position and surface data from CTD stations.

Table 4. Meteorological data from En-143. Approximately $1 / 3$ of the surface temperature data are interpolated.

Table 5. Heat flux calculations from En-143 using data from Table 4. The columns are sensible, latent and long-wave heat flux with positive values emanating from the sea surface. SWR is short-wave insolation, Tau is the wind stress in Pascals and mix is the mixing effect on the ocean surface in Watts $/ \mathrm{m}^{2}$.

Table 6. Listings of the CTD, hydrographic and chlorofluorocarbon (F-11, F-12) data obtained during R/V Endeavor cruise \# 143. The first listing for each station is the CTD data at standard pressures. Each temperature, salinity and oxygen value is the average of a 2 decibar segment of the water column. These observed values are followed by the derived values potential temperature, sigma-theta, sigma-1500 m, sigma-3000 m, dynamic height, Brunt Vaisala frequency and depth. The second listing presents the water sample values for salinity, oxygen and Freons F-11 and F-12 together with the CTD pressure and temperature at which the sample was collected. 


\section{ABSTRACT}

Hydrographic data collected during R/V Endeavor cruise 143 is presented as a preliminary study of subduction in the northeast Atlantic south of the Azores Front. The front is clearly defined at the northern end of CTD section \# 1 which also shows a layer of $16-18^{\circ} \mathrm{C}$ water subducted to the south. Section \# $2,280 \mathrm{~km}$ to the east, is dominated by a large cyclonic ring with characteristics similar to 'eastern' rings reported earlier. An anomalously salty parcel of Mediterranean water in this section is typical of highly saline lenses seen in the Canary Basin. 


\section{BACKGROUND}

$\mathrm{R} / \mathrm{V}$ Endeavor cruise \# 143 was the result of a proposal entitled 'An Exploratory Study of Oceanic Subduction' which was submitted to the National Science Foundation (NSF) by Drs. Thomas Keffer and Terrence M. Joyce in 1985. The long-term goal of this study is to determine the processes which control the potential vorticity of a water parcel as it is subducted from the mixed layer to the interior. Keffer's and Joyce's proposal is envisioned as the first of a two part program during which an exploratory study will examine the first principles of subduction and new instruments will be tested.

The field work during the Endeavor cruise was designed to gather data to determine the geometry of the mixed layer/thermocline connection in late winter, to assess the extent to which episodic events dominate this geometry, to learn whether potential vorticity is conserved within the water column as it leaves the near-surface, to define the 'upstream' conditions needed for subduction and learn how the initial 'capping' of the subducted water occurs, and finally to determine the scales of subduction in the eastern subtropical North Atlantic.

Two long sections of 15-16 CTD stations to $3000 \mathrm{db}$ (Figure 1), were planned to depict the late winter interconnection between the mixed layers and the thermocline. The sections were also designed to delineate the scales of subduction and to identify the 'age class' of the various water parcels at different stages of the subduction process. Previous work in the nearby 'beta-triangle' region by Jenkins (1987) suggested a direction for the 'age gradient' from $3 \mathrm{H} /{ }^{3} \mathrm{He}$ measurements in the thermocline. The two long sections were extended along this 'age gradient'.

Two small scale surveys consisting of about 30 shallow CTD ( $300 \mathrm{db}$ ) stations spaced about $18 \mathrm{~km}$ apart (Figure 1 ), were planned to map the mesoscale variability of temperature, salinity and thickness of the near surface mixed layers and to illustrate how the 'capping off' process occurs. At three selected levels, samples were collected from the rosette for chlorofluorocarbons (Freons) and $3_{\mathrm{H}} / 3_{\mathrm{He}}$ for meso-scale resolving tracer surveys.

Acoustic Doppler current measurements (Joyce et al., 1982) operating at $115 \mathrm{kHz}$ were planned to provide continuous velocity profiles to $300 \mathrm{~m}$ in the study area. Unfortunately, a failure of one component of the equipment resulted in the loss of this data set.

A complementary proposal was submitted to NSF by Dr. J.F. Price to test several variable buoyancy SOFAR floats (bobbers) which are 
capable of cycling between preset isotherms and/or pressure surfaces. To track the 'bobbers' two Real-time Link and Acquisition Yare System (RELAYS) buoys were designated for deployment within the study area.

An important component of this experiment is the measurement of Freon 11 and 12, helium and tritium which will enable ages to be assigned to the various water parcels. Drs. Jenkins and Bullister of WHOI are responsible for helium-tritium and Freon respectively.

R/V Endeavor cruise \# 143 was designed as a preliminary study of subduction and is expected to provide information useful in planning a major field experiment on subduction which is being formulated as part of the World Ocean Circulation Experiment.

\section{CRUISE NARRATIVE}

The following is a narrative of the event 1 og presented in Table 1. R/V Endeavor cruise \# 143 began at 1025 May 1, 1986 when the ship left the harbor at Punta Delgada, Azores. At 2140 that evening RELAYS drifter \# 02 was launched at $35^{\circ} 50.6^{\prime} \mathrm{N}, 26^{\circ} 52.3^{\prime} \mathrm{W}$ to provide for the tracking of 'bobber' floats to be tested later in the cruise. This operation was followed by a CTD station to $2000 \mathrm{~m}$ to test instrument \# 7. Following this station an XBT section was begun toward the SSW at 0248 May 2 to define the temperature structure in the upper $800 \mathrm{~m}$ of the water column along the northeastern edge of the survey area. Problems with the XBT deck launcher were encountered which resulted in the failure of several probes. The use of a hand held launcher solved this problem. At 0008 and again at 0344 on May 3, CTD \# 9 was tested at two $1000 \mathrm{~m}$ CTD stations after which the XBT section was continued. CTD station \# 4 was made to $1000 \mathrm{~m}$ at 0722 on May 3 at $30^{\circ} 52.5^{\prime} \mathrm{N}, 29^{\circ} 35.6^{\prime} \mathrm{W}$. At 0530 May 4 the second RELAYS buoy was deployed at $34^{\circ} 00.2^{\prime} \mathrm{N}$, $30^{\circ} 59.7^{\prime} \mathrm{W}$ near the site of CTD station \# 5 . This station marked the beginning of the first long section of 15 deep (3000 db) CTD stations made toward the SSW. CTD instrument \# 7 was used during these deep stations.

At 0952 May 6, 1986 the first test of a neutrally buoyant 'bobber' float was made at $31^{\circ} 10.0^{\prime} \mathrm{N}, 29^{\circ} 45.0^{\prime} \mathrm{W}$. This site marked the beginning of the first small-scale, shallow survey. Except during stations 16-18, CTD instrument \# 9 was used throughout this survey which consisted of approximately 30 CTD stations to $300 \mathrm{~m}$ on a grid with about $18 \mathrm{~km}$ spacing between stations. The northern of the two smal1-scale surveys was located immediately to the south of the Azores Front, (Kase and Siedler, 1982).

The study was centered near $32^{\circ} \mathrm{N}, 30.5^{\circ} \mathrm{W}$. CTD stations 46 and 47 
were made near the position of the second bobber float test at about $31^{\circ} 10^{\prime} \mathrm{N}, 29^{\circ} 45^{\prime} \mathrm{W}$. Station \# 46 was to $567 \mathrm{db}$ and \# 47 went to within $20 \mathrm{db}$ of the bottom at $3526 \mathrm{db}$. CTD stations 48-53 completed the long SSW section and were followed by the second, southern, small-scale survey centered near $29^{\circ} \mathrm{N}, 32^{\circ} \mathrm{W}$. Station \# 54 began this survey at 1310 May 11 and was followed by a test of a bobber. At 1306 May 12 another bobber test was made near the position of CTD station \# 68 at $29^{\circ} 10.1^{\prime} \mathrm{N}, 31^{\circ} 51.2^{\prime} \mathrm{W}$. This bobber was recovered the next day at 1043. CTD station \# 85 marked the beginning of the second long section at 1435 May 13 at $29^{\circ} 24^{\prime} \mathrm{N}, 30^{\circ} 03.1^{\prime} \mathrm{W}$. This section was composed of 16 CTD stations along a section toward the NNE at a nominal spacing of $32 \mathrm{~km}$ and a maximum depth of $3000 \mathrm{~m}$. One bobber was tested near CTD station \# 89 at 1038 May 15 and another near CTD station \# 90 at 1504 the same day. The latter was recovered the following day at 1535. CTD station \# 101 ended the section at 1127 May 18, 1986. One RELAYS buoy was recovered at 2012 that evening. The cruise ended when the ship returned to Punta Delgada at 1105 May 19, 1986.

\section{GENERAL DESCRIPTION OF WORK}

The following table lists the scientific participants of Endeavor Cruise \# 143 and notes each person's responsibility during the cruise. The asterisks indicate those individuals who, in addition to their regular duties, maintained a scientific watch throughout the cruise to launch XBTs, occupy CTD stations, etc.

\section{Scientific Party During En-143}

\begin{tabular}{lll} 
SCIENTIST & & RESPONSIBILITY \\
\hline Dr. James Price & $*$ & Chief Scientist \\
Dr. Terrence Joyce & $*$ & CTD, Acoustic Doppler \\
Dr. Thomas Keffer & $*$ & CTD \\
Dr. John Bullister & & Freon \\
Dr. David Musgrave & $*$ & CTD \\
Mr. Marvel Stalcup & $*$ & Salinity-Oxygen \\
Ms. Mary Woodgate-Jones & $*$ & CTD data processing \\
Ms. Siobhan Knutte1 & $*$ & CTD hardware \\
Mr. Jan Zelag & $*$ & URI Technician \\
Mr. Patrick O'Malley & & RELAYS Buoys \\
Mr. Douglas Webb & & SOFAR bobbing floats \\
Mr. Edward Mellinger & & RELAYS buoys \\
Mr. Scot Birdwhistell & $*$ & Helium-tritium
\end{tabular}


The equipment used during typical WHOI hydrographic cruises consists of a CTD, rosette and a pinger suspended from a $0.83 \mathrm{~cm}$ (0.326 in) conducting cable about $10,000 \mathrm{~m}$ long. The instruments are housed within a stainless steel cage to protect them from damage should the package hit the side of the ship. Other equipment may be attached to this package and their signals added to the normal data stream which is displayed and recorded in the ship's laboratory. The accuracy of the CTD is described by Millard (1982).

The General Oceanics Rosette water sampler permits the sequential tripping of 24 Niskin bottles. During typical WHOI hydrographic stations, 24 water samples of 1.2 liter volume are collected with the rosette and analysed for salinity and dissolved oxygen concentrations. These values are used to calibrate the respective sensors on the CTD. During the present cruise, 5 liter Niskin bottles were used to collect the water samples. The larger volume bottles were required to minimize Freon contamination contributed by some components of the Niskin bottles. Samples were also collected for the analysis of helium and tritium to permit dating of the various water masses.

The Oceanographic Instrument Systems bottom finding pinger emits a $12 \mathrm{kHz}$ sound once per second. This pulse, together with its echo from the bottom, is graphically recorded in the ship's laboratory to continuously monitor the distance between the instrument package and the ocean bottom. At typical hydrographic stations water samples are collected within $20 \mathrm{~m}$ of the bottom. During this cruise, however, most casts ended at either 300 or $3000 \mathrm{db}$.

To minimize the loss of oxygen due to biologic activity or other causes, oxygen samples are preferentially the first to be collected from the Niskin bottles. During En-143 cruise, however, it was necessary to change the normal procedure and collect the Freon and helium-tritium samples first. This resulted in a delay of about 30 minutes before the oxygen samples were drawn and reagents added. 


\section{MEASUREMENTS AND ACCURACIES}

Continuous measurements of pressure, temperature, conductivity and oxygen were made with a Mark-3 NBIS Conductivity- Temperature-Depth (CTD) instrument at each CTD station, (CTD instrument \# 7 was used during most $3000 \mathrm{db}$ stations while CTD \# 9 was employed at the 300 $\mathrm{db}$ stations). At each of the deep stations, 24 five liter water samples were collected at various depths to calibrate the CTD conductivity and oxygen sensors, define maxima and minima in the water column and provide samples for the analysis of Freon, helium and tritium. During most of the $300 \mathrm{db}$ stations three samples were collected from the five liter Niskin bottles, primarily for the analysis of Freon, helium and tritium. As discussed below, these samples could not be used to calibrate the CTD oxygen and salinity sensors. The ' $O$ ' rings in the 5 liter Niskin bottles were treated before the cruise to remove contaminants and plastic coated closure springs were installed in the bottles to minimize contamination of the Freon samples.

On1y those CTD data collected while the instrument was lowered are presented here. The placement of the pressure, conductivity and oxygen sensors near the bottom of the instrument ensures that relatively undisturbed water is encountered during lowering. With the sensors in this configuration gradients are measured more accurately during lowering than during retrieval of the CTD. The calibration water samples, however, are collected as the instrument is raised to the surface while pressure is decreasing and temperature is increasing. Water samples collected during retrieval expand due to the decreasing pressures and increasing temperatures. The change in volume forces water out of the Niskin bottles. If samples were collected while lowering the CTD, increased pressures and decreased temperatures would reduce the volume of the sample and sea water would enter the Niskin bottle. Contamination of this type has been observed during tests to develop a sampler suitable for use during the 'down' cast.

\section{Pressure and Temperature Measurements}

Some years ago the CTD pressure and temperature sensors were calibrated using data collected with deep sea, reversing thermometers. Over the years however, the stability and accuracy of these CTD sensors have proven superior to the thermometric measurements and this practice has been abandoned. The CTD pressure and temperature sensors are now calibrated before and after each cruise. Historically the stability of both sensors is quite good with an average drift of $1 \mathrm{db}$ and $0.01^{\circ} \mathrm{C}$ per year. During the present study the CTD pressures and temperatures from all of the deep (3000 db) and shallow (300 db) stations are believed accurate 
to $\pm 1 \mathrm{db}$ and $\pm 0.001^{\circ} \mathrm{C}$ (R.C. Millard, personal communication).

Salinity Measurements

Knapp and Stalcup (1987) have shown that shipboard salinity measurements presently made at WHOI are accurate to \pm 0.001 psu. Calibration of the conductivity sensor on CTD \# 7, used during the deep stations, with the water sample salinity data yields essentially the same accuracy. The quality of the salinity data from instrument \# 9, used during the shallow stations, suffers from the lack of deep calibration data. These salinities are believed accurate to $\pm 0.004 \mathrm{psu}$.

\section{Oxygen Measurements}

For a variety of reasons the Beckman oxygen sensor employed on the NBIS CTD is only marginally useful. The placement of the thermistor within the cell results in a long time constant and does not adequately measure the temperature of the Teflon membrane. Contamination of this membrane is also a problem since it changes the rate at which oxygen diffuses through the membrane and thus affects the electrical output of the cell. Bubbles within the oil filled, pressure compensation chamber may also cause problems. Poor or intermittent electrical contact between the sensor and its mount have also been observed. With all of these shortcomings however, it is possible to calibrate the CTD oxygen data to approximately the same accuracy as that of the water sample data (Owens and Millard, 1985). The CTD oxygen values from the deep stations made during this cruise are believed accurate to $\pm 0.04 \mathrm{ml} / 1$ (Knapp and Stalcup, 1987).

\section{Shallow (300db) Stations}

CTD instrument \# 9 was used during both shallow surveys. This instrument was calibrated with water sample data collected from only one deep station at the beginning and another at the end of the cruise. The stability of the pressure and temperature sensors was adequate to ensure that these data meet the standard accuracies. The conductivity sensor, although not generally as reliable as either the pressure or temperature sensors, appears to have been stable enough to produce CTD salinities to $\pm 0.004 \mathrm{psu}$. The calibration of the oxygen sensor, however, required special treatment.

Only three water samples were collected at each of the $300 \mathrm{db}$ CTD stations made during the shallow surveys. These samples were collected in a region which exhibits relatively large horizontal variations in temperature, salinity and dissolved oxygen in the upper $300 \mathrm{db}$. Since the CTD data are recorded during the 'down' cast and the water samples are collected during the 'up' cast, 
these horizontal variations produced large differences between the two observations at the same pressure and severely limited our ability to accurately calibrate the CTD oxygen sensor. This limitation, together with the poor stability and reliability of the oxygen sensor, forced us to use the following, non-standard calibration procedure. The differences between the water sample and CTD oxygen data from each survey were averaged and used to calculate a single set of calibration values for each survey. Using this calibration procedure we estimate that the overall accuracy of the CTD oxygen data is about $\pm 0.15 \mathrm{ml} / 1$ which is a factor of $3-4$ larger than for the deep cast.

\section{Freon Measurements}

A chlorofluorocarbon (CFC) analytical system built at WHOI was tested during this cruise. Vertical profiles of two dissolved $\mathrm{CFCs}-\mathrm{CCl}_{3} \mathrm{~F}(\mathrm{~F}-11)$ and $\mathrm{CCl}_{2} \mathrm{~F}_{2}(\mathrm{~F}-12)$ were obtained at a number of stations along the cruise track. The sampling procedure was designed to collect samples within thermostads and at various extrema within the water column. These data will be used to determine the 'age' of the various water masses. This age is the time interval since the water was ventilated by the atmosphere. The concentrations of $\mathrm{F}-11$ and $\mathrm{F}-12$ are presented in Table 6 in units of $\mathrm{pmol} / \mathrm{kg}$ ( $1 \mathrm{pmol} / \mathrm{kg}=10-12$ moles per kilogram seawater), and based on the Scripps Institution of Oceanography calibration scale (Bullister, 1984).

CFC contamination problems were encountered during this expedition which reduced the overall accuracy of the seawater measurements. This contamination was in part due to elevated levels of CFCs in shipboard air. At a number of stations seawater samples were contaminated by individual Niskin bottles. Efforts were made during the cruise to identify and eliminate these problems, and contamination episodes were less frequent and less severe during the second half of the cruise (stations 50-101). Standard deviations for measurements of replicate seawater samples (collected from 12 Niskin bottles closed at the same depth at station \# 90) were approximately 0.015 pmol $/ \mathrm{kg}$ for $\mathrm{F}-11$ and 0.027 pmol/kg for F-12. Questions regarding the Freon data should be addressed to Dr. John L. Bullister at WHOI.

Helium-tritium Measurement

Several years are required to process the helium and tritium samples. It is expected that these values will be available in about June 1988. Dr. William J. Jenkins is responsible for these data. Please direct enquiries to him at the Woods Hole Oceanographic Institution, Woods Hole, Massachusetts 02543. 


\section{DATA INTERPRETATION}

CTD SECTION \# 1

\section{Sha11ow Water}

Large horizontal gradients of temperature, salinity and potential density are apparent to a depth of $600 \mathrm{db}$ between stations 5 and 7 at the northern end of this section (Figure 3 ). Using a reference level of $1500 \mathrm{db}$ the density gradient indicates an easterly geostrophic flow near the surface of about $30 \mathrm{~cm} / \mathrm{sec}$. Kaese and Siedler (1982) reported a similar feature in the same region in 1982 and suggested that it represented "part of the gyre circulation, resulting from the branching of the North Atlantic Current". They report a baroclinic transport of $10 \mathrm{~Sv}$ in a $60 \mathrm{~km}$ jet which is in good agreement with the $10 \mathrm{~Sv}$ we measured between stations 5-7 which are $74 \mathrm{~km}$ apart. This strong upper level flow has been termed the Azores Current or Front (Käse et al., 1985).

As shown in Figure 3, to the north of the front the salinity anomaly relative to North Atlantic Central Water is negative and is approximately bounded by the 16 and $18^{\circ} \mathrm{C}$ isotherms (Figure 3a). It can be traced from its outcropping at the surface, near stations 5 and 6 , towards the south where it is subducted and capped by saltier surface water between stations 7-10. The layer thins markedly at about $32^{\circ} \mathrm{N}$ near station 12. As discussed in the Background section of this report, this feature appears to meet the criteria of subducted water. The dissolved oxygen content, however, seems slightly lower than expected at $4.75-5.00 \mathrm{ml} / 1$ or only about $90 \%$ saturated. Perhaps these low oxygen values will be explained when the age of this water has been determined from the tracer analyses.

The deepening of both the salinity anomaly and the dissolved oxygen isopleths beneath the subducted water at stations 7-9 suggest that the influence of this layer may reach as deep as $800 \mathrm{db}$. T. Mueller (personal communication) has pointed out that significant changes in the North Atlantic Deep Water $\mathrm{T} / \mathrm{S}$ properties are co-located with the upper level front.

The $200 \mathrm{db}$ thickness of the $17-19^{\circ} \mathrm{C}$ layer at stations $12-14$ and 48-49 contains water with a salinity between $36.4-36.7$ psu (Figure $3 \mathrm{~b}$ ) and appears to be 'eighteen degree water' as defined by Worthington (1959). His Figure 3 shows the salinity at $300 \mathrm{~m}$ in the North Atlantic and indicates that 'eighteen degree water' is formed to the west of the present study area. The general eastward shoaling of the isotherms within the North Atlantic results in 
$16^{\circ} \mathrm{C}, 36.3 \mathrm{psu}$ water at $300 \mathrm{~m}$ in this section. Our $18-19^{\circ} \mathrm{C}$ temperature modes are slightly warmer, saltier and less dense than the $18^{\circ} \mathrm{C}$ Sargasso Mode Water. To the east of our study region, Siedler et a1. (1987) have shown the dominant temperature mode to be $17-18^{\circ} \mathrm{C}$ and have called this 'Madeira Mode Water'. The $18-19^{\circ} \mathrm{C}$ water is both warmer and saltier than that found in the eastern basin by Siedler et al. (1987).

\section{Oxygen Minimum Layer}

As shown in Figure 3c, the oxygen minimum layer varies from less than $4.25 \mathrm{ml} / 1$ at $700 \mathrm{db}$ in the northern part of this section to less than $4.00 \mathrm{ml} / 1$ at $900 \mathrm{db}$ in the south. Using the 4.5 isopleth to define the boundary of this layer its thickness varies from about $200 \mathrm{db}$ beneath the capped, subducted water at stations 7-9 to $750 \mathrm{db}$ at station 52. The wedge shape of this feature, with lowest values in the south, suggests a source region in this direction. It is likely that the low oxygen characteristic associated with this layer is formed in the area of upwelling, high biologic productivity and low oxygen values found off the coast of Dakar (Bubnov, 1966).

\section{Mediterranean Water}

Large positive salinity anomalies (Figure $3 e$ ) beneath the oxygen minimum layer identify water with an admixture of Mediterranean water which enters the Atlantic over the sill at the Strait of Gibraltar. With salinity anomalies as great as $0.45 \mathrm{psu}$ and salinities near $35.52 \mathrm{psu}$ at a potential temperature of $8.8^{\circ} \mathrm{C}$., this water contains only a small percentage of Mediterranean overflow water. It will be interesting to compare the age of this water with that of the same layer in Section 2, $280 \mathrm{~km}$ to the east. The latter section shows higher salinities in this layer and presumably contains less diluted and younger Mediterranean water.

\section{Deep Water}

The nearly constant depth of the 27.8 potential density surface at $1500 \mathrm{db}$ (Figure 3d) suggests a level of minimum motion here. The slopes of the deeper isopycnals indicate a broad westward flow somewhat concentrated near the southern end of the section. Dissolved oxygen concentrations decrease towards the south while salinity anomalies increase.

Knapp and Stalcup (1987) discuss the accuracy of the salinity and oxygen analyses made during Endeavor cruise \# 143 and note slightly higher standard deviations than typically seen during WHOI cruises. They suggest that geographic variations in these variables at a potential temperature of $2.8^{\circ} \mathrm{C}$ might account for some of the scatter. They place an upper limit on the accuracy of the 
measurements made during this cruise of \pm 0.004 psu for salinity and $\pm 0.06 \mathrm{ml} / 1$ for dissolved oxygen. The calibrated CTD data at this temperature show that, from south to north, the salinity varies between 34.955 to 34.968 psu while the dissolved oxygen changes from 5.72 to $5.96 \mathrm{ml} / 1$. Such oceanic variability increases the standard deviations of the salinity and oxygen measurements at this potential temperature and indicates that the accuracy of these analyses is probably better than reported. As noted above, T/S variations have been noted by Mueller under the Azores Front.

\section{CTD SECTION \# 2}

\section{Shallow Water}

The distribution of the variables in the 0-400 db layer in Section 2 is quite different from that shown in Section 1. The shallow layers in this section are dominated by a large (400 $\mathrm{km}$ diameter) cyclonic ring with eastward flow at stations 85-86 and westward flow at stations 97-98. The doming of the isotherms (Figure 4a) in the center of the ring (near stations 92-93) marks its center and is persistent to a depth of nearly $1000 \mathrm{db}$. At this depth the distribution of the high salinity Mediterranean water (Figure 4b) at both the northern and southern ends of the section shows a symmetry which may be related to the ring dynamics. The ring is clearly shown in Figure 8 . Here the 17 and $18^{\circ} \mathrm{C}$ potential temperature surfaces at $150 \mathrm{~m}$ define the ring which may be a cast off remnant of the Azores Front.

McCartney et al. (1978) report observing several large cold core cyclonic current rings east of $60^{\circ} \mathrm{W}$ and discuss their possible origin near $40^{\circ} \mathrm{W}$. They state that these features are characterised as being larger than typical cold core rings found in the western Sargasso Sea with positive oxygen and negative salinity anomalies at temperatures greater than $13^{\circ} \mathrm{C}$. They used the $500 \mathrm{~m}$ depth of the $15^{\circ} \mathrm{C}$ isotherm to define ring diameter and noted that western rings average $100 \mathrm{~km}$ while eastern rings range from 180-230 km. Current meter measurements in the latter indicate a level of no motion near $2000 \mathrm{~m}$ and they appear to drift slightly south of west at speeds of $2-5 \mathrm{~km} / \mathrm{day}$.

Gould (1981) observed a ring in the process of formation near $33^{\circ} \mathrm{N}$, $33^{\circ} \mathrm{W}$. The ring had a diameter of $100-150 \mathrm{~km}$ with surface speeds near $25 \mathrm{~cm} / \mathrm{sec}$ and was drifting westward at a rate of $2.5 \mathrm{~km} /$ day. Using a reference level of $3400 \mathrm{~m}$ he calculates a transport of 10 Sv, above $1000 \mathrm{~m}$.

In the eastern Atlantic the $15^{\circ} \mathrm{C}$ isotherm rises to less than $500 \mathrm{~m}$ so that this criterion cannot be used to determine the diameter of the ring found in the present study. The ring seen in Section 2 is surrounded by a high salinity band which appears to be nearly symmetrical about its center. If this feature is used to define the 
edge of the ring, its diameter is about $400 \mathrm{~km}$. If the same technique is used to define the rings reported by McCartney et al. (1978, Figure 11), their rings have about the same diameter. The ring described here has salinity and oxygen anomalies similar to those shown by McCartney et al. (1978) in their Figure 10. It is east of the ring reported by Gould (1981) and thus may represent the easternmost of the eastern rings observed to date and places the formation region east of $30^{\circ} \mathrm{W}$.

The negative salinity anomalies (Figure 4e) in the shallow water near the center of the ring contrast with the positive anomalies found in the same temperature range in Section 1. This difference suggests that the source region for this water is different in each section. In Section 1 the $17-19^{\circ} \mathrm{C}$. layer appears to be 'eighteen degree water' which is formed to the west of this area. The same layer near the center of the ring in Section 2 is generally 0.10-0.25 psu fresher than in Section 1 and is probably formed locally. The thicker $17-18^{\circ} \mathrm{C}$ layer in the ring core has charcteristics similar to the Madeira Mode Water.

The high salinity water surrounding the ring is typical of that found along the southern edge of the study area. Levitus (1982) shows an area of maximum surface salinity near $25^{\circ} \mathrm{N}, 34^{\circ} \mathrm{W}$ in which average values exceed 37.2 psu. The northern margin of this region contains surface water with salinities identical to that found along the margin of the ring.

\section{Oxygen Minimum Layer}

In Figure $4 c$ the oxygen minimum layer (less than $4.50 \mathrm{ml} / 1$ ) is centered near $700 \mathrm{db}$ and is relatively uniform in thickness throughout this section with a slight thickening near the southern end where it is closest to its source. The thinning of the $<4.25$ $\mathrm{ml} / 1$ layer between stations $90-97$ may be related to the dynamics of the ring.

Mediterranean Water

The influence of the Mediterranean overflow is stronger here than in Section 1. Figure $4 \mathrm{e}$ shows a continuous layer of $>$ 0.40 psu salinity anomaly with an average thickness of almost 400 $\mathrm{db}$. High values of the anomaly, in excess of $0.50 \mathrm{psu}$, were found at the northern end of the section. Station 87 recorded a particularly anomalous layer of salty water $300 \mathrm{db}$ thick centered near $1000 \mathrm{db}$ with a maximum salinity of almost 35.8 psu and a potential density near 27.65. Figures $5 a-b$ are the potential temperature vs salinity curves for CTD stations made in section 1 (5a) and section 2 (5b). The influence of Mediterranean water is clearly seen at a density anomaly slightly greater than $27.6 \mathrm{~kg} / \mathrm{m}^{3}$ in Figure 5b. The variations in salinity seen at 27.6 in Figure $5 \mathrm{a}$ depict the large variability in the relative abundance of 
Mediterranean water along this section. Station \#. 52 contains the smallest admixture of this water type. The potential temperature vs salinity relationship on the same density anomaly surface in Figure $5 \mathrm{~b}$ shows considerably less variability and generally higher salinities than in $5 \mathrm{a}$. With the exception of station \# 87 described above, the variability in salinity between density anomalies $27.3-27.7$ is only about \pm 0.045 psu in $5 b$ compared with \pm 0.090 for 5 a.

McDowel1 and Rossby (1978) reported anomalously high salinity values in an eddy they observed near the Bahamas and coined the term 'Meddy' to indicate the Mediterranean source for the high salinities found in the core of the eddy.

Armi and Zenk (1984) surveyed three salt lenses in the Canary Basin with central salinities of almost 36.3 psu near $1100 \mathrm{~m}$. Small scale CTD surveys of these features together with moored current meter and temperature records indicated the lenses were about $100 \mathrm{~km}$ in diameter with a homogeneous center about $50 \mathrm{~km}$ across. The geostrophic velocity (at 1000 relative to $1900 \mathrm{db}$ ) at a radius of $20-40 \mathrm{~km}$, is $20-24 \mathrm{~cm} / \mathrm{sec}$. The current meter data indicated that these lenses drift at about $5 \mathrm{~cm} / \mathrm{sec}$. A plot of salinity on the sigma-theta surface 27.7, which used all of the high quality historical data between $30-36^{\circ} \mathrm{N}, 20-30^{\circ} \mathrm{W}$ between $1914-1972$, shows 27 of 97 stations with anomalous salinities, (Armi and Zenk, fig. $23)$. This ratio agrees well with their estimate of greater than .2 for the probability of finding salt lenses within the Canary Basin..

Amber and Howe (1979) discuss mixing in the Mediterranean outflow water and find an upper and lower core which they trace towards Cape St. Vincent where the fraction of Mediterranean water mixed with North Atlantic Central water is reduced to 50 and $38 \%$ respectively in each core. Near Cape St. Vincent, water with a potential density of about 27.6 is found within the lower core. Assuming isentropic mixing and using Amber's and Howe's value for the percentage of Mediterranean water within the lower core, the high salinity water seen at station \# 87 contains about $15 \%$ Mediterranean water.

Armi and Zenk (1984) note that the water mass characteristics within their lenses are similar to that found near Cape St. Vincent, $1000 \mathrm{~km}$ away. They esimate that the lenses they observed are 1-3 years old and are apparently long lived.

The evidence presented above indicates that the anomalous lens of highly saline water observed at station \# 87 during the present study may represent the edge of one of the Canary Basin lenses described by Armi and Zenk (1984). 
Deep Water

The distribution of variables within the deep water in Section \# 2 is similar to that seen in Section \# 1. However, the depths of the temperature and salinity isopleths in the former are generally greater than in the latter. With slightly greater densities present in the eastern section a general southward flow is indicated in the deep water between these sections relative to a mid-depth reference level.

\section{HORIZONTAL PROPERTY DISTRIBUTIONS}

The XBT and CTD observations at $150 \mathrm{~m}$ depth have been combined to show the large scale thermal structure during the cruise (Figure $6)$. The Azores Current, roughly defined by the $16-17^{\circ} \mathrm{C}$ temperature bounds, flows to the southeast and broadens as it encounters the seamounts. As we noted earlier, a cold cyclonic ring near $31.5^{\circ} \mathrm{N}$, $29^{\circ} \mathrm{W}$ was found to the south of the frontal zone. This feature is poorly defined on the eastern edge of our large scale survey. Two meso-scale surveys were made: one at the southern end of the frontal zone and another further to the south, away from the region of possible frontal subduction.

In the northern survey (Figure $7 a-d$ ) property maps have been made on four potential density surfaces: 26.2, in the seasonal thermocline; 26.3, within the region of low static stability or potential vorticity; 26.4 , at the base of the pycnocline; and 26.5, in the upper main pycnocline. According to seasonal climatological data, the 26.5 surface is ventilated in the northeastern portion of our survey area (Keffer, 1985). The topography of this lower surface (Figure 7d) clearly shows the southern edge of the Azores Current, with depths of $150 \mathrm{~m}$ in the northwest and $230 \mathrm{~m}$ in the southeast, with some weak but coherent pattern in the temperature and salinity fields. Dissolved oxygen variations of $0.2 \mathrm{ml} / 1$ exceed our estimate of the 'noise' in the oxygen probe of $\pm 0.15 \mathrm{ml} / 1$. The topography of the 26.3 and 26.4 surfaces is more complicated. Generally, when the surfaces are deeper, salinities and temperatures are higher and vice versa.

In the southern small-scale survey (Figure 8a-d) there is little or no trend to the topography of the density surfaces, only small eddies. The water masses, however, show a definite pattern with warmer, saltier water to the south and cooler, fresher water to the north. A tongue of warm, salty water extends northward and is most apparent at the potential density surface $26.3 \mathrm{~kg} / \mathrm{m}^{3}$. This suggestion of 'flow' is not mirrored in the dynamic height at the surface relative to $300 \mathrm{db}$ (Figure 9). 
Heat Flux

Shipboard observations of barometric pressure, cloud cover, wind speed, wet and dry bulb air temperature and sea surface temperature were made during the cruise at intervals of four hours (Table 4). Although about $30 \%$ of the sea surface temperatures are interpolated values, the absence of strong thermal fronts and uniform temperature gradients near the areas where interpolation was needed suggests that these values are probably accurate to $\pm 0.2^{\circ} \mathrm{C}$.

The meteorological data have been used to calculate the various heat flux components which are presented in Table 5 and Figure 10. These data indicate that winter conditions had passed at the time of the cruise and insolation was warming the surface water to produce the seasonal thermocline. Surface temperatures at CTD stations occupied near the end of the cruise were $1-2^{\circ} \mathrm{C}$ higher than those of XBTs taken near the same position at the beginning of the cruise. 


\section{DESCRIPTION OF TABULATED CTD AND HYDROGRAPHIC DATA}

The station data listings show the CTD and hydrographic observations in two formats. The first listing on each page contains the CTD temperature, salinity and oxygen values at standard pressures. These values are the $2 \mathrm{db}$ average of the calibrated measurements. The next seven columns contain the calculated variables: potential temperature, density relative to 0 , 1500 and $3000 \mathrm{db}$, dynamic height, Brunt-Váisälä period and depth.

Water sample data are shown in the second listing. The first two columns are the CTD pressures and temperatures at which each water sample was collected. These columns are followed by the water sample salinity, dissolved oxygen, Freon 11 and Freon 12. The final columns contain the calculated variables potential temperature, potential density relative to 0 and $3000 \mathrm{db}$ and depth. Missing values generally indicate that the measurement is believed erroneous and has been deleted. Freon samples were not collected from every Niskin bottle. The complete $2 \mathrm{db}$ CTD data set and the water sample data are on file with the National Oceanographic Data Center in Washington D.C.

\section{ACKNOWLEDGEMENTS}

We would like to thank H. Stommel, J. Luyten and R. Käse for sharing their preliminary data with us which helped significantly in planning the field work. We also acknowledge the excellent cooperation and fine seamanship exhibited by the officers and crew of the R/V Endeavor during this cruise. We are indebted to the University of Rhode Island's Graduate School of Oceanography Marine Technician group, under the direction of $\mathrm{Mr}$. William Hahn, for their assistance in preparing the ship for our observational program and for providing technical help during the cruise. This work was supported by the National Science Foundation under grants \# OCE85-15642 and OCE85-18372 to the Woods Hole Oceanographic Institution. 


\section{REFERENCES}

Armi, L. and W. Zenk. 1984. Large lenses of highly saline Mediterranean water. J. Phys. Ocean., 14, 1560-1576.

Amber, I. and M.R. Howe. 1979. Observations of the Mediterranean outflow-I Mixing in the Mediterranean outflow. Deep-Sea Res., 26A, $535-554$.

Bubnov, V.A. 1966. The distribution pattern of minimum oxygen concentration in the Atlantic. Oceanology, 6, 2, 193-201.

Bullister, J.L. 1984. Atmospheric chlorofluoromethanes as tracers of ocean circulation and mixing: Measurement and calibration techniques and studies in the Greenland and Norwegian Seas. PhD Thesis, Univ. Calif. San Diego. 172 p.

Gould, W.J. 1981. A front southwest of the Azores. Int. Counc. Exp1. Sea, CM 1981/C:16.

Jenkins, W.J. 1987. ${ }^{3} \mathrm{H}$ and $3 \mathrm{He}$ in the Beta triangle: Observations of gyre ventilation and oxygen utilization rates. J. Phys. Ocean., $17,6,763-783$.

Joyce, T.M., D.S. Bitterman, Jr. and K.E. Prada. 1982. Shipboard acoustic profiling of upper ocean currents. Deep-Sea Res., 29, 903-913.

Kăse, R.H. and G. Siedler. 1982. Meandering of the subtropical front south-east of the Azores. Nature, 300, 245-246.

Käse, R.H., W. Zenk, T.B. Sanford and W. Hiller. 1985. Currents, fronts and eddy fluxes in the Canary Basin. Prog. Oceanog., 14, $231-257$.

Keffer, T. 1985. The ventilation of the world's oceans: Maps of the potential vorticity field. J. Phys. Ocean., 15, 5, 509-523.

Knapp, G.P. and M.C. Stalcup. 1987. Progress in the measurement of salinity and oxygen at the Woods Hole Oceanographic Institution. Woods Hole Oceanogr. Tech. Rept. WHOI-87-4, 27 pp.

Levitus, S. 1982. Climatic atlas of the World Oceans. NOAA Professional Paper, 13, 173p.

McCartney, M.S., L.V. Worthington and W.J. Schmitz, Jr. 1978. Large cyclonic rings in the northeast Sargasso Sea. J. Geophys. Res., 83, C2, 901-914.

McDowel1, S.E. and H.T. Rossby. 1978. Mediterranean Water: An intense mesoscale eddy off the Bahamas. Science, 202, 1085-1087. 
Millard, R.C. 1982. Calibration and data processing techniques at WHOI using the 1978 practical salinity scale. Proc. Int. STD conference and workshop, La Jolla, Mar. Technol. Soc., 19 pp.

Owens, W.B. and R.C. Millard. 1985. A new algorithm for CTD oxygen calibration. J. Phys. Ocean., 15, 5, 621-631.

Siedler, G., A. Kuhl and W. Zenk. 1987. The Madeira mode water. J. Phys. Ocean. 17, 10, 1561-1570.

Worthington, L.V. 1959. The 18 degree water in the Sargasso Sea. Deep-Sea Res., 5, 297-305. 
Figure 1. Positions of CTD-02 stations occupied from May 1-19, 1987 during $R / V$ Endeavor cruise \# 143. The hatching define areas with less than 1000 and less than $3000 \mathrm{~m}$. The positions of several seamounts are noted.

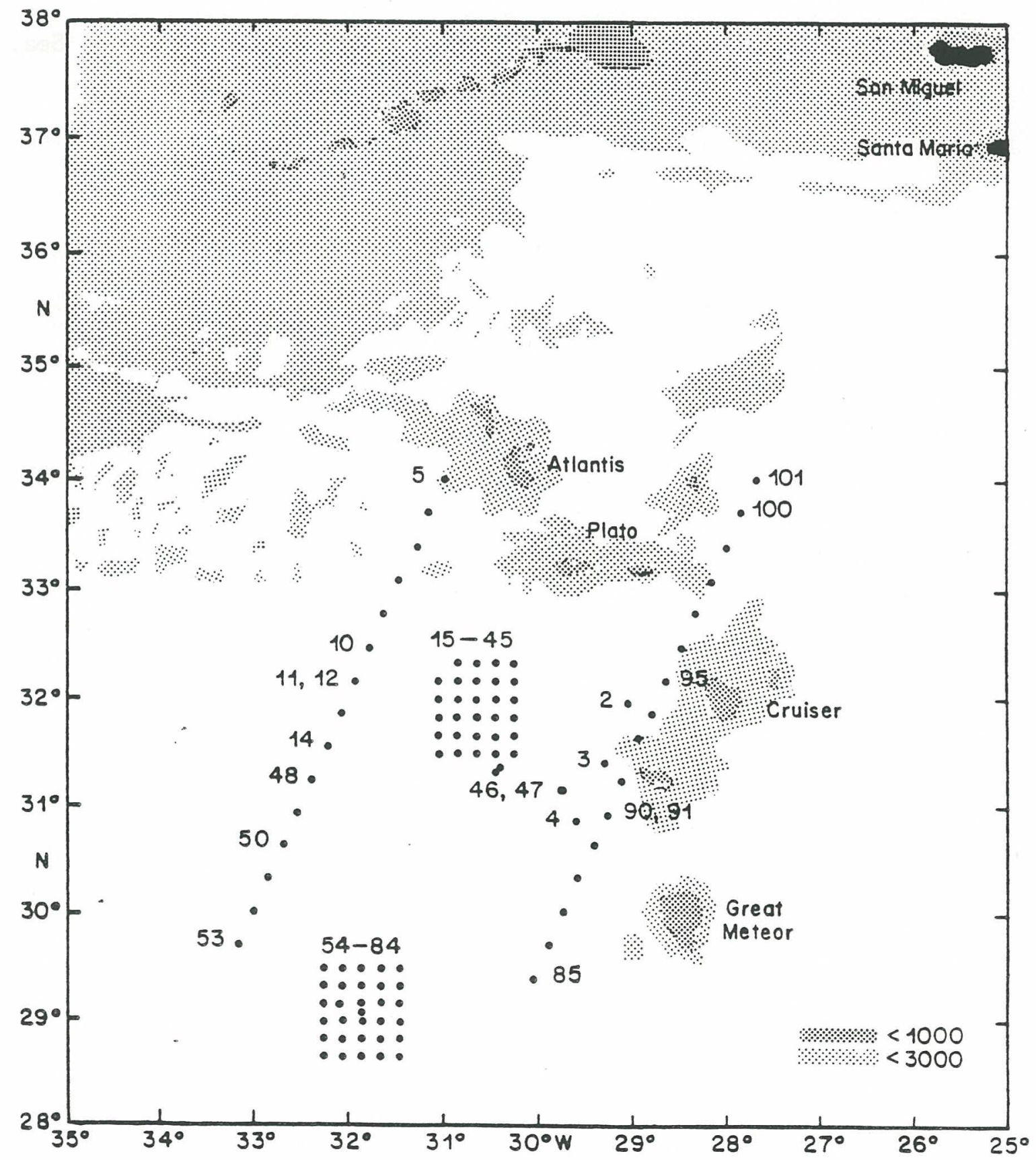


Figure 2. Positions of XBT observations made during R/V Endeavor cruise \# 143. Hatching as in Fig. 1.

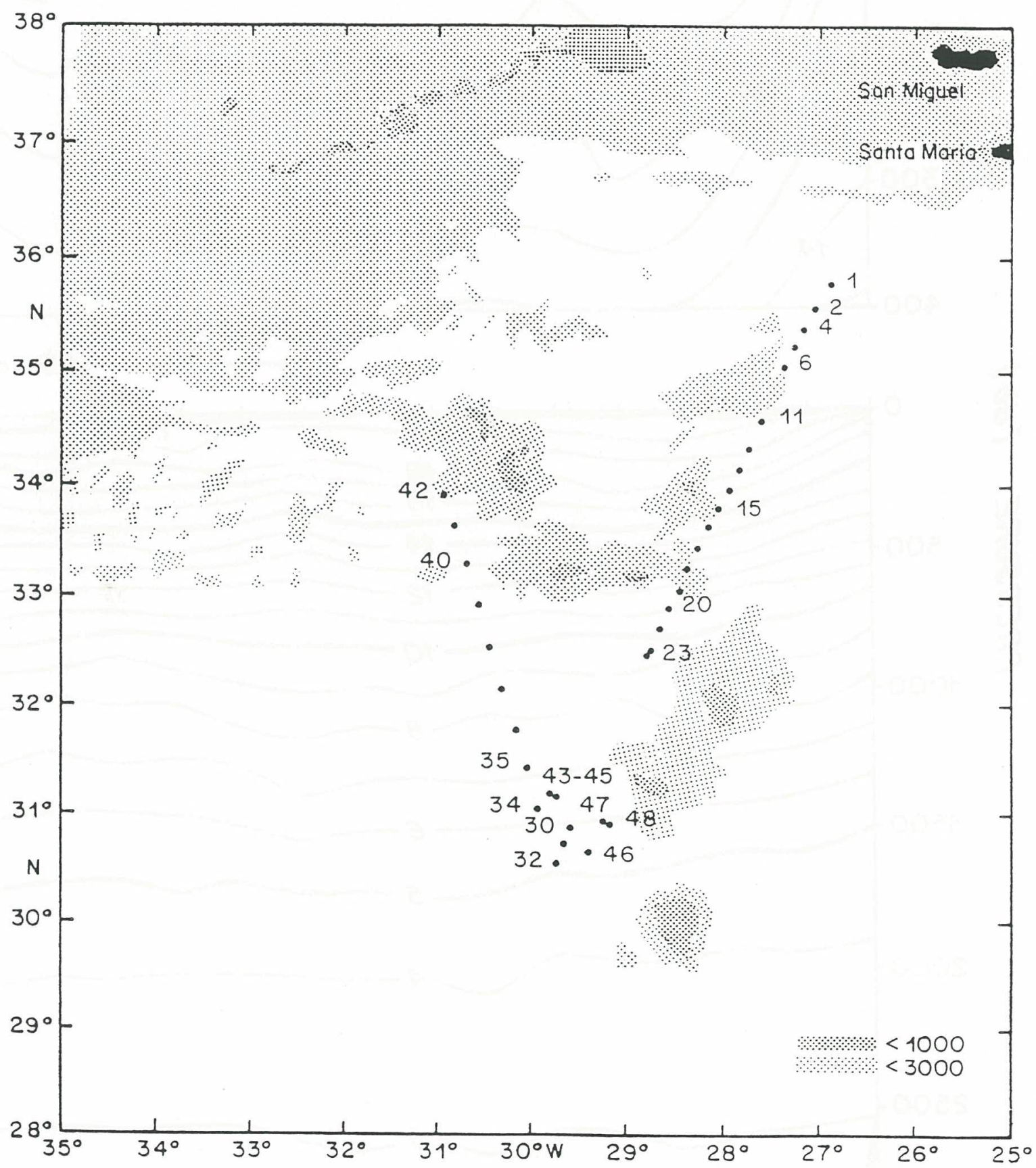


Figure 3a. Western section of CTD stations (5-14 \& 48-53). Pressure vs potential temperature. The shallow depth at station 5 marks the flank of Atlantis seamount.
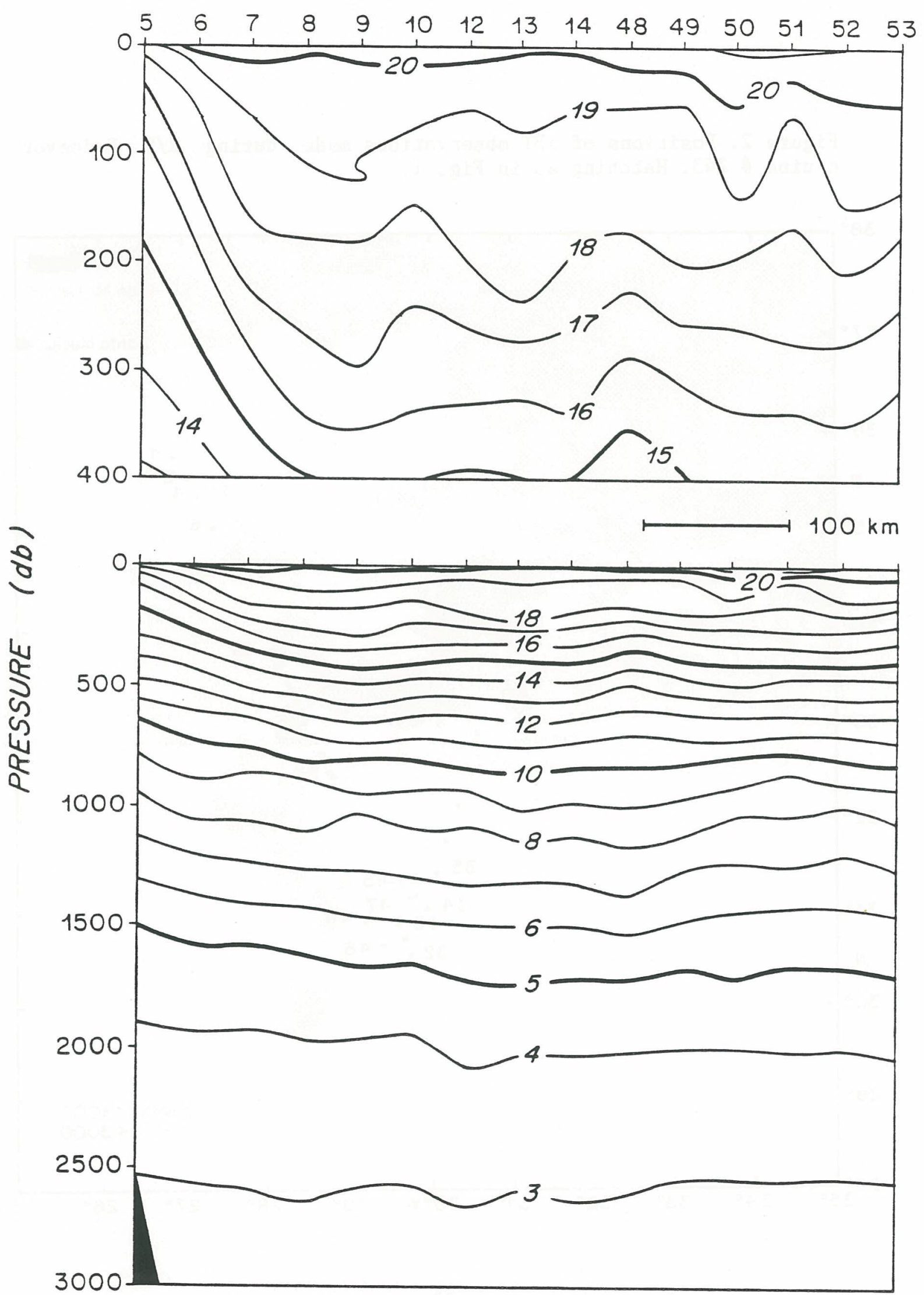
Figure 3b. Same as Fig. 3a except salinity (psu).
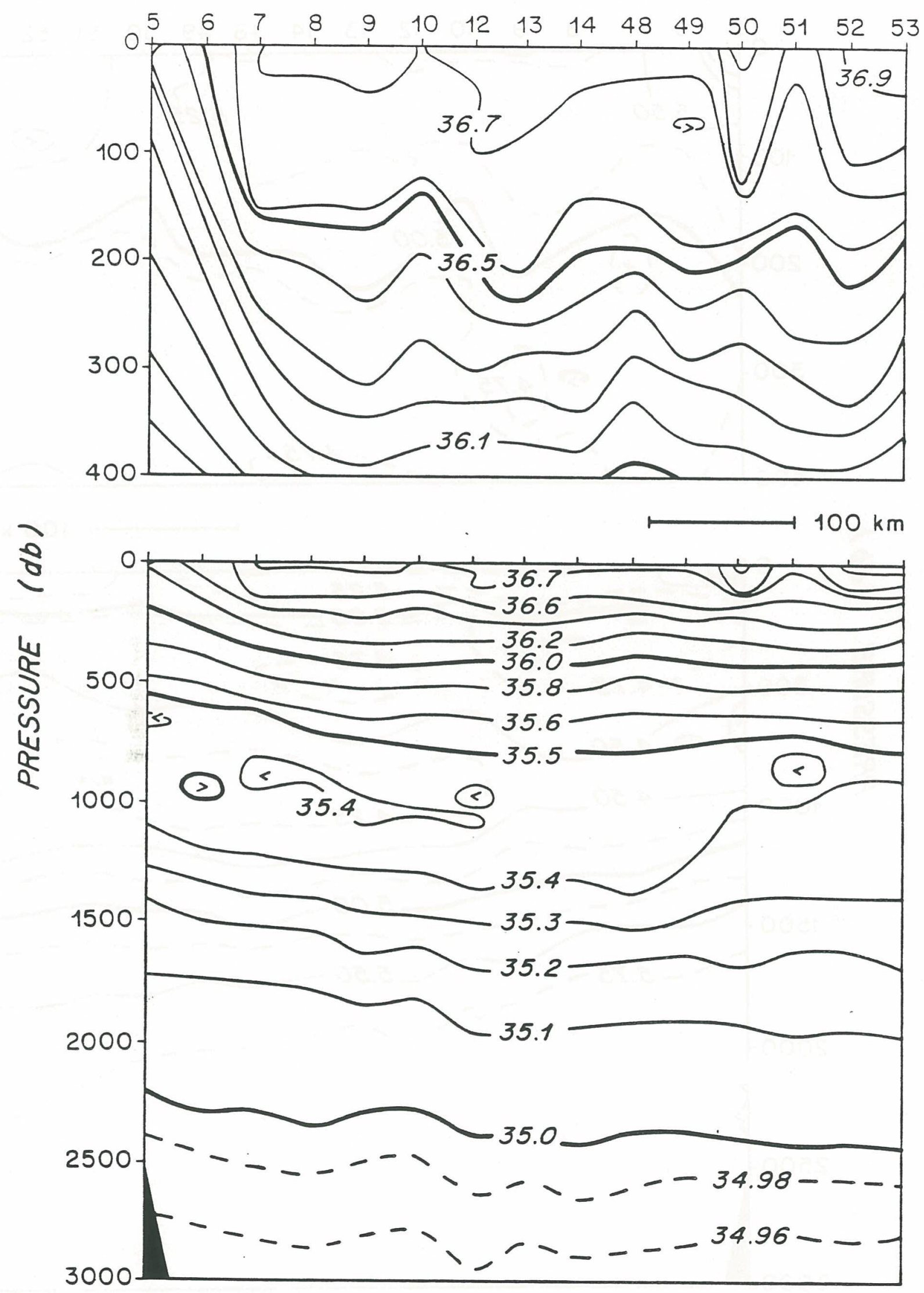
Figure 3c. Same as Fig. 3a except dissolved oxygen (m1/1).
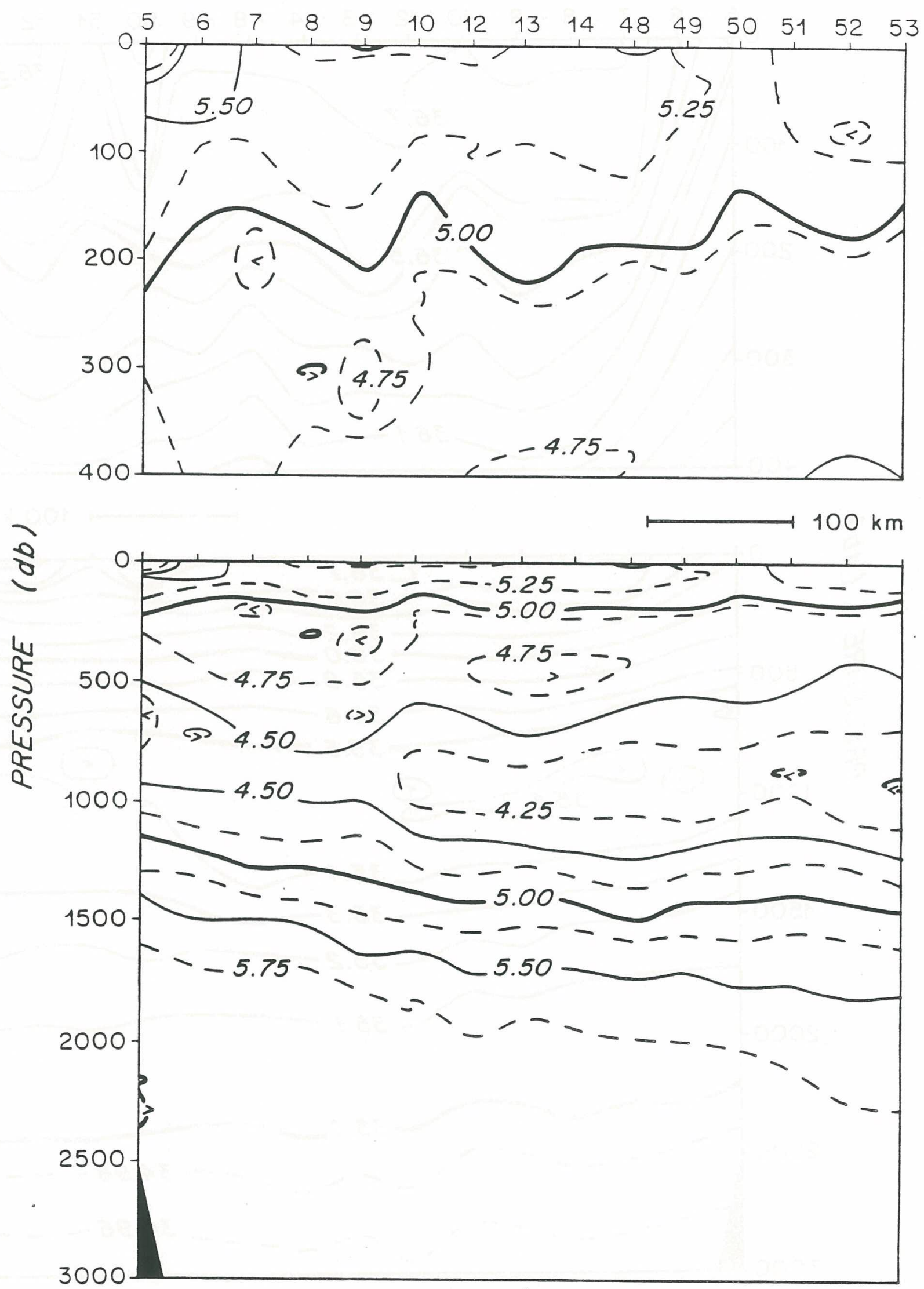
Figure 3d. Same as Fig. 3a except potential density $\left(\mathrm{kg} / \mathrm{m}^{3}\right)$
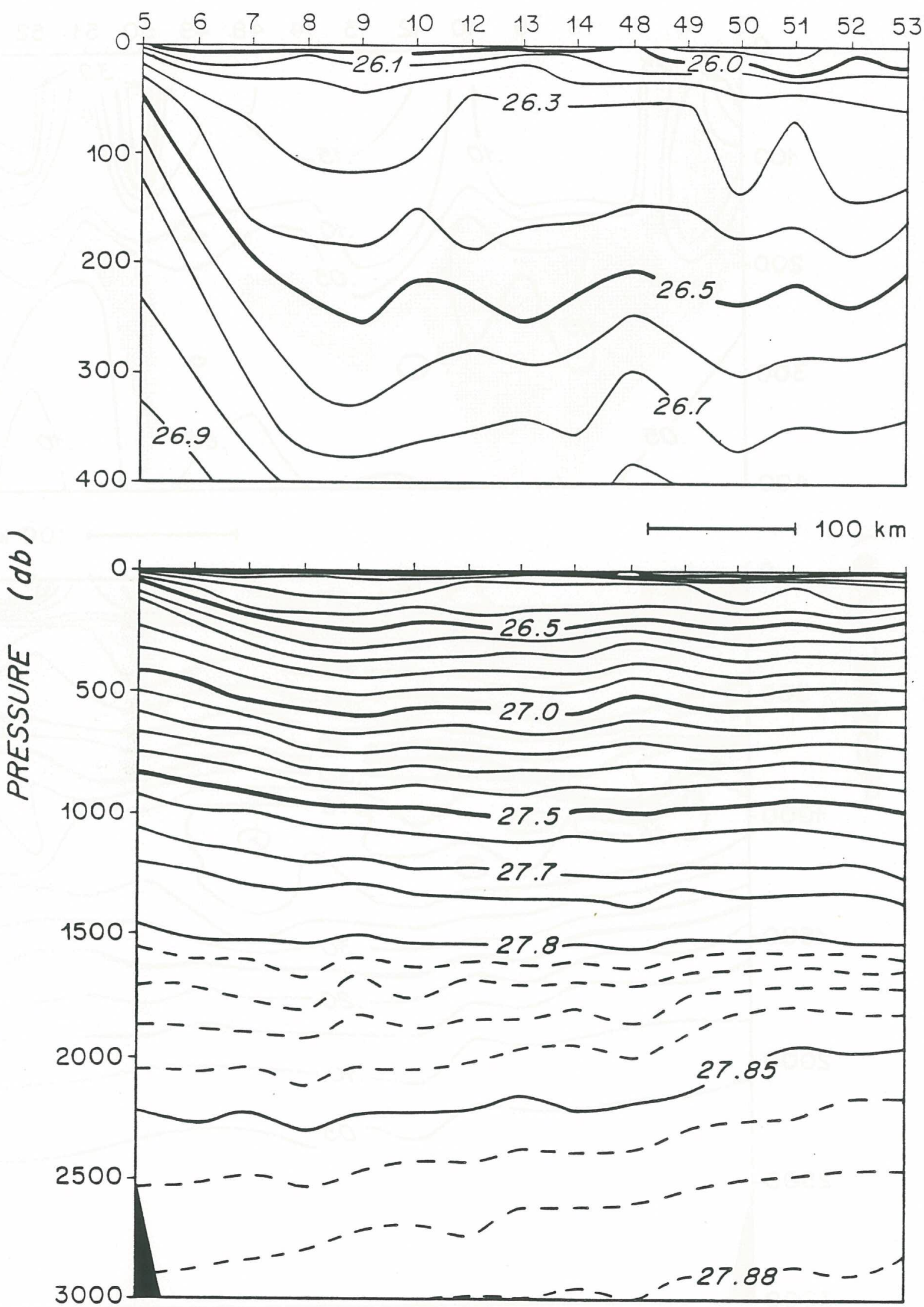
Figure 3e. Same as Fig. 3a except salinity anomaly (psu).
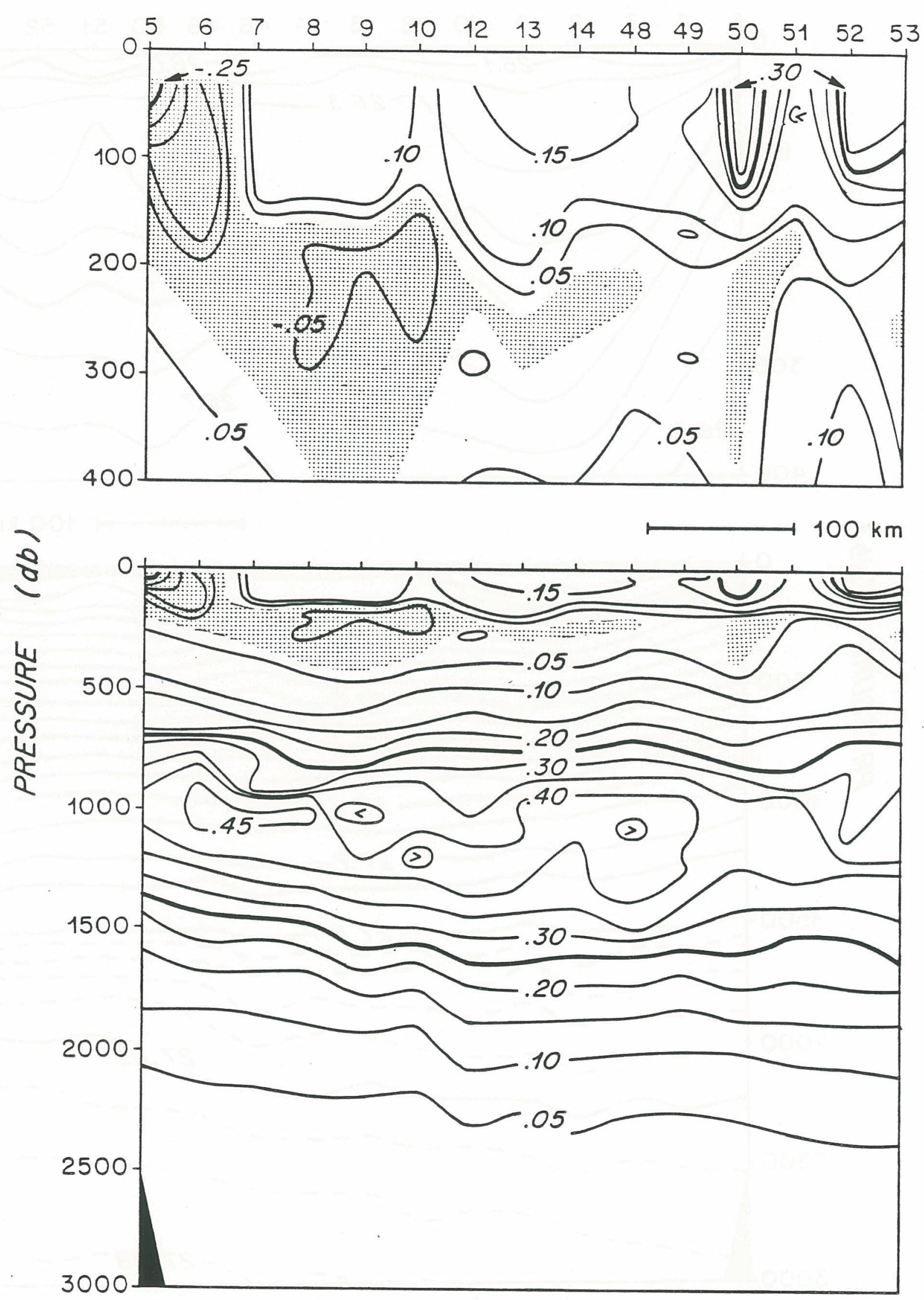
Figure 4a. Eastern section of CTD stations (85-101). The shallow depths at stations 93 and 98 are the flanks of Cruiser and Plato seamounts respectively.
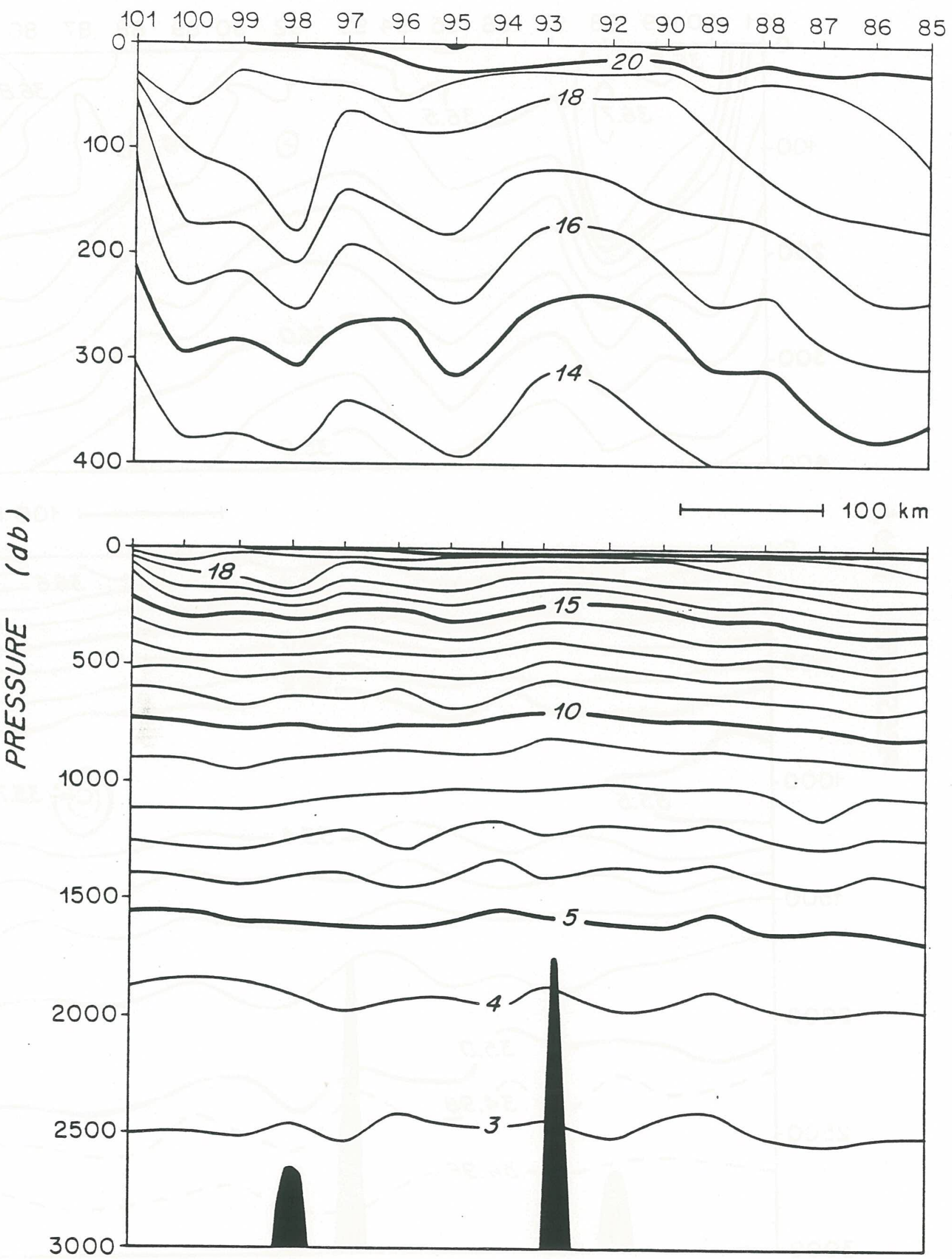
Figure 4b. Same as Fig. 4a except salinity (psu).
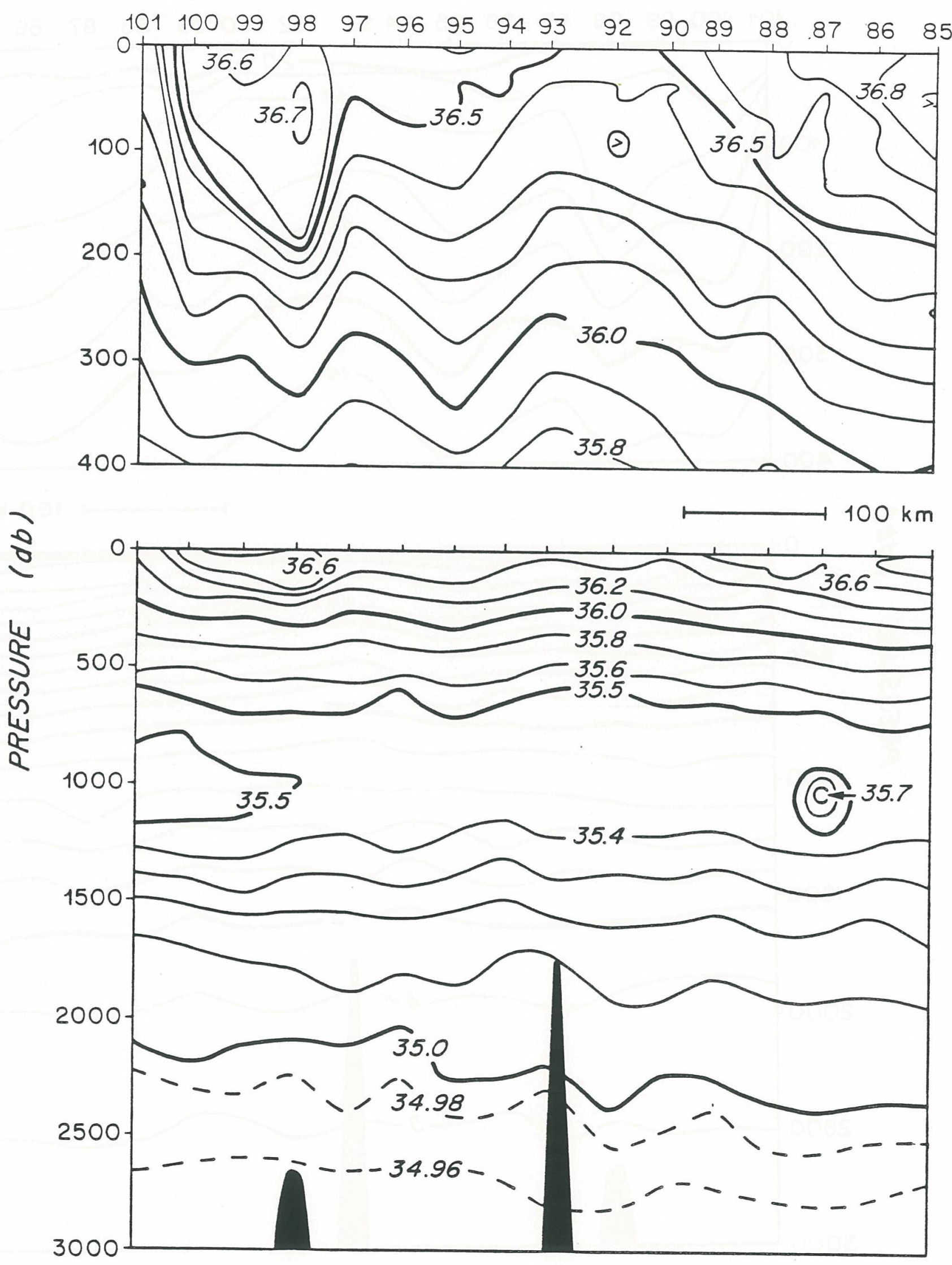
Figure 4c. Same as Fig. $4 a$ except dissolved oxygen (m1/1).

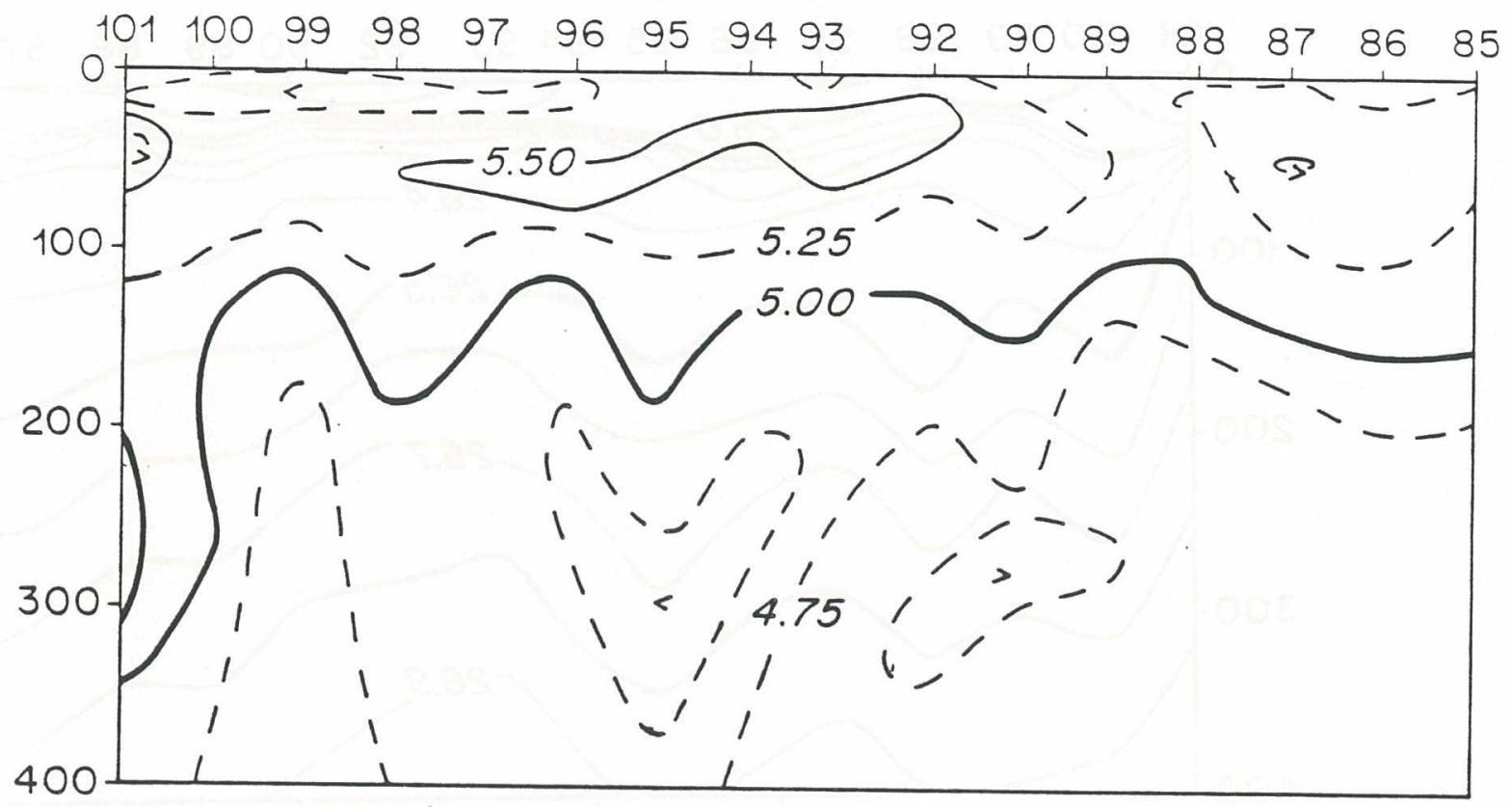

0
0
4
5
5
5
5
5

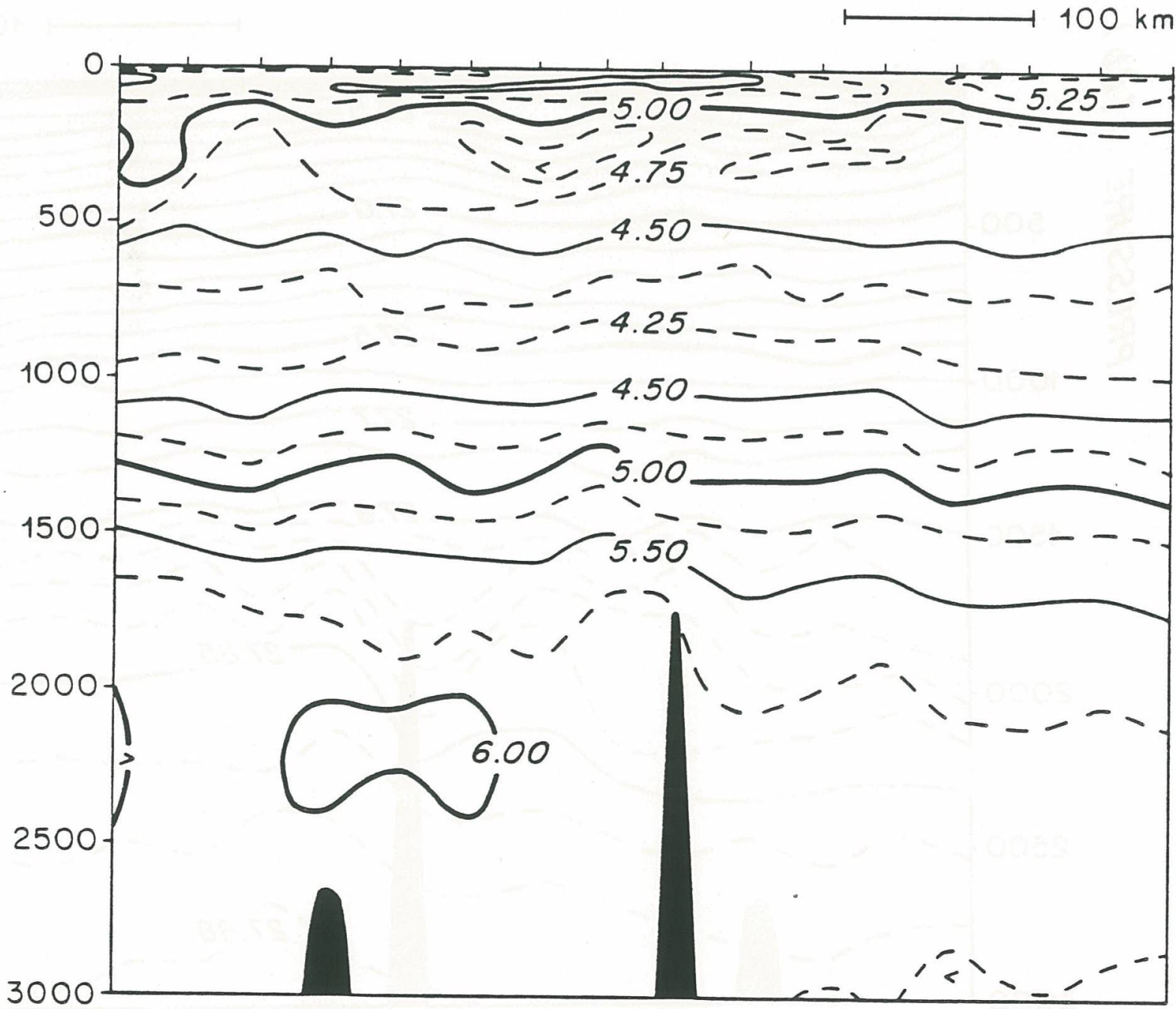


Figure $4 \mathrm{~d}$. Same as Fig. $4 a$ except potential density $\left(\mathrm{kg} / \mathrm{m}^{3}\right)$
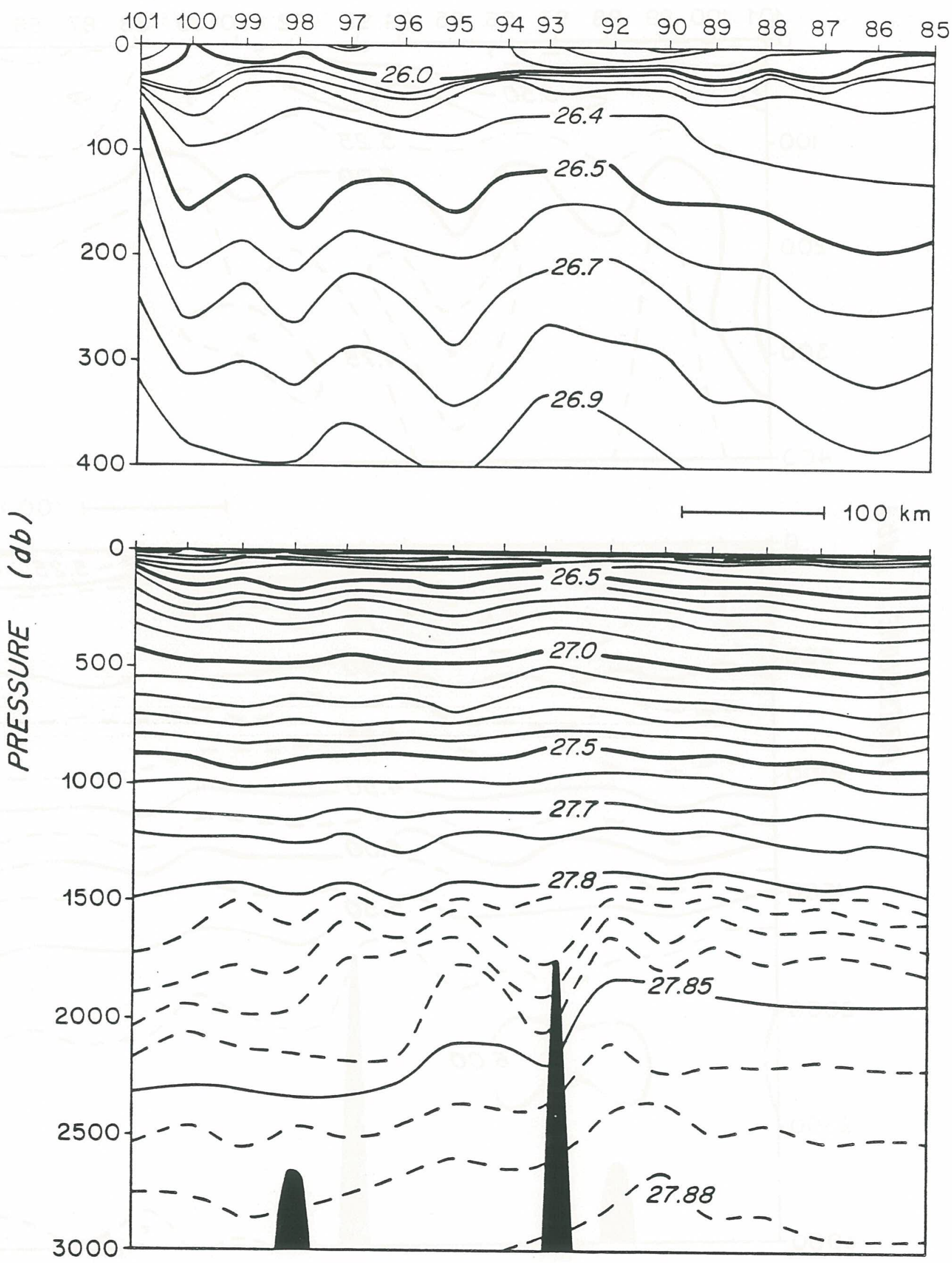
Figure 4e. Same as Fig. $4 a$ except salinity anomaly (psu).
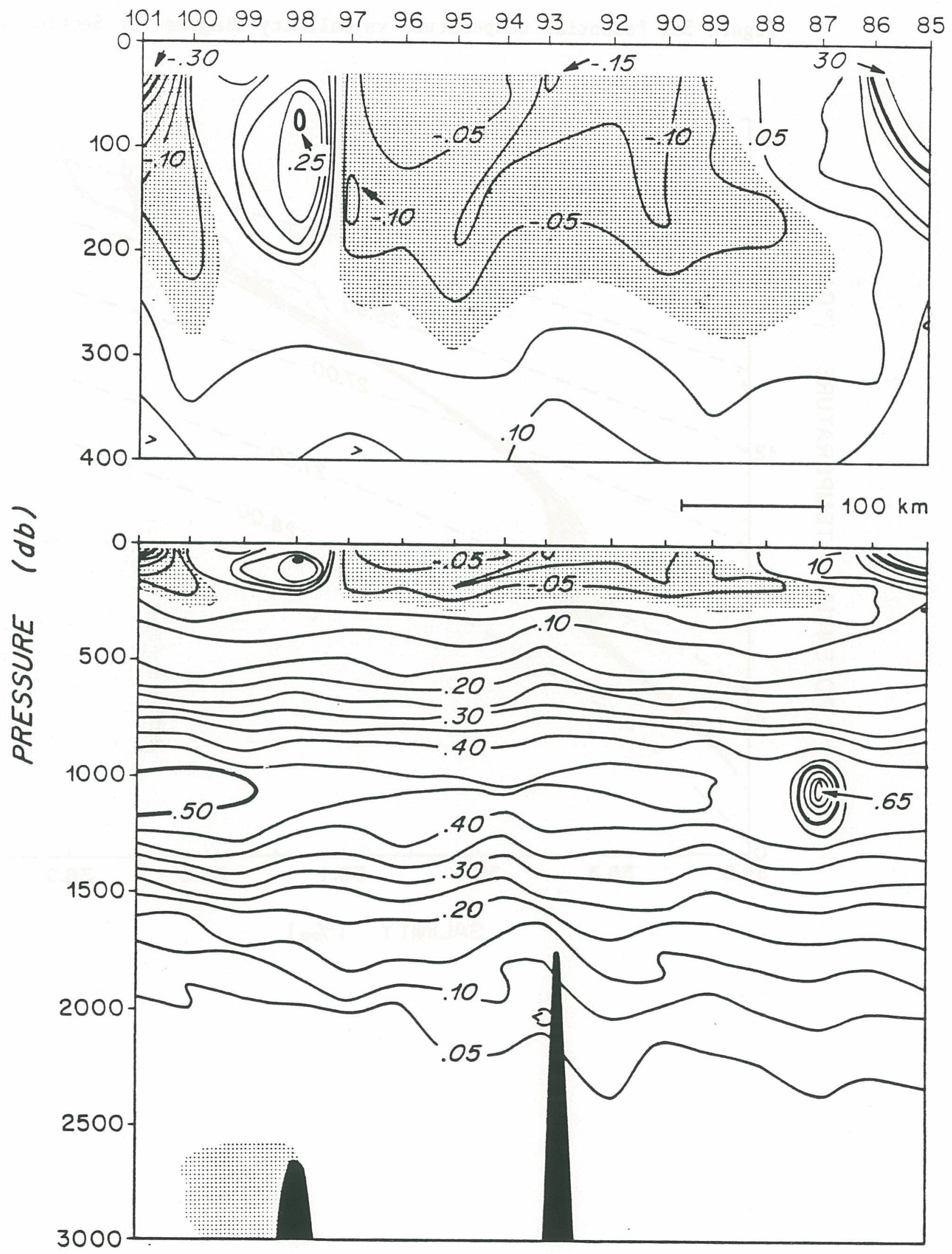
Figure fa. Potential temperature vs salinity diagram for Section 1.

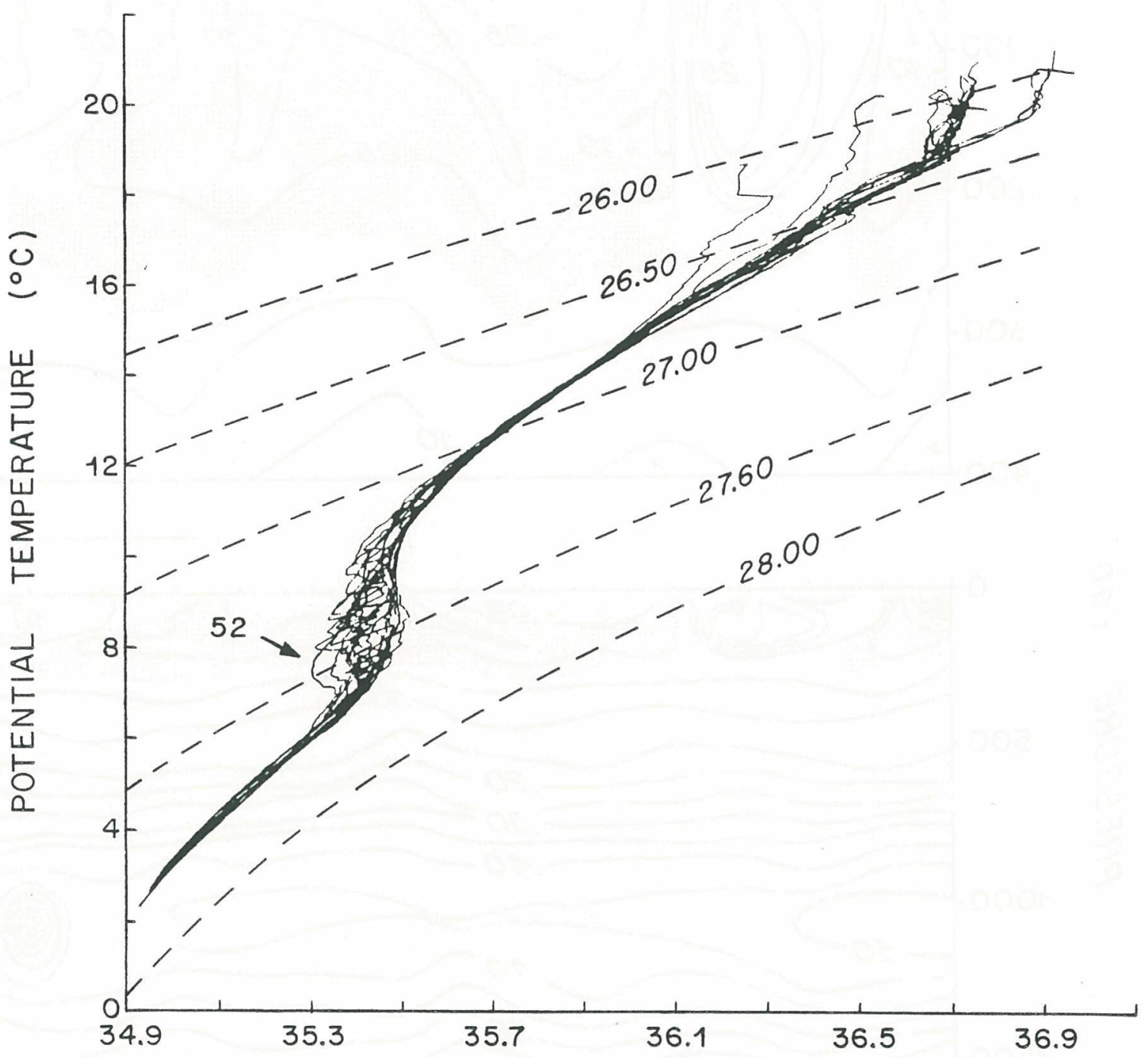

SALINITY (\%o)

34 
Figure sb. Potential temperature vs salinity diagram for Section 2.

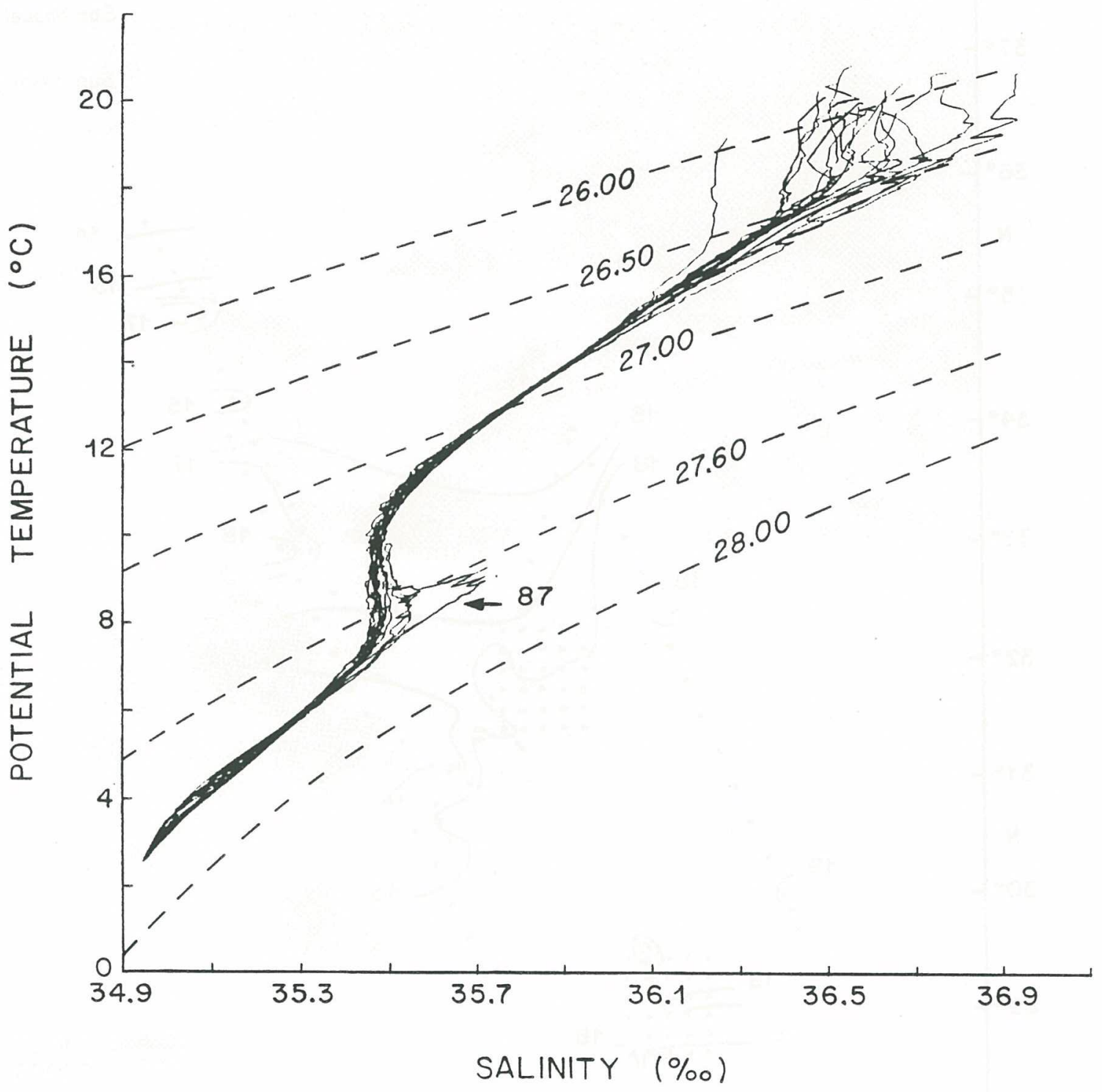

35 
Figure 6. Potential temperature at a depth of $150 \mathrm{~m}$ during En-143.

Data are from CTD and XBT measurements. Hatching as in Fig. 1.

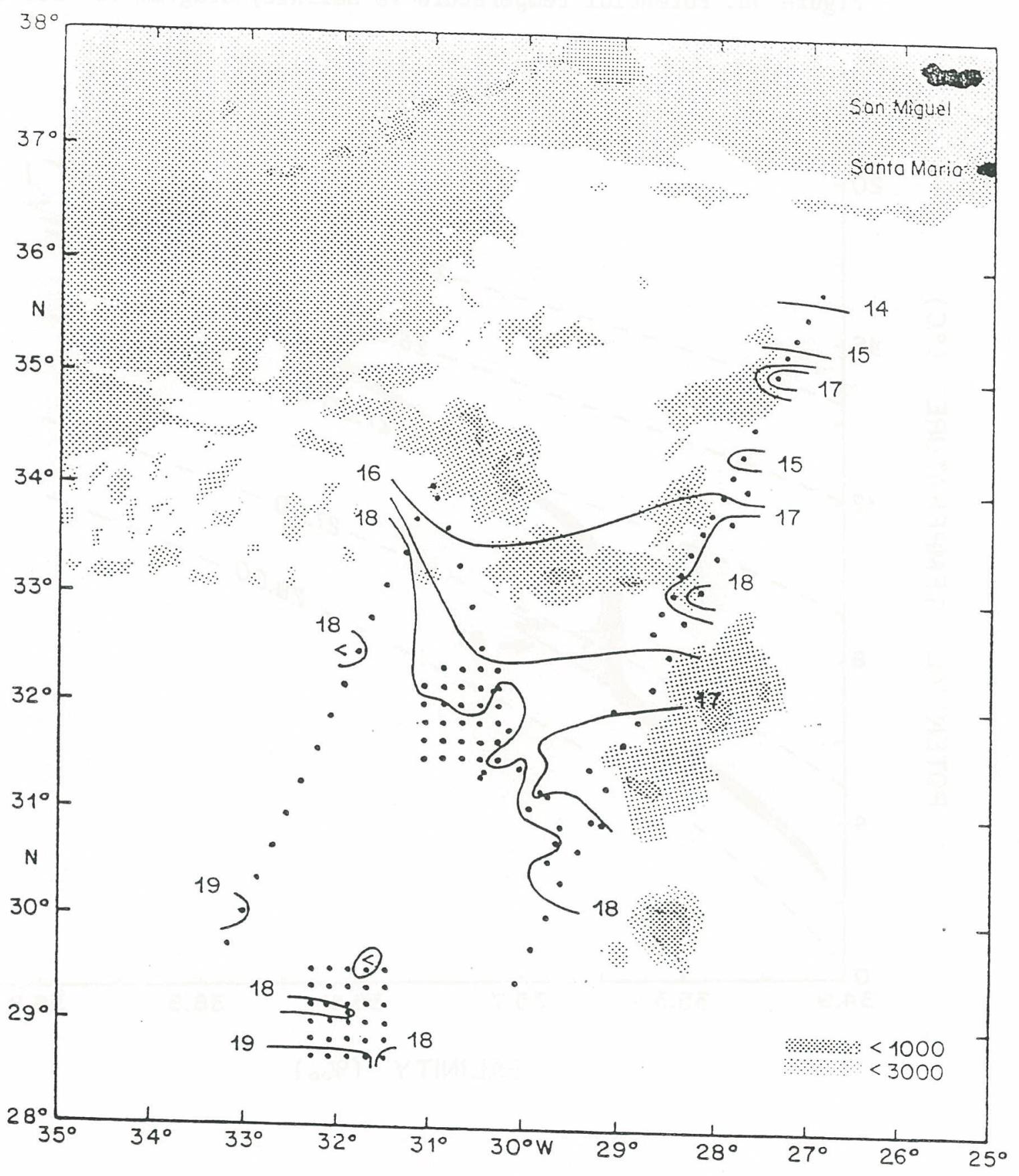


Figure 7a. Pressure, potential temperature, salinity and dissolved oxygen at a potential density of 26.2 during northern survey.
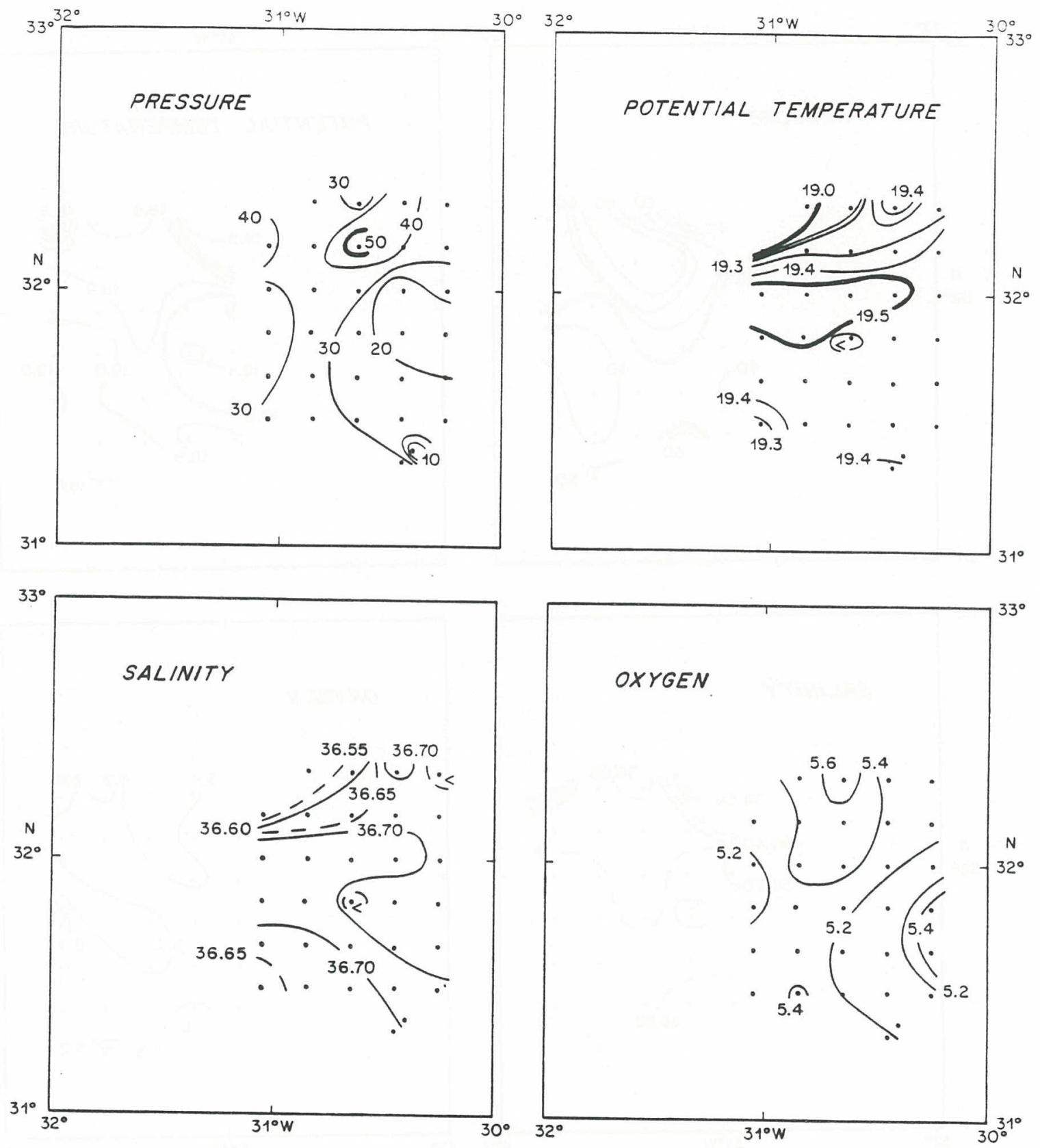

SIGMA THETA 26.2 
Figure 7b. Same as Fig. 7a except the potential density is 26.3.
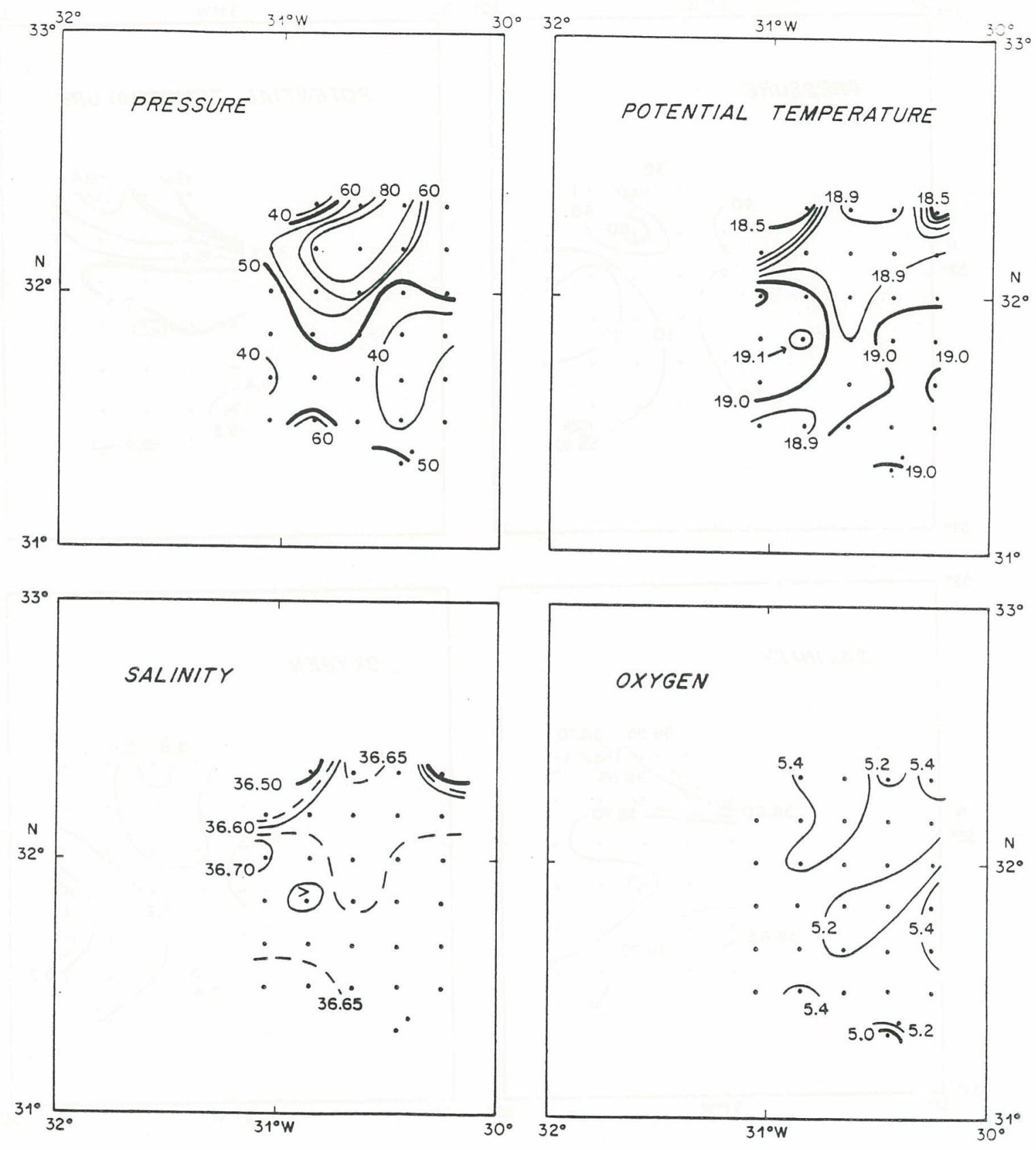

SIGMA THETA 26.3 
Figure 7c. Same as Fig. 7a except the potential density is 26.4.
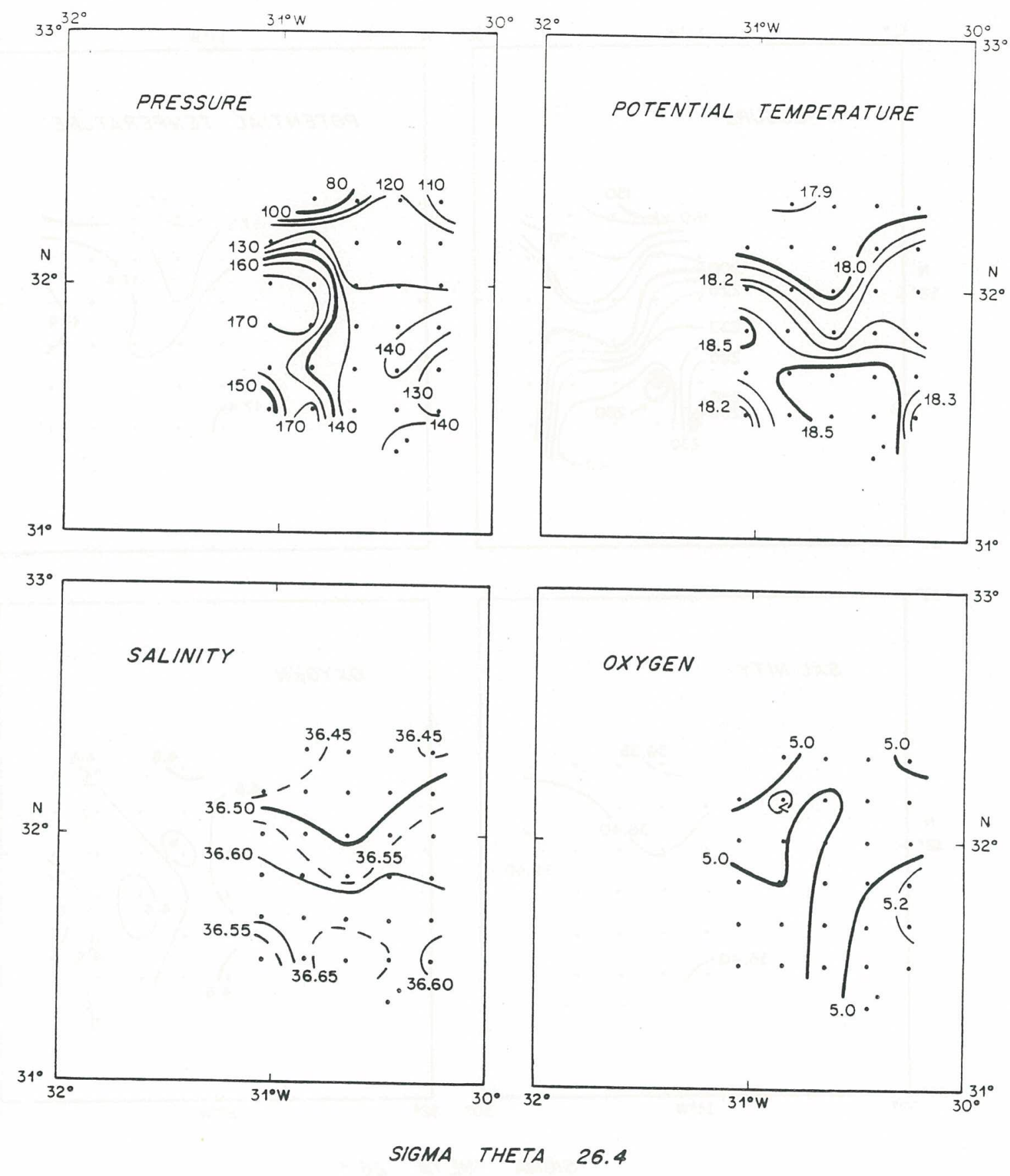
Figure 7d. Same as Fig. 7a except the potential density is 26.5.
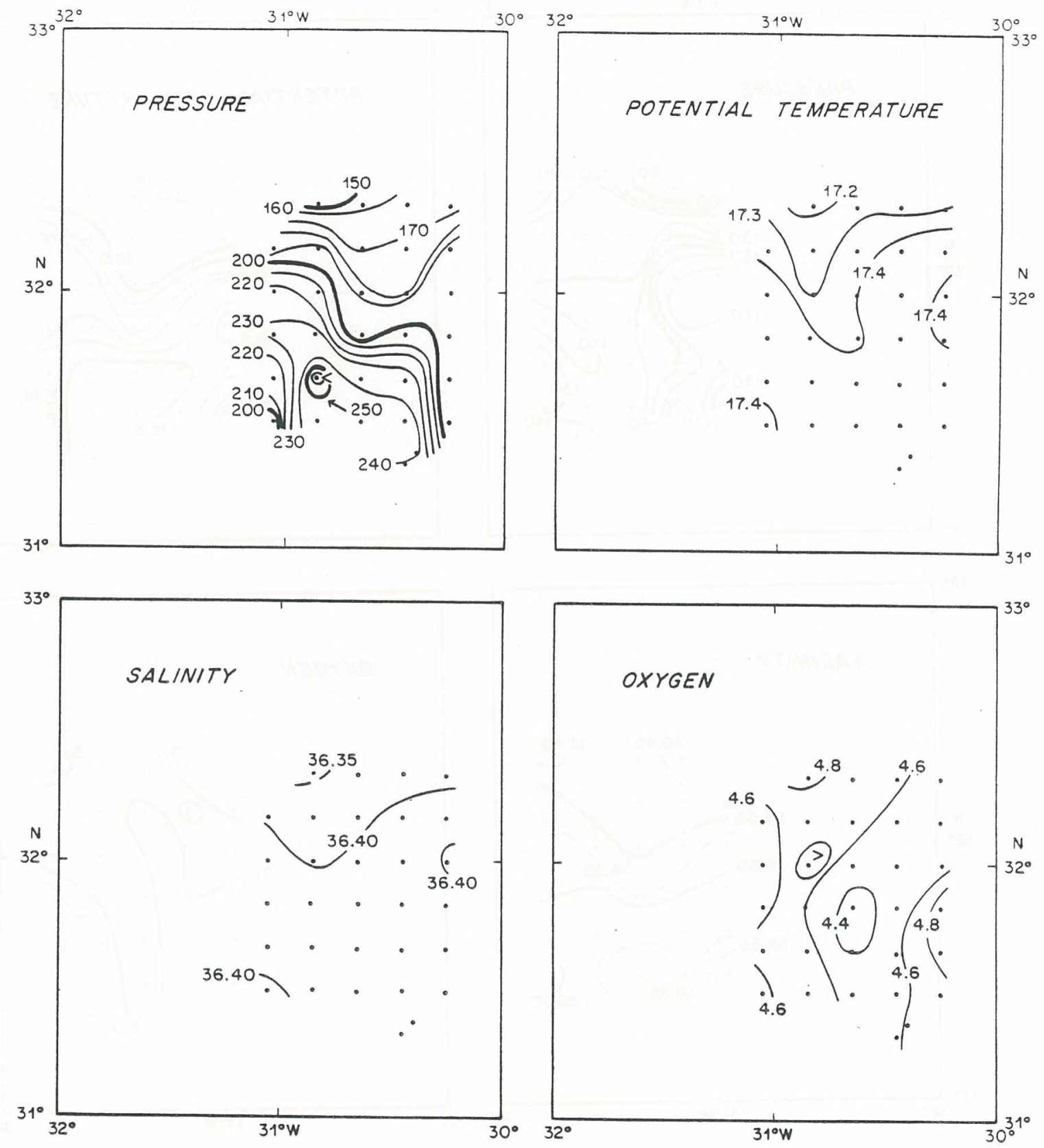

SIGMA THETA 26.5 
Figure 8a. Pressure, potential temperature, salinity and dissolved oxygen at a potential density of 26.2 during southern survey.
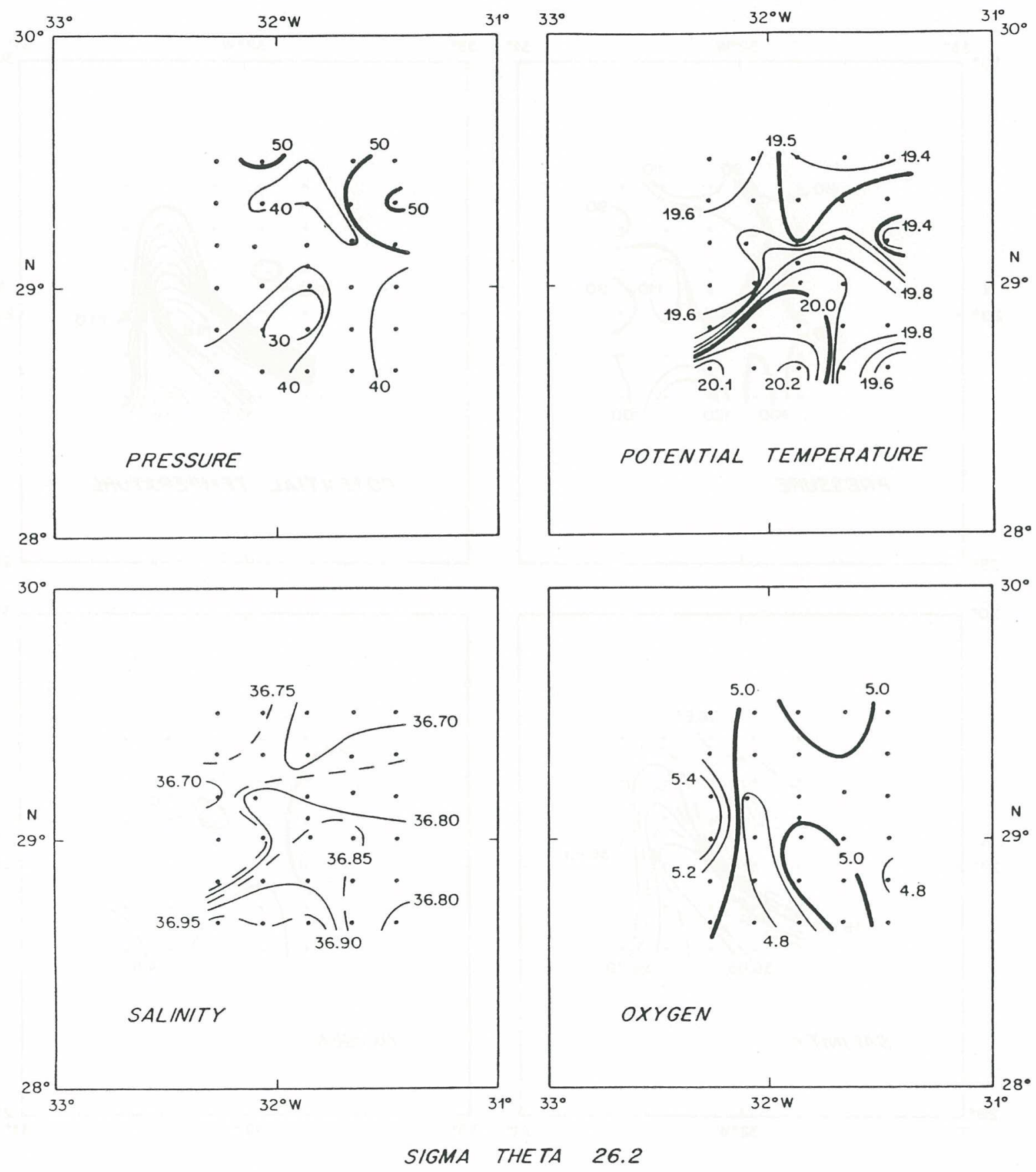
Figure 8b. Same as Fig. 8a except the potential density is 26.3.
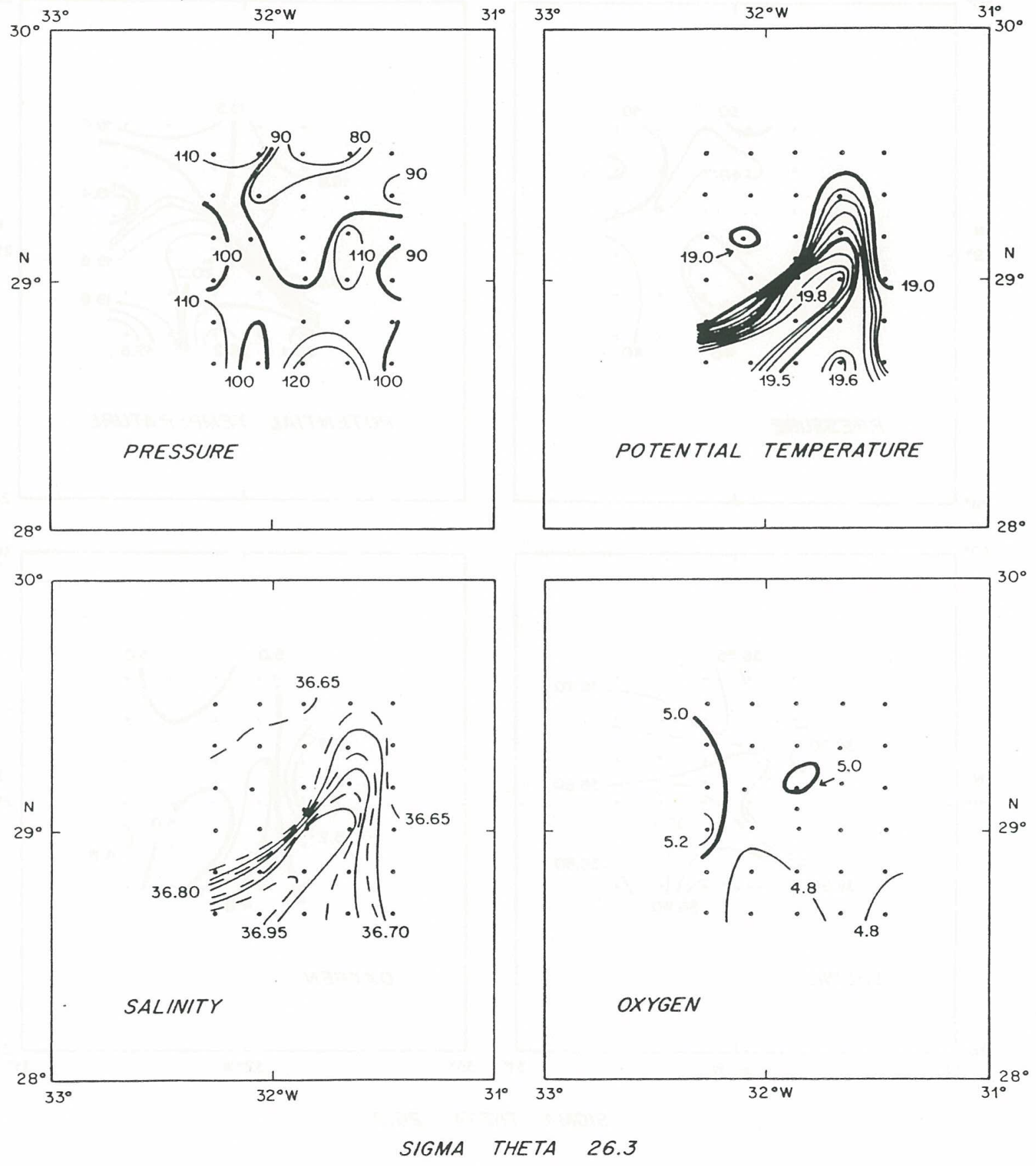
Figure $8 c$. Same as Fig. 8 a except the potential density is 26.4 .

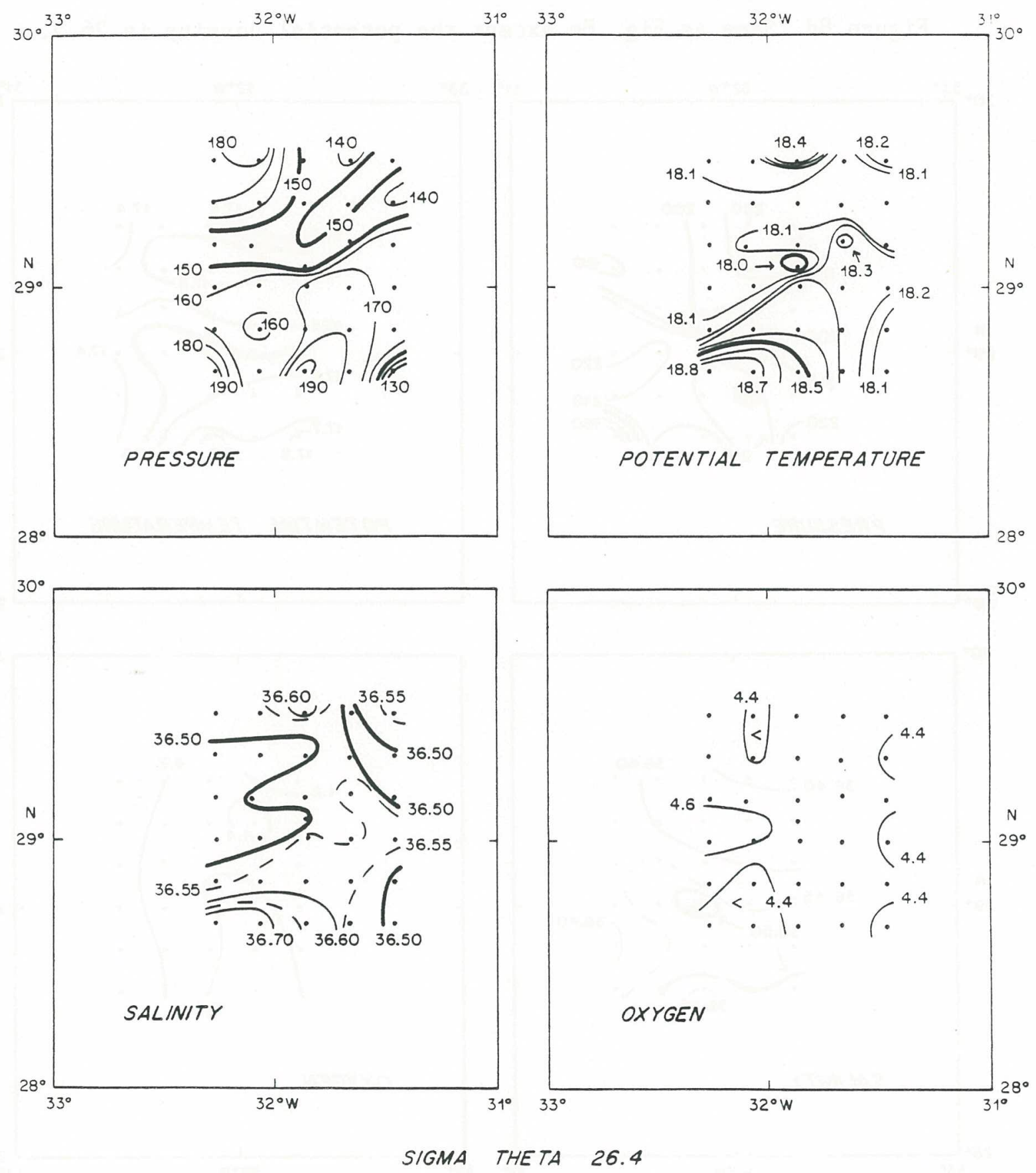


Figure 8d. Same as Fig. 8a except the potential density is 26.5 .
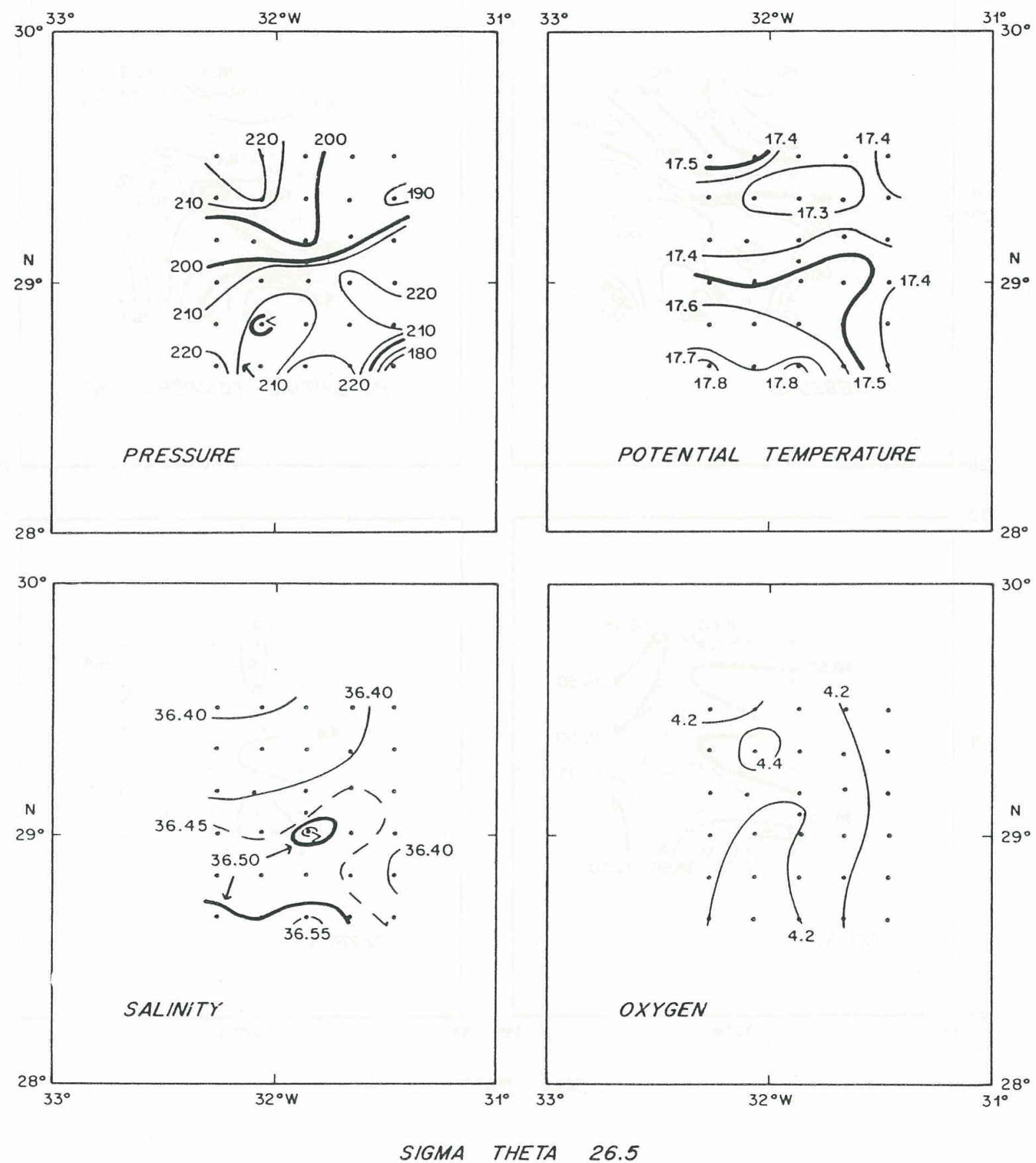
Figure 9. Dynamic height of the sea surface relative to $300 \mathrm{db}$ for the northern (a) and southern (b) small-scale surveys.
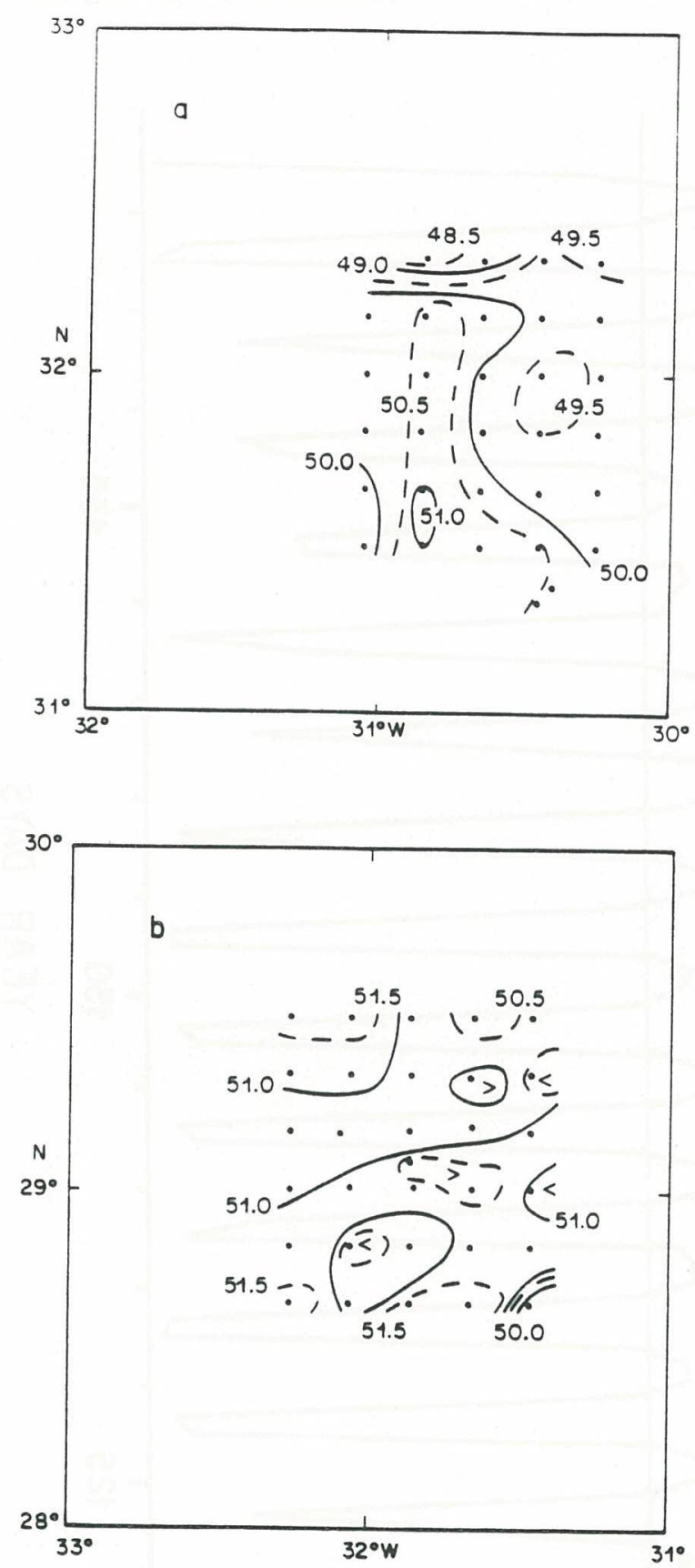
Figure 10. Total heat flux (watts $/ \mathrm{m}^{2}$ ) during En-143 using data from Table 5.

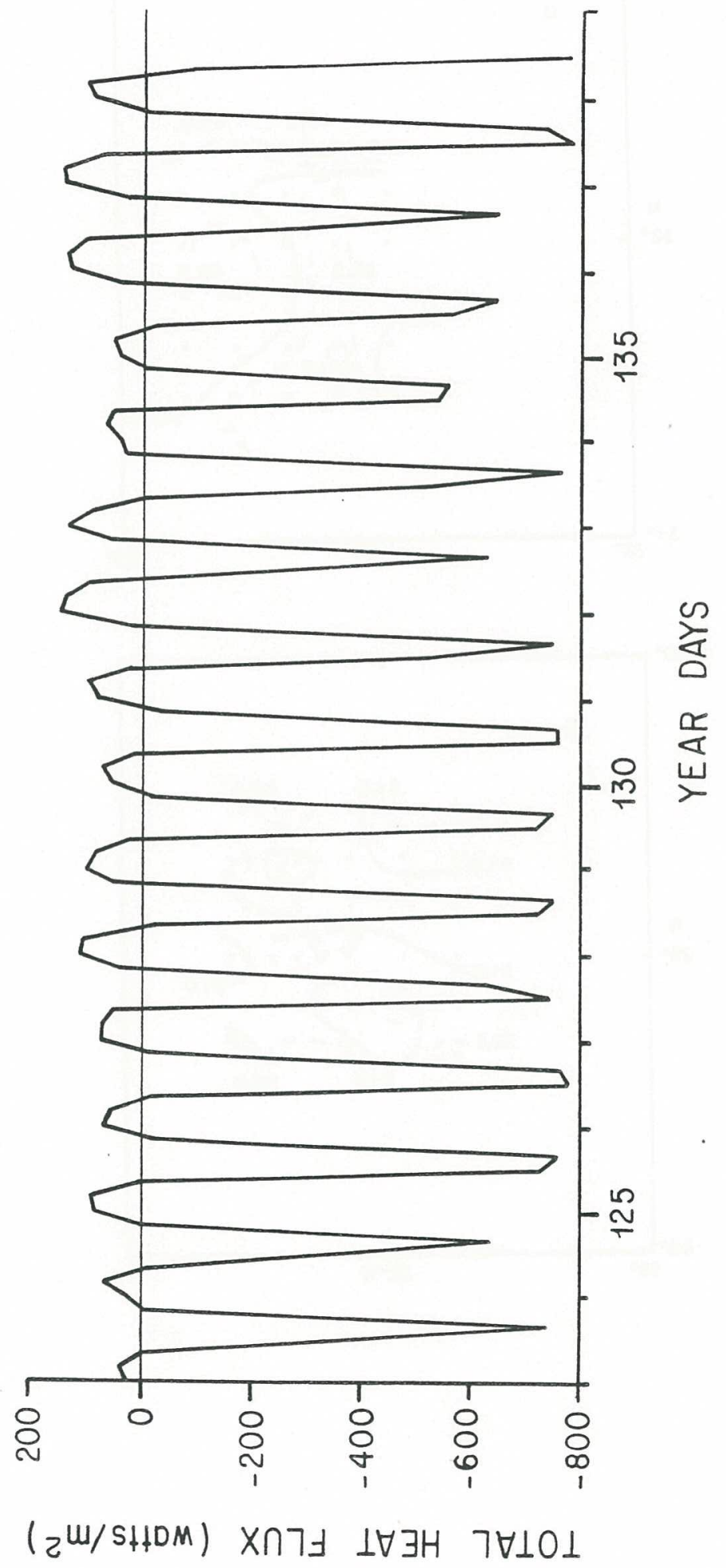


Table 1. Log of events occurring during En-143. Events are Conductivity-Temperature-Depth stations (C), Expendable Bathythermographs (X), 'bobber' floats and buoys (B) and other (O).

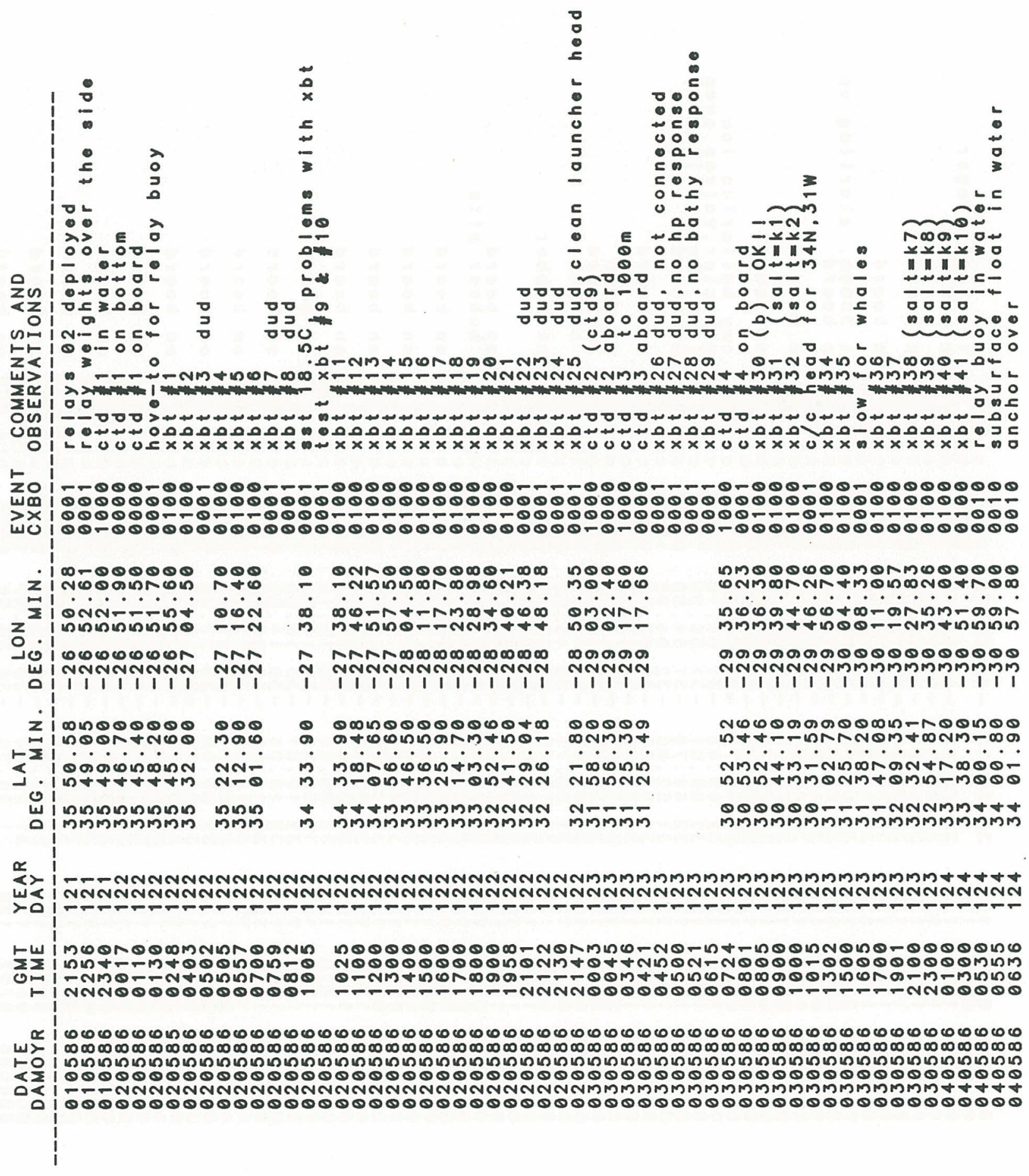




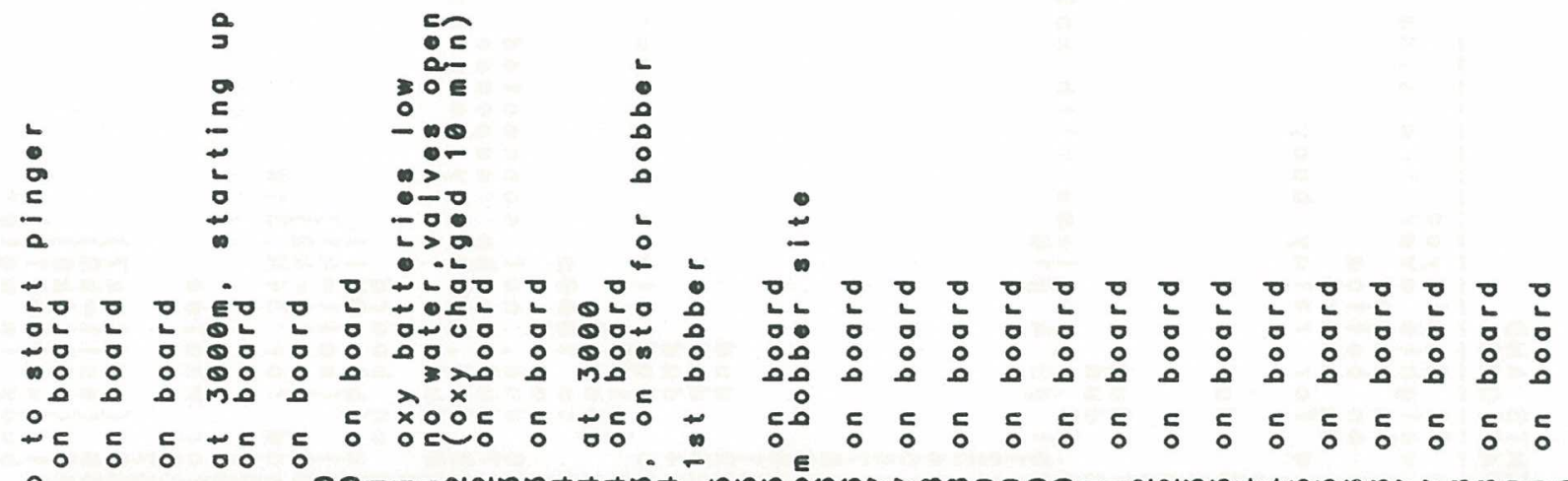
$00 \pi+-N N m m+t+m+$

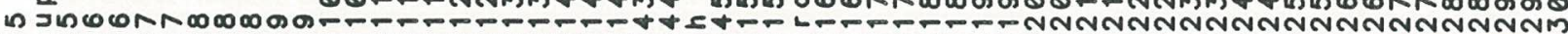
1. ס סףס

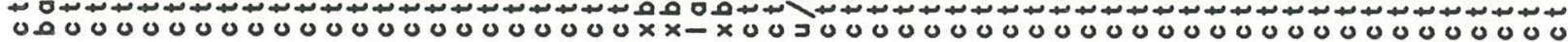

000000000000000000000000000000-00000000000000000000000000000

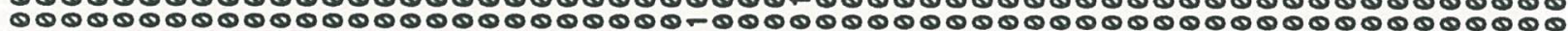

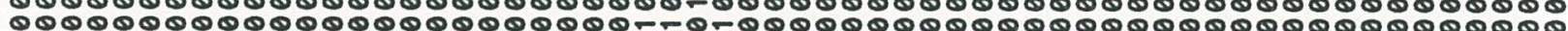
-

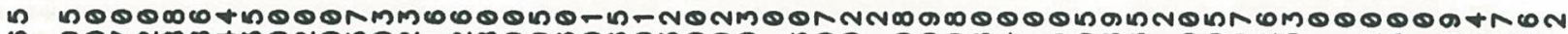

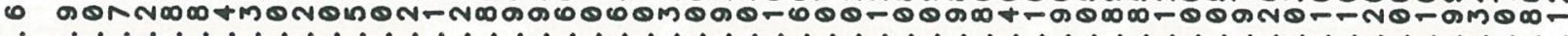

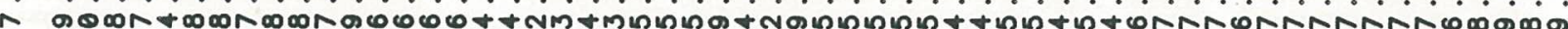
и מ-

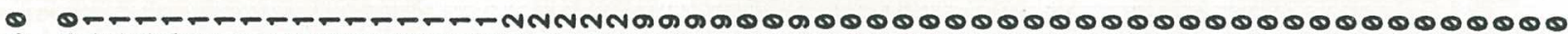

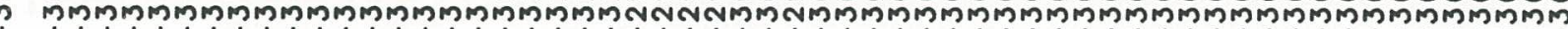
111111111111111111111111111111111111111111111111111111111

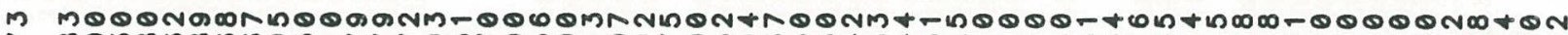

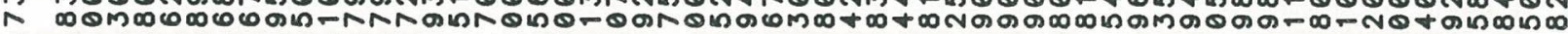

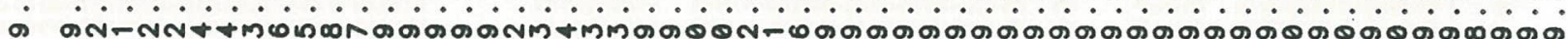

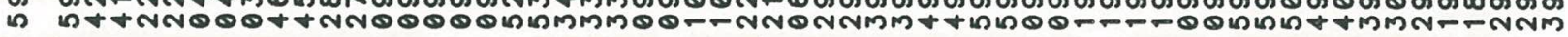

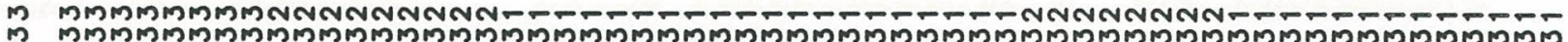

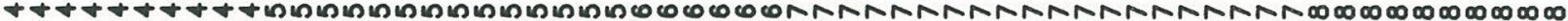

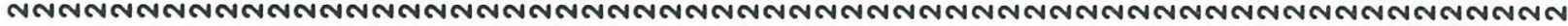

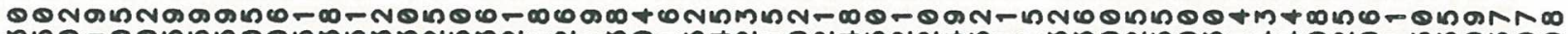

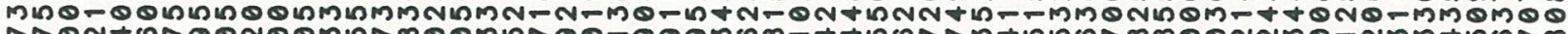
MONt

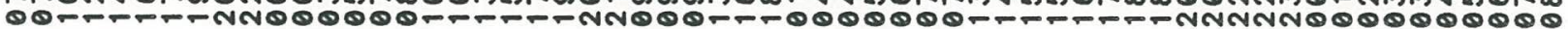

000000000000000000000000000000000000000000000000000000000000

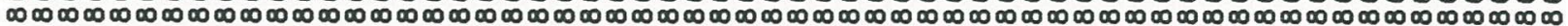

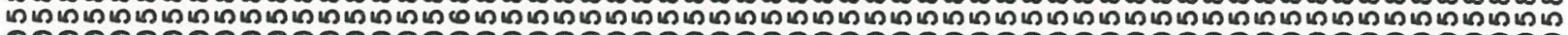
000000000000000000000000000000000000000000000000000000000

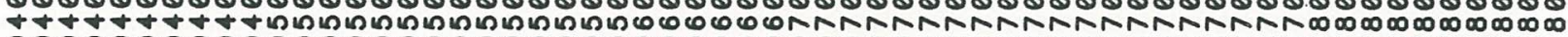
000000000000000000000000000000000000000000000000000000000 


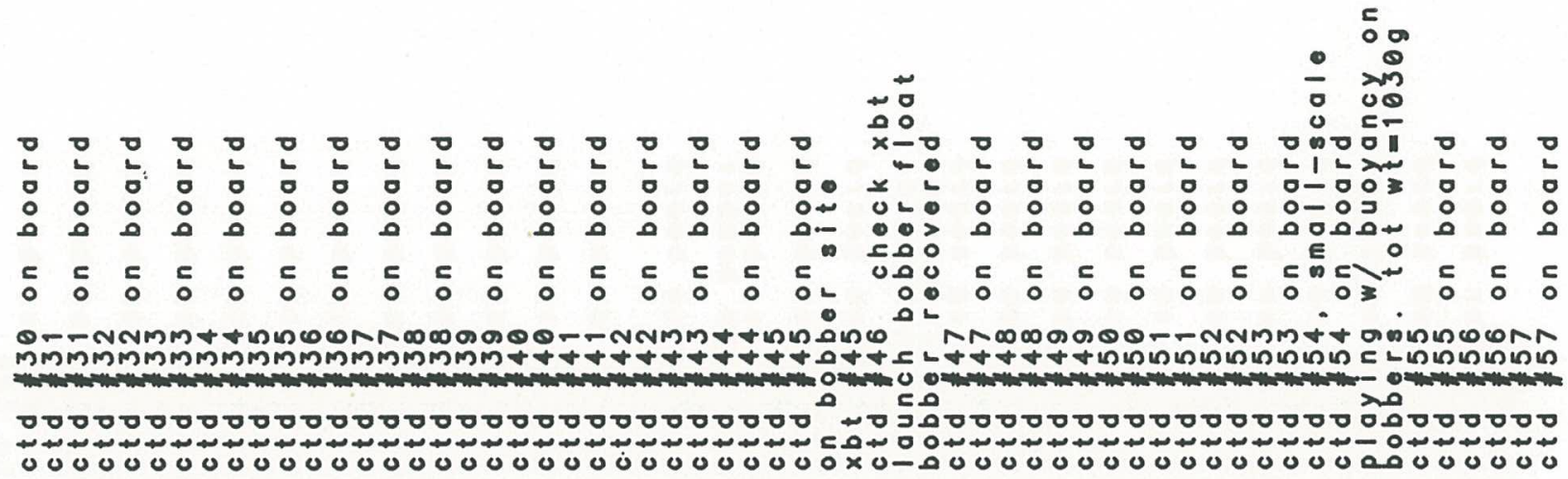

$0000000000000000000000000000000-000-000000000000000000000000$

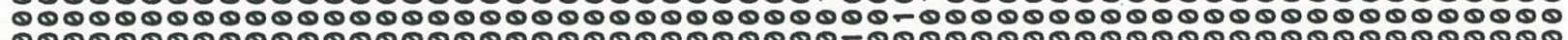

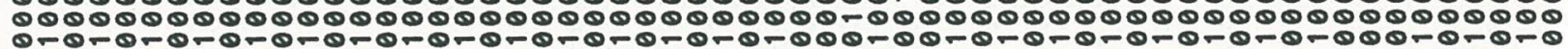

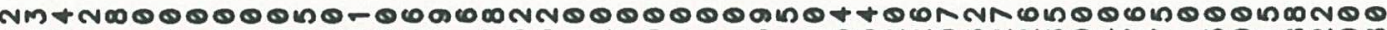

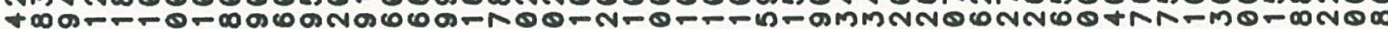
-

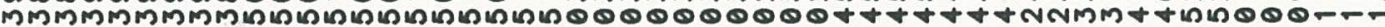

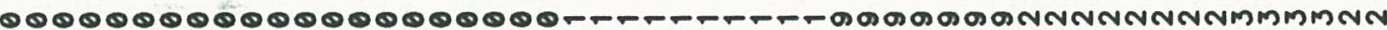

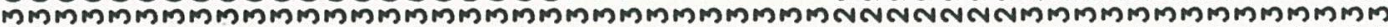

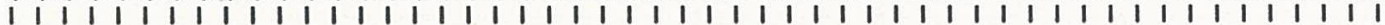

$\cos -\cos$ -iosoiिi⿻i土i r-m NONONO mMmmMm $\begin{array}{lllll}1 & 1 & 1 & 1\end{array}$

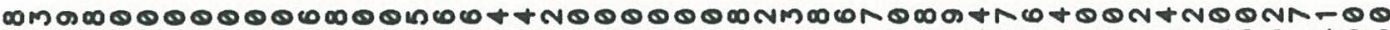

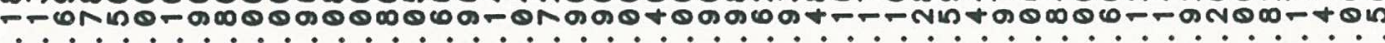

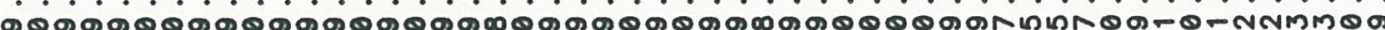
M

$-\infty 0 N m N$ NNT-OT OONOO Nor-

- - - NNNNNNNNN-_-

कosisas

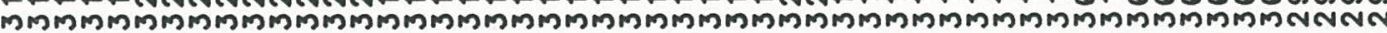
NNNNNN

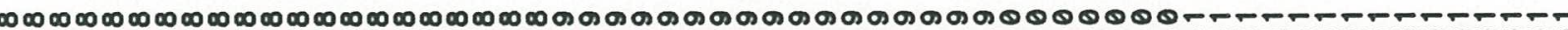

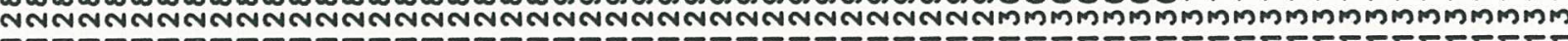

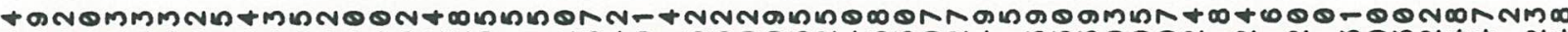

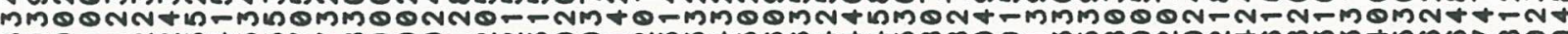

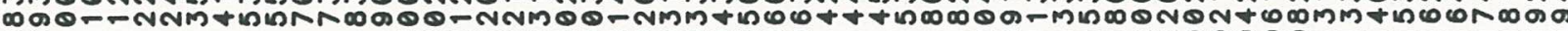
O0-6-

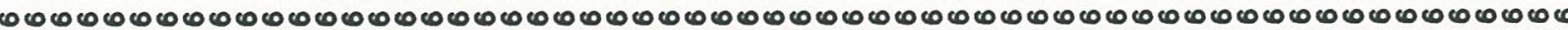

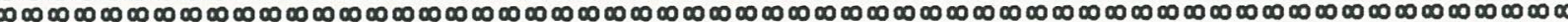
ம 00000000000000000000000000000000000000000000000000000000000

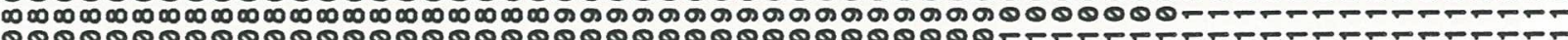




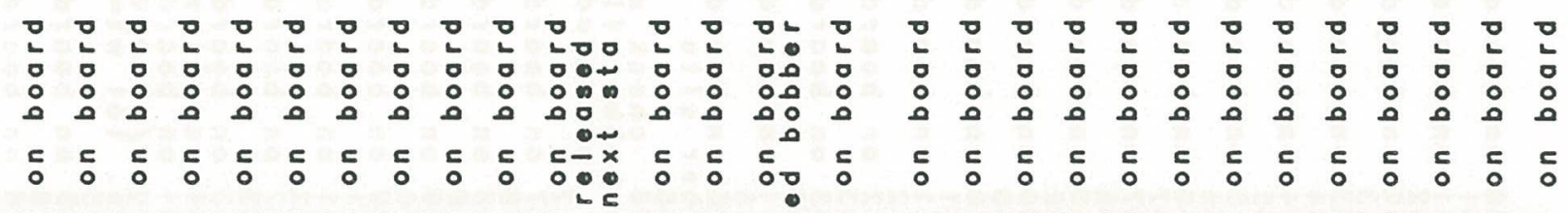

W மレாம 2.

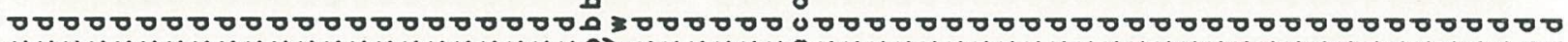

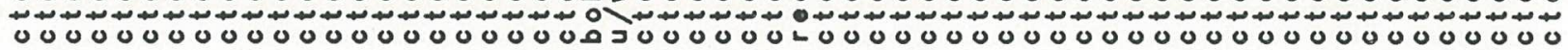

000000000000000000000000000000000000000000000000000000000000 $0000000000000000000000-0000000-00000000000000000000000000000$ 000000000000000000000000000000000000000000000000000000000000

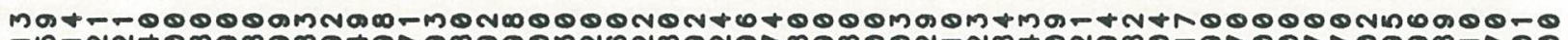

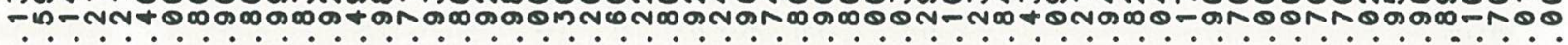
ம் -

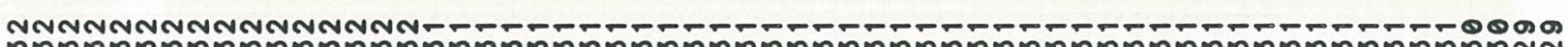

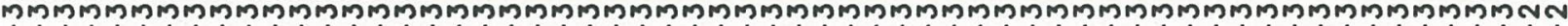

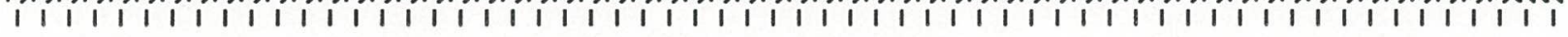

WN

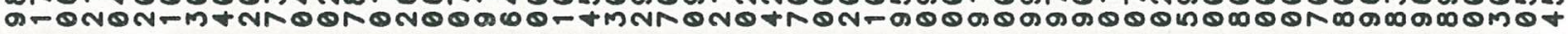

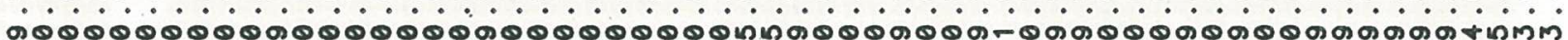

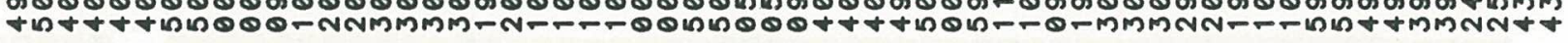

ஸ

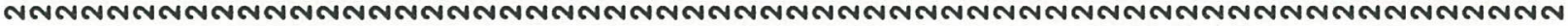

МMMMMMMMMMMMMMMM

-NOTMNO-MMMNOMN CMN"

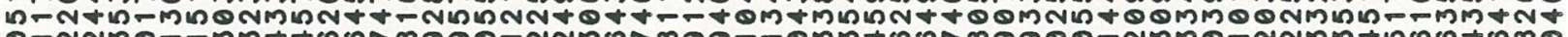
O-NNMO-FM+ NNNNNO0000000000007, -

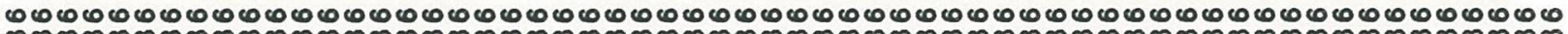

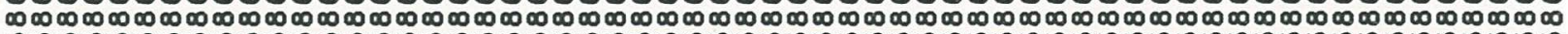

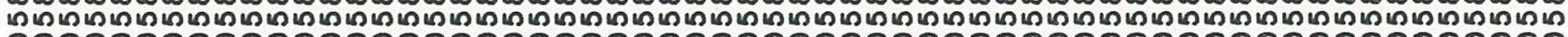
000000000000000000000000000000000000000000000000000000000000

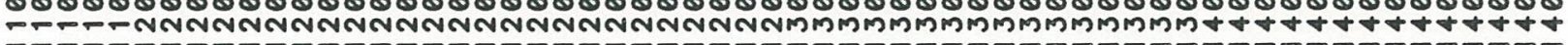




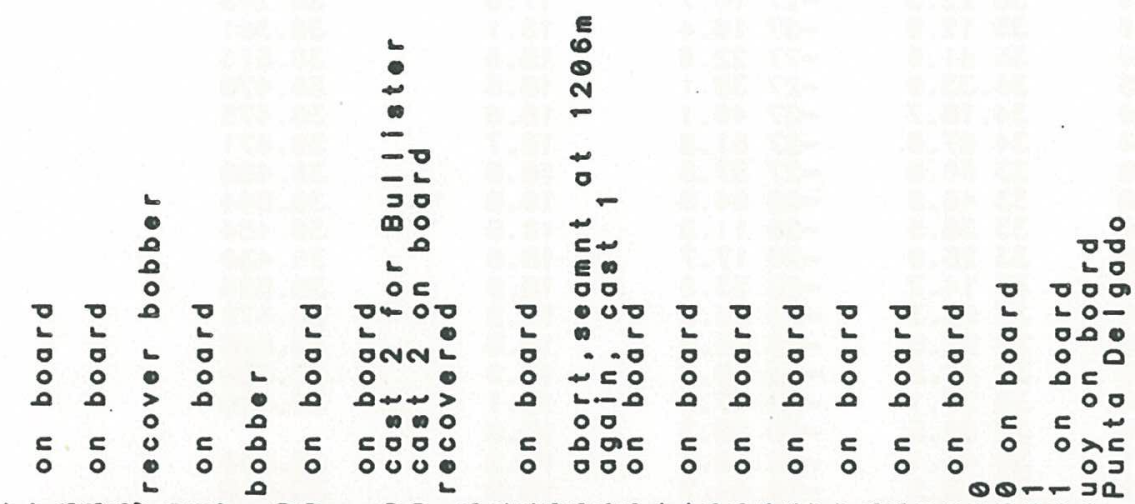

ANmmm

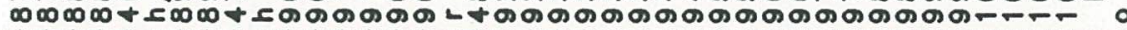

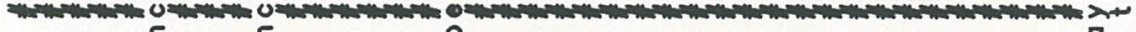
正

00000000000000000 $00000-000-000000-$ $0000-000-00000000$ $-0-000-000-0-0-00$

NOOM O6000NNOWOOO

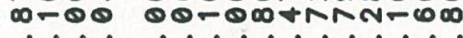

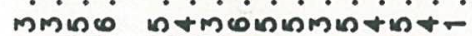
trmm NNNF-

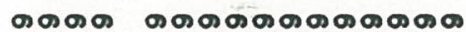
NNNN NNNNNNNNNNNN

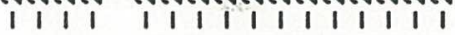

nOOM toOOOMாNMOOO

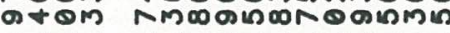

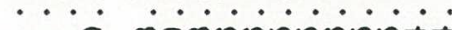

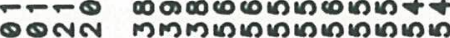

0000000000000000 МMMM МMMMMMMMMMMM

000000000000000000000000 $000000000000000000000-0$

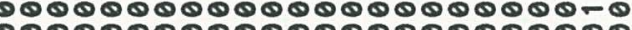
(1)

D-NOROONRNOOOG-

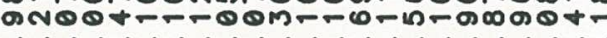

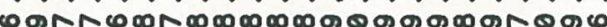

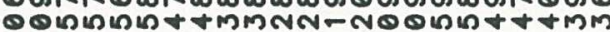

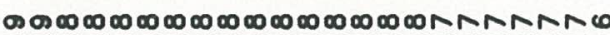
NNNNNNNNNNNNNNNNNNNNNNO $\begin{array}{lllllllllllllllll}1 & 1 & 1 & 1 & 1 & 1 & 1 & 1 & 1 & 1 & 1 & 1 & 1 & 1 & 1 & 1 & 1\end{array}$

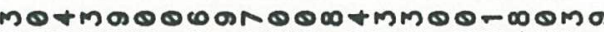
ONOOWM• • • • • • • . • • . . . -mMmt

-F-F- -NNNNNNMMMMMMMtL

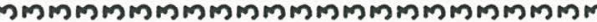

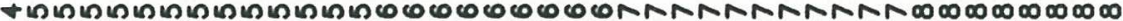

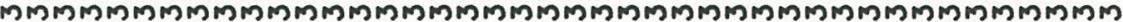

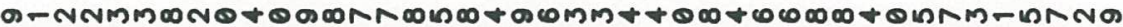

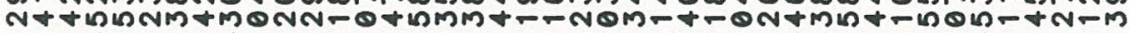
Nontonon No0000-

000000000000000000000000000000000000000000 p

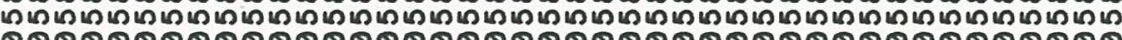
(10) + 
Table 2. Date, time, position and surface temperature and salinity values at XBT launch sites.

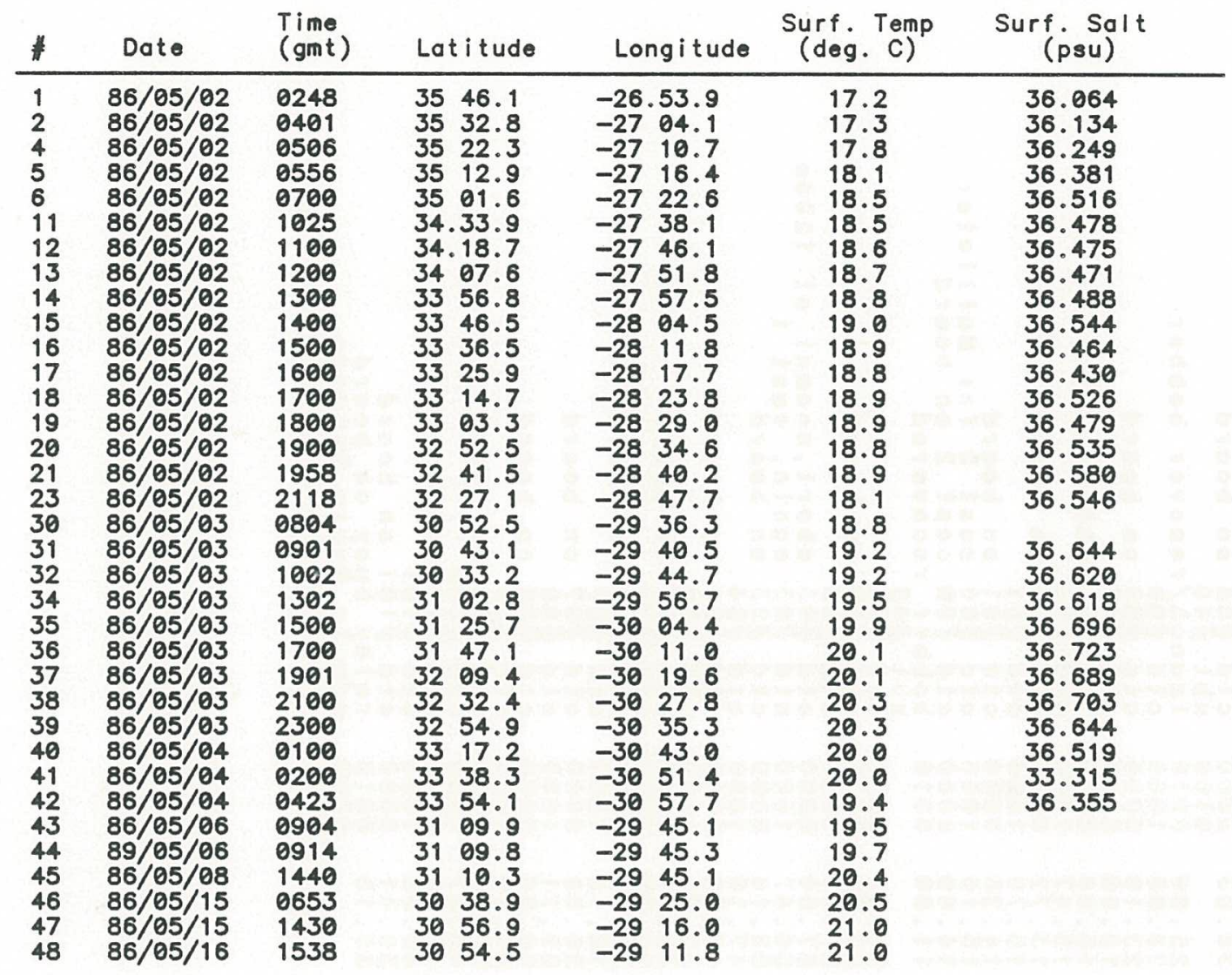


Table 3. Date, time, position and surface data from CTD stations.

\begin{tabular}{|c|c|c|c|c|c|c|c|c|}
\hline Sta. & Date & $\begin{array}{l}\text { Time } \\
(\operatorname{gnt})\end{array}$ & Latitude & Longitude & $\begin{array}{c}\text { Temp } \\
\left(\text { deg. }^{\prime} \dot{C}\right)\end{array}$ & $\begin{array}{c}\text { Surface } \\
\text { Salt } \\
\text { (psu) }\end{array}$ & $\begin{array}{c}\text { lalues } \\
\text { Ox } \\
(m \mid / 1)\end{array}$ & gen $_{\%}$ sot. \\
\hline$* 1$ & $86 / 05 / 01$ & 2339 & $3546.7 \mathrm{~N}$ & $-2651.6 W$ & & & & \\
\hline 2 & $86 / 05 / 03$ & 0008 & 3158.2 & $\begin{array}{l}-2902.6 \\
-29\end{array}$ & $\begin{array}{l}18.564 \\
18.774\end{array}$ & $\begin{array}{l}36.383 \\
36.418\end{array}$ & 5.29 & 100.5 \\
\hline $\begin{array}{l}3 \\
4\end{array}$ & $86 / 05 / 03$ & $\begin{array}{l}0344 \\
0722\end{array}$ & $\begin{array}{l}3125.3 \\
3052.5\end{array}$ & $\begin{array}{l}-2917.6 \\
-29\end{array}$ & 19.000 & 36.572 & $\begin{array}{l}3.41 \\
5.43\end{array}$ & $\begin{array}{l}103.2 \\
104.1\end{array}$ \\
\hline${ }^{*} 5$ & $86 / 05 / 04$ & 0730 & 3359.7 & -3059.6 & 18.721 & 36.240 & 5.18 & 98.6 \\
\hline$* 6$ & $86 / 05 / 04$ & 1218 & $\begin{array}{ll}33 & 42.0 \\
33 & 22.8\end{array}$ & 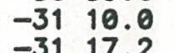 & 20.238 & 36.568 & 5.45 & 106.9 \\
\hline $\begin{array}{r}* 7 \\
\$ 8\end{array}$ & $\begin{array}{l}86 / 05 / 04 \\
86 / 05 / 04\end{array}$ & 1959 & $\begin{array}{ll}33 & 22.8 \\
33 & 04.9\end{array}$ & $\begin{array}{ll}-31 & 17.2 \\
-31 & 28.9\end{array}$ & 20.718 & 36.726 & 5.20 & 103.1 \\
\hline$\Rightarrow 9$ & $86 / 05 / 05$ & 0006 & 3247.0 & -3138.0 & 20.415 & 36.762 & 5.26 & 103.7 \\
\hline$* 10$ & $86 / 05 / 05$ & 0338 & $32 \quad 28.1$ & -3147.0 & 20. & 36.693 & 5.18 & 101.8 \\
\hline$* 11$ & $86 / 05 / 05$ & 0732 & 3209.8 & -3156.0 & 19.965 & 36.789 & 5.19 & 101.5 \\
\hline$* 12$ & $86 / 05 / 05$ & 1050 & 3209.5 & -3156.3 & 20.301 & 36.714 & 5.12 & 100.7 \\
\hline$* 13$ & $86 / 05 / 05$ & 1520 & 3152.0 & -3204.9 & 20.626 & 36.758 & 5.35 & 105.9 \\
\hline$* 14$ & $86 / 05 / 05$ & 1926 & 3133.1 & -3213.6 & 20.520 & 36.702 & 5.48 & 108.2 \\
\hline$* 15$ & $86 / 05 / 06$ & 1623 & $\begin{array}{ll}31 & 22.7\end{array}$ & -3024.0 & 19.822 & 36.710 & 5.30 & $103 . \overline{3}$ \\
\hline$* 16$ & $86 / 05 / 07$ & 0421 & 3129.8 & $\begin{array}{ll}-30 & 15.1\end{array}$ & 19.994 & 36.701 & 5.37 & 105.0 \\
\hline 17 & $86 / 05 / 07$ & 0550 & 3139.8 & -3015.1 & $19.930^{\circ}$ & 36.660 & 5.32 & 103.9 \\
\hline & $86 / 05 / 07$ & 0720 & 3149.8 & 15.1 & 19.896 & 36.714 & 5.45 & 106.4 \\
\hline & & 1353 & 3159.9 & 14.8 & 19.900 & 36.694 & 5.24 & 102.3 \\
\hline 20 & $86 / 05 / 07$ & 1515 & 3209.9 & $-30 \quad 15.1$ & 20.536 & 36.707 & 5.08 & 100.3 \\
\hline 21 & $86 / 05 / 07$ & 1636 & $32 \quad 19.8$ & -3015.1 & 20.489 & 36.710 & 5.15 & 101.6 \\
\hline 22 & $86 / 05 / 07$ & 1832 & 3220.0 & -3026.9 & 20. & 36. & & 99.6 \\
\hline 23 & $86 / 05 / 97$ & 2000 & 3210.0 & -3027 & 20. & 36.702 & 5.07 & 99.8 \\
\hline 24 & $86 / 05 / 07$ & 2214 & 3200.0 & -3027 & 20.352 & 36.764 & 4.99 & 98.2 \\
\hline 25 & $86 / 05 / 07$ & 2344 & 3150.1 & -3027.0 & 19.932 & 36.712 & 5.03 & 98.2 \\
\hline 26 & $86 / 05 / 08$ & 0147 & 3139.5 & $-30 \quad 27.1$ & 19.965 & 36.731 & 4.77 & 93.2 \\
\hline 27 & $5 / 08$ & 0312 & 3130.0 & -3027.0 & 20.049 & 36.720 & 4.94 & 96.7 \\
\hline 28 & $86 / 05 / 08$ & 0435 & $31 \quad 19.9$ & -3027.0 & 19.903 & $36.473 ?$ & 4.89 & 95.3 \\
\hline 29 & $86 / 05 / 08$ & 0637 & 3129.8 & -3039.1 & 19. & 36.700 & 5.06 & 98.7 \\
\hline$\leqslant 0$ & $86 / 05 / 08$ & 0808 & 3039.8 & -3039 & 20. & 36. & & 102.7 \\
\hline 3 & & 0939 & 3150.1 & -3038 & 20 . & 36. & & 103.3 \\
\hline 3 & & 1101 & 3159.8 & -3039.1 & 20. & 36. & 5.52 & 108.2 \\
\hline 3. & & 1210 & 3210.0 & $=0=1$ & 20.2 & 36.681 & & 102.8 \\
\hline 34 & & 1352 & $32 \quad 19.9$ & 1 & 20. & 36. & 88 & 113.4 \\
\hline 35 & $86 / 0$ & 1534 & 3220.2 & -3050.9 & 20.155 & 36.585 & 5.23 & 102.5 \\
\hline 36 & 86 & 1705 & 3210.0 & -3051.0 & 20.224 & 36.609 & 5.38 & 105.6 \\
\hline 37 & & 1830 & 3200.0 & -3050.9 & 20.3 & 36.723 & 5.47 & 107.4 \\
\hline 38 & 108 & 2002 & 3150.1 & -3051.7 & 20. & 36. & 5.09 & 99.9 \\
\hline 39 & $86 / 05 / 08$ & 2128 & 3140.0 & -3051.2 & 20. & 36. & 5.16 & 101.0 \\
\hline 40 & $86 / 0$ & 2251 & 3130.1 & -3051.0 & 20 . & & 5.15 & 100.8 \\
\hline 41 & 86 & 0020 & 3129.9 & -3103.1 & 20. & & & 98.7 \\
\hline 42 & 86 & 0142 & 3140.0 & -3103 & 20 & & & 99.8 \\
\hline 43 & 86 & 0314 & 3150.0 & -3103.1 & 19. & 36. & 5.05 & 98.7 \\
\hline 44 & $86 / 05 / 09$ & 0432 & 3200.0 & -3103.1 & 19.9 & 36. & 5.11 & 99.9 \\
\hline $\begin{array}{l}4 \\
4\end{array}$ & $5 / 09$ & $\begin{array}{l}0609 \\
1458\end{array}$ & $\begin{array}{ll}32 & 09.9 \\
31 & 10.1\end{array}$ & $\begin{array}{ll}-31 & 03.1\end{array}$ & 20.229 & 36.706 & $\begin{array}{l}4.97 \\
5.36\end{array}$ & $\begin{array}{r}97.6 \\
105.4\end{array}$ \\
\hline$\$ 47$ & 86 & 1826 & $\begin{array}{ll}31 & 10.1 \\
31 & 09.6\end{array}$ & $\begin{array}{l}-29 \\
-29 \\
44.2\end{array}$ & 20.423 & 36.647 & & 114.1 \\
\hline$* 48$ & & 0915 & 3115.1 & -3224.3 & 20. & 36.742 & 5.59 & 109.9 \\
\hline$* 49$ & $/ 10$ & 1330 & 3057.0 & -3233.0 & 20. & 36.725 & 5.45 & 108.1 \\
\hline$* 50$ & $86 / 05 / 10$ & 1803 & 3039.1 & -3241.8 & 21. & 36. & 5.04 & 101.1 \\
\hline$* 51$ & $86 / 95 / 10$ & 2207 & $\begin{array}{ll}30 & 20.9\end{array}$ & -3252.1 & 21. & 36. & 5.32 & 106.1 \\
\hline$* 52$ & $86 / 05 / 11$ & 0218 & 3002.0 & -3301 & & & 25 & 104.5 \\
\hline$\$ 53$ & $86 / 05 / 11$ & 0616 & 2943.2 & -3309.9 & 20. & 36 & & 108.2 \\
\hline 5 & $86 / 05 / 11$ & 1307 & 2930.0 & -3216.0 & 20.862 & 36.801 & & 104.4 \\
\hline$=$ & 1 & 1622 & 2920.2 & -3216.2 & 20.663 & 36.740 & & 102.8 \\
\hline 3 & & & 2009 & $\begin{array}{l}-3216 \\
-30\end{array}$ & & & .34 & $\begin{array}{l}106.2 \\
105.6\end{array}$ \\
\hline 5 & $86 / 05 / 11$ & $\begin{array}{l}1923 \\
2051\end{array}$ & $\begin{array}{ll}29 & 00.0 \\
28 & 50.0\end{array}$ & $\begin{array}{l}-3216.0 \\
-3216.1\end{array}$ & $\begin{array}{l}20.801 \\
20.840\end{array}$ & $\begin{array}{l}36.828 \\
36.889\end{array}$ & $\begin{array}{l}5.32 \\
4.86\end{array}$ & $\begin{array}{r}100.0 \\
96.6\end{array}$ \\
\hline $5 s$ & $86 / 05 / 11$ & 2220 & 2840.1 & -3216.1 & 20.960 & 36.964 & 4.84 & 96.5 \\
\hline , & 86 & 200 & 40.0 & -3204.1 & 21.018 & 36.982 & 4.83 & \\
\hline $\begin{array}{l}61 \\
62 \\
62\end{array}$ & $\begin{array}{l}86 / 05 / 12 \\
86 / 05 / 12\end{array}$ & $\begin{array}{l}0130 \\
0303\end{array}$ & 2850.1 & -3204.0 & 20.861 & 36.946 & 4.63 & $\begin{array}{l}92.1 \\
91.5\end{array}$ \\
\hline 6 & $86 / 05 / 12$ & 0433 & 2909.7 & -3206.0 & $\begin{array}{l}20.448 \\
20.638\end{array}$ & 36.859 & $\begin{array}{l}4.64 \\
4.68\end{array}$ & 92.7 \\
\hline 64 & $86 / 05 / 12$ & 0620 & 2920.0 & -3203.9 & 20.591 & & & 91.6 \\
\hline $\begin{array}{l}65 \\
66\end{array}$ & $\begin{array}{l}86 / 05 / 12 \\
86 / 05 / 12\end{array}$ & $\begin{array}{l}0747 \\
0928\end{array}$ & $\begin{array}{l}2930.0 \\
2930.0\end{array}$ & $\begin{array}{l}-3204.0 \\
-31 \\
-32.1\end{array}$ & $\begin{array}{l}20.776 \\
20.774\end{array}$ & $\begin{array}{l}36.831 \\
36.839\end{array}$ & $\begin{array}{l}4.70 \\
4.74\end{array}$ & $\begin{array}{l}93.3 \\
94.1\end{array}$ \\
\hline & & & & & & & & \\
\hline
\end{tabular}




\begin{tabular}{|c|c|c|c|c|c|c|c|c|c|c|}
\hline $\begin{array}{l}67 \\
68 \\
69 \\
70 \\
71 \\
72 \\
73 \\
74 \\
75 \\
76 \\
77 \\
78 \\
79 \\
80 \\
81 \\
82 \\
83 \\
84 \\
* 85 \\
* 86 \\
* 87 \\
* 88 \\
* 89 \\
* 90 \\
91 \\
* 92 \\
* 93 \\
* 94 \\
* 95 \\
* 96 \\
* 97 \\
* 98 \\
* 99 \\
* 100 \\
* 101\end{array}$ & $\begin{array}{l}86 / 05 / 12 \\
86 / 05 / 12 \\
86 / 05 / 12 \\
86 / 05 / 12 \\
86 / 05 / 12 \\
86 / 05 / 13 \\
86 / 05 / 13 \\
86 / 05 / 13 \\
86 / 05 / 13 \\
86 / 05 / 13 \\
86 / 05 / 13 \\
86 / 05 / 13 \\
86 / 05 / 13 \\
86 / 05 / 14 \\
86 / 05 / 14 \\
86 / 05 / 14 \\
86 / 05 / 14 \\
86 / 05 / 14 \\
86 / 05 / 14 \\
86 / 05 / 14 \\
86 / 05 / 14 \\
86 / 05 / 15 \\
86 / 05 / 15 \\
86 / 05 / 15 \\
86 / 05 / 15 \\
86 / 05 / 16 \\
86 / 05 / 16 \\
86 / 05 / 17 \\
86 / 05 / 17 \\
86 / 05 / 17 \\
86 / 05 / 17 \\
86 / 05 / 17 \\
86 / 05 / 18 \\
86 / 05 / 18 \\
86 / 05 / 18\end{array}$ & $\begin{array}{l}1055 \\
1225 \\
1746 \\
1912 \\
2104 \\
1327 \\
1458 \\
1628 \\
1740 \\
1907 \\
2025 \\
2144 \\
2309 \\
0039 \\
0202 \\
0330 \\
0451 \\
0610 \\
1437 \\
1828 \\
2229 \\
0240 \\
1037 \\
1620 \\
1918 \\
1744 \\
2306 \\
0314 \\
0718 \\
1226 \\
1638 \\
2044 \\
0055 \\
0453 \\
0845\end{array}$ & $\begin{array}{l}29 \\
29 \\
29 \\
28 \\
29 \\
28 \\
28 \\
28 \\
29 \\
29 \\
29 \\
29 \\
29 \\
29 \\
29 \\
29 \\
28 \\
28 \\
29 \\
29 \\
30 \\
30 \\
30 \\
30 \\
30 \\
31 \\
31 \\
31 \\
32 \\
32 \\
32 \\
33 \\
33 \\
33 \\
33\end{array}$ & $\begin{array}{l}19.9 \\
10.0 \\
00.3 \\
50.1 \\
05.0 \\
40.0 \\
40.0 \\
50.0 \\
00.0 \\
11.0 \\
19.5 \\
30.0 \\
30.0 \\
20.0 \\
10.0 \\
00.0 \\
50.0 \\
40.0 \\
24.0 \\
43.1 \\
02.0 \\
21.0 \\
39.4 \\
55.8 \\
56.0 \\
15.0 \\
38.7 \\
52.1 \\
10.2 \\
28.2 \\
47.2 \\
04.9 \\
23.1 \\
42.3 \\
59.8\end{array}$ & $\begin{array}{l}-31 \\
-31 \\
-31 \\
-31 \\
-31 \\
-31 \\
-31 \\
-31 \\
-31 \\
-31 \\
-31 \\
-31 \\
-31 \\
-31 \\
-31 \\
-31 \\
-31 \\
-31 \\
-30 \\
-29 \\
-29 \\
-29 \\
-29 \\
-29 \\
-29 \\
-29 \\
-28 \\
-28 \\
-28 \\
-28 \\
-28 \\
-28 \\
-27 \\
-27 \\
-27\end{array}$ & $\begin{array}{l}51.9 \\
52.0 \\
51.3 \\
51.9 \\
51.9 \\
51.9 \\
40.0 \\
40.0 \\
40.0 \\
39.8 \\
40.1 \\
39.4 \\
28.0 \\
27.9 \\
28.0 \\
27.7 \\
27.9 \\
28.0 \\
03.1 \\
53.0 \\
43.9 \\
35.0 \\
24.1 \\
15.5 \\
15.8 \\
07.0 \\
56.5 \\
47.1 \\
38.1 \\
28.3 \\
19.1 \\
09.1 \\
59.1 \\
49.9 \\
40.1\end{array}$ & $\begin{array}{l}20.551 \\
20.663 \\
21.062 \\
20.911 \\
20.803 \\
20.734 \\
20.845 \\
20.995 \\
21.155 \\
21.163 \\
20.970 \\
21.306 \\
20.970 \\
20.827 \\
20.689 \\
20.810 \\
20.538 \\
20.509 \\
20.929 \\
20.764 \\
20.871 \\
20.519 \\
20.703 \\
21.039 \\
20.931 \\
20.530 \\
20.337 \\
20.146 \\
19.989 \\
20.118 \\
20.624 \\
20.286 \\
19.870 \\
19.908 \\
19.421\end{array}$ & $\begin{array}{l}36.753 \\
36.839 \\
36.939 \\
36.967 \\
36.961 \\
36.871 \\
36.868 \\
36.870 \\
36.889 \\
36.889 \\
36.742 \\
36.830 \\
36.815 \\
36.782 \\
36.840 \\
36.874 \\
36.829 \\
36.812 \\
36.942 \\
36.800 \\
36.774 \\
36.641 \\
36.674 \\
36.557 \\
36.586 \\
36.474 \\
36.503 \\
36.584 \\
36.615 \\
36.571 \\
36.523 \\
36.625 \\
36.590 \\
36.622 \\
36.277\end{array}$ & $\begin{array}{l}4.79 \\
4.89 \\
5.22 \\
5.25 \\
4.65 \\
4.81 \\
5.17 \\
4.87 \\
4.70 \\
4.85 \\
4.54 \\
4.63 \\
4.61 \\
4.63 \\
4.68 \\
4.91 \\
4.74 \\
4.75 \\
5.28 \\
5.23 \\
5.25 \\
5.21 \\
4.78 \\
4.96 \\
5.50 \\
5.32 \\
5.16 \\
5.46 \\
5.42 \\
5.35 \\
5.31 \\
5.28 \\
5.25 \\
5.31 \\
5.44\end{array}$ & $\begin{array}{r}92 \\
97 . \\
93 . \\
93 . \\
105 . \\
103 . \\
104 . \\
102 . \\
94 . \\
98 . \\
109 . \\
104 . \\
101 . \\
107 . \\
105 . \\
104 . \\
104 . \\
103 . \\
102 . \\
103 . \\
105 .\end{array}$ \\
\hline
\end{tabular}

*CTD Instrument 7 was used.

CTD Instrument 9 was used at all other stations. 
Table 4. Meteorological data from En-143. Approximately $1 / 3$ of the surface temperature data are interpolated.

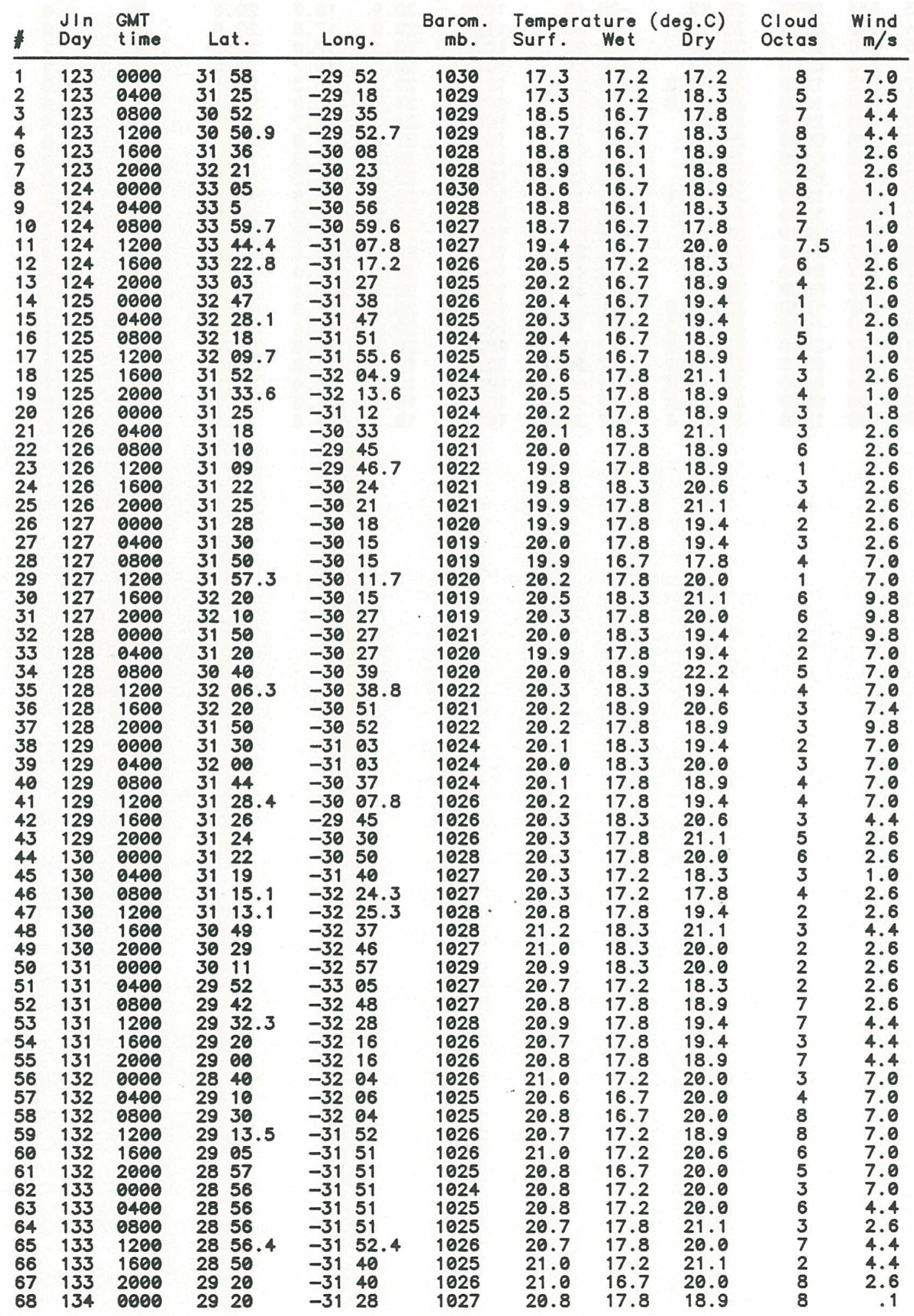




$\begin{array}{lllllllllllll}69 & 134 & 0400 & 29 & 00 & -31 & 28 & 1026 & 20.8 & 17.8 & 20.6 & 8 & 4.4 \\ 70 & 134 & 0800 & 29 & 04 & -31 & 30 & 1027 & 20.8 & 17.8 & 18.9 & 8 & 4.4 \\ 71 & 134 & 1200 & 29 & 08.1 & -30 & 31.5 & 1028 & 20.9 & 17.2 & 21.1 & 7 & 4.4 \\ 72 & 134 & 1600 & 29 & 34 & -29 & 58 & 1027 & 20.9 & 18.3 & 21.1 & 7 & 2.6 \\ 73 & 134 & 2000 & 29 & 52 & -29 & 48 & 1026 & 20.9 & 16.7 & 20.6 & 7 & 1.0 \\ 74 & 135 & 0000 & 30 & 10 & -29 & 39 & 1026 & 20.9 & 17.8 & 20.6 & 7 & 1.0 \\ 75 & 135 & 0400 & 30 & 30 & -29 & 29 & 1025 & 20.9 & 17.8 & 20.6 & 4 & .1 \\ 76 & 135 & 0800 & 30 & 34 & -29 & 26 & 1025 & 20.9 & 16.7 & 20.0 & 7 & .1 \\ 77 & 135 & 1200 & 30 & 38.8 & -29 & 23.5 & 1025 & 20.9 & 18.3 & 21.1 & 7 & 4.4 \\ 78 & 135 & 1600 & 30 & 55.8 & -29 & 15.5 & 1022 & 21.0 & 17.8 & 21.1 & 6 & 4.4 \\ 79 & 135 & 2000 & 30 & 58 & -29 & 15 & 1020 & 21.0 & 16.7 & 20.0 & 7 & 4.4 \\ 80 & 136 & 0000 & 30 & 57 & -29 & 14 & 1020 & 21.0 & 17.8 & 20.6 & 2 & 7.0 \\ 81 & 136 & 0400 & 30 & 56 & -29 & 13 & 1017 & 20.9 & 17.8 & 18.9 & 4 & 7.0 \\ 82 & 136 & 0800 & 30 & 55 & -29 & 12 & 1016 & 20.8 & 17.8 & 18.9 & 8 & 9.8 \\ 83 & 136 & 1200 & 30 & 54.1 & -29 & 11.2 & 1016 & 20.7 & 18.9 & 18.9 & 8 & 9.8 \\ 84 & 136 & 1600 & 31 & 09 & -29 & 07 & 1016 & 20.6 & 18.9 & 20.0 & 6 & 7.0 \\ 85 & 136 & 2000 & 31 & 24 & -29 & 101 & 1017 & 20.5 & 17.8 & 20.0 & 8 & 4.4 \\ 86 & 137 & 0000 & 31 & 39 & -28 & 56 & 1020 & 20.4 & 14.4 & 18.9 & 4 & 4.4 \\ 87 & 137 & 0400 & 31 & 52 & -28 & 47 & 1020 & 20.3 & 14.4 & 18.9 & 3 & 4.4 \\ 88 & 137 & 0800 & 32 & 10 & -28 & 38 & 1021 & 20.2 & 14.4 & 18.9 & 8 & 4.4 \\ 89 & 137 & 1200 & 32 & 27.9 & -28 & 28.2 & 1023 & 20.1 & 13.9 & 18.3 & 1 & .1 \\ 90 & 137 & 1600 & 32 & 47.2 & -28 & 19.1 & 1023 & 20.6 & 14.4 & 18.3 & 1 & .1 \\ 91 & 137 & 2000 & 33 & 04.9 & -28 & 09.1 & 1024 & 20.3 & 15.6 & 18.9 & 4 & .1 \\ 92 & 138 & 0000 & 33 & 23.1 & -27 & 59.1 & 1026 & 19.9 & 13.9 & 18.9 & 2 & .1 \\ 93 & 138 & 0400 & 33 & 42.3 & -27 & 49.9 & 1025 & 19.9 & 14.4 & 17.2 & 1 & .1 \\ 94 & 138 & 0800 & 33 & 59.8 & -27 & 40.1 & 1025 & 19.4 & 15.6 & 21.1 & 3 & 1.0 \\ 95 & 138 & 1200 & 34 & 05.4 & -27 & 35.9 & 1026 & 19.1 & 15.6 & 18.3 & 2 & 4.7\end{array}$


Table 5. Heat flux calculations from En-143 using data from Table 4. The columns are sensible, latent and long-wave heat flux with positive values emanating from the sea surface. SWR is short-wave insolation, Tau is the wind stress in Pascals and mix is the mixing effect on the ocean surface in Watts $/ \mathrm{m}^{2}$.

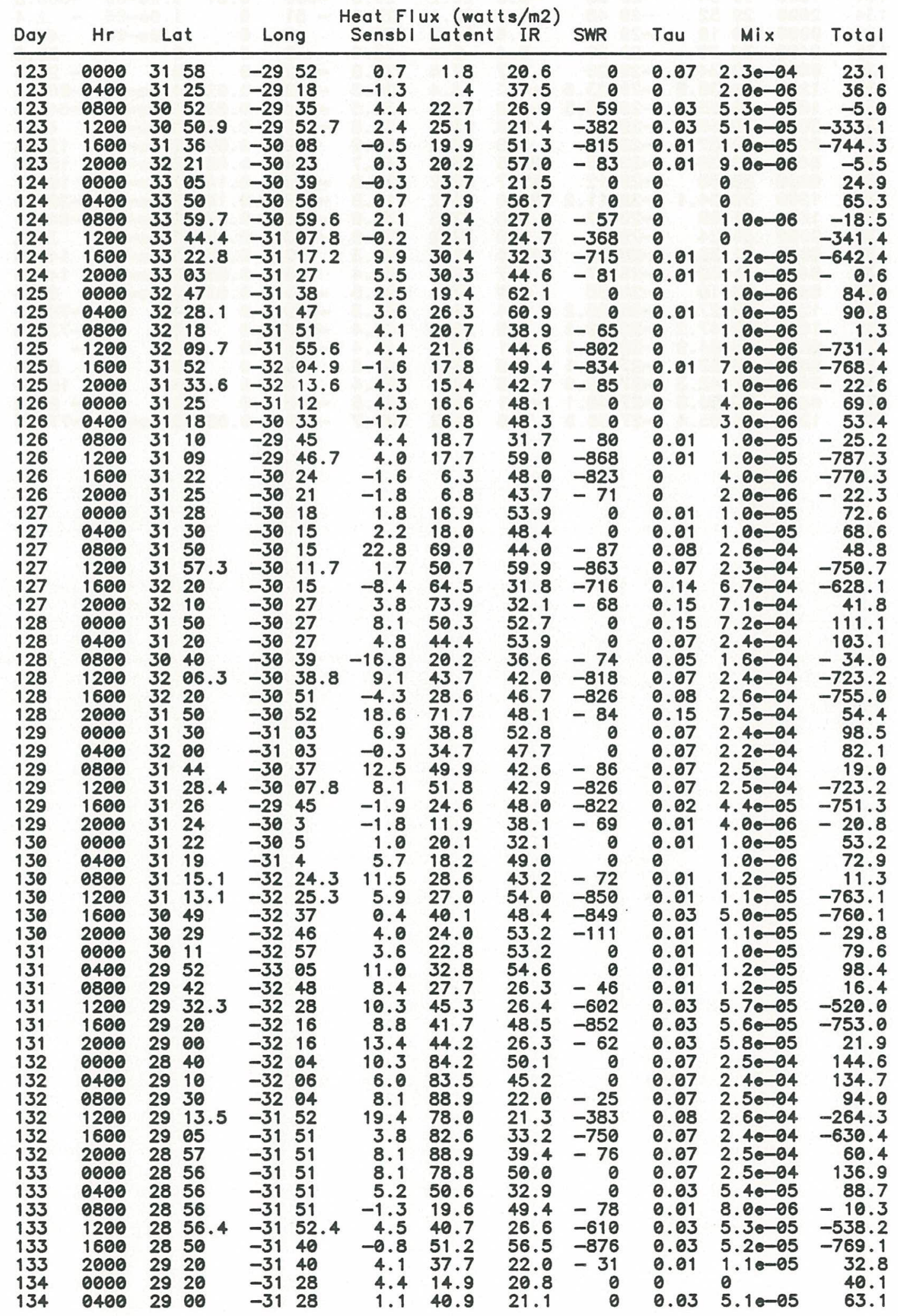




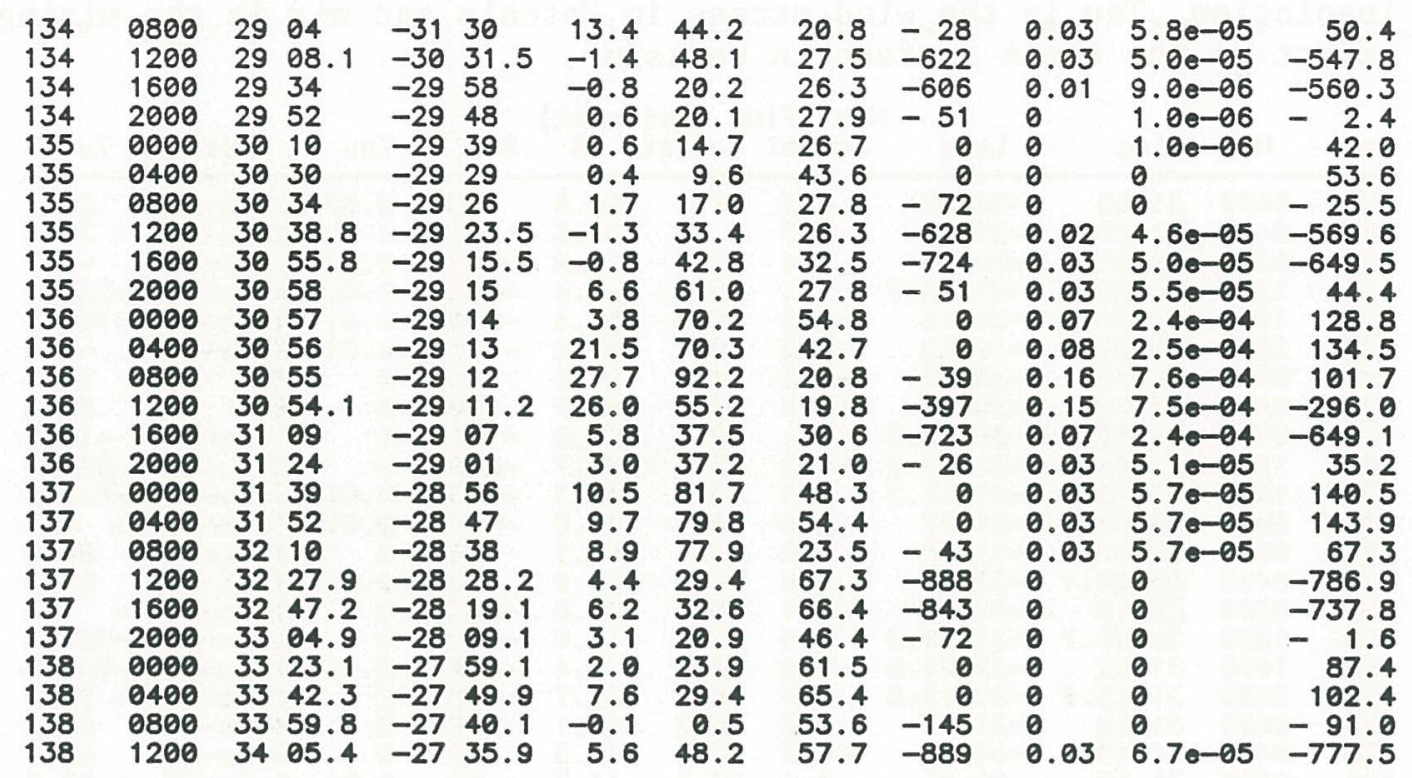




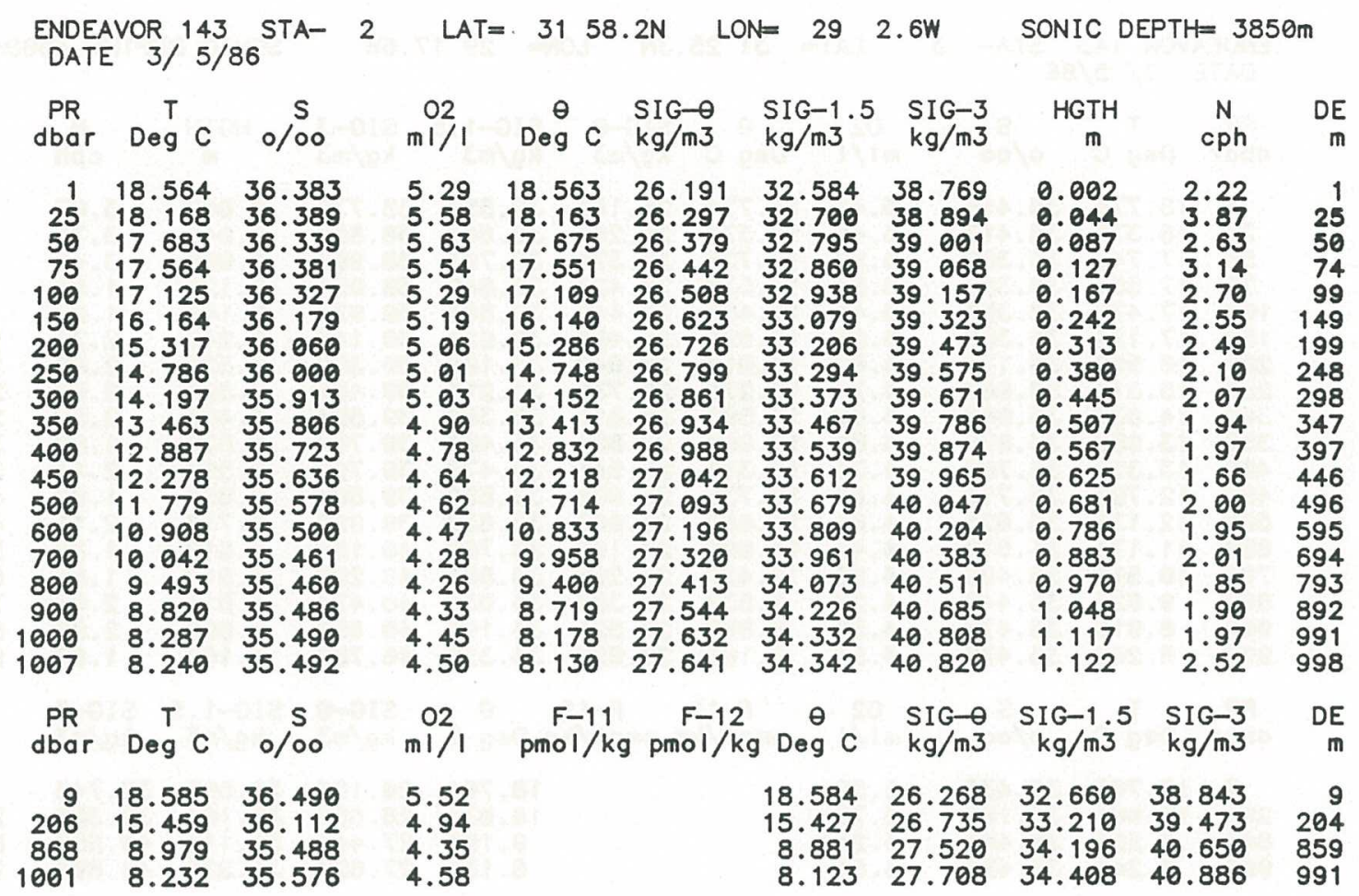

Table 6. Listings of the CTD, hydrographic and chlorofluorocarbon (F-11, F-12) data obtained during R/V Endeavor cruise \# 143. The first listing for each station is the CTD data at standard pressures. Each temperature, salinity and oxygen value is the average of a 2 decibar segment of the water column. These observed values are followed by the derived values potential temperature, sigma-theta, sigma-1500 m, sigma-3000 m, dynamic height, Brunt Vaisala frequency and depth. The second listing presents the water sample values for salinity, oxygen and Freons F-11 and F-12 together with the CTD pressure and temperature at which the sample was collected. 


\begin{tabular}{|c|c|c|c|c|c|c|c|c|c|c|}
\hline \multicolumn{3}{|c|}{$\begin{array}{l}\text { ENDEAVOR } 143 \text { STA- } \\
\text { DATE } 3 / 5 / 86\end{array}$} & LAT $=$ & \multicolumn{2}{|c|}{$3125.3 \mathrm{~N}$} & \multicolumn{2}{|c|}{$\mathrm{ON}=$} & SONIC & \multicolumn{2}{|c|}{$=4000 \mathrm{~m}$} \\
\hline $\begin{array}{l}\text { PR } \\
\text { dbar }\end{array}$ & $\stackrel{T}{\text { Deg } C}$ & $\stackrel{S}{0 / 00}$ & $\begin{array}{l}02 \\
\mathrm{ml} / \mathrm{l}\end{array}$ & $\stackrel{\theta}{\operatorname{Deg} C}$ & $\begin{array}{l}\mathrm{SIG}-\theta \\
\mathrm{kg} / \mathrm{m} 3\end{array}$ & $\begin{array}{l}\mathrm{SIG}-1.5 \\
\mathrm{~kg} / \mathrm{m3}\end{array}$ & $\begin{array}{l}\text { SIG-3 } \\
\mathrm{kg} / \mathrm{m} 3\end{array}$ & $\begin{array}{c}\text { HGTH } \\
\mathbf{m}\end{array}$ & $\underset{c p h}{N}$ & $D E$ \\
\hline $\begin{array}{r}1 \\
25 \\
50 \\
75 \\
100 \\
150 \\
200 \\
250 \\
300 \\
350 \\
400 \\
450 \\
500 \\
600 \\
700 \\
800 \\
900 \\
997\end{array}$ & $\begin{array}{r}18.774 \\
18.378 \\
17.747 \\
17.551 \\
17.470 \\
17.124 \\
16.050 \\
15.313 \\
14.638 \\
13.959 \\
13.370 \\
12.792 \\
12.130 \\
11.173 \\
10.516 \\
9.624 \\
8.910 \\
8.288\end{array}$ & $\begin{array}{l}36.418 \\
36.417 \\
36.359 \\
36.352 \\
36.350 \\
36.308 \\
36.172 \\
36.067 \\
35.969 \\
35.875 \\
35.789 \\
35.710 \\
35.625 \\
35.519 \\
35.496 \\
35.449 \\
35.474 \\
35.475\end{array}$ & $\begin{array}{l}5.46 \\
5.59 \\
5.53 \\
5.40 \\
5.08 \\
4.68 \\
4.74 \\
4.82 \\
4.89 \\
4.74 \\
4.68 \\
4.64 \\
4.45 \\
4.28 \\
4.27 \\
4.30 \\
4.54\end{array}$ & $\begin{array}{r}18.774 \\
18.373 \\
17.738 \\
17.538 \\
17.453 \\
17.099 \\
16.018 \\
15.275 \\
14.593 \\
13.908 \\
13.313 \\
12.730 \\
12.063 \\
11.096 \\
10.429 \\
9.530 \\
8.808 \\
8.180\end{array}$ & $\begin{array}{l}26.165 \\
26.265 \\
26.379 \\
26.423 \\
26.442 \\
26.496 \\
26.646 \\
26.735 \\
26.810 \\
26.884 \\
26.941 \\
26.998 \\
27.063 \\
27.162 \\
27.265 \\
27.383 \\
27.521 \\
27.620\end{array}$ & $\begin{array}{l}32.553 \\
32.663 \\
32.793 \\
32.842 \\
32.863 \\
32.926 \\
33.105 \\
33.215 \\
33.309 \\
33.493 \\
33.478 \\
33.552 \\
33.638 \\
33.768 \\
33.891 \\
34.038 \\
34.199 \\
34.320\end{array}$ & $\begin{array}{l}38 \\
38 \\
38 \\
39 \\
39 \\
39 \\
39 \\
39 \\
39 \\
39 \\
39 \\
39 \\
39 \\
40 \\
40 \\
40 \\
40 \\
40\end{array}$ & $\begin{array}{l}0.002 \\
0.045 \\
0.088 \\
0.129 \\
0.169 \\
0.249 \\
0.326 \\
0.396 \\
0.465 \\
0.529 \\
0.592 \\
0.652 \\
0.710 \\
0.819 \\
0.919 \\
1.011 \\
1.092 \\
1.160\end{array}$ & $\begin{array}{l}3.07 \\
3.75 \\
3.45 \\
1.59 \\
1.26 \\
2.74 \\
2.88 \\
2.07 \\
2.51\end{array}$ & $\begin{array}{r}1 \\
25 \\
50 \\
74 \\
99 \\
149 \\
199 \\
248 \\
298 \\
347 \\
397 \\
446 \\
496 \\
595 \\
694 \\
793 \\
892 \\
988\end{array}$ \\
\hline $\begin{array}{l}\text { PR } \\
\text { dbar }\end{array}$ & $\stackrel{T}{\operatorname{Deg} C}$ & $\stackrel{S}{1 / 00}$ & $\begin{array}{l}02 \\
\mathrm{ml} / \mathrm{l}\end{array}$ & $\begin{array}{c}\mathrm{F}-11 \\
\mathrm{pmol} / \mathrm{kg}\end{array}$ & $\begin{array}{c}\mathrm{F}-12 \\
\mathrm{pmol} / \mathrm{kg}\end{array}$ & $\stackrel{\theta}{\operatorname{Deg} C}$ & $\begin{array}{l}\mathrm{SIG}-\theta \\
\mathrm{kg} / \mathrm{m} 3\end{array}$ & $\underset{\mathrm{kg} / \mathrm{m} 3}{\mathrm{SIG}-1.5}$ & $\begin{array}{l}\mathrm{SIG}-3 \\
\mathrm{~kg} / \mathrm{m} 3\end{array}$ & $D E$ \\
\hline $\begin{array}{r}7 \\
202 \\
844 \\
993\end{array}$ & $\begin{array}{r}18.766 \\
16.064 \\
9.293 \\
8.245\end{array}$ & $\begin{array}{l}36.435 \\
36.181 \\
35.460 \\
35.475\end{array}$ & $\begin{array}{l}5.5 \\
4.7\end{array}$ & & & $\begin{array}{r}18.764 \\
16.031 \\
9.195 \\
8.137\end{array}$ & $\begin{array}{l}26.180 \\
26.650 \\
27.447 \\
27.627\end{array}$ & $\begin{array}{l}32.568 \\
33.109 \\
34.113 \\
34.328\end{array}$ & $\begin{array}{l}38.748 \\
39.356 \\
40.558 \\
40.806\end{array}$ & $\begin{array}{r}7 \\
200 \\
835 \\
983\end{array}$ \\
\hline
\end{tabular}




\begin{tabular}{|c|c|c|c|c|c|c|c|c|c|c|}
\hline \multicolumn{3}{|c|}{$\begin{array}{l}\text { ENDEAVOR } 143 \text { STA- } \\
\text { DATE } 3 / 5 / 86\end{array}$} & LAT $=$ & \multicolumn{2}{|c|}{$3052.5 \mathrm{~N}$} & \multicolumn{2}{|c|}{$O N=2935.6 \mathrm{~W}$} & \multicolumn{2}{|c|}{ SONIC DEPTH= } & \multirow{2}{*}{$\begin{array}{l}\text { Om } \\
\text { DE } \\
\text { m }\end{array}$} \\
\hline $\begin{array}{l}\text { PR } \\
\text { dbar }\end{array}$ & $\stackrel{T}{T} C$ & $\stackrel{5}{0 / 00}$ & $\begin{array}{ll}02 \\
\mathrm{ml} / \mathrm{l}\end{array}$ & $\stackrel{\theta}{\operatorname{Deg}} \mathrm{C}$ & $\begin{array}{l}\mathrm{SIG}-\theta \\
\mathrm{kg} / \mathrm{m} 3\end{array}$ & $\begin{array}{l}\mathrm{SIG}-1.5 \\
\mathrm{~kg} / \mathrm{m} 3\end{array}$ & & $\underset{m}{H G T H}$ & $\underset{\mathrm{cph}}{N}$ & \\
\hline $\begin{array}{r}5 \\
25 \\
50 \\
75 \\
100 \\
150 \\
200 \\
250 \\
300 \\
350 \\
400 \\
450 \\
500 \\
600 \\
700 \\
800 \\
900 \\
1990\end{array}$ & $\begin{array}{r}19.000 \\
18.973 \\
18.645 \\
18.543 \\
18.268 \\
17.798 \\
17.018 \\
16.369 \\
15.559 \\
14.839 \\
14.353 \\
13.702 \\
13.027 \\
11.877 \\
10.820 \\
10.161 \\
9.392 \\
8.565 \\
8.453\end{array}$ & $\begin{array}{l}36 \\
36 \\
36 \\
36 \\
36 \\
36 \\
36 \\
36 \\
35 \\
35 \\
35 \\
35 \\
35 \\
35 \\
35 \\
35\end{array}$ & 7 & $\begin{array}{l}18 . \\
18 . \\
18 . \\
18 . \\
17 . \\
16 . \\
16 . \\
15 . \\
14 . \\
14 . \\
13 . \\
12 . \\
11 . \\
10 . \\
10 . \\
9 . \\
8 . \\
8 .\end{array}$ & $\begin{array}{l}26.241 \\
26.337 \\
26.370 \\
26.389 \\
26.440 \\
26.531 \\
26.615 \\
26.719 \\
26.796 \\
26.850 \\
26.915 \\
26.984 \\
27.095 \\
27.210 \\
27.314 \\
27.461 \\
27.576 \\
27.590\end{array}$ & $\begin{array}{l}3 \\
3 \\
3 \\
3 \\
3 \\
3 \\
3 \\
3 \\
3 \\
3\end{array}$ & & & & $\begin{array}{r}5 \\
25 \\
50 \\
74 \\
99 \\
149 \\
199 \\
248 \\
298 \\
347 \\
397 \\
446 \\
496 \\
595 \\
694 \\
793 \\
892 \\
991 \\
1002\end{array}$ \\
\hline $\begin{array}{l}\text { PR } \\
\text { dbar }\end{array}$ & $\stackrel{T}{D} C$ & $\stackrel{5}{0 / 00}$ & $\mathrm{ml} / \mathrm{l}$ & $\begin{array}{c}\mathrm{F}-11 \\
\mathrm{pmol} / \mathrm{kg}\end{array}$ & $\begin{array}{c}\mathrm{F}-12 \\
\mathrm{pmol} / \mathrm{kg}\end{array}$ & $\stackrel{\theta}{\operatorname{Deg} C}$ & & $\underset{\mathrm{kg} / \mathrm{m} 3}{\mathrm{SIG}-1.5}$ & $\mathrm{~kg} / \mathrm{m} 3$ & \\
\hline $\begin{array}{r}5 \\
204 \\
849 \\
1005\end{array}$ & $\begin{array}{r}17.038 \\
9.711 \\
8.448\end{array}$ & $\begin{array}{l}36.33 \\
35.47 \\
35.46\end{array}$ & 5 & & & $\begin{array}{r}17.004 \\
9.610 \\
8.337\end{array}$ & $\begin{array}{l}26.541 \\
27.387 \\
27.588\end{array}$ & $\begin{array}{l}32.615 \\
32.973 \\
34.039 \\
34.282\end{array}$ & $\begin{array}{l}39.195 \\
40.471 \\
40.754\end{array}$ & $\begin{array}{l}202 \\
840 \\
994\end{array}$ \\
\hline
\end{tabular}




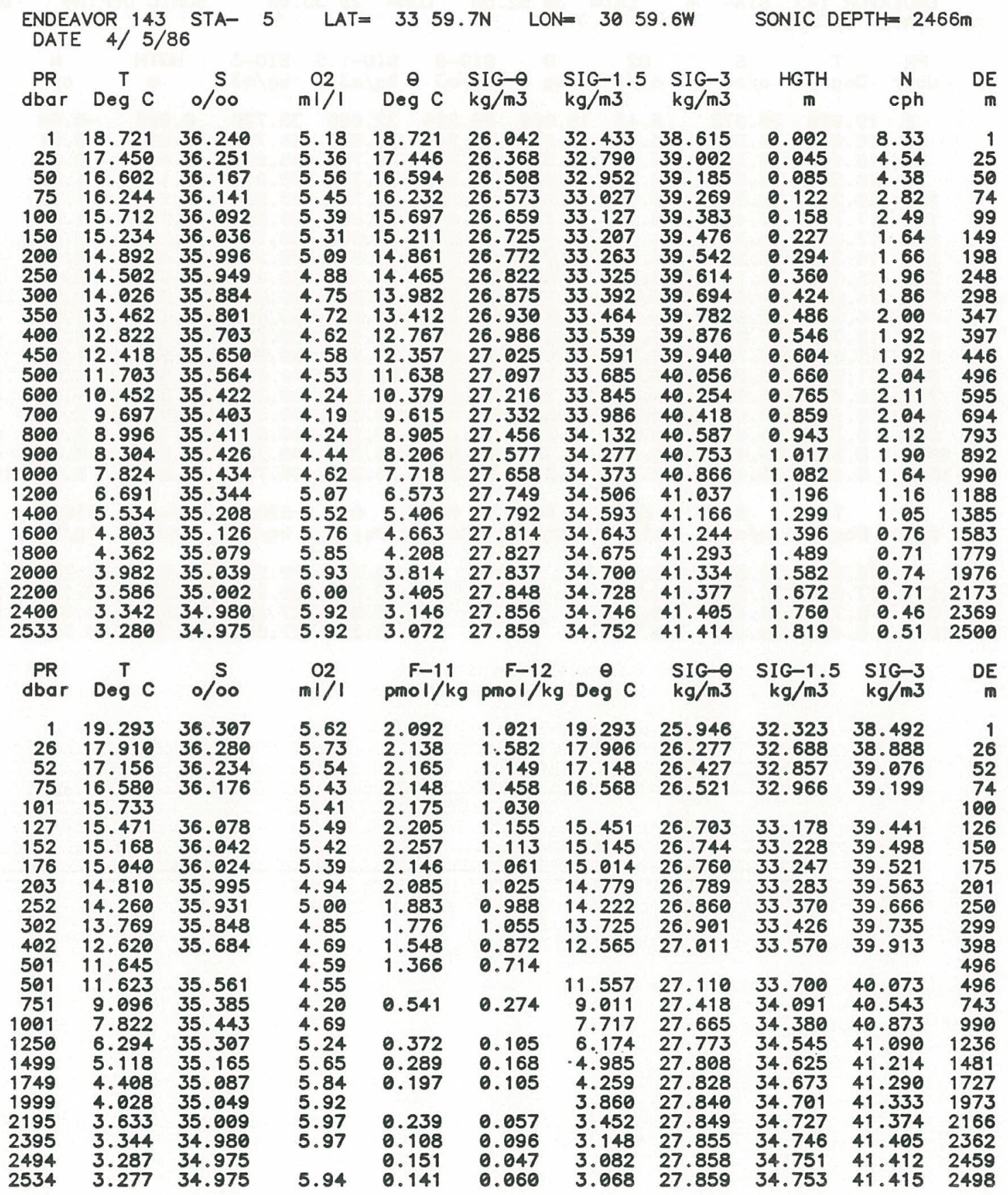




\begin{tabular}{|c|c|c|c|c|c|c|c|c|c|c|}
\hline \multicolumn{3}{|c|}{$\begin{array}{l}\text { ENDEAVOR } 143 \text { STA- } \\
\text { DATE } 4 / 5 / 86\end{array}$} & \multirow{2}{*}{$6 \begin{array}{l}\text { LAT }= \\
02 \\
\mathrm{ml} / \mathrm{l}\end{array}$} & \multicolumn{2}{|c|}{$3342.0 \mathrm{~N}$} & \multicolumn{2}{|c|}{$\mathrm{N}=31 \quad 10.0 \mathrm{~W}$} & \multicolumn{3}{|c|}{ SONIC DEPTH $=3550 \mathrm{~m}$} \\
\hline $\begin{array}{l}\text { PR } \\
\text { dbar }\end{array}$ & $\stackrel{T}{\text { Deg } C}$ & $\stackrel{S}{0 / 00}$ & & $\stackrel{\theta}{\operatorname{Deg} C}$ & $\begin{array}{l}\mathrm{SIG}-\theta \\
\mathrm{kg} / \mathrm{m} 3\end{array}$ & $\begin{array}{l}\mathrm{SIG}-1.5 \\
\mathrm{~kg} / \mathrm{m} 3\end{array}$ & $\begin{array}{l}\mathrm{SIG}-3 \\
\mathrm{~kg} / \mathrm{m3}\end{array}$ & $\begin{array}{l}\text { HGTH } \\
\mathrm{m}\end{array}$ & $\underset{c p h}{N}$ & $\begin{aligned} D E \\
m\end{aligned}$ \\
\hline $\begin{array}{r}1 \\
25 \\
50 \\
75 \\
100 \\
150 \\
200 \\
250 \\
300 \\
350 \\
400 \\
450 \\
500 \\
600 \\
700 \\
800 \\
900 \\
1000 \\
1200 \\
1400 \\
1600 \\
1800 \\
2000 \\
2200 \\
2400 \\
2600 \\
2800 \\
2995\end{array}$ & $\begin{array}{r}20.238 \\
18.870 \\
18.181 \\
17.775 \\
17.405 \\
16.674 \\
15.790 \\
15.208 \\
14.720 \\
14.224 \\
13.426 \\
12.783 \\
12.265 \\
11.198 \\
10.273 \\
9.543 \\
9.012 \\
8.487 \\
7.147 \\
5.911 \\
5.049 \\
4.410 \\
4.088 \\
3.689 \\
3.427 \\
3.168 \\
3.050 \\
2.920\end{array}$ & $\begin{array}{l}36.568 \\
36.488 \\
36.429 \\
36.396 \\
36.356 \\
36.238 \\
36.130 \\
36.044 \\
35.975 \\
35.908 \\
35.793 \\
35.698 \\
35.625 \\
35.513 \\
35.469 \\
35.481 \\
35.504 \\
35.502 \\
35.401 \\
35.256 \\
35.156 \\
35.083 \\
35.052 \\
35.013 \\
34.989 \\
34.966 \\
34.959 \\
34.950\end{array}$ & $\begin{array}{l}5.45 \\
5.66 \\
5.67 \\
5.49 \\
5.20 \\
5.17 \\
4.94 \\
4.85 \\
4.85 \\
4.81 \\
4.89 \\
4.77 \\
4.72 \\
4.52 \\
4.50 \\
4.44 \\
4.45 \\
4.52 \\
4.98 \\
5.41 \\
5.65 \\
5.83 \\
5.90 \\
5.96 \\
5.99 \\
5.98\end{array}$ & $\begin{array}{c}20.238 \\
18.866 \\
18.172 \\
17.762 \\
17.388 \\
16.649 \\
15.758 \\
15.170 \\
14.675 \\
14.172 \\
13.369 \\
12.721 \\
12.197 \\
11.122 \\
10.188 \\
9.450 \\
8.910 \\
8.377 \\
7.024 \\
5.779 \\
4.907 \\
4.256 \\
3.919 \\
3.507 \\
3.229 \\
2.954 \\
2.819 \\
2.671\end{array}$ & $\begin{array}{l}25.896 \\
26.195 \\
26.325 \\
26.402 \\
26.463 \\
26.549 \\
26.673 \\
26.741 \\
26.796 \\
26.854 \\
26.933 \\
26.991 \\
27.037 \\
27.154 \\
27.286 \\
27.422 \\
27.528 \\
27.611 \\
27.731 \\
27.784 \\
27.810 \\
27.825 \\
27.837 \\
27.847 \\
27.855 \\
27.863 \\
27.869 \\
27.875\end{array}$ & $\begin{array}{l}32.248 \\
32.580 \\
32.728 \\
32.814 \\
32.885 \\
32.991 \\
33.140 \\
33.223 \\
33.293 \\
33.365 \\
33.468 \\
33.546 \\
33.608 \\
33.758 \\
33.920 \\
34.079 \\
34.203 \\
34.303 \\
34.472 \\
34.570 \\
34.630 \\
34.670 \\
34.696 \\
34.722 \\
34.742 \\
34.761 \\
34.773 \\
34.785\end{array}$ & $\begin{array}{l}38.394 \\
38.757 \\
38.921 \\
39.017 \\
39.098 \\
39.222 \\
39.394 \\
39.493 \\
39.576 \\
39.662 \\
39.788 \\
39.884 \\
39.962 \\
40.144 \\
40.334 \\
40.516 \\
40.656 \\
40.774 \\
40.987 \\
41.130 \\
41.222 \\
41.287 \\
41.325 \\
41.368 \\
41.398 \\
41.428 \\
41.445 \\
41.463\end{array}$ & $\begin{array}{l}0.002 \\
0.050 \\
0.093 \\
0.135 \\
0.176 \\
0.254 \\
0.328 \\
0.398 \\
0.466 \\
0.532 \\
0.595 \\
0.656 \\
0.714 \\
0.825 \\
0.925 \\
1.015 \\
1.094 \\
1.165 \\
1.288 \\
1.395 \\
1.495 \\
1.590 \\
1.683 \\
1.775 \\
1.864 \\
1.953 \\
2.041 \\
2.127\end{array}$ & $\begin{array}{l}6.85 \\
5.42 \\
3.63 \\
2.41 \\
2.35 \\
2.59 \\
2.64 \\
1.73 \\
2.15 \\
1.97 \\
2.08 \\
2.01 \\
1.95 \\
1.94 \\
2.14 \\
2.07 \\
1.89 \\
1.73 \\
1.41 \\
1.01 \\
0.92 \\
0.73 \\
0.70 \\
0.68 \\
0.61 \\
0.56 \\
0.51 \\
0.57\end{array}$ & $\begin{array}{r}1 \\
25 \\
50 \\
74 \\
99 \\
149 \\
198 \\
248 \\
298 \\
347 \\
397 \\
446 \\
496 \\
595 \\
694 \\
793 \\
892 \\
991 \\
1188 \\
1385 \\
1583 \\
1780 \\
1976 \\
2173 \\
2369 \\
2566 \\
2762 \\
2953\end{array}$ \\
\hline $\begin{array}{l}\text { PR } \\
\text { dbar }\end{array}$ & $\stackrel{T}{\operatorname{Deg}} \mathrm{C}$ & $\stackrel{5}{0 / 00}$ & $\begin{array}{l}02 \\
\mathrm{ml} / \mathrm{l}\end{array}$ & $\begin{array}{c}\mathrm{F}-11 \\
\mathrm{pmol} / \mathrm{kg}\end{array}$ & $\begin{array}{c}\mathrm{F}-12 \\
\mathrm{pmol} / \mathrm{kg}\end{array}$ & $\stackrel{\theta}{g} \mathrm{Deg}$ & $\begin{array}{l}\mathrm{SIG}-\theta \\
\mathrm{kg} / \mathrm{m3}\end{array}$ & $\underset{\mathrm{kg} / \mathrm{m} 3}{\mathrm{SIG}-1.5}$ & $\begin{array}{l}\mathrm{SIG}-3 \\
\mathrm{~kg} / \mathrm{m} 3\end{array}$ & $\underset{m}{\mathrm{DE}}$ \\
\hline $\begin{array}{r}5 \\
29 \\
55 \\
77 \\
105 \\
127 \\
154 \\
176 \\
203 \\
253 \\
305 \\
403 \\
501 \\
501 \\
749 \\
1001 \\
1248 \\
1499 \\
1745\end{array}$ & $\begin{array}{r}19.727 \\
18.419 \\
17.763 \\
17.607 \\
16.940 \\
16.639 \\
16.204 \\
15.806 \\
15.307 \\
14.986 \\
14.312 \\
13.273 \\
12.209 \\
12.199 \\
10.038 \\
8.379 \\
6.587 \\
5.404 \\
4.553 \\
4.110 \\
3.602 \\
3.305 \\
3.079 \\
2.916\end{array}$ & $\begin{array}{l}36.545 \\
36.472 \\
36.407 \\
36.388 \\
36.291 \\
36.240 \\
36.176 \\
36.143 \\
36.072 \\
36.026 \\
35.929 \\
35.780 \\
35.624 \\
35.622 \\
35.474 \\
35.500 \\
35.340 \\
35.200 \\
35.098 \\
35.056 \\
35.004 \\
34.979 \\
34.960\end{array}$ & $\begin{array}{l}5.48 \\
5.56\end{array}$ & & & $\begin{array}{r}19.726 \\
18.414 \\
17.753 \\
17.594 \\
16.922 \\
16.618 \\
16.179 \\
15.778 \\
15.276 \\
14.947 \\
14.267 \\
13.216 \\
12.142 \\
12.132 \\
9.948 \\
8.269 \\
6.464 \\
5.267 \\
4.402 \\
3.942 \\
3.416 \\
3.100 \\
2.854 \\
2.667\end{array}$ & $\begin{array}{l}26.015 \\
26.297 \\
26.412 \\
26.437 \\
26.525 \\
26.558 \\
26.612 \\
26.679 \\
26.738 \\
26.776 \\
26.849 \\
26.054 \\
27.047 \\
27.048 \\
27.332 \\
27.626 \\
27.761 \\
27.802 \\
27.821 \\
27.837 \\
27.849 \\
27.859 \\
27.867 \\
27.872\end{array}$ & $\begin{array}{l}32.379 \\
32.693 \\
32.825 \\
32.854 \\
32.960 \\
33.001 \\
33.067 \\
33.145 \\
33.218 \\
33.265 \\
33.358 \\
33.494 \\
33.620 \\
33.620 \\
33.973 \\
34.322 \\
34.521 \\
34.608 \\
34.661 \\
34.695 \\
34.728 \\
34.751 \\
34.769 \\
34.782\end{array}$ & $\begin{array}{l}38.536 \\
38.881 \\
39.028 \\
39.061 \\
39.184 \\
39.233 \\
39.310 \\
39.398 \\
39.485 \\
39.541 \\
39.652 \\
39.818 \\
39.975 \\
39.976 \\
40.395 \\
40.796 \\
41.057 \\
41.187 \\
41.272 \\
41.324 \\
41.377 \\
41.413 \\
41.440 \\
41.461\end{array}$ & $\begin{array}{r}5 \\
28 \\
54 \\
77 \\
104 \\
126 \\
153 \\
174 \\
201 \\
251 \\
302 \\
400 \\
497 \\
496 \\
742 \\
990 \\
1234 \\
1481 \\
1724 \\
1967 \\
2211 \\
2459 \\
2701 \\
2953\end{array}$ \\
\hline
\end{tabular}




\begin{tabular}{|c|c|c|c|c|c|c|c|c|c|c|}
\hline \multicolumn{3}{|c|}{$\begin{array}{l}\text { ENDEAVOR } 143 \text { STA- } \\
\text { DATE } 4 / 5 / 86\end{array}$} & \multirow{2}{*}{$\begin{array}{l}\text { LAT }= \\
02 \\
\mathrm{ml} / \mathrm{l}\end{array}$} & \multicolumn{2}{|c|}{$3322.8 \mathrm{~N}$} & \multicolumn{2}{|c|}{ LON $=3117.2 \mathrm{~W}$} & \multicolumn{3}{|c|}{ SONIC DEPTH $=3350 \mathrm{~m}$} \\
\hline $\begin{array}{l}\text { PR } \\
\text { dbar }\end{array}$ & $\stackrel{T}{T} \mathrm{c}$ & $\stackrel{5}{0 / 00}$ & & $\stackrel{\theta}{\operatorname{Deg}} \mathrm{C}$ & $\begin{array}{l}\mathrm{SIG}-\theta \\
\mathrm{kg} / \mathrm{m3}\end{array}$ & $\begin{array}{l}\mathrm{SIG}-1.5 \\
\mathrm{~kg} / \mathrm{m} 3\end{array}$ & $\begin{array}{l}\text { SIG-3 } \\
\mathrm{kg} / \mathrm{m} 3\end{array}$ & $\begin{array}{l}\text { HGTH } \\
\mathbf{m}\end{array}$ & $\begin{array}{c}N \\
\text { cph }\end{array}$ & \\
\hline $\begin{array}{r}3 \\
25 \\
50 \\
75 \\
100 \\
150 \\
200 \\
250 \\
300 \\
350 \\
400 \\
450 \\
500 \\
600 \\
700 \\
800 \\
900 \\
1000 \\
1200 \\
1400 \\
1600 \\
1800 \\
2000 \\
2200 \\
2400 \\
2600 \\
2800 \\
2995\end{array}$ & $\begin{array}{r}20.451 \\
19.715 \\
19.160 \\
18.900 \\
18.810 \\
18.648 \\
17.359 \\
16.518 \\
15.777 \\
15.072 \\
14.464 \\
13.803 \\
13.232 \\
11.650 \\
10.478 \\
9.791 \\
8.528 \\
8.393 \\
7.329 \\
6.174 \\
5.086 \\
4.465 \\
4.014 \\
3.676 \\
3.428 \\
3.207 \\
3.070 \\
2.933\end{array}$ & $\begin{array}{l}36.668 \\
36.696 \\
36.672 \\
36.645 \\
36.647 \\
36.604 \\
36.397 \\
36.261 \\
36.139 \\
36.033 \\
35.946 \\
35.849 \\
35.765 \\
35.544 \\
35.466 \\
35.449 \\
35.345 \\
35.475 \\
35.412 \\
35.290 \\
35.160 \\
35.087 \\
35.044 \\
35.012 \\
34.991 \\
34.973 \\
34.961 \\
34.950\end{array}$ & $\begin{array}{l}5.08 \\
5.19 \\
5.29 \\
5.34 \\
5.23 \\
5.12 \\
4.72 \\
4.74 \\
4.79 \\
4.83 \\
4.87 \\
4.81 \\
4.79 \\
4.48 \\
4.57 \\
4.43 \\
4.40 \\
4.52 \\
4.85 \\
5.27 \\
5.64 \\
5.82 \\
5.87 \\
5.91 \\
5.92 \\
5.95 \\
5.97\end{array}$ & $\begin{array}{l}20.450 \\
19.711 \\
19.151 \\
18.887 \\
18.792 \\
18.622 \\
17.325 \\
16.477 \\
15.729 \\
15.019 \\
14.404 \\
13.738 \\
13.161 \\
11.571 \\
10.392 \\
9.696 \\
8.429 \\
8.283 \\
7.205 \\
6.039 \\
4.943 \\
4.311 \\
3.846 \\
3.493 \\
3.230 \\
2.993 \\
2.838 \\
2.684\end{array}$ & $\begin{array}{l}25.916 \\
26.134 \\
26.262 \\
26.309 \\
26.336 \\
26.346 \\
26.509 \\
26.608 \\
26.687 \\
26.765 \\
26.833 \\
26.899 \\
26.954 \\
27.094 \\
27.248 \\
27.355 \\
27.480 \\
27.604 \\
27.715 \\
27.777 \\
27.809 \\
27.823 \\
27.837 \\
27.848 \\
27.856 \\
27.864 \\
27.869 \\
27.874\end{array}$ & $\begin{array}{l}32.263 \\
32.497 \\
32.639 \\
32.693 \\
32.721 \\
32.736 \\
32.933 \\
33.054 \\
33.154 \\
33.252 \\
33.337 \\
33.423 \\
33.495 \\
33.684 \\
33.876 \\
34.005 \\
34.172 \\
34.300 \\
34.449 \\
34.554 \\
34.628 \\
34.666 \\
34.699 \\
34.724 \\
34.743 \\
34.761 \\
34.772 \\
34.784\end{array}$ & $\begin{array}{l}38.403 \\
38.653 \\
38.808 \\
38.868 \\
38.898 \\
38.917 \\
39.146 \\
39.289 \\
39.409 \\
39.526 \\
39.628 \\
39.733 \\
39.821 \\
40.057 \\
40.284 \\
40.435 \\
40.642 \\
40.774 \\
40.958 \\
41.104 \\
41.218 \\
41.281 \\
41.331 \\
41.370 \\
41.399 \\
41.426 \\
41.444 \\
41.461\end{array}$ & $\begin{array}{l}0.006 \\
0.049 \\
0.095 \\
0.138 \\
0.181 \\
0.268 \\
0.350 \\
0.427 \\
0.501 \\
0.572 \\
0.640 \\
0.705 \\
0.768 \\
0.887 \\
0.991 \\
1.085 \\
1.168 \\
1.240 \\
1.366 \\
1.478 \\
1.579 \\
1.675 \\
1.768 \\
1.859 \\
1.948 \\
2.037 \\
2.125 \\
2.211\end{array}$ & $\begin{array}{l}6.83 \\
4.83 \\
2.87 \\
2.33 \\
0.99 \\
2.89 \\
2.43 \\
2.59 \\
2.22 \\
2.46 \\
1.76 \\
2.04 \\
1.93 \\
2.58 \\
2.07 \\
2.15 \\
2.11 \\
1.62 \\
1.34 \\
1.23 \\
0.89 \\
0.79 \\
0.74 \\
0.69 \\
0.56 \\
0.58 \\
0.48 \\
0.57\end{array}$ & $\begin{array}{l}1188 \\
1385 \\
158 \\
178 \\
1976 \\
217 \\
236 \\
2560 \\
276 \\
295\end{array}$ \\
\hline $\begin{array}{l}\text { PR } \\
\text { dbar }\end{array}$ & $\stackrel{T}{T} C$ & o/00 & $\begin{array}{ll}02 \\
\mathrm{ml} / \mathrm{I}\end{array}$ & $\begin{array}{c}\mathrm{F}-11 \\
\mathrm{pmol} / \mathrm{kg}\end{array}$ & $\begin{array}{c}\mathrm{F}-12 \\
\mathrm{pmol} / \mathrm{kg}\end{array}$ & $\stackrel{\oplus}{\operatorname{Deg}} \mathrm{C}$ & $\begin{array}{l}\mathrm{SIG}-0 \\
\mathrm{~kg} / \mathrm{m} 3\end{array}$ & $\underset{\mathrm{kg} / \mathrm{m} 3}{\mathrm{SIG}-1.5}$ & $\begin{array}{l}\mathrm{SIG}-3 \\
\mathrm{~kg} / \mathrm{m} 3\end{array}$ & \\
\hline $\begin{array}{r}2 \\
25 \\
52 \\
76 \\
103 \\
127 \\
153 \\
175 \\
201 \\
251 \\
300 \\
401 \\
502 \\
500 \\
750 \\
898 \\
1248 \\
1504 \\
1746 \\
2003 \\
2246 \\
2495\end{array}$ & $\begin{array}{r}20.575 \\
19.400 \\
18.666 \\
18.837 \\
18.771 \\
18.578 \\
17.629 \\
17.341 \\
17.055 \\
16.235 \\
15.372 \\
14.241 \\
13.200 \\
13.206 \\
10.230 \\
8.565 \\
6.932 \\
5.496 \\
4.660 \\
4.049 \\
3.615 \\
3.307 \\
3.092 \\
2.932\end{array}$ & $\begin{array}{l}36.7 \\
36.6 \\
36.5 \\
36.6 \\
36.6 \\
36.6 \\
36.4 \\
36.4 \\
36.4 \\
36.2 \\
36.6 \\
35.9 \\
35.7 \\
35.7 \\
35.4 \\
35.3 \\
35.7 \\
35.2 \\
35.1 \\
35 .\end{array}$ & $\begin{array}{l}5.38 \\
5.36 \\
5.43 \\
5.34 \\
5.23 \\
5.20 \\
4.76 \\
4.70 \\
4.68 \\
4.75 \\
4.81 \\
4.94 \\
4.77 \\
4.84 \\
4.45 \\
4.33 \\
5.00\end{array}$ & $\begin{array}{l}1.527 \\
0.856 \\
0.470 \\
0.242\end{array}$ & $\begin{array}{l}0.711 \\
0.353 \\
0.190 \\
0.109\end{array}$ & $\begin{array}{l}20.5 \\
19.3 \\
18.6 \\
18.8 \\
18.7 \\
18.5 \\
17.6 \\
17.3 \\
17.0 \\
16.1 \\
15.3 \\
14.1 \\
13.1 \\
13.1 \\
10.1 \\
8.4 \\
6.8 \\
5.3 \\
4.5 \\
3.8 \\
3.4 \\
3.1 \\
2.8 \\
2.6\end{array}$ & $\begin{array}{l}25.925 \\
26.203 \\
26.296 \\
26.333 \\
26.348 \\
26.367 \\
26.474 \\
26.521 \\
26.558 \\
26.644 \\
26.737 \\
26.861 \\
26.961 \\
26.962 \\
27.292 \\
27.481 \\
27.742 \\
27.801 \\
27.819 \\
27.842 \\
27.851 \\
27.860 \\
27.868 \\
27.873\end{array}$ & $\begin{array}{l}32 . \\
32 . \\
32 . \\
32 . \\
32 . \\
32 . \\
32 . \\
32 . \\
33 . \\
33 . \\
33 . \\
33 . \\
33 . \\
33 . \\
34 .\end{array}$ & $\begin{array}{l}39 . \\
39 . \\
39 . \\
40 . \\
40 . \\
41 . \\
41 . \\
41 .\end{array}$ & $\begin{array}{l}1234 \\
1486 \\
1725 \\
1977 \\
2216 \\
2460 \\
2707 \\
2952\end{array}$ \\
\hline
\end{tabular}




\begin{tabular}{|c|c|c|c|c|c|c|c|c|c|c|}
\hline \multicolumn{3}{|c|}{$\begin{array}{l}\text { ENDEAVOR } 143 \text { STA- } \\
\text { DATE } 4 / 5 / 86\end{array}$} & \multirow{2}{*}{ 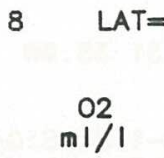 } & \multirow{2}{*}{$\begin{array}{c}=334 \\
\stackrel{\theta}{\text { Deg } C}\end{array}$} & \multirow{2}{*}{$\begin{array}{l}.9 \mathrm{~N} \\
\mathrm{SIG}-\mathrm{E} \\
\mathrm{kg} / \mathrm{m}^{3}\end{array}$} & \multicolumn{2}{|c|}{$N=3128.9 W$} & \multicolumn{3}{|c|}{ SONIC DEPTH= 3650m } \\
\hline $\begin{array}{l}\text { PR } \\
\text { dbar }\end{array}$ & $\stackrel{T}{\operatorname{Deg} C}$ & $\stackrel{S}{0 / 00}$ & & & & $\begin{array}{l}\mathrm{SIG}-1.5 \\
\mathrm{~kg} / \mathrm{m} 3\end{array}$ & $\begin{array}{l}S I G-3 \\
\mathrm{~kg} / \mathrm{m} 3\end{array}$ & $\underset{m}{\mathrm{HGTH}}$ & $\begin{array}{c}N \\
\text { cph }\end{array}$ & $D E$ \\
\hline $\begin{array}{r}3 \\
25 \\
50 \\
75 \\
100 \\
150 \\
200 \\
250 \\
300 \\
350 \\
400 \\
450 \\
500 \\
600 \\
700 \\
800 \\
900 \\
1000 \\
1200 \\
1400 \\
1600 \\
1800 \\
2000 \\
2200 \\
2400 \\
2600 \\
2800 \\
2997\end{array}$ & $\begin{array}{r}20.718 \\
19.657 \\
19.246 \\
19.156 \\
19.074 \\
18.602 \\
17.670 \\
17.214 \\
16.698 \\
15.947 \\
14.951 \\
14.371 \\
13.724 \\
12.393 \\
11.167 \\
10.333 \\
8.998 \\
8.838 \\
7.585 \\
6.275 \\
5.216 \\
4.558 \\
4.101 \\
3.746 \\
3.487 \\
3.268 \\
3.086 \\
2.919\end{array}$ & $\begin{array}{l}36.726 \\
36.709 \\
36.687 \\
36.687 \\
36.678 \\
36.584 \\
36.419 \\
36.358 \\
36.277 \\
36.161 \\
36.005 \\
35.926 \\
35.830 \\
35.640 \\
35.512 \\
35.460 \\
35.359 \\
35.492 \\
35.431 \\
35.302 \\
35.173 \\
35.096 \\
35.053 \\
35.019 \\
34.995 \\
34.978 \\
34.964 \\
34.952\end{array}$ & $\begin{array}{l}5.20 \\
5.31 \\
5.31 \\
5.33 \\
5.36 \\
5.19 \\
4.94 \\
4.93 \\
5.00 \\
4.77 \\
4.80 \\
4.80 \\
4.76 \\
4.61 \\
4.64 \\
4.50 \\
4.35 \\
4.49 \\
4.81 \\
5.20 \\
5.62 \\
5.83 \\
5.92 \\
5.95 \\
5.97\end{array}$ & $\begin{array}{c}20.717 \\
19.653 \\
19.237 \\
19.143 \\
19.056 \\
18.575 \\
17.636 \\
17.172 \\
16.649 \\
15.891 \\
14.890 \\
14.303 \\
13.652 \\
12.311 \\
11.077 \\
10.234 \\
8.896 \\
8.725 \\
7.459 \\
6.139 \\
5.071 \\
4.402 \\
3.932 \\
3.563 \\
3.288 \\
3.052 \\
2.854 \\
2.671 \\
2\end{array}$ & $\begin{array}{l}25.887 \\
26.159 \\
26.251 \\
26.275 \\
26.292 \\
26.343 \\
26.450 \\
26.516 \\
26.579 \\
26.667 \\
26.772 \\
26.839 \\
26.903 \\
27.027 \\
27.161 \\
27.271 \\
27.417 \\
27.548 \\
27.693 \\
27.773 \\
27.805 \\
27.820 \\
27.836 \\
27.846 \\
27.854 \\
27.863 \\
27.870 \\
27.877\end{array}$ & $\begin{array}{l}32.227 \\
32.524 \\
32.625 \\
32.652 \\
32.670 \\
32.734 \\
32.866 \\
32.944 \\
33.021 \\
33.130 \\
33.263 \\
33.347 \\
33.430 \\
33.594 \\
33.767 \\
33.904 \\
34.094 \\
34.229 \\
34.418 \\
34.546 \\
34.618 \\
34.659 \\
34.694 \\
34.720 \\
34.739 \\
34.757 \\
34.772 \\
34.787\end{array}$ & $\begin{array}{l}38.362 \\
38.681 \\
38.792 \\
38.821 \\
38.841 \\
38.916 \\
39.072 \\
39.162 \\
39.252 \\
39.380 \\
39.541 \\
39.640 \\
39.742 \\
39.944 \\
40.154 \\
40.317 \\
40.549 \\
40.688 \\
40.919 \\
41.093 \\
41.204 \\
41.270 \\
41.323 \\
41.363 \\
41.392 \\
41.420 \\
41.443 \\
41.465\end{array}$ & $\begin{array}{l}0.006 \\
0.049 \\
0.094 \\
0.138 \\
0.182 \\
0.269 \\
0.354 \\
0.434 \\
0.513 \\
0.589 \\
0.662 \\
0.730 \\
0.796 \\
0.920 \\
1.034 \\
1.137 \\
1.228 \\
1.308 \\
1.442 \\
1.556 \\
1.659 \\
1.756 \\
1.851 \\
1.943 \\
2.033 \\
2.123 \\
2.212 \\
2.299\end{array}$ & $\begin{array}{l}9.20 \\
3.96 \\
2.30 \\
1.41 \\
1.64 \\
2.27 \\
2.62 \\
1.82 \\
2.23 \\
2.30 \\
2.71 \\
1.99 \\
2.13 \\
2.06 \\
2.14 \\
2.10 \\
2.18 \\
1.93 \\
1.56 \\
1.19 \\
0.91 \\
0.77 \\
0.75 \\
0.66 \\
0.63 \\
0.62 \\
0.54 \\
0.59\end{array}$ & $\begin{array}{r}3 \\
25 \\
50 \\
74 \\
99 \\
149 \\
198 \\
248 \\
298 \\
347 \\
397 \\
446 \\
496 \\
595 \\
694 \\
793 \\
892 \\
991 \\
1188 \\
1385 \\
1583 \\
1780 \\
1976 \\
2173 \\
2370 \\
2566 \\
2762 \\
2955\end{array}$ \\
\hline $\begin{array}{l}\text { PR } \\
\text { dbar }\end{array}$ & $\stackrel{T}{\operatorname{Deg}} \mathrm{C}$ & $\stackrel{5}{0 / 00}$ & $\begin{array}{l}02 \\
\mathrm{ml} / \mathrm{I}\end{array}$ & $\begin{array}{c}\mathrm{F}-11 \\
\mathrm{pmol} / \mathrm{kg}\end{array}$ & $\begin{array}{c}\mathrm{F}-12 \\
\mathrm{pmol} / \mathrm{kg}\end{array}$ & $\stackrel{\theta}{\operatorname{Deg} C}$ & $\mathrm{~kg} / \mathrm{m3}$ & $\mathrm{kg} / \mathrm{m} 3$ & $\begin{array}{l}\mathrm{SIG}-3 \\
\mathrm{~kg} / \mathrm{m} 3\end{array}$ & DE \\
\hline $\begin{array}{r}3 \\
27 \\
52 \\
77 \\
102 \\
125 \\
149 \\
176 \\
201 \\
250 \\
296 \\
401 \\
497 \\
495 \\
749 \\
999 \\
1249 \\
1499 \\
1747 \\
1997 \\
2247\end{array}$ & $\begin{array}{l}19 . \\
19 . \\
19 . \\
19 . \\
18 . \\
18 . \\
17 . \\
17 . \\
17 . \\
16 . \\
15 . \\
13 . \\
13 . \\
10 . \\
8 . \\
7 . \\
5 . \\
4 . \\
4 . \\
3 . \\
3 . \\
3 .\end{array}$ & $\begin{array}{l}36.745 \\
36.723 \\
36.710 \\
36.670 \\
36.683 \\
36.654 \\
36.543 \\
36.472 \\
36.423 \\
36.354 \\
36.283 \\
36.076 \\
35.868 \\
35.868 \\
35.479 \\
35.495 \\
35.412 \\
35.226\end{array}$ & $\begin{array}{l}8 \\
7 \\
7\end{array}$ & & & $\begin{array}{r}20.610 \\
19.676 \\
19.389 \\
19.061 \\
19.001 \\
18.841 \\
18.343 \\
17.942 \\
17.593 \\
17.088 \\
16.646 \\
15.285 \\
13.878 \\
13.881 \\
10.556 \\
8.629 \\
7.124 \\
5.490 \\
4.483 \\
3.924 \\
3.486 \\
3.153 \\
2.902 \\
2.669\end{array}$ & $\begin{array}{l}25.931 \\
26.164 \\
26.229 \\
26.284 \\
26.309 \\
26.328 \\
26.370 \\
26.415 \\
26.464 \\
26.534 \\
26.585 \\
26.739 \\
26.885 \\
26.884 \\
27.229 \\
27.566 \\
27.726 \\
27.796 \\
27.818 \\
27.840 \\
27.850 \\
27.858 \\
27.870 \\
27.879\end{array}$ & $\begin{array}{l}32.274 \\
32.528 \\
32.600 \\
32.663 \\
32.689 \\
32.712 \\
32.767 \\
32.823 \\
32.881 \\
32.964 \\
33.027 \\
33.219 \\
33.405 \\
33.404 \\
33.851 \\
34.250 \\
34.463 \\
34.593 \\
34.655 \\
34.698 \\
34.726 \\
34.748 \\
34.770 \\
34.789\end{array}$ & $\begin{array}{l}38.410 \\
38.685 \\
38.763 \\
38.833 \\
38.861 \\
38.888 \\
38.955 \\
39.021 \\
39.087 \\
39.183 \\
39.257 \\
39.485 \\
39.710 \\
39.709 \\
40.254 \\
40.712 \\
40.975 \\
41.164 \\
41.263 \\
41.327 \\
41.372 \\
41.407 \\
41.439 \\
41.467\end{array}$ & $\begin{array}{r}3 \\
27 \\
51 \\
77 \\
101 \\
124 \\
148 \\
175 \\
199 \\
248 \\
293 \\
397 \\
492 \\
490 \\
742 \\
988 \\
1235 \\
1482 \\
1725 \\
1972 \\
2217 \\
2461 \\
2679 \\
2954\end{array}$ \\
\hline
\end{tabular}




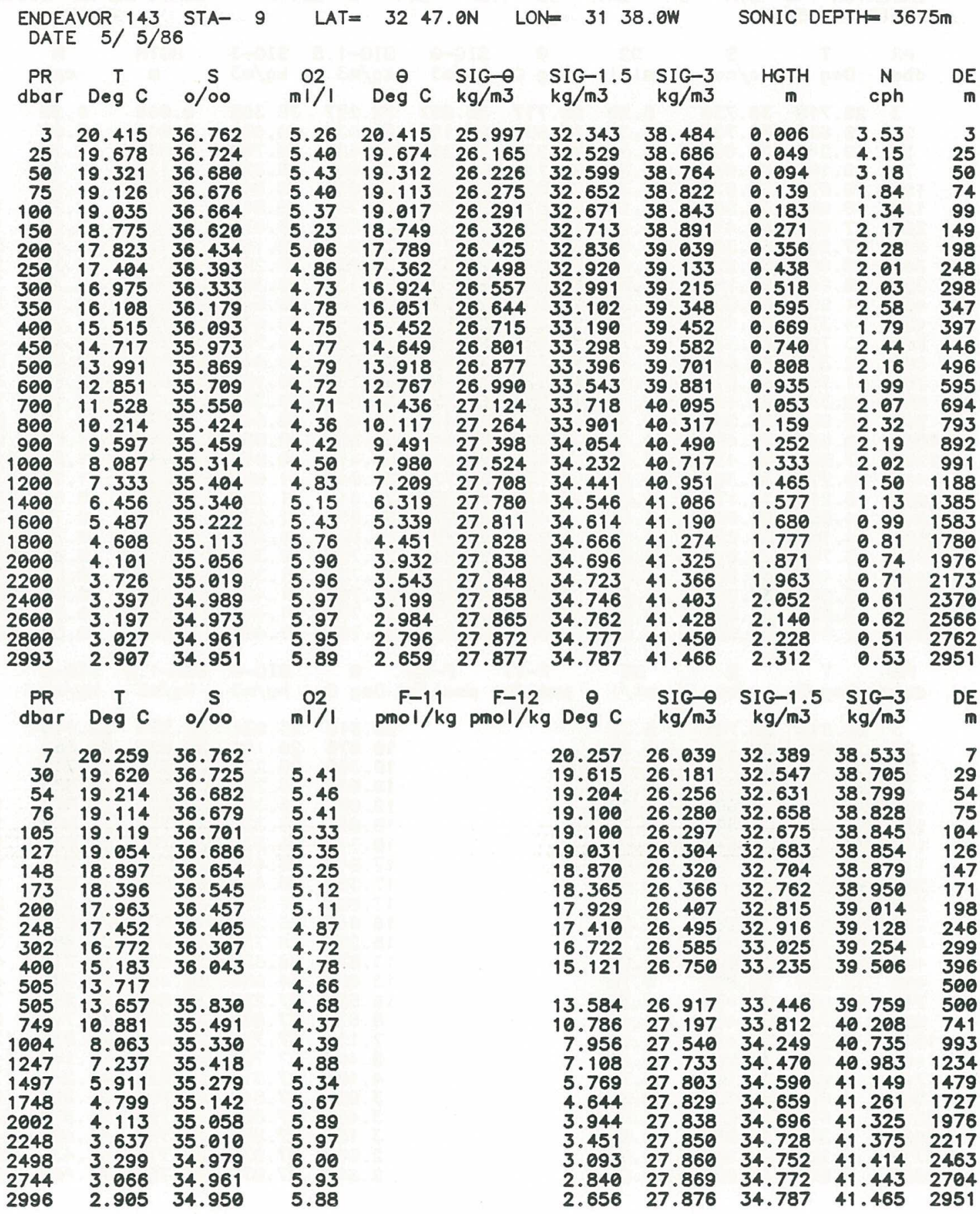




\begin{tabular}{|c|c|c|c|c|c|c|c|c|c|c|}
\hline \multicolumn{3}{|c|}{$\begin{array}{l}\text { ENDEAVOR } 143 \text { STA- } 10 \\
\text { DATE } 5 / 5 / 86\end{array}$} & LAT $=$ & \multicolumn{2}{|c|}{$3228.1 \mathrm{~N}$} & \multicolumn{2}{|c|}{$\mathrm{ON}=3147.0 \mathrm{~W}$} & \multicolumn{3}{|c|}{ SONIC DEPTH $=4000 \mathrm{~m}$} \\
\hline $\begin{array}{l}\text { PR } \\
\text { dbar }\end{array}$ & $\stackrel{T}{\operatorname{Deg}} \mathrm{C}$ & $\stackrel{5}{0 / 00}$ & $\begin{array}{ll}02 \\
\mathrm{ml} / \mathrm{I}\end{array}$ & $\stackrel{\ominus}{\operatorname{Deg} C}$ & $\begin{array}{l}\mathrm{SIG}-\theta \\
\mathrm{kg} / \mathrm{m3}\end{array}$ & $\begin{array}{l}\mathrm{SIG}-1.5 \\
\mathrm{~kg} / \mathrm{m} 3\end{array}$ & $\begin{array}{l}\mathrm{SIG}-3 \\
\mathrm{~kg} / \mathrm{m} 3\end{array}$ & $\begin{array}{l}\text { HGTH } \\
\text { m }\end{array}$ & $\underset{c p h}{N}$ & $\begin{aligned} \mathrm{DE} \\
\mathrm{m}\end{aligned}$ \\
\hline $\begin{array}{r}1 \\
25 \\
50 \\
75 \\
100 \\
150 \\
200 \\
250 \\
300 \\
350 \\
400 \\
450 \\
500 \\
600 \\
700 \\
800 \\
900 \\
1000 \\
1200 \\
1400 \\
1600 \\
1800 \\
2000 \\
2200 \\
2400 \\
2600 \\
2800 \\
2995\end{array}$ & $\begin{array}{r}20.284 \\
19.436 \\
19.135 \\
19.010 \\
18.933 \\
17.973 \\
17.442 \\
16.957 \\
16.523 \\
15.881 \\
15.191 \\
14.429 \\
13.741 \\
12.480 \\
11.340 \\
10.298 \\
9.436 \\
8.686 \\
7.899 \\
6.566 \\
5.450 \\
4.609 \\
4.062 \\
3.693 \\
3.374 \\
3.179 \\
3.000 \\
2.888\end{array}$ & $\begin{array}{l}36.693 \\
36.657 \\
36.647 \\
36.648 \\
36.642 \\
36.454 \\
36.388 \\
36.317 \\
36.252 \\
36.155 \\
36.057 \\
35.948 \\
35.845 \\
35.663 \\
35.552 \\
35.483 \\
35.457 \\
35.424 \\
35.476 \\
35.346 \\
35.209 \\
35.108 \\
35.047 \\
35.015 \\
34.987 \\
34.973 \\
34.959 \\
34.950\end{array}$ & $\begin{array}{l}5.18 \\
5.35 \\
5.35 \\
5.28 \\
5.20 \\
4.91 \\
4.83 \\
4.72 \\
4.83 \\
4.64 \\
4.70 \\
4.73 \\
4.66 \\
4.51 \\
4.41 \\
4.22 \\
4.11 \\
4.15 \\
4.56 \\
5.05 \\
5.42 \\
5.73 \\
5.85 \\
5.87 \\
5.93 \\
5.92 \\
5.88 \\
5.88\end{array}$ & $\begin{array}{l}20.284 \\
19.432 \\
19.126 \\
18.997 \\
18.915 \\
17.947 \\
17.408 \\
16.915 \\
16.474 \\
15.825 \\
15.129 \\
14.361 \\
13.668 \\
12.398 \\
11.249 \\
10.200 \\
9.331 \\
8.574 \\
7.770 \\
6.428 \\
5.303 \\
4.452 \\
3.893 \\
3.510 \\
3.177 \\
2.966 \\
2.770 \\
2.640\end{array}$ & $\begin{array}{l}25.979 \\
26.177 \\
26.250 \\
26.284 \\
26.300 \\
26.400 \\
26.482 \\
26.547 \\
26.602 \\
26.678 \\
26.759 \\
26.844 \\
26.911 \\
27.028 \\
27.160 \\
27.295 \\
27.422 \\
27.518 \\
27.683 \\
27.770 \\
27.805 \\
27.824 \\
27.835 \\
27.848 \\
27.858 \\
27.867 \\
27.873 \\
27.878\end{array}$ & $\begin{array}{l}32.329 \\
32.548 \\
32.627 \\
32.664 \\
32.683 \\
32.808 \\
32.904 \\
32.981 \\
33.048 \\
33.142 \\
33.243 \\
33.349 \\
33.437 \\
33.592 \\
33.760 \\
33.928 \\
34.084 \\
34.205 \\
34.397 \\
34.532 \\
34.610 \\
34.662 \\
34.695 \\
34.724 \\
34.747 \\
34.764 \\
34.779 \\
34.789\end{array}$ & $\begin{array}{l}38.473 \\
38.710 \\
38.797 \\
38.837 \\
38.857 \\
39.006 \\
39.115 \\
39.205 \\
39.283 \\
39.394 \\
39.514 \\
39.641 \\
39.748 \\
39.940 \\
40.142 \\
40.342 \\
40.525 \\
40.670 \\
40.887 \\
41.068 \\
41.187 \\
41.270 \\
41.326 \\
41.369 \\
41.405 \\
41.431 \\
41.453 \\
41.468\end{array}$ & $\begin{array}{l}0.002 \\
0.049 \\
0.094 \\
0.138 \\
0.182 \\
0.268 \\
0.350 \\
0.430 \\
0.508 \\
0.583 \\
0.655 \\
0.724 \\
0.790 \\
0.914 \\
1.027 \\
1.129 \\
1.220 \\
1.300 \\
1.438 \\
1.555 \\
1.660 \\
1.758 \\
1.852 \\
1.943 \\
2.032 \\
2.120 \\
2.207 \\
2.291\end{array}$ & $\begin{array}{l}4.56 \\
4.67 \\
2.03 \\
2.08 \\
1.58 \\
2.86 \\
2.05 \\
1.94 \\
1.97 \\
2.45 \\
2.16 \\
2.53 \\
2.09 \\
2.08 \\
2.13 \\
2.26 \\
2.03 \\
1.80 \\
1.50 \\
1.23 \\
1.02 \\
0.82 \\
0.77 \\
0.73 \\
0.64 \\
0.60 \\
0.54 \\
0.35\end{array}$ & $\begin{array}{r}1 \\
25 \\
50 \\
74 \\
99 \\
149 \\
199 \\
248 \\
298 \\
347 \\
397 \\
446 \\
496 \\
595 \\
694 \\
793 \\
892 \\
991 \\
1188 \\
1386 \\
1583 \\
1780 \\
1977 \\
2173 \\
2370 \\
2566 \\
2762 \\
2953\end{array}$ \\
\hline $\begin{array}{l}\text { PR } \\
\text { dbar }\end{array}$ & $\stackrel{T}{\text { Deg }} C$ & $\stackrel{S}{\circ / 00}$ & $\begin{array}{l}02 \\
\mathrm{ml} / 1\end{array}$ & $\begin{array}{c}\mathrm{F}-11 \\
\mathrm{pmol} / \mathrm{kg}\end{array}$ & $\begin{array}{c}\mathrm{F}-12 \\
\mathrm{pmol} / \mathrm{kg}\end{array}$ & $\stackrel{\theta}{\operatorname{Deg} C}$ & $\begin{array}{l}\mathrm{SIG}-0 \\
\mathrm{~kg} / \mathrm{m} 3\end{array}$ & $\underset{\mathrm{kg} / \mathrm{m} 3}{\mathrm{SIG}-1.5}$ & $\begin{array}{l}\mathrm{sig}-3 \\
\mathrm{~kg} / \mathrm{m} 3\end{array}$ & $\begin{array}{c}\mathrm{DE} \\
\mathrm{m}\end{array}$ \\
\hline $\begin{array}{r}1 \\
26 \\
54 \\
75 \\
101 \\
128 \\
156 \\
157 \\
203 \\
252 \\
301 \\
400 \\
505 \\
504 \\
752 \\
1000 \\
1250 \\
1497\end{array}$ & $\begin{array}{r}20.300 \\
19.261 \\
19.065 \\
18.954 \\
18.863 \\
18.046 \\
17.747 \\
17.718 \\
17.247 \\
16.858 \\
16.373 \\
15.030 \\
13.636 \\
13.650 \\
10.656 \\
8.808 \\
7.754 \\
6.167 \\
4.841 \\
4.113 \\
3.657 \\
3.284 \\
3.044 \\
2.883\end{array}$ & $\begin{array}{l}36.695 \\
36.659 \\
36.655 \\
36.652 \\
36.634 \\
36.474 \\
36.436 \\
36.434 \\
36.365 \\
36.310 \\
36.238 \\
36.043 \\
35.833 \\
35.832 \\
35.496 \\
35.431 \\
35.473 \\
35.302 \\
35.135 \\
35.054 \\
35.010 \\
34.979\end{array}$ & $\begin{array}{l}5.33 \\
5.38 \\
5.29 \\
5.27 \\
5.21 \\
4.97 \\
4.93 \\
4.95 \\
4.85 \\
4.82 \\
4.74 \\
4.74 \\
4.73 \\
4.70 \\
4.23 \\
4.07 \\
4.62 \\
5.16 \\
5.88 \\
5.93 \\
5.99 \\
5.87\end{array}$ & & & $\begin{array}{r}20.300 \\
19.256 \\
19.055 \\
18.941 \\
18.844 \\
18.024 \\
17.720 \\
17.691 \\
17.213 \\
16.816 \\
16.324 \\
14.969 \\
13.563 \\
13.577 \\
10.563 \\
8.695 \\
7.620 \\
6.022 \\
4.686 \\
3.943 \\
3.470 \\
3.079 \\
2.819 \\
2.635\end{array}$ & $\begin{array}{l}25.976 \\
26.225 \\
26.274 \\
26.301 \\
26.312 \\
26.397 \\
26.443 \\
26.448 \\
26.512 \\
26.565 \\
26.626 \\
26.784 \\
26.924 \\
26.920 \\
27.241 \\
27.505 \\
27.703 \\
27.789 \\
27.819 \\
27.835 \\
27.848 \\
27.861 \\
27.871 \\
27.876\end{array}$ & $\begin{array}{l}32.326 \\
32.599 \\
32.653 \\
32.683 \\
32.696 \\
32.802 \\
32.856 \\
32.863 \\
32.939 \\
33.002 \\
33.076 \\
33.272 \\
33.453 \\
33.449 \\
33.863 \\
34.188 \\
34.421 \\
34.566 \\
34.647 \\
34.693 \\
34.725 \\
34.754 \\
34.775 \\
34.787\end{array}$ & $\begin{array}{l}38.470 \\
38.766 \\
38.824 \\
38.856 \\
38.872 \\
38.998 \\
39.060 \\
39.067 \\
39.155 \\
39.229 \\
39.316 \\
39.548 \\
39.767 \\
39.762 \\
40.266 \\
40.649 \\
40.916 \\
41.117 \\
41.247 \\
41.322 \\
41.372 \\
41.416 \\
41.447 \\
41.467\end{array}$ & $\begin{array}{r}1 \\
25 \\
54 \\
74 \\
100 \\
127 \\
154 \\
155 \\
201 \\
250 \\
298 \\
396 \\
500 \\
500 \\
744 \\
989 \\
1236 \\
1480 \\
1726 \\
1973 \\
2216 \\
2460 \\
2707 \\
2954\end{array}$ \\
\hline
\end{tabular}




\begin{tabular}{|c|c|c|c|c|c|c|c|c|c|c|}
\hline$D A$ & $\begin{array}{l}R \\
5\end{array}$ & & & & & & & & $=$ & \\
\hline $\begin{array}{l}\text { PR } \\
\text { dbar }\end{array}$ & $\stackrel{T}{\operatorname{Deg} C}$ & $\stackrel{S}{0 / 00}$ & $\begin{array}{c}02 \\
\mathrm{ml} / \mathrm{l}\end{array}$ & $\stackrel{\ominus}{\operatorname{Deg} C}$ & $\begin{array}{l}\mathrm{SIG}-0 \\
\mathrm{~kg} / \mathrm{m3}\end{array}$ & $\begin{array}{l}S I G-1.5 \\
\mathrm{~kg} / \mathrm{m} 3\end{array}$ & $\begin{array}{l}\mathrm{SIG}-3 \\
\mathrm{~kg} / \mathrm{m} 3\end{array}$ & $\underset{m}{H G T H}$ & $\underset{c p h}{N}$ & $D E$ \\
\hline $\begin{array}{r}1 \\
25 \\
50 \\
75 \\
100 \\
150 \\
200 \\
250 \\
300 \\
350 \\
400 \\
450 \\
500 \\
600 \\
700 \\
800 \\
900 \\
1000 \\
1200 \\
1400\end{array}$ & $\begin{array}{r}19.965 \\
19.279 \\
18.960 \\
18.884 \\
18.852 \\
18.464 \\
17.489 \\
16.952 \\
16.434 \\
15.660 \\
14.885 \\
14.221 \\
13.600 \\
12.412 \\
11.400 \\
10.494 \\
9.651 \\
8.753 \\
7.941 \\
6.776 \\
5.651 \\
4.731 \\
4.257 \\
3.776 \\
3.463 \\
3.210 \\
3.050 \\
2.911 \\
2.900\end{array}$ & $\begin{array}{l}36 \\
36 \\
36 \\
36 \\
36 \\
36 \\
36 \\
36 \\
36 \\
36 \\
35 \\
35 \\
35 \\
35 \\
35 \\
35 \\
35 \\
35 \\
35 \\
35 \\
35 \\
35 \\
35 \\
34 \\
34 \\
35\end{array}$ & $\begin{array}{l}.18 \\
.89 \\
.92 \\
.93 \\
.60 \\
.64 \\
.64 \\
.69 \\
.75 \\
.78 \\
.68 \\
.58 \\
.43\end{array}$ & $\begin{array}{r}19.965 \\
19.275 \\
18.951 \\
18.870 \\
18.834 \\
18.437 \\
17.455 \\
16.910 \\
16.385 \\
15.604 \\
14.824 \\
14.155 \\
13.528 \\
12.330 \\
11.310 \\
10.395 \\
9.545 \\
8.641 \\
7.812 \\
6.635 \\
5.501 \\
4.573 \\
4.086 \\
3.592 \\
3.264 \\
2.996 \\
2.819 \\
2.662 \\
2.649\end{array}$ & $\begin{array}{l}26.138 \\
26.254 \\
26.335 \\
26.356 \\
26.363 \\
26.392 \\
26.500 \\
26.573 \\
26.642 \\
26.714 \\
26.796 \\
26.865 \\
26.927 \\
27.043 \\
27.156 \\
27.270 \\
27.379 \\
27.475 \\
27.669 \\
27.768 \\
27.811 \\
27.828 \\
27.841 \\
27.851 \\
27.860 \\
27.869 \\
27.877 \\
27.881 \\
27.880\end{array}$ & $\begin{array}{l}32.495 \\
32.628 \\
32.716 \\
32.739 \\
32.747 \\
32.786 \\
32.920 \\
33.007 \\
33.091 \\
33.185 \\
33.288 \\
33.377 \\
33.457 \\
33.610 \\
33.754 \\
33.897 \\
34.034 \\
34.161 \\
34.381 \\
34.523 \\
34.607 \\
34.661 \\
34.694 \\
34.724 \\
34.745 \\
34.765 \\
34.781 \\
34.791 \\
34.791\end{array}$ & $\begin{array}{l}38.644 \\
38.793 \\
38.889 \\
38.914 \\
38.923 \\
38.972 \\
39.130 \\
39.231 \\
39.328 \\
39.442 \\
39.567 \\
39.675 \\
39.772 \\
39.959 \\
40.134 \\
40.305 \\
40.468 \\
40.624 \\
40.870 \\
41.052 \\
41.177 \\
41.265 \\
41.316 \\
41.365 \\
41.400 \\
41.430 \\
41.452 \\
41.469 \\
41.470\end{array}$ & $\begin{array}{l}0.002 \\
0.046 \\
0.089 \\
0.132 \\
0.174 \\
0.259 \\
0.341 \\
0.419 \\
0.495 \\
0.569 \\
0.639 \\
0.706 \\
0.771 \\
0.893 \\
1.006 \\
1.110 \\
1.204 \\
1.289 \\
1.433 \\
1.553 \\
1.659 \\
1.757 \\
1.851 \\
1.944 \\
2.033 \\
2.121 \\
2.208 \\
2.295 \\
2.302\end{array}$ & $\begin{array}{r}-0.69 \\
4.90 \\
2.08 \\
1.33 \\
0.70 \\
2.05 \\
2.63 \\
2.23 \\
2.06 \\
2.34 \\
2.04 \\
2.22 \\
2.05 \\
2.02 \\
1.95 \\
2.00 \\
1.92 \\
1.98 \\
1.62 \\
1.37 \\
1.06 \\
0.82 \\
0.72 \\
0.75 \\
0.68 \\
0.57 \\
0.54 \\
0.46 \\
-0.25\end{array}$ & $\begin{array}{r}1 \\
25 \\
50 \\
74 \\
99 \\
149 \\
199 \\
248 \\
298 \\
347 \\
397 \\
446 \\
496 \\
595 \\
694 \\
793 \\
892 \\
991 \\
1188 \\
1386 \\
1583 \\
1780 \\
1977 \\
2173 \\
2370 \\
2566 \\
2762 \\
2958 \\
2973\end{array}$ \\
\hline
\end{tabular}




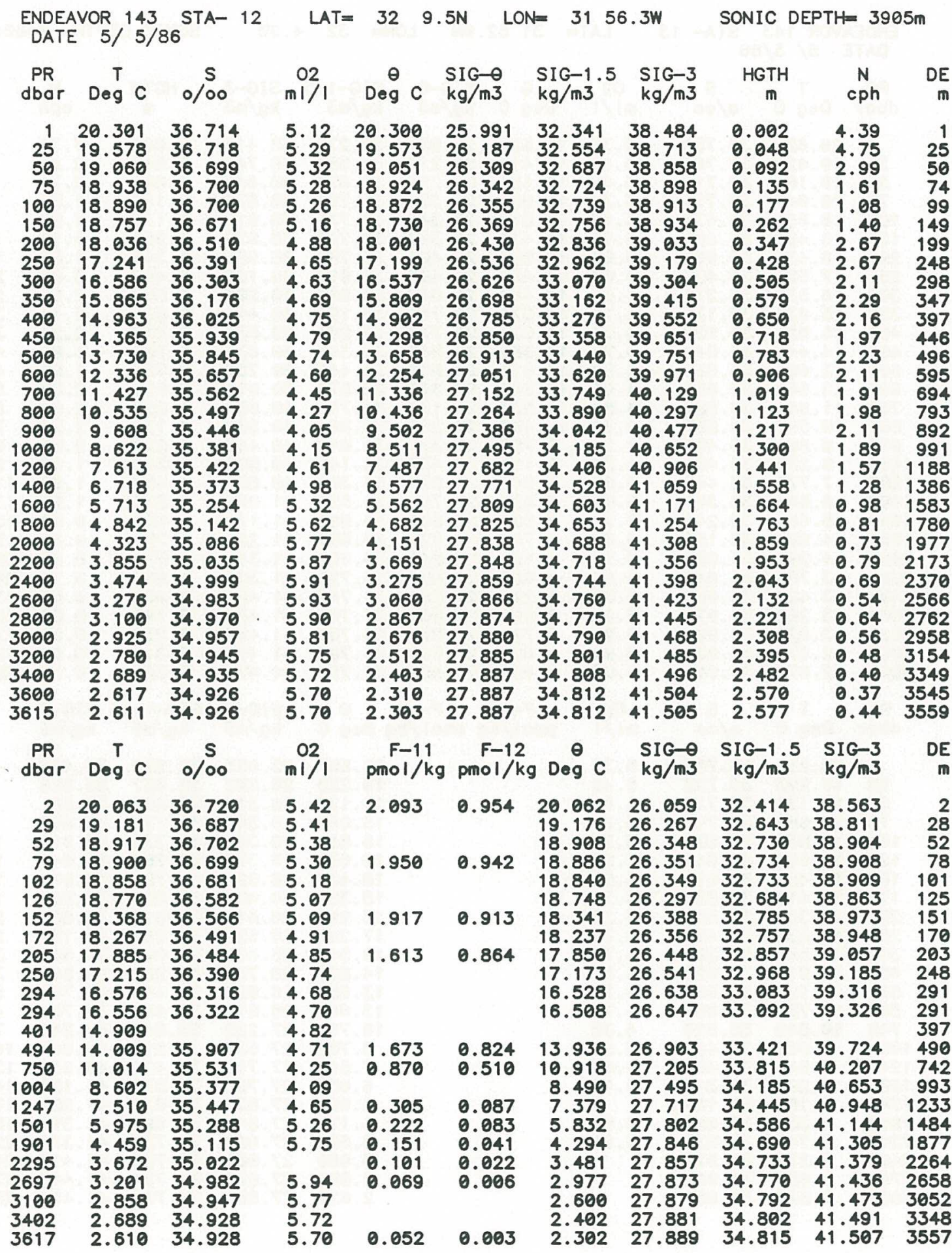




\begin{tabular}{|c|c|c|c|c|c|c|c|c|c|c|}
\hline \multicolumn{3}{|c|}{$\begin{array}{l}\text { ENDEAVOR } 143 \\
\text { DATE } 5 / 5 / 86\end{array}$} & \multicolumn{3}{|c|}{ LAT $=3152.0 \mathrm{~N}$} & \multicolumn{2}{|c|}{$\mathrm{ON}=$} & SONIC & \multicolumn{2}{|c|}{$=4400 m$} \\
\hline $\begin{array}{l}\text { PR } \\
\text { dbar }\end{array}$ & $\stackrel{T}{\operatorname{Deg} C}$ & $\stackrel{S}{0 / 00}$ & $\begin{array}{l}02 \\
\mathrm{ml} / \mathrm{l}\end{array}$ & $\stackrel{\theta}{\operatorname{Deg} C}$ & $\begin{array}{l}I G-\theta \\
g / m 3\end{array}$ & $\begin{array}{l}\mathrm{SIG}-1.5 \\
\mathrm{~kg} / \mathrm{m} 3\end{array}$ & $\begin{array}{l}\mathrm{SIG}-3 \\
\mathrm{~kg} / \mathrm{m3}\end{array}$ & $\begin{array}{c}\text { HGTH } \\
\mathbf{m}\end{array}$ & $\underset{c p h}{N}$ & DE \\
\hline $\begin{array}{r}1 \\
25 \\
50 \\
75 \\
100 \\
150 \\
200 \\
250 \\
300 \\
350 \\
400 \\
450 \\
500 \\
600 \\
700 \\
800 \\
900 \\
1000 \\
1200 \\
1400 \\
1600 \\
1800 \\
2000 \\
2200 \\
2400 \\
2600 \\
2800 \\
3000 \\
3005\end{array}$ & $\begin{array}{r}20.626 \\
19.422 \\
19.164 \\
19.041 \\
18.835 \\
18.457 \\
18.433 \\
17.525 \\
16.536 \\
15.632 \\
15.053 \\
14.449 \\
13.691 \\
12.662 \\
11.649 \\
10.597 \\
9.861 \\
9.235 \\
7.770 \\
6.664 \\
5.683 \\
4.908 \\
4.299 \\
3.784 \\
3.422 \\
3.208 \\
3.023 \\
2.876 \\
2.874\end{array}$ & $\begin{array}{l}36.758 \\
36.703 \\
36.718 \\
36.714 \\
36.674 \\
36.609 \\
36.606 \\
36.427 \\
36.275 \\
36.135 \\
36.039 \\
35.949 \\
35.838 \\
35.690 \\
35.582 \\
35.505 \\
35.482 \\
35.482 \\
35.443 \\
35.361 \\
35.246 \\
35.155 \\
35.087 \\
35.030 \\
34.996 \\
34.979 \\
34.964 \\
34.952 \\
34.951\end{array}$ & $\begin{array}{l}5.42 \\
5.34 \\
5.18 \\
5.15 \\
5.09 \\
4.68 \\
4.61 \\
4.70 \\
4.74 \\
4.79 \\
4.82 \\
4.65 \\
4.56 \\
4.36 \\
4.24 \\
4.22 \\
4.52 \\
5.02 \\
5.38 \\
5.65 \\
5.79 \\
5.89 \\
5.93 \\
5.92 \\
5.91\end{array}$ & $\begin{array}{c}20.626 \\
19.417 \\
19.155 \\
19.028 \\
18.817 \\
18.430 \\
18.397 \\
17.482 \\
16.487 \\
15.576 \\
14.992 \\
14.381 \\
13.618 \\
12.580 \\
11.557 \\
10.498 \\
9.753 \\
9.119 \\
7.642 \\
6.524 \\
5.533 \\
4.747 \\
4.127 \\
3.600 \\
3.224 \\
2.994 \\
2.793 \\
2.628 \\
2.625\end{array}$ & $\begin{array}{l}25.937 \\
26.217 \\
26.296 \\
26.326 \\
26.349 \\
26.398 \\
26.404 \\
26.495 \\
26.616 \\
26.719 \\
26.776 \\
26.840 \\
26.916 \\
27.013 \\
27.126 \\
27.260 \\
27.371 \\
27.477 \\
27.676 \\
27.769 \\
27.806 \\
27.827 \\
27.842 \\
27.851 \\
27.861 \\
27.869 \\
27.875 \\
27.880 \\
27.880\end{array}$ & $\begin{array}{l}32.279 \\
32.587 \\
32.672 \\
32.705 \\
32.734 \\
32.792 \\
32.799 \\
32.914 \\
33.062 \\
33.190 \\
33.264 \\
33.345 \\
33.443 \\
33.572 \\
33.717 \\
33.884 \\
34.019 \\
34.145 \\
34.394 \\
34.527 \\
34.602 \\
34.653 \\
34.692 \\
34.723 \\
34.748 \\
34.766 \\
34.780 \\
34.792 \\
34.792\end{array}$ & $\begin{array}{l}38.749 \\
38.840 \\
38.876 \\
38.910 \\
38.978 \\
38.986 \\
39.123 \\
39.297 \\
39.449 \\
39.538 \\
39.636 \\
39.756 \\
39.914 \\
40.089 \\
40.288 \\
40.446 \\
40.592 \\
40.889 \\
41.060 \\
41.170 \\
41.251 \\
41.314 \\
41.364 \\
41.404 \\
41.431 \\
41.453 \\
41.471 \\
41.472\end{array}$ & $\begin{array}{l}0.002 \\
0.046 \\
0.091 \\
0.134 \\
0.176 \\
0.261 \\
0.345 \\
0.429 \\
0.507 \\
0.581 \\
0.652 \\
0.720 \\
0.786 \\
0.911 \\
1.026 \\
1.132 \\
1.227 \\
1.313 \\
1.457 \\
1.575 \\
1.681 \\
1.781 \\
1.876 \\
1.968 \\
2.058 \\
2.145 \\
2.232 \\
2.319 \\
2.321\end{array}$ & $\begin{array}{l}7.71 \\
2.81 \\
2.74 \\
1.80 \\
1.80 \\
1.17 \\
1.00 \\
3.06 \\
2.61 \\
2.14 \\
2.24 \\
1.87 \\
1.92 \\
2.07 \\
2.04 \\
2.22 \\
2.05 \\
1.97 \\
1.69 \\
1.28 \\
0.99 \\
0.86 \\
0.85 \\
0.74 \\
0.65 \\
0.59 \\
0.52 \\
0.58 \\
0.35\end{array}$ & $\begin{array}{r}1 \\
25 \\
50 \\
74 \\
99 \\
149 \\
199 \\
248 \\
298 \\
347 \\
397 \\
446 \\
496 \\
595 \\
694 \\
793 \\
892 \\
991 \\
1188 \\
1386 \\
1583 \\
1780 \\
1977 \\
2173 \\
2370 \\
2566 \\
2762 \\
2958 \\
2963\end{array}$ \\
\hline $\begin{array}{l}\text { PR } \\
\text { dbar }\end{array}$ & $\stackrel{T}{D e g} C$ & $\stackrel{5}{0}$ & 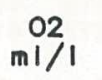 & $\begin{array}{c}\mathrm{F}-11 \\
\mathrm{pmol} / \mathrm{kg}\end{array}$ & $\begin{array}{c}\mathrm{F}-12 \\
\mathrm{pmol} / \mathrm{kg}\end{array}$ & $\stackrel{\theta}{\operatorname{Deg}} C$ & $\underset{\mathrm{kg} / \mathrm{m} 3}{S I G-\theta}$ & $\underset{\mathrm{kg} / \mathrm{m} 3}{S I G-1}$ & $\begin{array}{l}\mathrm{SIG}-3 \\
\mathrm{~kg} / \mathrm{m3}\end{array}$ & DE \\
\hline $\begin{array}{r}2 \\
28 \\
55 \\
78 \\
103 \\
129 \\
155 \\
177 \\
202 \\
252 \\
302 \\
402 \\
505 \\
503 \\
749 \\
1051 \\
1247 \\
1501 \\
1746 \\
1992 \\
2247 \\
2497 \\
0718\end{array}$ & $\begin{array}{r}20.558 \\
19.225 \\
19.136 \\
18.982 \\
18.838 \\
18.662 \\
18.481 \\
18.419 \\
18.331 \\
17.330 \\
16.565 \\
14.917 \\
13.725 \\
13.733 \\
10.849 \\
8.908 \\
7.648 \\
6.201 \\
5.180 \\
4.352 \\
3.741 \\
3.274 \\
3.055 \\
2.872\end{array}$ & $\begin{array}{l}36 . \\
36 \\
36 \\
36 . \\
36 . \\
36 . \\
36 . \\
36 . \\
36 . \\
36 \\
36 \\
36 \\
35 . \\
35 . \\
35 . \\
35 \\
35 \\
35\end{array}$ & $\begin{array}{l}5.42 \\
5.41 \\
5.39 \\
5.33 \\
5.28 \\
5.23 \\
5.22 \\
5.26 \\
4.76 \\
4.72 \\
4.81 \\
4.73 \\
4.73\end{array}$ & . & & $\begin{array}{r}19.220 \\
19.126 \\
18.968 \\
18.819 \\
18.639 \\
18.454 \\
18.387 \\
18.296 \\
17.288 \\
16.515 \\
14.855 \\
13.652 \\
13.660 \\
10.754 \\
8.788 \\
7.516 \\
6.055 \\
5.021 \\
4.179 \\
3.553 \\
3.069 \\
2.829 \\
2.623\end{array}$ & $\begin{array}{l}25.955 \\
26.282 \\
26.316 \\
26.345 \\
26.351 \\
26.373 \\
26.398 \\
26.408 \\
26.414 \\
26.527 \\
26.623 \\
26.794 \\
26.923 \\
26.919 \\
27.229 \\
27.531 \\
27.712 \\
27.795 \\
27.821 \\
27.844 \\
27.859 \\
27.861 \\
27.871 \\
27.883\end{array}$ & $\begin{array}{l}32.657 \\
32.693 \\
32.726 \\
32.735 \\
32.762 \\
32.791 \\
32.804 \\
32.812 \\
32.951 \\
33.069 \\
33.285 \\
33.449 \\
33.445 \\
33.844 \\
34.210 \\
34.434 \\
34.571 \\
34.637 \\
34.692 \\
34.733 \\
34.755 \\
34.774 \\
34.794\end{array}$ & $\begin{array}{l}38.911 \\
38.942 \\
38.977 \\
38.990 \\
39.001 \\
39.166 \\
39.302 \\
39.564 \\
39.761 \\
39.756 \\
40.241 \\
40.667 \\
40.932 \\
41.120 \\
41.224 \\
41.311 \\
41.376 \\
41.417 \\
41.446 \\
41.474\end{array}$ & $\begin{array}{r}2 \\
27 \\
54 \\
77 \\
102 \\
128 \\
154 \\
176 \\
200 \\
250 \\
300 \\
399 \\
500 \\
498 \\
741 \\
1039 \\
1233 \\
1483 \\
1725 \\
1966 \\
2216 \\
2462 \\
2708 \\
2962\end{array}$ \\
\hline
\end{tabular}




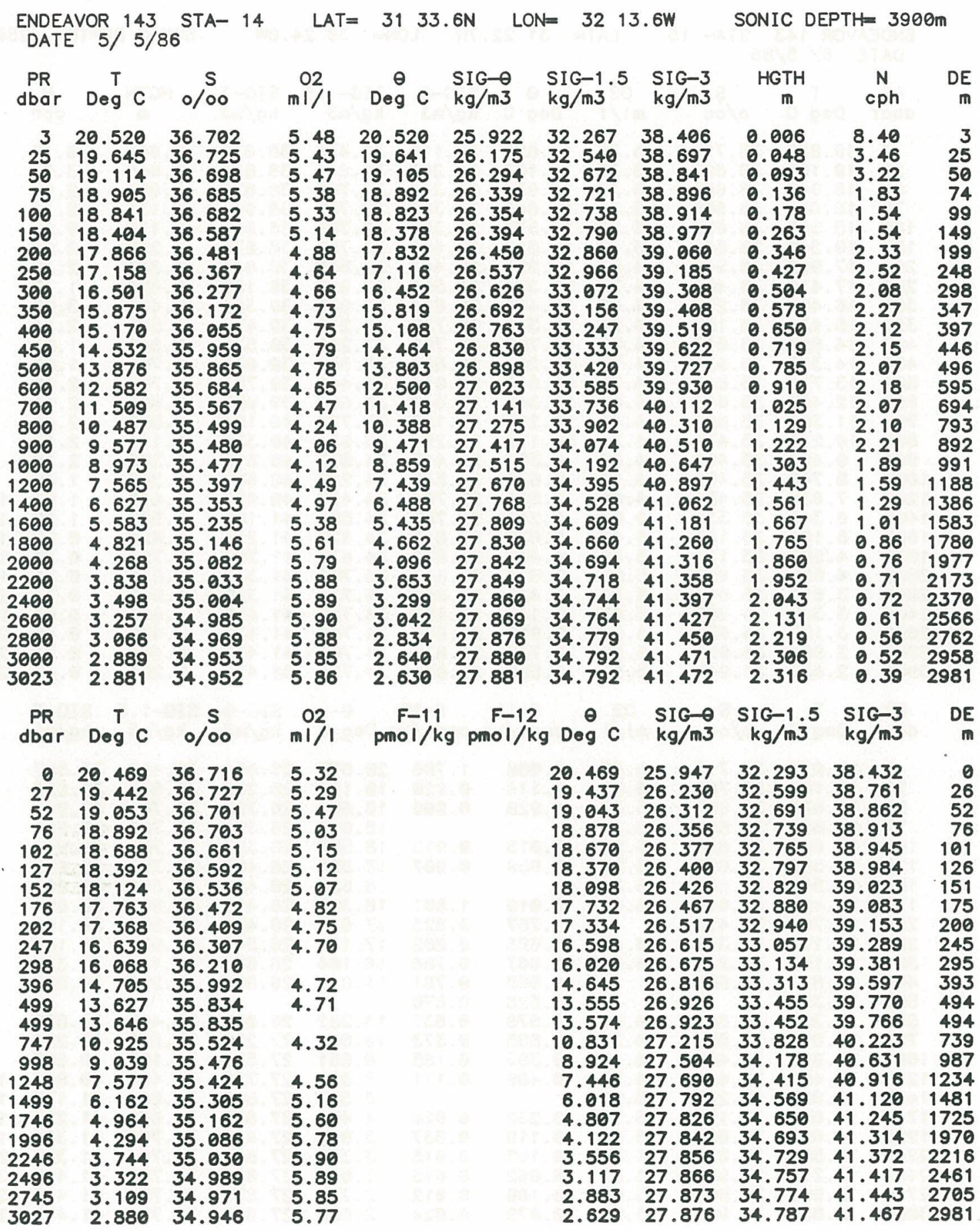




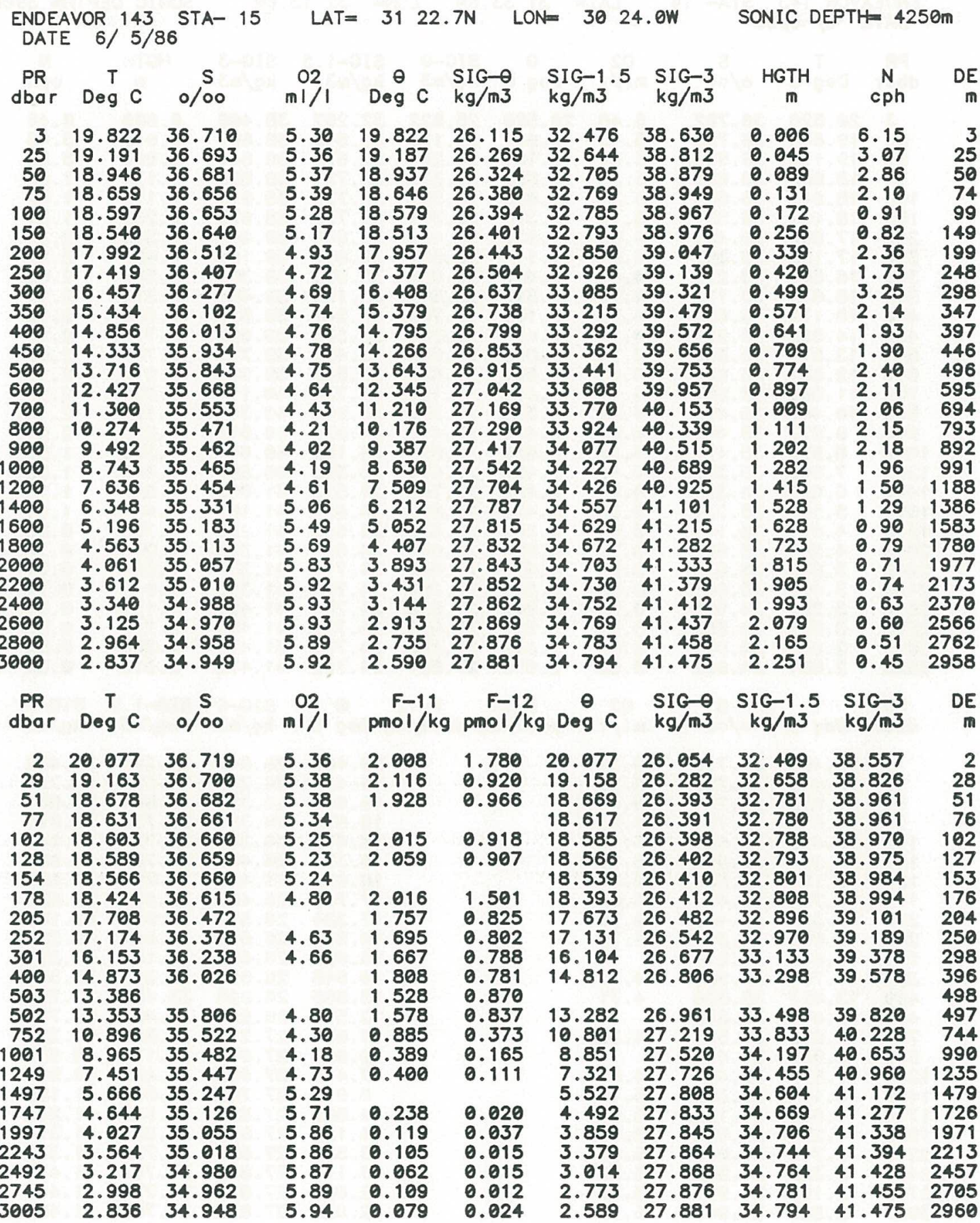


ENDEAVOR 143 STA- 16
DATE $7 / 5 / 86$

\begin{tabular}{|c|c|c|c|c|c|c|c|c|c|c|}
\hline $\begin{array}{l}\text { PR } \\
\text { dbar }\end{array}$ & $\stackrel{T}{T} C$ & $\frac{S}{0 / 00}$ & $\begin{array}{c}02 \\
\mathrm{ml} / \mathrm{l}\end{array}$ & $\stackrel{\theta}{\operatorname{Deg} C}$ & $\begin{array}{l}\mathrm{SIG}-0 \\
\mathrm{~kg} / \mathrm{m} 3\end{array}$ & $\begin{array}{l}\mathrm{SIG}-1.5 \\
\mathrm{~kg} / \mathrm{m} 3\end{array}$ & $\begin{array}{l}\mathrm{SIG}-3 \\
\mathrm{~kg} / \mathrm{m} 3\end{array}$ & $\underset{m}{H G T H}$ & $\underset{c p h}{N}$ & $\begin{array}{r}\mathrm{DE} \\
\mathbf{m}\end{array}$ \\
\hline $\begin{array}{r}1 \\
25 \\
50 \\
75 \\
100 \\
150 \\
200 \\
250 \\
300\end{array}$ & $\begin{array}{l}19.994 \\
19.406 \\
19.024 \\
18.919 \\
18.777 \\
17.890 \\
17.394 \\
16.683 \\
15.950\end{array}$ & $\begin{array}{l}36.701 \\
36.702 \\
36.686 \\
36.689 \\
36.669 \\
36.475 \\
36.408 \\
36.303 \\
36.185\end{array}$ & $\begin{array}{l}5.37 \\
5.18 \\
5.32 \\
5.29 \\
5.27 \\
4.96 \\
4.70 \\
4.68 \\
4.74\end{array}$ & $\begin{array}{l}19.994 \\
19.401 \\
19.015 \\
18.906 \\
18.759 \\
17.864 \\
17.360 \\
16.642 \\
15.902\end{array}$ & $\begin{array}{l}26.063 \\
26.220 \\
26.308 \\
26.338 \\
26.360 \\
26.437 \\
26.509 \\
26.601 \\
26.683\end{array}$ & $\begin{array}{l}32.420 \\
32.591 \\
32.687 \\
32.720 \\
32.746 \\
32.847 \\
32.932 \\
33.043 \\
33.145\end{array}$ & $\begin{array}{l}38.570 \\
38.754 \\
38.859 \\
38.894 \\
38.924 \\
39.047 \\
39.144 \\
39.273 \\
39.395\end{array}$ & $\begin{array}{l}0.002 \\
0.048 \\
0.092 \\
0.135 \\
0.177 \\
0.261 \\
0.342 \\
0.419 \\
0.494\end{array}$ & $\begin{array}{r}-2.11 \\
5.65 \\
2.54 \\
1.44 \\
2.02 \\
2.18 \\
2.51 \\
2.42 \\
2.45\end{array}$ & $\begin{array}{r}1 \\
25 \\
50 \\
74 \\
99 \\
149 \\
199 \\
248 \\
298\end{array}$ \\
\hline $\begin{array}{l}\text { PR } \\
\text { dbar }\end{array}$ & $\stackrel{T}{\text { Deg } C}$ & $\begin{array}{c}S \\
0 / 00\end{array}$ & $\begin{array}{c}02 \\
\mathrm{ml} / \mathrm{l}\end{array}$ & $\begin{array}{c}\mathrm{F}-11 \\
\mathrm{pmol} / \mathrm{kg}\end{array}$ & $\begin{array}{c}\mathrm{F}-12 \\
\mathrm{pmol} / \mathrm{kg}\end{array}$ & $\stackrel{\theta}{\operatorname{Deg}} C$ & $\begin{array}{l}\mathrm{SIG}-0 \\
\mathrm{~kg} / \mathrm{m} 3\end{array}$ & $\underset{\mathrm{kg} / \mathrm{m} 3}{\mathrm{SIG}-1} .5$ & $\begin{array}{l}\mathrm{SIG}-3 \\
\mathrm{~kg} / \mathrm{m} 3\end{array}$ & E \\
\hline $\begin{array}{r}78 \\
173 \\
211\end{array}$ & $\begin{array}{l}18.906 \\
17.719 \\
17.157\end{array}$ & $\begin{array}{l}36.698 \\
36.454 \\
36.404\end{array}$ & $\begin{array}{l}5.35 \\
4.92 \\
4.74\end{array}$ & & & $\begin{array}{l}18.892 \\
17.689 \\
17.122\end{array}$ & $\begin{array}{l}26.349 \\
26.464 \\
26.564\end{array}$ & $\begin{array}{l}32.731 \\
32.878 \\
32.993\end{array}$ & $\begin{array}{l}38.906 \\
39.083 \\
39.211\end{array}$ & $\begin{array}{r}77 \\
171 \\
209\end{array}$ \\
\hline
\end{tabular}

\begin{tabular}{|c|c|c|c|c|c|c|c|c|c|c|}
\hline \multicolumn{3}{|c|}{$\begin{array}{l}\text { ENDEAVOR } 143 \text { STA- } 17 \\
\text { DATE } 7 / 5 / 86\end{array}$} & LAT $=$ & \multicolumn{2}{|c|}{$3139.8 \mathrm{~N}$} & \multicolumn{2}{|c|}{ LONE } & \multicolumn{2}{|c|}{ SONIC DEPTH } & \multirow{2}{*}{$\begin{array}{l}\text { Om } \\
\text { DE } \\
\text { m }\end{array}$} \\
\hline $\begin{array}{l}\text { PR } \\
\text { dbar }\end{array}$ & $\stackrel{T}{\text { Deg } C}$ & $\stackrel{5}{0 / 00}$ & $\begin{array}{l}02 \\
\mathrm{ml} / \mathrm{l}\end{array}$ & $\stackrel{\theta}{D e g} \mathrm{C}$ & $\begin{array}{l}\text { SIG-O } \\
\mathrm{kg} / \mathrm{m3}\end{array}$ & $\begin{array}{l}\mathrm{SIG}-1.5 \\
\mathrm{~kg} / \mathrm{m} 3\end{array}$ & $\begin{array}{l}\mathrm{SIG}-3 \\
\mathrm{~kg} / \mathrm{m} 3\end{array}$ & $\underset{m}{\mathrm{HGTH}}$ & $\underset{c p h}{N}$ & \\
\hline $\begin{array}{r}5 \\
25 \\
50 \\
75 \\
100 \\
150 \\
200 \\
250 \\
300\end{array}$ & $\begin{array}{l}19.930 \\
19.232 \\
18.965 \\
18.685 \\
18.685 \\
18.102 \\
17.384 \\
16.936 \\
16.263\end{array}$ & $\begin{array}{l}36.6 \\
36.6 \\
36.6 \\
36.6 \\
36.6 \\
36.5 \\
36.4 \\
36.3 \\
36.2\end{array}$ & $\begin{array}{l}5.32 \\
5.50 \\
5.50 \\
5.40 \\
5.33 \\
5.04 \\
4.80 \\
4.75\end{array}$ & $\begin{array}{l}19.929 \\
19.228 \\
18.956 \\
18.672 \\
18.667 \\
18.076 \\
17.350 \\
16.895 \\
16.214\end{array}$ & $\begin{array}{l}26.049 \\
26.246 \\
26.303 \\
26.344 \\
26.372 \\
26.432 \\
26.513 \\
26.572 \\
26.649\end{array}$ & $\begin{array}{l}32.408 \\
32.621 \\
32.684 \\
32.732 \\
32.760 \\
32.835 \\
32.936 \\
33.007 \\
33.103\end{array}$ & $\begin{array}{l}559 \\
788 \\
858 \\
912 \\
940 \\
030 \\
148 \\
231 \\
344\end{array}$ & & & $\begin{array}{r}5 \\
25 \\
50 \\
74 \\
99 \\
149 \\
199 \\
248 \\
298\end{array}$ \\
\hline $\begin{array}{l}\text { PR } \\
\text { dbar }\end{array}$ & $\stackrel{T}{T} C$ & $\begin{array}{l}S \\
0 / 00\end{array}$ & $\begin{array}{l}02 \\
\mathrm{ml} / \mathrm{I}\end{array}$ & $\begin{array}{c}\mathrm{F}-11 \\
\mathrm{pmol} / \mathrm{kg}\end{array}$ & $\begin{array}{c}\mathrm{F}-12 \\
\mathrm{pmol} / \mathrm{kg}\end{array}$ & Deg & $\begin{array}{l}\text { SIG-O } \\
\mathrm{kg} / \mathrm{m} 3\end{array}$ & $\underset{\mathrm{kg} / \mathrm{m} 3}{\mathrm{SIG}-1.5}$ & $\begin{array}{l}\mathrm{SIG} \\
\mathrm{kg} / \mathrm{n}\end{array}$ & DE \\
\hline $\begin{array}{r}87 \\
175 \\
220\end{array}$ & $\begin{array}{l}18.667 \\
17.676 \\
17.247\end{array}$ & $\begin{array}{l}36.619 \\
36.467 \\
36.389\end{array}$ & $\begin{array}{l}5.32 \\
4.94 \\
4.76\end{array}$ & & & $\begin{array}{l}17.646 \\
17.210\end{array}$ & $\begin{array}{l}26.350 \\
26.485 \\
26.531\end{array}$ & $\begin{array}{l}32.739 \\
32.900 \\
32.958\end{array}$ & $\begin{array}{l}39.105 \\
39.174\end{array}$ & $\begin{array}{r}86 \\
174 \\
218\end{array}$ \\
\hline
\end{tabular}

$L A T=3149.8 \mathrm{~N} \quad$ LON= $3015.1 \mathrm{~W}$

$02 \theta \theta$ SIG- $\theta$ SIG-1.5 SIG-3 $\mathrm{ml} / \mathrm{l}$ Deg C $\mathrm{kg} / \mathrm{m3} \quad \mathrm{kg} / \mathrm{m} 3 \quad \mathrm{~kg} / \mathrm{m3}$

$5.45 \quad 19.896$

$5.52 \quad 19.175$

5.4518 .906

$5.42 \quad 18.874$

$\begin{array}{ll}5.37 & 18.673 \\ 5.20 & 18.155\end{array}$

$4.82 \quad 17.375$

$\begin{array}{ll}4.80 & 16.818 \\ 4.83 & 16.298\end{array}$

26.099

$26.264 \quad 32.640$

$26.333 \quad 32.715$

26.33932 .722

$26.363 \quad 32.752$

$26.405 \quad 32.807$

$26.505 \quad 32.927$

$26.571 \quad 33.008$

$26.646 \quad 33.097$

F-11

$F-12$

$\mathrm{ml} / 1$

5.27

5.03
4.73
38.610

38.808

38.889

38.897

38.931

39.000

39.140

39.234

39.337
SONIC DEPTH $\quad$ Om

$\begin{array}{crr}\begin{array}{c}\text { HGTH } \\ \mathrm{m}\end{array} & \begin{array}{c}\mathrm{N} \\ \text { cph }\end{array} & \begin{array}{r}\text { DE } \\ \mathrm{m}\end{array} \\ 0.006 & 4.57 & 3 \\ 0.046 & 3.59 & 25 \\ 0.089 & 1.62 & 50 \\ 0.132 & 0.91 & 74 \\ 0.174 & 1.98 & 99 \\ 0.258 & 2.33 & 149 \\ 0.340 & 2.24 & 199 \\ 0.418 & 2.43 & 248 \\ 0.494 & 1.85 & 298\end{array}$

(1)

m

122 176 211 


\begin{tabular}{|c|c|c|c|c|c|c|c|c|c|c|}
\hline \multicolumn{3}{|c|}{$\begin{array}{l}\text { ENDEAVOR } 143 \text { STA- } 19 \\
\text { DATE } 7 / 5 / 86\end{array}$} & LAT $=$ & \multicolumn{2}{|c|}{$=3159.9 \mathrm{~N}$} & \multicolumn{2}{|c|}{$O N=3014.8 \mathrm{~W}$} & \multicolumn{2}{|c|}{ SONIC DEPTH= } & \multirow{2}{*}{$\begin{array}{l}\text { Om } \\
\text { DE } \\
\mathrm{m}\end{array}$} \\
\hline $\begin{array}{l}\text { PR } \\
\text { dbar }\end{array}$ & $\stackrel{T}{\operatorname{Deg}} \mathrm{C}$ & $\stackrel{5}{0 / 00}$ & $\begin{array}{l}02 \\
\mathrm{ml} / \mathrm{l}\end{array}$ & $\stackrel{\theta}{\operatorname{Deg} C}$ & $\begin{array}{l}\mathrm{SIG}-\mathrm{O} \\
\mathrm{kg} / \mathrm{m} 3\end{array}$ & $\begin{array}{l}\mathrm{SIG}-1.5 \\
\mathrm{~kg} / \mathrm{m3}\end{array}$ & $\begin{array}{l}\mathrm{SIG}-3 \\
\mathrm{~kg} / \mathrm{m3}\end{array}$ & $\underset{m}{\mathrm{HGTH}}$ & $\underset{c p h}{N}$ & \\
\hline $\begin{array}{r}1 \\
25 \\
50 \\
75 \\
100 \\
150 \\
200 \\
250 \\
300\end{array}$ & $\begin{array}{l}19.900 \\
19.404 \\
19.003 \\
18.930 \\
18.859 \\
18.079 \\
17.336 \\
16.649 \\
16.084\end{array}$ & $\begin{array}{l}36.694 \\
36.687 \\
36.664 \\
36.664 \\
36.673 \\
36.514 \\
36.389 \\
36.288 \\
36.213\end{array}$ & $\begin{array}{l}5.14 \\
5.11 \\
5.14 \\
5.20 \\
5.13 \\
4.72 \\
4.45 \\
4.47 \\
4.48\end{array}$ & $\begin{array}{l}19.899 \\
19.400 \\
18.994 \\
18.917 \\
18.841 \\
18.053 \\
17.302 \\
16.607 \\
16.036\end{array}$ & $\begin{array}{l}26.083 \\
26.209 \\
26.297 \\
26.316 \\
26.342 \\
26.420 \\
26.509 \\
26.598 \\
26.673\end{array}$ & $\begin{array}{l}32.442 \\
32.580 \\
32.677 \\
32.699 \\
32.726 \\
32.825 \\
32.933 \\
33.041 \\
33.132\end{array}$ & $\begin{array}{l}38.594 \\
38.743 \\
38.850 \\
38.873 \\
38.902 \\
39.020 \\
39.147 \\
39.272 \\
39.378\end{array}$ & $\begin{array}{l}0.002 \\
0.047 \\
0.091 \\
0.134 \\
0.177 \\
0.261 \\
0.342 \\
0.420 \\
0.494\end{array}$ & $\begin{array}{l}3.40 \\
4.90 \\
2.11 \\
1.46 \\
2.44 \\
1.54 \\
2.53 \\
2.09 \\
2.36\end{array}$ & $\begin{array}{r}1 \\
25 \\
50 \\
74 \\
99 \\
149 \\
199 \\
248 \\
298\end{array}$ \\
\hline $\begin{array}{l}P R \\
\text { dbar }\end{array}$ & $\stackrel{T}{D e g} C$ & $\stackrel{5}{0 / 00}$ & $\begin{array}{l}02 \\
\mathrm{ml} / \mathrm{l}\end{array}$ & $\begin{array}{c}\mathrm{F}-11 \\
\mathrm{pmol} / \mathrm{kg}\end{array}$ & $\begin{array}{c}\mathrm{F}-12 \\
\mathrm{pmol} / \mathrm{kg}\end{array}$ & $\stackrel{\theta}{\operatorname{Deg} C}$ & $\begin{array}{l}\mathrm{SIG}-\mathrm{O} \\
\mathrm{kg} / \mathrm{m} 3\end{array}$ & $\underset{\mathrm{kg} / \mathrm{m} 3}{\mathrm{SIG}-1.5}$ & $\begin{array}{l}\mathrm{SIG}-3 \\
\mathrm{~kg} / \mathrm{m} 3\end{array}$ & $\begin{array}{c}\mathrm{DE} \\
\mathbf{m}\end{array}$ \\
\hline $\begin{array}{r}91 \\
150 \\
198\end{array}$ & $\begin{array}{l}18.861 \\
18.060 \\
17.279\end{array}$ & $\begin{array}{l}36.683 \\
36.527 \\
36.394\end{array}$ & $\begin{array}{l}5.29 \\
4.98 \\
4.76\end{array}$ & & & $\begin{array}{l}18.845 \\
18.034 \\
17.245\end{array}$ & $\begin{array}{l}26.349 \\
26.435 \\
26.527\end{array}$ & $\begin{array}{l}32.733 \\
32.840 \\
32.952\end{array}$ & $\begin{array}{l}38.909 \\
39.035 \\
39.167\end{array}$ & $\begin{array}{r}90 \\
148 \\
196\end{array}$ \\
\hline \multicolumn{2}{|c|}{$\begin{array}{l}\text { ENDEAVOR } 143 \\
\text { DATE } 7 / 5 / 86\end{array}$} & ${ }_{66}^{S T A-20}$ & LAT $=$ & 329.8 & LON & \multicolumn{2}{|c|}{ LON $=3015.1 \mathrm{~W}$} & \multicolumn{2}{|c|}{ SONIC DEPTH= } & $\theta m$ \\
\hline $\begin{array}{l}\text { PR } \\
\text { dbar }\end{array}$ & $\stackrel{T}{D} C$ & $\stackrel{5}{0 / 00}$ & $\begin{array}{l}02 \\
\mathrm{ml} / \mathrm{l}\end{array}$ & $\stackrel{\theta}{\operatorname{Deg}} \subset \vec{k}$ & $\begin{array}{l}\mathrm{SIG}-\mathrm{O} \\
\mathrm{kg} / \mathrm{m3}\end{array}$ & $\begin{array}{l}\mathrm{SIG}-1.5 \\
\mathrm{~kg} / \mathrm{m3}\end{array}$ & $\begin{array}{l}\mathrm{SIG}-3 \\
\mathrm{~kg} / \mathrm{m3}\end{array}$ & $\underset{m}{\text { HGTH }}$ & $\underset{\text { cph }}{N}$ & $\underset{m}{D E}$ \\
\hline $\begin{array}{r}1 \\
25 \\
50 \\
75 \\
100 \\
150 \\
200 \\
250 \\
299\end{array}$ & $\begin{array}{l}20.536 \\
19.995 \\
18.982 \\
18.885 \\
18.734 \\
18.072 \\
17.412 \\
16.518 \\
15.751\end{array}$ & $\begin{array}{l}36.707 \\
36.704 \\
36.646 \\
36.661 \\
36.647 \\
36.517 \\
36.412 \\
36.282 \\
36.145\end{array}$ & $\begin{array}{l}5.08 \\
5.11 \\
5.31 \\
5.15 \\
5.02 \\
4.81 \\
4.60 \\
4.42 \\
4.43\end{array}$ & $\begin{array}{l}20.536 \\
19.990 \\
18.973 \\
18.872 \\
18.716 \\
18.046 \\
17.378 \\
16.477 \\
15.703\end{array}$ & $\begin{array}{l}25.922 \\
26.066 \\
26.288 \\
26.326 \\
26.355 \\
26.424 \\
26.508 \\
26.624 \\
26.698\end{array}$ & $\begin{array}{l}32.267 \\
32.423 \\
32.669 \\
32.709 \\
32.742 \\
32.829 \\
32.930 \\
33.070 \\
33.165\end{array}$ & $\begin{array}{l}38.405 \\
38.573 \\
38.842 \\
38.884 \\
38.921 \\
39.024 \\
39.142 \\
39.305 \\
39.421\end{array}$ & $\begin{array}{l}0.002 \\
0.051 \\
0.097 \\
0.140 \\
0.182 \\
0.266 \\
0.347 \\
0.424 \\
0.495\end{array}$ & $\begin{array}{l}1.62 \\
6.88 \\
3.70 \\
2.16 \\
1.62 \\
1.81 \\
2.83 \\
2.52 \\
1.71\end{array}$ & $\begin{array}{r}1 \\
25 \\
50 \\
74 \\
99 \\
149 \\
199 \\
248 \\
297\end{array}$ \\
\hline $\begin{array}{l}P R \\
\text { dbar }\end{array}$ & $\stackrel{T}{D e g} C$ & $\stackrel{s}{0 / 00}$ & $\begin{array}{l}02 \\
\mathrm{~m} I / 1\end{array}$ & $\begin{array}{c}\mathrm{F}-11 \\
\mathrm{pmol} / \mathrm{kg}\end{array}$ & $\begin{array}{c}\mathrm{F}-12 \\
\mathrm{pmol} / \mathrm{kg}\end{array}$ & $\stackrel{\ominus}{\operatorname{Deg} C}$ & $\begin{array}{l}\mathrm{SIG}-\Theta \\
\mathrm{kg} / \mathrm{m3}\end{array}$ & $\underset{\mathrm{kg} / \mathrm{m} 3}{\mathrm{SIG}-1} .5$ & $\begin{array}{l}\mathrm{SIG}-3 \\
\mathrm{~kg} / \mathrm{m} 3\end{array}$ & $\underset{m}{D E}$ \\
\hline $\begin{array}{r}84 \\
146 \\
205\end{array}$ & $\begin{array}{l}18.831 \\
18.072 \\
17.183\end{array}$ & $\begin{array}{l}36.681 \\
36.529 \\
36.417\end{array}$ & $\begin{array}{l}5.35 \\
5.01 \\
4.76\end{array}$ & & & $\begin{array}{l}18.816 \\
18.047 \\
17.149\end{array}$ & $\begin{array}{l}26.355 \\
26.433 \\
26.568\end{array}$ & $\begin{array}{l}32.740 \\
32.838 \\
32.995\end{array}$ & $\begin{array}{l}38.916 \\
39.033 \\
39.213\end{array}$ & $\begin{array}{r}83 \\
145 \\
203\end{array}$ \\
\hline \multicolumn{2}{|c|}{$\begin{array}{l}\text { ENDEAVOR } 143 \\
\text { DATE } 7 / 5 / 86\end{array}$} & ${ }_{6}$ STA- 21 & LAT $=$ & \multicolumn{2}{|c|}{$3219.8 \mathrm{~N}$} & \multicolumn{2}{|c|}{$3015.1 w$} & \multicolumn{2}{|c|}{ SONIC DEPTH } & Om \\
\hline $\begin{array}{l}P R \\
\text { dbar }\end{array}$ & $\stackrel{T}{\operatorname{Deg}} \mathrm{C}$ & $\stackrel{5}{0 / 00}$ & $\begin{array}{l}02 \\
\mathrm{ml} / \mathrm{I}\end{array}$ & $\stackrel{\theta}{\operatorname{Deg} C}$ & $\begin{array}{l}S I G-\theta \\
\mathrm{kg} / \mathrm{m} 3\end{array}$ & $\underset{\mathrm{kg} / \mathrm{m} 3}{S I G-1} .5$ & $\begin{array}{l}S I G-3 \\
\mathrm{~kg} / \mathrm{m} 3\end{array}$ & $\underset{\mathrm{m}}{\mathrm{HGTH}}$ & $\underset{c p h}{N}$ & $\begin{array}{c}\mathrm{DE} \\
\text { m }\end{array}$ \\
\hline $\begin{array}{r}3 \\
25 \\
50 \\
75 \\
100 \\
150 \\
200 \\
250 \\
300 \\
309\end{array}$ & $\begin{array}{l}20.489 \\
19.631 \\
18.530 \\
18.214 \\
17.947 \\
17.458 \\
16.950 \\
16.438 \\
15.628 \\
15.430\end{array}$ & $\begin{array}{l}36.710 \\
36.680 \\
36.494 \\
36.472 \\
36.443 \\
36.393 \\
36.314 \\
36.234 \\
36.108 \\
36.080\end{array}$ & $\begin{array}{l}5.15 \\
5.33 \\
5.48 \\
5.43 \\
5.12 \\
4.65 \\
4.55 \\
4.59 \\
4.43 \\
4.36\end{array}$ & $\begin{array}{l}20.489 \\
19.626 \\
18.521 \\
18.201 \\
17.930 \\
17.433 \\
16.916 \\
16.397 \\
15.581 \\
15.382\end{array}$ & $\begin{array}{l}25.937 \\
26.144 \\
26.287 \\
26.351 \\
26.396 \\
26.480 \\
26.545 \\
26.606 \\
26.697 \\
26.721\end{array}$ & $\begin{array}{l}32.282 \\
32.510 \\
32.680 \\
32.753 \\
32.804 \\
32.901 \\
32.979 \\
33.055 \\
33.168 \\
33.197\end{array}$ & $\begin{array}{l}38.422 \\
38.668 \\
38.865 \\
38.945 \\
39.003 \\
39.112 \\
39.203 \\
39.292 \\
39.427 \\
39.462\end{array}$ & $\begin{array}{l}0.006 \\
0.050 \\
0.095 \\
0.138 \\
0.180 \\
0.261 \\
0.339 \\
0.416 \\
0.490 \\
0.503\end{array}$ & $\begin{array}{l}2.90 \\
5.78 \\
3.53 \\
2.23 \\
2.70 \\
2.27 \\
1.95 \\
2.17 \\
2.69 \\
3.22\end{array}$ & $\begin{array}{r}3 \\
25 \\
50 \\
74 \\
99 \\
149 \\
199 \\
248 \\
298 \\
307\end{array}$ \\
\hline $\begin{array}{l}\text { PR } \\
\text { dbar }\end{array}$ & $\stackrel{T}{\operatorname{Deg}} \mathrm{C}$ & $\begin{array}{c}S \\
0 / 00\end{array}$ & $\begin{array}{c}02 \\
\mathrm{ml} / \mathrm{l}\end{array}$ & $\begin{array}{c}\mathrm{F}-11 \\
\mathrm{pmol} / \mathrm{kg}\end{array}$ & $\begin{array}{c}\mathrm{F}-12 \\
\mathrm{pmol} / \mathrm{kg}\end{array}$ & $\stackrel{\theta}{\operatorname{Deg} C}$ & $\begin{array}{l}\mathrm{SIG}-0 \\
\mathrm{~kg} / \mathrm{m} 3\end{array}$ & $\underset{\mathrm{kg} / \mathrm{m} 3}{\mathrm{SIG}-1.5}$ & $\begin{array}{l}S I G-3 \\
\mathrm{~kg} / \mathrm{m} 3\end{array}$ & $\underset{m}{D E}$ \\
\hline $\begin{array}{r}86 \\
160 \\
308\end{array}$ & $\begin{array}{l}18.133 \\
17.267 \\
15.345\end{array}$ & $\begin{array}{l}36.478 \\
36.383 \\
36.079\end{array}$ & $\begin{array}{l}5.47 \\
5.91 \\
4.67\end{array}$ & & & $\begin{array}{l}18.118 \\
17.240 \\
15.297\end{array}$ & $\begin{array}{l}26.376 \\
26.519 \\
26.739\end{array}$ & $\begin{array}{l}32.779 \\
32.945 \\
33.218\end{array}$ & $\begin{array}{l}38.974 \\
39.161 \\
39.484\end{array}$ & $\begin{array}{r}85 \\
159 \\
305\end{array}$ \\
\hline
\end{tabular}




\begin{tabular}{|c|c|c|c|c|c|c|c|c|c|c|}
\hline \multicolumn{3}{|c|}{$\begin{array}{l}\text { ENDEAVOR } 143 \text { STA- } 22 \\
\text { DATE } 7 / 5 / 86\end{array}$} & \multirow{2}{*}{$\begin{array}{l}\text { LAT }= \\
02 \\
\mathrm{ml} / 1\end{array}$} & \multicolumn{2}{|c|}{$3220.0 \mathrm{~N}$} & \multicolumn{2}{|c|}{$O N=3026.9 \mathrm{~W}$} & \multicolumn{2}{|c|}{ SONIC DEPTH= } & \multirow{2}{*}{$\begin{array}{l}\text { Om } \\
\text { DE } \\
m\end{array}$} \\
\hline $\begin{array}{l}\text { PR } \\
\text { dbar }\end{array}$ & $\stackrel{T}{\text { Deg } C}$ & $\stackrel{5}{0 / 00}$ & & $\stackrel{\theta}{\operatorname{Deg} C}$ & $\begin{array}{l}\mathrm{SIG}-\theta \\
\mathrm{kg} / \mathrm{m} 3\end{array}$ & $\begin{array}{l}\mathrm{SIG}-1.5 \\
\mathrm{~kg} / \mathrm{m} 3\end{array}$ & $\begin{array}{l}\mathrm{SIG}-3 \\
\mathrm{~kg} / \mathrm{m} 3\end{array}$ & $\begin{array}{c}\text { HGTH } \\
\text { m }\end{array}$ & $\underset{c p h}{N}$ & \\
\hline $\begin{array}{r}1 \\
25 \\
50 \\
75 \\
100 \\
150 \\
200 \\
250 \\
300 \\
307\end{array}$ & $\begin{array}{l}20.540 \\
20.122 \\
19.391 \\
19.134 \\
18.456 \\
17.533 \\
16.800 \\
16.366 \\
15.704 \\
15.566\end{array}$ & $\begin{array}{l}36.747 \\
36.725 \\
36.707 \\
36.685 \\
36.547 \\
36.392 \\
36.275 \\
36.224 \\
36.119 \\
36.096\end{array}$ & $\begin{array}{l}5.04 \\
5.13 \\
5.30 \\
5.25 \\
5.01 \\
4.85 \\
4.95 \\
4.67 \\
4.50 \\
4.50\end{array}$ & $\begin{array}{l}20.540 \\
20.117 \\
19.382 \\
19.121 \\
18.438 \\
17.507 \\
16.767 \\
16.325 \\
15.656 \\
15.517\end{array}$ & $\begin{array}{l}25.951 \\
26.048 \\
26.229 \\
26.279 \\
26.349 \\
26.461 \\
26.550 \\
26.615 \\
26.689 \\
26.703\end{array}$ & $\begin{array}{l}32.296 \\
32.402 \\
32.600 \\
32.657 \\
32.744 \\
32.880 \\
32.989 \\
33.066 \\
33.158 \\
33.176\end{array}$ & $\begin{array}{l}38.433 \\
38.549 \\
38.763 \\
38.826 \\
38.930 \\
39.089 \\
39.217 \\
39.305 \\
39.415 \\
39.436\end{array}$ & $\begin{array}{l}0.002 \\
0.051 \\
0.097 \\
0.142 \\
0.185 \\
0.268 \\
0.347 \\
0.423 \\
0.497 \\
0.507\end{array}$ & $\begin{array}{r}-0.51 \\
5.92 \\
3.23 \\
2.24 \\
2.95 \\
2.56 \\
2.28 \\
2.14 \\
2.30 \\
2.52\end{array}$ & $\begin{array}{r}1 \\
25 \\
50 \\
74 \\
99 \\
149 \\
199 \\
248 \\
298 \\
305\end{array}$ \\
\hline $\begin{array}{l}P R \\
\text { dbar }\end{array}$ & $\stackrel{T}{D e g} C$ & $\stackrel{5}{0 / 00}$ & $\begin{array}{l}02 \\
\mathrm{ml} / \mathrm{l}\end{array}$ & $\begin{array}{c}\mathrm{F}-11 \\
\mathrm{pmol} / \mathrm{kg}\end{array}$ & $\begin{array}{c}\mathrm{F}-12 \\
\mathrm{pmol} / \mathrm{kg}\end{array}$ & $\stackrel{\theta}{\operatorname{Deg} C}$ & $\begin{array}{l}\text { SIG-O } \\
\mathrm{kg} / \mathrm{m3}\end{array}$ & $\underset{\mathrm{kg} / \mathrm{m} 3}{\mathrm{SIG}-1.5}$ & $\begin{array}{l}\mathrm{SIG}-3 \\
\mathrm{~kg} / \mathrm{m} 3\end{array}$ & $\begin{array}{c}\mathrm{DE} \\
\mathbf{m}\end{array}$ \\
\hline $\begin{array}{r}87 \\
115 \\
180\end{array}$ & $\begin{array}{l}19.021 \\
18.112 \\
17.232\end{array}$ & $\begin{array}{l}36.675 \\
36.487 \\
36.362\end{array}$ & $\begin{array}{l}5.33 \\
5.03 \\
4.87\end{array}$ & & & $\begin{array}{l}19.005 \\
18.092 \\
17.202\end{array}$ & $\begin{array}{l}26.302 \\
26.390 \\
26.513\end{array}$ & $\begin{array}{l}32.682 \\
32.794 \\
32.940\end{array}$ & $\begin{array}{l}38.854 \\
38.988 \\
39.156\end{array}$ & $\begin{array}{r}86 \\
114 \\
178\end{array}$ \\
\hline
\end{tabular}

\begin{tabular}{|c|c|c|c|c|c|c|c|c|c|c|}
\hline \multicolumn{3}{|c|}{$\begin{array}{l}\text { ENDEAVOR } 143 \text { STA- } 23 \\
\text { DATE } 7 / 5 / 86\end{array}$} & \multirow{2}{*}{$\begin{array}{l}\text { LAT }= \\
02 \\
\mathrm{ml} / 1\end{array}$} & \multicolumn{2}{|c|}{$3210.0 \mathrm{~N}$} & \multicolumn{2}{|c|}{$3027.0 \mathrm{~W}$} & \multicolumn{2}{|c|}{ SONIC DEPTH } & \multirow{2}{*}{$\begin{array}{l}\text { Om } \\
\underset{m}{\mathrm{DE}} \\
\mathbf{m}\end{array}$} \\
\hline $\begin{array}{l}\text { PR } \\
\text { dbar }\end{array}$ & $\stackrel{T}{T} C$ & $\stackrel{5}{0 / 00}$ & & $\stackrel{\theta}{\operatorname{Deg} C}$ & $\begin{array}{l}\mathrm{SIG}-\theta \\
\mathrm{kg} / \mathrm{m} 3\end{array}$ & $\begin{array}{l}\mathrm{SIG}-1.5 \\
\mathrm{~kg} / \mathrm{m3}\end{array}$ & $\begin{array}{l}\mathrm{SIG}-3 \\
\mathrm{~kg} / \mathrm{m} 3\end{array}$ & $\underset{m}{\text { HGTH }}$ & $\underset{\text { cph }}{N}$ & \\
\hline $\begin{array}{r}1 \\
25 \\
50 \\
75 \\
100 \\
150 \\
200 \\
250 \\
300 \\
305\end{array}$ & $\begin{array}{l}20.338 \\
19.976 \\
19.023 \\
18.857 \\
18.697 \\
17.730 \\
17.080 \\
16.488 \\
15.779 \\
15.631\end{array}$ & $\begin{array}{l}36.702 \\
36.699 \\
36.623 \\
36.618 \\
36.596 \\
36.445 \\
36.357 \\
36.268 \\
36.156 \\
36.136\end{array}$ & $\begin{array}{l}5.07 \\
5.12 \\
5.39 \\
5.23 \\
5.21 \\
4.60 \\
4.40 \\
4.38 \\
4.52 \\
4.54\end{array}$ & $\begin{array}{l}20.337 \\
19.972 \\
19.014 \\
18.844 \\
18.679 \\
17.704 \\
17.046 \\
16.447 \\
15.732 \\
15.583\end{array}$ & $\begin{array}{l}25.972 \\
26.067 \\
26.260 \\
26.300 \\
26.325 \\
26.454 \\
26.546 \\
26.620 \\
26.700 \\
26.718\end{array}$ & $\begin{array}{l}32.321 \\
32.425 \\
32.640 \\
32.684 \\
32.714 \\
32.867 \\
32.977 \\
33.067 \\
33.167 \\
33.189\end{array}$ & $\begin{array}{l}38.463 \\
38.575 \\
38.813 \\
38.860 \\
38.894 \\
39.071 \\
39.197 \\
39.303 \\
39.421 \\
39.448\end{array}$ & $\begin{array}{l}0.002 \\
0.050 \\
0.096 \\
0.140 \\
0.183 \\
0.267 \\
0.346 \\
0.422 \\
0.496 \\
0.503\end{array}$ & $\begin{array}{l}1.14 \\
5.69 \\
3.72 \\
1.84 \\
2.66 \\
2.36 \\
2.47 \\
1.88 \\
2.42 \\
3.61\end{array}$ & $\begin{array}{r}1 \\
25 \\
50 \\
74 \\
99 \\
149 \\
199 \\
248 \\
298 \\
303\end{array}$ \\
\hline $\begin{array}{l}\text { PR } \\
\text { dbar }\end{array}$ & $\stackrel{T}{T} c$ & $\stackrel{5}{0 / 00}$ & $\begin{array}{ll}02 \\
\mathrm{ml} / \mathrm{I}\end{array}$ & $\begin{array}{c}\mathrm{F}-11 \\
\mathrm{pmol} / \mathrm{kg}\end{array}$ & $\begin{array}{c}\mathrm{F}-12 \\
\mathrm{pmol} / \mathrm{kg}\end{array}$ & $\stackrel{\theta}{\operatorname{Deg} C}$ & $\begin{array}{l}\mathrm{SIG}-\theta \\
\mathrm{kg} / \mathrm{m} 3\end{array}$ & $\underset{\mathrm{kg} / \mathrm{m} 3}{\mathrm{SIG}-1.5}$ & $\begin{array}{l}\mathrm{SIG}-3 \\
\mathrm{~kg} / \mathrm{m} 3\end{array}$ & $\begin{array}{c}\mathrm{DE} \\
\mathrm{m}\end{array}$ \\
\hline $\begin{array}{r}83 \\
103 \\
198\end{array}$ & $\begin{array}{l}18.786 \\
18.515 \\
17.067\end{array}$ & 36.617 & $\begin{array}{l}5.35 \\
4.70\end{array}$ & & & 18.496 & 26.388 & 32.780 & 38.964 & $\begin{array}{r}82 \\
103 \\
196\end{array}$ \\
\hline
\end{tabular}

ENDEAVOR 143 STA- 24
DATE $7 / 5 / 86$

SONIC DEPTH $4020 m$

$L A T=320.0 N \quad L O N=3027.0 W$

$02 \theta \theta \quad$ SIG- $\theta$ SIG-1.5 SIG-3 $\mathrm{ml} / \mathrm{l}$

$4.99 \quad 20.351$

$5.31 \quad 19.372$

$5.13 \quad 18.782$

$5.06 \quad 18.722$

$4.71 \quad 17.872$

$\begin{array}{ll}4.52 & 17.177\end{array}$

4.3716 .516

$\begin{array}{ll}4.46 & 15.757\end{array}$

$4.53 \quad 15.722$

26.015
26.241
26.316
26.339
26.359
26.439
26.541
26.621
26.702
26.706

$32.363 \quad 38.505$

$32.612 \quad 38.776$

$32.700 \quad 38.875$

$32.724 \quad 38.902$

$32.746 \quad 38.925$

$32.848 \quad 39.048$

$32.968 \quad 39.185$

$33.066 \quad 39.300$

$33.168 \quad 39.422$

$33.173 \quad 39.428$
HGTH $N$ DE

0.002

0.047

0.091

0.134
0.176

0.260

0.339

0.415

0.489

0.496

5.57

3.67

2.22

1.81

1.81

$2.63 \quad 149$

$2.43 \quad 199$

1.97248

$2.70 \quad 298$

1.80303

\begin{tabular}{|c|c|c|c|c|c|c|c|c|c|c|}
\hline $\begin{array}{l}\text { PR } \\
\text { dbar }\end{array}$ & $\stackrel{T}{D e g} C$ & $\stackrel{S}{0 / 00}$ & $\begin{array}{c}02 \\
\mathrm{ml} / \mathrm{l}\end{array}$ & $\begin{array}{c}\mathrm{F}-11 \\
\mathrm{pmol} / \mathrm{kg}\end{array}$ & $\begin{array}{c}\mathrm{F}-12 \\
\mathrm{pmol} / \mathrm{kg}\end{array}$ & $\stackrel{\theta}{D e g} C$ & $\begin{array}{l}\mathrm{SIG}-0 \\
\mathrm{~kg} / \mathrm{m3}\end{array}$ & $\underset{\mathrm{kg} / \mathrm{m} 3}{\mathrm{SIG}-1} .5$ & $\begin{array}{l}S I G-3 \\
\mathrm{~kg} / \mathrm{m} 3\end{array}$ & D \\
\hline $\begin{array}{r}79 \\
149 \\
204\end{array}$ & $\begin{array}{l}18.804 \\
18.013 \\
17.149\end{array}$ & $\begin{array}{l}36.639 \\
36.504 \\
36.391\end{array}$ & $\begin{array}{l}5.34 \\
4.92 \\
4.68\end{array}$ & & & $\begin{array}{l}18.790 \\
17.987 \\
17.114\end{array}$ & $\begin{array}{l}26.330 \\
26.429 \\
26.556\end{array}$ & $\begin{array}{l}32.715 \\
32.835 \\
32.985\end{array}$ & $\begin{array}{l}38.892 \\
39.032 \\
39.203\end{array}$ & $\begin{array}{r}78 \\
148 \\
202\end{array}$ \\
\hline
\end{tabular}




\begin{tabular}{|c|c|c|c|c|c|c|c|c|c|c|}
\hline \multicolumn{3}{|c|}{$\begin{array}{l}\text { ENDEAVOR } 143 \text { STA- } 25 \\
\text { DATE } 7 / 5 / 86\end{array}$} & LAT $=$ & \multicolumn{2}{|c|}{$=3150.1 \mathrm{~N}$} & \multicolumn{2}{|c|}{$\mathrm{DN}=$} & \multicolumn{2}{|c|}{ SONIC DEPTH= } & \multirow[t]{2}{*}{$\theta m$} \\
\hline $\begin{array}{l}\text { PR } \\
\text { dbar }\end{array}$ & $\stackrel{T}{\operatorname{Deg} C}$ & $\stackrel{5}{0 / 00}$ & $\begin{array}{l}02 \\
\mathrm{ml} / \mathrm{l}\end{array}$ & $\stackrel{\theta}{\operatorname{Deg} C}$ & $\begin{array}{l}\mathrm{SIG}-0 \\
\mathrm{~kg} / \mathrm{m3}\end{array}$ & $\underset{\mathrm{kg} / \mathrm{m} 3}{\mathrm{SIG}-1.5}$ & $\begin{array}{l}\mathrm{SIG}-3 \\
\mathrm{~kg} / \mathrm{m} 3\end{array}$ & $\underset{m}{\text { HGTH }}$ & $\underset{\mathrm{cph}}{\mathrm{N}}$ & \\
\hline $\begin{array}{r}1 \\
25 \\
50 \\
75 \\
100 \\
150 \\
200 \\
250 \\
300\end{array}$ & $\begin{array}{l}19.932 \\
19.204 \\
18.963 \\
18.771 \\
18.619 \\
18.347 \\
17.545 \\
16.990 \\
16.279\end{array}$ & $\begin{array}{l}36.712 \\
36.692 \\
36.697 \\
36.675 \\
36.651 \\
36.590 \\
36.435 \\
36.359 \\
36.241\end{array}$ & $\begin{array}{l}5.03 \\
5.16 \\
5.00 \\
5.09 \\
5.06 \\
4.91 \\
4.51 \\
4.35 \\
4.36\end{array}$ & $\begin{array}{l}19.932 \\
19.199 \\
18.954 \\
18.757 \\
18.601 \\
18.321 \\
17.510 \\
16.948 \\
16.231\end{array}$ & $\begin{array}{l}26.087 \\
26.265 \\
26.332 \\
26.365 \\
26.387 \\
26.411 \\
26.493 \\
26.571 \\
26.650\end{array}$ & $\begin{array}{l}32.446 \\
32.640 \\
32.713 \\
32.751 \\
32.777 \\
32.809 \\
32.912 \\
33.005 \\
33.103\end{array}$ & $\begin{array}{l}38.597 \\
38.808 \\
38.886 \\
38.929 \\
38.958 \\
38.997 \\
39.121 \\
39.227 \\
39.345\end{array}$ & $\begin{array}{l}0.002 \\
0.046 \\
0.090 \\
0.132 \\
0.174 \\
0.257 \\
0.339 \\
0.418 \\
0.493\end{array}$ & $\begin{array}{l}1.54 \\
4.46 \\
2.52 \\
1.78 \\
1.11 \\
1.80 \\
2.52 \\
2.17 \\
2.59\end{array}$ & $\begin{array}{l}14 \\
19 \\
24 \\
29\end{array}$ \\
\hline $\begin{array}{l}\text { PR } \\
\text { dbar }\end{array}$ & $\stackrel{T}{\operatorname{Deg}} C$ & $\stackrel{S}{0 / 00}$ & $\begin{array}{l}02 \\
\mathrm{ml} / \mathrm{I}\end{array}$ & $\begin{array}{c}\mathrm{F}-11 \\
\mathrm{pmol} / \mathrm{kg}\end{array}$ & $\begin{array}{c}\mathrm{F}-12 \\
\mathrm{pmol} / \mathrm{kg}\end{array}$ & $\stackrel{\theta}{\operatorname{\theta eg} C}$ & $\begin{array}{l}\mathrm{SIG}-\mathrm{O} \\
\mathrm{kg} / \mathrm{m} 3\end{array}$ & $\underset{\mathrm{kg} / \mathrm{m} 3}{\mathrm{SIG}-1.5}$ & $\begin{array}{l}\mathrm{SIG}-3 \\
\mathrm{~kg} / \mathrm{m} 3\end{array}$ & \\
\hline $\begin{array}{r}74 \\
122 \\
239\end{array}$ & $\begin{array}{l}18.754 \\
18.582 \\
17.043\end{array}$ & $\begin{array}{l}36.695 \\
36.653 \\
36.411\end{array}$ & $\begin{array}{l}5.29 \\
5.19 \\
4.69\end{array}$ & & & $\begin{array}{l}18.741 \\
18.560 \\
17.003\end{array}$ & $\begin{array}{l}26.385 \\
26.399 \\
26.598\end{array}$ & $\begin{array}{l}32.771 \\
32.790 \\
33.029\end{array}$ & $\begin{array}{l}38.949 \\
38.972 \\
39.250\end{array}$ & $\begin{array}{l}12 \\
23\end{array}$ \\
\hline
\end{tabular}

ENDEAVOR $143 \quad$ STA- $26 \quad$ LAT $=3139.5 \mathrm{~N} \quad$ LON= $3027.1 \mathrm{~W} \quad$ SONIC DEPTH= Om DATE $8 / 5 / 86$

\begin{tabular}{|c|c|c|c|c|c|c|c|c|c|}
\hline $\begin{array}{l}\text { PR } \\
\text { dbar }\end{array}$ & $\stackrel{T}{T} C$ & $\stackrel{5}{0 / 00}$ & $\begin{array}{c}02 \\
\mathrm{ml} / \mathrm{I}\end{array}$ & $\stackrel{\ominus}{\operatorname{Deg} C}$ & $\begin{array}{l}\mathrm{SIG}-0 \\
\mathrm{~kg} / \mathrm{m} 3\end{array}$ & $\underset{\mathrm{kg} / \mathrm{m} 3}{\mathrm{SIG}-1.5}$ & $\begin{array}{l}\text { SIG-3 } \\
\mathrm{kg} / \mathrm{m} 3\end{array}$ & $\underset{\mathrm{m}}{\mathrm{HGTH}}$ & $\underset{\text { cph }}{N}$ \\
\hline $\begin{array}{r}1 \\
25 \\
50 \\
75 \\
100 \\
150 \\
200 \\
250 \\
295\end{array}$ & $\begin{array}{l}19.965 \\
19.372 \\
18.789 \\
18.631 \\
18.599 \\
18.547 \\
18.490 \\
17.234 \\
16.337\end{array}$ & $\begin{array}{l}36.731 \\
36.694 \\
36.659 \\
36.649 \\
36.649 \\
36.642 \\
36.629 \\
36.388 \\
36.246\end{array}$ & $\begin{array}{l}4.77 \\
5.16 \\
5.24 \\
5.18 \\
5.12 \\
5.07 \\
4.99 \\
4.48 \\
4.51\end{array}$ & $\begin{array}{l}19.964 \\
19.367 \\
18.780 \\
18.618 \\
18.582 \\
18.520 \\
18.454 \\
17.192 \\
16.289\end{array}$ & $\begin{array}{l}26.093 \\
26.223 \\
26.348 \\
26.381 \\
26.390 \\
26.401 \\
26.408 \\
26.535 \\
26.640\end{array}$ & $\begin{array}{l}32.451 \\
32.594 \\
32.733 \\
32.771 \\
32.781 \\
32.793 \\
32.801 \\
32.962 \\
33.091\end{array}$ & $\begin{array}{l}38.601 \\
38.758 \\
38.911 \\
38.952 \\
38.962 \\
38.976 \\
38.986 \\
39.178 \\
39.331\end{array}$ & $\begin{array}{l}0.002 \\
0.047 \\
0.091 \\
0.132 \\
0.174 \\
0.258 \\
0.342 \\
0.424 \\
0.492\end{array}$ & $\begin{array}{r}-0.93 \\
5.35 \\
3.04 \\
1.27 \\
0.87 \\
0.67 \\
2.07 \\
3.15 \\
2.41\end{array}$ \\
\hline $\begin{array}{l}P R \\
\text { dbar }\end{array}$ & ${ }_{\text {Deg }}^{T} C$ & $\stackrel{5}{0 / 00}$ & $\begin{array}{l}02 \\
\mathrm{ml} / \mathrm{I}\end{array}$ & $\begin{array}{c}\mathrm{F}-11 \\
\mathrm{pmol} / \mathrm{kg}\end{array}$ & $\begin{array}{c}\mathrm{F}-12 \\
\mathrm{pmol} / \mathrm{kg}\end{array}$ & $\stackrel{\theta}{\operatorname{Deg} C}$ & $\begin{array}{l}S I G-0 \\
\mathrm{~kg} / \mathrm{m3}\end{array}$ & $\underset{\mathrm{kg} / \mathrm{m} 3}{\mathrm{SIG}-1.5}$ & $\begin{array}{l}S I G-3 \\
\mathrm{~kg} / \mathrm{m} 3\end{array}$ \\
\hline $\begin{array}{l}168 \\
215 \\
244\end{array}$ & $\begin{array}{l}18.546 \\
17.889 \\
17.239\end{array}$ & $\begin{array}{l}36.692 \\
36.626 \\
36.445\end{array}$ & $\begin{array}{l}5.20 \\
4.89 \\
4.70\end{array}$ & & & $\begin{array}{l}18.516 \\
17.852 \\
17.198\end{array}$ & $\begin{array}{l}26.440 \\
26.556 \\
26.577\end{array}$ & $\begin{array}{l}32.832 \\
32.964 \\
33.003\end{array}$ & $\begin{array}{l}39.015 \\
39.163 \\
39.219\end{array}$ \\
\hline
\end{tabular}

\begin{tabular}{|c|c|c|c|c|c|c|c|c|c|c|}
\hline \multicolumn{3}{|c|}{$\begin{array}{l}\text { ENDEAVOR } 143 \text { STA- } 27 \\
\text { DATE } 8 / 5 / 86\end{array}$} & \multirow{2}{*}{$\begin{array}{l}\text { LAT }= \\
02 \\
\mathrm{ml} / \mathrm{l}\end{array}$} & \multicolumn{2}{|c|}{$=3130.0 \mathrm{~N}$} & \multicolumn{2}{|c|}{$3027.0 \mathrm{w}$} & \multicolumn{2}{|c|}{ SONIC DEPTH } & \multirow{2}{*}{$\begin{array}{l}O m \\
D E \\
m\end{array}$} \\
\hline $\begin{array}{l}\text { PR } \\
\text { dbar }\end{array}$ & $\stackrel{T}{T} C$ & $\stackrel{5}{0 / 00}$ & & $\stackrel{\theta}{\operatorname{Deg} C}$ & $\begin{array}{l}\mathrm{SIG}-\Theta \\
\mathrm{kg} / \mathrm{m} 3\end{array}$ & $\begin{array}{l}\mathrm{SIG}-1.5 \\
\mathrm{~kg} / \mathrm{m} 3\end{array}$ & $\begin{array}{l}\mathrm{SIG}-3 \\
\mathrm{~kg} / \mathrm{m} 3\end{array}$ & $\underset{\mathrm{m}}{\mathrm{HGTH}}$ & $\underset{\mathrm{cph}}{N}$ & \\
\hline $\begin{array}{r}1 \\
25 \\
50 \\
75 \\
100 \\
150 \\
200 \\
250 \\
300\end{array}$ & $\begin{array}{l}20.049 \\
19.673 \\
18.839 \\
18.688 \\
18.641 \\
18.568 \\
18.483 \\
17.415 \\
16.709\end{array}$ & $\begin{array}{l}36.720 \\
36.707 \\
36.664 \\
36.663 \\
36.663 \\
36.650 \\
36.626 \\
36.412 \\
36.304\end{array}$ & $\begin{array}{l}4.94 \\
5.14 \\
5.27 \\
5.20 \\
5.13 \\
4.92 \\
4.84 \\
4.46 \\
4.38\end{array}$ & $\begin{array}{l}20.049 \\
19.668 \\
18.830 \\
18.675 \\
18.624 \\
18.541 \\
18.447 \\
17.372 \\
16.659\end{array}$ & $\begin{array}{l}26.063 \\
26.154 \\
26.339 \\
26.377 \\
26.391 \\
26.401 \\
26.407 \\
26.509 \\
26.598\end{array}$ & $\begin{array}{l}32.418 \\
32.518 \\
32.723 \\
32.765 \\
32.780 \\
32.793 \\
32.801 \\
32.932 \\
33.039\end{array}$ & $\begin{array}{l}38.567 \\
38.675 \\
38.899 \\
38.945 \\
38.961 \\
38.975 \\
38.986 \\
39.144 \\
39.269\end{array}$ & $\begin{array}{l}0.002 \\
0.048 \\
0.092 \\
0.134 \\
0.175 \\
0.259 \\
0.343 \\
0.426 \\
0.504\end{array}$ & $\begin{array}{r}-0.66 \\
6.34 \\
2.43 \\
1.62 \\
0.98 \\
0.57 \\
1.45 \\
2.85 \\
2.31\end{array}$ & $\begin{array}{r}1 \\
25 \\
50 \\
74 \\
99 \\
149 \\
199 \\
248 \\
298\end{array}$ \\
\hline $\begin{array}{l}\text { PR } \\
\text { dbar }\end{array}$ & $\stackrel{T}{T} C$ & $\stackrel{5}{0 / 00}$ & $\begin{array}{l}02 \\
\mathrm{ml} / \mathrm{I}\end{array}$ & $\begin{array}{c}\mathrm{F}-11 \\
\mathrm{pmol} / \mathrm{kg}\end{array}$ & $\begin{array}{c}\mathrm{F}-12 \\
\mathrm{gmol} / \mathrm{kg}\end{array}$ & $\stackrel{\theta}{\operatorname{Deg} C}$ & $\begin{array}{l}\mathrm{SIG}-\theta \\
\mathrm{kg} / \mathrm{m} 3\end{array}$ & $\underset{\mathrm{kg} / \mathrm{m} 3}{\mathrm{SIG}-1.5}$ & $\begin{array}{l}\mathrm{SIG}-3 \\
\mathrm{~kg} / \mathrm{m3}\end{array}$ & $\begin{array}{l}\mathrm{DE} \\
\mathrm{m}\end{array}$ \\
\hline $\begin{array}{l}100 \\
173 \\
221 \\
256\end{array}$ & $\begin{array}{l}18.629 \\
18.519 \\
18.045 \\
17.184\end{array}$ & $\begin{array}{l}36.673 \\
36.642 \\
36.536 \\
36.409\end{array}$ & $\begin{array}{l}5.27 \\
5.21 \\
4.93 \\
4.70\end{array}$ & & & $\begin{array}{l}18.611 \\
18.489 \\
18.006 \\
17.141\end{array}$ & $\begin{array}{l}26.401 \\
26.409 \\
26.449 \\
26.563\end{array}$ & $\begin{array}{l}32.791 \\
32.801 \\
32.854 \\
32.991\end{array}$ & $\begin{array}{l}38.972 \\
38.985 \\
39.050 \\
39.209\end{array}$ & $\begin{array}{r}99 \\
171 \\
219 \\
254\end{array}$ \\
\hline
\end{tabular}




\begin{tabular}{|c|c|c|c|c|c|c|c|c|c|c|}
\hline \multicolumn{3}{|c|}{$\begin{array}{l}\text { ENDEAVOR } 143 \text { STA- } 28 \\
\text { DATE } 8 / 5 / 86\end{array}$} & \multirow{2}{*}{$\begin{array}{l}\text { LAT }= \\
02 \\
\mathrm{~m} / / 1\end{array}$} & \multicolumn{2}{|c|}{$=3119.9 \mathrm{~N}$} & \multicolumn{2}{|c|}{$3027.0 \mathrm{~W}$} & \multicolumn{2}{|c|}{ SONIC DEPTH= } & \multirow{2}{*}{$\begin{array}{l}\text { On } \\
\underset{m}{\mathrm{DE}}\end{array}$} \\
\hline $\begin{array}{l}\text { PR } \\
\text { dbar }\end{array}$ & $\stackrel{\text { T }}{\text { Deg } C}$ & $\stackrel{S}{0 / 00}$ & & $\stackrel{\theta}{\operatorname{Deg} C}$ & $\begin{array}{l}\mathrm{SIG}-\theta \\
\mathrm{kg} / \mathrm{m} 3\end{array}$ & $\begin{array}{l}\mathrm{SIG}-1.5 \\
\mathrm{~kg} / \mathrm{m} 3\end{array}$ & $\begin{array}{l}\mathrm{SIG}-3 \\
\mathrm{~kg} / \mathrm{m3}\end{array}$ & $\begin{array}{l}\text { HGTH } \\
m\end{array}$ & $\underset{c p h}{N}$ & \\
\hline $\begin{array}{r}1 \\
25 \\
50 \\
75 \\
100 \\
150 \\
200 \\
250 \\
300 \\
309\end{array}$ & $\begin{array}{l}19.903 \\
19.847 \\
19.024 \\
18.697 \\
18.611 \\
18.555 \\
18.189 \\
17.120 \\
16.547 \\
16.400\end{array}$ & $\begin{array}{l}36.7 \\
36.6 \\
36.6 \\
36.6 \\
36.6 \\
36.6 \\
36.5 \\
36.3 \\
36.2 \\
36.2\end{array}$ & $\begin{array}{l}4.89 \\
4.95 \\
4.98 \\
5.22 \\
5.05 \\
5.05 \\
4.82 \\
4.46 \\
4.44\end{array}$ & $\begin{array}{l}19.903 \\
19.843 \\
19.015 \\
18.683 \\
18.593 \\
18.529 \\
18.154 \\
17.078 \\
16.498 \\
16.349\end{array}$ & $\begin{array}{l}26.088 \\
26.096 \\
26.292 \\
26.369 \\
26.391 \\
26.400 \\
26.429 \\
26.547 \\
26.619 \\
26.636\end{array}$ & $\begin{array}{l}32.447 \\
32.457 \\
32.672 \\
32.757 \\
32.781 \\
32.792 \\
32.831 \\
32.978 \\
33.065 \\
33.085\end{array}$ & $\begin{array}{l}38.599 \\
38.610 \\
38.844 \\
38.937 \\
38.963 \\
38.975 \\
39.023 \\
39.197 \\
39.299 \\
39.323\end{array}$ & & $\begin{array}{r}-1.61 \\
5.08 \\
3.09 \\
2.58 \\
0.88 \\
0.80 \\
2.35 \\
2.60 \\
2.13 \\
2.49\end{array}$ & $\begin{array}{r}1 \\
25 \\
50 \\
74 \\
99 \\
149 \\
199 \\
248 \\
298 \\
307\end{array}$ \\
\hline $\begin{array}{l}\text { PR } \\
\text { dbor }\end{array}$ & $\stackrel{T}{D} C$ & $\stackrel{5}{5}$ & $\begin{array}{l}02 \\
\mathrm{ml} / \mathrm{l}\end{array}$ & $\begin{array}{c}\mathrm{F}-11 \\
\mathrm{pmol} / \mathrm{kg}\end{array}$ & $\begin{array}{c}\mathrm{F}-12 \\
\mathrm{pmol} / \mathrm{kg}\end{array}$ & $\stackrel{\theta}{D e g} C$ & $\begin{array}{l}\mathrm{SIG}-0 \\
\mathrm{~kg} / \mathrm{m3}\end{array}$ & $\underset{\mathrm{kg} / \mathrm{m} 3}{\mathrm{SIG}-1.5}$ & $\begin{array}{l}\text { SIG-3 } \\
\mathrm{kg} / \mathrm{m3}\end{array}$ & $E$ \\
\hline $\begin{array}{r}45 \\
93 \\
203 \\
243\end{array}$ & $\begin{array}{l}19.078 \\
18.626 \\
18.039 \\
17.226\end{array}$ & $\begin{array}{l}36.684 \\
36.658 \\
36.535 \\
36.389\end{array}$ & $\begin{array}{l}5.41 \\
5.27 \\
4.92 \\
5.15\end{array}$ & & & $\begin{array}{l}19.069 \\
18.610 \\
18.004 \\
17.185\end{array}$ & $\begin{array}{l}26.292 \\
26.390 \\
26.448 \\
26.537\end{array}$ & $\begin{array}{l}32.671 \\
32.780 \\
32.854 \\
32.965\end{array}$ & $\begin{array}{l}38.841 \\
38.961 \\
39.050 \\
39.181\end{array}$ & $\begin{array}{r}45 \\
92 \\
202 \\
241\end{array}$ \\
\hline
\end{tabular}

\begin{tabular}{|c|c|c|c|c|c|c|c|c|c|c|}
\hline$\sigma$ & $\begin{array}{l}D R \\
8 / 14\end{array}$ & STA- 29 & LA & 312 & LON & 30 & $1 W$ & SONIC & $\mathrm{TH}=$ & Om \\
\hline $\begin{array}{l}\text { PR } \\
\text { dbar }\end{array}$ & $\stackrel{T}{\text { Deg } C}$ & $\stackrel{5}{0 / 00}$ & $\begin{array}{l}02 \\
\mathrm{ml} / \mathrm{l}\end{array}$ & $\stackrel{\theta}{D e g} \mathrm{C}$ & $\begin{array}{l}\mathrm{SIG}-0 \\
\mathrm{~kg} / \mathrm{m3}\end{array}$ & $\begin{array}{l}\mathrm{SIG}-1.5 \\
\mathrm{~kg} / \mathrm{m3}\end{array}$ & $\begin{array}{l}\mathrm{SIG}-3 \\
\mathrm{~kg} / \mathrm{m3}\end{array}$ & $\underset{m}{\text { HGTH }}$ & $\underset{\mathrm{cph}}{\mathrm{N}}$ & $\begin{array}{r}\mathrm{DE} \\
\mathrm{m}\end{array}$ \\
\hline $\begin{array}{r}1 \\
25 \\
50 \\
75 \\
100 \\
150 \\
200 \\
250 \\
300 \\
307\end{array}$ & $\begin{array}{l}19.837 \\
19.653 \\
18.914 \\
18.644 \\
18.606 \\
18.554 \\
18.482 \\
17.428 \\
16.822 \\
16.652\end{array}$ & $\begin{array}{l}36.700 \\
36.695 \\
36.675 \\
36.659 \\
36.656 \\
36.646 \\
36.624 \\
36.408 \\
36.325 \\
36.294\end{array}$ & $\begin{array}{l}5.06 \\
5.15 \\
5.25 \\
5.21 \\
5.12 \\
4.97 \\
4.84 \\
4.44 \\
4.37 \\
4.42\end{array}$ & $\begin{array}{l}19.837 \\
19.648 \\
18.905 \\
18.631 \\
18.588 \\
18.528 \\
18.446 \\
17.386 \\
16.772 \\
16.602\end{array}$ & $\begin{array}{l}26.104 \\
26.150 \\
26.328 \\
26.386 \\
26.394 \\
26.402 \\
26.406 \\
26.503 \\
26.587 \\
26.604\end{array}$ & $\begin{array}{l}32.464 \\
32.515 \\
32.710 \\
32.775 \\
32.784 \\
32.794 \\
32.800 \\
32.925 \\
33.025 \\
33.047\end{array}$ & $\begin{array}{l}38.618 \\
38.672 \\
38.885 \\
38.955 \\
38.966 \\
38.977 \\
38.985 \\
39.137 \\
39.252 \\
39.278\end{array}$ & $\begin{array}{l}0.002 \\
0.047 \\
0.091 \\
0.133 \\
0.175 \\
0.258 \\
0.342 \\
0.425 \\
0.504 \\
0.515\end{array}$ & $\begin{array}{l}1.25 \\
4.94 \\
3.91 \\
1.73 \\
0.57 \\
0.62 \\
1.53 \\
2.16 \\
2.63 \\
2.99\end{array}$ & $\begin{array}{r}1 \\
25 \\
50 \\
74 \\
99 \\
149 \\
199 \\
248 \\
298 \\
305\end{array}$ \\
\hline $\begin{array}{l}\text { PR } \\
\text { dbar }\end{array}$ & $\stackrel{T}{\text { Deg } C}$ & $\stackrel{5}{0 / 00}$ & $\begin{array}{l}02 \\
\mathrm{ml} / \mathrm{l}\end{array}$ & $\begin{array}{c}\mathrm{F}-11 \\
\mathrm{pmol} / \mathrm{kg}\end{array}$ & $\begin{array}{c}\mathrm{F}-12 \\
\mathrm{pmol} / \mathrm{kg}\end{array}$ & $\operatorname{Deg}^{\ominus} \mathrm{C}$ & $\begin{array}{l}\mathrm{SIG}-0 \\
\mathrm{~kg} / \mathrm{m} 3\end{array}$ & $\underset{\mathrm{kg} / \mathrm{m} 3}{\mathrm{SIG}-1.5}$ & $\begin{array}{l}\text { SIG-3 } \\
\mathrm{kg} / \mathrm{m} 3\end{array}$ & $\mathrm{E}$ \\
\hline $\begin{array}{r}53 \\
119 \\
214 \\
269\end{array}$ & $\begin{array}{l}18.755 \\
18.596 \\
18.008 \\
17.216\end{array}$ & $\begin{array}{l}36.686 \\
36.667 \\
36.528 \\
36.389\end{array}$ & $\begin{array}{l}5.39 \\
5.24 \\
4.96 \\
4.71\end{array}$ & & & $\begin{array}{l}18.746 \\
18.574 \\
17.970 \\
17.171\end{array}$ & $\begin{array}{l}26.377 \\
26.406 \\
26.451 \\
26.541\end{array}$ & $\begin{array}{l}32.763 \\
32.796 \\
32.858 \\
32.968\end{array}$ & $\begin{array}{l}38.941 \\
38.978 \\
39.055 \\
39.185\end{array}$ & $\begin{array}{r}53 \\
118 \\
212 \\
267\end{array}$ \\
\hline
\end{tabular}

\begin{tabular}{|c|c|c|c|c|c|c|c|c|c|c|}
\hline \multicolumn{3}{|c|}{$\begin{array}{l}\text { ENDEAVOR } 143 \text { STA- } 30 \\
\text { DATE } 8 / 5 / 86\end{array}$} & LAT $=$ & \multicolumn{2}{|c|}{$3139.8 \mathrm{~N}$} & \multicolumn{2}{|c|}{ ON= $3039.1 W$} & \multicolumn{2}{|c|}{ SONIC DEPTH } & \multirow{2}{*}{$\begin{array}{l}\theta m \\
D E \\
m\end{array}$} \\
\hline $\begin{array}{l}\text { PR } \\
\text { dbar }\end{array}$ & $\stackrel{T}{\operatorname{Dog}} \mathrm{C}$ & $\begin{array}{l}S \\
0 / 00\end{array}$ & $\begin{array}{l}02 \\
\mathrm{ml} / 1\end{array}$ & $\stackrel{\theta}{\operatorname{Deg} C}$ & $\begin{array}{l}\mathrm{SIG}-0 \\
\mathrm{~kg} / \mathrm{m} 3\end{array}$ & $\underset{\mathrm{kg} / \mathrm{m} 3}{\operatorname{SIG}-1.5}$ & $\begin{array}{l}\mathrm{SIG}-3 \\
\mathrm{~kg} / \mathrm{m} 3\end{array}$ & $\underset{\mathrm{m}}{\mathrm{HGTH}}$ & $\underset{c p h}{N}$ & \\
\hline $\begin{array}{r}1 \\
25 \\
50 \\
75 \\
100 \\
150 \\
200 \\
250 \\
300 \\
309\end{array}$ & $\begin{array}{l}20.004 \\
19.295 \\
18.851 \\
18.627 \\
18.581 \\
18.550 \\
18.376 \\
17.244 \\
16.508 \\
16.404\end{array}$ & $\begin{array}{l}36.734 \\
36.692 \\
36.656 \\
36.648 \\
36.648 \\
36.643 \\
36.602 \\
36.392 \\
36.275 \\
36.258\end{array}$ & $\begin{array}{l}5.25 \\
5.17 \\
5.15 \\
5.08 \\
5.04 \\
4.92 \\
4.76 \\
4.42 \\
4.36 \\
4.43\end{array}$ & $\begin{array}{l}20.004 \\
19.291 \\
18.842 \\
18.614 \\
18.563 \\
18.524 \\
18.341 \\
17.202 \\
16.458 \\
16.354\end{array}$ & $\begin{array}{l}26.085 \\
26.241 \\
26.329 \\
26.382 \\
26.394 \\
26.401 \\
26.415 \\
26.535 \\
26.623 \\
26.634\end{array}$ & $\begin{array}{l}32.441 \\
32.614 \\
32.713 \\
32.771 \\
32.785 \\
32.793 \\
32.812 \\
32.962 \\
33.070 \\
33.084\end{array}$ & $\begin{array}{l}38.591 \\
38.780 \\
38.889 \\
38.952 \\
38.967 \\
38.976 \\
38.999 \\
39.178 \\
39.305 \\
39.322\end{array}$ & $\begin{array}{l}0.002 \\
0.047 \\
0.091 \\
0.132 \\
0.174 \\
0.257 \\
0.341 \\
0.424 \\
0.501 \\
0.514\end{array}$ & $\begin{array}{l}0.69 \\
4.72 \\
3.65 \\
1.78 \\
0.89 \\
0.54 \\
1.66 \\
3.03 \\
2.00 \\
2.11\end{array}$ & $\begin{array}{r}1 \\
25 \\
50 \\
74 \\
99 \\
149 \\
199 \\
248 \\
298 \\
307\end{array}$ \\
\hline $\begin{array}{l}\text { PR } \\
\text { dbar }\end{array}$ & $\stackrel{T}{\operatorname{Deg} C}$ & $\stackrel{5}{0 / 00}$ & $\mathrm{O} 2$ & $\begin{array}{c}\mathrm{F}-11 \\
\mathrm{pmol} / \mathrm{kg}\end{array}$ & $\begin{array}{c}\mathrm{F}-12 \\
\mathrm{pmol} / \mathrm{kg}\end{array}$ & $\stackrel{\theta}{\mathrm{Deg}} \mathrm{C}$ & $\begin{array}{l}\mathrm{SIG}-\theta \\
\mathrm{kg} / \mathrm{m3}\end{array}$ & $\underset{\mathrm{kg} / \mathrm{m} 3}{S I G-1.5}$ & $\begin{array}{l}S I G-3 \\
\mathrm{~kg} / \mathrm{m} 3\end{array}$ & $\underset{m}{D E}$ \\
\hline $\begin{array}{l}109 \\
220 \\
254\end{array}$ & $\begin{array}{l}18.575 \\
18.148 \\
17.078\end{array}$ & $\begin{array}{l}36.650 \\
36.556 \\
36.419\end{array}$ & $\begin{array}{l}5.26 \\
4.99 \\
4.75\end{array}$ & & & $\begin{array}{l}18.556 \\
18.109 \\
17.036\end{array}$ & $\begin{array}{l}26.398 \\
26.438 \\
26.596\end{array}$ & $\begin{array}{l}32.789 \\
32.841 \\
33.027\end{array}$ & $\begin{array}{l}38.971 \\
39.035 \\
39.247\end{array}$ & $\begin{array}{l}108 \\
218 \\
252\end{array}$ \\
\hline
\end{tabular}


ENDEAVOR 143 STA- 31
DATE $8 / 5 / 86$

$L A T=3150.1 \mathrm{~N} \quad$ LON= $3038.8 \mathrm{~W}$

SONIC DEPTH=

$\theta m$

\begin{tabular}{|c|c|c|c|c|c|c|c|c|c|c|}
\hline $\begin{array}{l}\text { PR } \\
\text { dbar }\end{array}$ & $\stackrel{T}{\operatorname{Deg} C}$ & $\begin{array}{c}S \\
0 / 00\end{array}$ & $\begin{array}{c}02 \\
\mathrm{ml} / \mathrm{l}\end{array}$ & $\stackrel{\theta}{\operatorname{Deg} C}$ & $\begin{array}{l}\mathrm{SIG}-0 \\
\mathrm{~kg} / \mathrm{m} 3\end{array}$ & $\underset{\mathrm{kg} / \mathrm{m} 3}{\mathrm{SIG}-1.5}$ & $\begin{array}{l}S I G-3 \\
\mathrm{~kg} / \mathrm{m} 3\end{array}$ & $\underset{\mathbf{m}}{\mathrm{HGTH}}$ & $\underset{c p h}{N}$ & $\mathrm{DE}$ \\
\hline $\begin{array}{r}1 \\
25 \\
50 \\
75 \\
100 \\
150 \\
200 \\
250 \\
300 \\
307\end{array}$ & $\begin{array}{l}20.161 \\
19.435 \\
18.904 \\
18.729 \\
18.686 \\
18.127 \\
17.282 \\
16.706 \\
15.966 \\
15.835\end{array}$ & $\begin{array}{l}36.642 \\
36.650 \\
36.637 \\
36.621 \\
36.626 \\
36.524 \\
36.381 \\
36.314 \\
36.196 \\
36.175\end{array}$ & $\begin{array}{l}5.27 \\
5.28 \\
5.17 \\
5.23 \\
5.03 \\
4.67 \\
4.40 \\
4.40 \\
4.37 \\
4.34\end{array}$ & $\begin{array}{l}20.161 \\
19.431 \\
18.895 \\
18.716 \\
18.669 \\
18.101 \\
17.249 \\
16.665 \\
15.918 \\
15.787\end{array}$ & $\begin{array}{l}25.973 \\
26.173 \\
26.301 \\
26.335 \\
26.351 \\
26.416 \\
26.516 \\
26.605 \\
26.688 \\
26.701\end{array}$ & $\begin{array}{l}32.327 \\
32.543 \\
32.684 \\
32.722 \\
32.739 \\
32.819 \\
32.941 \\
33.046 \\
33.149 \\
33.167\end{array}$ & $\begin{array}{l}38.474 \\
38.706 \\
38.859 \\
38.901 \\
38.919 \\
39.013 \\
39.157 \\
39.276 \\
39.399 \\
39.420\end{array}$ & $\begin{array}{l}0.002 \\
0.050 \\
0.094 \\
0.137 \\
0.179 \\
0.264 \\
0.345 \\
0.422 \\
0.496 \\
0.506\end{array}$ & $\begin{array}{l}1.79 \\
6.67 \\
2.42 \\
1.55 \\
1.47 \\
2.46 \\
2.48 \\
2.65 \\
2.00 \\
2.41\end{array}$ & $\begin{array}{r}1 \\
25 \\
50 \\
74 \\
99 \\
149 \\
199 \\
248 \\
298 \\
305\end{array}$ \\
\hline $\begin{array}{l}\text { PR } \\
\text { dbar }\end{array}$ & $\stackrel{T}{\operatorname{Deg} C}$ & $\frac{S}{0 / 00}$ & $\begin{array}{c}02 \\
\mathrm{ml} / \mathrm{l}\end{array}$ & $\begin{array}{c}\mathrm{F}-11 \\
\mathrm{pmol} / \mathrm{kg}\end{array}$ & $\begin{array}{c}\mathrm{F}-12 \\
\mathrm{gmol} / \mathrm{kg}\end{array}$ & $\stackrel{\theta}{\operatorname{Deg} C}$ & $\begin{array}{l}S I G-\theta \\
\mathrm{kg} / \mathrm{m} 3\end{array}$ & $\underset{\mathrm{kg} / \mathrm{m} 3}{\mathrm{SIG}-1.5}$ & $\begin{array}{l}S I G-3 \\
\mathrm{~kg} / \mathrm{m} 3\end{array}$ & \\
\hline $\begin{array}{l}101 \\
158 \\
220\end{array}$ & $\begin{array}{l}18.686 \\
17.894 \\
17.115\end{array}$ & $\begin{array}{l}36.634 \\
36.499 \\
36.376\end{array}$ & $\begin{array}{l}5.38 \\
5.03 \\
4.81\end{array}$ & & & $\begin{array}{l}18.668 \\
17.867 \\
17.078\end{array}$ & $\begin{array}{l}26.357 \\
26.455 \\
26.553\end{array}$ & $\begin{array}{l}32.746 \\
32.864 \\
32.983\end{array}$ & $\begin{array}{l}38.926 \\
39.064 \\
39.203\end{array}$ & \\
\hline
\end{tabular}

\begin{tabular}{|c|c|c|c|c|c|c|c|c|c|c|}
\hline \multicolumn{3}{|c|}{$\begin{array}{l}\text { ENDEAVOR } 143 \text { STA- } 32 \\
\text { DATE } 8 / 5 / 86\end{array}$} & LAT $=$ & \multicolumn{2}{|c|}{$=3159.8 \mathrm{~N}$} & \multicolumn{2}{|c|}{ ON= } & \multicolumn{2}{|c|}{ SONIC DEPTH } & \multirow{2}{*}{$\begin{array}{l}\theta \mathrm{m} \\
\underset{\mathrm{m}}{\mathrm{D}}\end{array}$} \\
\hline $\begin{array}{l}\text { PR } \\
\text { dbar }\end{array}$ & $\stackrel{T}{D e g} C$ & $\stackrel{S}{0 / 00}$ & $\begin{array}{l}02 \\
\mathrm{ml} / \mathrm{l}\end{array}$ & $\stackrel{\theta}{D e g} \mathrm{C}$ & $\begin{array}{l}\mathrm{SIG}-\mathrm{O} \\
\mathrm{kg} / \mathrm{m3}\end{array}$ & $\mathrm{SIG}_{\mathrm{kg} / \mathrm{m} 3}$ & $\begin{array}{l}S I G-3 \\
\mathrm{~kg} / \mathrm{m} 3\end{array}$ & $\underset{m}{\text { HGTH }}$ & $\underset{\text { cph }}{N}$ & \\
\hline $\begin{array}{r}5 \\
25 \\
50 \\
75 \\
100 \\
150 \\
200 \\
250 \\
300 \\
311\end{array}$ & $\begin{array}{l}20.157 \\
19.683 \\
19.416 \\
18.981 \\
18.419 \\
17.642 \\
17.154 \\
16.599 \\
15.969 \\
15.794\end{array}$ & $\begin{array}{l}36.678 \\
36.718 \\
36.728 \\
36.642 \\
36.532 \\
36.414 \\
36.363 \\
36.272 \\
36.188 \\
36.163\end{array}$ & $\begin{array}{l}5.52 \\
5.38 \\
5.41 \\
5.30 \\
5.23 \\
4.76 \\
4.47 \\
4.43 \\
4.47 \\
4.51\end{array}$ & $\begin{array}{l}20.156 \\
19.678 \\
19.407 \\
18.967 \\
18.402 \\
17.616 \\
17.120 \\
16.558 \\
15.921 \\
15.745\end{array}$ & $\begin{array}{l}26.002 \\
26.159 \\
26.239 \\
26.286 \\
26.346 \\
26.452 \\
26.533 \\
26.597 \\
26.681 \\
26.702\end{array}$ & $\begin{array}{l}32.356 \\
32.523 \\
32.609 \\
32.668 \\
32.742 \\
32.868 \\
32.962 \\
33.041 \\
33.142 \\
33.168\end{array}$ & $\begin{array}{l}38.502 \\
38.680 \\
38.771 \\
38.841 \\
38.929 \\
39.074 \\
39.181 \\
39.274 \\
39.392 \\
39.422\end{array}$ & $\begin{array}{l}0.010 \\
0.049 \\
0.095 \\
0.139 \\
0.182 \\
0.265 \\
0.345 \\
0.422 \\
0.497 \\
0.513\end{array}$ & $\begin{array}{l}1.20 \\
4.97 \\
2.55 \\
2.94 \\
2.51 \\
2.32 \\
2.25 \\
2.20 \\
2.08 \\
2.20\end{array}$ & $\begin{array}{r}5 \\
25 \\
50 \\
74 \\
99 \\
149 \\
199 \\
248 \\
298 \\
309\end{array}$ \\
\hline $\begin{array}{l}\text { PR } \\
\text { dbar }\end{array}$ & $\stackrel{T}{\text { Deg }} \mathrm{C}$ & $\begin{array}{l}S \\
0 / 00\end{array}$ & $\begin{array}{l}02 \\
\mathrm{ml} / \mathrm{I}\end{array}$ & $\begin{array}{c}\mathrm{F}-11 \\
\mathrm{pmol} / \mathrm{kg}\end{array}$ & $\begin{array}{c}\mathrm{F}-12 \\
\mathrm{pmol} / \mathrm{kg}\end{array}$ & $\stackrel{\theta}{\operatorname{Deg} C}$ & $\begin{array}{l}\mathrm{SIG}-\Theta \\
\mathrm{kg} / \mathrm{m3}\end{array}$ & $\underset{\mathrm{kg} / \mathrm{m} 3}{\mathrm{SIG}-1.5}$ & $\begin{array}{l}\mathrm{SIG}-3 \\
\mathrm{~kg} / \mathrm{m} 3\end{array}$ & $\begin{aligned} \mathrm{DE} \\
\mathrm{m}\end{aligned}$ \\
\hline $\begin{array}{r}90 \\
158 \\
193\end{array}$ & $\begin{array}{l}18.645 \\
17.600 \\
17.180\end{array}$ & $\begin{array}{l}36.581 \\
36.451 \\
36.370\end{array}$ & $\begin{array}{l}5.39 \\
4.98 \\
4.68\end{array}$ & & & $\begin{array}{l}18.629 \\
17.573 \\
17.148\end{array}$ & $\begin{array}{l}26.326 \\
26.491 \\
26.532\end{array}$ & $\begin{array}{l}32.716 \\
32.907 \\
32.960\end{array}$ & $\begin{array}{l}38.898 \\
39.114 \\
39.178\end{array}$ & $\begin{array}{r}89 \\
156 \\
191\end{array}$ \\
\hline
\end{tabular}

ENDEAVOR 143 STA- 33
DATE $8 / 5 / 86$

(1) 


\begin{tabular}{|c|c|c|c|c|c|c|c|c|c|c|}
\hline \multicolumn{3}{|c|}{$\begin{array}{l}\text { ENDEAVOR } 143 \text { STA- } 34 \\
\text { DATE } 8 / 5 / 86\end{array}$} & \multirow{2}{*}{$\begin{array}{l}\text { LAT }= \\
02 \\
\mathrm{ml} / 1\end{array}$} & \multicolumn{2}{|c|}{$=3219.9 \mathrm{~N}$} & \multicolumn{2}{|c|}{$O N=3039.1 W$} & \multicolumn{2}{|c|}{ SONIC DEPTH= } & \multirow{2}{*}{$\begin{array}{l}\text { Om } \\
\text { DE } \\
m\end{array}$} \\
\hline $\begin{array}{l}\text { PR } \\
\text { dbar }\end{array}$ & $\stackrel{T}{\operatorname{Deg}} \mathrm{C}$ & $\stackrel{S}{\circ / 00}$ & & $\stackrel{\theta}{\operatorname{Deg} C}$ & $\begin{array}{l}\mathrm{SIG}-\theta \\
\mathrm{kg} / \mathrm{m3}\end{array}$ & $\begin{array}{l}\mathrm{SIG}-1.5 \\
\mathrm{~kg} / \mathrm{m} 3\end{array}$ & $\begin{array}{l}\mathrm{SIG}-3 \\
\mathrm{~kg} / \mathrm{m} 3\end{array}$ & $\begin{array}{c}\text { HGTH } \\
\mathbf{m}\end{array}$ & $\underset{c p h}{N}$ & \\
\hline $\begin{array}{r}1 \\
25 \\
50 \\
75 \\
100 \\
150 \\
200 \\
250 \\
300 \\
305\end{array}$ & $\begin{array}{l}20.226 \\
19.072 \\
19.106 \\
18.901 \\
18.264 \\
17.372 \\
16.812 \\
16.193 \\
15.432 \\
15.407\end{array}$ & $\begin{array}{l}36.594 \\
36.578 \\
36.678 \\
36.649 \\
36.514 \\
36.379 \\
36.300 \\
36.200 \\
36.085 \\
36.081\end{array}$ & $\begin{array}{l}5.78 \\
5.79 \\
5.49 \\
5.38 \\
5.09 \\
4.81 \\
4.77 \\
4.63 \\
4.50 \\
4.51\end{array}$ & $\begin{array}{l}20.226 \\
19.067 \\
19.097 \\
18.888 \\
18.246 \\
17.347 \\
16.779 \\
16.152 \\
15.385 \\
15.359\end{array}$ & $\begin{array}{l}25.919 \\
26.211 \\
26.281 \\
26.312 \\
26.372 \\
26.490 \\
26.566 \\
26.637 \\
26.724 \\
26.726\end{array}$ & $\begin{array}{l}32.272 \\
32.591 \\
32.659 \\
32.695 \\
32.771 \\
32.914 \\
33.004 \\
33.092 \\
33.200 \\
33.203\end{array}$ & $\begin{array}{l}38.417 \\
38.763 \\
38.829 \\
38.870 \\
38.962 \\
39.127 \\
39.232 \\
39.336 \\
39.464 \\
39.468\end{array}$ & $\begin{array}{l}0.002 \\
0.050 \\
0.094 \\
0.137 \\
0.180 \\
0.261 \\
0.339 \\
0.415 \\
0.487 \\
0.494\end{array}$ & $\begin{array}{l}2.49 \\
6.44 \\
0.97 \\
2.30 \\
3.14 \\
2.38 \\
2.12 \\
2.34 \\
2.67 \\
0.90\end{array}$ & $\begin{array}{r}1 \\
25 \\
50 \\
74 \\
99 \\
149 \\
199 \\
248 \\
298 \\
303\end{array}$ \\
\hline $\begin{array}{l}\text { PR } \\
\text { dbar }\end{array}$ & $\stackrel{T}{T} C$ & 1 100 & $\begin{array}{ll}02 \\
m ! / 1\end{array}$ & $\begin{array}{c}\mathrm{F}-11 \\
\mathrm{pmol} / \mathrm{kg}\end{array}$ & $\begin{array}{c}\mathrm{F}-12 \\
\mathrm{pmol} / \mathrm{kg}\end{array}$ & $\stackrel{\theta}{\operatorname{Deg} C}$ & $\begin{array}{l}\mathrm{SIG}-0 \\
\mathrm{~kg} / \mathrm{m3}\end{array}$ & $\underset{\mathrm{kg} / \mathrm{m} 3}{\mathrm{SIG}-1.5}$ & $\begin{array}{l}\mathrm{SIG}-3 \\
\mathrm{~kg} / \mathrm{m} 3\end{array}$ & \\
\hline $\begin{array}{r}81 \\
105 \\
177\end{array}$ & $\begin{array}{l}18.860 \\
18.026 \\
17.163\end{array}$ & $\begin{array}{l}36.651 \\
36.482 \\
36.361\end{array}$ & $\begin{array}{l}5.27 \\
5.04 \\
4.72\end{array}$ & & & $\begin{array}{l}18.846 \\
18.007 \\
17.133\end{array}$ & $\begin{array}{l}26.325 \\
26.407 \\
26.528\end{array}$ & $\begin{array}{l}32.709 \\
32.813 \\
32.957\end{array}$ & $\begin{array}{l}38.885 \\
39.009 \\
39.175\end{array}$ & $\begin{array}{r}80 \\
104 \\
175\end{array}$ \\
\hline
\end{tabular}

\begin{tabular}{|c|c|c|c|c|c|c|c|c|c|c|}
\hline \multicolumn{3}{|c|}{$\begin{array}{l}\text { ENDEAVOR } 143 \text { STA- } 35 \\
\text { DATE } 8 / 5 / 86\end{array}$} & \multirow{2}{*}{$\begin{array}{l}\text { LAT }= \\
02 \\
\mathrm{ml} / \mathrm{l}\end{array}$} & \multicolumn{2}{|c|}{$3220.2 \mathrm{~N}$} & \multicolumn{2}{|c|}{ ON= } & \multicolumn{2}{|c|}{ SONIC DEPTH } & \multirow{2}{*}{$\begin{array}{l}\text { Om } \\
\text { DE } \\
\text { m }\end{array}$} \\
\hline $\begin{array}{l}\text { PR } \\
\text { dbar }\end{array}$ & $\stackrel{T}{\text { Deg } C}$ & $\stackrel{5}{0 / 00}$ & & $\stackrel{\theta}{\operatorname{Deg} C}$ & $\begin{array}{l}\text { SIG-O } \\
\mathrm{kg} / \mathrm{m3}\end{array}$ & $\begin{array}{l}\mathrm{SIG}-1.5 \\
\mathrm{~kg} / \mathrm{m} 3\end{array}$ & $\begin{array}{l}\mathrm{SIG}-3 \\
\mathrm{~kg} / \mathrm{m} 3\end{array}$ & $\underset{m}{\text { HGTH }}$ & $\underset{c p h}{N}$ & \\
\hline $\begin{array}{r}1 \\
25 \\
50 \\
75 \\
100 \\
150 \\
200 \\
250 \\
300 \\
305\end{array}$ & $\begin{array}{l}20.155 \\
19.429 \\
18.223 \\
17.884 \\
17.563 \\
17.087 \\
16.751 \\
16.185 \\
15.501 \\
15.455\end{array}$ & $\begin{array}{l}36.585 \\
36.549 \\
36.477 \\
36.431 \\
36.379 \\
36.317 \\
36.294 \\
36.208 \\
36.093 \\
36.085\end{array}$ & $\begin{array}{l}5.23 \\
5.37 \\
5.50 \\
5.19 \\
5.05 \\
4.85 \\
4.50 \\
4.52 \\
4.54 \\
4.52\end{array}$ & $\begin{array}{l}20.155 \\
19.425 \\
18.214 \\
17.871 \\
17.546 \\
17.062 \\
16.718 \\
16.144 \\
15.454 \\
15.407\end{array}$ & $\begin{array}{l}25.932 \\
26.097 \\
26.351 \\
26.401 \\
26.442 \\
26.512 \\
26.576 \\
26.644 \\
26.714 \\
26.719\end{array}$ & $\begin{array}{l}32.286 \\
32.468 \\
32.752 \\
32.811 \\
32.860 \\
32.943 \\
33.016 \\
33.100 \\
33.189 \\
33.195\end{array}$ & $\begin{array}{l}38.433 \\
38.632 \\
38.944 \\
39.011 \\
39.069 \\
39.163 \\
39.245 \\
39.344 \\
39.451 \\
39.458\end{array}$ & $\begin{array}{l}0.002 \\
0.051 \\
0.095 \\
0.136 \\
0.177 \\
0.256 \\
0.333 \\
0.408 \\
0.481 \\
0.488\end{array}$ & $\begin{array}{l}1.00 \\
7.35 \\
3.24 \\
2.20 \\
2.21 \\
2.14 \\
1.87 \\
2.08 \\
2.20 \\
1.70\end{array}$ & $\begin{array}{r}1 \\
25 \\
50 \\
74 \\
99 \\
149 \\
199 \\
248 \\
298 \\
303\end{array}$ \\
\hline $\begin{array}{l}P R \\
\text { dbar }\end{array}$ & $\stackrel{T}{\operatorname{Deg} C}$ & $\stackrel{5}{0 / 00}$ & $\begin{array}{l}02 \\
\mathrm{ml} / \mathrm{l}\end{array}$ & $\begin{array}{c}\mathrm{F}-11 \\
\mathrm{pmol} / \mathrm{kg}\end{array}$ & $\begin{array}{c}\mathrm{F}-12 \\
\mathrm{pmol} / \mathrm{kg}\end{array}$ & $\stackrel{\theta}{\operatorname{Deg} C}$ & $\begin{array}{l}\mathrm{SIG}-0 \\
\mathrm{~kg} / \mathrm{m} 3\end{array}$ & $\underset{\mathrm{kg} / \mathrm{m} 3}{\mathrm{SIG}-1} .^{5}$ & $\begin{array}{l}\mathrm{SIG}-3 \\
\mathrm{~kg} / \mathrm{m3}\end{array}$ & $\underset{m}{\mathrm{DE}}$ \\
\hline $\begin{array}{r}33 \\
51 \\
141\end{array}$ & $\begin{array}{l}18.690 \\
18.181 \\
17.140\end{array}$ & $\begin{array}{l}36.518 \\
36.471 \\
36.329\end{array}$ & $\begin{array}{l}5.54 \\
5.48 \\
5.09\end{array}$ & & & $\begin{array}{l}18.684 \\
18.172 \\
17.116\end{array}$ & $\begin{array}{l}26.264 \\
26.357 \\
26.508\end{array}$ & $\begin{array}{l}32.653 \\
32.759 \\
32.937\end{array}$ & $\begin{array}{l}38.834 \\
38.952 \\
39.156\end{array}$ & $\begin{array}{r}33 \\
51 \\
140\end{array}$ \\
\hline
\end{tabular}

\begin{tabular}{|c|c|c|c|c|c|c|c|c|c|c|}
\hline \multicolumn{3}{|c|}{$\begin{array}{l}\text { ENDEAVOR } 143 \text { STA- } 36 \\
\text { DATE } 8 / 5 / 86\end{array}$} & LAT $=$ & \multicolumn{2}{|c|}{$3210.0 \mathrm{~N}$} & \multicolumn{2}{|c|}{ ONE } & \multicolumn{2}{|c|}{ SONIC DEPTH } & \multirow{2}{*}{$\begin{array}{l}\text { Om } \\
\underset{m}{\mathrm{D}}\end{array}$} \\
\hline $\begin{array}{l}\text { PR } \\
\text { dbar }\end{array}$ & $\stackrel{T}{\text { Deg } C}$ & $\stackrel{S}{0 / 00}$ & $\begin{array}{l}02 \\
\mathrm{ml} / \mathrm{I}\end{array}$ & $\stackrel{\theta}{\operatorname{Deg}} \mathrm{C}$ & $\begin{array}{l}\mathrm{SIG}-0 \\
\mathrm{~kg} / \mathrm{m} 3\end{array}$ & $\mathrm{SIG}_{\mathrm{kg} / \mathrm{m} 3}^{-1} \cdot 5$ & $\begin{array}{l}\mathrm{SIG}-3 \\
\mathrm{~kg} / \mathrm{m} 3\end{array}$ & $\underset{m}{\text { HGTH }}$ & $\underset{c p h}{N}$ & \\
\hline $\begin{array}{r}1 \\
25 \\
50 \\
75 \\
100 \\
150 \\
200 \\
250 \\
300\end{array}$ & $\begin{array}{l}20.224 \\
20.030 \\
19.124 \\
18.926 \\
18.784 \\
17.785 \\
17.283 \\
16.788 \\
16.243\end{array}$ & $\begin{array}{l}36.609 \\
36.645 \\
36.638 \\
36.631 \\
36.607 \\
36.428 \\
36.366 \\
36.297 \\
36.215\end{array}$ & $\begin{array}{l}5.38 \\
5.17 \\
5.43 \\
5.27 \\
5.07 \\
4.74 \\
4.70 \\
4.60\end{array}$ & $\begin{array}{l}20.224 \\
20.026 \\
19.115 \\
18.912 \\
18.766 \\
17.759 \\
17.250 \\
16.747 \\
16.194\end{array}$ & $\begin{array}{l}25.931 \\
26.012 \\
26.245 \\
26.292 \\
26.312 \\
26.427 \\
26.504 \\
26.571 \\
26.638\end{array}$ & $\begin{array}{l}32.283 \\
32.368 \\
32.623 \\
32.675 \\
32.698 \\
32.840 \\
32.930 \\
33.010 \\
33.093\end{array}$ & $\begin{array}{l}38.429 \\
38.518 \\
38.793 \\
38.849 \\
38.876 \\
39.043 \\
39.145 \\
39.239 \\
39.335\end{array}$ & $\begin{array}{l}0.002 \\
0.051 \\
0.097 \\
0.141 \\
0.185 \\
0.270 \\
0.351 \\
0.429 \\
0.505\end{array}$ & $\begin{array}{l}2.48 \\
5.76 \\
3.39 \\
1.58 \\
2.33 \\
2.59 \\
2.12 \\
2.05 \\
2.02\end{array}$ & $\begin{array}{r}1 \\
25 \\
50 \\
74 \\
99 \\
149 \\
199 \\
248 \\
298\end{array}$ \\
\hline $\begin{array}{l}\text { PR } \\
\text { dbar }\end{array}$ & $\begin{array}{r}T \\
\text { Deg }\end{array}$ & $\stackrel{S}{1 / 00}$ & $\begin{array}{l}02 \\
\mathrm{ml} / \mathrm{l}\end{array}$ & $\begin{array}{c}\mathrm{F}-11 \\
\mathrm{pmol} / \mathrm{kg}\end{array}$ & $\begin{array}{c}\mathrm{F}-12 \\
\mathrm{pmol} / \mathrm{kg}\end{array}$ & $\stackrel{\theta}{D e g} C$ & $\begin{array}{l}\mathrm{SIG}-\theta \\
\mathrm{kg} / \mathrm{m} 3\end{array}$ & $\underset{\mathrm{kg} / \mathrm{m} 3}{\mathrm{SIG}-1.5}$ & $\begin{array}{l}\mathrm{SIG}-3 \\
\mathrm{~kg} / \mathrm{m} 3\end{array}$ & $\begin{aligned} \mathrm{DE} \\
\mathrm{m}\end{aligned}$ \\
\hline $\begin{array}{l}105 \\
128 \\
213\end{array}$ & $\begin{array}{l}18.594 \\
18.059 \\
17.041\end{array}$ & $\begin{array}{l}36.581 \\
36.482 \\
36.329\end{array}$ & $\begin{array}{l}5.10 \\
4.96 \\
4.89\end{array}$ & & & $\begin{array}{l}18.575 \\
18.037 \\
17.005\end{array}$ & $\begin{array}{l}26.340 \\
26.400 \\
26.535\end{array}$ & $\begin{array}{l}32.731 \\
32.805 \\
32.967\end{array}$ & $\begin{array}{l}38.914 \\
39.001 \\
39.188\end{array}$ & $\begin{array}{l}105 \\
126 \\
211\end{array}$ \\
\hline
\end{tabular}




\begin{tabular}{|c|c|c|c|c|c|c|c|c|c|c|}
\hline \multicolumn{3}{|c|}{$\begin{array}{l}\text { ENDEAVOR } 143 \text { STA- } 37 \\
\text { DATE } 8 / 5 / 86\end{array}$} & \multicolumn{2}{|c|}{ LAT $=$} & $0.0 \mathrm{~N}$ & \multicolumn{2}{|c|}{$N=3050.9 W$} & \multicolumn{2}{|c|}{ SONIC DEPTH= } & \multirow[t]{2}{*}{$\theta m$} \\
\hline $\begin{array}{l}\text { PR } \\
\text { dbar }\end{array}$ & $\stackrel{T}{T} \mathrm{C}$ & $\stackrel{5}{0 / 00}$ & $\begin{array}{l}02 \\
\mathrm{ml} / \mathrm{l}\end{array}$ & $\stackrel{\theta}{\operatorname{Deg} C}$ & $\begin{array}{l}\mathrm{SIG}-0 \\
\mathrm{~kg} / \mathrm{m3}\end{array}$ & $\begin{array}{l}\mathrm{SIG}-1.5 \\
\mathrm{~kg} / \mathrm{m3}\end{array}$ & $\begin{array}{l}\mathrm{SIG}-3 \\
\mathrm{~kg} / \mathrm{m3}\end{array}$ & $\underset{m}{\mathrm{HGTH}}$ & $\underset{c p h}{N}$ & \\
\hline $\begin{array}{r}1 \\
25 \\
50 \\
75 \\
100 \\
150 \\
200 \\
250 \\
300 \\
311\end{array}$ & $\begin{array}{l}20.231 \\
19.995 \\
19.163 \\
19.053 \\
18.751 \\
18.878 \\
17.399 \\
16.888 \\
16.421 \\
16.234\end{array}$ & $\begin{array}{l}36.723 \\
36.710 \\
36.658 \\
36.709 \\
36.631 \\
36.697 \\
36.387 \\
36.313 \\
36.261 \\
36.232\end{array}$ & $\begin{array}{l}5.47 \\
5.54 \\
5.58 \\
5.48 \\
5.34 \\
5.29 \\
4.89 \\
4.75 \\
4.60 \\
4.58\end{array}$ & $\begin{array}{l}20.231 \\
19.991 \\
19.154 \\
19.040 \\
18.733 \\
18.851 \\
17.365 \\
16.847 \\
16.372 \\
16.184\end{array}$ & $\begin{array}{l}26.016 \\
26.071 \\
26.250 \\
26.319 \\
26.338 \\
26.359 \\
26.492 \\
26.560 \\
26.633 \\
26.654\end{array}$ & $\begin{array}{l}32.368 \\
32.428 \\
32.627 \\
32.698 \\
32.725 \\
32.742 \\
32.915 \\
32.997 \\
33.082 \\
33.108\end{array}$ & $\begin{array}{l}38.512 \\
38.577 \\
38.796 \\
38.869 \\
38.903 \\
38.918 \\
39.127 \\
39.222 \\
39.319 \\
39.351\end{array}$ & $\begin{array}{l}0.002 \\
0.049 \\
0.095 \\
0.139 \\
0.182 \\
0.267 \\
0.351 \\
0.430 \\
0.506 \\
0.523\end{array}$ & $\begin{array}{l}1.94 \\
5.04 \\
3.18 \\
2.19 \\
1.32 \\
1.98 \\
2.19 \\
2.12 \\
2.14 \\
2.82\end{array}$ & $\begin{array}{r}1 \\
25 \\
50 \\
74 \\
99 \\
149 \\
199 \\
248 \\
298 \\
309\end{array}$ \\
\hline $\begin{array}{l}\text { PR } \\
\text { dbar }\end{array}$ & Deg $c$ & $\begin{array}{l}S \\
0 / 00\end{array}$ & 02 & $\begin{array}{l}\mathrm{F}-1 \\
\mathrm{pmol}\end{array}$ & $\begin{array}{c}\mathrm{F}-12 \\
\mathrm{pmol} / \mathrm{kg}\end{array}$ & $\stackrel{\theta}{D} \mathrm{C}$ & $\begin{array}{l}\mathrm{SIG}-9 \\
\mathrm{~kg} / \mathrm{m3}\end{array}$ & $\underset{\mathrm{kg} / \mathrm{m} 3}{\mathrm{SIG}-1.5}$ & $\begin{array}{l}\mathrm{sIg} \\
\mathrm{kg} /\end{array}$ & DE \\
\hline $\begin{array}{r}79 \\
126 \\
145 \\
217\end{array}$ & $\begin{array}{l}18.921 \\
18.675 \\
18.856 \\
17.250\end{array}$ & $\begin{array}{l}36.686 \\
36.626 \\
36.692 \\
36.375\end{array}$ & $\begin{array}{l}5.34 \\
5.13 \\
5.28 \\
4.89\end{array}$ & & & $\begin{array}{l}18.906 \\
18.652 \\
18.830 \\
17.214\end{array}$ & $\begin{array}{l}26.336 \\
26.355 \\
26.360 \\
26.520\end{array}$ & $\begin{array}{l}32.718 \\
32.744 \\
32.744 \\
32.946\end{array}$ & $\begin{array}{l}38.892 \\
38.924 \\
38.920 \\
39.162\end{array}$ & $\begin{array}{r}78 \\
125 \\
144 \\
215\end{array}$ \\
\hline
\end{tabular}

\begin{tabular}{|c|c|c|c|c|c|c|c|c|c|c|}
\hline $\begin{array}{l}\text { ENDEA } \\
\text { DATE }\end{array}$ & $\begin{array}{l}\text { AVOR } 143 \\
=8 / 5\end{array}$ & STA- 38 & LAT & 3150. & $.1 N$ & 3051 & $1.7 W$ & SONIC DE & PTH & $\theta m$ \\
\hline $\begin{array}{l}\text { PR } \\
\text { dbar }\end{array}$ & $\stackrel{T}{D e g} C$ & $\stackrel{S}{0 / 00}$ & $\begin{array}{l}02 \\
\mathrm{ml} / \mathrm{l}\end{array}$ & $\stackrel{\theta}{D e g} C$ & $\begin{array}{l}\mathrm{SIG}-0 \\
\mathrm{~kg} / \mathrm{m} 3\end{array}$ & $\mathrm{SIG}_{\mathrm{kg} / \mathrm{m} 3} \cdot 5$ & $\begin{array}{l}S I G-3 \\
\mathrm{~kg} / \mathrm{m} 3\end{array}$ & $\underset{\mathbf{m}}{\mathrm{HGTH}}$ & $\underset{c p h}{N}$ & $\begin{array}{r}\mathrm{DE} \\
\mathrm{m}\end{array}$ \\
\hline $\begin{array}{r}1 \\
25 \\
50 \\
75 \\
100 \\
150 \\
200 \\
250 \\
300 \\
307\end{array}$ & $\begin{array}{l}20.176 \\
19.899 \\
19.154 \\
18.964 \\
18.859 \\
18.800 \\
17.865 \\
17.224 \\
16.516 \\
16.497\end{array}$ & $\begin{array}{l}36.736 \\
36.730 \\
36.712 \\
36.704 \\
36.692 \\
36.685 \\
36.474 \\
36.379 \\
36.285 \\
36.282\end{array}$ & $\begin{array}{l}5.09 \\
5.25 \\
5.35 \\
5.33 \\
5.27 \\
5.15 \\
4.78 \\
4.58 \\
4.58 \\
4.58\end{array}$ & $\begin{array}{l}20.175 \\
19.894 \\
19.145 \\
18.951 \\
18.841 \\
18.773 \\
17.830 \\
17.182 \\
16.467 \\
16.446\end{array}$ & $\begin{array}{l}26.041 \\
26.111 \\
26.294 \\
26.338 \\
26.357 \\
26.369 \\
26.445 \\
26.531 \\
26.629 \\
26.631\end{array}$ & $\begin{array}{l}32.393 \\
32.470 \\
32.670 \\
32.719 \\
32.741 \\
32.755 \\
32.855 \\
32.958 \\
33.075 \\
33.078\end{array}$ & $\begin{array}{l}38.539 \\
38.622 \\
38.839 \\
38.892 \\
38.917 \\
38.932 \\
39.056 \\
39.175 \\
39.310 \\
39.314\end{array}$ & $\begin{array}{l}0.002 \\
0.049 \\
0.094 \\
0.137 \\
0.179 \\
0.264 \\
0.349 \\
0.430 \\
0.506 \\
0.517\end{array}$ & $\begin{array}{l}0.94 \\
4.88 \\
3.70 \\
1.82 \\
0.99 \\
1.50 \\
2.48 \\
2.64 \\
1.98 \\
1.00\end{array}$ & $\begin{array}{r}1 \\
25 \\
50 \\
74 \\
99 \\
149 \\
199 \\
248 \\
298 \\
305\end{array}$ \\
\hline $\begin{array}{l}\text { PR } \\
\text { dbar }\end{array}$ & $\stackrel{T}{D e g} C$ & $\begin{array}{c}S \\
0 / 00\end{array}$ & $\begin{array}{l}02 \\
\mathrm{ml} / \mathrm{l}\end{array}$ & $\begin{array}{c}\mathrm{F}-11 \\
\mathrm{pmol} / \mathrm{kg}\end{array}$ & $\begin{array}{c}\mathrm{F}-12 \\
\mathrm{pmol} / \mathrm{kg}\end{array}$ & $\stackrel{\theta}{\operatorname{Deg} C}$ & $\begin{array}{l}S I G-\theta \\
\mathrm{kg} / \mathrm{m} 3\end{array}$ & $\underset{\mathrm{kg} / \mathrm{m} 3}{\mathrm{SIG}-1.5}$ & $\begin{array}{l}S I G-3 \\
\mathrm{~kg} / \mathrm{m} 3\end{array}$ & $\begin{array}{r}\mathrm{DE} \\
\mathbf{m}\end{array}$ \\
\hline $\begin{array}{l}128 \\
161 \\
249\end{array}$ & $\begin{array}{l}18.818 \\
18.494 \\
17.099\end{array}$ & $\begin{array}{l}36.693 \\
36.621 \\
36.363\end{array}$ & $\begin{array}{l}5.27 \\
5.16 \\
4.70\end{array}$ & & & $\begin{array}{l}18.795 \\
18.466 \\
17.057\end{array}$ & $\begin{array}{l}26.370 \\
26.398 \\
26.548\end{array}$ & $\begin{array}{l}32.755 \\
32.792 \\
32.979\end{array}$ & $\begin{array}{l}38.931 \\
38.977 \\
39.199\end{array}$ & $\begin{array}{l}127 \\
160 \\
247\end{array}$ \\
\hline
\end{tabular}

\begin{tabular}{|c|c|c|c|c|c|c|c|c|c|c|}
\hline $\begin{array}{l}\text { END } \\
\text { DA }\end{array}$ & $\begin{array}{l}\text { OR } 143 \\
8 / 5\end{array}$ & STA- 39 & LA & 3140. & LON & 3051 & $2 W$ & SONIC DE & $\mathrm{TH}=$ & $\theta m$ \\
\hline $\begin{array}{l}\text { PR } \\
\text { dbar }\end{array}$ & $\stackrel{T}{T} C$ & $\begin{array}{c}S \\
0 / 00\end{array}$ & $\begin{array}{c}02 \\
\mathrm{ml} / \mathrm{l}\end{array}$ & $\stackrel{\ominus}{\operatorname{Deg}} \mathrm{C}$ & $\begin{array}{l}S I G-0 \\
\mathrm{~kg} / \mathrm{m} 3\end{array}$ & $\underset{\mathrm{kg} / \mathrm{m} 3}{\mathrm{SIG}-1.5}$ & $\begin{array}{l}\mathrm{SIG}-3 \\
\mathrm{~kg} / \mathrm{m3}\end{array}$ & $\underset{\mathrm{m}}{\text { HGTH }}$ & $\underset{c p h}{N}$ & $D E$ \\
\hline $\begin{array}{r}3 \\
25 \\
50 \\
75 \\
100 \\
150 \\
200 \\
250 \\
300 \\
309\end{array}$ & $\begin{array}{l}20.034 \\
19.798 \\
18.945 \\
18.708 \\
18.622 \\
18.567 \\
18.496 \\
17.793 \\
16.833 \\
16.697\end{array}$ & $\begin{array}{l}36.723 \\
36.709 \\
36.667 \\
36.656 \\
36.652 \\
36.649 \\
36.633 \\
36.480 \\
36.325 \\
36.302\end{array}$ & $\begin{array}{l}5.16 \\
5.22 \\
5.37 \\
5.32 \\
5.28 \\
5.17 \\
5.10 \\
4.72 \\
4.53 \\
4.55\end{array}$ & $\begin{array}{l}20.034 \\
19.793 \\
18.936 \\
18.695 \\
18.605 \\
18.541 \\
18.461 \\
17.750 \\
16.783 \\
16.646\end{array}$ & $\begin{array}{l}26.069 \\
26.122 \\
26.313 \\
26.367 \\
26.387 \\
26.401 \\
26.409 \\
26.469 \\
26.584 \\
26.600\end{array}$ & $\begin{array}{l}32.425 \\
32.483 \\
32.695 \\
32.754 \\
32.777 \\
32.792 \\
32.802 \\
32.882 \\
33.022 \\
33.041\end{array}$ & $\begin{array}{l}38.574 \\
38.638 \\
38.869 \\
38.934 \\
38.958 \\
38.975 \\
38.987 \\
39.084 \\
39.249 \\
39.272\end{array}$ & $\begin{array}{l}0.006 \\
0.048 \\
0.093 \\
0.135 \\
0.177 \\
0.261 \\
0.345 \\
0.429 \\
0.509 \\
0.523\end{array}$ & $\begin{array}{l}0.62 \\
4.69 \\
3.62 \\
2.45 \\
1.05 \\
0.84 \\
0.61 \\
2.98 \\
2.31 \\
2.58\end{array}$ & $\begin{array}{r}3 \\
25 \\
50 \\
74 \\
99 \\
149 \\
199 \\
248 \\
298 \\
307\end{array}$ \\
\hline $\begin{array}{l}\text { PR } \\
\text { dbar }\end{array}$ & $\stackrel{T}{D e g} C$ & $\stackrel{S}{\circ}$ & $\begin{array}{l}02 \\
\mathrm{ml} / \mathrm{l}\end{array}$ & $\begin{array}{c}\mathrm{F}-1 \mathrm{q} \\
\mathrm{pmol} / \mathrm{kg}\end{array}$ & $\begin{array}{c}\mathrm{F}-12 \\
\mathrm{pmol} / \mathrm{kg}\end{array}$ & $\stackrel{\theta}{\operatorname{Deg}} \mathrm{C}$ & $\begin{array}{l}\mathrm{SIG}-0 \\
\mathrm{~kg} / \mathrm{m} 3\end{array}$ & $\underset{\mathrm{kg} / \mathrm{m} 3}{\mathrm{SIG}-1.5}$ & $\begin{array}{l}S I G-3 \\
\mathrm{~kg} / \mathrm{m} 3\end{array}$ & \\
\hline $\begin{array}{l}143 \\
235 \\
272\end{array}$ & $\begin{array}{l}18.561 \\
18.217 \\
17.227\end{array}$ & $\begin{array}{l}36.650 \\
36.594 \\
36.392\end{array}$ & $\begin{array}{l}5.24 \\
5.14 \\
4.69\end{array}$ & & & $\begin{array}{l}18.535 \\
18.175 \\
17.181\end{array}$ & $\begin{array}{l}26.403 \\
26.451 \\
26.541\end{array}$ & $\begin{array}{l}32.795 \\
32.852 \\
32.968\end{array}$ & $\begin{array}{l}38.977 \\
39.043 \\
39.185\end{array}$ & $\begin{array}{l}142 \\
233 \\
270\end{array}$ \\
\hline
\end{tabular}




\begin{tabular}{|c|c|c|c|c|c|c|c|c|c|c|}
\hline \multicolumn{3}{|c|}{$\begin{array}{l}\text { ENDEAVOR } 143 \text { STA- } 40 \\
\text { DATE } 8 / 5 / 86\end{array}$} & LAT $=$ & \multicolumn{2}{|c|}{$=3130.1 \mathrm{~N}$} & \multicolumn{2}{|c|}{$\mathrm{ON}=3051.0 \mathrm{~W}$} & \multicolumn{2}{|c|}{ SONIC DEPTH= } & \multirow{2}{*}{$\begin{array}{l}\text { Om } \\
\underset{m}{\mathrm{DE}}\end{array}$} \\
\hline $\begin{array}{l}\text { PR } \\
\text { dbar }\end{array}$ & $\stackrel{T}{T} C$ & $\stackrel{S}{0 / 00}$ & $\begin{array}{l}02 \\
\mathrm{ml} / \mathrm{l}\end{array}$ & $\stackrel{\ominus}{\operatorname{Deg} C}$ & $\begin{array}{l}\mathrm{SIG}-\theta \\
\mathrm{kg} / \mathrm{m} 3\end{array}$ & $\begin{array}{l}\mathrm{SIG}-1.5 \\
\mathrm{~kg} / \mathrm{m} 3\end{array}$ & $\begin{array}{l}\mathrm{SIG}-3 \\
\mathrm{~kg} / \mathrm{m} 3\end{array}$ & $\underset{m}{H G T H}$ & $\underset{c p h}{N}$ & \\
\hline $\begin{array}{r}1 \\
25 \\
50 \\
75 \\
100 \\
150 \\
200 \\
250 \\
300 \\
309\end{array}$ & $\begin{array}{l}20.072 \\
19.823 \\
19.099 \\
18.766 \\
18.691 \\
18.561 \\
18.251 \\
17.455 \\
16.737 \\
16.668\end{array}$ & $\begin{array}{l}36.694 \\
36.699 \\
36.654 \\
36.642 \\
36.666 \\
36.641 \\
36.564 \\
36.424 \\
36.312 \\
36.300\end{array}$ & $\begin{array}{l}5.15 \\
5.33 \\
5.46 \\
5.38 \\
5.26 \\
5.21 \\
5.06 \\
4.70 \\
4.63 \\
4.64\end{array}$ & $\begin{array}{l}20.071 \\
19.819 \\
19.090 \\
18.752 \\
18.673 \\
18.534 \\
18.215 \\
17.413 \\
16.687 \\
16.617\end{array}$ & $\begin{array}{l}26.037 \\
26.108 \\
26.264 \\
26.342 \\
26.380 \\
26.396 \\
26.418 \\
26.509 \\
26.598 \\
26.604\end{array}$ & $\begin{array}{l}32.392 \\
32.469 \\
32.642 \\
32.728 \\
32.768 \\
32.788 \\
32.818 \\
32.930 \\
33.038 \\
33.047\end{array}$ & $\begin{array}{l}38.540 \\
38.623 \\
38.813 \\
38.906 \\
38.948 \\
38.971 \\
39.009 \\
39.141 \\
39.268 \\
39.278\end{array}$ & $\begin{array}{l}0.002 \\
0.049 \\
0.094 \\
0.137 \\
0.180 \\
0.263 \\
0.348 \\
0.430 \\
0.509 \\
0.522\end{array}$ & $\begin{array}{l}0.65 \\
5.33 \\
2.88 \\
2.80 \\
1.33 \\
0.88 \\
1.87 \\
2.41 \\
2.43 \\
1.54\end{array}$ & $\begin{array}{r}1 \\
25 \\
50 \\
74 \\
99 \\
149 \\
199 \\
248 \\
298 \\
307\end{array}$ \\
\hline $\begin{array}{l}\text { PR } \\
\text { dbar }\end{array}$ & $\stackrel{T}{\text { Deg } C}$ & $\stackrel{5}{0 / 00}$ & $\begin{array}{l}02 \\
\mathrm{ml} / \mathrm{l}\end{array}$ & $\begin{array}{c}\mathrm{F}-11 \\
\mathrm{pmol} / \mathrm{kg}\end{array}$ & $\begin{array}{c}\mathrm{F}-12 \\
\mathrm{pmol} / \mathrm{kg}\end{array}$ & $\stackrel{\theta}{\text { Deg } C}$ & $\begin{array}{l}\mathrm{SIG}-0 \\
\mathrm{~kg} / \mathrm{m3}\end{array}$ & $\underset{\mathrm{kg} / \mathrm{m} 3}{\mathrm{SIG}-1.5}$ & $\begin{array}{l}\mathrm{SIG}-3 \\
\mathrm{~kg} / \mathrm{m} 3\end{array}$ & DE \\
\hline $\begin{array}{r}77 \\
160 \\
265\end{array}$ & $\begin{array}{l}18.676 \\
18.526 \\
17.250\end{array}$ & $\begin{array}{l}36.638 \\
36.634 \\
36.394\end{array}$ & $\begin{array}{l}5.36 \\
5.19 \\
5.00\end{array}$ & & & $\begin{array}{l}18.662 \\
18.497 \\
17.205\end{array}$ & $\begin{array}{l}26.362 \\
26.400 \\
26.536\end{array}$ & $\begin{array}{l}32.750 \\
32.793 \\
32.963\end{array}$ & $\begin{array}{l}38.930 \\
38.977 \\
39.179\end{array}$ & $\begin{array}{r}76 \\
158 \\
263\end{array}$ \\
\hline
\end{tabular}

ENDEAVOR 143 STA- 41
DATE $9 / 5 / 86$

\begin{tabular}{|c|c|c|c|c|c|c|c|c|c|c|}
\hline $\begin{array}{c}P R \\
\text { dbar }\end{array}$ & $\stackrel{T}{\text { Deg }} C$ & $\begin{array}{c}S \\
0 / 00\end{array}$ & $\begin{array}{c}02 \\
\mathrm{ml} / \mathrm{l}\end{array}$ & $\stackrel{\theta}{\text { Deg } C}$ & $\begin{array}{l}\mathrm{SIG}-\Theta \\
\mathrm{kg} / \mathrm{m3}\end{array}$ & $\underset{\mathrm{kg} / \mathrm{m} 3}{\mathrm{SIG}-1.5}$ & $\begin{array}{l}\mathrm{SIG}-3 \\
\mathrm{~kg} / \mathrm{m} 3\end{array}$ & $\underset{\mathbf{m}}{\mathrm{HGTH}}$ & $\begin{array}{c}N \\
c p h\end{array}$ & \\
\hline $\begin{array}{r}1 \\
25 \\
50 \\
75 \\
100 \\
150 \\
200 \\
250 \\
300 \\
305\end{array}$ & $\begin{array}{l}20.079 \\
19.825 \\
18.797 \\
18.672 \\
18.709 \\
18.192 \\
17.325 \\
16.836 \\
16.209 \\
16.085\end{array}$ & $\begin{array}{l}36.722 \\
36.687 \\
36.614 \\
36.621 \\
36.656 \\
36.527 \\
36.376 \\
36.312 \\
36.233 \\
36.211\end{array}$ & $\begin{array}{l}5.04 \\
5.20 \\
5.36 \\
5.30 \\
5.18 \\
5.13 \\
4.55 \\
4.43 \\
4.48 \\
4.50\end{array}$ & $\begin{array}{l}20.079 \\
19.820 \\
18.789 \\
18.659 \\
18.692 \\
18.166 \\
17.292 \\
16.795 \\
16.161 \\
16.036\end{array}$ & $\begin{array}{l}26.057 \\
26.099 \\
26.311 \\
26.349 \\
26.368 \\
26.402 \\
26.502 \\
26.572 \\
26.660 \\
26.672\end{array}$ & $\begin{array}{l}32.411 \\
32.460 \\
32.696 \\
32.738 \\
32.756 \\
32.804 \\
32.926 \\
33.010 \\
33.115 \\
33.130\end{array}$ & $\begin{array}{l}38.559 \\
38.613 \\
38.874 \\
38.918 \\
38.935 \\
38.996 \\
39.141 \\
39.236 \\
39.358 \\
39.377\end{array}$ & $\begin{array}{l}0.002 \\
0.049 \\
0.093 \\
0.136 \\
0.178 \\
0.263 \\
0.344 \\
0.423 \\
0.498 \\
0.506\end{array}$ & $\begin{array}{r}-0.97 \\
5.34 \\
3.32 \\
1.72 \\
1.28 \\
2.23 \\
2.55 \\
2.02 \\
2.69 \\
2.80\end{array}$ & $\begin{array}{l}1 \\
1 \\
2 \\
2 \\
3\end{array}$ \\
\hline $\begin{array}{c}\text { PR } \\
\text { dbar }\end{array}$ & $\stackrel{T}{D e g} C$ & $\begin{array}{c}S \\
0 / 00\end{array}$ & $\begin{array}{c}02 \\
\mathrm{ml} / \mathrm{l}\end{array}$ & $\begin{array}{c}\mathrm{F}-11 \\
\mathrm{pmol} / \mathrm{kg}\end{array}$ & $\begin{array}{c}\mathrm{F}-12 \\
\mathrm{pmol} / \mathrm{kg}\end{array}$ & $\stackrel{\theta}{\operatorname{Deg} C}$ & $\begin{array}{l}\mathrm{SIG}-\theta \\
\mathrm{kg} / \mathrm{m} 3\end{array}$ & $\underset{\mathrm{kg} / \mathrm{m} 3}{S I G-1} 5$ & $\begin{array}{l}S I G-3 \\
\mathrm{~kg} / \mathrm{m3}\end{array}$ & \\
\hline $\begin{array}{r}57 \\
156 \\
211\end{array}$ & $\begin{array}{l}18.746 \\
18.036 \\
17.159\end{array}$ & $\begin{array}{l}36.617 \\
36.501 \\
36.352\end{array}$ & $\begin{array}{l}5.46 \\
5.18 \\
4.78\end{array}$ & & & $\begin{array}{l}18.735 \\
18.009 \\
17.124\end{array}$ & $\begin{array}{l}26.327 \\
26.421 \\
26.524\end{array}$ & $\begin{array}{l}32.714 \\
32.827 \\
32.953\end{array}$ & $\begin{array}{l}38.892 \\
39.023 \\
39.171\end{array}$ & \\
\hline
\end{tabular}

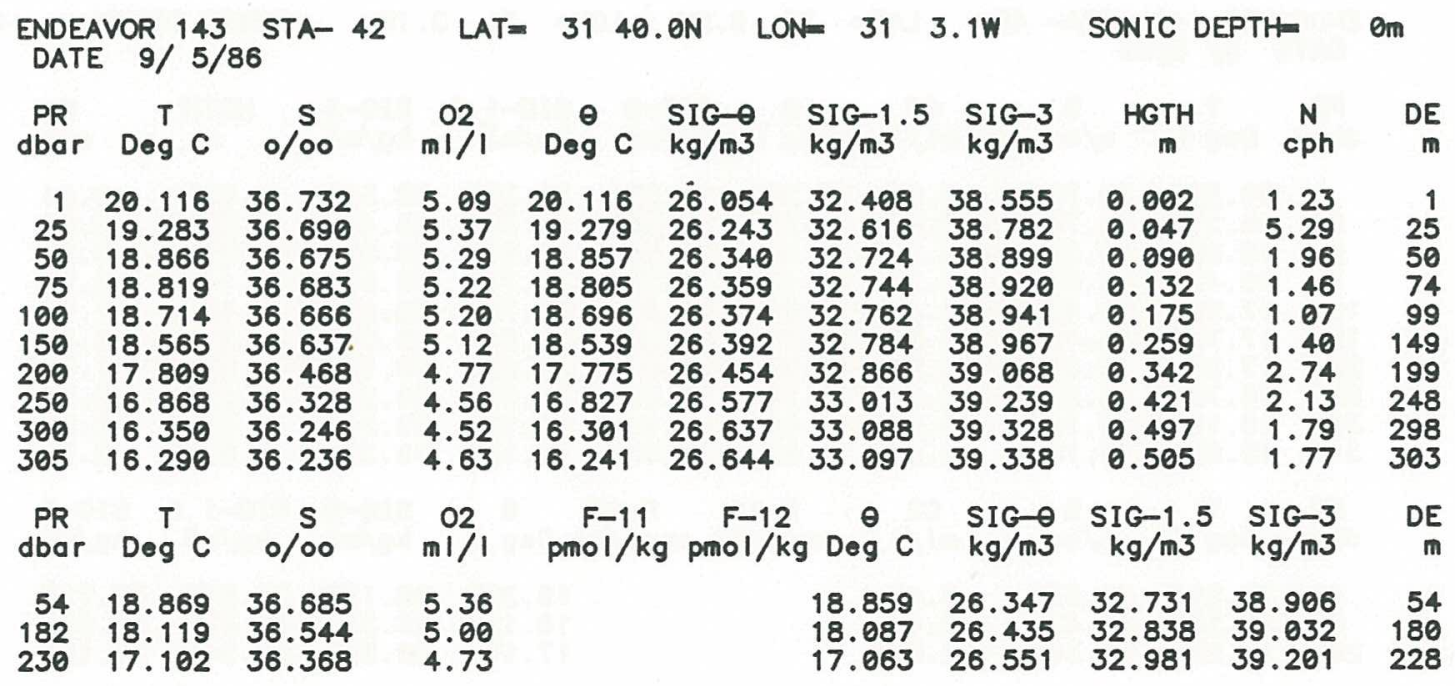




\begin{tabular}{|c|c|c|c|c|c|c|c|c|c|c|}
\hline \multicolumn{3}{|c|}{$\begin{array}{l}\text { ENDEAVOR } 143 \text { STA- } 43 \\
\text { DATE } 9 / 5 / 86\end{array}$} & LAT $=$ & \multicolumn{2}{|c|}{$=3150.0 \mathrm{~N}$} & \multicolumn{2}{|c|}{$\mathrm{ON}=$} & \multicolumn{2}{|c|}{ SONIC DEPTH= } & \multirow{2}{*}{$\begin{array}{l}\text { Om } \\
\underset{m}{\mathrm{DE}}\end{array}$} \\
\hline $\begin{array}{l}\text { PR } \\
\text { dbar }\end{array}$ & $\stackrel{T}{\operatorname{Teg} C}$ & $\frac{5}{0 / 00}$ & $\mathrm{O} 2$ & $\stackrel{\theta}{\operatorname{Deg} C}$ & $\begin{array}{l}\mathrm{SIG}-\theta \\
\mathrm{kg} / \mathrm{m} 3\end{array}$ & $\begin{array}{l}\mathrm{SIG}-1.5 \\
\mathrm{~kg} / \mathrm{m} 3\end{array}$ & $\begin{array}{l}\mathrm{SIG}-3 \\
\mathrm{~kg} / \mathrm{m} 3\end{array}$ & $\underset{m}{\mathrm{HGTH}}$ & $\underset{\mathrm{cph}}{\mathrm{N}}$ & \\
\hline $\begin{array}{r}3 \\
25 \\
50 \\
75 \\
100 \\
150 \\
200 \\
250 \\
300\end{array}$ & $\begin{array}{l}19.980 \\
19.674 \\
19.014 \\
18.811 \\
18.709 \\
18.615 \\
18.170 \\
17.147 \\
16.342\end{array}$ & $\begin{array}{l}36.731 \\
36.715 \\
36.675 \\
36.671 \\
36.665 \\
36.658 \\
36.551 \\
36.376 \\
36.253\end{array}$ & $\begin{array}{l}5.05 \\
5.09 \\
5.25 \\
5.22 \\
5.17 \\
5.11 \\
4.77 \\
4.50 \\
4.51\end{array}$ & $\begin{array}{l}19.979 \\
19.669 \\
19.005 \\
18.798 \\
18.691 \\
18.588 \\
18.135 \\
17.105 \\
16.293\end{array}$ & $\begin{array}{l}26.089 \\
26.159 \\
26.302 \\
26.352 \\
26.375 \\
26.395 \\
26.428 \\
26.547 \\
26.645\end{array}$ & $\begin{array}{l}32.446 \\
32.524 \\
32.682 \\
32.737 \\
32.763 \\
32.786 \\
32.830 \\
32.976 \\
33.096\end{array}$ & $\begin{array}{l}38.596 \\
38.680 \\
38.854 \\
38.914 \\
38.942 \\
38.967 \\
39.023 \\
39.195 \\
39.336\end{array}$ & $\begin{array}{l}0.006 \\
0.048 \\
0.092 \\
0.135 \\
0.177 \\
0.261 \\
0.345 \\
0.426 \\
0.502\end{array}$ & $\begin{array}{l}0.61 \\
5.50 \\
2.56 \\
2.33 \\
1.38 \\
0.93 \\
2.35 \\
2.75 \\
2.58\end{array}$ & $\begin{array}{r}3 \\
25 \\
50 \\
74 \\
99 \\
149 \\
199 \\
248 \\
298\end{array}$ \\
\hline $\begin{array}{l}P R \\
\text { dbar }\end{array}$ & $\stackrel{T}{\text { Deg }} \mathrm{C}$ & $1 / 00$ & $\begin{array}{ll}02 \\
m i / 1\end{array}$ & $\begin{array}{c}\mathrm{F}-11 \\
\mathrm{pmol} / \mathrm{kg}\end{array}$ & $\begin{array}{c}\mathrm{F}-12 \\
\mathrm{pmol} / \mathrm{kg}\end{array}$ & $\stackrel{\theta}{\operatorname{Deg} C}$ & $\underset{\mathrm{kg} / \mathrm{m} 3}{\mathrm{SIG}-\theta}$ & $\underset{\mathrm{kg} / \mathrm{m} 3}{\mathrm{SIG}-1.5}$ & $\begin{array}{l}\mathrm{SIG}-3 \\
\mathrm{~kg} / \mathrm{m} 3\end{array}$ & \\
\hline $\begin{array}{r}87 \\
201 \\
245\end{array}$ & $\begin{array}{l}18.736 \\
18.117 \\
17.172\end{array}$ & $\begin{array}{l}36.669 \\
36.548 \\
36.382\end{array}$ & $\begin{array}{l}5.38 \\
4.99 \\
4.69\end{array}$ & & & $\begin{array}{l}18.721 \\
18.082 \\
17.131\end{array}$ & $\begin{array}{l}26.370 \\
26.439 \\
26.545\end{array}$ & $\begin{array}{l}32.757 \\
32.842 \\
32.974\end{array}$ & $\begin{array}{l}38.936 \\
39.037 \\
39.192\end{array}$ & $\begin{array}{r}86 \\
199 \\
242\end{array}$ \\
\hline
\end{tabular}

\begin{tabular}{|c|c|c|c|c|c|c|c|c|c|c|}
\hline $\begin{array}{r}\text { END } \\
\text { DA }\end{array}$ & $\begin{array}{c}\text { OR } 143 \\
9 / 5\end{array}$ & STA- 44 & LAT: & 32 & LON & 31 & $3.1 w$ & SONIC DE & $\mathrm{TH}$ & $\theta m$ \\
\hline $\begin{array}{l}\text { PR } \\
\text { dbar }\end{array}$ & $\stackrel{T}{\text { Deg }} C$ & $\begin{array}{c}5 \\
0 / 00\end{array}$ & $\begin{array}{l}02 \\
\mathrm{ml} / \mathrm{l}\end{array}$ & $\stackrel{\theta}{\operatorname{Deg}} \mathrm{C}$ & $\begin{array}{l}S I G-\theta \\
\mathrm{kg} / \mathrm{m} 3\end{array}$ & $\underset{\mathrm{kg} / \mathrm{m3}}{\mathrm{SIG}-1.5}$ & $\begin{array}{l}\mathrm{SIG}-3 \\
\mathrm{~kg} / \mathrm{m} 3\end{array}$ & $\begin{array}{c}\text { HGTH } \\
\text { m }\end{array}$ & $\underset{c p h}{N}$ & $\begin{array}{c}\mathrm{DE} \\
\mathrm{m}\end{array}$ \\
\hline $\begin{array}{r}5 \\
25 \\
50 \\
75 \\
100 \\
150 \\
200 \\
250 \\
300 \\
305\end{array}$ & $\begin{array}{l}19.972 \\
19.761 \\
19.060 \\
18.944 \\
18.888 \\
18.769 \\
17.942 \\
17.058 \\
16.518 \\
16.432\end{array}$ & $\begin{array}{l}36.751 \\
36.734 \\
36.707 \\
36.708 \\
36.704 \\
36.676 \\
36.503 \\
36.361 \\
36.284 \\
36.269\end{array}$ & $\begin{array}{l}5.11 \\
5.16 \\
5.27 \\
5.23 \\
5.16 \\
5.09 \\
4.78 \\
4.57 \\
4.55 \\
4.53\end{array}$ & $\begin{array}{l}19.971 \\
19.757 \\
19.051 \\
18.931 \\
18.870 \\
18.743 \\
17.908 \\
17.017 \\
16.469 \\
16.383\end{array}$ & $\begin{array}{l}26.107 \\
26.151 \\
26.315 \\
26.347 \\
26.359 \\
26.370 \\
26.448 \\
26.557 \\
26.627 \\
26.636\end{array}$ & $\begin{array}{l}32.464 \\
32.513 \\
32.694 \\
32.728 \\
32.742 \\
32.756 \\
32.856 \\
32.988 \\
33.073 \\
33.085\end{array}$ & $\begin{array}{l}38.614 \\
38.668 \\
38.864 \\
38.902 \\
38.917 \\
38.934 \\
39.055 \\
39.209 \\
39.309 \\
39.322\end{array}$ & $\begin{array}{l}0.009 \\
0.047 \\
0.092 \\
0.134 \\
0.177 \\
0.262 \\
0.346 \\
0.426 \\
0.502 \\
0.510\end{array}$ & $\begin{array}{r}-0.83 \\
5.24 \\
3.03 \\
1.52 \\
0.89 \\
1.40 \\
2.70 \\
2.50 \\
1.89 \\
2.85\end{array}$ & $\begin{array}{r}5 \\
25 \\
50 \\
74 \\
99 \\
149 \\
199 \\
248 \\
298 \\
303\end{array}$ \\
\hline $\begin{array}{c}\text { PR } \\
\text { dbar }\end{array}$ & $\stackrel{T}{\text { Deg } C}$ & $\stackrel{S}{0 / 00}$ & $\begin{array}{c}02 \\
\mathrm{ml} / \mathrm{l}\end{array}$ & $\begin{array}{c}\mathrm{F}-11 \\
\mathrm{pmol} / \mathrm{kg}\end{array}$ & $\begin{array}{c}\mathrm{F}-12 \\
\mathrm{pmol} / \mathrm{kg}\end{array}$ & $\stackrel{\theta}{\operatorname{Deg} C}$ & $\begin{array}{l}S I G-\Theta \\
\mathrm{kg} / \mathrm{m3}\end{array}$ & $\underset{\mathrm{kg} / \mathrm{m} 3}{S I G-1} 5$ & $\begin{array}{l}S I G-3 \\
\mathrm{~kg} / \mathrm{m} 3\end{array}$ & $D E$ \\
\hline $\begin{array}{l}104 \\
188 \\
241\end{array}$ & $\begin{array}{l}18.874 \\
18.107 \\
17.212\end{array}$ & $\begin{array}{l}36.703 \\
36.536 \\
36.385\end{array}$ & $\begin{array}{l}5.29 \\
4.96 \\
4.73\end{array}$ & & & $\begin{array}{l}18.856 \\
18.074 \\
17.172\end{array}$ & $\begin{array}{l}26.362 \\
26.432 \\
26.537\end{array}$ & $\begin{array}{l}32.745 \\
32.835 \\
32.965\end{array}$ & $\begin{array}{l}38.920 \\
39.030 \\
39.182\end{array}$ & $\begin{array}{l}103 \\
186 \\
239\end{array}$ \\
\hline
\end{tabular}

\begin{tabular}{|c|c|c|c|c|c|c|c|c|c|c|}
\hline $\begin{array}{r}\text { END } \\
\text { DA }\end{array}$ & $\begin{array}{c}\text { OR } 143 \\
9 / 5\end{array}$ & STA- 45 & LA & 329. & LON & 31 & $3.1 w$ & SONIC DE & PTH & $\theta m$ \\
\hline $\begin{array}{l}\text { PR } \\
\text { dbar }\end{array}$ & $\stackrel{T}{\text { Deg } C}$ & $\stackrel{5}{0 / 00}$ & $\begin{array}{l}02 \\
\mathrm{ml} / \mathrm{l}\end{array}$ & $\stackrel{\theta}{\operatorname{Deg} C}$ & $\begin{array}{l}S I G-\theta \\
\mathrm{kg} / \mathrm{m} 3\end{array}$ & $\underset{\mathrm{kg} / \mathrm{m} 3}{\mathrm{SIG}-1} \cdot 5$ & $\begin{array}{l}S I G-3 \\
\mathrm{~kg} / \mathrm{m} 3\end{array}$ & $\underset{\text { m }}{\text { HGTH }}$ & $\underset{\text { cph }}{N}$ & $\underset{m}{D E}$ \\
\hline $\begin{array}{r}1 \\
25 \\
50 \\
75 \\
100 \\
150 \\
200 \\
250 \\
300 \\
313\end{array}$ & $\begin{array}{l}20.229 \\
20.227 \\
18.697 \\
18.429 \\
17.961 \\
17.757 \\
17.298 \\
16.782 \\
16.141 \\
15.936\end{array}$ & $\begin{array}{l}36.706 \\
36.701 \\
36.509 \\
36.522 \\
36.430 \\
36.421 \\
36.383 \\
36.284 \\
36.193 \\
36.163\end{array}$ & $\begin{array}{l}4.97 \\
5.08 \\
5.31 \\
5.22 \\
5.36 \\
4.95 \\
4.51 \\
4.80 \\
4.59 \\
4.55\end{array}$ & $\begin{array}{l}20.229 \\
20.222 \\
18.688 \\
18.416 \\
17.944 \\
17.731 \\
17.264 \\
16.741 \\
16.093 \\
15.886\end{array}$ & $\begin{array}{l}26.004 \\
26.002 \\
26.256 \\
26.335 \\
26.383 \\
26.429 \\
26.513 \\
26.563 \\
26.645 \\
26.670\end{array}$ & $\begin{array}{l}32.355 \\
32.354 \\
32.645 \\
32.730 \\
32.790 \\
32.842 \\
32.939 \\
33.002 \\
33.102 \\
33.132\end{array}$ & $\begin{array}{l}38.500 \\
38.498 \\
38.826 \\
38.917 \\
38.989 \\
39.045 \\
39.154 \\
39.231 \\
39.348 \\
39.383\end{array}$ & $\begin{array}{l}0.002 \\
0.050 \\
0.098 \\
0.141 \\
0.183 \\
0.266 \\
0.347 \\
0.425 \\
0.501 \\
0.520\end{array}$ & $\begin{array}{r}-0.84 \\
3.06 \\
5.11 \\
2.70 \\
1.77 \\
2.34 \\
1.69 \\
2.16 \\
2.39 \\
2.70\end{array}$ & $\begin{array}{r}1 \\
25 \\
50 \\
74 \\
99 \\
149 \\
199 \\
248 \\
298 \\
311\end{array}$ \\
\hline $\begin{array}{l}\text { PR } \\
\text { dbar }\end{array}$ & $\stackrel{T}{T} C$ & $\stackrel{5}{0 / 00}$ & $\begin{array}{c}02 \\
\mathrm{ml} / \mathrm{l}\end{array}$ & $\begin{array}{c}\mathrm{F}-11 \\
\mathrm{pmol} / \mathrm{kg}\end{array}$ & $\begin{array}{c}\mathrm{F}-12 \\
\mathrm{gmol} / \mathrm{kg}\end{array}$ & $\stackrel{\ominus}{D e g} \mathrm{C}$ & $\begin{array}{l}S I G-\theta \\
\mathrm{kg} / \mathrm{m} 3\end{array}$ & $\underset{\mathrm{kg} / \mathrm{m} 3}{S I G-1.5}$ & $\begin{array}{l}S I G-3 \\
\mathrm{~kg} / \mathrm{m} 3\end{array}$ & $\begin{array}{l}\mathrm{DE} \\
\mathrm{m}\end{array}$ \\
\hline $\begin{array}{r}41 \\
82 \\
201\end{array}$ & $\begin{array}{l}19.214 \\
18.137 \\
17.209\end{array}$ & $\begin{array}{l}36.573 \\
36.472 \\
36.362\end{array}$ & $\begin{array}{l}5.49 \\
5.49 \\
4.83\end{array}$ & & & $\begin{array}{l}19.207 \\
18.123 \\
17.175\end{array}$ & $\begin{array}{l}26.172 \\
26.370 \\
26.519\end{array}$ & $\begin{array}{l}32.548 \\
32.774 \\
32.947\end{array}$ & $\begin{array}{l}38.717 \\
38.968 \\
39.164\end{array}$ & $\begin{array}{r}40 \\
82 \\
199\end{array}$ \\
\hline
\end{tabular}




\begin{tabular}{|c|c|c|c|c|c|c|c|c|c|c|}
\hline \multicolumn{3}{|c|}{$\begin{array}{l}\text { ENDEAVOR } 143 \text { STA- } 46 \\
\text { DATE } 9 / 5 / 86\end{array}$} & \multicolumn{3}{|c|}{ LAT $=3110.1 \mathrm{~N}$} & \multicolumn{2}{|c|}{$O N=$} & \multicolumn{2}{|c|}{ SONIC DEPTH= } & $\theta m$ \\
\hline $\begin{array}{l}\text { PR } \\
\text { dbar }\end{array}$ & $\stackrel{T}{\operatorname{Deg}} \mathrm{C}$ & $\stackrel{5}{0 / 00}$ & $\begin{array}{l}02 \\
\mathrm{ml} / \mathrm{l}\end{array}$ & $\stackrel{\theta}{\operatorname{Deg} C}$ & $\begin{array}{l}\mathrm{SIG}-\theta \\
\mathrm{kg} / \mathrm{m} 3\end{array}$ & $\begin{array}{l}\mathrm{SIG}-1.5 \\
\mathrm{~kg} / \mathrm{m} 3\end{array}$ & $\begin{array}{l}\mathrm{SIG}-3 \\
\mathrm{~kg} / \mathrm{m} 3\end{array}$ & $\underset{\mathbf{m}}{\mathrm{HGTH}}$ & $\underset{c p h}{N}$ & $\begin{aligned} \mathrm{DE} \\
\mathbf{m}\end{aligned}$ \\
\hline $\begin{array}{r}1 \\
25 \\
50 \\
75 \\
100 \\
150 \\
200 \\
250 \\
300 \\
350 \\
400 \\
450 \\
500 \\
571\end{array}$ & $\begin{array}{l}20.311 \\
19.497 \\
18.245 \\
17.971 \\
17.632 \\
16.947 \\
16.345 \\
15.658 \\
15.105 \\
14.693 \\
13.925 \\
13.084 \\
12.532 \\
11.0799\end{array}$ & $\begin{array}{l}36.638 \\
36.560 \\
36.479 \\
36.459 \\
36.412 \\
36.305 \\
36.220 \\
36.117 \\
36.056 \\
35.988 \\
35.875 \\
35.749 \\
35.680 \\
35.596\end{array}$ & $\begin{array}{l}5.36 \\
5.84 \\
5.96 \\
5.60 \\
5.37 \\
5.26 \\
5.16 \\
5.03 \\
4.93 \\
4.87 \\
4.84 \\
4.73 \\
4.68\end{array}$ & $\begin{array}{l}20.311 \\
19.493 \\
18.236 \\
17.958 \\
17.615 \\
16.923 \\
16.313 \\
15.618 \\
15.059 \\
14.640 \\
13.866 \\
13.020 \\
12.464 \\
11.724\end{array}$ & $\begin{array}{l}25.930 \\
26.087 \\
26.348 \\
26.401 \\
26.451 \\
26.536 \\
26.615 \\
26.696 \\
26.774 \\
26.814 \\
26.893 \\
26.970 \\
27.028 \\
27.105\end{array}$ & $\begin{array}{l}32.280 \\
32.457 \\
32.748 \\
32.809 \\
32.867 \\
32.971 \\
33.066 \\
33.166 \\
33.260 \\
33.312 \\
33.413 \\
33.516 \\
33.590 \\
33.691\end{array}$ & $\begin{array}{l}38.424 \\
38.619 \\
38.939 \\
39.007 \\
39.073 \\
39.195 \\
39.305 \\
39.424 \\
39.533 \\
39.596 \\
39.718 \\
39.845 \\
39.936 \\
40.058\end{array}$ & $\begin{array}{l}0.002 \\
0.050 \\
0.094 \\
0.135 \\
0.176 \\
0.255 \\
0.330 \\
0.403 \\
0.472 \\
0.540 \\
0.606 \\
0.668 \\
0.727 \\
0.807\end{array}$ & $\begin{array}{l}4.94 \\
6.44 \\
3.12 \\
2.13 \\
2.63 \\
2.17 \\
2.13 \\
2.49 \\
1.75 \\
1.77 \\
2.51 \\
1.99 \\
1.95 \\
1.87\end{array}$ & $\begin{array}{r}1 \\
25 \\
50 \\
74 \\
99 \\
149 \\
199 \\
248 \\
298 \\
347 \\
397 \\
446 \\
496 \\
566\end{array}$ \\
\hline $\begin{array}{l}\text { PR } \\
\text { dbar }\end{array}$ & $\stackrel{T}{D e g} C$ & $\stackrel{S}{0 / 00}$ & $\begin{array}{l}02 \\
\mathrm{ml} / \mathrm{l}\end{array}$ & $\begin{array}{c}\mathrm{F}-11 \\
\mathrm{pmol} / \mathrm{kg}\end{array}$ & $\begin{array}{c}\mathrm{F}-12 \\
\mathrm{pmol} / \mathrm{kg}\end{array}$ & $\stackrel{\theta}{\operatorname{Deg} C}$ & $\begin{array}{l}\mathrm{SIG}-\mathrm{O} \\
\mathrm{kg} / \mathrm{m} 3\end{array}$ & $\underset{\mathrm{kg} / \mathrm{m} 3}{\mathrm{SIG}-1.5}$ & $\begin{array}{l}\mathrm{SIG}-3 \\
\mathrm{~kg} / \mathrm{m3}\end{array}$ & DE \\
\hline $\begin{array}{r}4 \\
355 \\
458 \\
568\end{array}$ & $\begin{array}{l}20.227 \\
14.644 \\
12.963 \\
11.771\end{array}$ & $\begin{array}{l}36.654 \\
35.981 \\
35.737 \\
35.599\end{array}$ & $\begin{array}{l}5.36 \\
4.75 \\
4.65 \\
4.48\end{array}$ & & & $\begin{array}{l}20.226 \\
14.590 \\
12.899 \\
11.696\end{array}$ & $\begin{array}{l}25.965 \\
26.820 \\
26.985 \\
27.113\end{array}$ & $\begin{array}{l}32.317 \\
33.319 \\
33.534 \\
33.699\end{array}$ & $\begin{array}{l}38.462 \\
39.604 \\
39.867 \\
40.068\end{array}$ & $\begin{array}{r}4 \\
352 \\
454 \\
562\end{array}$ \\
\hline
\end{tabular}




\begin{tabular}{|c|c|c|c|c|c|c|c|c|c|c|}
\hline $\begin{array}{l}\text { END } \\
\text { DA }\end{array}$ & $\begin{array}{l}R \quad 14 \\
9 / 5\end{array}$ & STA- 47 & LA & 1 & LON & 29 & ow & ONIC DE & $\mathrm{TH}=$ & $\theta m$ \\
\hline $\begin{array}{c}\text { PR } \\
\text { dbar }\end{array}$ & $\stackrel{T}{\text { Deg } C}$ & $\begin{array}{c}S \\
0 / 00\end{array}$ & $\begin{array}{l}02 \\
\mathrm{ml} / \mathrm{l}\end{array}$ & $\stackrel{\theta}{\text { Deg } C}$ & $\begin{array}{l}S I G-\theta \\
\mathrm{kg} / \mathrm{m} 3\end{array}$ & $\underset{\mathrm{kg} / \mathrm{m} 3}{\mathrm{SIG}-1.5}$ & $\begin{array}{l}S I G-3 \\
\mathrm{~kg} / \mathrm{m} 3\end{array}$ & $\underset{\mathbf{m}}{\mathrm{HGTH}}$ & $\underset{c p h}{N}$ & $\mathrm{DE}$ \\
\hline $\begin{array}{r}1 \\
25 \\
50 \\
75 \\
100 \\
150 \\
200 \\
250 \\
300 \\
350 \\
400 \\
450 \\
500 \\
600 \\
700 \\
800 \\
900 \\
1000 \\
1200 \\
1400 \\
1600 \\
1800 \\
2000 \\
2200 \\
2400 \\
2600 \\
2800 \\
3000 \\
3200 \\
3400 \\
3551\end{array}$ & $\begin{array}{r}20.423 \\
19.758 \\
18.254 \\
18.039 \\
17.782 \\
17.148 \\
16.671 \\
16.072 \\
15.346 \\
14.840 \\
14.027 \\
13.311 \\
12.749 \\
11.470 \\
10.393 \\
9.505 \\
8.842 \\
8.347 \\
7.069 \\
5.961 \\
5.096 \\
4.497 \\
3.931 \\
3.561 \\
3.305 \\
3.094 \\
2.912 \\
2.798 \\
2.711 \\
2.653 \\
2.609\end{array}$ & $\begin{array}{l}36.647 \\
36.593 \\
36.477 \\
36.468 \\
36.431 \\
36.337 \\
36.263 \\
36.187 \\
36.080 \\
36.010 \\
35.890 \\
35.782 \\
35.710 \\
35.550 \\
35.470 \\
35.451 \\
35.465 \\
35.468 \\
35.407 \\
35.290 \\
35.195 \\
35.133 \\
35.064 \\
35.024 \\
34.998 \\
34.978 \\
34.962 \\
34.950 \\
34.940 \\
34.932 \\
34.926\end{array}$ & $\begin{array}{l}5.79 \\
5.63 \\
5.90 \\
5.62 \\
5.40 \\
5.20 \\
5.20 \\
4.94 \\
5.09 \\
4.90 \\
4.96 \\
4.86 \\
4.73 \\
4.50 \\
4.32 \\
4.26 \\
4.28 \\
4.50 \\
5.00 \\
5.24 \\
5.59 \\
5.71 \\
5.85 \\
5.87 \\
5.89 \\
5.87 \\
5.83 \\
5.81 \\
5.80 \\
5.81\end{array}$ & $\begin{array}{l}20.423 \\
19.753 \\
18.245 \\
18.026 \\
17.765 \\
17.123 \\
16.638 \\
16.032 \\
15.299 \\
14.787 \\
13.968 \\
13.247 \\
12.680 \\
11.392 \\
10.307 \\
9.412 \\
8.740 \\
8.238 \\
6.947 \\
5.829 \\
4.953 \\
4.342 \\
3.764 \\
3.380 \\
3.109 \\
2.882 \\
2.683 \\
2.551 \\
2.445 \\
2.367 \\
2.308\end{array}$ & $\begin{array}{l}25.907 \\
26.044 \\
26.343 \\
26.391 \\
26.428 \\
26.512 \\
26.572 \\
26.655 \\
26.739 \\
26.799 \\
26.883 \\
26.949 \\
27.008 \\
27.132 \\
27.266 \\
27.404 \\
27.524 \\
27.606 \\
27.748 \\
27.804 \\
27.836 \\
27.855 \\
27.862 \\
27.868 \\
27.873 \\
27.879 \\
27.884 \\
27.886 \\
27.886 \\
27.887 \\
27.887\end{array}$ & $\begin{array}{l}32.254 \\
32.407 \\
32.744 \\
32.797 \\
32.840 \\
32.941 \\
33.014 \\
33.114 \\
33.218 \\
33.292 \\
33.400 \\
33.488 \\
33.564 \\
33.728 \\
33.897 \\
34.063 \\
34.205 \\
34.304 \\
34.490 \\
34.588 \\
34.653 \\
34.697 \\
34.727 \\
34.749 \\
34.765 \\
34.780 \\
34.793 \\
34.800 \\
34.806 \\
34.809 \\
34.812\end{array}$ & $\begin{array}{l}38.395 \\
38.564 \\
38.935 \\
38.993 \\
39.043 \\
39.160 \\
39.245 \\
39.360 \\
39.484 \\
39.572 \\
39.703 \\
39.811 \\
39.903 \\
40.106 \\
40.307 \\
40.501 \\
40.664 \\
40.779 \\
41.009 \\
41.146 \\
41.243 \\
41.310 \\
41.362 \\
41.399 \\
41.426 \\
41.449 \\
41.470 \\
41.483 \\
41.493 \\
41.499 \\
41.505\end{array}$ & $\begin{array}{l}0.002 \\
0.051 \\
0.096 \\
0.137 \\
0.178 \\
0.258 \\
0.335 \\
0.410 \\
0.482 \\
0.550 \\
0.616 \\
0.679 \\
0.740 \\
0.853 \\
0.956 \\
1.047 \\
1.126 \\
1.197 \\
1.318 \\
1.422 \\
1.518 \\
1.609 \\
1.697 \\
1.782 \\
1.867 \\
1.952 \\
2.036 \\
2.119 \\
2.204 \\
2.290 \\
2.357\end{array}$ & $\begin{array}{l}4.85 \\
5.73 \\
3.76 \\
2.18 \\
2.50 \\
1.97 \\
2.14 \\
2.32 \\
2.35 \\
2.03 \\
1.97 \\
2.06 \\
2.14 \\
2.08 \\
2.18 \\
2.22 \\
1.74 \\
1.84 \\
1.41 \\
1.08 \\
0.94 \\
0.76 \\
0.74 \\
0.62 \\
0.60 \\
0.55 \\
0.52 \\
0.42 \\
0.36 \\
0.34 \\
0.37\end{array}$ & $\begin{array}{r}1 \\
25 \\
50 \\
74 \\
99 \\
149 \\
199 \\
248 \\
298 \\
347 \\
397 \\
446 \\
496 \\
595 \\
694 \\
793 \\
892 \\
991 \\
1188 \\
1386 \\
1583 \\
1780 \\
1977 \\
2173 \\
2370 \\
2566 \\
2762 \\
2958 \\
3154 \\
3350 \\
3497\end{array}$ \\
\hline $\begin{array}{l}\text { PR } \\
\text { dbar }\end{array}$ & $\stackrel{T}{\operatorname{Deg}} \mathrm{C}$ & $\stackrel{S}{0 / 00}$ & $\mathrm{ml} / \mathrm{l}$ & $\begin{array}{c}\mathrm{F}-11 \\
\mathrm{pmol} / \mathrm{kg}\end{array}$ & $\begin{array}{c}\mathrm{F}-12 \\
\mathrm{pmol} / \mathrm{kg}\end{array}$ & $\stackrel{\theta}{\operatorname{Deg} C}$ & $\begin{array}{l}S I G-\theta \\
\mathrm{kg} / \mathrm{m} 3\end{array}$ & $\underset{\mathrm{kg} / \mathrm{m} 3}{\mathrm{SIG}-1.5}$ & $\begin{array}{l}S I G-3 \\
\mathrm{~kg} / \mathrm{m} 3\end{array}$ & E \\
\hline $\begin{array}{r}3 \\
32 \\
58 \\
78 \\
130 \\
152 \\
181\end{array}$ & $\begin{array}{l}20.363 \\
18.731 \\
18.150 \\
17.974 \\
17.329 \\
17.189 \\
16.871\end{array}$ & $\begin{array}{l}36.653 \\
36.523 \\
36.490 \\
36.469 \\
36.370 \\
36.367\end{array}$ & $\begin{array}{l}5.58 \\
5.86 \\
5.80 \\
5.63 \\
5.31 \\
5.14\end{array}$ & & & $\begin{array}{l}20.362 \\
18.725 \\
18.140 \\
17.961 \\
17.307 \\
17.164\end{array}$ & $\begin{array}{l}25.928 \\
26.257 \\
26.380 \\
26.409 \\
26.493 \\
26.526\end{array}$ & $\begin{array}{l}32.277 \\
32.646 \\
32.783 \\
32.816 \\
32.918 \\
32.953\end{array}$ & $\begin{array}{l}8.419 \\
8.825 \\
8.976 \\
9.014 \\
9.132 \\
9.171\end{array}$ & $\begin{array}{r}3 \\
32 \\
57 \\
77 \\
129 \\
151\end{array}$ \\
\hline $\begin{array}{l}203 \\
250 \\
305 \\
403\end{array}$ & $\begin{array}{l}16.612 \\
16.070 \\
15.240 \\
13.953 \\
12.872 \\
10.254\end{array}$ & $\begin{array}{l}36.267 \\
36.197 \\
36.083 \\
35.885\end{array}$ & $\begin{array}{l}5.20 \\
5.07 \\
5.04 \\
4.93\end{array}$ & & & $\begin{array}{l}16.579 \\
16.030 \\
15.193 \\
13.894\end{array}$ & $\begin{array}{l}26.588 \\
26.663 \\
26.765 \\
26.895\end{array}$ & $\begin{array}{l}33.032 \\
33.121 \\
33.247 \\
33.414\end{array}$ & $\begin{array}{l}39.265 \\
39.368 \\
39.516 \\
39.719\end{array}$ & $\begin{array}{l}201 \\
248 \\
303 \\
399\end{array}$ \\
\hline $\begin{array}{r}998 \\
1197 \\
1400 \\
1596 \\
1800\end{array}$ & $\begin{array}{r}16.254 \\
8.400 \\
7.272 \\
5.856 \\
5.105 \\
4.541\end{array}$ & $\begin{array}{l}35.468 \\
35.439 \\
35.278 \\
35.204\end{array}$ & $\begin{array}{l}4.51 \\
4.91 \\
5.41 \\
5.64\end{array}$ & & & $\begin{array}{l}8.290 \\
7.148 \\
5.724 \\
4.963\end{array}$ & $\begin{array}{l}27.598 \\
27.744 \\
27.808 \\
27.842\end{array}$ & $\begin{array}{l}34.294 \\
34.480 \\
34.596 \\
34.659\end{array}$ & $\begin{array}{l}40.767 \\
40.991 \\
41.158 \\
41.248\end{array}$ & $\begin{array}{r}987 \\
1184 \\
1384 \\
1577 \\
1778\end{array}$ \\
\hline $\begin{array}{l}2003 \\
2203 \\
2603 \\
3001\end{array}$ & $\begin{array}{l}3.825 \\
3.518 \\
3.082 \\
2.770 \\
2.645 \\
2.605\end{array}$ & $\begin{array}{l}35.052 \\
35.019 \\
34.977 \\
34.944 \\
34.927 \\
34.925\end{array}$ & $\begin{array}{l}6.13 \\
5.94 \\
5.90 \\
5.73 \\
5.71 \\
5.69\end{array}$ & & & $\begin{array}{l}3.659 \\
3.338 \\
2.870 \\
2.524 \\
2.359 \\
2.306\end{array}$ & $\begin{array}{l}27.863 \\
27.868 \\
27.879 \\
27.883 \\
27.884 \\
27.887\end{array}$ & $\begin{array}{l}34.732 \\
34.751 \\
34.780 \\
34.799 \\
34.807 \\
34.812\end{array}$ & $\begin{array}{l}41.371 \\
41.402 \\
41.450 \\
41.483 \\
41.497 \\
41.504\end{array}$ & $\begin{array}{l}1977 \\
2174 \\
2566 \\
2956 \\
3356 \\
3475\end{array}$ \\
\hline
\end{tabular}




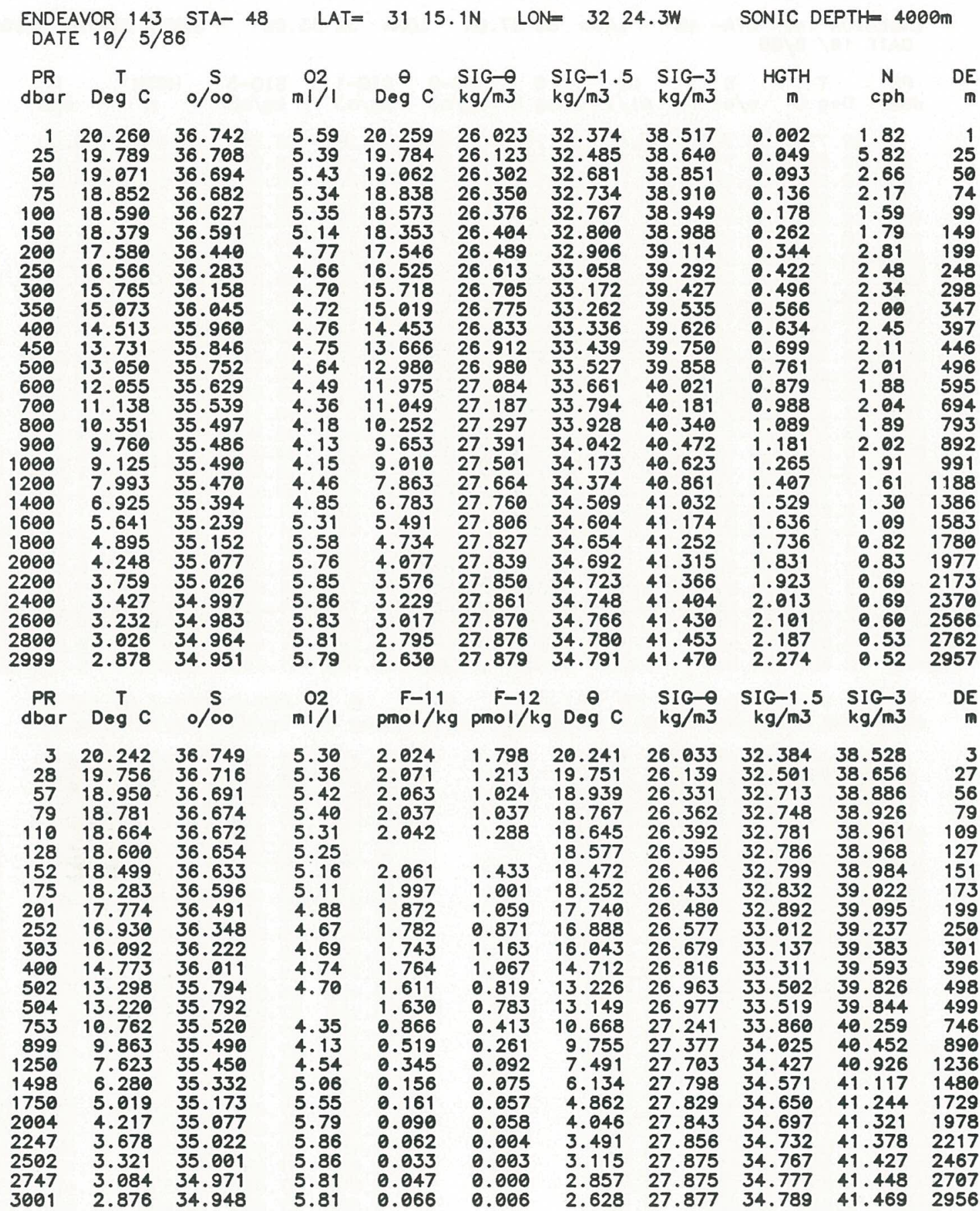




\begin{tabular}{|c|c|c|c|c|c|c|c|c|c|c|}
\hline \multicolumn{3}{|c|}{$\begin{array}{l}\text { ENDEAVOR } 143 \text { STA- } 49 \\
\text { DATE } 10 / 5 / 86\end{array}$} & \multicolumn{3}{|c|}{$L A T=3057.0 \mathrm{~N}$} & \multicolumn{2}{|c|}{$\mathrm{ON}=3233.0 \mathrm{~W}$} & \multicolumn{3}{|c|}{ SONIC DEPTH $=4200 \mathrm{~m}$} \\
\hline $\begin{array}{l}\text { PR } \\
\text { dbar }\end{array}$ & $\stackrel{T}{T} \mathrm{C}$ & $\stackrel{S}{0 / 00}$ & $\begin{array}{l}02 \\
\mathrm{ml} / \mathrm{l}\end{array}$ & $\stackrel{\theta}{\operatorname{Deg} C}$ & $\begin{array}{l}\mathrm{SIG}-\theta \\
\mathrm{kg} / \mathrm{m} 3\end{array}$ & $\begin{array}{l}\mathrm{SIG}-1.5 \\
\mathrm{~kg} / \mathrm{m} 3\end{array}$ & $\begin{array}{l}\mathrm{SIG}-3 \\
\mathrm{~kg} / \mathrm{m} 3\end{array}$ & $\underset{\mathbf{m}}{\mathrm{HGTH}}$ & $\underset{c p h}{N}$ & DE \\
\hline $\begin{array}{r}3 \\
25 \\
50 \\
75 \\
100 \\
150 \\
200 \\
250 \\
300 \\
350 \\
400 \\
450 \\
500 \\
600 \\
700 \\
800 \\
900 \\
1000 \\
1200 \\
1400 \\
1600 \\
1800 \\
2000 \\
2200 \\
2400 \\
2600 \\
2800 \\
3000\end{array}$ & $\begin{array}{r}20.778 \\
19.863 \\
19.971 \\
18.949 \\
18.679 \\
18.440 \\
18.066 \\
17.120 \\
16.326 \\
15.555 \\
14.909 \\
14.282 \\
13.626 \\
12.211 \\
11.348 \\
10.317 \\
9.540 \\
8.844 \\
7.464 \\
6.511 \\
5.440 \\
4.691 \\
4.178 \\
3.705 \\
3.394 \\
3.165 \\
2.988 \\
2.851\end{array}$ & $\begin{array}{l}36.725 \\
36.712 \\
36.680 \\
36.699 \\
36.645 \\
36.605 \\
36.530 \\
36.381 \\
36.251 \\
36.119 \\
36.017 \\
35.927 \\
35.833 \\
35.642 \\
35.553 \\
35.480 \\
35.480 \\
35.467 \\
35.400 \\
35.342 \\
35.218 \\
35.131 \\
35.072 \\
35.024 \\
34.996 \\
34.977 \\
34.962 \\
34.947\end{array}$ & $\begin{array}{l}5.45 \\
5.34 \\
5.34 \\
5.21 \\
5.15 \\
5.07 \\
4.90 \\
4.64 \\
4.62 \\
4.65 \\
4.69 \\
4.71 \\
4.61 \\
4.42 \\
4.35 \\
4.21 \\
4.13 \\
4.17 \\
4.50 \\
4.96 \\
5.34 \\
5.59 \\
5.75 \\
5.82 \\
5.89 \\
5.88 \\
5.86\end{array}$ & $\begin{array}{l}20.778 \\
19.859 \\
19.062 \\
18.935 \\
18.662 \\
18.413 \\
18.032 \\
17.078 \\
16.277 \\
15.500 \\
14.848 \\
14.215 \\
13.554 \\
12.130 \\
11.258 \\
10.219 \\
9.434 \\
8.731 \\
7.339 \\
6.373 \\
5.293 \\
4.533 \\
4.008 \\
3.522 \\
3.196 \\
2.952 \\
2.758 \\
2.603\end{array}$ & $\begin{array}{l}25.870 \\
26.107 \\
26.291 \\
26.338 \\
26.367 \\
26.400 \\
26.437 \\
26.557 \\
26.647 \\
26.724 \\
26.791 \\
26.858 \\
26.925 \\
27.064 \\
27.159 \\
27.289 \\
27.423 \\
27.528 \\
27.686 \\
27.774 \\
27.813 \\
27.833 \\
27.843 \\
27.855 \\
27.864 \\
27.871 \\
27.877 \\
27.879\end{array}$ & $\begin{array}{l}32.209 \\
32.467 \\
32.670 \\
32.720 \\
32.755 \\
32.795 \\
32.842 \\
32.987 \\
33.098 \\
33.197 \\
33.283 \\
33.368 \\
33.455 \\
33.637 \\
33.759 \\
33.922 \\
34.081 \\
34.209 \\
34.415 \\
34.538 \\
34.618 \\
34.668 \\
34.698 \\
34.730 \\
34.752 \\
34.770 \\
34.784 \\
34.792\end{array}$ & $\begin{array}{l}38.342 \\
38.620 \\
38.841 \\
38.893 \\
38.935 \\
38.981 \\
39.038 \\
39.206 \\
39.339 \\
39.458 \\
39.561 \\
39.664 \\
39.769 \\
39.992 \\
40.141 \\
40.335 \\
40.518 \\
40.668 \\
40.921 \\
41.077 \\
41.196 \\
41.273 \\
41.324 \\
41.374 \\
41.409 \\
41.436 \\
41.458 \\
41.472\end{array}$ & $\begin{array}{l}0.006 \\
0.050 \\
0.095 \\
0.138 \\
0.181 \\
0.265 \\
0.349 \\
0.429 \\
0.505 \\
0.578 \\
0.648 \\
0.716 \\
0.780 \\
0.902 \\
1.013 \\
1.116 \\
1.207 \\
1.288 \\
1.425 \\
1.539 \\
1.643 \\
1.740 \\
1.833 \\
1.924 \\
2.012 \\
2.099 \\
2.185 \\
2.271\end{array}$ & $\begin{array}{l}5.30 \\
5.96 \\
3.25 \\
1.61 \\
1.76 \\
1.25 \\
2.57 \\
2.65 \\
2.38 \\
2.00 \\
2.15 \\
2.27 \\
2.00 \\
2.04 \\
1.89 \\
2.21 \\
2.01 \\
1.90 \\
1.56 \\
1.11 \\
1.04 \\
0.80 \\
0.77 \\
0.78 \\
0.66 \\
0.56 \\
0.52 \\
0.49\end{array}$ & $\begin{array}{r}3 \\
25 \\
50 \\
74 \\
99 \\
149 \\
199 \\
248 \\
298 \\
347 \\
397 \\
446 \\
496 \\
595 \\
694 \\
793 \\
892 \\
991 \\
1188 \\
1386 \\
1583 \\
1780 \\
1977 \\
2173 \\
2370 \\
2566 \\
2762 \\
2958\end{array}$ \\
\hline $\begin{array}{l}\text { PR } \\
\text { dbar }\end{array}$ & $\stackrel{T}{T} c$ & $\stackrel{5}{5 / 00}$ & $\begin{array}{l}02 \\
\mathrm{ml} / \mathrm{l}\end{array}$ & $\begin{array}{c}\mathrm{F}-11 \\
\mathrm{pmol} / \mathrm{kg}\end{array}$ & $\begin{array}{c}\mathrm{F}-12 \\
\mathrm{pmol} / \mathrm{kg}\end{array}$ & $\stackrel{\theta}{\operatorname{Deg} C}$ & $\begin{array}{l}\mathrm{SIG}-\theta \\
\mathrm{kg} / \mathrm{m3}\end{array}$ & $\underset{\mathrm{kg} / \mathrm{m} 3}{\mathrm{SIG}-1.5}$ & $\begin{array}{l}\mathrm{SIG}-3 \\
\mathrm{~kg} / \mathrm{m} 3\end{array}$ & DE \\
\hline $\begin{array}{r}6 \\
30 \\
52 \\
78 \\
104 \\
127 \\
155 \\
176 \\
202 \\
252 \\
302 \\
402 \\
499 \\
600 \\
798 \\
1000 \\
1249 \\
1499 \\
1749 \\
1994 \\
2247 \\
2500 \\
2746\end{array}$ & $\begin{array}{r}20.471 \\
19.387 \\
18.998 \\
18.861 \\
18.654 \\
18.574 \\
18.460 \\
18.397 \\
17.744 \\
16.877 \\
16.236 \\
14.779 \\
13.564 \\
12.239 \\
10.224 \\
8.907 \\
7.611 \\
5.955 \\
4.812 \\
4.176 \\
3.658 \\
3.257 \\
3.038 \\
2.846\end{array}$ & $\begin{array}{l}36 \\
36 \\
36 \\
36 \\
36 \\
36 \\
36 \\
36 \\
36 \\
36 \\
36 \\
35 \\
35 \\
35 \\
35 \\
35 \\
35 \\
35 \\
35\end{array}$ & $\begin{array}{l}5.42 \\
5.44 \\
5.36 \\
5.38 \\
5.26 \\
5.18 \\
5.17 \\
4.85 \\
4.66 \\
4.69 \\
4.76 \\
4.60 \\
4.46 \\
4.20 \\
4.11 \\
4.60 \\
5.23 \\
5.65\end{array}$ & & & $\begin{array}{r}20.470 \\
19.381 \\
18.988 \\
18.847 \\
18.635 \\
18.552 \\
18.433 \\
18.366 \\
17.709 \\
16.835 \\
16.187 \\
14.717 \\
13.492 \\
12.158 \\
10.127 \\
8.793 \\
7.479 \\
5.812 \\
4.657 \\
4.006 \\
3.472 \\
3.052 \\
2.813 \\
2.598\end{array}$ & $\begin{array}{l}25 \\
26 \\
26 \\
26 \\
26 \\
26 \\
26 \\
26 \\
26 \\
26 \\
26 \\
26 \\
26 \\
27 \\
27 \\
27 \\
27 \\
27 \\
27 \\
27 \\
27 \\
27 \\
27 \\
27\end{array}$ & $\begin{array}{l}32.307 \\
32.605 \\
32.694 \\
32.733 \\
32.774 \\
32.789 \\
32.806 \\
32.816 \\
32.899 \\
33.019 \\
33.115 \\
33.303 \\
33.477 \\
33.638 \\
33.942 \\
34.198 \\
34.431 \\
34.583 \\
34.668 \\
34.702 \\
34.733 \\
34.759 \\
34.778 \\
34.801\end{array}$ & $\begin{array}{l}38.446 \\
38.768 \\
38.866 \\
38.908 \\
38.954 \\
38.972 \\
38.992 \\
39.003 \\
39.103 \\
39.244 \\
39.358 \\
39.585 \\
39.793 \\
39.993 \\
40.358 \\
40.656 \\
40.930 \\
41.142 \\
41.269 \\
41.327 \\
41.379 \\
41.422 \\
41.451 \\
41.481\end{array}$ & $\begin{array}{r}6 \\
30 \\
52 \\
78 \\
103 \\
126 \\
154 \\
174 \\
200 \\
250 \\
299 \\
399 \\
495 \\
594 \\
790 \\
989 \\
1235 \\
1481 \\
1728 \\
1968 \\
2217 \\
2465 \\
2705 \\
2958\end{array}$ \\
\hline
\end{tabular}




\begin{tabular}{|c|c|c|c|c|c|c|c|c|c|c|}
\hline \multicolumn{3}{|c|}{$\begin{array}{l}\text { ENDEAVOR } 143 \text { STA- } 50 \\
\text { DATE } 10 / 5 / 86\end{array}$} & $-A T=$ & \multicolumn{2}{|c|}{$039.1 \mathrm{~N}$} & \multicolumn{2}{|c|}{$\mathrm{ON}=3241.8 \mathrm{~W}$} & \multicolumn{3}{|c|}{ SONIC DEPTH= 4420m } \\
\hline $\begin{array}{l}\text { PR } \\
\text { dbar }\end{array}$ & $\stackrel{T}{\operatorname{Deg} C}$ & $\stackrel{5}{0 / 00}$ & $\mathrm{ml} / \mathrm{l}$ & $\stackrel{\theta}{\operatorname{Deg} C}$ & $\begin{array}{l}S I G-\theta \\
\mathrm{kg} / \mathrm{m} 3\end{array}$ & $\begin{array}{l}\mathrm{SIG}-1.5 \\
\mathrm{~kg} / \mathrm{m}^{3}\end{array}$ & $\begin{array}{l}\mathrm{SIG}-3 \\
\mathrm{~kg} / \mathrm{m} 3\end{array}$ & $\underset{m}{\text { HGTH }}$ & $\underset{c p h}{N}$ & $D E$ \\
\hline $\begin{array}{r}3 \\
25 \\
50 \\
75 \\
100 \\
150 \\
200 \\
250 \\
300 \\
350 \\
400 \\
450 \\
500 \\
600 \\
700 \\
800 \\
900 \\
1000 \\
1200 \\
1400 \\
1600 \\
1800 \\
2000 \\
2200 \\
2400 \\
2600 \\
2800 \\
3000 \\
3005\end{array}$ & $\begin{array}{r}21.333 \\
20.331 \\
20.026 \\
19.894 \\
19.804 \\
18.707 \\
17.855 \\
17.218 \\
16.518 \\
15.899 \\
15.182 \\
14.639 \\
14.049 \\
12.518 \\
11.199 \\
10.028 \\
9.152 \\
8.496 \\
7.311 \\
6.301 \\
5.477 \\
4.791 \\
4.164 \\
3.691 \\
3.397 \\
3.141 \\
2.965 \\
2.835 \\
2.832\end{array}$ & $\begin{array}{l}36.8 \\
36.8 \\
36.8 \\
36.8 \\
36.8 \\
36.4 \\
36.8 \\
36.2 \\
36.1 \\
36.8 \\
35.9 \\
35.8 \\
35.6 \\
35.5 \\
35.4 \\
35.4 \\
35.4 \\
35.9 \\
35.9 \\
35.2 \\
35.1 \\
35.8 \\
35.8 \\
35.8 \\
34.9 \\
34.9 \\
34.9 \\
34.9\end{array}$ & $\begin{array}{l}5.04 \\
5.17 \\
5.19 \\
5.20 \\
5.21 \\
4.81 \\
4.70 \\
4.64 \\
4.61 \\
4.60 \\
4.65 \\
4.69 \\
4.70 \\
4.49 \\
4.29 \\
4.15 \\
4.14 \\
4.21 \\
4.57 \\
4.97 \\
5.31 \\
5.53 \\
5.72 \\
5.83 \\
5.85 \\
5.85 \\
5.84 \\
5.84\end{array}$ & $\begin{array}{l}21.332 \\
20.327 \\
20.016 \\
19.880 \\
19.786 \\
18.680 \\
17.820 \\
17.176 \\
16.469 \\
15.843 \\
15.120 \\
14.571 \\
13.976 \\
12.436 \\
11.109 \\
9.932 \\
9.049 \\
8.385 \\
7.188 \\
6.165 \\
5.330 \\
4.632 \\
3.994 \\
3.508 \\
3.200 \\
2.929 \\
2.736 \\
2.588 \\
2.584\end{array}$ & $\begin{array}{l}25.876 \\
26.119 \\
26.206 \\
26.231 \\
26.248 \\
26.367 \\
26.442 \\
26.522 \\
26.602 \\
26.672 \\
26.751 \\
26.814 \\
26.879 \\
27.029 \\
27.166 \\
27.292 \\
27.441 \\
27.533 \\
27.690 \\
27.771 \\
27.816 \\
27.839 \\
27.849 \\
27.858 \\
27.866 \\
27.872 \\
27.877 \\
27.881 \\
27.881\end{array}$ & $\begin{array}{l}32.201 \\
32.466 \\
32.561 \\
32.589 \\
32.608 \\
32.756 \\
32.852 \\
32.949 \\
33.049 \\
33.136 \\
33.235 \\
33.314 \\
33.396 \\
33.592 \\
33.771 \\
33.935 \\
34.113 \\
34.227 \\
34.425 \\
34.543 \\
34.619 \\
34.669 \\
34.705 \\
34.733 \\
34.755 \\
34.772 \\
34.785 \\
34.795 \\
34.795\end{array}$ & $\begin{array}{l}38.320 \\
38.607 \\
38.708 \\
38.740 \\
38.761 \\
38.935 \\
39.053 \\
39.167 \\
39.284 \\
39.388 \\
39.507 \\
39.600 \\
39.698 \\
39.939 \\
40.157 \\
40.357 \\
40.562 \\
40.698 \\
40.935 \\
41.089 \\
41.195 \\
41.271 \\
41.331 \\
41.378 \\
41.411 \\
41.439 \\
41.460 \\
41.476 \\
41.476\end{array}$ & $\begin{array}{l}0.006 \\
0.050 \\
0.096 \\
0.141 \\
0.187 \\
0.275 \\
0.360 \\
0.441 \\
0.518 \\
0.594 \\
0.666 \\
0.736 \\
0.803 \\
0.928 \\
1.042 \\
1.145 \\
1.234 \\
1.313 \\
1.447 \\
1.561 \\
1.664 \\
1.761 \\
1.854 \\
1.944 \\
2.031 \\
2.118 \\
2.203 \\
2.289 \\
2.291\end{array}$ & $\begin{array}{l}6.99 \\
4.85 \\
1.78 \\
1.69 \\
1.41 \\
2.80 \\
2.43 \\
2.33 \\
2.11 \\
2.13 \\
2.18 \\
2.01 \\
2.40 \\
2.20 \\
2.06 \\
2.33 \\
2.08 \\
1.89 \\
1.48 \\
1.20 \\
1.00 \\
0.91 \\
0.79 \\
0.70 \\
0.65 \\
0.58 \\
0.50 \\
0.47 \\
-0.30\end{array}$ & $\begin{array}{r}3 \\
25 \\
50 \\
74 \\
99 \\
149 \\
199 \\
248 \\
298 \\
347 \\
397 \\
446 \\
496 \\
595 \\
694 \\
793 \\
892 \\
991 \\
1188 \\
1386 \\
1583 \\
1780 \\
1977 \\
2173 \\
2370 \\
2566 \\
2762 \\
2958 \\
2963\end{array}$ \\
\hline $\begin{array}{l}\text { PR } \\
\text { dbar }\end{array}$ & $\stackrel{T}{\operatorname{Deg}} \mathrm{C}$ & $\stackrel{5}{0 / 00}$ & $\begin{array}{l}02 \\
\mathrm{ml} / \mathrm{I}\end{array}$ & $\begin{array}{c}\mathrm{F}-11 \\
\mathrm{pmol} / \mathrm{kg}\end{array}$ & $\begin{array}{c}\mathrm{F}-12 \\
\mathrm{pmol} / \mathrm{kg}\end{array}$ & $\stackrel{\theta}{\operatorname{Deg}} c$ & $\begin{array}{l}\mathrm{SIG}-\Theta \\
\mathrm{kg} / \mathrm{m} 3\end{array}$ & $\underset{\mathrm{kg} / \mathrm{m} 3}{S I G-1.5}$ & $\begin{array}{l}S I G-3 \\
\mathrm{~kg} / \mathrm{m} 3\end{array}$ & \\
\hline $\begin{array}{r}2 \\
27 \\
52 \\
77 \\
103 \\
127 \\
151 \\
176 \\
202 \\
250 \\
301 \\
400 \\
499 \\
599 \\
802 \\
999 \\
1250 \\
1503 \\
1744 \\
1997 \\
2249 \\
2497\end{array}$ & $\begin{array}{r}20.1 \\
19.8 \\
19.7 \\
19.5 \\
19.0 \\
18.6 \\
17.7 \\
17.4 \\
16.7 \\
16.1 \\
14.9 \\
13.7 \\
12.3 \\
9.9 \\
8.4 \\
6.9 \\
5.8 \\
4.9 \\
4.2 \\
3.6 \\
3.2 \\
3.8 \\
2.8\end{array}$ & $\begin{array}{l}3 \\
3 \\
3 \\
3 \\
3 \\
3 \\
3 \\
3 \\
3 \\
3 \\
3 \\
3 \\
3 \\
3 \\
3 \\
3\end{array}$ & $\begin{array}{l}5.19 \\
5.30 \\
5.27 \\
5.28 \\
5.20 \\
4.98 \\
4.78 \\
4.80 \\
4.72 \\
4.66 \\
4.63 \\
4.74 \\
4.64 \\
4.47 \\
4.11 \\
4.22 \\
4.67 \\
5.15 \\
5.10\end{array}$ & & . & $\begin{array}{r}20.09 \\
19.8 \\
19.7 \\
19.5 \\
19.06 \\
18.58 \\
17.7 \\
17.4 \\
16.6 \\
16.1 \\
14.88 \\
13.6 \\
12.26 \\
9.88 \\
8.3 \\
6.8 \\
5.7\end{array}$ & $\begin{array}{l}25 \\
26 \\
26 \\
26 \\
26 \\
26 \\
26 \\
26 \\
26 \\
26 \\
26 \\
26 \\
26 \\
27 \\
27 \\
27 \\
27 \\
27\end{array}$ & $\begin{array}{l}32.232 \\
32.528 \\
32.584 \\
32.609 \\
32.640 \\
32.728 \\
32.779 \\
32.866 \\
32.917 \\
33.032 \\
33.099 \\
33.273 \\
33.442 \\
33.623 \\
33.944 \\
34.236 \\
34.474 \\
34.591\end{array}$ & $\begin{array}{l}8.353 \\
.675 \\
8.735 \\
8.763 \\
8.798 \\
8.898 \\
0.961 \\
9.068 \\
9.128 \\
9.262 \\
9.343 \\
9.550 \\
9.755 \\
9.975 \\
0.368 \\
0.707 \\
0.998 \\
1.153\end{array}$ & $\begin{array}{r}2 \\
26 \\
51 \\
77 \\
102 \\
126 \\
150 \\
174 \\
201 \\
248 \\
299\end{array}$ \\
\hline
\end{tabular}




\begin{tabular}{|c|c|c|c|c|c|c|c|c|c|c|}
\hline \multicolumn{3}{|c|}{$\begin{array}{l}\text { ENDEAVOR } 143 \text { STA- } 51 \\
\text { DATE } 10 / 5 / 86\end{array}$} & \multicolumn{3}{|c|}{$L A T=3020.9 \mathrm{~N}$} & \multicolumn{2}{|c|}{ LON $=3252.1 \mathrm{~W}$} & SONIC & \multicolumn{2}{|c|}{$=4250 m$} \\
\hline $\begin{array}{l}\text { PR } \\
\text { dbar }\end{array}$ & $\stackrel{T}{T} C$ & $\stackrel{5}{0 / 00}$ & 02 & $\stackrel{\theta}{\operatorname{Deg} C}$ & $\begin{array}{l}\mathrm{SIG}-\theta \\
\mathrm{kg} / \mathrm{m3}\end{array}$ & $\begin{array}{l}\mathrm{SIG}-1.5 \\
\mathrm{~kg} / \mathrm{m} 3\end{array}$ & $\begin{array}{l}\text { SIG-3 } \\
\mathrm{kg} / \mathrm{m} 3\end{array}$ & $\underset{m}{H G T H}$ & $\underset{c p h}{N}$ & $D E$ \\
\hline $\begin{array}{r}1 \\
25 \\
50 \\
75 \\
100 \\
150 \\
200 \\
250 \\
300 \\
350 \\
400 \\
450 \\
500 \\
600 \\
700 \\
800 \\
900 \\
1000 \\
200 \\
400 \\
1600 \\
1800 \\
2000 \\
2200 \\
2400 \\
2600 \\
2800 \\
2997\end{array}$ & $\begin{array}{r}21.074 \\
20.314 \\
19.155 \\
18.892 \\
18.813 \\
18.606 \\
17.744 \\
17.407 \\
16.636 \\
15.946 \\
15.173 \\
14.415 \\
13.631 \\
12.376 \\
11.154 \\
9.713 \\
8.736 \\
8.379 \\
7.326 \\
6.229 \\
5.367 \\
4.737 \\
4.232 \\
3.707 \\
3.414 \\
3.154 \\
2.964 \\
2.803\end{array}$ & $\begin{array}{l}36.774 \\
36.734 \\
36.652 \\
36.647 \\
36.644 \\
36.611 \\
36.483 \\
36.464 \\
36.318 \\
36.211 \\
36.075 \\
35.949 \\
35.830 \\
35.655 \\
35.530 \\
35.402 \\
35.348 \\
35.408 \\
35.382 \\
35.296 \\
35.210 \\
35.147 \\
35.091 \\
35.028 \\
35.002 \\
34.978 \\
34.960 \\
34.947\end{array}$ & $\begin{array}{l}5.32 \\
5.33 \\
5.34 \\
5.35 \\
5.12 \\
5.12 \\
4.65 \\
4.64 \\
4.56 \\
4.62 \\
4.59 \\
4.53 \\
4.51 \\
4.39 \\
4.24 \\
4.04 \\
4.01 \\
4.29 \\
4.63 \\
5.02 \\
5.31 \\
5.53\end{array}$ & $\begin{array}{r}21.073 \\
20.309 \\
19.146 \\
18.879 \\
18.795 \\
18.579 \\
17.710 \\
17.365 \\
16.587 \\
15.890 \\
15.111 \\
14.348 \\
13.559 \\
12.294 \\
11.065 \\
9.619 \\
8.636 \\
8.270 \\
7.202 \\
6.094 \\
5.221 \\
4.579 \\
4.061 \\
3.524 \\
3.216 \\
2.941 \\
2.735 \\
2.557\end{array}$ & $\begin{array}{l}25.827 \\
26.003 \\
26.248 \\
26.313 \\
26.332 \\
26.362 \\
26.481 \\
26.552 \\
26.626 \\
26.706 \\
26.777 \\
26.848 \\
26.922 \\
27.042 \\
27.177 \\
27.331 \\
27.450 \\
27.554 \\
27.692 \\
27.775 \\
27.816 \\
27.840 \\
27.853 \\
27.857 \\
27.867 \\
27.873 \\
27.878 \\
27.882\end{array}$ & $\begin{array}{l}32.159 \\
32.353 \\
32.625 \\
32.696 \\
32.717 \\
32.753 \\
32.895 \\
32.973 \\
33.069 \\
33.168 \\
33.261 \\
33.354 \\
33.452 \\
33.610 \\
33.783 \\
33.984 \\
34.135 \\
34.251 \\
34.426 \\
34.550 \\
34.623 \\
34.673 \\
34.706 \\
34.732 \\
34.754 \\
34.772 \\
34.785 \\
34.797\end{array}$ & $\begin{array}{l}38.285 \\
38.496 \\
38.794 \\
38.872 \\
38.894 \\
38.935 \\
39.098 \\
39.185 \\
39.301 \\
39.418 \\
39.532 \\
39.646 \\
39.766 \\
39.961 \\
40.171 \\
40.416 \\
40.599 \\
40.726 \\
40.936 \\
41.098 \\
41.203 \\
41.277 \\
41.329 \\
41.377 \\
41.410 \\
41.439 \\
41.460 \\
41.479\end{array}$ & $\begin{array}{l}0.002 \\
0.052 \\
0.099 \\
0.143 \\
0.186 \\
0.272 \\
0.355 \\
0.434 \\
0.512 \\
0.585 \\
0.656 \\
0.725 \\
0.790 \\
0.912 \\
1.025 \\
1.124 \\
1.211 \\
1.287 \\
1.420 \\
1.534 \\
1.636 \\
1.732 \\
1.824 \\
1.914 \\
2.001 \\
2.088 \\
2.173 \\
2.257\end{array}$ & $\begin{array}{l}5.19 \\
5.74 \\
3.79 \\
2.51 \\
1.28 \\
2.39 \\
2.40 \\
2.02 \\
2.42 \\
2.27 \\
2.12 \\
2.46 \\
2.06 \\
2.11 \\
2.26 \\
2.30 \\
2.05 \\
1.70 \\
1.50 \\
1.27 \\
0.99 \\
0.84 \\
0.79 \\
0.69 \\
0.62 \\
0.60 \\
0.59 \\
0.50\end{array}$ & $\begin{array}{r}1 \\
25 \\
50 \\
74 \\
99 \\
149 \\
199 \\
248 \\
298 \\
347 \\
397 \\
446 \\
496 \\
595 \\
694 \\
793 \\
892 \\
991 \\
1188 \\
1386 \\
1583 \\
1780 \\
1977 \\
2174 \\
2370 \\
2566 \\
2762 \\
2955\end{array}$ \\
\hline $\begin{array}{l}\text { PR } \\
\text { dbar }\end{array}$ & $\stackrel{T}{T} C$ & $\stackrel{5}{1 / 00}$ & ו & $\begin{array}{c}\mathrm{F}-11 \\
\mathrm{pmol} / \mathrm{kg}\end{array}$ & $\begin{array}{c}\mathrm{F}-12 \\
\mathrm{pmol} / \mathrm{kg}\end{array}$ & $\stackrel{\theta}{D e g} C$ & $\begin{array}{l}\mathrm{SIG}-\theta \\
\mathrm{kg} / \mathrm{m} 3\end{array}$ & $\underset{\mathrm{kg} / \mathrm{m} 3}{\mathrm{SIG}-1.5}$ & $\begin{array}{l}\mathrm{SIG}-3 \\
\mathrm{~kg} / \mathrm{m} 3\end{array}$ & $\begin{array}{c}D E \\
\text { m }\end{array}$ \\
\hline $\begin{array}{r}3 \\
28 \\
56 \\
79 \\
104 \\
127 \\
148 \\
174 \\
204 \\
251 \\
303 \\
403 \\
505 \\
598 \\
798 \\
1001 \\
1248 \\
1498 \\
1750 \\
2003 \\
2251 \\
2498 \\
2698 \\
2997\end{array}$ & $\begin{array}{r}21.027 \\
19.807 \\
18.990 \\
18.834 \\
18.805 \\
18.755 \\
18.673 \\
17.898 \\
17.669 \\
17.288 \\
16.424 \\
15.024 \\
13.571 \\
12.352 \\
9.829 \\
8.328 \\
6.992 \\
5.585 \\
4.806 \\
4.166 \\
3.618 \\
3.242 \\
3.047\end{array}$ & $\begin{array}{l}36.731 \\
36.673 \\
36.660 \\
36.645 \\
36.652 \\
36.632 \\
36.494 \\
36.450 \\
36.471 \\
36.299 \\
36.066 \\
35.828 \\
35.659 \\
35.410 \\
35.410 \\
35.375 \\
35.234 \\
35.155 \\
35.086 \\
35.022 \\
34.985\end{array}$ & $\begin{array}{l}5.25 \\
4.72 \\
4.66 \\
4.64 \\
4.61 \\
4.50 \\
4.38 \\
3.99\end{array}$ & $\begin{array}{l}0.273 \\
0.276 \\
0.096 \\
0.079 \\
0.038 \\
0.035\end{array}$ & $\begin{array}{l}0.165 \\
0.092 \\
0.069 \\
0.063 \\
0.039 \\
0.000 \\
0.036\end{array}$ & $\begin{array}{r}21.027 \\
19.802 \\
18.980 \\
18.820 \\
18.787 \\
18.732 \\
18.647 \\
17.868 \\
17.634 \\
17.246 \\
16.374 \\
14.962 \\
13.498 \\
12.271 \\
9.734 \\
8.219 \\
6.866 \\
5.447 \\
4.652 \\
3.996 \\
3.431 \\
3.038 \\
2.826 \\
2.556\end{array}$ & $\begin{array}{l}25.873 \\
26.137 \\
26.307 \\
26.338 \\
26.335 \\
26.354 \\
26.361 \\
26.451 \\
26.475 \\
26.586 \\
26.661 \\
26.804 \\
26.933 \\
27.049 \\
27.318 \\
27.563 \\
27.733 \\
27.807 \\
27.838 \\
27.855 \\
27.862 \\
27.870 \\
27.877 \\
27.884\end{array}$ & $\begin{array}{l}32.206 \\
32.498 \\
32.688 \\
32.723 \\
32.721 \\
32.741 \\
32.750 \\
32.860 \\
32.890 \\
33.010 \\
33.110 \\
33.292 \\
33.464 \\
33.617 \\
33.967 \\
34.262 \\
34.479 \\
34.606 \\
34\end{array}$ & $\begin{array}{l}38.333 \\
38.652 \\
38.860 \\
38.899 \\
38.898 \\
38.920 \\
38.930 \\
39.060 \\
39.096 \\
39.225 \\
39.347 \\
39.567 \\
39.780 \\
39.969 \\
40.396 \\
40.738 \\
41.001 \\
41.178 \\
41.269 \\
41.337 \\
41.388 \\
41.428 \\
41.452 \\
41.481\end{array}$ & $\begin{array}{r}3 \\
27 \\
55 \\
79 \\
103 \\
126 \\
147 \\
173 \\
202 \\
249 \\
301 \\
399 \\
500 \\
592 \\
790 \\
990 \\
1234 \\
1480 \\
1729 \\
1977 \\
2221 \\
2463 \\
2659 \\
2952\end{array}$ \\
\hline
\end{tabular}




\begin{tabular}{|c|c|c|c|c|c|c|c|c|c|c|}
\hline \multicolumn{3}{|c|}{$\begin{array}{l}\text { ENDEAVOR } 143 \text { STA- } 52 \\
\text { DATE } 11 / 5 / 86\end{array}$} & \multicolumn{2}{|c|}{ LAT $=30$} & $.0 N$ & \multicolumn{2}{|c|}{$1.0 W$} & \multicolumn{3}{|c|}{ SONIC DEPTH $=4330 \mathrm{~m}$} \\
\hline $\begin{array}{l}\text { PR } \\
\text { dbar }\end{array}$ & $\stackrel{T}{\operatorname{Deg} C}$ & $\stackrel{5}{0 / 00}$ & $\begin{array}{l}02 \\
\mathrm{ml} / \mathrm{l}\end{array}$ & $\stackrel{\theta}{\operatorname{Deg}} \mathrm{C}$ & $\begin{array}{l}\mathrm{SIG}-\theta \\
\mathrm{kg} / \mathrm{m3}\end{array}$ & $\begin{array}{l}\mathrm{SIG}-1.5 \\
\mathrm{~kg} / \mathrm{m} 3\end{array}$ & $\begin{array}{l}\mathrm{SIG}-3 \\
\mathrm{~kg} / \mathrm{m} 3\end{array}$ & $\underset{\mathrm{m}}{\mathrm{HGTH}}$ & $\underset{\mathrm{cph}}{\mathrm{N}}$ & $\underset{\mathrm{m}}{\mathrm{DE}}$ \\
\hline $\begin{array}{r}1 \\
25 \\
50 \\
75 \\
100 \\
150 \\
200 \\
250 \\
300 \\
350 \\
400 \\
450 \\
500 \\
600 \\
700 \\
800 \\
900 \\
1000 \\
1200 \\
1400 \\
1600 \\
1800 \\
2000 \\
2200 \\
2400 \\
2600 \\
2800 \\
2997\end{array}$ & $\begin{array}{r}20.898 \\
20.416 \\
20.003 \\
19.849 \\
19.697 \\
18.993 \\
18.233 \\
17.507 \\
16.775 \\
16.064 \\
15.219 \\
14.359 \\
13.624 \\
12.525 \\
11.216 \\
10.228 \\
9.183 \\
8.081 \\
7.116 \\
6.250 \\
5.349 \\
4.808 \\
4.196 \\
3.813 \\
3.427 \\
3.131 \\
2.970 \\
2.851\end{array}$ & $\begin{array}{l}36.928 \\
36.899 \\
36.883 \\
36.877 \\
36.839 \\
36.670 \\
36.564 \\
36.474 \\
36.357 \\
36.232 \\
36.088 \\
35.945 \\
35.836 \\
35.686 \\
35.549 \\
35.474 \\
35.421 \\
35.327 \\
35.353 \\
35.296 \\
35.205 \\
35.155 \\
35.086 \\
35.046 \\
35.005 \\
34.976 \\
34.962 \\
34.950\end{array}$ & $\begin{array}{l}5.25 \\
5.24 \\
5.27 \\
5.22 \\
5.23 \\
5.20 \\
4.70 \\
4.62 \\
4.57 \\
4.51 \\
4.51 \\
4.50 \\
4.46 \\
4.40 \\
4.27 \\
4.09 \\
4.02 \\
4.08 \\
4.59 \\
4.98 \\
5.30 \\
5.49 \\
5.65 \\
5.72 \\
5.78 \\
5.81 \\
5.81 \\
5.80\end{array}$ & $\begin{array}{c}20.897 \\
20.412 \\
19.994 \\
19.836 \\
19.679 \\
18.966 \\
18.198 \\
17.465 \\
16.726 \\
16.007 \\
15.157 \\
14.292 \\
13.551 \\
12.443 \\
11.126 \\
10.131 \\
9.079 \\
7.973 \\
6.994 \\
6.114 \\
5.203 \\
4.649 \\
4.025 \\
3.628 \\
3.229 \\
2.919 \\
2.741 \\
2.604\end{array}$ & $\begin{array}{l}25.992 \\
26.102 \\
26.202 \\
26.239 \\
26.251 \\
26.308 \\
26.422 \\
26.535 \\
26.623 \\
26.695 \\
26.777 \\
26.856 \\
26.929 \\
27.037 \\
27.181 \\
27.300 \\
27.436 \\
27.535 \\
27.698 \\
27.772 \\
27.814 \\
27.839 \\
27.852 \\
27.861 \\
27.868 \\
27.874 \\
27.878 \\
27.881\end{array}$ & $\begin{array}{l}32.327 \\
32.448 \\
32.557 \\
32.598 \\
32.614 \\
32.689 \\
32.823 \\
32.954 \\
33.062 \\
33.154 \\
33.260 \\
33.364 \\
33.458 \\
33.600 \\
33.785 \\
33.936 \\
34.106 \\
34.244 \\
34.440 \\
34.546 \\
34.622 \\
34.669 \\
34.707 \\
34.731 \\
34.755 \\
34.773 \\
34.785 \\
34.794\end{array}$ & $\begin{array}{l}38.455 \\
38.587 \\
38.705 \\
38.750 \\
38.770 \\
38.862 \\
39.014 \\
39.163 \\
39.290 \\
39.401 \\
39.529 \\
39.658 \\
39.772 \\
39.946 \\
40.170 \\
40.352 \\
40.555 \\
40.729 \\
40.957 \\
41.094 \\
41.203 \\
41.270 \\
41.332 \\
41.372 \\
41.410 \\
41.441 \\
41.460 \\
41.474\end{array}$ & $\begin{array}{l}0.002 \\
0.049 \\
0.096 \\
0.141 \\
0.186 \\
0.275 \\
0.362 \\
0.443 \\
0.521 \\
0.595 \\
0.666 \\
0.734 \\
0.799 \\
0.922 \\
1.034 \\
1.135 \\
1.224 \\
1.302 \\
1.435 \\
1.549 \\
1.651 \\
1.747 \\
1.840 \\
1.930 \\
2.018 \\
2.104 \\
2.190 \\
2.274\end{array}$ & $\begin{array}{l}3.33 \\
4.61 \\
2.73 \\
1.57 \\
1.85 \\
1.81 \\
3.08 \\
2.82 \\
2.15 \\
2.47 \\
2.10 \\
2.33 \\
1.87 \\
2.19 \\
2.22 \\
2.15 \\
2.22 \\
1.73 \\
1.48 \\
1.21 \\
0.99 \\
0.83 \\
0.80 \\
0.69 \\
0.66 \\
0.56 \\
0.49 \\
0.38\end{array}$ & $\begin{array}{r}1 \\
25 \\
50 \\
74 \\
99 \\
149 \\
199 \\
248 \\
298 \\
347 \\
397 \\
446 \\
496 \\
595 \\
694 \\
793 \\
892 \\
991 \\
1188 \\
1386 \\
1583 \\
1780 \\
1977 \\
2174 \\
2370 \\
2566 \\
2763 \\
2956\end{array}$ \\
\hline $\begin{array}{l}\text { PR } \\
\text { dbar }\end{array}$ & $\stackrel{T}{\operatorname{Deg}} \mathrm{C}$ & $\stackrel{S}{0 / 00}$ & $\begin{array}{l}02 \\
\mathrm{ml} / \mathrm{l}\end{array}$ & $\begin{array}{c}\mathrm{F}-11 \\
\mathrm{pmol} / \mathrm{kg}\end{array}$ & $\begin{array}{c}\mathrm{F}-12 \\
\mathrm{pmol} / \mathrm{kg}\end{array}$ & $\stackrel{\theta}{\operatorname{Deg} C}$ & $\begin{array}{l}\mathrm{SIG}-0 \\
\mathrm{~kg} / \mathrm{m} 3\end{array}$ & $\underset{\mathrm{kg} / \mathrm{m} 3}{\mathrm{SIG}-1.5}$ & $\begin{array}{l}\mathrm{SIG}-3 \\
\mathrm{~kg} / \mathrm{m} 3\end{array}$ & $D E$ \\
\hline $\begin{array}{r}2 \\
26 \\
53 \\
76 \\
106 \\
127 \\
153 \\
176 \\
205 \\
253 \\
303 \\
405 \\
501 \\
602 \\
800 \\
1002 \\
1248 \\
1499 \\
1749 \\
1998 \\
2249 \\
2502 \\
2747\end{array}$ & $\begin{array}{r}20.803 \\
20.182 \\
19.867 \\
19.802 \\
19.331 \\
19.034 \\
18.813 \\
18.616 \\
18.073 \\
17.337 \\
16.753 \\
15.297 \\
13.612 \\
12.506 \\
10.115 \\
8.059 \\
7.049 \\
5.824 \\
5.002 \\
4.317 \\
3.738 \\
3.289 \\
3.013 \\
2.846\end{array}$ & $\begin{array}{l}36.919 \\
36.897 \\
36.883 \\
36.882 \\
36.801 \\
36.691 \\
36.647 \\
36.611 \\
36.548 \\
36.451 \\
36.366 \\
36.105 \\
35.838 \\
35.688 \\
35.470 \\
35.329 \\
35.359 \\
35.257 \\
35.178 \\
35.100 \\
35.037 \\
34.992\end{array}$ & $\begin{array}{l}5.21 \\
5.27 \\
5.25 \\
5.24 \\
5.22 \\
5.26 \\
5.19 \\
5.06 \\
4.73 \\
4.72 \\
4.68 \\
4.60 \\
4.53 \\
4.41 \\
4.04\end{array}$ & & & $\begin{array}{r}20.803 \\
20.177 \\
19.857 \\
19.788 \\
19.312 \\
19.011 \\
18.785 \\
18.585 \\
18.037 \\
17.294 \\
16.703 \\
15.234 \\
13.540 \\
12.424 \\
10.018 \\
7.952 \\
6.922 \\
5.682 \\
4.845 \\
4.145 \\
3.550 \\
3.084 \\
2.788 \\
2.598\end{array}$ & $\begin{array}{l}26.011 \\
26.164 \\
26.238 \\
26.256 \\
26.319 \\
26.313 \\
26.337 \\
26.361 \\
26.450 \\
26.558 \\
26.635 \\
26.773 \\
26.932 \\
27.042 \\
27.316 \\
27.540 \\
27.713 \\
27.796 \\
27.835 \\
27.851 \\
27.862 \\
27.871 \\
27.876 \\
27.882\end{array}$ & $\begin{array}{l}32.348 \\
32.515 \\
32.597 \\
32.616 \\
32.691 \\
32.692 \\
32.723 \\
32.751 \\
32.855 \\
32.982 \\
33.075 \\
33.253 \\
33.462 \\
33.605 \\
33.956 \\
34.249 \\
34.457 \\
34.586 \\
34.657 \\
34.700 \\
34.736 \\
34.764 \\
34.781 \\
34.794\end{array}$ & $\begin{array}{l}38.478 \\
38.659 \\
38.748 \\
38.769 \\
38.855 \\
38.864 \\
38.900 \\
38.933 \\
39.050 \\
39.196 \\
39.303 \\
39.521 \\
39.777 \\
39.952 \\
40.375 \\
40.735 \\
40.977 \\
41.150 \\
41.251 \\
41.321 \\
41.379 \\
41.425 \\
41.454 \\
41.475\end{array}$ & $\begin{array}{r}2 \\
25 \\
52 \\
76 \\
105 \\
126 \\
152 \\
174 \\
204 \\
251 \\
300 \\
401 \\
496 \\
596 \\
792 \\
991 \\
1234 \\
1481 \\
1728 \\
1972 \\
2218 \\
2466 \\
2707 \\
2954\end{array}$ \\
\hline
\end{tabular}




\begin{tabular}{|c|c|c|c|c|c|c|c|c|c|c|}
\hline \multicolumn{3}{|c|}{$\begin{array}{l}\text { ENDEAVOR } 143 \text { STA- } 53 \\
\text { DATE } 11 / 5 / 86\end{array}$} & LAT $=$ & \multicolumn{2}{|c|}{$2943.2 \mathrm{~N}$} & \multicolumn{2}{|c|}{$O N=$} & \multicolumn{3}{|c|}{ SONIC DEPTH $=4200 \mathrm{~m}$} \\
\hline $\begin{array}{l}\text { PR } \\
\text { dbar }\end{array}$ & $\stackrel{T}{\text { Deg } C}$ & $\stackrel{5}{0 / 00}$ & $\begin{array}{l}02 \\
\mathrm{ml} / \mathrm{I}\end{array}$ & $\stackrel{\theta}{\operatorname{Deg} C}$ & $\begin{array}{l}\mathrm{SIG}-0 \\
\mathrm{~kg} / \mathrm{m3}\end{array}$ & $\begin{array}{l}\mathrm{SIG}-1.5 \\
\mathrm{~kg} / \mathrm{m3}\end{array}$ & $\begin{array}{l}S I G-3 \\
\mathrm{~kg} / \mathrm{m} 3\end{array}$ & $\underset{m}{H G T H}$ & $\underset{c p h}{N}$ & DE \\
\hline $\begin{array}{r}1 \\
25 \\
50 \\
75 \\
100 \\
150 \\
200 \\
250 \\
300 \\
350 \\
400 \\
450 \\
500 \\
600 \\
700 \\
800 \\
900 \\
1000\end{array}$ & $\begin{array}{r}20.759 \\
20.452 \\
20.077 \\
19.716 \\
19.381 \\
18.621 \\
17.593 \\
16.956 \\
16.259 \\
15.651 \\
14.949 \\
14.360 \\
13.693 \\
12.424 \\
11.312 \\
10.459 \\
9.304 \\
8.486 \\
7.401 \\
6.332 \\
5.580 \\
4.806 \\
4.263 \\
3.795 \\
3.430 \\
3.149 \\
2.946 \\
2.831\end{array}$ & $\begin{array}{l}36.981 \\
36.888 \\
36.880 \\
36.842 \\
36.764 \\
36.633 \\
36.447 \\
36.340 \\
36.231 \\
36.130 \\
36.031 \\
35.946 \\
35.843 \\
35.675 \\
35.563 \\
35.484 \\
35.407 \\
35.387 \\
35.359 \\
35.299 \\
35.239 \\
35.155 \\
35.095 \\
35.044 \\
35.006 \\
34.979 \\
34.961 \\
34.949\end{array}$ & $\begin{array}{l}5.45 \\
5.47 \\
5.36 \\
5.30 \\
5.29 \\
4.93 \\
4.66 \\
4.60 \\
4.59 \\
4.63 \\
4.57 \\
4.52 \\
4.52 \\
4.36 \\
4.25 \\
4.09 \\
3.99 \\
4.07 \\
4.47 \\
4.88 \\
5.25 \\
5.50 \\
5.65 \\
5.74 \\
5.78 \\
5.78 \\
5.78 \\
5.77\end{array}$ & $\begin{array}{c}20.758 \\
20.447 \\
20.067 \\
19.702 \\
19.363 \\
18.595 \\
17.559 \\
16.914 \\
16.211 \\
15.596 \\
14.887 \\
14.293 \\
13.620 \\
12.342 \\
11.222 \\
10.360 \\
9.200 \\
8.376 \\
7.276 \\
6.195 \\
5.431 \\
4.647 \\
4.091 \\
3.611 \\
3.232 \\
2.937 \\
2.717 \\
2.584\end{array}$ & $\begin{array}{l}26.071 \\
26.084 \\
26.180 \\
26.248 \\
26.277 \\
26.375 \\
26.491 \\
26.565 \\
26.647 \\
26.711 \\
26.793 \\
26.857 \\
26.919 \\
27.048 \\
27.174 \\
27.268 \\
27.495 \\
27.520 \\
27.663 \\
27.764 \\
27.813 \\
27.839 \\
27.852 \\
27.861 \\
27.868 \\
27.875 \\
27.880 \\
27.882\end{array}$ & $\begin{array}{l}32.408 \\
32.429 \\
32.533 \\
32.610 \\
32.648 \\
32.765 \\
32.908 \\
33.000 \\
33.100 \\
33.181 \\
33.284 \\
33.364 \\
33.447 \\
33.614 \\
33.775 \\
33.897 \\
34.071 \\
34.214 \\
34.395 \\
34.535 \\
34.613 \\
34.669 \\
34.704 \\
34.732 \\
34.754 \\
34.773 \\
34.787 \\
34.796\end{array}$ & $\begin{array}{l}38.539 \\
38.567 \\
38.680 \\
38.765 \\
38.811 \\
38.947 \\
39.115 \\
39.223 \\
39.342 \\
39.440 \\
39.561 \\
39.658 \\
39.759 \\
39.963 \\
40.157 \\
40.305 \\
40.517 \\
40.686 \\
40.902 \\
41.080 \\
41.185 \\
41.270 \\
41.326 \\
41.373 \\
41.410 \\
41.441 \\
41.463 \\
41.477\end{array}$ & $\begin{array}{l}0.002 \\
0.050 \\
0.097 \\
0.142 \\
0.187 \\
0.274 \\
0.357 \\
0.435 \\
0.511 \\
0.585 \\
0.655 \\
0.723 \\
0.788 \\
0.910 \\
1.022 \\
1.125 \\
1.218 \\
1.298 \\
1.438 \\
1.556 \\
1.660 \\
1.758 \\
1.850 \\
1.940 \\
2.029 \\
2.115 \\
2.200 \\
2.285\end{array}$ & $\begin{array}{r}-4.05 \\
5.28 \\
3.01 \\
2.42 \\
1.70 \\
3.16 \\
2.20 \\
2.19 \\
2.27 \\
2.34 \\
2.22 \\
2.00 \\
2.12 \\
2.14 \\
1.88 \\
2.09 \\
2.29 \\
1.75 \\
1.53 \\
1.34 \\
1.03 \\
0.90 \\
0.74 \\
0.69 \\
0.67 \\
0.62 \\
0.56 \\
0.44\end{array}$ & $\begin{array}{r}1 \\
25 \\
50 \\
74 \\
99 \\
149 \\
199 \\
248 \\
298 \\
347 \\
397 \\
446 \\
496 \\
595 \\
694 \\
793 \\
892 \\
991 \\
1188 \\
1386 \\
1583 \\
1780 \\
1977 \\
2174 \\
2370 \\
2566 \\
2763 \\
2958\end{array}$ \\
\hline $\begin{array}{l}\text { PR } \\
\text { dbar }\end{array}$ & $\stackrel{T}{T} C$ & $\stackrel{5}{5}$ & $\begin{array}{l}02 \\
\mathrm{ml} / \mathrm{l}\end{array}$ & $\begin{array}{c}\mathrm{F}-11 \\
\mathrm{pmol} / \mathrm{kg}\end{array}$ & $\begin{array}{c}\mathrm{F}-12 \\
\mathrm{pmol} / \mathrm{kg}\end{array}$ & $\stackrel{\ominus}{\operatorname{Deg} C}$ & $\begin{array}{l}\mathrm{SIG}-\Theta \\
\mathrm{kg} / \mathrm{m3}\end{array}$ & $\underset{\mathrm{kg} / \mathrm{m} 3}{\mathrm{SIG}-1}$ & $\begin{array}{l}\mathrm{SIG}-3 \\
\mathrm{~kg} / \mathrm{m} 3\end{array}$ & \\
\hline $\begin{array}{r}3 \\
28 \\
54 \\
78 \\
104 \\
127 \\
151 \\
163 \\
205 \\
255 \\
302 \\
402 \\
504 \\
749\end{array}$ & $\begin{array}{r}20.095 \\
19.852 \\
19.700 \\
19.708 \\
19.455 \\
18.303 \\
18.080 \\
17.441 \\
16.863 \\
16.222 \\
15.008 \\
13.527 \\
10.865 \\
8.290 \\
7.282 \\
6.036 \\
5.018 \\
4.278 \\
3.662 \\
3.283 \\
3.015 \\
2.831\end{array}$ & $\begin{array}{l}36 . \\
36 . \\
36 . \\
36 . \\
36 . \\
36 . \\
36 . \\
36 . \\
36 . \\
36 . \\
36 . \\
35 . \\
35 . \\
35 . \\
35 \\
35 . \\
35 . \\
35 . \\
35 . \\
35 . \\
34 . \\
34 .\end{array}$ & $\begin{array}{l}5.30 \\
5.31 \\
5.27 \\
5.23 \\
5.13 \\
4.84 \\
4.70 \\
4.67 \\
4.64 \\
4.66 \\
4.62\end{array}$ & $\begin{array}{l}1.908 \\
1.963 \\
1.943 \\
1.916 \\
1.961 \\
1.835 \\
1.790 \\
1.766 \\
1.714 \\
1.690 \\
1.660 \\
1.470 \\
0.697 \\
0.151 \\
0.172 \\
0.084 \\
0.062 \\
0.031 \\
0.023 \\
0.022 \\
0.020 \\
0.022\end{array}$ & $\begin{array}{l}1.169 \\
1.059 \\
1.156 \\
1.251 \\
1.255 \\
1.159 \\
1.170 \\
1.147 \\
0.981 \\
0.954 \\
0.902 \\
0.810 \\
0.733 \\
0.337 \\
0.099 \\
0.059 \\
0.055 \\
0.031 \\
0.010 \\
0.000 \\
0.000 \\
0.008 \\
0.000\end{array}$ & $\begin{array}{r}20.902 \\
20.090 \\
19.842 \\
19.686 \\
19.689 \\
19.431 \\
18.277 \\
18.052 \\
17.406 \\
16.821 \\
16.174 \\
14.946 \\
13.455 \\
10.771 \\
8.181 \\
7.153 \\
5.892 \\
4.861 \\
4.106 \\
3.475 \\
3.077 \\
2.790 \\
2.584\end{array}$ & $\begin{array}{l}991 \\
143 \\
250 \\
300 \\
285 \\
317 \\
414 \\
447 \\
521 \\
579 \\
658 \\
792 \\
945 \\
226 \\
539 \\
689 \\
793 \\
838 \\
852 \\
862 \\
879 \\
876 \\
881\end{array}$ & $\begin{array}{l}32.325 \\
32.497 \\
32.609 \\
32.662 \\
32.647 \\
32.685 \\
32.812 \\
32.851 \\
32.942 \\
33.016 \\
33.112 \\
33.281 \\
33.478 \\
33.841 \\
34.240 \\
34.425 \\
34.575 \\
34.659 \\
34.704 \\
34.739 \\
34.772 \\
34.781 \\
34.795\end{array}$ & $\begin{array}{l}38.454 \\
38.643 \\
38.760 \\
38.817 \\
38.802 \\
38.846 \\
39.002 \\
39.046 \\
39.153 \\
39.242 \\
39.355 \\
39.557 \\
39.795 \\
40.237 \\
40.718 \\
40.937 \\
41.130 \\
41.252 \\
41.325 \\
41.385 \\
41.433 \\
41.454 \\
41.476\end{array}$ & $\begin{array}{r}27 \\
53 \\
77 \\
103 \\
125 \\
150 \\
162 \\
203 \\
252 \\
300 \\
398 \\
499 \\
741 \\
991 \\
1235 \\
1479 \\
1726 \\
1973 \\
2217 \\
2463 \\
2705 \\
2956\end{array}$ \\
\hline
\end{tabular}




\begin{tabular}{|c|c|c|c|c|c|c|c|c|c|c|}
\hline \multicolumn{3}{|c|}{$\begin{array}{l}\text { ENDEAVOR } 143 \text { STA- } 54 \\
\text { DATE } 11 / 5 / 86\end{array}$} & LAT $=$ & \multicolumn{2}{|c|}{$2930.0 \mathrm{~N}$} & \multicolumn{2}{|c|}{$\mathrm{ON}=$} & \multicolumn{2}{|c|}{ SONIC DEPTH= } & \multirow{2}{*}{$\begin{array}{l}\text { Om } \\
\text { DE } \\
\text { m }\end{array}$} \\
\hline $\begin{array}{l}\text { PR } \\
\text { dbar }\end{array}$ & $\stackrel{T}{T} \mathrm{C}$ & $\stackrel{S}{100}$ & $\begin{array}{l}02 \\
\mathrm{ml} / \mathrm{l}\end{array}$ & $\stackrel{\ominus}{\operatorname{Deg} C}$ & $\begin{array}{l}\mathrm{SIG}-0 \\
\mathrm{~kg} / \mathrm{m} 3\end{array}$ & $\underset{\mathrm{kg} / \mathrm{m} 3}{\mathrm{SIG}-1.5}$ & $\begin{array}{l}\mathrm{SIG}-3 \\
\mathrm{~kg} / \mathrm{m} 3\end{array}$ & $\underset{m}{\mathrm{HGTH}}$ & $\underset{c p h}{N}$ & \\
\hline $\begin{array}{r}3 \\
25 \\
50 \\
75 \\
100 \\
150 \\
200 \\
250 \\
300 \\
311\end{array}$ & $\begin{array}{l}20.862 \\
20.343 \\
19.604 \\
19.219 \\
19.075 \\
18.760 \\
17.803 \\
17.263 \\
16.378 \\
16.170\end{array}$ & $\begin{array}{l}36.801 \\
36.788 \\
36.754 \\
36.693 \\
36.673 \\
36.625 \\
36.469 \\
36.423 \\
36.248 \\
36.213\end{array}$ & $\begin{array}{l}5.25 \\
5.11 \\
5.10 \\
5.09 \\
5.01 \\
4.83 \\
4.33 \\
4.21 \\
4.16 \\
4.21\end{array}$ & $\begin{array}{l}20.861 \\
20.338 \\
19.595 \\
19.206 \\
19.057 \\
18.733 \\
17.768 \\
17.221 \\
16.329 \\
16.120\end{array}$ & $\begin{array}{l}25.905 \\
26.037 \\
26.209 \\
26.264 \\
26.287 \\
26.333 \\
26.456 \\
26.555 \\
26.632 \\
26.655\end{array}$ & $\begin{array}{l}32.242 \\
32.385 \\
32.574 \\
32.639 \\
32.666 \\
32.720 \\
32.868 \\
32.981 \\
33.083 \\
33.111\end{array}$ & $\begin{array}{l}38.372 \\
38.527 \\
38.733 \\
38.807 \\
38.837 \\
38.899 \\
39.070 \\
39.197 \\
39.321 \\
39.355\end{array}$ & $\begin{array}{l}0.006 \\
0.052 \\
0.098 \\
0.143 \\
0.187 \\
0.275 \\
0.360 \\
0.440 \\
0.516 \\
0.533\end{array}$ & $\begin{array}{l}2.71 \\
6.13 \\
3.17 \\
2.05 \\
1.68 \\
2.03 \\
2.69 \\
2.36 \\
2.48 \\
2.30\end{array}$ & $\begin{array}{r}3 \\
25 \\
50 \\
74 \\
99 \\
149 \\
199 \\
248 \\
298 \\
309\end{array}$ \\
\hline $\begin{array}{l}\text { PR } \\
\text { dbar }\end{array}$ & $\stackrel{T}{\text { Deg } C}$ & $\stackrel{5}{0 / 00}$ & $\begin{array}{l}02 \\
\mathrm{ml} / \mathrm{I}\end{array}$ & $\begin{array}{c}\mathrm{F}-11 \\
\mathrm{pmol} / \mathrm{kg}\end{array}$ & $\begin{array}{c}\mathrm{F}-12 \\
\mathrm{pmol} / \mathrm{kg}\end{array}$ & $\stackrel{\theta}{D} C$ & $\begin{array}{l}\mathrm{SIG}-\mathrm{O} \\
\mathrm{kg} / \mathrm{m3}\end{array}$ & $\underset{\mathrm{kg} / \mathrm{m} 3}{\mathrm{SIG}-1.5}$ & $\begin{array}{l}S I G-3 \\
\mathrm{~kg} / \mathrm{m} 3\end{array}$ & \\
\hline $\begin{array}{r}30 \\
45 \\
106 \\
226\end{array}$ & $\begin{array}{l}19.865 \\
19.676 \\
18.995 \\
17.424\end{array}$ & $\begin{array}{l}36.782 \\
36.691 \\
36.437\end{array}$ & $\begin{array}{l}5.44 \\
5.44 \\
4.78\end{array}$ & & & $\begin{array}{l}19.667 \\
18.976 \\
17.385\end{array}$ & $\begin{array}{l}26.211 \\
26.322 \\
26.526\end{array}$ & $\begin{array}{l}32.575 \\
32.702 \\
32.947\end{array}$ & $\begin{array}{l}38.731 \\
38.875 \\
39.159\end{array}$ & $\begin{array}{r}29 \\
44 \\
105 \\
224\end{array}$ \\
\hline
\end{tabular}

\begin{tabular}{|c|c|c|c|c|c|c|c|c|c|c|}
\hline \multicolumn{3}{|c|}{$\begin{array}{l}\text { ENDEAVOR } 143 \text { STA- } 55 \\
\text { DATE } 11 / 5 / 86\end{array}$} & $L A T=$ & \multicolumn{2}{|c|}{$2920.2 \mathrm{~N}$} & \multicolumn{2}{|c|}{$\mathrm{ON}=$} & \multicolumn{3}{|c|}{ SONIC DEPTH $4950 \mathrm{~m}$} \\
\hline $\begin{array}{l}\text { PR } \\
\text { dbar }\end{array}$ & $\stackrel{T}{D} C$ & \$ & $\begin{array}{l}02 \\
\mathrm{ml} / \mathrm{l}\end{array}$ & $\stackrel{\theta}{\operatorname{Deg} C}$ & $\begin{array}{l}\mathrm{SIG}-0 \\
\mathrm{~kg} / \mathrm{m} 3\end{array}$ & $\underset{\mathrm{kg} / \mathrm{m} 3}{\mathrm{SIG}-1.5}$ & $\begin{array}{l}\mathrm{SIG}-3 \\
\mathrm{~kg} / \mathrm{m} 3\end{array}$ & $\underset{m}{H G T H}$ & $\underset{\text { cph }}{N}$ & DE \\
\hline $\begin{array}{r}1 \\
25 \\
50 \\
75 \\
100 \\
150 \\
200 \\
250 \\
300\end{array}$ & $\begin{array}{l}20.663 \\
20.289 \\
19.590 \\
19.210 \\
19.029 \\
18.687 \\
17.587 \\
16.925 \\
16.068\end{array}$ & $\begin{array}{l}36.740 \\
36.779 \\
36.760 \\
36.690 \\
36.668 \\
36.612 \\
36.423 \\
36.327 \\
36.193\end{array}$ & $\begin{array}{l}5.19 \\
5.13 \\
5.17 \\
5.16 \\
5.09 \\
4.92 \\
4.31 \\
4.24 \\
4.09\end{array}$ & $\begin{array}{l}20.663 \\
20.284 \\
19.581 \\
19.197 \\
19.011 \\
18.660 \\
17.553 \\
16.883 \\
16.020\end{array}$ & $\begin{array}{l}25.913 \\
26.045 \\
26.217 \\
26.264 \\
26.295 \\
26.343 \\
26.474 \\
26.562 \\
26.662\end{array}$ & $\begin{array}{l}32.254 \\
32.394 \\
32.583 \\
32.640 \\
32.675 \\
32.731 \\
32.892 \\
32.997 \\
33.121\end{array}$ & $\begin{array}{l}38.389 \\
38.537 \\
38.741 \\
38.807 \\
38.847 \\
38.912 \\
39.099 \\
39.222 \\
39.368\end{array}$ & $\begin{array}{l}0.002 \\
0.051 \\
0.097 \\
0.142 \\
0.186 \\
0.274 \\
0.357 \\
0.436 \\
0.512\end{array}$ & $\begin{array}{l}4.11 \\
5.13 \\
2.66 \\
2.50 \\
1.79 \\
2.40 \\
2.66 \\
2.24 \\
2.59\end{array}$ & $\begin{array}{r}1 \\
25 \\
50 \\
74 \\
99 \\
149 \\
199 \\
248 \\
298\end{array}$ \\
\hline $\begin{array}{l}\text { PR } \\
\text { dbar }\end{array}$ & $\stackrel{T}{D e g} C$ & $\stackrel{5}{0 / 00}$ & $\begin{array}{l}02 \\
m 1 / 1\end{array}$ & $\begin{array}{c}\mathrm{F}-11 \\
\mathrm{pmol} / \mathrm{kg}\end{array}$ & $\begin{array}{c}\mathrm{F}-12 \\
\mathrm{pmol} / \mathrm{kg}\end{array}$ & $\stackrel{\theta}{\operatorname{Deg}} \mathrm{C}$ & $\begin{array}{l}\mathrm{SIG}-\mathrm{O} \\
\mathrm{kg} / \mathrm{m3}\end{array}$ & $\underset{\mathrm{kg} / \mathrm{m} 3}{\mathrm{SIG}-1.5}$ & $\begin{array}{l}\text { SIG } \\
\mathrm{kg} /\end{array}$ & $D E$ \\
\hline $\begin{array}{r}33 \\
35 \\
98 \\
209\end{array}$ & $\begin{array}{l}19.873 \\
19.767 \\
18.996 \\
17.479\end{array}$ & $\begin{array}{l}36.782 \\
36.773 \\
36.666 \\
36.417\end{array}$ & $\begin{array}{l}5.37 \\
5.38 \\
5.35 \\
4.67\end{array}$ & & & $\begin{array}{l}19.867 \\
19.760 \\
18.978 \\
17.443\end{array}$ & $\begin{array}{l}26.158 \\
26.180 \\
26.302 \\
26.496\end{array}$ & $\begin{array}{l}32.518 \\
32.541 \\
32.683 \\
32.917\end{array}$ & $\begin{array}{l}38.670 \\
38.696 \\
38.855 \\
39.127\end{array}$ & $\begin{array}{r}32 \\
35 \\
97 \\
207\end{array}$ \\
\hline
\end{tabular}

\begin{tabular}{|c|c|c|c|c|c|c|c|c|c|c|}
\hline \multicolumn{3}{|c|}{$\begin{array}{l}\text { ENDEAVOR } 143 \text { STA- } 56 \\
\text { DATE } 11 / 5 / 86\end{array}$} & \multicolumn{3}{|c|}{ LAT $=2910.1 \mathrm{~N}$} & \multicolumn{2}{|c|}{ LON= } & \multicolumn{3}{|c|}{ SONIC DEPTH $4280 \mathrm{~m}$} \\
\hline $\begin{array}{l}\text { PR } \\
\text { dbar }\end{array}$ & $\stackrel{T}{\text { Deg } C}$ & $\stackrel{5}{0 / 00}$ & $\begin{array}{l}02 \\
\mathrm{ml} / \mathrm{l}\end{array}$ & $\stackrel{\theta}{D e g} \mathrm{C}$ & $\begin{array}{l}\mathrm{SIG}-\theta \\
\mathrm{kg} / \mathrm{m3}\end{array}$ & $\mathrm{SIG}_{\mathrm{kg} / \mathrm{m} 3}^{-1.5}$ & $\begin{array}{l}\mathrm{SIG}-3 \\
\mathrm{~kg} / \mathrm{m} 3\end{array}$ & $\underset{m}{\mathrm{HGTH}}$ & $\underset{\text { cph }}{N}$ & $\begin{array}{l}\mathrm{DE} \\
\text { m }\end{array}$ \\
\hline $\begin{array}{r}1 \\
25 \\
50 \\
75 \\
100 \\
150 \\
200 \\
250 \\
300\end{array}$ & $\begin{array}{l}20.873 \\
20.116 \\
19.428 \\
19.075 \\
18.875 \\
18.017 \\
17.365 \\
16.585 \\
15.959\end{array}$ & $\begin{array}{l}36.787 \\
36.750 \\
36.688 \\
36.652 \\
36.635 \\
36.477 \\
36.392 \\
36.264 \\
36.179\end{array}$ & $\begin{array}{l}5.34 \\
5.44 \\
5.41 \\
5.22 \\
5.09 \\
4.55 \\
4.25 \\
4.28 \\
4.14\end{array}$ & $\begin{array}{l}20.873 \\
20.111 \\
19.419 \\
19.062 \\
18.857 \\
17.991 \\
17.331 \\
16.544 \\
15.911\end{array}$ & $\begin{array}{l}25.891 \\
26.069 \\
26.204 \\
26.270 \\
26.310 \\
26.407 \\
26.505 \\
26.594 \\
26.676\end{array}$ & $\begin{array}{l}32.228 \\
32.423 \\
32.575 \\
32.649 \\
32.694 \\
32.813 \\
32.928 \\
33.039 \\
33.138\end{array}$ & $\begin{array}{l}38.358 \\
38.570 \\
38.737 \\
38.820 \\
38.869 \\
39.010 \\
39.141 \\
39.272 \\
39.388\end{array}$ & $\begin{array}{l}0 . \\
0 . \\
0 . \\
0 . \\
0 . \\
0 . \\
0 . \\
0 . \\
0 .\end{array}$ & $\begin{array}{l}3.32 \\
5.69 \\
3.07 \\
2.83 \\
2.13 \\
2.89 \\
2.19 \\
2.57 \\
2.36\end{array}$ & $\begin{array}{r}1 \\
25 \\
50 \\
74 \\
99 \\
149 \\
199 \\
248 \\
298\end{array}$ \\
\hline $\begin{array}{l}\text { PR } \\
\text { dbor }\end{array}$ & ${ }_{\text {Deg }}^{T}$ & $\stackrel{S}{0 / 00}$ & $\begin{array}{l}02 \\
\mathrm{ml} / \mathrm{I}\end{array}$ & $\begin{array}{c}\mathrm{F}-11 \\
\mathrm{pmol} / \mathrm{kg}\end{array}$ & $\begin{array}{c}\mathrm{F}-12 \\
\mathrm{~mol} / \mathrm{kg}\end{array}$ & $\stackrel{\theta}{\operatorname{Deg}} \mathrm{C}$ & $\begin{array}{l}\mathrm{SIG}-\theta \\
\mathrm{kg} / \mathrm{m} 3\end{array}$ & $\underset{\mathrm{kg} / \mathrm{r}}{\mathrm{SIG}}$ & $\begin{array}{l}\text { SIG-3 } \\
\mathrm{kg} / \mathrm{m3}\end{array}$ & $\begin{array}{l}\mathrm{DE} \\
\mathrm{m}\end{array}$ \\
\hline $\begin{array}{r}39 \\
88 \\
189\end{array}$ & $\begin{array}{l}19.735 \\
18.954 \\
17.479\end{array}$ & $\begin{array}{l}36.763 \\
36.652 \\
36.444\end{array}$ & $\begin{array}{l}5.38 \\
5.37 \\
4.67\end{array}$ & & & $\begin{array}{l}19.728 \\
18.938 \\
17.447\end{array}$ & $\begin{array}{l}26.181 \\
26.302 \\
26.516\end{array}$ & $\begin{array}{l}32.543 \\
32.684 \\
32.936\end{array}$ & $\begin{array}{l}38.698 \\
38.857 \\
39.146\end{array}$ & $\begin{array}{r}38 \\
87 \\
187\end{array}$ \\
\hline
\end{tabular}




\begin{tabular}{|c|c|c|c|c|c|c|c|c|c|c|}
\hline \multicolumn{3}{|c|}{$\begin{array}{l}\text { ENDEAVOR } 143 \text { STA- } 57 \\
\text { DATE } 11 / 5 / 86\end{array}$} & \multicolumn{2}{|c|}{$L A T=29$} & $0.0 \mathrm{~N}$ & \multicolumn{2}{|c|}{$3216.0 \mathrm{w}$} & \multicolumn{2}{|c|}{ SONIC DEPTH= } & \multirow{2}{*}{$\begin{array}{l}\text { Om } \\
\text { DE } \\
\text { m }\end{array}$} \\
\hline $\begin{array}{l}\text { PR } \\
\text { dbar }\end{array}$ & $\stackrel{T}{\operatorname{Deg}} \mathrm{C}$ & $\stackrel{5}{0 / 00}$ & $\begin{array}{l}02 \\
\mathrm{ml} / \mathrm{l}\end{array}$ & $\stackrel{\theta}{\operatorname{Deg} C}$ & $\begin{array}{l}S I G-\theta \\
\mathrm{kg} / \mathrm{m} 3\end{array}$ & $\begin{array}{l}\mathrm{SIG}-1.5 \\
\mathrm{~kg} / \mathrm{m} 3\end{array}$ & $\begin{array}{l}\mathrm{SIG}-3 \\
\mathrm{~kg} / \mathrm{m} 3\end{array}$ & $\underset{m}{\text { HGTH }}$ & $\underset{\mathrm{cph}}{\mathrm{N}}$ & \\
\hline $\begin{array}{r}1 \\
25 \\
50 \\
75 \\
100 \\
150 \\
200 \\
250 \\
300 \\
305\end{array}$ & $\begin{array}{l}20.801 \\
19.880 \\
19.305 \\
19.051 \\
18.925 \\
18.244 \\
17.594 \\
16.876 \\
16.276 \\
16.170\end{array}$ & $\begin{array}{l}36.828 \\
36.738 \\
36.675 \\
36.650 \\
36.643 \\
36.517 \\
36.440 \\
36.324 \\
36.238 \\
36.217\end{array}$ & $\begin{array}{l}5.32 \\
5.46 \\
5.43 \\
5.35 \\
5.24 \\
4.82 \\
4.36 \\
4.24 \\
4.24 \\
4.25\end{array}$ & $\begin{array}{l}20.800 \\
19.876 \\
19.296 \\
19.038 \\
18.907 \\
18.218 \\
17.560 \\
16.834 \\
16.227 \\
16.121\end{array}$ & $\begin{array}{l}25.942 \\
26.122 \\
26.226 \\
26.275 \\
26.303 \\
26.381 \\
26.485 \\
26.572 \\
26.648 \\
26.657\end{array}$ & $\begin{array}{l}32.280 \\
32.481 \\
32.600 \\
32.654 \\
32.686 \\
32.781 \\
32.903 \\
33.009 \\
33.101 \\
33.113\end{array}$ & $\begin{array}{l}38.411 \\
38.634 \\
38.765 \\
38.826 \\
38.860 \\
38.973 \\
39.110 \\
39.234 \\
39.343 \\
39.357\end{array}$ & $\begin{array}{l}0.002 \\
0.050 \\
0.096 \\
0.140 \\
0.184 \\
0.270 \\
0.353 \\
0.432 \\
0.507 \\
0.515\end{array}$ & $\begin{array}{l}4.04 \\
4.68 \\
3.29 \\
2.02 \\
1.88 \\
2.74 \\
2.37 \\
2.34 \\
2.12 \\
2.26\end{array}$ & $\begin{array}{r}1 \\
25 \\
50 \\
74 \\
99 \\
149 \\
199 \\
248 \\
298 \\
303\end{array}$ \\
\hline $\begin{array}{l}P R \\
\text { dbar }\end{array}$ & $\stackrel{T}{\operatorname{Deg}} C$ & $\stackrel{5}{0 / 00}$ & $\begin{array}{ll}02 \\
m ! / 1\end{array}$ & $\begin{array}{c}\mathrm{F}-11 \\
\mathrm{pmol} / \mathrm{kg}\end{array}$ & $\begin{array}{c}\mathrm{F}-12 \\
\mathrm{pmol} / \mathrm{kg}\end{array}$ & $\stackrel{\theta}{\operatorname{Deg} C}$ & $\begin{array}{l}\mathrm{SIG}-\theta \\
\mathrm{kg} / \mathrm{m} 3\end{array}$ & $\underset{\mathrm{kg} / \mathrm{m} 3}{\mathrm{SIG}-1.5}$ & $\begin{array}{l}\mathrm{SIG}-3 \\
\mathrm{~kg} / \mathrm{m} 3\end{array}$ & $\underset{m}{\mathrm{DE}}$ \\
\hline $\begin{array}{r}35 \\
85 \\
212\end{array}$ & $\begin{array}{l}19.722 \\
19.008 \\
17.474\end{array}$ & $\begin{array}{l}36.748 \\
36.654 \\
36.450\end{array}$ & $\begin{array}{l}5.35 \\
5.33 \\
4.69\end{array}$ & & & $\begin{array}{l}19.716 \\
18.993 \\
17.438\end{array}$ & $\begin{array}{l}26.172 \\
26.289 \\
26.523\end{array}$ & $\begin{array}{l}32.535 \\
32.670 \\
32.943\end{array}$ & $\begin{array}{l}38.691 \\
38.842 \\
39.153\end{array}$ & $\begin{array}{r}35 \\
84 \\
210\end{array}$ \\
\hline
\end{tabular}

ENDEAVOR 143 STA- 58
DATE $11 / 5 / 86$

\begin{tabular}{|c|c|c|c|c|c|c|c|c|c|c|}
\hline $\begin{array}{c}\text { PR } \\
\text { dbar }\end{array}$ & $\stackrel{T}{D e g} C$ & $\stackrel{S}{0 / 00}$ & $\begin{array}{c}02 \\
\mathrm{ml} / \mathrm{l}\end{array}$ & $\stackrel{\theta}{\operatorname{Deg} C}$ & $\begin{array}{l}S I G-O \\
\mathrm{~kg} / \mathrm{m} 3\end{array}$ & $\underset{\mathrm{kg} / \mathrm{m} 3}{\mathrm{SIG}-1.5}$ & $\begin{array}{l}S I G-3 \\
\mathrm{~kg} / \mathrm{m} 3\end{array}$ & $\underset{\mathbf{m}}{\text { HGTH }}$ & $\underset{\text { cph }}{N}$ & \\
\hline $\begin{array}{r}1 \\
25 \\
50 \\
75 \\
100 \\
150 \\
200 \\
250 \\
300\end{array}$ & $\begin{array}{l}20.840 \\
20.028 \\
19.479 \\
19.299 \\
19.112 \\
18.521 \\
17.697 \\
17.217 \\
16.589\end{array}$ & $\begin{array}{l}36.889 \\
36.805 \\
36.723 \\
36.716 \\
36.679 \\
36.571 \\
36.459 \\
36.425 \\
36.321\end{array}$ & $\begin{array}{l}4.86 \\
5.06 \\
5.13 \\
5.10 \\
4.95 \\
4.70 \\
4.16 \\
4.18 \\
4.10\end{array}$ & $\begin{array}{l}20.840 \\
20.023 \\
19.470 \\
19.285 \\
19.094 \\
18.494 \\
17.662 \\
17.175 \\
16.540\end{array}$ & $\begin{array}{l}25.978 \\
26.134 \\
26.218 \\
26.261 \\
26.282 \\
26.353 \\
26.475 \\
26.567 \\
26.639\end{array}$ & $\begin{array}{l}32.314 \\
32.490 \\
32.586 \\
32.634 \\
32.660 \\
32.746 \\
32.889 \\
32.994 \\
33.084\end{array}$ & $\begin{array}{l}38.444 \\
38.638 \\
38.748 \\
38.799 \\
38.830 \\
38.931 \\
39.094 \\
39.211 \\
39.317\end{array}$ & $\begin{array}{l}0.002 \\
0.050 \\
0.096 \\
0.141 \\
0.185 \\
0.272 \\
0.356 \\
0.435 \\
0.512\end{array}$ & $\begin{array}{l}1.13 \\
5.25 \\
2.97 \\
1.93 \\
1.91 \\
2.53 \\
2.44 \\
2.61 \\
1.92\end{array}$ & $\begin{array}{l}2 \\
29\end{array}$ \\
\hline $\begin{array}{l}\text { PR } \\
\text { dbar }\end{array}$ & $\stackrel{T}{\operatorname{Deg}} \mathrm{C}$ & $\begin{array}{c}S \\
0 / 00\end{array}$ & $\begin{array}{l}02 \\
\mathrm{ml} / \mathrm{l}\end{array}$ & $\begin{array}{c}\mathrm{F}-11 \\
\mathrm{pmol} / \mathrm{kg}\end{array}$ & $\begin{array}{c}\mathrm{F}-12 \\
\mathrm{pmol} / \mathrm{kg}\end{array}$ & $\stackrel{\theta}{D e g} C$ & $\begin{array}{l}\mathrm{SIG}-0 \\
\mathrm{~kg} / \mathrm{m3}\end{array}$ & $\underset{\mathrm{kg} / \mathrm{m} 3}{\mathrm{SIG}-1.5}$ & $\begin{array}{l}\mathrm{SIG}-3 \\
\mathrm{~kg} / \mathrm{m} 3\end{array}$ & \\
\hline $\begin{array}{r}62 \\
128 \\
244\end{array}$ & $\begin{array}{l}19.419 \\
19.153 \\
17.486\end{array}$ & $\begin{array}{l}36.733 \\
36.732 \\
36.477\end{array}$ & $\begin{array}{l}5.35 \\
5.20 \\
4.70\end{array}$ & & & $\begin{array}{l}19.407 \\
19.130 \\
17.444\end{array}$ & $\begin{array}{l}26.242 \\
26.313 \\
26.542\end{array}$ & $\begin{array}{l}32.612 \\
32.690 \\
32.962\end{array}$ & $\begin{array}{l}38.775 \\
38.859 \\
39.172\end{array}$ & \\
\hline
\end{tabular}

ENDEAVOR 143 STA- 59
DATE $11 / 5 / 86$

$\begin{array}{rcc}\begin{array}{c}\text { PR } \\ \text { dbar }\end{array} & \begin{array}{c}T \\ \text { Deg C }\end{array} & \begin{array}{c}\mathrm{S} \\ 0 / 00\end{array} \\ 1 & 20.960 & 36.964 \\ 25 & 20.474 & 36.997 \\ 50 & 20.228 & 36.999 \\ 75 & 20.096 & 36.999 \\ 100 & 20.044 & 37.011 \\ 150 & 19.901 & 36.987 \\ 200 & 18.526 & 36.670 \\ 250 & 17.512 & 36.491 \\ 300 & 16.643 & 36.336 \\ 305 & 16.578 & 36.325\end{array}$

$\begin{array}{cccc}\begin{array}{c}\text { PR } \\ \text { dbar }\end{array} & \begin{array}{c}T \\ \text { Deg }\end{array} & \begin{array}{c}\mathrm{S} \\ 0 / 00\end{array} & \begin{array}{c}02 \\ \mathrm{ml} / \mathrm{I}\end{array} \\ 75 & 20.063 & 36.987 & 5.23 \\ 133 & 19.911 & 37.000 & 5.16 \\ 190 & 19.033 & 36.794 & 4.92 \\ 254 & 17.402 & 36.483 & 4.65\end{array}$

LAT $=2840.1 \mathrm{~N}$ LON= $3216.1 \mathrm{~W}$

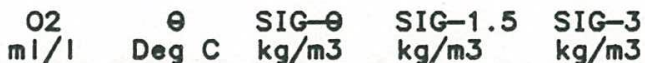

$\begin{array}{llllll}4.81 & 20.959 & 26.003 & 32.336 & 38.463\end{array}$

$\begin{array}{lllll}4.98 & 20.469 & 26.161 & 32.505 & 38.642\end{array}$

$\begin{array}{lllll}4.98 & 20.219 & 26.230 & 32.579 & 38.722\end{array}$

$\begin{array}{lllll}4.95 & 20.082 & 26.267 & 32.619 & 38.764\end{array}$

$\begin{array}{lllll}4.89 & 20.025 & 26.291 & 32.645 & 38.791\end{array}$

$\begin{array}{llllll}4.78 & 19.873 & 26.314 & 32.671 & 38.821\end{array}$

$\begin{array}{llllll}4.29 & 18.491 & 26.430 & 32.822 & 39.006\end{array}$

$\begin{array}{lllll}4.18 & 17.470 & 26.546 & 32.965 & 39.175\end{array}$

$\begin{array}{lllll}4.14 & 16.593 & 26.638 & 33.081 & 39.312\end{array}$

$\begin{array}{lllll}4.20 & 16.528 & 26.645 & 33.089 & 39.323\end{array}$

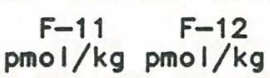
$\mathrm{pmol} / \mathrm{kg} \mathrm{pmol} / \mathrm{kg}$

(a)

SONIC DEPTH $4700 \mathrm{~m}$

$\underset{\mathrm{m}}{\mathrm{HGTH}} \underset{\mathrm{cph}}{\mathrm{N}} \quad \mathrm{DE}$

$0.002-0.47$

$\begin{array}{lll}0.049 & 5.70 \quad 25\end{array}$

$\begin{array}{lll}0.095 & 2.36 \quad 50\end{array}$

$\begin{array}{lll}0.139 & 2.05 \quad 74\end{array}$

$0.184 \quad 1.38 \quad 99$

$0.272 \quad 1.33 \quad 149$

$0.358 \quad 3.42 \quad 199$

$0.439 \quad 2.45 \quad 248$

$\begin{array}{lll}0.515 & 2.35 & 298\end{array}$

$\begin{array}{lll}0.523 & 2.63 \quad 303\end{array}$

DE

$\begin{array}{llr}\mathrm{kg} / \mathrm{m} 3 & \mathrm{Kg} / \mathrm{m} 3 & \mathrm{~m}\end{array}$

$\begin{array}{lllll}20.049 & 26.267 & 32.620 & 38.766 & 74\end{array}$

$\begin{array}{lllll}19.886 & 26.320 & 32.676 & 38.826 & 131\end{array}$

$\begin{array}{lllll}18.998 & 26.395 & 32.774 & 38.945 & 188\end{array}$

$\begin{array}{lllll}17.359 & 26.567 & 32.989 & 39.201 & 252\end{array}$ 


\begin{tabular}{|c|c|c|c|c|c|c|c|c|c|c|}
\hline \multicolumn{3}{|c|}{$\begin{array}{l}\text { ENDEAVOR } 143 \text { STA- } 60 \\
\text { DATE } 11 / 5 / 86\end{array}$} & LAT $=$ & \multicolumn{2}{|c|}{$2840.0 \mathrm{~N}$} & $O N=$ & $4.1 W$ & \multicolumn{2}{|c|}{ SONIC DEPTH= } & $\theta m$ \\
\hline $\begin{array}{l}\text { PR } \\
\text { dbar }\end{array}$ & $\stackrel{T}{\operatorname{Deg} C}$ & $\stackrel{5}{0 / 00}$ & $\mathrm{O} 2$ & $\stackrel{\theta}{\operatorname{Deg} C}$ & $\begin{array}{l}\mathrm{SIG}-0 \\
\mathrm{~kg} / \mathrm{m3}\end{array}$ & $\begin{array}{l}\mathrm{SIG}-1.5 \\
\mathrm{~kg} / \mathrm{m} 3\end{array}$ & $\begin{array}{l}\mathrm{SIG}-3 \\
\mathrm{~kg} / \mathrm{m} 3\end{array}$ & $\underset{m}{H G T H}$ & $\underset{c p h}{N}$ & $\begin{aligned} D E \\
m\end{aligned}$ \\
\hline $\begin{array}{r}1 \\
25 \\
50 \\
75 \\
100 \\
150 \\
200 \\
250 \\
300 \\
317\end{array}$ & $\begin{array}{l}21.018 \\
20.926 \\
20.090 \\
19.903 \\
19.847 \\
19.371 \\
17.775 \\
17.213 \\
16.245 \\
15.969\end{array}$ & $\begin{array}{l}36.982 \\
37.018 \\
36.965 \\
36.953 \\
36.955 \\
36.840 \\
36.507 \\
36.441 \\
36.261 \\
36.212\end{array}$ & $\begin{array}{l}4.83 \\
4.82 \\
4.97 \\
4.92 \\
4.74 \\
4.53 \\
4.16 \\
4.08 \\
4.09 \\
4.15\end{array}$ & $\begin{array}{l}21.017 \\
20.921 \\
20.081 \\
19.889 \\
19.828 \\
19.344 \\
17.740 \\
17.171 \\
16.196 \\
15.919\end{array}$ & $\begin{array}{l}26.000 \\
26.054 \\
26.241 \\
26.283 \\
26.301 \\
26.341 \\
26.492 \\
26.581 \\
26.674 \\
26.700\end{array}$ & $\begin{array}{l}32.331 \\
32.387 \\
32.594 \\
32.640 \\
32.659 \\
32.711 \\
32.905 \\
33.008 \\
33.127 \\
33.161\end{array}$ & $\begin{array}{l}38.457 \\
38.515 \\
38.740 \\
38.790 \\
38.810 \\
38.874 \\
39.107 \\
39.224 \\
39.369 \\
39.411\end{array}$ & $\begin{array}{l}0.002 \\
0.049 \\
0.095 \\
0.140 \\
0.184 \\
0.271 \\
0.354 \\
0.433 \\
0.508 \\
0.532\end{array}$ & $\begin{array}{l}2.36 \\
5.02 \\
2.79 \\
2.04 \\
1.11 \\
3.06 \\
2.62 \\
2.44 \\
2.64 \\
2.12\end{array}$ & $\begin{array}{r}1 \\
25 \\
50 \\
74 \\
99 \\
149 \\
199 \\
248 \\
298 \\
315\end{array}$ \\
\hline $\begin{array}{l}\text { PR } \\
\text { dbar }\end{array}$ & $\stackrel{T}{T} \mathrm{C}$ & $\stackrel{5}{0 / 00}$ & $\begin{array}{l}02 \\
\mathrm{ml} / 1\end{array}$ & $\begin{array}{c}\mathrm{F}-11 \\
\mathrm{pmol} / \mathrm{kg}\end{array}$ & $\begin{array}{c}\mathrm{F}-12 \\
\mathrm{pmol} / \mathrm{kg}\end{array}$ & $\stackrel{\theta}{D e g} C$ & $\begin{array}{l}S I G-\theta \\
\mathrm{kg} / \mathrm{m}^{3}\end{array}$ & $\underset{\mathrm{kg} / \mathrm{m} 3}{\mathrm{SIG}-1.5}$ & $\begin{array}{l}\text { SIG-3 } \\
\mathrm{kg} / \mathrm{m}^{3}\end{array}$ & $D E$ \\
\hline $\begin{array}{l}109 \\
157 \\
217\end{array}$ & $\begin{array}{l}19.857 \\
19.210 \\
17.444\end{array}$ & $\begin{array}{l}36.978 \\
36.816 \\
36.472\end{array}$ & $\begin{array}{l}5.20 \\
5.03 \\
4.64\end{array}$ & & & $\begin{array}{l}19.836 \\
19.182 \\
17.407\end{array}$ & $\begin{array}{l}26 \cdot 316 \\
26 \cdot 364 \\
26.547\end{array}$ & $\begin{array}{l}32.674 \\
32.739 \\
32.968\end{array}$ & $\begin{array}{l}38.825 \\
38.906 \\
39.179\end{array}$ & $\begin{array}{l}108 \\
156 \\
215\end{array}$ \\
\hline
\end{tabular}

ENDEAVOR 143 STA- $61 \quad$ LAT $=2850.1 \mathrm{~N} \quad$ LON= $32 \quad 4.0 \mathrm{~W} \quad$ SONIC DEPTH= Om DATE $12 / 5 / 86$

\begin{tabular}{|c|c|c|c|c|c|c|c|c|c|c|}
\hline $\begin{array}{l}\text { PR } \\
\text { dbar }\end{array}$ & $\stackrel{T}{D e g} C$ & $\stackrel{5}{0}$ & $\begin{array}{l}02 \\
\mathrm{~m} 1 / 1\end{array}$ & $\stackrel{\theta}{D e g} C$ & $\begin{array}{l}S I G-\theta \\
\mathrm{kg} / \mathrm{m3}\end{array}$ & $\underset{\mathrm{kg} / \mathrm{m} 3}{\mathrm{SIG}-1} .5$ & $\begin{array}{l}S I G-3 \\
\mathrm{~kg} / \mathrm{m} 3\end{array}$ & $\underset{\mathrm{m}}{\mathrm{HGTH}}$ & $\underset{c p h}{N}$ & \\
\hline $\begin{array}{r}3 \\
25 \\
50 \\
75 \\
100 \\
150 \\
200 \\
250 \\
300 \\
313\end{array}$ & $\begin{array}{l}20.861 \\
20.164 \\
19.665 \\
19.284 \\
19.442 \\
18.327 \\
17.568 \\
17.018 \\
16.377 \\
16.078\end{array}$ & $\begin{array}{l}36.946 \\
36.907 \\
36.818 \\
36.726 \\
36.814 \\
36.548 \\
36.465 \\
36.403 \\
36.286 \\
36.231\end{array}$ & $\begin{array}{l}4.63 \\
4.75 \\
4.74 \\
4.73 \\
4.60 \\
4.43 \\
4.17 \\
4.06 \\
4.10 \\
4.14\end{array}$ & $\begin{array}{l}20.860 \\
20.160 \\
19.656 \\
19.271 \\
19.424 \\
18.301 \\
17.534 \\
16.976 \\
16.328 \\
16.028\end{array}$ & $\begin{array}{l}26.016 \\
26.176 \\
26.242 \\
26.272 \\
26.299 \\
26.384 \\
26.510 \\
26.598 \\
26.662 \\
26.689\end{array}$ & $\begin{array}{l}32.351 \\
32.527 \\
32.606 \\
32.646 \\
32.669 \\
32.782 \\
32.928 \\
33.031 \\
33.112 \\
33.148\end{array}$ & $\begin{array}{l}38.480 \\
38.672 \\
38.762 \\
38.811 \\
38.830 \\
38.971 \\
39.136 \\
39.252 \\
39.351 \\
39.394\end{array}$ & $\begin{array}{l}0.006 \\
0.049 \\
0.094 \\
0.138 \\
0.182 \\
0.268 \\
0.350 \\
0.428 \\
0.503 \\
0.522\end{array}$ & $\begin{array}{l}0.87 \\
5.11 \\
2.08 \\
1.83 \\
2.25 \\
2.84 \\
2.30 \\
2.07 \\
2.26 \\
2.82\end{array}$ & $\begin{array}{l}9 \\
19 \\
19 \\
2 \\
29 \\
3\end{array}$ \\
\hline $\begin{array}{l}\text { PR } \\
\text { dbar }\end{array}$ & $\stackrel{T}{D e g} C$ & $\stackrel{5}{0 / 00}$ & $\begin{array}{l}02 \\
\mathrm{ml} / \mathrm{l}\end{array}$ & $\begin{array}{c}\mathrm{F}-11 \\
\mathrm{pmol} / \mathrm{kg}\end{array}$ & $\begin{array}{c}\mathrm{F}-12 \\
\mathrm{pmol} / \mathrm{kg}\end{array}$ & $\stackrel{\theta}{\operatorname{Deg} C}$ & $\begin{array}{l}\mathrm{SIG}-0 \\
\mathrm{~kg} / \mathrm{m} 3\end{array}$ & $\underset{\mathrm{kg} / \mathrm{m} 3}{S I G-1} .^{5}$ & $\begin{array}{l}\mathrm{SIG}-3 \\
\mathrm{~kg} / \mathrm{m} 3\end{array}$ & \\
\hline $\begin{array}{r}48 \\
112 \\
212\end{array}$ & $\begin{array}{l}19.769 \\
19.021 \\
17.392\end{array}$ & $\begin{array}{l}36.855 \\
36.729 \\
36.446\end{array}$ & $\begin{array}{l}5.35 \\
4.98 \\
4.64\end{array}$ & & & $\begin{array}{l}19.760 \\
19.001 \\
17.356\end{array}$ & $\begin{array}{l}26.242 \\
26.344 \\
26.540\end{array}$ & $\begin{array}{l}32.603 \\
32.724 \\
32.962\end{array}$ & $\begin{array}{l}38.757 \\
38.896 \\
39.174\end{array}$ & \\
\hline
\end{tabular}

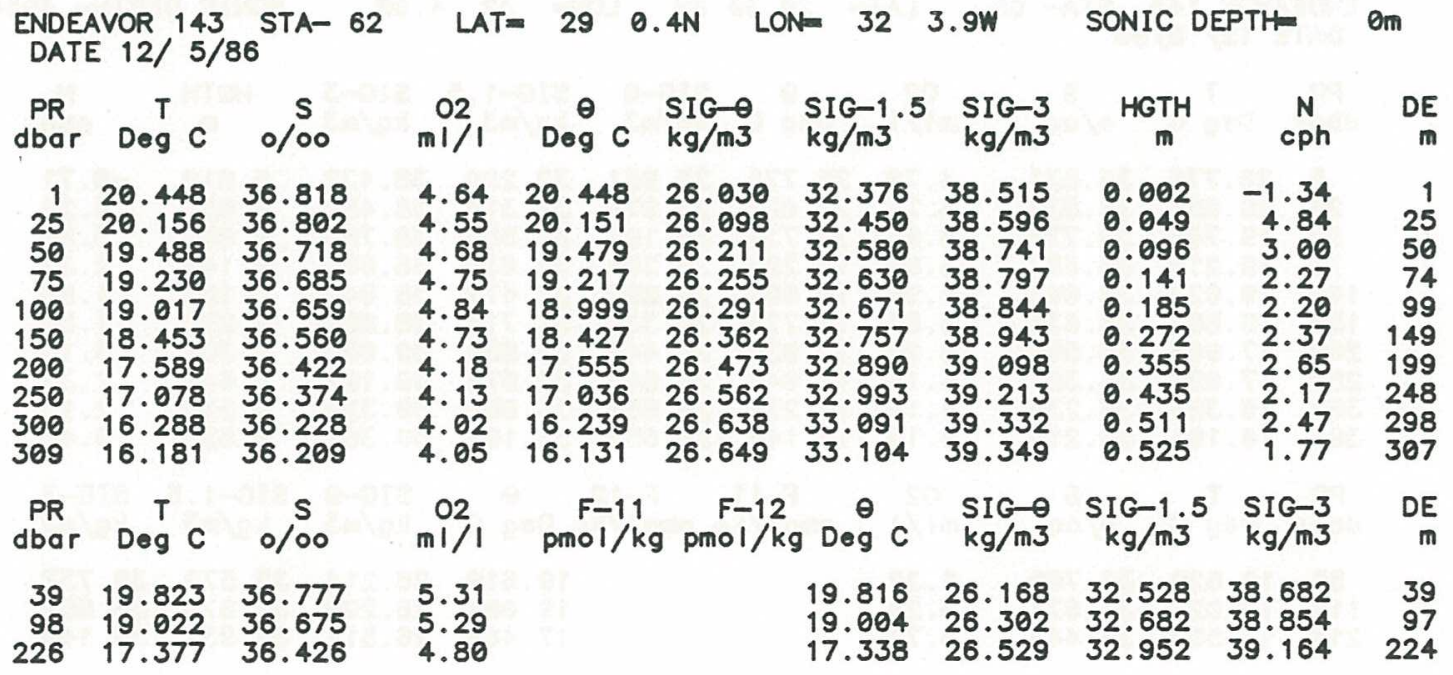




\begin{tabular}{|c|c|c|c|c|c|c|c|c|c|c|}
\hline $\begin{array}{l}\text { END } \\
\text { DA }\end{array}$ & $\begin{array}{l}\mathrm{DR}, 14 \\
12 / 5\end{array}$ & STA- 63 & L & 29 & LON & 32 & $6.0 \mathrm{w}$ & IC & $H=$ & \\
\hline $\begin{array}{l}\text { PR } \\
\text { dbar }\end{array}$ & $\stackrel{T}{\text { Deg }} C$ & $\stackrel{S}{0 / 00}$ & $\begin{array}{l}02 \\
\mathrm{~m} / / 1\end{array}$ & $\stackrel{\theta}{\operatorname{Deg}} C$ & $\begin{array}{l}\mathrm{SIG}-0 \\
\mathrm{~kg} / \mathrm{m3}\end{array}$ & $\underset{\mathrm{kg} / \mathrm{m} 3}{\mathrm{SIG}-1.5}$ & $\begin{array}{l}\text { SIG-3 } \\
\mathrm{kg} / \mathrm{m} 3\end{array}$ & $\underset{\mathbf{m}}{\mathrm{HGTH}}$ & $\underset{c p h}{N}$ & $D E$ \\
\hline $\begin{array}{r}5 \\
25 \\
50 \\
75 \\
100 \\
150 \\
200 \\
250 \\
300\end{array}$ & $\begin{array}{l}20.638 \\
20.034 \\
19.831 \\
19.641 \\
19.064 \\
18.057 \\
17.324 \\
16.901 \\
16.305\end{array}$ & $\begin{array}{l}36.859 \\
36.829 \\
36.835 \\
36.819 \\
36.664 \\
36.501 \\
36.384 \\
36.354 \\
36.255\end{array}$ & $\begin{array}{l}4.68 \\
4.81 \\
4.81 \\
4.81 \\
4.95 \\
4.37 \\
4.24 \\
4.00 \\
4.04\end{array}$ & $\begin{array}{l}20.637 \\
20.030 \\
19.822 \\
19.628 \\
19.046 \\
18.031 \\
17.291 \\
16.859 \\
16.256\end{array}$ & $\begin{array}{l}26.010 \\
26.151 \\
26.211 \\
26.250 \\
26.283 \\
26.416 \\
26.508 \\
26.588 \\
26.655\end{array}$ & $\begin{array}{l}32.351 \\
32.506 \\
32.570 \\
32.614 \\
32.663 \\
32.821 \\
32.932 \\
33.024 \\
33.107\end{array}$ & $\begin{array}{l}38.486 \\
38.654 \\
38.723 \\
38.771 \\
38.834 \\
39.017 \\
39.147 \\
39.249 \\
39.348\end{array}$ & $\begin{array}{l}0.010 \\
0.049 \\
0.095 \\
0.140 \\
0.184 \\
0.270 \\
0.351 \\
0.429 \\
0.504\end{array}$ & $\begin{array}{l}2.08 \\
4.11 \\
2.73 \\
1.57 \\
2.83 \\
2.70 \\
2.30 \\
2.25 \\
1.82\end{array}$ & $\begin{array}{r}14 \\
99 \\
149 \\
199 \\
248 \\
298\end{array}$ \\
\hline $\begin{array}{l}\text { PR } \\
\text { dbar }\end{array}$ & $\stackrel{T}{\text { Deg }} C$ & $\stackrel{5}{0 / 00}$ & $\begin{array}{c}02 \\
\mathrm{ml} / 1\end{array}$ & $\begin{array}{c}\mathrm{F}-11 \\
\mathrm{pmol} / \mathrm{kg}\end{array}$ & $\begin{array}{c}\mathrm{F}-12 \\
\mathrm{pmol} / \mathrm{kg}\end{array}$ & $\operatorname{Deg}^{\ominus} \mathrm{C}$ & $\begin{array}{l}S I G-\theta \\
\mathrm{kg} / \mathrm{m3}\end{array}$ & $\underset{\mathrm{kg} / \mathrm{m} 3}{S I G-1.5}$ & $\begin{array}{l}\text { SIG-3 } \\
\mathrm{kg} / \mathrm{m} 3\end{array}$ & \\
\hline $\begin{array}{r}84 \\
118 \\
207\end{array}$ & $\begin{array}{l}19.574 \\
19.195 \\
17.302\end{array}$ & $\begin{array}{l}36.807 \\
36.757 \\
36.382\end{array}$ & $\begin{array}{l}5.27 \\
5.19 \\
4.72\end{array}$ & & & $\begin{array}{l}19.558 \\
19.173 \\
17.267\end{array}$ & $\begin{array}{l}26.259 \\
26.321 \\
26.512\end{array}$ & $\begin{array}{l}32.625 \\
32.697 \\
32.937\end{array}$ & $\begin{array}{l}38.783 \\
38.864 \\
39.152\end{array}$ & $\begin{array}{l}117 \\
206\end{array}$ \\
\hline
\end{tabular}

ENDEAVOR 143 STA- $64 \quad$ LAT $=2920.0 \mathrm{~N} \quad$ LON= $32 \quad 3.9 \mathrm{~W}$ SONIC DEPTH= 5070m DATE 12/5/86

\begin{tabular}{|c|c|c|c|c|c|c|c|c|c|c|}
\hline $\begin{array}{l}\text { PR } \\
\text { dbar }\end{array}$ & $\stackrel{T}{\operatorname{Deg}} \mathrm{C}$ & $\stackrel{5}{1 / 00}$ & $\begin{array}{l}02 \\
\mathrm{ml} / \mathrm{l}\end{array}$ & $\stackrel{\theta}{D e g} C$ & $\begin{array}{l}\text { SIG-O } \\
\mathrm{kg} / \mathrm{m} 3\end{array}$ & $\underset{\mathrm{kg} / \mathrm{m} 3}{\mathrm{SIG}-1} .5$ & $\begin{array}{l}\mathrm{SIG}-3 \\
\mathrm{~kg} / \mathrm{m} 3\end{array}$ & $\underset{\mathrm{m}}{\mathrm{HGTH}}$ & $\underset{c p h}{N}$ & \\
\hline $\begin{array}{r}3 \\
25 \\
50 \\
75 \\
100 \\
150 \\
200 \\
250 \\
300 \\
305\end{array}$ & $\begin{array}{l}20.591 \\
19.750 \\
19.399 \\
18.995 \\
18.882 \\
18.390 \\
17.559 \\
16.920 \\
16.204 \\
16.109\end{array}$ & $\begin{array}{l}36.766 \\
36.705 \\
36.715 \\
36.651 \\
36.642 \\
36.549 \\
36.408 \\
36.317 \\
36.215 \\
36.200\end{array}$ & $\begin{array}{l}4.63 \\
4.93 \\
4.93 \\
4.94 \\
4.90 \\
4.57 \\
4.33 \\
4.20 \\
4.14 \\
4.14\end{array}$ & $\begin{array}{l}20.590 \\
19.745 \\
19.390 \\
18.982 \\
18.864 \\
18.363 \\
17.525 \\
16.878 \\
16.155 \\
16.060\end{array}$ & $\begin{array}{l}25.952 \\
26.132 \\
26.233 \\
26.290 \\
26.313 \\
26.369 \\
26.469 \\
26.556 \\
26.647 \\
26.658\end{array}$ & $\begin{array}{l}32.295 \\
32.495 \\
32.604 \\
32.671 \\
32.697 \\
32.766 \\
32.888 \\
32.991 \\
33.102 \\
33.116\end{array}$ & $\begin{array}{l}38.432 \\
38.650 \\
38.767 \\
38.843 \\
38.872 \\
38.954 \\
39.096 \\
39.216 \\
39.346 \\
39.362\end{array}$ & $\begin{array}{l}0.006 \\
0.050 \\
0.096 \\
0.140 \\
0.184 \\
0.270 \\
0.353 \\
0.433 \\
0.509 \\
0.517\end{array}$ & $\begin{array}{r}-0.91 \\
5.86 \\
2.74 \\
2.27 \\
1.41 \\
2.60 \\
2.14 \\
2.24 \\
2.78 \\
2.77\end{array}$ & \\
\hline $\begin{array}{c}\text { PR } \\
\text { dbar }\end{array}$ & ${ }^{\top}{ }^{\top} C$ & $\stackrel{S}{0 / 00}$ & $\begin{array}{l}02 \\
\mathrm{ml} / \mathrm{I}\end{array}$ & $\begin{array}{c}\mathrm{F}-11 \\
\mathrm{pmol} / \mathrm{kg}\end{array}$ & $\begin{array}{c}\mathrm{F}-12 \\
\mathrm{pmol} / \mathrm{kg}\end{array}$ & $\stackrel{\theta}{\operatorname{Deg}} \mathrm{C}$ & $\begin{array}{l}\mathrm{SIG}-\Theta \\
\mathrm{kg} / \mathrm{m3}\end{array}$ & $\underset{\mathrm{kg} / \mathrm{m} 3}{\mathrm{SIG}-1.5}$ & $\begin{array}{l}S I G-3 \\
\mathrm{~kg} / \mathrm{m} 3\end{array}$ & \\
\hline $\begin{array}{r}47 \\
91 \\
208\end{array}$ & $\begin{array}{l}19.533 \\
18.905 \\
17.407\end{array}$ & $\begin{array}{l}36.775 \\
36.650 \\
36.393\end{array}$ & $\begin{array}{l}5.04 \\
5.31 \\
4.77\end{array}$ & & & $\begin{array}{l}19.524 \\
18.888 \\
17.372\end{array}$ & $\begin{array}{l}26.243 \\
26.313 \\
26.495\end{array}$ & $\begin{array}{l}32.611 \\
32.696 \\
32.917\end{array}$ & $\begin{array}{l}38.770 \\
38.871 \\
39.130\end{array}$ & \\
\hline
\end{tabular}

\begin{tabular}{|c|c|c|c|c|c|c|c|c|c|c|}
\hline \multicolumn{3}{|c|}{$\begin{array}{l}\text { ENDEAVOR } 143 \text { STA- } 65 \\
\text { DATE } 12 / 5 / 86\end{array}$} & LAT $=$ & \multicolumn{2}{|c|}{$2930.0 \mathrm{~N}$} & \multicolumn{2}{|c|}{$\mathrm{ON}=$} & \multicolumn{3}{|c|}{ SONIC DEPTH $=4960 \mathrm{~m}$} \\
\hline $\begin{array}{l}\text { PR } \\
\text { dbar }\end{array}$ & $\stackrel{T}{T} C$ & $\stackrel{5}{0 / 00}$ & $\begin{array}{l}02 \\
\mathrm{ml} / 1\end{array}$ & $\stackrel{\theta}{\operatorname{Deg} C}$ & $\begin{array}{l}\mathrm{SIG}-\theta \\
\mathrm{kg} / \mathrm{m} 3\end{array}$ & $\underset{\mathrm{kg} / \mathrm{m} 3}{\mathrm{SIG}-1.5}$ & $\begin{array}{l}\mathrm{SIG}-3 \\
\mathrm{~kg} / \mathrm{m3}\end{array}$ & $\underset{m}{\text { HGTH }}$ & $\underset{\text { cph }}{N}$ & DE \\
\hline $\begin{array}{r}5 \\
25 \\
50 \\
75 \\
100 \\
150 \\
200 \\
250 \\
300 \\
305\end{array}$ & $\begin{array}{l}20.776 \\
20.695 \\
19.740 \\
19.216 \\
19.027 \\
18.802 \\
17.965 \\
17.090 \\
16.324 \\
16.191\end{array}$ & $\begin{array}{l}36.831 \\
36.836 \\
36.777 \\
36.687 \\
36.663 \\
36.635 \\
36.508 \\
36.357 \\
36.238 \\
36.216\end{array}$ & $\begin{array}{l}4.70 \\
4.78 \\
4.95 \\
4.99 \\
4.96 \\
4.85 \\
4.26 \\
4.14 \\
4.14\end{array}$ & $\begin{array}{l}20.775 \\
20.691 \\
19.731 \\
19.202 \\
19.009 \\
18.775 \\
17.930 \\
17.048 \\
16.275 \\
16.142\end{array}$ & $\begin{array}{l}25.951 \\
25.978 \\
26.191 \\
26.260 \\
26.292 \\
26.330 \\
26.446 \\
26.545 \\
26.637 \\
26.652\end{array}$ & $\begin{array}{l}32.290 \\
32.318 \\
32.553 \\
32.635 \\
32.672 \\
32.716 \\
32.854 \\
32.976 \\
33.089 \\
33.107\end{array}$ & $\begin{array}{l}38.422 \\
38.452 \\
38.708 \\
38.803 \\
38.844 \\
38.894 \\
39.052 \\
39.197 \\
39.329 \\
39.351\end{array}$ & $\begin{array}{l}0.010 \\
0.051 \\
0.099 \\
0.144 \\
0.188 \\
0.276 \\
0.361 \\
0.441 \\
0.518 \\
0.525\end{array}$ & $\begin{array}{r}-0.71 \\
5.26 \\
3.60 \\
2.37 \\
1.53 \\
1.83 \\
3.10 \\
2.37 \\
2.12 \\
3.48\end{array}$ & $\begin{array}{r}5 \\
25 \\
50 \\
74 \\
99 \\
149 \\
199 \\
248 \\
298 \\
303\end{array}$ \\
\hline $\begin{array}{l}\text { PR } \\
\text { dbar }\end{array}$ & $\stackrel{\mathrm{T}}{\mathrm{Deg}} \mathrm{C}$ & $\stackrel{S}{\circ / 00}$ & $\begin{array}{l}02 \\
\mathrm{ml} / \mathrm{l}\end{array}$ & $\begin{array}{c}\mathrm{F}-11 \\
\mathrm{pmol} / \mathrm{kg}\end{array}$ & $\begin{array}{c}\mathrm{F}-12 \\
\mathrm{pmol} / \mathrm{kg}\end{array}$ & $\stackrel{\theta}{\operatorname{Deg}} \mathrm{C}$ & $\begin{array}{l}\mathrm{SIG}-\mathrm{O} \\
\mathrm{kg} / \mathrm{m3}\end{array}$ & $\underset{\mathrm{kg} / \mathrm{m}}{\mathrm{SIG}}$ & $\begin{array}{l}\mathrm{SIG}-3 \\
\mathrm{~kg} / \mathrm{m3}\end{array}$ & \\
\hline $\begin{array}{r}55 \\
110 \\
216\end{array}$ & $\begin{array}{l}19.629 \\
19.024 \\
17.500\end{array}$ & $\begin{array}{l}36.769 \\
36.671 \\
36.443\end{array}$ & $\begin{array}{l}5.32 \\
5.29 \\
4.70\end{array}$ & & & $\begin{array}{l}19.619 \\
19.004 \\
17.464\end{array}$ & $\begin{array}{l}26.214 \\
26.299 \\
26.511\end{array}$ & $\begin{array}{l}32.579 \\
32.679 \\
32.931\end{array}$ & $\begin{array}{l}38.737 \\
38.852 \\
39.140\end{array}$ & $\begin{array}{r}54 \\
109 \\
214\end{array}$ \\
\hline
\end{tabular}




\begin{tabular}{|c|c|c|c|c|c|c|c|c|c|c|}
\hline \multicolumn{3}{|c|}{$\begin{array}{l}\text { ENDEAVOR } 143 \text { STA- } 66 \\
\text { DATE } 12 / 5 / 86\end{array}$} & LAT $=$ & \multicolumn{2}{|c|}{$=2930.0 \mathrm{~N}$} & \multicolumn{2}{|c|}{$O N=$} & \multicolumn{3}{|c|}{ SONIC DEPTH $=4250 \mathrm{~m}$} \\
\hline $\begin{array}{l}\text { PR } \\
\text { dbar }\end{array}$ & $\stackrel{T}{\operatorname{Deg} C}$ & $\stackrel{S}{0 / 00}$ & $\begin{array}{l}02 \\
\mathrm{ml} / \mathrm{l}\end{array}$ & $\stackrel{\theta}{\operatorname{Deg} C}$ & $\begin{array}{l}\mathrm{SIG}-\theta \\
\mathrm{kg} / \mathrm{m3}\end{array}$ & $\begin{array}{l}\mathrm{SIG}-1.5 \\
\mathrm{~kg} / \mathrm{m} 3\end{array}$ & $\begin{array}{l}\mathrm{SIG}-3 \\
\mathrm{~kg} / \mathrm{m3}\end{array}$ & $\underset{m}{H G T H}$ & $\underset{c p h}{N}$ & $\begin{array}{r}\mathrm{DE} \\
\mathrm{m}\end{array}$ \\
\hline $\begin{array}{r}3 \\
25 \\
50 \\
75 \\
100 \\
150 \\
200 \\
250 \\
300 \\
313\end{array}$ & $\begin{array}{l}20.774 \\
20.624 \\
19.188 \\
18.931 \\
18.750 \\
18.430 \\
17.462 \\
16.663 \\
16.151 \\
16.044\end{array}$ & $\begin{array}{l}36.839 \\
36.820 \\
36.660 \\
36.649 \\
36.627 \\
36.605 \\
36.398 \\
36.272 \\
36.197 \\
36.183\end{array}$ & $\begin{array}{l}4.74 \\
4.83 \\
5.09 \\
4.97 \\
4.85 \\
4.72 \\
4.30 \\
4.22 \\
4.12 \\
4.13\end{array}$ & $\begin{array}{l}20.774 \\
20.619 \\
19.179 \\
18.918 \\
18.733 \\
18.403 \\
17.428 \\
16.621 \\
16.1 .03 \\
15.994\end{array}$ & $\begin{array}{l}25.958 \\
25.985 \\
26.246 \\
26.305 \\
26.335 \\
26.402 \\
26.485 \\
26.582 \\
26.646 \\
26.661\end{array}$ & $\begin{array}{l}32.296 \\
32.327 \\
32.622 \\
32.687 \\
32.722 \\
32.797 \\
32.906 \\
33.025 \\
33.103 \\
33.120\end{array}$ & $\begin{array}{l}38.428 \\
38.462 \\
38.790 \\
38.862 \\
38.900 \\
38.983 \\
39.117 \\
39.256 \\
39.348 \\
39.368\end{array}$ & $\begin{array}{l}0.006 \\
0.051 \\
0.098 \\
0.142 \\
0.185 \\
0.270 \\
0.352 \\
0.430 \\
0.506 \\
0.525\end{array}$ & $\begin{array}{r}-0.76 \\
5.69 \\
3.56 \\
2.18 \\
1.76 \\
2.03 \\
2.37 \\
2.21 \\
2.05 \\
2.20\end{array}$ & $\begin{array}{r}3 \\
25 \\
50 \\
74 \\
99 \\
149 \\
199 \\
248 \\
298 \\
311\end{array}$ \\
\hline $\begin{array}{l}P R \\
\text { dbar }\end{array}$ & $\stackrel{T}{T} \mathrm{C}$ & $\stackrel{S}{1 / 00}$ & $\begin{array}{l}02 \\
\mathrm{ml} / \mathrm{I}\end{array}$ & $\begin{array}{c}\mathrm{F}-11 \\
\mathrm{pmol} / \mathrm{kg}\end{array}$ & $\begin{array}{c}\mathrm{F}-12 \\
\mathrm{pmol} / \mathrm{kg}\end{array}$ & $\stackrel{\theta}{\operatorname{Deg} C}$ & $\begin{array}{l}\mathrm{SIG}-0 \\
\mathrm{~kg} / \mathrm{m3}\end{array}$ & $\underset{\mathrm{kg} / \mathrm{m} 3}{\mathrm{SIG}-1.5}$ & $\begin{array}{l}\mathrm{SIG}-3 \\
\mathrm{~kg} / \mathrm{m3}\end{array}$ & $\begin{array}{l}\mathrm{DE} \\
\mathbf{m}\end{array}$ \\
\hline $\begin{array}{r}63 \\
93 \\
191\end{array}$ & $\begin{array}{l}18.956 \\
18.751 \\
17.501\end{array}$ & $\begin{array}{l}36.654 \\
36.634 \\
36.409\end{array}$ & $\begin{array}{l}5.38 \\
5.22 \\
4.78\end{array}$ & & & $\begin{array}{l}18.945 \\
18.734 \\
17.469\end{array}$ & $\begin{array}{l}26.301 \\
26.340 \\
26.484\end{array}$ & $\begin{array}{l}32.683 \\
32.727 \\
32.904\end{array}$ & $\begin{array}{l}38.857 \\
38.906 \\
39.114\end{array}$ & $\begin{array}{r}63 \\
92 \\
189\end{array}$ \\
\hline
\end{tabular}

\section{ENDEAVOR 143 STA- 67
DATE $12 / 5 / 86$}

\begin{tabular}{|c|c|c|c|c|c|c|c|c|c|c|}
\hline $\begin{array}{l}\text { PR } \\
\text { dbar }\end{array}$ & $\stackrel{T}{\operatorname{Deg}} \mathrm{C}$ & $\stackrel{5}{5}$ & $\begin{array}{c}02 \\
\mathrm{ml} / \mathrm{l}\end{array}$ & $\stackrel{\theta}{\operatorname{Deg} C}$ & $\begin{array}{l}\mathrm{SIG}-\theta \\
\mathrm{kg} / \mathrm{m} 3\end{array}$ & $\underset{\mathrm{kg} / \mathrm{m} 3}{\mathrm{SIG}-1} \cdot 5$ & $\begin{array}{l}\text { SIG-3 } \\
\mathrm{kg} / \mathrm{m} 3\end{array}$ & $\underset{m}{\text { HGTH }}$ & $\underset{c p h}{N}$ & $\underset{m}{\mathrm{DE}}$ \\
\hline $\begin{array}{r}3 \\
25 \\
50 \\
75 \\
100 \\
150 \\
200 \\
250 \\
300 \\
305\end{array}$ & $\begin{array}{l}20.551 \\
20.001 \\
19.280 \\
19.069 \\
18.935 \\
18.025 \\
17.326 \\
16.782 \\
16.228 \\
16.123\end{array}$ & $\begin{array}{l}36.753 \\
36.779 \\
36.666 \\
36.654 \\
36.646 \\
36.475 \\
36.365 \\
36.289 \\
36.217 \\
36.203\end{array}$ & $\begin{array}{l}4.79 \\
4.91 \\
4.86 \\
4.90 \\
4.88 \\
4.45 \\
4.31 \\
4.11 \\
4.00 \\
4.05\end{array}$ & $\begin{array}{l}20.550 \\
19.996 \\
19.271 \\
19.056 \\
18.917 \\
17.999 \\
17.292 \\
16.741 \\
16.179 \\
16.074\end{array}$ & $\begin{array}{l}25.953 \\
26.121 \\
26.227 \\
26.273 \\
26.302 \\
26.404 \\
26.493 \\
26.567 \\
26.644 \\
26.657\end{array}$ & $\begin{array}{l}32.297 \\
32.478 \\
32.601 \\
32.652 \\
32.685 \\
32.810 \\
32.917 \\
33.006 \\
33.098 \\
33.114\end{array}$ & $\begin{array}{l}38.434 \\
38.627 \\
38.767 \\
38.823 \\
38.859 \\
39.007 \\
39.132 \\
39.234 \\
39.341 \\
39.360\end{array}$ & $\begin{array}{l}0.006 \\
0.050 \\
0.096 \\
0.141 \\
0.185 \\
0.271 \\
0.352 \\
0.431 \\
0.507 \\
0.515\end{array}$ & $\begin{array}{l}0.61 \\
5.96 \\
2.78 \\
2.02 \\
1.95 \\
3.06 \\
2.16 \\
1.95 \\
2.52 \\
3.47\end{array}$ & $\begin{array}{r}3 \\
25 \\
50 \\
74 \\
99 \\
149 \\
199 \\
248 \\
298 \\
303\end{array}$ \\
\hline $\begin{array}{l}R \\
\text { ar }\end{array}$ & $\stackrel{T}{\mathrm{~T}} \mathrm{C}$ & $\begin{array}{c}S \\
0 / 00\end{array}$ & $\begin{array}{c}02 \\
\mathrm{ml} / \mathrm{I}\end{array}$ & $\begin{array}{c}\mathrm{F}-11 \\
\mathrm{pmol} / \mathrm{kg}\end{array}$ & $\begin{array}{c}\mathrm{F}-12 \\
\mathrm{pmol} / \mathrm{kg}\end{array}$ & $\stackrel{\ominus}{g} \mathrm{Deg} C$ & $\begin{array}{l}\text { SIG-O } \\
\mathrm{kg} / \mathrm{m3}\end{array}$ & $\underset{\mathrm{kg} / \mathrm{m} 3}{\mathrm{SIG}-1} .5$ & $\begin{array}{l}\mathrm{SIG}-3 \\
\mathrm{~kg} / \mathrm{m} 3\end{array}$ & \\
\hline 95 & $\begin{array}{l}19.567 \\
18.933 \\
17.328\end{array}$ & $\begin{array}{l}36.716 \\
36.653 \\
36.371\end{array}$ & $\begin{array}{l}5.34 \\
5.28 \\
4.89\end{array}$ & & & $\begin{array}{l}19.561 \\
18.916 \\
17.295\end{array}$ & $\begin{array}{l}26.189 \\
26.308 \\
26.497\end{array}$ & $\begin{array}{l}32.555 \\
32.691 \\
32.921\end{array}$ & $\begin{array}{l}38.715 \\
38.865 \\
39.136\end{array}$ & $\begin{array}{r}31 \\
94 \\
194\end{array}$ \\
\hline
\end{tabular}

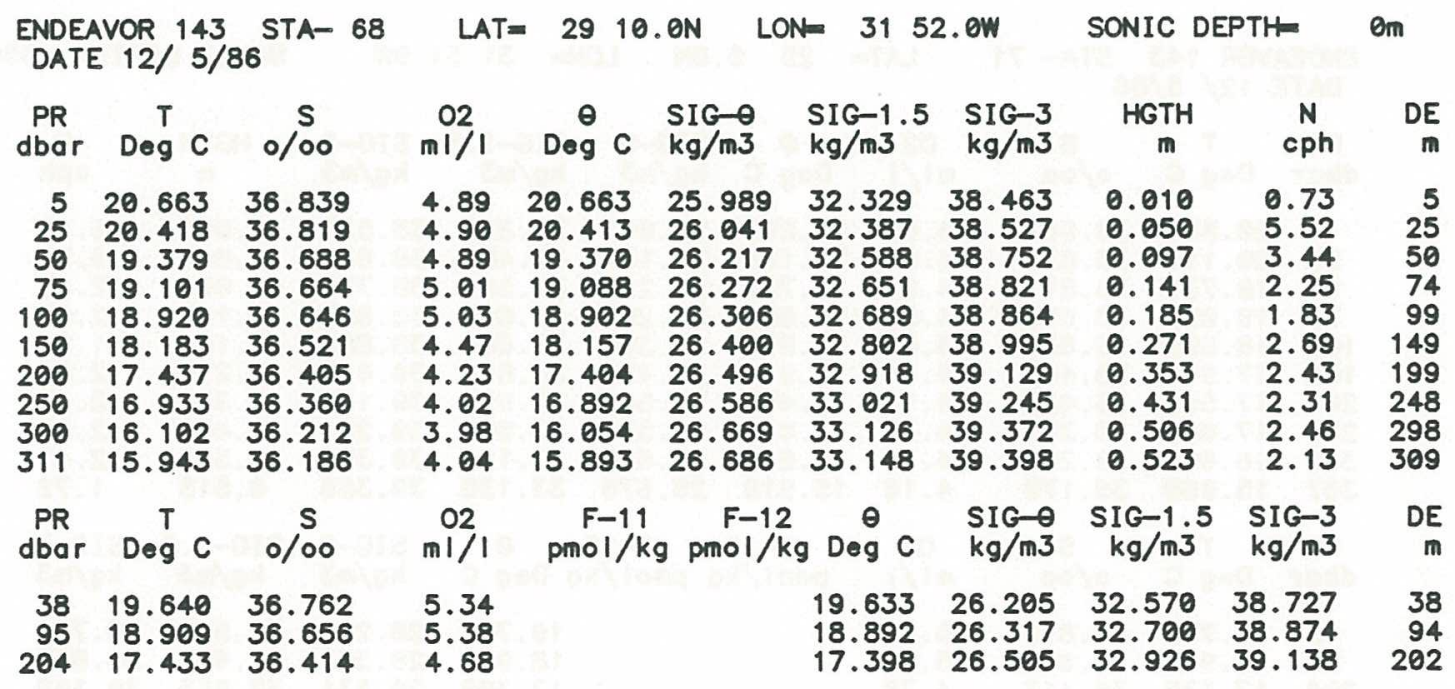




\begin{tabular}{|c|c|c|c|c|c|c|c|c|c|c|}
\hline \multicolumn{3}{|c|}{$\begin{array}{l}\text { ENDEAVOR } 143 \text { STA- } 69 \\
\text { DATE } 12 / 5 / 86\end{array}$} & LAT $=$ & \multicolumn{2}{|c|}{$=290.3 \mathrm{~N}$} & \multicolumn{2}{|c|}{$\mathrm{DN}=3151.3 \mathrm{~W}$} & \multicolumn{3}{|c|}{ SONIC DEPTH $=4500 \mathrm{~m}$} \\
\hline $\begin{array}{l}\text { PR } \\
\text { dbar }\end{array}$ & $\stackrel{T}{T} C$ & $\stackrel{S}{0 / 00}$ & $\begin{array}{l}02 \\
\mathrm{ml} / \mathrm{l}\end{array}$ & $\stackrel{\theta}{\operatorname{Deg} C}$ & $\begin{array}{l}\mathrm{SIG}-\theta \\
\mathrm{kg} / \mathrm{m} 3\end{array}$ & $\begin{array}{l}\mathrm{SIG}-1.5 \\
\mathrm{~kg} / \mathrm{m3}\end{array}$ & $\begin{array}{l}\mathrm{SIG}-3 \\
\mathrm{~kg} / \mathrm{m} 3\end{array}$ & $\underset{m}{\text { HGTH }}$ & $\underset{c p h}{N}$ & $\begin{aligned} \mathrm{DE} \\
\mathrm{m}\end{aligned}$ \\
\hline $\begin{array}{r}3 \\
25 \\
50 \\
75 \\
100 \\
150 \\
200 \\
250 \\
300 \\
305\end{array}$ & $\begin{array}{l}21.062 \\
20.244 \\
19.676 \\
19.695 \\
19.634 \\
18.773 \\
18.083 \\
17.304 \\
16.531 \\
16.417\end{array}$ & $\begin{array}{l}36.989 \\
36.913 \\
36.830 \\
36.883 \\
36.886 \\
36.633 \\
36.576 \\
36.447 \\
36.311 \\
36.292\end{array}$ & $\begin{array}{l}5.22 \\
5.17 \\
5.17 \\
5.09 \\
5.00 \\
4.95 \\
4.34 \\
4.27 \\
4.23 \\
4.23\end{array}$ & $\begin{array}{l}21.061 \\
20.239 \\
19.667 \\
19.681 \\
19.615 \\
18.746 \\
18.048 \\
17.262 \\
16.482 \\
16.367\end{array}$ & $\begin{array}{l}25.994 \\
26.159 \\
26.248 \\
26.285 \\
26.304 \\
26.337 \\
26.469 \\
26.563 \\
26.645 \\
26.657\end{array}$ & $\begin{array}{l}32.324 \\
32.508 \\
32.611 \\
32.647 \\
32.668 \\
32.723 \\
32.873 \\
32.988 \\
33.090 \\
33.106\end{array}$ & $\begin{array}{l}38.449 \\
38.651 \\
38.767 \\
38.802 \\
38.825 \\
38.902 \\
39.068 \\
39.202 \\
39.325 \\
39.343\end{array}$ & $\begin{array}{l}0.006 \\
0.049 \\
0.094 \\
0.139 \\
0.182 \\
0.270 \\
0.354 \\
0.434 \\
0.510 \\
0.517\end{array}$ & $\begin{array}{l}1.97 \\
5.59 \\
2.80 \\
1.76 \\
0.95 \\
2.20 \\
2.87 \\
2.50 \\
1.97 \\
2.54\end{array}$ & $\begin{array}{r}3 \\
25 \\
50 \\
74 \\
99 \\
149 \\
199 \\
248 \\
298 \\
303\end{array}$ \\
\hline $\begin{array}{l}\text { PR } \\
\text { dbar }\end{array}$ & $\stackrel{T}{T} C$ & $\stackrel{5}{0 / 00}$ & $\begin{array}{ll}02 \\
m 1 / 1\end{array}$ & $\begin{array}{c}\mathrm{F}-11 \\
\mathrm{pmol} / \mathrm{kg}\end{array}$ & $\begin{array}{l}\mathrm{F}-12 \\
\mathrm{pmol} / \mathrm{kg}\end{array}$ & $\stackrel{\theta}{D e g} C$ & $\begin{array}{l}\mathrm{SIG}-0 \\
\mathrm{~kg} / \mathrm{m3}\end{array}$ & $\underset{\mathrm{kg} / \mathrm{m} 3}{\mathrm{SIG}-1.5}$ & $\begin{array}{l}\mathrm{SIG}-3 \\
\mathrm{~kg} / \mathrm{m} 3\end{array}$ & $\begin{aligned} \mathrm{DE} \\
\mathrm{m}\end{aligned}$ \\
\hline $\begin{array}{r}94 \\
121 \\
239\end{array}$ & $\begin{array}{l}19.458 \\
18.944 \\
17.415\end{array}$ & & & & & & & & & $\begin{array}{r}93 \\
120 \\
237\end{array}$ \\
\hline
\end{tabular}

\begin{tabular}{|c|c|c|c|c|c|c|c|c|c|c|}
\hline \multicolumn{3}{|c|}{$\begin{array}{l}\text { ENDEAVOR } 143 \text { STA- } 70 \\
\text { DATE } 12 / 5 / 86\end{array}$} & LAT $\equiv$ & \multicolumn{2}{|c|}{$2850.1 \mathrm{~N}$} & \multicolumn{2}{|c|}{ ON= $3151.9 W$} & \multicolumn{2}{|c|}{ SONIC DEPTH= } & \multirow[t]{2}{*}{$\theta m$} \\
\hline $\begin{array}{l}\text { PR } \\
\text { dbar }\end{array}$ & $\stackrel{T}{\text { Deg } C}$ & $\begin{array}{c}S \\
0 / 00\end{array}$ & 02 & $\stackrel{\theta}{\operatorname{Deg} C}$ & $\begin{array}{l}\mathrm{SIG}-0 \\
\mathrm{~kg} / \mathrm{m} 3\end{array}$ & $\operatorname{SIG}_{\mathrm{kg} / \mathrm{m} 3}^{-1.5}$ & $\begin{array}{l}\mathrm{SIG}-3 \\
\mathrm{~kg} / \mathrm{m3}\end{array}$ & $\underset{m}{\text { HGTH }}$ & $\underset{\mathrm{cph}}{\mathrm{N}}$ & \\
\hline $\begin{array}{r}5 \\
25 \\
50 \\
75 \\
100 \\
150 \\
200 \\
250 \\
300 \\
329\end{array}$ & $\begin{array}{l}20.911 \\
19.948 \\
19.833 \\
19.863 \\
19.836 \\
18.951 \\
17.763 \\
16.949 \\
16.219 \\
15.734\end{array}$ & $\begin{array}{l}36.967 \\
36.880 \\
36.911 \\
36.944 \\
36.947 \\
36.711 \\
36.498 \\
36.389 \\
36.255 \\
36.171\end{array}$ & $\begin{array}{l}5.25 \\
5.06 \\
5.04 \\
4.96 \\
4.91 \\
4.73 \\
4.28 \\
4.21 \\
4.17 \\
4.15\end{array}$ & $\begin{array}{l}20.910 \\
19.944 \\
19.824 \\
19.849 \\
19.817 \\
18.924 \\
17.729 \\
16.907 \\
16.171 \\
15.682\end{array}$ & $\begin{array}{l}26.019 \\
26.213 \\
26.268 \\
26.287 \\
26.297 \\
26.350 \\
26.488 \\
26.604 \\
26.675 \\
26.722\end{array}$ & $\begin{array}{l}32.353 \\
32.569 \\
32.627 \\
32.645 \\
32.656 \\
32.732 \\
32.901 \\
33.038 \\
33.129 \\
33.190\end{array}$ & $\begin{array}{l}38.480 \\
38.719 \\
38.779 \\
38.796 \\
38.808 \\
38.906 \\
39.104 \\
39.262 \\
39.372 \\
39.446\end{array}$ & $\begin{array}{l}0.010 \\
0.048 \\
0.093 \\
0.136 \\
0.180 \\
0.268 \\
0.351 \\
0.429 \\
0.504 \\
0.546\end{array}$ & $\begin{array}{l}3.79 \\
4.13 \\
2.23 \\
1.13 \\
1.21 \\
2.81 \\
2.81 \\
2.38 \\
2.15 \\
2.55\end{array}$ & $\begin{array}{r}5 \\
25 \\
50 \\
74 \\
99 \\
149 \\
199 \\
248 \\
298 \\
327\end{array}$ \\
\hline $\begin{array}{l}\text { PR } \\
\text { dbar }\end{array}$ & $\stackrel{T}{\text { Deg } C}$ & $\stackrel{5}{0 / 00}$ & $\begin{array}{l}02 \\
\mathrm{ml} / \mathrm{l}\end{array}$ & $\begin{array}{c}\mathrm{F}-11 \\
\mathrm{pmol} / \mathrm{kg}\end{array}$ & $\begin{array}{c}\mathrm{F}-12 \\
\mathrm{gmol} / \mathrm{kg}\end{array}$ & $\stackrel{\theta}{D e g} C$ & $\begin{array}{l}\mathrm{SIG}-\mathrm{O} \\
\mathrm{kg} / \mathrm{m3}\end{array}$ & $\underset{\mathrm{kg} / \mathrm{m} 3}{\mathrm{SIG}-1.5}$ & $\begin{array}{l}S I G-3 \\
\mathrm{~kg} / \mathrm{m} 3\end{array}$ & $D E$ \\
\hline $\begin{array}{l}114 \\
150 \\
217\end{array}$ & $\begin{array}{l}19.862 \\
19.383 \\
17.489\end{array}$ & $\begin{array}{l}36.982 \\
36.868 \\
36.469\end{array}$ & $\begin{array}{l}5.24 \\
5.14 \\
4.75\end{array}$ & & & $\begin{array}{l}19.841 \\
19.356 \\
17.452\end{array}$ & $\begin{array}{l}26.318 \\
26.359 \\
26.534\end{array}$ & $\begin{array}{l}32.676 \\
32.729 \\
32.954\end{array}$ & $\begin{array}{l}38.827 \\
38.891 \\
39.163\end{array}$ & $\begin{array}{l}113 \\
148 \\
215\end{array}$ \\
\hline
\end{tabular}

\begin{tabular}{|c|c|c|c|c|c|c|c|c|c|c|}
\hline \multicolumn{3}{|c|}{$\begin{array}{l}\text { ENDEAVOR } 143 \text { STA- } 71 \\
\text { DATE } 12 / 5 / 86\end{array}$} & LAT $=$ & 295.0 & LON= & \multicolumn{2}{|c|}{$3151.9 w$} & \multicolumn{3}{|c|}{ SONIC DEPTH $=4350 \mathrm{~m}$} \\
\hline $\begin{array}{l}\text { PR } \\
\text { dbar }\end{array}$ & $\stackrel{T}{\text { Deg }} C$ & $\stackrel{5}{0 / 00}$ & $\begin{array}{l}02 \\
\mathrm{ml} / 1\end{array}$ & $\stackrel{\theta}{\text { Deg } C}$ & $\begin{array}{l}\mathrm{SIG}-0 \\
\mathrm{~kg} / \mathrm{m3}\end{array}$ & $\begin{array}{l}\mathrm{SIG}-1.5 \\
\mathrm{~kg} / \mathrm{m} 3\end{array}$ & $\begin{array}{l}\mathrm{SIG}-3 \\
\mathrm{~kg} / \mathrm{m} 3\end{array}$ & $\underset{m}{\text { HGTH }}$ & $\underset{\text { cph }}{N}$ & $\underset{m}{\mathrm{DE}}$ \\
\hline $\begin{array}{r}1 \\
25 \\
50 \\
75 \\
100 \\
150 \\
200 \\
250 \\
300 \\
307\end{array}$ & $\begin{array}{l}20.803 \\
20.113 \\
19.731 \\
19.098 \\
18.895 \\
17.940 \\
17.505 \\
17.084 \\
16.084 \\
15.968\end{array}$ & $\begin{array}{l}36.961 \\
36.830 \\
36.811 \\
36.655 \\
36.630 \\
36.449 \\
36.433 \\
36.390 \\
36.202 \\
36.179\end{array}$ & $\begin{array}{l}4.65 \\
4.87 \\
4.81 \\
4.97 \\
4.82 \\
4.41 \\
4.16 \\
4.17 \\
4.16 \\
4.16\end{array}$ & $\begin{array}{l}20.803 \\
20.109 \\
19.722 \\
19.084 \\
18.877 \\
17.914 \\
17.471 \\
17.042 \\
16.036 \\
15.919\end{array}$ & $\begin{array}{l}26.043 \\
26.131 \\
26.219 \\
26.267 \\
26.300 \\
26.405 \\
26.501 \\
26.573 \\
26.666 \\
26.675\end{array}$ & $\begin{array}{l}32.379 \\
32.484 \\
32.581 \\
32.645 \\
32.684 \\
32.814 \\
32.921 \\
33.004 \\
33.124 \\
33.136\end{array}$ & $\begin{array}{l}38.510 \\
38.630 \\
38.736 \\
38.815 \\
38.859 \\
39.013 \\
39.131 \\
39.224 \\
39.370 \\
39.386\end{array}$ & $\begin{array}{l}0.002 \\
0.049 \\
0.094 \\
0.139 \\
0.183 \\
0.270 \\
0.351 \\
0.430 \\
0.505 \\
0.515\end{array}$ & $\begin{array}{r}-1.12 \\
4.73 \\
2.48 \\
2.50 \\
1.31 \\
2.85 \\
2.42 \\
2.26 \\
2.47 \\
1.72\end{array}$ & $\begin{array}{r}1 \\
25 \\
50 \\
74 \\
99 \\
149 \\
199 \\
248 \\
298 \\
305\end{array}$ \\
\hline $\begin{array}{l}\text { PR } \\
\text { dbar }\end{array}$ & $\stackrel{T}{D e g} C$ & $\stackrel{5}{0 / 00}$ & $\mathrm{O} 2$ & $\begin{array}{c}F_{-11} \\
\mathrm{pmol} / \mathrm{kg}\end{array}$ & $\begin{array}{c}\mathrm{F}-12 \\
\mathrm{pmol} / \mathrm{kg}\end{array}$ & $\stackrel{\theta}{\operatorname{Deg} C}$ & $\begin{array}{l}\mathrm{SIG}-\mathrm{O} \\
\mathrm{kg} / \mathrm{m} 3\end{array}$ & $\underset{\mathrm{kg} / \mathrm{m} 3}{\mathrm{SIG}-1.5}$ & $\begin{array}{l}\mathrm{SIG}-3 \\
\mathrm{~kg} / \mathrm{m} 3\end{array}$ & $\underset{m}{D E}$ \\
\hline $\begin{array}{r}43 \\
89 \\
206\end{array}$ & $\begin{array}{l}19.755 \\
18.942 \\
17.335\end{array}$ & $\begin{array}{l}36.819 \\
36.647 \\
36.417\end{array}$ & $\begin{array}{l}5.43 \\
5.54 \\
4.78\end{array}$ & & & $\begin{array}{l}19.747 \\
18.926 \\
17.300\end{array}$ & $\begin{array}{l}26.219 \\
26.301 \\
26.531\end{array}$ & $\begin{array}{l}32.580 \\
32.683 \\
32.955\end{array}$ & $\begin{array}{l}38.734 \\
38.857 \\
39.169\end{array}$ & $\begin{array}{r}43 \\
88 \\
204\end{array}$ \\
\hline
\end{tabular}




\begin{tabular}{|c|c|c|c|c|c|c|c|c|c|c|}
\hline \multicolumn{3}{|c|}{$\begin{array}{l}\text { ENDEAVOR } 143 \text { STA- } 72 \\
\text { DATE } 13 / 5 / 86\end{array}$} & \multirow{2}{*}{$\begin{array}{l}\text { LAT }= \\
02 \\
\mathrm{ml} / \mathrm{l}\end{array}$} & \multicolumn{2}{|c|}{$28 \quad 40.0 \mathrm{~N}$} & \multicolumn{2}{|c|}{$O N=3151.9 \mathrm{~W}$} & \multicolumn{3}{|c|}{ SONIC DEPTH $=4770 \mathrm{~m}$} \\
\hline $\begin{array}{l}\text { PR } \\
\text { dbar }\end{array}$ & $\stackrel{T}{\text { Deg } C}$ & $\stackrel{5}{0 / 00}$ & & $\stackrel{\theta}{\operatorname{Deg} C}$ & $\begin{array}{l}\mathrm{SIG}-\theta \\
\mathrm{kg} / \mathrm{m3}\end{array}$ & $\begin{array}{l}\mathrm{SIG}-1.5 \\
\mathrm{~kg} / \mathrm{m} 3\end{array}$ & $\begin{array}{l}\mathrm{SIG}-3 \\
\mathrm{~kg} / \mathrm{m} 3\end{array}$ & $\begin{array}{l}\text { HGTH } \\
\text { m }\end{array}$ & $\underset{c p h}{N}$ & DE \\
\hline $\begin{array}{r}1 \\
25 \\
50 \\
75 \\
100 \\
150 \\
200 \\
250 \\
300 \\
309\end{array}$ & $\begin{array}{l}20.734 \\
20.562 \\
20.217 \\
19.784 \\
19.520 \\
19.355 \\
18.469 \\
17.568 \\
16.786 \\
16.698\end{array}$ & $\begin{array}{l}36.871 \\
36.883 \\
36.971 \\
36.871 \\
36.827 \\
36.799 \\
36.649 \\
36.498 \\
36.361 \\
36.346\end{array}$ & $\begin{array}{l}4.81 \\
4.69 \\
4.71 \\
4.86 \\
4.81 \\
4.83 \\
4.39 \\
4.23 \\
4.17 \\
4.15\end{array}$ & $\begin{array}{l}20.734 \\
20.557 \\
20.208 \\
19.770 \\
19.502 \\
19.328 \\
18.434 \\
17.525 \\
16.736 \\
16.647\end{array}$ & $\begin{array}{l}25.993 \\
26.051 \\
26.212 \\
26.252 \\
26.289 \\
26.313 \\
26.428 \\
26.538 \\
26.624 \\
26.633\end{array}$ & $\begin{array}{l}32.332 \\
32.393 \\
32.561 \\
32.613 \\
32.656 \\
32.684 \\
32.822 \\
32.956 \\
33.062 \\
33.074\end{array}$ & $\begin{array}{l}38.465 \\
38.529 \\
38.704 \\
38.766 \\
38.816 \\
38.848 \\
39.007 \\
39.164 \\
39.290 \\
39.304\end{array}$ & $\begin{array}{l}0.002 \\
0.049 \\
0.096 \\
0.141 \\
0.186 \\
0.274 \\
0.360 \\
0.441 \\
0.518 \\
0.532\end{array}$ & $\begin{array}{l}3.67 \\
3.35 \\
2.72 \\
2.19 \\
1.46 \\
1.69 \\
3.11 \\
2.37 \\
2.29 \\
1.45\end{array}$ & $\begin{array}{r}20 \\
50 \\
74 \\
99 \\
149 \\
199 \\
248 \\
298 \\
307\end{array}$ \\
\hline $\begin{array}{l}\text { PR } \\
\text { dbar }\end{array}$ & $\stackrel{T}{\text { Deg } C}$ & $\stackrel{5}{0 / 00}$ & $\begin{array}{l}02 \\
\mathrm{ml} / \mathrm{I}\end{array}$ & $\begin{array}{c}\mathrm{F}-11 \\
\mathrm{pmol} / \mathrm{kg}\end{array}$ & $\begin{array}{c}\mathrm{F}-12 \\
\mathrm{pmol} / \mathrm{kg}\end{array}$ & $\stackrel{\theta}{\operatorname{Dog} C}$ & $\begin{array}{l}\mathrm{SIG}-0 \\
\mathrm{~kg} / \mathrm{m} 3\end{array}$ & $\underset{\mathrm{kg} / \mathrm{m} 3}{\mathrm{SIG}-1.5}$ & $\begin{array}{l}S I G-3 \\
\mathrm{~kg} / \mathrm{m} 3\end{array}$ & \\
\hline $\begin{array}{l}121 \\
170 \\
252\end{array}$ & $\begin{array}{l}19.445 \\
19.021 \\
17.437\end{array}$ & $\begin{array}{l}36.830 \\
36.734 \\
36.497\end{array}$ & $\begin{array}{l}5.27 \\
5.13 \\
4.76\end{array}$ & & & $\begin{array}{l}19.423 \\
18.990 \\
17.394\end{array}$ & $\begin{array}{l}26.312 \\
26.351 \\
26.569\end{array}$ & $\begin{array}{l}32.681 \\
32.731 \\
32.990\end{array}$ & $\begin{array}{l}38.842 \\
38.903 \\
39.201\end{array}$ & $\begin{array}{l}120 \\
169 \\
250\end{array}$ \\
\hline
\end{tabular}

\begin{tabular}{|c|c|c|c|c|c|c|c|c|c|c|}
\hline \multicolumn{3}{|c|}{$\begin{array}{l}\text { ENDEAVOR } 143 \text { STA- } 73 \\
\text { DATE } 13 / 5 / 86\end{array}$} & LAT $=$ & \multicolumn{2}{|c|}{$2840.0 \mathrm{~N}$} & \multicolumn{2}{|c|}{$3140.0 w$} & \multicolumn{2}{|c|}{ SONIC DEPTH } & \multirow{2}{*}{$\begin{array}{l}\text { Om } \\
\text { DE } \\
\text { m }\end{array}$} \\
\hline $\begin{array}{l}\text { PR } \\
\text { dbar }\end{array}$ & $\stackrel{T}{T} C$ & 1/00 & $\begin{array}{l}02 \\
\mathrm{ml} / \mathrm{l}\end{array}$ & $\stackrel{\theta}{\operatorname{Deg} C}$ & $\begin{array}{l}\mathrm{SIG}-\theta \\
\mathrm{kg} / \mathrm{m} 3\end{array}$ & $\begin{array}{l}\mathrm{SIG}-1.5 \\
\mathrm{~kg} / \mathrm{m} 3\end{array}$ & $\begin{array}{l}\text { SIG-3 } \\
\mathrm{kg} / \mathrm{m} 3\end{array}$ & $\underset{m}{\text { HGTH }}$ & $\underset{\mathrm{cph}}{\mathrm{N}}$ & \\
\hline $\begin{array}{r}1 \\
25 \\
50 \\
75 \\
100 \\
150 \\
200 \\
250 \\
300 \\
309\end{array}$ & $\begin{array}{l}20.845 \\
20.422 \\
19.781 \\
19.643 \\
19.569 \\
19.195 \\
17.936 \\
17.302 \\
16.546 \\
16.264\end{array}$ & $\begin{array}{l}36.868 \\
36.846 \\
36.807 \\
36.829 \\
36.829 \\
36.763 \\
36.513 \\
36.435 \\
36.305 \\
36.254\end{array}$ & $\begin{array}{l}5.17 \\
5.09 \\
5.14 \\
4.99 \\
4.96 \\
4.72 \\
4.23 \\
4.11 \\
3.98\end{array}$ & $\begin{array}{l}20.845 \\
20.417 \\
19.771 \\
19.629 \\
19.551 \\
19.168 \\
17.902 \\
17.260 \\
16.496 \\
16.214\end{array}$ & $\begin{array}{l}25.961 \\
26.060 \\
26.203 \\
26.257 \\
26.277 \\
26.327 \\
26.457 \\
26.555 \\
26.637 \\
26.664\end{array}$ & $\begin{array}{l}32.297 \\
32.406 \\
32.564 \\
32.621 \\
32.643 \\
32.703 \\
32.865 \\
32.979 \\
33.083 \\
33.117\end{array}$ & $\begin{array}{l}38.427 \\
38.545 \\
38.718 \\
38.778 \\
38.802 \\
38.870 \\
39.064 \\
39.194 \\
39.317 \\
39.359\end{array}$ & $\begin{array}{l}0.002 \\
0.050 \\
0.096 \\
0.142 \\
0.186 \\
0.274 \\
0.359 \\
0.439 \\
0.515 \\
0.528\end{array}$ & $\begin{array}{l}4 . \\
5 . \\
2 . \\
2 . \\
1 . \\
2 . \\
2 . \\
2 . \\
1 .\end{array}$ & $\begin{array}{r}1 \\
25 \\
50 \\
74 \\
99 \\
149 \\
199 \\
248 \\
298 \\
307\end{array}$ \\
\hline $\begin{array}{l}\text { PR } \\
\text { dbar }\end{array}$ & $\stackrel{T}{D} C$ & $\stackrel{s}{0 / 00}$ & $\begin{array}{l}02 \\
\mathrm{ml} / \mathrm{I}\end{array}$ & $\begin{array}{c}\mathrm{F}-11 \\
\mathrm{pmol} / \mathrm{kg}\end{array}$ & $\begin{array}{c}\mathrm{F}-12 \\
\mathrm{pmol} / \mathrm{kg}\end{array}$ & $\stackrel{\theta}{\operatorname{Deg} C}$ & $\begin{array}{l}\mathrm{SIG}-\mathrm{O} \\
\mathrm{kg} / \mathrm{m} 3\end{array}$ & $\underset{\mathrm{kg} / \mathrm{m} 3}{\mathrm{SIG}-1.5}$ & $\begin{array}{l}\mathrm{SIG}-3 \\
\mathrm{~kg} / \mathrm{m3}\end{array}$ & DE \\
\hline $\begin{array}{r}82 \\
117 \\
150 \\
240\end{array}$ & $\begin{array}{l}19.632 \\
19.696 \\
19.138 \\
17.397\end{array}$ & $\begin{array}{l}36.879 \\
36.898 \\
36.780 \\
36.463\end{array}$ & $\begin{array}{l}5.29 \\
5.23 \\
5.11 \\
4.70\end{array}$ & & & $\begin{array}{l}19.617 \\
19.674 \\
19.111 \\
17.357\end{array}$ & $\begin{array}{l}26.299 \\
26.298 \\
26.355 \\
26.553\end{array}$ & $\begin{array}{l}32.663 \\
32.660 \\
32.732 \\
32.975\end{array}$ & $\begin{array}{l}38.819 \\
38.816 \\
38.900 \\
39.187\end{array}$ & $\begin{array}{r}82 \\
116 \\
148 \\
238\end{array}$ \\
\hline
\end{tabular}

\begin{tabular}{|c|c|c|c|c|c|c|c|c|c|c|}
\hline \multicolumn{3}{|c|}{$\begin{array}{l}\text { ENDEAVOR } 143 \text { STA- } 74 \\
\text { DATE } 13 / 5 / 86\end{array}$} & $L A T=$ & \multicolumn{2}{|c|}{$2850.0 \mathrm{~N}$} & \multicolumn{2}{|c|}{ LON= } & \multicolumn{2}{|c|}{ SONIC DEPTH } & \multirow{2}{*}{$\begin{array}{l}\text { Om } \\
\underset{m}{\text { DE }} \\
\text { n }\end{array}$} \\
\hline $\begin{array}{l}\text { PR } \\
\text { dbar }\end{array}$ & $\stackrel{T}{\text { Deg }} \mathrm{C}$ & $\stackrel{5}{\circ / 00}$ & $\begin{array}{l}02 \\
\mathrm{ml} / 1\end{array}$ & $\stackrel{\theta}{\text { Deg } C}$ & $\begin{array}{l}\mathrm{SIG}-\mathrm{O} \\
\mathrm{kg} / \mathrm{m3}\end{array}$ & $\begin{array}{l}\mathrm{SIG}-1.5 \\
\mathrm{~kg} / \mathrm{m3}\end{array}$ & $\begin{array}{l}S I G-3 \\
\mathrm{~kg} / \mathrm{m3}\end{array}$ & $\underset{m}{\operatorname{HGTH}}$ & $\underset{\text { cph }}{N}$ & \\
\hline $\begin{array}{r}1 \\
25 \\
50 \\
75 \\
100 \\
150 \\
200 \\
250 \\
300\end{array}$ & $\begin{array}{l}20.995 \\
20.229 \\
19.845 \\
19.635 \\
19.545 \\
18.768 \\
17.778 \\
17.267 \\
16.509\end{array}$ & $\begin{array}{l}36.8 \\
36.8 \\
36.8 \\
36.8 \\
36.8 \\
36.6 \\
36.4 \\
36.4 \\
36.3\end{array}$ & $\begin{array}{l}4.87 \\
4.98 \\
5.02 \\
4.99 \\
4.92 \\
4.76 \\
4.28 \\
4.24 \\
4.19\end{array}$ & $\begin{array}{l}20.995 \\
20.225 \\
19.836 \\
19.621 \\
19.527 \\
18.741 \\
17.744 \\
17 . .225 \\
16.460\end{array}$ & $\begin{array}{l}25.921 \\
26.113 \\
26.220 \\
26.261 \\
26.292 \\
26.348 \\
26.460 \\
26.552 \\
26.649\end{array}$ & $\begin{array}{l}32.254 \\
32.464 \\
32.579 \\
32.625 \\
32.658 \\
32.735 \\
32.873 \\
32.978 \\
33.095\end{array}$ & $\begin{array}{l}38.381 \\
38.607 \\
38.731 \\
38.782 \\
38.817 \\
38.913 \\
39.076 \\
39.193 \\
39.330\end{array}$ & $\begin{array}{l}0.002 \\
0.050 \\
0.096 \\
0.141 \\
0.185 \\
0.272 \\
0.357 \\
0.436 \\
0.512\end{array}$ & $\begin{array}{l}4.91 \\
5.55 \\
2.56 \\
2.10 \\
1.61 \\
2.39 \\
2.98 \\
2.30 \\
2.47\end{array}$ & $\begin{array}{r}1 \\
25 \\
50 \\
74 \\
99 \\
149 \\
199 \\
248 \\
298\end{array}$ \\
\hline $\begin{array}{l}\text { PR } \\
\text { dbar }\end{array}$ & $\stackrel{T}{\text { Deg } C}$ & $\stackrel{5}{1 / 00}$ & $\begin{array}{l}02 \\
\mathrm{ml} / \mathrm{l}\end{array}$ & $\begin{array}{c}\mathrm{F}-11 \\
\mathrm{pmol} / \mathrm{kg}\end{array}$ & $\begin{array}{c}\mathrm{F}-12 \\
\mathrm{pmol} / \mathrm{kg}\end{array}$ & $\stackrel{\theta}{D} C$ & $\begin{array}{l}\mathrm{SIG}-\theta \\
\mathrm{kg} / \mathrm{m} 3\end{array}$ & $\underset{\mathrm{kg} / \mathrm{m} 3}{\mathrm{SIG}-1.5}$ & $\begin{array}{l}\mathrm{SIG}-3 \\
\mathrm{~kg} / \mathrm{m} 3\end{array}$ & $\underset{\mathbf{m}}{D E}$ \\
\hline $\begin{array}{r}83 \\
138 \\
221\end{array}$ & $\begin{array}{l}19.550 \\
19.075 \\
17.427\end{array}$ & $\begin{array}{l}36.826 \\
36.740 \\
36.453\end{array}$ & $\begin{array}{l}5.27 \\
5.11 \\
4.69\end{array}$ & & & $\begin{array}{l}19.535 \\
19.050 \\
17.389\end{array}$ & $\begin{array}{l}26.280 \\
26.340 \\
26.537\end{array}$ & $\begin{array}{l}32.646 \\
32.719 \\
32.958\end{array}$ & $\begin{array}{l}38.805 \\
38.889 \\
39.170\end{array}$ & $\begin{array}{r}82 \\
137 \\
219\end{array}$ \\
\hline
\end{tabular}




\begin{tabular}{|c|c|c|c|c|c|c|c|c|c|c|}
\hline \multicolumn{3}{|c|}{$\begin{array}{l}\text { ENDEAVOR } 143 \text { STA- } 75 \\
\text { DATE } 13 / 5 / 86\end{array}$} & \multirow{2}{*}{$\begin{array}{l}\text { LAT }= \\
02 \\
\mathrm{ml} / 1\end{array}$} & \multirow{2}{*}{$\begin{array}{c}=290 . \\
\operatorname{Deg}^{\theta} \mathrm{C}\end{array}$} & $. O N \quad L O N=$ & \multicolumn{2}{|c|}{$3140.0 W$} & \multicolumn{2}{|c|}{ SONIC DEPTH } & \multirow{2}{*}{$\begin{array}{l}\text { Om } \\
\text { DE } \\
m\end{array}$} \\
\hline $\begin{array}{l}P R \\
\text { dbar }\end{array}$ & $\stackrel{T}{T} \mathrm{C}$ & $\begin{array}{l}S \\
0 / 00\end{array}$ & & & $\begin{array}{l}\mathrm{SIG}-\theta \\
\mathrm{kg} / \mathrm{m3}\end{array}$ & $\begin{array}{l}\mathrm{SIG}-1.5 \\
\mathrm{~kg} / \mathrm{m} 3\end{array}$ & $\begin{array}{l}\mathrm{SIG}-3 \\
\mathrm{~kg} / \mathrm{m} 3\end{array}$ & $\underset{m}{\text { HGTH }}$ & $\underset{c p h}{N}$ & \\
\hline $\begin{array}{r}1 \\
25 \\
50 \\
75 \\
100 \\
150 \\
200 \\
250 \\
300 \\
305\end{array}$ & $\begin{array}{l}21.155 \\
20.662 \\
19.833 \\
19.997 \\
19.937 \\
19.787 \\
18.180 \\
17.284 \\
16.395 \\
16.282\end{array}$ & $\begin{array}{l}36.889 \\
36.894 \\
36.840 \\
36.968 \\
36.970 \\
36.946 \\
36.579 \\
36.433 \\
36.284 \\
36.263\end{array}$ & $\begin{array}{l}4.70 \\
4.79 \\
4.97 \\
4.89 \\
4.90 \\
4.75 \\
4.34 \\
4.19 \\
4.15 \\
4.14\end{array}$ & $\begin{array}{l}21.155 \\
20.658 \\
19.824 \\
19.983 \\
19.918 \\
19.759 \\
18.145 \\
17.241 \\
16.346 \\
16.232\end{array}$ & $\begin{array}{l}25.892 \\
26.031 \\
26.214 \\
26.270 \\
26.288 \\
26.312 \\
26.447 \\
26.558 \\
26.656 \\
26.667\end{array}$ & $\begin{array}{l}32.221 \\
32.371 \\
32.574 \\
32.624 \\
32.644 \\
32.672 \\
32.848 \\
32.983 \\
33.106 \\
33.120\end{array}$ & $\begin{array}{l}38 . \\
38 . \\
38 . \\
38 . \\
38 . \\
38 . \\
39 . \\
39 . \\
39 . \\
39 .\end{array}$ & $\begin{array}{l}0.002 \\
0.050 \\
0.097 \\
0.142 \\
0.186 \\
0.274 \\
0.359 \\
0.440 \\
0.515 \\
0.523\end{array}$ & $\begin{array}{l}5.40 \\
4.52 \\
2.98 \\
1.83 \\
1.60 \\
2.18 \\
2.84 \\
2.67 \\
2.06 \\
3.10\end{array}$ & $\begin{array}{r}1 \\
25 \\
50 \\
74 \\
99 \\
149 \\
199 \\
248 \\
298 \\
303\end{array}$ \\
\hline $\begin{array}{l}\text { PR } \\
\text { dbar }\end{array}$ & $\stackrel{T}{\text { Deg } C}$ & $\stackrel{5}{0 / 00}$ & $\begin{array}{l}02 \\
\mathrm{ml} / \mathrm{l}\end{array}$ & $\begin{array}{c}\mathrm{F}-11 \\
\mathrm{pmol} / \mathrm{kg}\end{array}$ & $\begin{array}{c}\mathrm{F}-12 \\
\mathrm{pmol} / \mathrm{kg}\end{array}$ & $\stackrel{\theta}{\operatorname{Deg} C}$ & $\begin{array}{l}S I G-\theta \\
\mathrm{kg} / \mathrm{m} 3\end{array}$ & $\underset{\mathrm{kg} / \mathrm{m} 3}{\mathrm{SIG}-1.5}$ & $\begin{array}{l}\mathrm{SIG}-3 \\
\mathrm{~kg} / \mathrm{m} 3\end{array}$ & E \\
\hline $\begin{array}{l}141 \\
162 \\
191\end{array}$ & $\begin{array}{l}19.800 \\
19.065 \\
18.022 \\
17.440\end{array}$ & $\begin{array}{l}36.879 \\
36.466 \\
36.826 \\
36.740\end{array}$ & $\begin{array}{l}5.29 \\
4.79 \\
5.27 \\
5.11\end{array}$ & & & $\begin{array}{l}19.774 \\
19.035 \\
17.989 \\
17.400\end{array}$ & $\begin{array}{l}26.257 \\
26.134 \\
26.675 \\
26.755\end{array}$ & $\begin{array}{l}32.617 \\
32.516 \\
33.078 \\
33.173\end{array}$ & $\begin{array}{l}38.771 \\
38.689 \\
39.272 \\
39.381\end{array}$ & $\begin{array}{l}140 \\
160 \\
189 \\
233\end{array}$ \\
\hline
\end{tabular}

ENDEAVOR 143 STA- 76 LAT $=2911.0 \mathrm{~N} \quad$ LON= $3139.8 \mathrm{~W} \quad$ SONIC DEPTH= Om DATE $13 / 5 / 86$

\begin{tabular}{|c|c|c|c|c|c|c|c|c|c|}
\hline $\begin{array}{l}\text { PR } \\
\text { dbar }\end{array}$ & $\stackrel{T}{\operatorname{Deg}} C$ & $\stackrel{5}{0 / 00}$ & $\begin{array}{c}02 \\
\mathrm{ml} / \mathrm{l}\end{array}$ & $\stackrel{\theta}{\text { Deg }} \mathrm{C}$ & $\begin{array}{l}\text { SIG-O } \\
\mathrm{kg} / \mathrm{m3}\end{array}$ & $\begin{array}{l}\mathrm{SIG}-1 \\
\mathrm{~kg} / \mathrm{m3}\end{array}$ & $\begin{array}{l}\text { SIG-3 } \\
\mathrm{kg} / \mathrm{m} 3\end{array}$ & $\begin{array}{c}\text { HGTH } \\
\text { m }\end{array}$ & $\underset{\text { cph }}{N}$ \\
\hline $\begin{array}{r}1 \\
25 \\
50 \\
75 \\
100 \\
150 \\
200 \\
250 \\
300 .\end{array}$ & $\begin{array}{l}21.163 \\
20.358 \\
19.605 \\
19.260 \\
19.516 \\
18.322 \\
17.477 \\
16.846 \\
16.072\end{array}$ & $\begin{array}{l}36.889 \\
36.846 \\
36.778 \\
36.712 \\
36.827 \\
36.568 \\
36.434 \\
36.338 \\
36.208\end{array}$ & $\begin{array}{l}4.85 \\
4.85 \\
4.95 \\
4.95 \\
4.89 \\
4.50 \\
4.21 \\
4.12 \\
4.13\end{array}$ & $\begin{array}{l}21.163 \\
20.354 \\
19.596 \\
19.246 \\
19.498 \\
18.296 \\
17.443 \\
16.805 \\
16.024\end{array}$ & $\begin{array}{l}25.889 \\
26.077 \\
26.227 \\
26.268 \\
26.290 \\
26.401 \\
26.509 \\
26.590 \\
26.673\end{array}$ & $\begin{array}{l}32.218 \\
32.425 \\
32.593 \\
32.642 \\
32.657 \\
32.799 \\
32.929 \\
33.027 \\
33.132\end{array}$ & $\begin{array}{l}38.341 \\
38.566 \\
38.750 \\
38.809 \\
38.817 \\
38.988 \\
39.140 \\
39.253 \\
39.378\end{array}$ & $\begin{array}{l}0.002 \\
0.051 \\
0.096 \\
0.141 \\
0.185 \\
0.272 \\
0.353 \\
0.431 \\
0.506\end{array}$ & $\begin{array}{l}5.34 \\
5.56 \\
2.65 \\
1.78 \\
1.75 \\
3.01 \\
2.20 \\
2.29 \\
2.67\end{array}$ \\
\hline $\begin{array}{l}\text { PR } \\
\text { dbar }\end{array}$ & $\stackrel{T}{\text { Deg } C}$ & $\stackrel{S}{\circ / 00}$ & $\begin{array}{l}02 \\
\mathrm{ml} / \mathrm{l}\end{array}$ & $\begin{array}{c}\mathrm{F}-11 \\
\mathrm{pmol} / \mathrm{kg}\end{array}$ & $\begin{array}{c}\mathrm{F}-12 \\
\mathrm{pmol} / \mathrm{kg}\end{array}$ & $\operatorname{Deg}^{\theta} \mathrm{C}$ & $\begin{array}{l}S I G-\theta \\
\mathrm{kg} / \mathrm{m3}\end{array}$ & $\underset{\mathrm{kg} / \mathrm{m} 3}{\mathrm{SIG}-1.5}$ & $\begin{array}{l}\mathrm{SIG}-3 \\
\mathrm{~kg} / \mathrm{m} 3\end{array}$ \\
\hline $\begin{array}{r}97 \\
124 \\
206\end{array}$ & $\begin{array}{l}19.564 \\
19.045 \\
17.385\end{array}$ & $\begin{array}{l}36.971 \\
36.795 \\
36.536\end{array}$ & $\begin{array}{l}5.14 \\
4.97 \\
4.79\end{array}$ & & & $\begin{array}{l}19.546 \\
19.023 \\
17.350\end{array}$ & $\begin{array}{l}26.388 \\
26.389 \\
26.610\end{array}$ & $\begin{array}{l}32.752 \\
32.768 \\
33.032\end{array}$ & $\begin{array}{l}38.910 \\
38.938 \\
39.243\end{array}$ \\
\hline
\end{tabular}

\begin{tabular}{|c|c|c|c|c|c|c|c|c|c|c|}
\hline \multicolumn{3}{|c|}{$\begin{array}{l}\text { ENDEAVOR } 143 \text { STA- } 77 \\
\text { DATE } 13 / 5 / 86\end{array}$} & LAT $=$ & \multicolumn{2}{|c|}{$2919.5 \mathrm{~N}$} & \multicolumn{2}{|c|}{ ON= $3140.1 \mathrm{~W}$} & \multicolumn{3}{|c|}{ SONIC DEPTH $=4350 \mathrm{~m}$} \\
\hline $\begin{array}{l}\text { PR } \\
\text { dbar }\end{array}$ & $\stackrel{T}{\text { Deg } C}$ & $\stackrel{5}{0 / 00}$ & $\begin{array}{ll}02 \\
\mathrm{ml} / \mathrm{l}\end{array}$ & $\stackrel{\theta}{\operatorname{Deg} C}$ & $\begin{array}{l}\mathrm{SIG}-\theta \\
\mathrm{kg} / \mathrm{m} 3\end{array}$ & $\underset{\mathrm{kg} / \mathrm{m} 3}{\mathrm{SIG}-1.5}$ & $\begin{array}{l}\mathrm{SIG}-3 \\
\mathrm{~kg} / \mathrm{m} 3\end{array}$ & $\underset{m}{\text { HGTH }}$ & $\underset{c p h}{N}$ & $\begin{aligned} \mathrm{DE} \\
\mathbf{m}\end{aligned}$ \\
\hline $\begin{array}{r}1 \\
25 \\
50 \\
75 \\
100 \\
150 \\
200 \\
250 \\
300\end{array}$ & $\begin{array}{l}20.970 \\
20.508 \\
19.652 \\
19.366 \\
19.147 \\
18.146 \\
17.298 \\
16.726 \\
15.837\end{array}$ & $\begin{array}{l}36.742 \\
36.755 \\
36.735 \\
36.747 \\
36.717 \\
36.507 \\
36.371 \\
36.294 \\
36.152\end{array}$ & $\begin{array}{l}4.54 \\
4.84 \\
4.99 \\
4.99 \\
4.94 \\
4.50 \\
4.24 \\
4.13 \\
4.18\end{array}$ & $\begin{array}{l}20.970 \\
20.503 \\
19.643 \\
19.352 \\
19.129 \\
18.120 \\
17.264 \\
16.684 \\
15.789\end{array}$ & $\begin{array}{l}25.830 \\
25.967 \\
26.182 \\
26.267 \\
26.302 \\
26.398 \\
26.504 \\
26.584 \\
26.684\end{array}$ & $\begin{array}{l}32.165 \\
32.312 \\
32.547 \\
32.638 \\
32.679 \\
32.801 \\
32.929 \\
33.025 \\
33.149\end{array}$ & $\begin{array}{l}38.294 \\
38.451 \\
38.704 \\
38.802 \\
38.848 \\
38.995 \\
39.144 \\
39.255 \\
39.402\end{array}$ & $\begin{array}{l}0.002 \\
0.053 \\
0.100 \\
0.145 \\
0.189 \\
0.275 \\
0.357 \\
0.435 \\
0.510\end{array}$ & $\begin{array}{l}1 . \\
1 . \\
2 . \\
2 . \\
2 .\end{array}$ & $\begin{array}{r}1 \\
25 \\
50 \\
74 \\
99 \\
149 \\
199 \\
248 \\
298\end{array}$ \\
\hline $\begin{array}{l}\text { PR } \\
\text { dbar }\end{array}$ & $\stackrel{T}{\text { Deg } C}$ & $\stackrel{5}{1}$ & $\begin{array}{l}02 \\
\mathrm{ml} / 1\end{array}$ & $\begin{array}{c}\mathrm{F}-11 \\
\mathrm{pmol} / \mathrm{kg}\end{array}$ & $\begin{array}{c}\mathrm{F}-12 \\
\mathrm{pmol} / \mathrm{kg}\end{array}$ & $\stackrel{\theta}{\operatorname{Deg} C}$ & $\begin{array}{l}\mathrm{SIG}-0 \\
\mathrm{~kg} / \mathrm{m3}\end{array}$ & $\underset{\mathrm{kg} / \mathrm{m} 3}{\mathrm{SIG}-1.5}$ & $\begin{array}{l}\mathrm{SIG} \\
\mathrm{kg} /\end{array}$ & $\mathrm{DE}$ \\
\hline $\begin{array}{r}66 \\
105 \\
179\end{array}$ & $\begin{array}{l}19.394 \\
19.027 \\
17.490\end{array}$ & $\begin{array}{l}36.767 \\
36.702 \\
36.405\end{array}$ & $\begin{array}{l}5.46 \\
5.31 \\
4.87\end{array}$ & & & $\begin{array}{l}19.382 \\
19.008 \\
17.460\end{array}$ & $\begin{array}{l}26.275 \\
26.322 \\
26.483\end{array}$ & $\begin{array}{l}32.645 \\
32.702 \\
32.903\end{array}$ & $\begin{array}{l}38.808 \\
38.873 \\
39.113\end{array}$ & $\begin{array}{r}65 \\
104 \\
178\end{array}$ \\
\hline
\end{tabular}




\begin{tabular}{|c|c|c|c|c|c|c|c|c|c|c|}
\hline \multicolumn{3}{|c|}{$\begin{array}{l}\text { ENDEAVOR } 143 \text { STA- } 78 \\
\text { DATE } 13 / 5 / 86\end{array}$} & LAT $=$ & \multicolumn{2}{|c|}{$2930.0 \mathrm{~N}$} & \multicolumn{2}{|c|}{$O N=3139.4 W$} & \multicolumn{3}{|c|}{ SONIC DEPTH $=4380 \mathrm{~m}$} \\
\hline $\begin{array}{l}\text { PR } \\
\text { dbar }\end{array}$ & $\stackrel{T}{\text { Deg } C}$ & $\stackrel{S}{\circ}$ & $\mathrm{O} 2$ & $\stackrel{\theta}{\operatorname{Deg} C}$ & $\begin{array}{l}\mathrm{SIG}-\Theta \\
\mathrm{kg} / \mathrm{m} 3\end{array}$ & $\begin{array}{l}\mathrm{SIG}-1.5 \\
\mathrm{~kg} / \mathrm{m} 3\end{array}$ & $\begin{array}{l}\mathrm{SIG}-3 \\
\mathrm{~kg} / \mathrm{m} 3\end{array}$ & $\underset{\mathbf{m}}{\mathrm{HGTH}}$ & $\underset{c p h}{N}$ & $\begin{aligned} \mathrm{DE} \\
\mathbf{m}\end{aligned}$ \\
\hline $\begin{array}{r}1 \\
25 \\
50 \\
75 \\
100 \\
150 \\
200 \\
250 \\
300 \\
305\end{array}$ & $\begin{array}{l}21.306 \\
20.601 \\
19.230 \\
18.924 \\
18.781 \\
17.796 \\
17.220 \\
16.522 \\
15.721 \\
15.667\end{array}$ & $\begin{array}{l}36.830 \\
36.807 \\
36.652 \\
36.644 \\
36.628 \\
36.443 \\
36.362 \\
36.254 \\
36.139 \\
36.132\end{array}$ & $\begin{array}{l}4.63 \\
4.79 \\
5.03 \\
4.94 \\
4.87 \\
4.26 \\
4.17 \\
4.10 \\
4.09 \\
4.10\end{array}$ & $\begin{array}{l}21.306 \\
20.597 \\
19.221 \\
18.910 \\
18.763 \\
17.771 \\
17.187 \\
16.481 \\
15.673 \\
15.619\end{array}$ & $\begin{array}{l}25.805 \\
25.982 \\
26.228 \\
26.303 \\
26.328 \\
26.436 \\
26.516 \\
26.602 \\
26.700 \\
26.707\end{array}$ & $\begin{array}{l}32.132 \\
32.324 \\
32.604 \\
32.685 \\
32.715 \\
32.848 \\
32.944 \\
33.048 \\
33.168 \\
33.177\end{array}$ & $\begin{array}{l}38.253 \\
38.460 \\
38.771 \\
38.860 \\
38.892 \\
39.051 \\
39.161 \\
39.283 \\
39.424 \\
39.434\end{array}$ & $\begin{array}{l}0.002 \\
0.053 \\
0.099 \\
0.144 \\
0.187 \\
0.271 \\
0.352 \\
0.429 \\
0.503 \\
0.510\end{array}$ & $\begin{array}{l}5.98 \\
6.31 \\
3.91 \\
2.25 \\
2.09 \\
2.27 \\
2.53 \\
2.40 \\
2.57 \\
1.09\end{array}$ & $\begin{array}{r}1 \\
25 \\
50 \\
74 \\
99 \\
149 \\
199 \\
248 \\
298 \\
303\end{array}$ \\
\hline $\begin{array}{l}\text { PR } \\
\text { dbar }\end{array}$ & $\stackrel{T}{\text { Deg } C}$ & $\stackrel{5}{\$}$ & $\begin{array}{l}02 \\
\mathrm{~m} 1 / 1\end{array}$ & $\begin{array}{c}\mathrm{F}-11 \\
\mathrm{pmol} / \mathrm{kg}\end{array}$ & $\begin{array}{c}\mathrm{F}-12 \\
\mathrm{pmol} / \mathrm{kg}\end{array}$ & $\stackrel{\theta}{\operatorname{Deg} C}$ & $\begin{array}{l}\mathrm{SIG}-0 \\
\mathrm{~kg} / \mathrm{m} 3\end{array}$ & $\underset{\mathrm{kg} / \mathrm{m} 3}{\mathrm{SIG}-1.5}$ & $\begin{array}{l}\mathrm{SIG}-3 \\
\mathrm{~kg} / \mathrm{m} 3\end{array}$ & $\begin{aligned} D E \\
m\end{aligned}$ \\
\hline $\begin{array}{r}64 \\
80 \\
177\end{array}$ & $\begin{array}{l}19.014 \\
18.838 \\
17.453\end{array}$ & $\begin{array}{l}36.662 \\
36.645 \\
36.400\end{array}$ & $\begin{array}{l}5.37 \\
5.38 \\
4.84\end{array}$ & & & $\begin{array}{l}19.002 \\
18.823 \\
17.423\end{array}$ & $\begin{array}{l}26.293 \\
26.326 \\
26.488\end{array}$ & $\begin{array}{l}32.673 \\
32.710 \\
32.909\end{array}$ & $\begin{array}{l}38.845 \\
38.887 \\
39.120\end{array}$ & $\begin{array}{r}64 \\
80 \\
175\end{array}$ \\
\hline
\end{tabular}

\begin{tabular}{|c|c|c|c|c|c|c|c|c|c|c|}
\hline \multicolumn{3}{|c|}{$\begin{array}{l}\text { ENDEAVOR } 143 \text { STA- } 79 \\
\text { DATE } 13 / 5 / 86\end{array}$} & $L A T=$ & \multicolumn{2}{|c|}{$2930.0 \mathrm{~N}$} & \multicolumn{2}{|c|}{ ON= } & \multicolumn{2}{|c|}{ SONIC DEPTH } & \multirow{2}{*}{$\begin{array}{l}\text { Om } \\
\underset{\text { m }}{\text { m }}\end{array}$} \\
\hline $\begin{array}{l}\text { PR } \\
\text { dbar }\end{array}$ & $\stackrel{T}{\text { Deg } C}$ & $\stackrel{S}{0 / 00}$ & $\begin{array}{l}02 \\
\mathrm{ml} / \mathrm{l}\end{array}$ & $\stackrel{\theta}{\operatorname{Deg}} \mathrm{C}$ & $\begin{array}{l}\mathrm{SIG}-0 \\
\mathrm{~kg} / \mathrm{m3}\end{array}$ & $\mathrm{SIG}_{\mathrm{kg} / \mathrm{m} 3}^{-1.5}$ & $\begin{array}{l}\mathrm{SIG}-3 \\
\mathrm{~kg} / \mathrm{m} 3\end{array}$ & $\underset{m}{\text { HGTH }}$ & $\underset{\text { cph }}{N}$ & \\
\hline $\begin{array}{r}1 \\
25 \\
50 \\
75 \\
100 \\
150 \\
200 \\
250 \\
300 \\
307\end{array}$ & $\begin{array}{l}20.970 \\
20.362 \\
19.562 \\
19.076 \\
18.913 \\
18.435 \\
17.431 \\
16.440 \\
15.794 \\
15.709\end{array}$ & $\begin{array}{l}36.815 \\
36.776 \\
36.699 \\
36.639 \\
36.646 \\
36.586 \\
36.417 \\
36.256 \\
36.167 \\
36.150\end{array}$ & $\begin{array}{l}4.61 \\
4.58 \\
4.78 \\
4.87 \\
4.78 \\
4.45 \\
4.16 \\
4.08 \\
4.09 \\
4.00\end{array}$ & $\begin{array}{l}20.970 \\
20.357 \\
19.553 \\
19.062 \\
18.895 \\
18.409 \\
17.397 \\
16.399 \\
15.746 \\
15.661\end{array}$ & $\begin{array}{l}25.886 \\
26.023 \\
26.178 \\
26.260 \\
26.308 \\
26.386 \\
26.508 \\
26.622 \\
26.705 \\
26.711\end{array}$ & $\begin{array}{l}32.220 \\
32.371 \\
32.545 \\
32.639 \\
32.691 \\
32.781 \\
32.929 \\
33.071 \\
33.171 \\
33.180\end{array}$ & $\begin{array}{l}38.348 \\
38.512 \\
38.705 \\
38.810 \\
38.866 \\
38.967 \\
39.141 \\
39.308 \\
39.425 \\
39.437\end{array}$ & $\begin{array}{l}0.002 \\
0.051 \\
0.099 \\
0.144 \\
0.188 \\
0.274 \\
0.356 \\
0.433 \\
0.507 \\
0.517\end{array}$ & $\begin{array}{l}4.52 \\
5.01 \\
3.45 \\
2.59 \\
2.09 \\
2.57 \\
2.94 \\
2.39 \\
2.58 \\
2.19\end{array}$ & $\begin{array}{r}1 \\
25 \\
50 \\
74 \\
99 \\
149 \\
199 \\
248 \\
298 \\
305\end{array}$ \\
\hline $\begin{array}{l}\text { PR } \\
\text { dbar }\end{array}$ & $\stackrel{T}{\text { Deg } C}$ & $\stackrel{S}{0 / 00}$ & $\begin{array}{l}02 \\
\mathrm{ml} / \mathrm{l}\end{array}$ & $\begin{array}{c}\mathrm{F}-11 \\
\mathrm{pmol} / \mathrm{kg}\end{array}$ & $\begin{array}{c}\mathrm{F}-12 \\
\mathrm{pmol} / \mathrm{kg}\end{array}$ & $\stackrel{\theta}{\operatorname{Deg} C}$ & $\begin{array}{l}\mathrm{SIG}-0 \\
\mathrm{~kg} / \mathrm{m3}\end{array}$ & $\underset{\mathrm{kg} / \mathrm{m} 3}{\mathrm{SIG}-1.5}$ & $\begin{array}{l}\mathrm{SIG}-3 \\
\mathrm{~kg} / \mathrm{m} 3\end{array}$ & DE \\
\hline $\begin{array}{r}45 \\
86 \\
187\end{array}$ & $\begin{array}{l}19.510 \\
18.928 \\
17.492\end{array}$ & $\begin{array}{l}36.725 \\
36.644 \\
36.455\end{array}$ & $\begin{array}{l}5.36 \\
5.40 \\
4.95\end{array}$ & & & $\begin{array}{l}19.501 \\
18.912 \\
17.460\end{array}$ & $\begin{array}{l}26.211 \\
26.302 \\
26.521\end{array}$ & $\begin{array}{l}32.579 \\
32.685 \\
32.941\end{array}$ & $\begin{array}{l}38.740 \\
38.859 \\
39.150\end{array}$ & $\begin{array}{r}45 \\
86 \\
186\end{array}$ \\
\hline
\end{tabular}

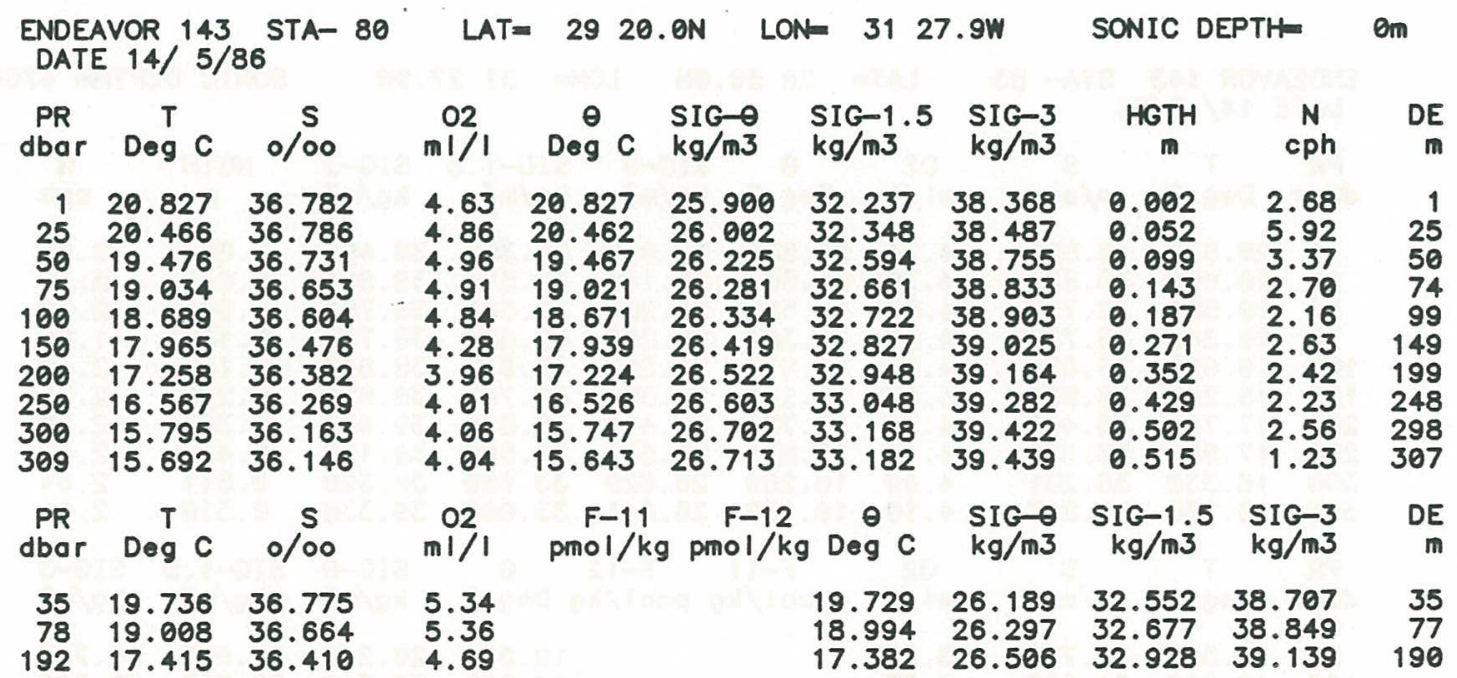




\begin{tabular}{|c|c|c|c|c|c|c|c|c|c|c|}
\hline DA & $\begin{array}{l}\text { OR } 1 \\
14 / 5\end{array}$ & STA- & $L A$ & 9 & $N$ & 1 & ow & IC & $\mathrm{TH}=$ & \\
\hline $\begin{array}{c}\text { PR } \\
\text { dbar }\end{array}$ & $\stackrel{{ }^{\top}}{\operatorname{Deg}} \mathrm{C}$ & $\begin{array}{c}S \\
0 / 00\end{array}$ & $\begin{array}{l}02 \\
\mathrm{ml} / \mathrm{l}\end{array}$ & $\stackrel{\theta}{\operatorname{Deg} C}$ & $\underset{\mathrm{kg} / \mathrm{m} 3}{\mathrm{SIG}-0}$ & $\begin{array}{l}\mathrm{SIG}-1.5 \\
\mathrm{~kg} / \mathrm{m} 3\end{array}$ & $\begin{array}{l}S I G-3 \\
\mathrm{~kg} / \mathrm{m} 3\end{array}$ & $\underset{\mathrm{m}}{\mathrm{HGTH}}$ & $\underset{c p h}{N}$ & $\underset{m}{\mathrm{DE}}$ \\
\hline $\begin{array}{r}3 \\
25 \\
50 \\
75 \\
100 \\
150 \\
200 \\
250 \\
300 \\
305\end{array}$ & $\begin{array}{l}20.689 \\
20.505 \\
19.717 \\
19.146 \\
18.946 \\
18.429 \\
17.574 \\
16.968 \\
16.103 \\
16.072\end{array}$ & $\begin{array}{l}36.840 \\
36.854 \\
36.721 \\
36.653 \\
36.641 \\
36.554 \\
36.414 \\
36.355 \\
36.206 \\
36.201\end{array}$ & $\begin{array}{l}4.68 \\
4.74 \\
4.89 \\
4.94 \\
4.89 \\
4.59 \\
4.22 \\
4.00 \\
3.98 \\
4.00\end{array}$ & $\begin{array}{l}20.688 \\
20.501 \\
19.707 \\
19.133 \\
18.928 \\
18.403 \\
17.540 \\
16.926 \\
16.055 \\
16.023\end{array}$ & $\begin{array}{l}25.982 \\
26.044 \\
26.154 \\
26.252 \\
26.296 \\
26.363 \\
26.470 \\
26.573 \\
26.664 \\
26.667\end{array}$ & $\begin{array}{l}32.322 \\
32.388 \\
32.518 \\
32.630 \\
32.678 \\
32.759 \\
32.888 \\
33.007 \\
33.122 \\
33.126\end{array}$ & $\begin{array}{l}38.456 \\
38.525 \\
38.674 \\
38.799 \\
38.852 \\
38.946 \\
39.096 \\
39.231 \\
39.368 \\
39.373\end{array}$ & $\begin{array}{l}0.006 \\
0.050 \\
0.098 \\
0.143 \\
0.188 \\
0.275 \\
0.358 \\
0.437 \\
0.513 \\
0.520\end{array}$ & $\begin{array}{l}4.06 \\
2.31 \\
4.04 \\
3.03 \\
1.74 \\
2.62 \\
2.46 \\
2.54 \\
2.46 \\
1.17\end{array}$ & $\begin{array}{r}3 \\
25 \\
50 \\
74 \\
99 \\
149 \\
199 \\
248 \\
298 \\
303\end{array}$ \\
\hline $\begin{array}{l}\text { PR } \\
\text { dbar }\end{array}$ & $\stackrel{T}{\text { Deg } C}$ & $\begin{array}{c}S \\
0 / 00\end{array}$ & $\begin{array}{l}02 \\
\mathrm{ml} / \mathrm{l}\end{array}$ & $\begin{array}{c}\mathrm{F}-11 \\
\mathrm{pmol} / \mathrm{kg}\end{array}$ & $\begin{array}{c}\mathrm{F}-12 \\
\mathrm{pmol} / \mathrm{kg}\end{array}$ & $\stackrel{\theta}{\operatorname{Deg}} \mathrm{C}$ & $\begin{array}{l}S I G-0 \\
\mathrm{~kg} / \mathrm{m} 3\end{array}$ & $\underset{\mathrm{kg} / \mathrm{m} 3}{S I G-1.5}$ & $\begin{array}{l}S I G-3 \\
\mathrm{~kg} / \mathrm{m} 3\end{array}$ & 0 \\
\hline $\begin{array}{r}48 \\
92 \\
211\end{array}$ & $\begin{array}{l}19.943 \\
18.952 \\
17.424\end{array}$ & $\begin{array}{l}36.823 \\
36.648 \\
36.423\end{array}$ & $\begin{array}{l}5.31 \\
5.38 \\
4.71\end{array}$ & & & $\begin{array}{l}19.934 \\
18.935 \\
17.388\end{array}$ & $\begin{array}{l}26.172 \\
26.299 \\
26.514\end{array}$ & $\begin{array}{l}32.529 \\
32.681 \\
32.936\end{array}$ & $\begin{array}{l}38.679 \\
38.855 \\
39.147\end{array}$ & $\begin{array}{r}47 \\
91 \\
209\end{array}$ \\
\hline
\end{tabular}

ENDEAVOR 143 STA- 82
DATE $14 / 5 / 86$

\begin{tabular}{|c|c|c|c|c|c|c|c|c|c|c|}
\hline $\begin{array}{l}\text { PR } \\
\text { dbar }\end{array}$ & ${ }_{\operatorname{Deg}}^{T} \mathrm{C}$ & $\begin{array}{c}S \\
0 / 00\end{array}$ & $\begin{array}{l}02 \\
\mathrm{ml} / 1\end{array}$ & $\stackrel{\theta}{\operatorname{Deg} C}$ & $\begin{array}{l}\mathrm{SIG}-0 \\
\mathrm{~kg} / \mathrm{m} 3\end{array}$ & $\underset{\mathrm{kg} / \mathrm{m} 3}{\mathrm{SIG}-1.5}$ & $\begin{array}{l}\mathrm{SIG}-3 \\
\mathrm{~kg} / \mathrm{m} 3\end{array}$ & $\underset{\mathbf{m}}{\mathrm{HGTH}}$ & $\underset{\text { cph }}{N}$ & \\
\hline $\begin{array}{r}1 \\
25 \\
50 \\
75 \\
100 \\
150 \\
200 \\
250 \\
300 \\
305\end{array}$ & $\begin{array}{l}20.810 \\
20.092 \\
19.323 \\
18.983 \\
18.960 \\
18.563 \\
17.530 \\
16.867 \\
16.186 \\
16.131\end{array}$ & $\begin{array}{l}36.874 \\
36.821 \\
36.723 \\
36.657 \\
36.659 \\
36.581 \\
36.437 \\
36.325 \\
36.210 \\
36.204\end{array}$ & $\begin{array}{l}4.91 \\
4.74 \\
4.88 \\
4.90 \\
4.86 \\
4.70 \\
4.10 \\
4.04 \\
3.99 \\
3.95\end{array}$ & $\begin{array}{l}20.810 \\
20.087 \\
19.314 \\
18.969 \\
18.942 \\
18.537 \\
17.496 \\
16.825 \\
16.137 \\
16.082\end{array}$ & $\begin{array}{l}25.975 \\
26.130 \\
26.259 \\
26.297 \\
26.306 \\
26.350 \\
26.498 \\
26.575 \\
26.648 \\
26.656\end{array}$ & $\begin{array}{l}32.312 \\
32.483 \\
32.631 \\
32.679 \\
32.688 \\
32.742 \\
32.917 \\
33.011 \\
33.104 \\
33.113\end{array}$ & $\begin{array}{l}38.443 \\
38.630 \\
38.796 \\
38.852 \\
38.861 \\
38.925 \\
39.126 \\
39.237 \\
39.348 \\
39.359\end{array}$ & $\begin{array}{l}0.002 \\
0.049 \\
0.094 \\
0.138 \\
0.182 \\
0.269 \\
0.352 \\
0.430 \\
0.506 \\
0.514\end{array}$ & $\begin{array}{l}4.49 \\
5.53 \\
2.75 \\
1.22 \\
0.94 \\
2.79 \\
2.76 \\
1.98 \\
2.63 \\
1.46\end{array}$ & $\begin{array}{l}149 \\
199 \\
248 \\
298\end{array}$ \\
\hline $\begin{array}{l}\text { PR } \\
\text { dbar }\end{array}$ & $\stackrel{T}{\operatorname{Deg}} C$ & $\begin{array}{c}S \\
0 / 00\end{array}$ & $\begin{array}{l}02 \\
\mathrm{ml} / \mathrm{l}\end{array}$ & $\begin{array}{c}\mathrm{F}-11 \\
\mathrm{pmol} / \mathrm{kg}\end{array}$ & $\begin{array}{c}\mathrm{F}-12 \\
\mathrm{pmol} / \mathrm{kg}\end{array}$ & $\stackrel{\theta}{\operatorname{Deg}} \mathrm{C}$ & $\begin{array}{l}\mathrm{SIG}-\Theta \\
\mathrm{kg} / \mathrm{m} 3\end{array}$ & $\underset{\mathrm{kg} / \mathrm{m} 3}{S I G-1.5}$ & $\begin{array}{l}S I G-3 \\
\mathrm{~kg} / \mathrm{m} 3\end{array}$ & \\
\hline $\begin{array}{r}90 \\
227 \\
263\end{array}$ & $\begin{array}{l}18.965 \\
17.205 \\
16.654\end{array}$ & $\begin{array}{l}36.667 \\
36.404 \\
36.288\end{array}$ & $\begin{array}{l}5.34 \\
4.70 \\
4.69\end{array}$ & & & $\begin{array}{l}18.948 \\
17.167 \\
16.611\end{array}$ & $\begin{array}{l}26.310 \\
26.553 \\
26.597\end{array}$ & $\begin{array}{l}32.692 \\
32.981 \\
33.040\end{array}$ & $\begin{array}{l}38.865 \\
39.198 \\
39.271\end{array}$ & \\
\hline
\end{tabular}

ENDEAVOR 143 STA- 83
DATE $14 / 5 / 86$

$\begin{array}{rcc}\begin{array}{c}P R \\ \text { dbar }\end{array} & \begin{array}{c}T \\ \text { Deg } C\end{array} & \begin{array}{c}S \\ \text { o } 00\end{array} \\ 1 & 20.538 & 36.829 \\ 25 & 20.068 & 36.852 \\ 50 & 19.591 & 36.774 \\ 75 & 19.365 & 36.733 \\ 100 & 19.090 & 36.686 \\ 150 & 18.286 & 36.531 \\ 200 & 17.755 & 36.440 \\ 250 & 17.065 & 36.339 \\ 300 & 16.338 & 36.231 \\ 305 & 16.238 & 36.217\end{array}$

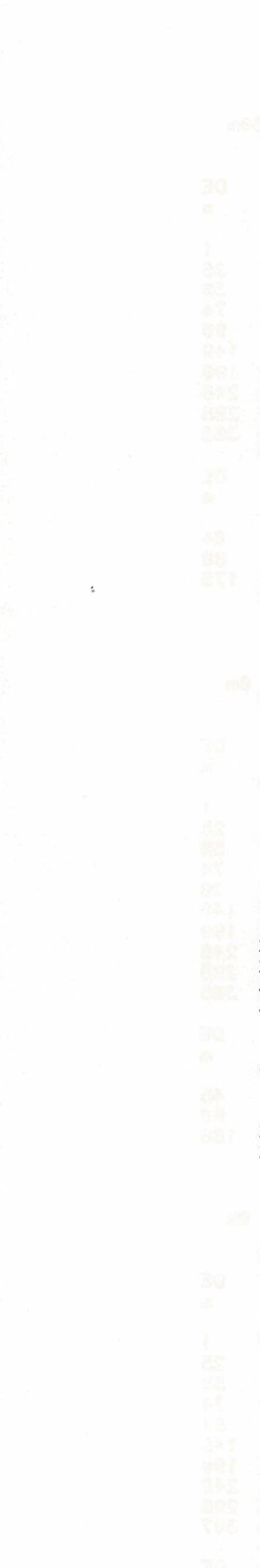

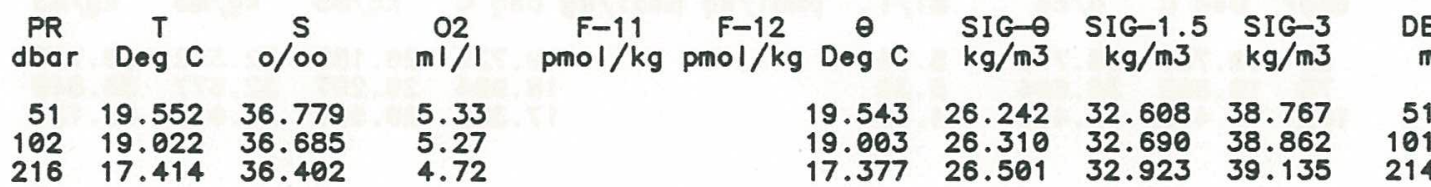




\begin{tabular}{|c|c|c|c|c|c|c|c|c|c|c|}
\hline \multicolumn{3}{|c|}{$\begin{array}{l}\text { ENDEAVOR } 143 \text { STA- } 84 \\
\text { DATE } 14 / 5 / 86\end{array}$} & \multirow{2}{*}{$\begin{array}{l}\text { LAT }= \\
\mathrm{ml} / \mathrm{l}\end{array}$} & \multicolumn{2}{|c|}{$=2840.0 \mathrm{~N}$} & \multicolumn{2}{|c|}{$\mathrm{N}=3128.0 \mathrm{~W}$} & \multicolumn{3}{|c|}{ SONIC DEPTH $=4560 \mathrm{~m}$} \\
\hline $\begin{array}{l}\text { PR } \\
\text { dbar }\end{array}$ & $\stackrel{T}{\operatorname{Deg} C}$ & $\begin{array}{c}S \\
0 / 00\end{array}$ & & $\stackrel{\ominus}{\operatorname{Deg}} \mathrm{C}$ & $\begin{array}{l}\mathrm{SIG}-\theta \\
\mathrm{kg} / \mathrm{m} 3\end{array}$ & $\underset{\mathrm{kg} / \mathrm{m} 3}{\mathrm{SIG}-1.5}$ & $\begin{array}{l}\mathrm{SIG}-3 \\
\mathrm{~kg} / \mathrm{m} 3\end{array}$ & $\underset{m}{H G T H}$ & $\underset{c p h}{N}$ & $\mathrm{DE}$ \\
\hline $\begin{array}{r}5 \\
25 \\
50 \\
75 \\
100 \\
150 \\
200 \\
250 \\
300\end{array}$ & $\begin{array}{l}20.509 \\
20.146 \\
19.438 \\
19.239 \\
18.924 \\
17.721 \\
17.205 \\
16.405 \\
15.750\end{array}$ & $\begin{array}{l}36.8 \\
36.7 \\
36.7 \\
36.7 \\
36.9 \\
36.9 \\
36.9 \\
36.2 \\
36 .\end{array}$ & $\begin{array}{l}4.75 \\
4.83 \\
4.83 \\
4.69 \\
4.69 \\
4.19 \\
3.97\end{array}$ & $\begin{array}{l}20.508 \\
20.141 \\
19.429 \\
19.225 \\
18.906 \\
17.696 \\
17.172 \\
16.364 \\
15.703\end{array}$ & $\begin{array}{l}26 . \\
26 . \\
26 . \\
26 . \\
26 . \\
26 . \\
26 . \\
26 . \\
26 .\end{array}$ & $\begin{array}{l}32.354 \\
32.437 \\
32.613 \\
32.656 \\
32.700 \\
32.860 \\
32.960 \\
33.072 \\
33.163\end{array}$ & $\begin{array}{l}.492 \\
.583 \\
.775 \\
.823 \\
.874 \\
.065 \\
.177 \\
.310 \\
.419\end{array}$ & & $\begin{array}{l}3.16 \\
4.89 \\
3.13 \\
1.32 \\
3.01 \\
2.64\end{array}$ & $\begin{array}{r}5 \\
25 \\
50 \\
74 \\
99 \\
149 \\
199 \\
248 \\
298\end{array}$ \\
\hline $\begin{array}{l}\text { PR } \\
\text { dbar }\end{array}$ & Deg $c$ & $\stackrel{S}{0 / 00}$ & $\begin{array}{l}02 \\
\mathrm{ml} / \mathrm{I}\end{array}$ & $\begin{array}{c}\mathrm{F}-11 \\
\mathrm{pmol} / \mathrm{kg}\end{array}$ & $\begin{array}{c}\mathrm{F}-12 \\
\mathrm{pmol} / \mathrm{kg}\end{array}$ & $\stackrel{\theta}{\operatorname{Deg} C}$ & $\begin{array}{l}\mathrm{SIG}-\Theta \\
\mathrm{kg} / \mathrm{m} 3\end{array}$ & $\begin{array}{l}\text { SIG- } \\
\mathrm{kg}\end{array}$ & $\begin{array}{l}\mathrm{SIG}-3 \\
\mathrm{~kg} / \mathrm{m} 3\end{array}$ & $\mathrm{DE}$ \\
\hline $\begin{array}{r}40 \\
112 \\
191\end{array}$ & 9.628 & $\begin{array}{l}36.7 \\
36.7 \\
36.4\end{array}$ & $\begin{array}{l}5.37 \\
5.29 \\
4.71\end{array}$ & & & $\begin{array}{l}19.621 \\
19.028 \\
17.387\end{array}$ & $\begin{array}{l}26.191 \\
26.318 \\
26.507\end{array}$ & $\begin{array}{l}32.556 \\
32.698 \\
32.929\end{array}$ & $\begin{array}{l}38.713 \\
38.869 \\
39.140\end{array}$ & $\begin{array}{r}39 \\
111 \\
189\end{array}$ \\
\hline
\end{tabular}




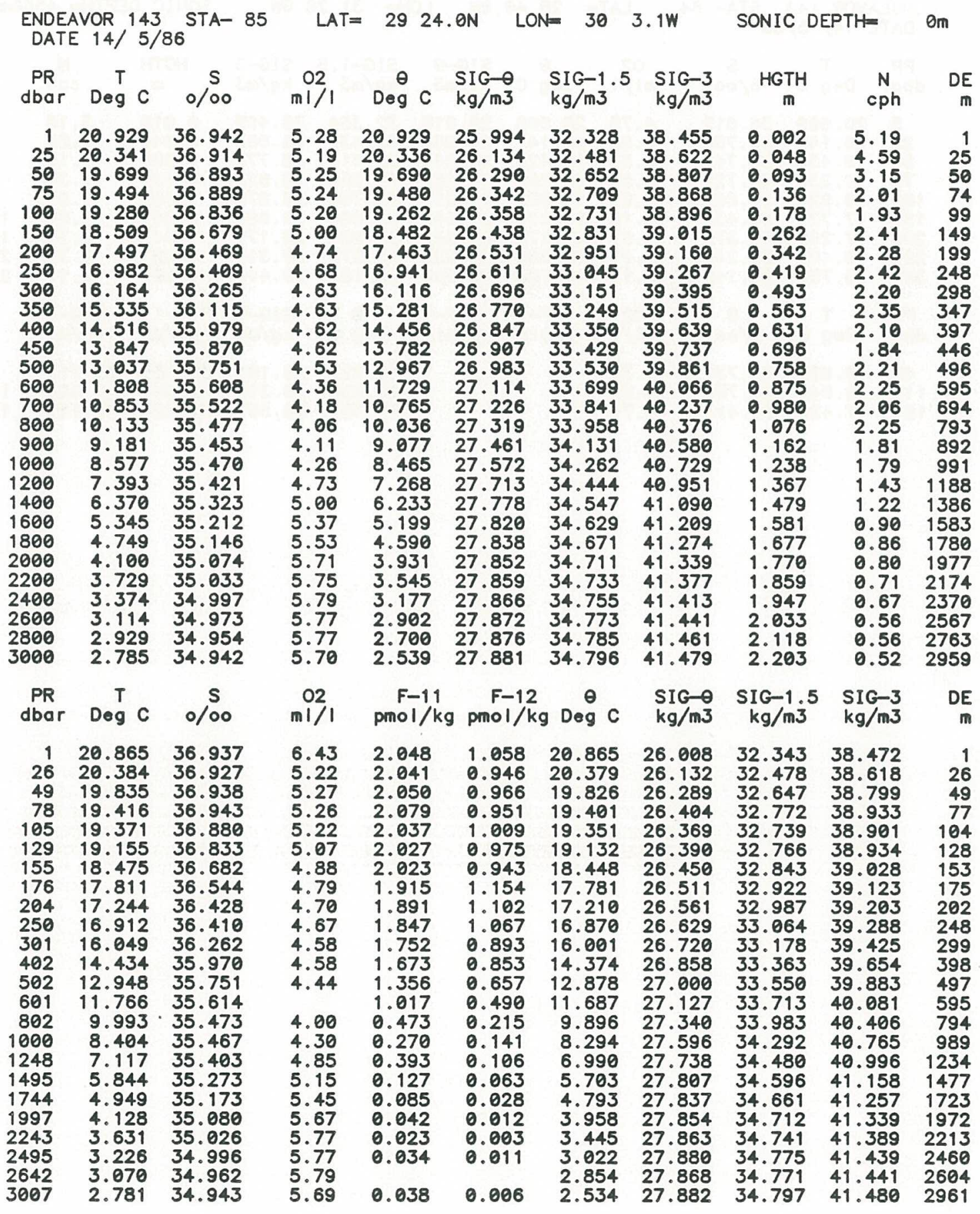




\begin{tabular}{|c|c|c|c|c|c|c|c|c|c|c|}
\hline \multicolumn{2}{|c|}{$\begin{array}{l}\text { ENDEAVOR } 143 \\
\text { DATE } 3 / 2 / 2\end{array}$} & STA- 86 & $L_{A}$ & 294 & N & 95 & & \multicolumn{3}{|c|}{ SONIC DEPTH $=4550 \mathrm{~m}$} \\
\hline $\begin{array}{l}\text { PR } \\
\text { dbar }\end{array}$ & $\operatorname{Deg}^{T} C$ & $\begin{array}{c}S \\
0 / 00\end{array}$ & $\begin{array}{l}02 \\
\mathrm{ml} / \mathrm{I}\end{array}$ & $\stackrel{\theta}{\operatorname{Deg}} \mathrm{C}$ & $\begin{array}{l}\mathrm{SIG}-0 \\
\mathrm{~kg} / \mathrm{m} 3\end{array}$ & $\begin{array}{l}\mathrm{SIG}-1.5 \\
\mathrm{~kg} / \mathrm{m} 3\end{array}$ & $\begin{array}{l}\text { SIG-3 } \\
\mathrm{kg} / \mathrm{m} 3\end{array}$ & $\underset{m}{\text { HGTH }}$ & $\underset{c p h}{N}$ & $\begin{array}{c}D E \\
m\end{array}$ \\
\hline $\begin{array}{r}3 \\
25 \\
50 \\
75 \\
100 \\
150 \\
200 \\
250 \\
300 \\
350 \\
400 \\
450 \\
500 \\
600 \\
700 \\
800 \\
900 \\
1000 \\
1200 \\
1400 \\
1600 \\
1800 \\
2000 \\
2200 \\
2400 \\
2600 \\
2800 \\
2997\end{array}$ & $\begin{array}{r}20.764 \\
19.978 \\
19.476 \\
18.930 \\
18.848 \\
18.222 \\
17.522 \\
16.954 \\
16.207 \\
15.355 \\
14.751 \\
14.124 \\
13.437 \\
12.146 \\
11.147 \\
10.296 \\
9.343 \\
8.490 \\
7.287 \\
6.096 \\
5.262 \\
4.620 \\
4.096 \\
3.702 \\
3.352 \\
3.128 \\
2.970 \\
2.815\end{array}$ & $\begin{array}{l}36.8 \\
36.7 \\
36.7 \\
36.6 \\
36.7 \\
36.5 \\
36.4 \\
36.0 \\
36.2 \\
36.0 \\
36.0 \\
35.9 \\
35.8 \\
35.6 \\
35.5 \\
35.4 \\
35.4 \\
35.4 \\
35.4 \\
35.2 \\
35.2 \\
35.1 \\
35.0 \\
35.0 \\
34.9 \\
34\end{array}$ & $\begin{array}{l}.23 \\
.34 \\
.41 \\
.39 \\
.28 \\
.02 \\
.73 \\
.69 \\
.70 \\
.70 \\
.67 \\
.65 \\
.58 \\
.43 \\
.29 \\
.21 \\
.15 \\
.28 \\
.69 \\
.13 \\
.43 \\
.61 \\
.74 \\
.77 \\
.79 \\
.78 \\
.76 \\
.73\end{array}$ & $\begin{array}{l}20.763 \\
19.974 \\
19.467 \\
18.917 \\
18.831 \\
18.196 \\
17.488 \\
16.912 \\
16.159 \\
15.300 \\
14.690 \\
14.058 \\
13.365 \\
12.065 \\
11.057 \\
10.198 \\
9.239 \\
8.379 \\
7.163 \\
5.962 \\
5.117 \\
4.463 \\
3.927 \\
3.519 \\
3.156 \\
2.916 \\
2.741 \\
2.568\end{array}$ & $\begin{array}{l}25.931 \\
26.141 \\
26.274 \\
26.334 \\
26.372 \\
26.423 \\
26.510 \\
26.585 \\
26.664 \\
26.753 \\
26.822 \\
26.880 \\
26.947 \\
27.079 \\
27.191 \\
27.289 \\
27.438 \\
27.579 \\
27.724 \\
27.794 \\
27.825 \\
27.842 \\
27.851 \\
27.860 \\
27.867 \\
27.872 \\
27.877 \\
27.881\end{array}$ & $\begin{array}{l}32.270 \\
32.497 \\
32.642 \\
32.716 \\
32.756 \\
32.823 \\
32.929 \\
33.020 \\
33.119 \\
33.232 \\
33.318 \\
33.395 \\
33.482 \\
33.654 \\
33.797 \\
33.922 \\
34.103 \\
34.272 \\
34.459 \\
34.573 \\
34.637 \\
34.679 \\
34.710 \\
34.735 \\
34.757 \\
34.772 \\
34.784 \\
34.795\end{array}$ & $\begin{array}{l}38.403 \\
38.647 \\
38.803 \\
38.890 \\
38.932 \\
39.015 \\
39.138 \\
39.243 \\
39.362 \\
39.498 \\
39.600 \\
39.695 \\
39.802 \\
40.011 \\
40.184 \\
40.336 \\
40.547 \\
40.742 \\
40.970 \\
41.126 \\
41.220 \\
41.287 \\
41.339 \\
41.379 \\
41.416 \\
41.440 \\
41.459 \\
41.477\end{array}$ & $\begin{array}{l}0.006 \\
0.049 \\
0.094 \\
0.137 \\
0.180 \\
0.263 \\
0.344 \\
0.422 \\
0.498 \\
0.570 \\
0.638 \\
0.705 \\
0.768 \\
0.888 \\
0.997 \\
1.098 \\
1.188 \\
1.265 \\
1.392 \\
1.501 \\
1.599 \\
1.693 \\
1.785 \\
1.874 \\
1.961 \\
2.047 \\
2.133 \\
2.217\end{array}$ & $\begin{array}{l}6.42 \\
5.24 \\
3.08 \\
2.43 \\
2.04 \\
1.95 \\
2.11 \\
2.30 \\
2.50 \\
2.40 \\
2.15 \\
1.95 \\
2.24 \\
2.00 \\
1.92 \\
2.23 \\
2.25 \\
1.92 \\
1.48 \\
1.12 \\
0.92 \\
0.77 \\
0.73 \\
0.71 \\
0.61 \\
0.57 \\
0.50 \\
0.52\end{array}$ & $\begin{array}{r}3 \\
25 \\
50 \\
74 \\
99 \\
149 \\
199 \\
248 \\
298 \\
347 \\
397 \\
446 \\
496 \\
595 \\
694 \\
793 \\
892 \\
991 \\
1188 \\
1386 \\
1583 \\
1780 \\
1977 \\
2174 \\
2370 \\
2566 \\
2763 \\
2956\end{array}$ \\
\hline $\begin{array}{c}\text { PR } \\
\text { dbar }\end{array}$ & $\stackrel{T}{\operatorname{Teg}} C$ & $\begin{array}{c}5 \\
0 / 00\end{array}$ & $\begin{array}{l}02 \\
\mathrm{~m} / 1 / 1\end{array}$ & $\begin{array}{c}\mathrm{F}-11 \\
\mathrm{pmol} / \mathrm{kg}\end{array}$ & $\begin{array}{c}\mathrm{F}-12 \\
\mathrm{gmol} / \mathrm{kg}\end{array}$ & $\operatorname{Deg}^{\theta} \mathrm{C}$ & $\begin{array}{l}\mathrm{SIG}-\theta \\
\mathrm{kg} / \mathrm{m} 3\end{array}$ & $\underset{\mathrm{kg} / \mathrm{m} 3}{\mathrm{SIG}-1.5}$ & $\begin{array}{l}\text { SIG-3 } \\
\mathrm{kg} / \mathrm{m3}\end{array}$ & $\begin{array}{c}D E \\
\mathbf{m}\end{array}$ \\
\hline $\begin{array}{r}24 \\
49 \\
76 \\
100 \\
126 \\
151 \\
177 \\
202 \\
252 \\
302 \\
402 \\
502 \\
604 \\
798 \\
999 \\
1252 \\
1501 \\
1744 \\
1987 \\
2246 \\
2496\end{array}$ & $\begin{array}{r}20.124 \\
19.452 \\
19.036 \\
18.994 \\
18.704 \\
18.212 \\
17.685 \\
17.515 \\
16.670 \\
15.852 \\
14.432 \\
13.187 \\
11.889 \\
9.846 \\
8.465 \\
7.068 \\
5.677 \\
4.813 \\
4.095 \\
3.644 \\
3.203 \\
2.996 \\
2.810\end{array}$ & $\begin{array}{l}171 \\
969 \\
781 \\
624 \\
460 \\
464 \\
418 \\
258 \\
158 \\
071 \\
026 \\
982\end{array}$ & $\begin{array}{l}8 \\
1 \\
75 \\
88 \\
56 \\
55\end{array}$ & & & $\begin{array}{r}19.443 \\
19.022 \\
18.976 \\
18.682 \\
18.186 \\
17.655 \\
17.481 \\
16.629 \\
15.804 \\
14.372 \\
13.116 \\
11.809 \\
9.751 \\
8.354 \\
6.940 \\
5.537 \\
4.659 \\
3.927 \\
3.457 \\
2.999 \\
2.772 \\
2.563\end{array}$ & $\begin{array}{l}279 \\
332 \\
371 \\
395 \\
.441 \\
.494 \\
.528 \\
.619 \\
.695 \\
.857 \\
.975 \\
.111 \\
.354 \\
.584 \\
.757 \\
.815 \\
.840 \\
.850 \\
.862 \\
.871 \\
.876 \\
.880\end{array}$ & $\begin{array}{l}.460 \\
.648 \\
.711 \\
.751 \\
.783 \\
.841 \\
.909 \\
.947 \\
.061 \\
.159 \\
.363 \\
.518 \\
.694 \\
.002 \\
.278 \\
.500 \\
.611 \\
.669 \\
.709 \\
.740 \\
.767 \\
.782 \\
.794\end{array}$ & $\begin{array}{l}38.606 \\
38.882 \\
38.923 \\
38.962 \\
39.033 \\
39.113 \\
39.156 \\
39.292 \\
39.412 \\
39.654 \\
39.845 \\
40.058 \\
40.430 \\
40.750 \\
41.018 \\
41.179 \\
41.270 \\
41.338 \\
41.387 \\
41.432 \\
41.456 \\
41.476\end{array}$ & $\begin{array}{r}24 \\
49 \\
75 \\
99 \\
125 \\
150 \\
175 \\
201 \\
250 \\
300 \\
398 \\
498 \\
598 \\
790 \\
989 \\
1238 \\
1483 \\
1722 \\
1961 \\
2216 \\
2461 \\
2704 \\
2955\end{array}$ \\
\hline
\end{tabular}




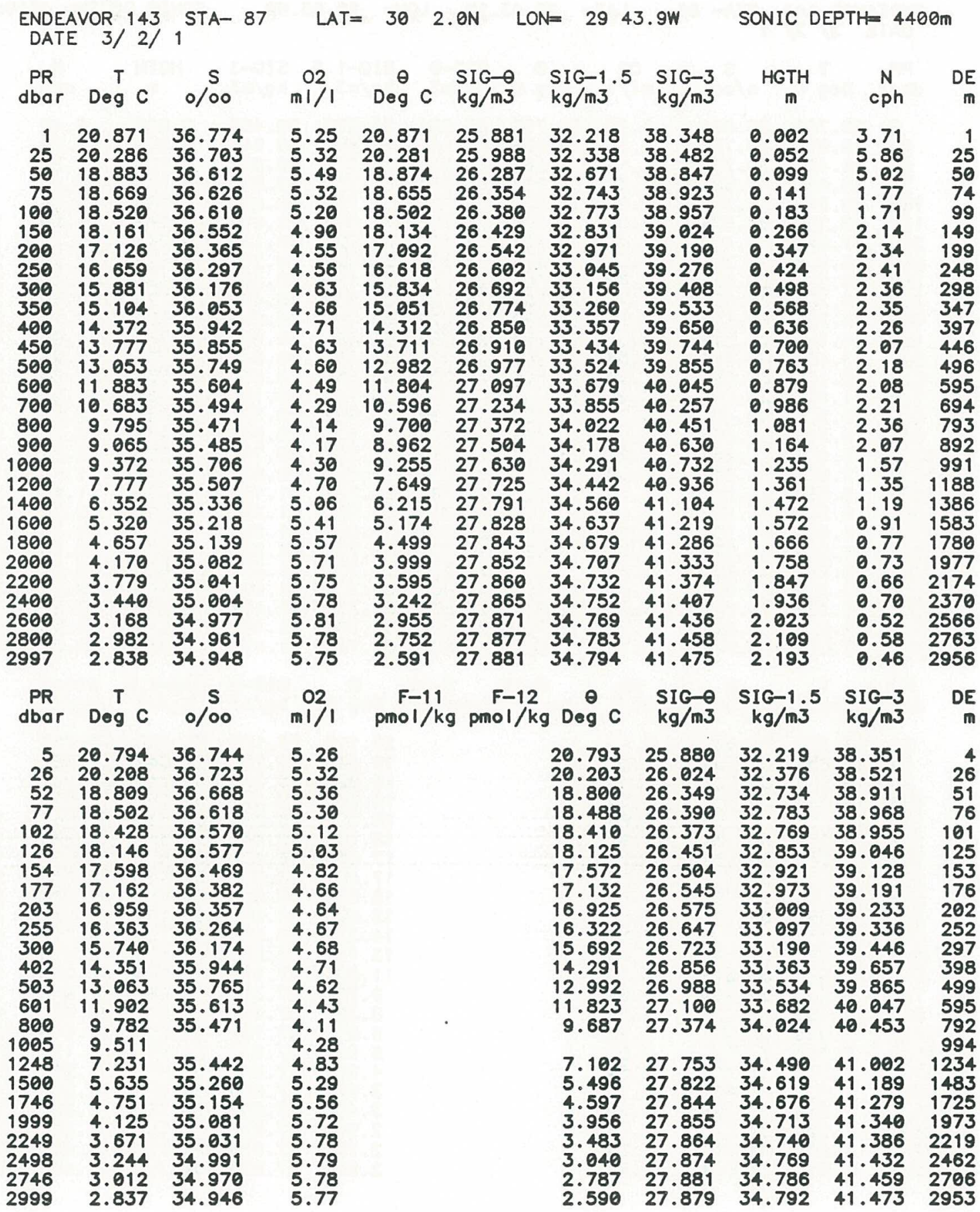




\begin{tabular}{|c|c|c|c|c|c|c|c|c|c|c|}
\hline \multicolumn{3}{|c|}{$\begin{array}{l}\text { ENDEAVOR } 143 \text { STA- } 88 \\
\text { DATE } 15 / 5 / 86\end{array}$} & \multicolumn{3}{|c|}{$L A T=3021.0 \mathrm{~N}$} & \multicolumn{2}{|c|}{ LON $=2935.0 \mathrm{~W}$} & \multicolumn{3}{|c|}{ SONIC DEPTH $=4180 \mathrm{~m}$} \\
\hline $\begin{array}{l}\text { PR } \\
\text { dbar }\end{array}$ & $\stackrel{T}{\operatorname{Deg}} \mathrm{C}$ & $\stackrel{5}{0 / 00}$ & $\mathrm{ml} / \mathrm{l}$ & $\stackrel{\theta}{\operatorname{Deg} C}$ & $\begin{array}{l}\mathrm{SIG}-\theta \\
\mathrm{kg} / \mathrm{m3}\end{array}$ & $\begin{array}{l}\mathrm{SIG}-1.5 \\
\mathrm{~kg} / \mathrm{m} 3\end{array}$ & $\begin{array}{l}\text { SIG-3 } \\
\mathrm{kg} / \mathrm{m} 3\end{array}$ & $\underset{\mathbf{m}}{\mathrm{HGTH}}$ & $\underset{c p h}{N}$ & DE \\
\hline $\begin{array}{r}1 \\
25 \\
50 \\
75 \\
100 \\
150 \\
200 \\
250 \\
300 \\
350 \\
400 \\
450 \\
500 \\
600 \\
700 \\
800 \\
900 \\
1000 \\
1200 \\
1400 \\
1600 \\
1800 \\
2000 \\
2200 \\
2400 \\
2600 \\
2800 \\
3000\end{array}$ & $\begin{array}{r}20.519 \\
19.527 \\
18.843 \\
18.551 \\
18.443 \\
17.355 \\
16.718 \\
16.003 \\
15.174 \\
14.647 \\
14.035 \\
13.395 \\
12.670 \\
11.609 \\
10.727 \\
9.875 \\
8.984 \\
8.354 \\
7.289 \\
6.238 \\
5.322 \\
4.611 \\
4.072 \\
3.648 \\
3.337 \\
3.123 \\
2.933 \\
2.807\end{array}$ & $\begin{array}{l}36.641 \\
36.612 \\
36.631 \\
36.600 \\
36.597 \\
36.374 \\
36.284 \\
36.178 \\
36.048 \\
35.980 \\
35.890 \\
35.794 \\
35.686 \\
35.564 \\
35.495 \\
35.468 \\
35.444 \\
35.454 \\
35.416 \\
35.327 \\
35.216 \\
35.132 \\
35.072 \\
35.024 \\
34.995 \\
34.977 \\
34.958 \\
34.946\end{array}$ & $\begin{array}{l}5.21 \\
5.27 \\
5.20 \\
5.20 \\
5.01 \\
4.79 \\
4.57 \\
4.51 \\
4.59 \\
4.64 \\
4.65 \\
4.64 \\
4.57 \\
4.39 \\
4.35 \\
4.14 \\
4.21 \\
4.35 \\
4.58 \\
5.04 \\
5.38 \\
5.59 \\
5.69 \\
5.78 \\
5.80 \\
5.75 \\
5.75 \\
5.71\end{array}$ & $\begin{array}{l}20.519 \\
19.523 \\
18.834 \\
18.537 \\
18.425 \\
17.330 \\
16.685 \\
15.963 \\
15.128 \\
14.594 \\
13.976 \\
13.331 \\
12.601 \\
11.531 \\
10.640 \\
9.780 \\
8.882 \\
8.245 \\
7.166 \\
6.103 \\
5.176 \\
4.454 \\
3.903 \\
3.466 \\
3.140 \\
2.911 \\
2.704 \\
2.560\end{array}$ & $\begin{array}{l}25.876 \\
26.119 \\
26.312 \\
26.364 \\
26.390 \\
26.491 \\
26.577 \\
26.664 \\
26.753 \\
26.817 \\
26.881 \\
26.941 \\
27.005 \\
27.117 \\
27.227 \\
27.355 \\
27.485 \\
27.594 \\
27.723 \\
27.798 \\
27.826 \\
27.843 \\
27.854 \\
27.860 \\
27.868 \\
27.875 \\
27.879 \\
27.881\end{array}$ & $\begin{array}{l}32.222 \\
32.488 \\
32.696 \\
32.756 \\
32.785 \\
32.914 \\
33.018 \\
33.124 \\
33.237 \\
33.316 \\
33.398 \\
33.477 \\
33.564 \\
33.708 \\
33.846 \\
34.002 \\
34.162 \\
34.291 \\
34.459 \\
34.572 \\
34.635 \\
34.680 \\
34.714 \\
34.737 \\
34.759 \\
34.775 \\
34.787 \\
34.796\end{array}$ & $\begin{array}{l}38.361 \\
38.649 \\
38.873 \\
38.940 \\
38.971 \\
39.128 \\
39.247 \\
39.373 \\
39.508 \\
39.602 \\
39.701 \\
39.798 \\
39.905 \\
40.082 \\
40.247 \\
40.429 \\
40.616 \\
40.766 \\
40.969 \\
41.119 \\
41.217 \\
41.289 \\
41.343 \\
41.383 \\
41.418 \\
41.443 \\
41.464 \\
41.478\end{array}$ & $\begin{array}{l}0.002 \\
0.051 \\
0.095 \\
0.138 \\
0.180 \\
0.261 \\
0.339 \\
0.414 \\
0.484 \\
0.552 \\
0.618 \\
0.681 \\
0.742 \\
0.857 \\
0.962 \\
1.058 \\
1.142 \\
1.215 \\
1.341 \\
1.449 \\
1.548 \\
1.642 \\
1.733 \\
1.821 \\
1.908 \\
1.993 \\
2.078 \\
2.163\end{array}$ & $\begin{array}{l}4.88 \\
7.00 \\
3.27 \\
2.07 \\
1.78 \\
2.72 \\
2.25 \\
2.49 \\
2.09 \\
2.11 \\
2.06 \\
2.18 \\
1.83 \\
2.05 \\
2.00 \\
2.12 \\
2.19 \\
1.72 \\
1.47 \\
1.13 \\
0.90 \\
0.81 \\
0.78 \\
0.66 \\
0.62 \\
0.57 \\
0.57 \\
0.44\end{array}$ & $\begin{array}{r}1 \\
25 \\
50 \\
74 \\
99 \\
149 \\
199 \\
248 \\
298 \\
347 \\
397 \\
446 \\
496 \\
595 \\
694 \\
793 \\
892 \\
991 \\
1188 \\
1386 \\
1583 \\
1780 \\
1977 \\
2174 \\
2370 \\
2566 \\
2762 \\
2958\end{array}$ \\
\hline $\begin{array}{l}\text { PR } \\
\text { dbar }\end{array}$ & $\stackrel{T}{\operatorname{Deg}} \mathrm{C}$ & $\begin{array}{l}S \\
0 / 00\end{array}$ & $\begin{array}{l}02 \\
m 1 / 1\end{array}$ & $\begin{array}{c}\mathrm{F}-11 \\
\mathrm{pmol} / \mathrm{kg}\end{array}$ & $\begin{array}{c}\mathrm{F}-12 \\
\mathrm{pmol} / \mathrm{kg}\end{array}$ & $\stackrel{\theta}{\operatorname{Deg} C}$ & $\begin{array}{l}\text { SIG-O } \\
\mathrm{kg} / \mathrm{m3}\end{array}$ & $\underset{\mathrm{kg} / \mathrm{m} 3}{\mathrm{SIG}-1.5}$ & $\begin{array}{l}\mathrm{SIG}-3 \\
\mathrm{~kg} / \mathrm{m} 3\end{array}$ & DE \\
\hline $\begin{array}{r}3 \\
29 \\
54 \\
78 \\
101 \\
127 \\
155 \\
177 \\
205 \\
230 \\
299 \\
402 \\
502 \\
601 \\
804 \\
999 \\
1252 \\
1500\end{array}$ & $\begin{array}{r}20.498 \\
19.188 \\
18.846 \\
18.629 \\
18.397 \\
18.201 \\
17.698 \\
17.327 \\
16.909 \\
16.621 \\
15.461 \\
14.199 \\
12.730 \\
11.510 \\
9.772 \\
8.339 \\
7.089 \\
5.667 \\
4.782 \\
4.082 \\
3.548 \\
3.225 \\
2.986 \\
2.805\end{array}$ & $\begin{array}{l}36.644 \\
36.651 \\
36.648 \\
36.639 \\
36.581 \\
36.561 \\
36.443 \\
36.372 \\
36.318 \\
36.289 \\
36.092 \\
35.921 \\
35.700 \\
35.560 \\
35.470 \\
35.459 \\
35.417 \\
35.254 \\
35.156 \\
35.075 \\
35.013\end{array}$ & $\begin{array}{l}5.27 \\
5.41 \\
5.40 \\
5.39 \\
5.20 \\
5.08 \\
4.99 \\
5.02 \\
4.69 \\
4.65 \\
4.65 \\
4.70 \\
4.56 \\
4.38 \\
4.09 \\
4.33 \\
4.69 \\
5.31 \\
5.55 \\
5.71 \\
5.80\end{array}$ & $\begin{array}{l}1.728 \\
1.189 \\
0.560 \\
0.288 \\
0.265 \\
0.162 \\
0.081 \\
0.050 \\
0.023 \\
0.028\end{array}$ & $\begin{array}{l}0.781 \\
0.560 \\
0.240 \\
0.148 \\
0.068 \\
0.073 \\
0.031 \\
0.005 \\
0.006 \\
0.000\end{array}$ & $\begin{array}{r}20.498 \\
19.182 \\
18.836 \\
18.615 \\
18.380 \\
18.179 \\
17.671 \\
17.298 \\
16.875 \\
16.583 \\
15.414 \\
14.140 \\
12.661 \\
11.432 \\
9.677 \\
8.230 \\
6.962 \\
5.528 \\
4.629 \\
3.914 \\
3.363 \\
3.021\end{array}$ & $\begin{array}{l}25.884 \\
26.238 \\
26.325 \\
26.374 \\
26.389 \\
26.425 \\
26.460 \\
26.497 \\
26.557 \\
26.604 \\
26.722 \\
26.870 \\
27.004 \\
27.132 \\
27.375 \\
27.600 \\
27.753 \\
27.813 \\
27.842 \\
27.855 \\
27.861 \\
27.870\end{array}$ & $\begin{array}{l}32.230 \\
32.614 \\
32.709 \\
32.764 \\
32.785 \\
32.826 \\
32.875 \\
32.921 \\
32.993 \\
33.048 \\
33.198 \\
33.382 \\
33.561 \\
33.727 \\
34.025 \\
34.298 \\
34.495 \\
34.609 \\
34.672 \\
34.714 \\
34.743 \\
34.766\end{array}$ & $\begin{array}{l}38.370 \\
38.782 \\
38.885 \\
38.945 \\
38.973 \\
39.018 \\
39.080 \\
39.136 \\
39.218 \\
39.280 \\
39.461 \\
39.680 \\
39.901 \\
40.103 \\
40.455 \\
40.773 \\
41.013 \\
41.178 \\
41.274 \\
41.343 \\
41.393 \\
41.430\end{array}$ & $\begin{array}{r}3 \\
28 \\
53 \\
77 \\
101 \\
126 \\
153 \\
175 \\
203 \\
228 \\
296 \\
398 \\
497 \\
595 \\
796 \\
988 \\
1238 \\
1482 \\
1723 \\
1968 \\
2217 \\
2462 \\
2706 \\
2959\end{array}$ \\
\hline
\end{tabular}




\begin{tabular}{|c|c|c|c|c|c|c|c|c|c|c|}
\hline \multicolumn{3}{|c|}{$\begin{array}{l}\text { ENDEAVOR } 143 \text { STA- } 89 \\
\text { DATE } 15 / 5 / 86\end{array}$} & \multicolumn{3}{|c|}{$L A T=3039.4 \mathrm{~N}$} & \multicolumn{2}{|c|}{$O N=2924.1 W$} & \multicolumn{3}{|c|}{ SONIC DEPTH $=4300 \mathrm{~m}$} \\
\hline $\begin{array}{l}\text { PR } \\
\text { dbar }\end{array}$ & $\stackrel{T}{\text { Deg } C}$ & $\stackrel{5}{0 / 00}$ & $\begin{array}{ll}02 \\
\mathrm{ml} / \mathrm{I}\end{array}$ & $\stackrel{\theta}{\operatorname{Deg} C}$ & $\begin{array}{l}\mathrm{SIG}-\theta \\
\mathrm{kg} / \mathrm{m} 3\end{array}$ & $\begin{array}{l}\mathrm{SIG}-1.5 \\
\mathrm{~kg} / \mathrm{m} 3\end{array}$ & $\begin{array}{l}\mathrm{SIG}-3 \\
\mathrm{~kg} / \mathrm{m}^{3}\end{array}$ & $\underset{\mathbf{m}}{\mathrm{HGTH}}$ & $\begin{array}{c}N \\
c p h\end{array}$ & \\
\hline $\begin{array}{r}1 \\
25 \\
50 \\
75 \\
100 \\
150 \\
200 \\
250 \\
300 \\
350 \\
400 \\
450 \\
500 \\
600 \\
700 \\
800 \\
900 \\
1000 \\
200 \\
400 \\
600 \\
800 \\
2000 \\
200 \\
2400 \\
2600 \\
2800 \\
2997\end{array}$ & $\begin{array}{r}20.703 \\
20.297 \\
18.825 \\
18.131 \\
18.050 \\
17.273 \\
16.672 \\
16.039 \\
15.120 \\
14.639 \\
14.176 \\
13.500 \\
12.903 \\
11.546 \\
10.415 \\
9.527 \\
8.859 \\
8.224 \\
6.992 \\
5.911 \\
5.045 \\
4.445 \\
3.923 \\
3.539 \\
3.221 \\
3.080 \\
2.900 \\
2.783\end{array}$ & $\begin{array}{l}36.674 \\
36.629 \\
36.538 \\
36.469 \\
36.482 \\
36.361 \\
36.279 \\
36.178 \\
36.035 \\
35.968 \\
35.905 \\
35.812 \\
35.725 \\
35.553 \\
35.488 \\
35.455 \\
35.471 \\
35.473 \\
35.399 \\
35.280 \\
35.185 \\
35.115 \\
35.052 \\
35.011 \\
34.980 \\
34.973 \\
34.955 \\
34.945\end{array}$ & $\begin{array}{l}4.78 \\
4.95 \\
5.30 \\
5.14 \\
5.03 \\
4.72 \\
4.67 \\
4.69 \\
4.95 \\
4.81 \\
4.80 \\
4.57 \\
4.58 \\
4.39 \\
4.23 \\
4.13 \\
4.29 \\
4.46 \\
4.85 \\
5.20 \\
5.48 \\
5.66 \\
5.80 \\
5.85 \\
5.87 \\
5.79 \\
5.81\end{array}$ & $\begin{array}{c}20.703 \\
20.292 \\
18.816 \\
18.118 \\
18.032 \\
17.247 \\
16.639 \\
15.999 \\
15.074 \\
14.586 \\
14.117 \\
13.436 \\
12.833 \\
11.468 \\
10.329 \\
9.434 \\
8.757 \\
8.115 \\
6.870 \\
5.779 \\
4.903 \\
4.290 \\
3.756 \\
3.359 \\
3.026 \\
2.869 \\
2.671 \\
2.537\end{array}$ & $\begin{array}{l}25.851 \\
25.928 \\
26.246 \\
26.370 \\
26.400 \\
26.501 \\
26.583 \\
26.655 \\
26.755 \\
26.811 \\
26.863 \\
26.934 \\
26.989 \\
27.120 \\
27.276 \\
27.404 \\
27.526 \\
27.629 \\
27.752 \\
27.803 \\
27.833 \\
27.847 \\
27.853 \\
27.860 \\
27.867 \\
27.876 \\
27.879 \\
27.883\end{array}$ & $\begin{array}{l}32.193 \\
32.279 \\
32.632 \\
32.773 \\
32.806 \\
32.926 \\
33.025 \\
33.115 \\
33.240 \\
33.310 \\
33.376 \\
33.467 \\
33.540 \\
33.713 \\
33.905 \\
34.062 \\
34.207 \\
34.330 \\
34.498 \\
34.589 \\
34.653 \\
34.691 \\
34.719 \\
34.741 \\
34.762 \\
34.777 \\
34.789 \\
34.798\end{array}$ & $\begin{array}{l}38.327 \\
38.423 \\
38.809 \\
38.967 \\
39.002 \\
39.142 \\
39.256 \\
39.362 \\
39.513 \\
39.596 \\
39.675 \\
39.785 \\
39.875 \\
40.089 \\
40.315 \\
40.500 \\
40.665 \\
40.809 \\
41.018 \\
41.149 \\
41.245 \\
41.306 \\
41.354 \\
41.392 \\
41.426 \\
41.447 \\
41.466 \\
41.481\end{array}$ & $\begin{array}{l}0.002 \\
0.053 \\
0.101 \\
0.144 \\
0.186 \\
0.267 \\
0.344 \\
0.418 \\
0.489 \\
0.557 \\
0.623 \\
0.687 \\
0.749 \\
0.864 \\
0.967 \\
1.058 \\
1.137 \\
1.206 \\
1.325 \\
1.429 \\
1.524 \\
1.615 \\
1.704 \\
1.791 \\
1.877 \\
1.961 \\
2.045 \\
2.129\end{array}$ & $\begin{array}{l}1.37 \\
5.73 \\
5.59 \\
2.80 \\
2.25 \\
2.35 \\
2.34 \\
2.48 \\
2.22 \\
1.91 \\
2.00 \\
1.92 \\
1.98 \\
2.30 \\
2.04 \\
2.15 \\
2.00 \\
1.60 \\
1.38 \\
1.10 \\
0.80 \\
0.79 \\
0.70 \\
0.66 \\
0.63 \\
0.52 \\
0.50 \\
0.48\end{array}$ & $\begin{array}{r}1 \\
25 \\
50 \\
74 \\
99 \\
149 \\
199 \\
248 \\
298 \\
347 \\
397 \\
446 \\
496 \\
595 \\
694 \\
793 \\
892 \\
991 \\
1188 \\
1386 \\
1583 \\
1780 \\
1977 \\
2173 \\
2370 \\
2566 \\
2762 \\
2955\end{array}$ \\
\hline $\begin{array}{l}\text { PR } \\
\text { dbar }\end{array}$ & $\stackrel{T}{D} C$ & $\stackrel{S}{0 / 00}$ & $\mathrm{ml} / \mathrm{l}$ & $\mathrm{pmol} / \mathrm{kg}$ & $\begin{array}{c}\mathrm{F}-12 \\
\mathrm{pmol} / \mathrm{kg}\end{array}$ & $\stackrel{\theta}{\operatorname{Deg} C}$ & $\begin{array}{l}\mathrm{SIG}-0 \\
\mathrm{~kg} / \mathrm{m} 3\end{array}$ & $\underset{\mathrm{kg} / \mathrm{m} 3}{\mathrm{SIG}-1.5}$ & $\mathrm{~kg} / \mathrm{m} 3$ & E \\
\hline $\begin{array}{r}5 \\
28 \\
53 \\
76 \\
102 \\
129 \\
153 \\
175 \\
202 \\
250 \\
300 \\
399 \\
500 \\
602 \\
800 \\
998 \\
1248 \\
1500 \\
1744 \\
1998 \\
2247 \\
2498\end{array}$ & $\begin{array}{r}20.677 \\
20.258 \\
18.785 \\
18.313 \\
18.007 \\
17.548 \\
17.183 \\
16.921 \\
16.747 \\
16.291 \\
15.443 \\
14.294 \\
12.925 \\
11.576 \\
9.628 \\
8.300 \\
6.767 \\
5.509 \\
4.626 \\
3.930 \\
3.450 \\
3.115 \\
2.943\end{array}$ & $\begin{array}{l}36.6 \\
36.6 \\
36.5 \\
36.5 \\
36.4 \\
36.4 \\
36.3 \\
36.3 \\
36.2 \\
36.2 \\
36.0 \\
35.9 \\
35.7 \\
35.5 \\
35.4 \\
35.4 \\
35.3 \\
35.2 \\
35.4\end{array}$ & $\begin{array}{l}5.3 \\
5.3 \\
5.5 \\
5.3 \\
5.3 \\
5.1 \\
4.9 \\
5.8 \\
4.9 \\
4.7 \\
5.1 \\
4.7 \\
4.5 \\
4.3 \\
4.1 \\
4.2 \\
4.5 \\
5.3 \\
5.8 \\
5.7 \\
5.8 \\
5 .\end{array}$ & & & $\begin{array}{r}20.253 \\
18.776 \\
18.300 \\
17.990 \\
17.526 \\
17.157 \\
16.892 \\
16.714 \\
16.250 \\
15.396 \\
14.235 \\
12.855 \\
11.497 \\
9.534 \\
8.191 \\
6.643 \\
5.371 \\
4.474 \\
3.763 \\
3.267 \\
2.913 \\
2.721 \\
2.532\end{array}$ & $\begin{array}{l}862 \\
.939 \\
.274 \\
.367 \\
.410 \\
.475 \\
.529 \\
.541 \\
.567 \\
.642 \\
.720 \\
861 \\
.996 \\
.140 \\
.388 \\
.616 \\
.768 \\
.831 \\
.844 \\
.852 \\
.858 \\
.869 \\
882 \\
.882\end{array}$ & $\begin{array}{l}32.2 \\
32.6 \\
32.7 \\
32.8 \\
32.8 \\
32.9 \\
32.9 \\
33.8 \\
33.8 \\
33.1 \\
33 . \\
33.5 \\
33.7 \\
34.8 \\
34.9 \\
34.5 \\
34.6\end{array}$ & $\begin{array}{l}38.339 \\
38.436 \\
38.838 \\
38.955 \\
39.013 \\
39.102 \\
39.174 \\
39.201 \\
39.236 \\
39.335 \\
39.460 \\
39.665 \\
39.881 \\
40.107 \\
40.477 \\
40.792 \\
41.051 \\
41.207 \\
41.289 \\
41 . .352 \\
41.398 \\
41.437 \\
41.465 \\
41.481\end{array}$ & $\begin{array}{r}5 \\
27 \\
52 \\
75 \\
101 \\
128 \\
152 \\
173 \\
200 \\
248 \\
297 \\
395 \\
496 \\
596 \\
792 \\
987 \\
1234 \\
1482 \\
1722 \\
1972 \\
2217 \\
2463 \\
2699 \\
2954\end{array}$ \\
\hline
\end{tabular}




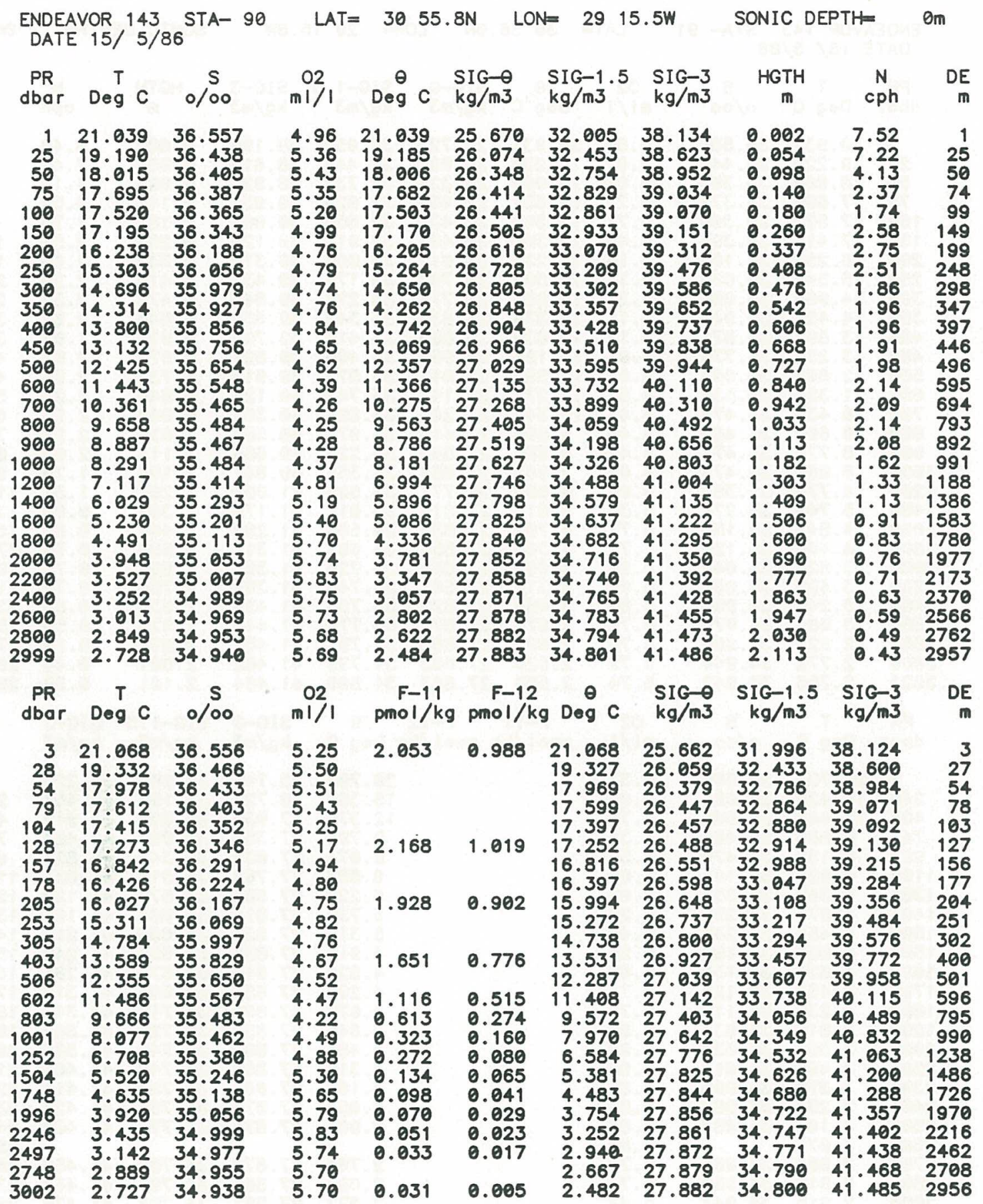




\begin{tabular}{|c|c|c|c|c|c|c|c|c|c|c|}
\hline \multicolumn{3}{|c|}{$\begin{array}{l}\text { ENDEAVOR } 143 \text { STA- } 91 \\
\text { DATE } 15 / 5 / 86\end{array}$} & LAT $=$ & \multicolumn{2}{|c|}{$=3056.0 \mathrm{~N}$} & \multicolumn{2}{|c|}{$\mathrm{N}=2915.8 \mathrm{~W}$} & \multicolumn{2}{|c|}{ SONIC DEPTH } & \multirow{2}{*}{$\begin{array}{l}\text { Om } \\
\text { DE } \\
m\end{array}$} \\
\hline $\begin{array}{l}\text { PR } \\
\text { dbar }\end{array}$ & $\stackrel{T}{\operatorname{Deg}} \mathrm{C}$ & $\stackrel{S}{0 / 00}$ & $\begin{array}{l}02 \\
\mathrm{~m} 1 / 1\end{array}$ & $\stackrel{\theta}{\operatorname{Deg} C}$ & $\begin{array}{l}I G-\theta \\
g / m 3\end{array}$ & $\begin{array}{l}\mathrm{SIG}-1.5 \\
\mathrm{~kg} / \mathrm{m3}\end{array}$ & $\begin{array}{l}S I G-3 \\
\mathrm{~kg} / \mathrm{m} 3\end{array}$ & $\underset{m}{\text { HGTH }}$ & $\underset{c p h}{N}$ & \\
\hline $\begin{array}{r}3 \\
25 \\
50 \\
75 \\
100 \\
150 \\
200 \\
250 \\
300 \\
350 \\
400 \\
450 \\
500 \\
600 \\
700 \\
800 \\
900 \\
1000 \\
200 \\
400 \\
600 \\
800 \\
2000 \\
2200 \\
2400 \\
2600 \\
2800 \\
3000 \\
3023\end{array}$ & $\begin{array}{r}20.931 \\
19.225 \\
18.069 \\
17.669 \\
17.522 \\
17.415 \\
16.259 \\
15.541 \\
14.908 \\
14.426 \\
13.897 \\
13.256 \\
12.662 \\
11.354 \\
10.433 \\
9.600 \\
8.770 \\
8.069 \\
6.778 \\
5.746 \\
4.842 \\
4.460 \\
3.826 \\
3.496 \\
3.249 \\
3.086 \\
2.921 \\
2.772 \\
2.756\end{array}$ & $\begin{array}{l}36.586 \\
36.440 \\
36.395 \\
36.372 \\
36.360 \\
36.394 \\
36.193 \\
36.092 \\
36.005 \\
35.944 \\
35.871 \\
35.775 \\
35.693 \\
35.536 \\
35.475 \\
35.485 \\
35.473 \\
35.472 \\
35.387 \\
35.274 \\
35.154 \\
35.126 \\
35.042 \\
35.008 \\
34.990 \\
34.975 \\
34.960 \\
34.944 \\
34.942\end{array}$ & $\begin{array}{l}5.50 \\
5.91 \\
6.06 \\
5.77 \\
5.71 \\
5.49 \\
5.15 \\
5.11 \\
5.21 \\
5.11 \\
5.17 \\
4.93 \\
5.02 \\
4.52 \\
4.43 \\
4.40 \\
4.42 \\
4.62 \\
4.99 \\
5.38 \\
5.77 \\
5.72 \\
5.96 \\
5.95 \\
5.82 \\
5.79 \\
5.74\end{array}$ & $\begin{array}{r}20.931 \\
19.220 \\
18.060 \\
17.657 \\
17.505 \\
17.390 \\
16.226 \\
15.502 \\
14.862 \\
14.374 \\
13.839 \\
13.192 \\
12.593 \\
11.277 \\
10.347 \\
9.506 \\
8.669 \\
7.962 \\
6.658 \\
5.616 \\
4.702 \\
4.305 \\
3.661 \\
3.317 \\
3.054 \\
2.874 \\
2.692 \\
2.526 \\
2.508\end{array}$ & $\begin{array}{l}25.723 \\
26.066 \\
26.327 \\
26.409 \\
26.437 \\
26.491 \\
26.615 \\
26.703 \\
26.779 \\
26.838 \\
26.895 \\
26.956 \\
27.012 \\
27.143 \\
27.263 \\
27.415 \\
27.542 \\
27.651 \\
27.771 \\
27.819 \\
27.832 \\
27.854 \\
27.854 \\
27.862 \\
27.872 \\
27.877 \\
27.882 \\
27.883 \\
27.883\end{array}$ & $\begin{array}{l}32.059 \\
32.444 \\
32.732 \\
32.825 \\
32.856 \\
32.913 \\
33.068 \\
33.176 \\
33.270 \\
33.343 \\
33.416 \\
33.496 \\
33.571 \\
33.742 \\
33.892 \\
34.071 \\
34.225 \\
34.358 \\
34.525 \\
34.611 \\
34.660 \\
34.697 \\
34.724 \\
34.745 \\
34.766 \\
34.779 \\
34.791 \\
34.799 \\
34.800\end{array}$ & $\begin{array}{l}38.190 \\
38.613 \\
38.928 \\
39.031 \\
39.066 \\
39.125 \\
39.310 \\
39.437 \\
39.548 \\
39.635 \\
39.723 \\
39.821 \\
39.913 \\
40.124 \\
40.301 \\
40.506 \\
40.686 \\
40.841 \\
41.053 \\
41.176\end{array}$ & $\begin{array}{l}0.007 \\
0.054 \\
0.098 \\
0.140 \\
0.181 \\
0.261 \\
0.337 \\
0.410 \\
0.479 \\
0.546 \\
0.610 \\
0.673 \\
0.733 \\
0.846 \\
0.948 \\
1.038 \\
1.116 \\
1.184 \\
1.297 \\
1.397 \\
1.490 \\
1.580 \\
1.668 \\
1.754 \\
1.839 \\
1.923 \\
2.007 \\
2.091 \\
2.101\end{array}$ & $\begin{array}{l}6.40 \\
7.46 \\
4.10 \\
2.59 \\
1.78 \\
2.51 \\
2.60 \\
2.29 \\
2.32 \\
2.07 \\
1.67 \\
2.01 \\
2.06 \\
2.23 \\
2.23 \\
2.23 \\
2.07 \\
1.70 \\
1.31 \\
0.98 \\
0.84 \\
0.75 \\
0.72 \\
0.70 \\
0.53 \\
0.56 \\
0.49 \\
0.44 \\
0.59\end{array}$ & $\begin{array}{r}3 \\
25 \\
50 \\
74 \\
99 \\
149 \\
199 \\
248 \\
298 \\
347 \\
397 \\
446 \\
496 \\
595 \\
694 \\
793 \\
892 \\
991 \\
1188 \\
1386 \\
1583 \\
1780 \\
1977 \\
2173 \\
2370 \\
2566 \\
2762 \\
2958 \\
2981\end{array}$ \\
\hline $\begin{array}{l}\text { PR } \\
\text { dbar }\end{array}$ & $\stackrel{T}{\text { Deg } C}$ & $\stackrel{5}{0 / 00}$ & $\begin{array}{l}02 \\
n 1 / 1\end{array}$ & $\begin{array}{c}\mathrm{F}-11 \\
\mathrm{pmol} / \mathrm{kg}\end{array}$ & $\begin{array}{c}\mathrm{F}-12 \\
\mathrm{pmol} / \mathrm{kg}\end{array}$ & $\stackrel{\theta}{\operatorname{\theta eg}} \mathrm{C}$ & $\begin{array}{l}S I G-\theta \\
\mathrm{kg} / \mathrm{m3}\end{array}$ & $\underset{\mathrm{kg} / \mathrm{m} 3}{\mathrm{SIG}-1.5}$ & $\begin{array}{l}\mathrm{SIG}-3 \\
\mathrm{~kg} / \mathrm{m} 3\end{array}$ & DE \\
\hline $\begin{array}{r}2 \\
249 \\
498 \\
747 \\
997 \\
1199\end{array}$ & $\begin{array}{r}20.796 \\
15.428 \\
12.446 \\
9.884 \\
8.182 \\
6.928 \\
6.348 \\
5.871 \\
5.456 \\
5.056 \\
4.670 \\
4.452 \\
4.233 \\
3.811 \\
3.653 \\
3.496\end{array}$ & $\begin{array}{l}36.569 \\
36.081 \\
35.668 \\
35.464 \\
35.479 \\
35.401 \\
35.359 \\
35.291 \\
35.246 \\
35.192 \\
35.139 \\
35.125 \\
35.110 \\
35.037 \\
35.030 \\
35.013 \\
34.995 \\
34.988 \\
34.983\end{array}$ & $\begin{array}{l}5 . \\
4 . \\
4 . \\
4 . \\
4 . \\
5 . \\
5 . \\
5 . \\
5 . \\
5 . \\
5 .\end{array}$ & & & $\begin{array}{r}20.795 \\
15.389 \\
12.379 \\
9.795 \\
8.074 \\
6.807 \\
6.222 \\
5.739 \\
5.319 \\
4.914 \\
4.522 \\
4.298 \\
4.072 \\
3.646 \\
3.481 \\
3.316 \\
3.183 \\
3.057 \\
2.963\end{array}$ & $\begin{array}{l}26 \\
27 \\
27 \\
27 \\
27 \\
27 \\
27 \\
27 \\
27 \\
27 \\
27 \\
27 \\
27 \\
27 \\
27 \\
27 \\
27 \\
27\end{array}$ & $\begin{array}{l}33 \\
33 \\
33\end{array}$ & $\begin{array}{l}39 . \\
39 . \\
40 . \\
40 . \\
41 . \\
41 . \\
41 . \\
41 . \\
41 . \\
41 . \\
41 . \\
41 . \\
41 . \\
41 . \\
41 . \\
41 . \\
41 . \\
41 .\end{array}$ & $\begin{array}{r}1 \\
247 \\
493 \\
740 \\
986 \\
1185 \\
1286 \\
1384 \\
1483 \\
1580 \\
1679 \\
1776 \\
1873 \\
1973 \\
2071 \\
2171 \\
2269 \\
2368 \\
2467\end{array}$ \\
\hline 30 & ? & 34 & & & & $\begin{array}{l}2.764 \\
2.683 \\
2.577 \\
2.505\end{array}$ & $\begin{array}{l}27.876 \\
27.881 \\
27.881 \\
27.890\end{array}$ & $\begin{array}{l}34.702 \\
34.791 \\
34.795 \\
34.806\end{array}$ & $\begin{array}{l}41.456 \\
41.468 \\
41.476 \\
41.491\end{array}$ & $\begin{array}{l}2762 \\
2863 \\
2966\end{array}$ \\
\hline
\end{tabular}




\begin{tabular}{|c|c|c|c|c|c|c|c|c|c|c|}
\hline \multicolumn{3}{|c|}{$\begin{array}{l}\text { ENDEAVOR } 143 \text { STA- } 92 \\
\text { DATE } 16 / 5 / 86\end{array}$} & $L A T=$ & \multicolumn{2}{|c|}{$3115.0 \mathrm{~N}$} & \multicolumn{2}{|c|}{ LON $=$} & \multicolumn{3}{|c|}{ SONIC DEPTH $=4100 \mathrm{~m}$} \\
\hline $\begin{array}{l}\text { PR } \\
\text { dbar }\end{array}$ & $\stackrel{T}{T} C$ & $\begin{array}{l}S \\
0 / 00\end{array}$ & $\begin{array}{l}02 \\
\mathrm{ml} / \mathrm{l}\end{array}$ & $\stackrel{\theta}{\operatorname{Deg} C}$ & $\begin{array}{l}\text { SIG-O } \\
\mathrm{kg} / \mathrm{m3}\end{array}$ & $\begin{array}{l}\mathrm{SIG}-1.5 \\
\mathrm{~kg} / \mathrm{m} 3\end{array}$ & $\begin{array}{l}\text { SIG-3 } \\
\mathrm{kg} / \mathrm{m} 3\end{array}$ & $\underset{m}{H G T H}$ & $\underset{c p h}{N}$ & $\begin{aligned} D E \\
m\end{aligned}$ \\
\hline $\begin{array}{r}3 \\
25 \\
50 \\
75 \\
100 \\
150 \\
200 \\
250 \\
300 \\
350 \\
400 \\
450 \\
500 \\
600 \\
700 \\
800 \\
900 \\
1000 \\
1200 \\
1400 \\
1600 \\
1800 \\
2000 \\
2200 \\
2400 \\
2600 \\
2800 \\
2995\end{array}$ & $\begin{array}{r}20.530 \\
19.323 \\
18.042 \\
17.685 \\
17.590 \\
16.489 \\
15.616 \\
14.945 \\
14.452 \\
13.864 \\
13.353 \\
12.795 \\
12.194 \\
11.028 \\
10.116 \\
9.373 \\
8.721 \\
8.186 \\
7.038 \\
5.999 \\
5.159 \\
4.581 \\
4.109 \\
3.647 \\
3.379 \\
3.116 \\
2.963 \\
2.831\end{array}$ & $\begin{array}{l}36.4 \\
36.4 \\
36.4 \\
36.3 \\
36.4 \\
36.2 \\
36.1 \\
36.0 \\
35.9 \\
35.8 \\
35.7 \\
35.7 \\
35.6 \\
35.5 \\
35.4 \\
35.4 \\
35.4 \\
35.4 \\
35.4 \\
35.2 \\
35.2 \\
35.1 \\
35.0 \\
35.0 \\
35.0 \\
34.9 \\
34.9 \\
34.9\end{array}$ & $\begin{array}{l}5.32 \\
5.57 \\
5.44 \\
5.20 \\
5.13 \\
4.81 \\
4.71 \\
4.72 \\
4.76 \\
4.72 \\
4.72 \\
4.65 \\
4.56 \\
4.31 \\
4.16 \\
4.19 \\
4.33 \\
4.42 \\
4.75 \\
5.12 \\
5.41 \\
5.61\end{array}$ & $\begin{array}{c}20.529 \\
19.319 \\
18.033 \\
17.672 \\
17.573 \\
16.465 \\
15.584 \\
14.907 \\
14.408 \\
13.813 \\
13.296 \\
12.733 \\
12.127 \\
10.953 \\
10.032 \\
9.281 \\
8.621 \\
8.078 \\
6.916 \\
5.866 \\
5.015 \\
4.425 \\
3.939 \\
3.466 \\
3.182 \\
2.904 \\
2.734 \\
2.585\end{array}$ & $\begin{array}{l}25.746 \\
26.046 \\
26.339 \\
26.415 \\
26.455 \\
26.587 \\
26.697 \\
26.775 \\
26.835 \\
26.897 \\
26.947 \\
26.999 \\
27.055 \\
27.177 \\
27.314 \\
27.433 \\
27.543 \\
27.644 \\
27.756 \\
27.804 \\
27.835 \\
27.849 \\
27.856 \\
27.863 \\
27.870 \\
27.876 \\
27.879 \\
27.883\end{array}$ & $\begin{array}{l}32.093 \\
32.421 \\
32.745 \\
32.830 \\
32.872 \\
33.034 \\
33.168 \\
33.265 \\
33.339 \\
33.418 \\
33.484 \\
33.553 \\
33.628 \\
33.787 \\
33.953 \\
34.097 \\
34.228 \\
34.347 \\
34.500 \\
34.587 \\
34.650 \\
34.688 \\
34.714 \\
34.740 \\
34.758 \\
34.776 \\
34.787 \\
34.796\end{array}$ & $\begin{array}{l}38.233 \\
38.588 \\
38.942 \\
39.035 \\
39.080 \\
39.270 \\
39.427 \\
39.542 \\
39.630 \\
39.726 \\
39.806 \\
39.891 \\
39.984 \\
40.178 \\
40.371 \\
40.539 \\
40.691 \\
40.827 \\
41.019 \\
41.143 \\
41.238 \\
41.297 \\
41.343 \\
41.386 \\
41.416 \\
41.445 \\
41.462 \\
41.477\end{array}$ & $\begin{array}{l}0.007 \\
0.054 \\
0.098 \\
0.140 \\
0.180 \\
0.258 \\
0.330 \\
0.399 \\
0.466 \\
0.529 \\
0.591 \\
0.651 \\
0.709 \\
0.818 \\
0.916 \\
1.004 \\
1.081 \\
1.148 \\
1.265 \\
1.369 \\
1.465 \\
1.557 \\
1.647 \\
1.735 \\
1.822 \\
1.907 \\
1.992 \\
2.075\end{array}$ & $\begin{array}{l}4.25 \\
7.14 \\
3.98 \\
2.25 \\
2.84 \\
2.73 \\
2.37 \\
1.97 \\
2.38 \\
1.81 \\
1.81 \\
2.14 \\
1.84 \\
2.15 \\
1.95 \\
2.10 \\
1.91 \\
1.64 \\
1.27 \\
1.02 \\
0.91 \\
0.70 \\
0.74 \\
0.62 \\
0.68 \\
0.58 \\
0.51 \\
0.49\end{array}$ & $\begin{array}{r}3 \\
25 \\
50 \\
74 \\
99 \\
149 \\
199 \\
248 \\
298 \\
347 \\
397 \\
446 \\
496 \\
595 \\
694 \\
793 \\
892 \\
991 \\
1188 \\
1386 \\
1583 \\
1780 \\
1977 \\
2173 \\
2370 \\
2566 \\
2762 \\
2953\end{array}$ \\
\hline $\begin{array}{l}\text { PR } \\
\text { dbar }\end{array}$ & $\stackrel{T}{T} C$ & $\stackrel{5}{\circ}$ & $\begin{aligned} 02 \\
\mathrm{ml} /\end{aligned}$ & $\begin{array}{c}\mathrm{F}-11 \\
\mathrm{pmol} / \mathrm{kg}\end{array}$ & $\begin{array}{c}\mathrm{F}-12 \\
\mathrm{pmol} / \mathrm{kg}\end{array}$ & $\stackrel{\theta}{\operatorname{Deg} C}$ & $\mathrm{~kg} / \mathrm{m} 3$ & kg. & $\mathrm{kg} / \mathrm{l}$ & \\
\hline $\begin{array}{r}4 \\
25 \\
50 \\
75 \\
101 \\
127 \\
153 \\
200 \\
249 \\
303 \\
405 \\
503 \\
602 \\
703 \\
801 \\
1001 \\
1250 \\
1502 \\
1742 \\
2000 \\
2248 \\
2497 \\
2747\end{array}$ & $\begin{array}{r}20.428 \\
18.674 \\
17.913 \\
17.575 \\
17.497 \\
16.982 \\
16.503 \\
15.491 \\
14.811 \\
14.389 \\
13.066 \\
12.004 \\
10.824 \\
10.040 \\
9.334 \\
8.246 \\
6.690 \\
5.561 \\
4.754 \\
4.179 \\
3.594 \\
3.233 \\
2.989 \\
2.830\end{array}$ & $\begin{array}{l}36 \\
36 \\
36 \\
36 \\
36 \\
36 \\
36 \\
36 \\
35 \\
35 \\
35 \\
35 \\
35 \\
35 \\
35\end{array}$ & $\begin{array}{l}5 \\
5 \\
5 \\
5 \\
5 \\
4 \\
4\end{array}$ & $\begin{array}{l}2.166 \\
2.179 \\
2.103 \\
2.151 \\
2.023 \\
2.015 \\
1.960 \\
1.828 \\
1.771 \\
1.658 \\
1.405 \\
0.993 \\
0.655 \\
0.515 \\
0.279 \\
0.157 \\
0.126 \\
0.075 \\
0.054 \\
0.042 \\
0.104 \\
0.025 \\
0.022\end{array}$ & $\begin{array}{l}1.071 \\
1.007 \\
1.010 \\
1.003 \\
0.989 \\
1.150 \\
1.273 \\
1.169 \\
0.978 \\
0.857 \\
0.669 \\
0.526 \\
0.315 \\
0.259 \\
0.145 \\
0.079 \\
0.060 \\
0.040 \\
0.007 \\
0.007 \\
0.004 \\
0.000 \\
0.000\end{array}$ & $\begin{array}{r}20.4 \\
18.6 \\
17.9 \\
17.5 \\
17.4 \\
16.9 \\
16.4 \\
15.4 \\
14.7 \\
14.3 \\
13.0 \\
11.9 \\
10.7 \\
9.9 \\
9.2 \\
8.1 \\
6.5 \\
5.4 \\
4.6 \\
4.0 \\
3.4 \\
3.0 \\
2.7 \\
2.5\end{array}$ & $\begin{array}{l}10 \\
1 \\
2 \\
9 \\
1 \\
10 \\
9 \\
1 \\
3 \\
1 \\
1 \\
5 \\
8 \\
19 \\
20 \\
38 \\
18 \\
1 \\
25 \\
14 \\
19 \\
35 \\
1 \\
1\end{array}$ & $\begin{array}{l}32.129 \\
32.601 \\
32.781 \\
32.857 \\
32.891 \\
32.973 \\
33.036 \\
33.185 \\
33.297 \\
33.347 \\
33.521 \\
33.657 \\
33.816 \\
33.962 \\
34.102 \\
34.348 \\
34.528 \\
34.624 \\
34.676 \\
34.715 \\
34.744 \\
34.766 \\
34\end{array}$ & $\begin{array}{l}38.271 \\
38.783 \\
38.981 \\
39.065 \\
39.100 \\
39.196 \\
39.271 \\
39.447 \\
39.577 \\
39.640 \\
39.851 \\
40.018 \\
40.213 \\
40.383 \\
40.546 \\
40.826 \\
41.059 \\
41.197 \\
41.279 \\
41.340 \\
41.393 \\
41.429 \\
41.461 \\
41.474\end{array}$ & $\begin{array}{r}4 \\
25 \\
50 \\
75 \\
100 \\
126 \\
151 \\
198 \\
247 \\
300 \\
401 \\
498 \\
596 \\
696 \\
792 \\
990 \\
1236 \\
1485 \\
1720 \\
1974 \\
2217 \\
2462 \\
2707 \\
2953\end{array}$ \\
\hline
\end{tabular}




\begin{tabular}{|c|c|c|c|c|c|c|c|c|c|c|}
\hline \multicolumn{3}{|c|}{$\begin{array}{l}\text { ENDEAVOR } 143 \text { STA- } 93 \\
\text { DATE } 16 / 5 / 86\end{array}$} & \multicolumn{3}{|c|}{$L A T=3138.7 \mathrm{~N}$} & \multicolumn{2}{|c|}{$O N=2856.5 W$} & SONIC & \multicolumn{2}{|c|}{$2980 m$} \\
\hline $\begin{array}{l}\text { PR } \\
\text { dbar }\end{array}$ & $\stackrel{T}{\operatorname{Deg} C} C$ & $\underset{0 / 00}{S}$ & $\mathrm{ml} / \mathrm{l}$ & $\stackrel{\ominus}{\operatorname{Deg}} \mathrm{C}$ & $\begin{array}{l}\mathrm{SIG}-\theta \\
\mathrm{kg} / \mathrm{m} 3\end{array}$ & $\begin{array}{l}\mathrm{SIG}-1.5 \\
\mathrm{~kg} / \mathrm{m3}\end{array}$ & $\begin{array}{l}\mathrm{SIG}-3 \\
\mathrm{~kg} / \mathrm{m} 3\end{array}$ & $\begin{array}{l}\text { HGTH } \\
\text { m }\end{array}$ & $\underset{\text { cph }}{N}$ & $D E$ \\
\hline $\begin{array}{r}3 \\
25 \\
50 \\
75 \\
100 \\
150 \\
200 \\
250 \\
300 \\
350 \\
400 \\
450 \\
500 \\
600 \\
700 \\
800 \\
900 \\
1000 \\
1200 \\
1400 \\
1600 \\
1800 \\
2000 \\
2200 \\
2400 \\
2600 \\
2800 \\
2999\end{array}$ & $\begin{array}{r}20.337 \\
19.186 \\
18.028 \\
17.603 \\
17.482 \\
16.474 \\
15.660 \\
14.963 \\
14.366 \\
13.598 \\
13.109 \\
12.481 \\
11.810 \\
10.745 \\
10.098 \\
9.297 \\
8.732 \\
8.225 \\
7.231 \\
6.167 \\
4.935 \\
4.365 \\
3.884 \\
3.573 \\
3.242 \\
3.096 \\
2.990 \\
2.851\end{array}$ & $\begin{array}{l}36.503 \\
36.425 \\
36.391 \\
36.377 \\
36.371 \\
36.229 \\
36.114 \\
36.015 \\
35.934 \\
35.829 \\
35.759 \\
35.683 \\
35.598 \\
35.502 \\
35.476 \\
35.464 \\
35.474 \\
35.474 \\
35.425 \\
35.309 \\
35.146 \\
35.074 \\
35.024 \\
35.002 \\
34.975 \\
34.967 \\
34.961 \\
34.949\end{array}$ & $\begin{array}{l}5.16 \\
5.45 \\
5.56 \\
5.34 \\
5.23 \\
4.93 \\
4.81 \\
4.77 \\
4.73 \\
4.69 \\
4.64 \\
4.54 \\
4.49 \\
4.33 \\
4.25 \\
4.22 \\
4.32 \\
4.45 \\
4.80 \\
5.15 \\
5.63 \\
5.85 \\
5.94 \\
5.91 \\
5.89 \\
5.88 \\
5.78 \\
5.71\end{array}$ & $\begin{array}{l}20.337 \\
19.181 \\
18.019 \\
17.590 \\
17.465 \\
16.450 \\
15.629 \\
14.925 \\
14.321 \\
13.548 \\
13.053 \\
12.420 \\
11.744 \\
10.671 \\
10.014 \\
9.205 \\
8.631 \\
8.116 \\
7.108 \\
6.033 \\
4.794 \\
4.211 \\
3.718 \\
3.392 \\
3.047 \\
2.884 \\
2.760 \\
2.604\end{array}$ & $\begin{array}{l}25.820 \\
26.066 \\
26.334 \\
26.430 \\
26.455 \\
26.590 \\
26.691 \\
26.772 \\
26.842 \\
26.923 \\
26.971 \\
27.038 \\
27.103 \\
27.226 \\
27.322 \\
27.448 \\
27.549 \\
27.629 \\
27.739 \\
27.793 \\
27.815 \\
27.823 \\
27.835 \\
27.849 \\
27.861 \\
27.869 \\
27.876 \\
27.880\end{array}$ & $\begin{array}{l}32.171 \\
32.444 \\
32.740 \\
32.847 \\
32.876 \\
33.037 \\
33.160 \\
33.262 \\
33.349 \\
33.453 \\
33.516 \\
33.602 \\
33.688 \\
33.845 \\
33.961 \\
34.114 \\
34.233 \\
34.331 \\
34.476 \\
34.570 \\
34.639 \\
34.670 \\
34.702 \\
34.730 \\
34.756 \\
34.771 \\
34.782 \\
34.793\end{array}$ & $\begin{array}{l}38.315 \\
38.614 \\
38.938 \\
39.054 \\
39.086 \\
39.273 \\
39.418 \\
39.538 \\
39.642 \\
39.768 \\
39.845 \\
39.949 \\
40.055 \\
40.244 \\
40.381 \\
40.558 \\
40.695 \\
40.810 \\
40.989 \\
41.120 \\
41.235 \\
41.289 \\
41.339 \\
41.379 \\
41.419 \\
41.440 \\
41.456 \\
41.473\end{array}$ & $\begin{array}{l}0.007 \\
0.053 \\
0.098 \\
0.139 \\
0.180 \\
0.257 \\
0.329 \\
0.398 \\
0.464 \\
0.526 \\
0.587 \\
0.645 \\
0.701 \\
0.805 \\
0.899 \\
0.986 \\
1.061 \\
1.130 \\
1.252 \\
1.359 \\
1.458 \\
1.553 \\
1.645 \\
1.735 \\
1.822 \\
1.907 \\
1.993 \\
2.079\end{array}$ & $\begin{array}{l}0.83 \\
8.26 \\
4.02 \\
2.73 \\
2.71 \\
2.71 \\
2.16 \\
2.61 \\
2.02 \\
2.11 \\
1.99 \\
2.05 \\
2.10 \\
2.19 \\
1.68 \\
2.22 \\
1.69 \\
1.61 \\
1.34 \\
1.15 \\
0.87 \\
0.80 \\
0.73 \\
0.75 \\
0.60 \\
0.50 \\
0.48 \\
0.60\end{array}$ & $\begin{array}{r}3 \\
25 \\
50 \\
74 \\
99 \\
149 \\
199 \\
248 \\
298 \\
347 \\
397 \\
446 \\
496 \\
595 \\
694 \\
793 \\
892 \\
991 \\
1188 \\
1386 \\
1583 \\
1780 \\
1977 \\
2173 \\
2370 \\
2566 \\
2762 \\
2957\end{array}$ \\
\hline $\begin{array}{l}\text { PR } \\
\text { dbar }\end{array}$ & $\stackrel{T}{\mathrm{~T}} \mathrm{C}$ & $\stackrel{5}{100}$ & $\begin{array}{l}02 \\
\mathrm{ml} / \mathrm{I}\end{array}$ & $\begin{array}{c}\mathrm{F}-11 \\
\mathrm{pmol} / \mathrm{kg}\end{array}$ & $\begin{array}{c}\mathrm{F}-12 \\
\mathrm{pmol} / \mathrm{kg}\end{array}$ & $\stackrel{\theta}{\operatorname{Deg} C}$ & $\begin{array}{l}\mathrm{SIG}-\theta \\
\mathrm{kg} / \mathrm{m} 3\end{array}$ & $\underset{\mathrm{kg} / \mathrm{m} 3}{\mathrm{SIG}-1.5}$ & $\begin{array}{l}\mathrm{SIG}-3 \\
\mathrm{~kg} / \mathrm{m} 3\end{array}$ & $D E$ \\
\hline $\begin{array}{r}3 \\
26 \\
50 \\
77 \\
103 \\
114 \\
151 \\
177 \\
203 \\
251 \\
300 \\
399 \\
500 \\
601 \\
801 \\
1000 \\
1247 \\
1498 \\
1747 \\
1996 \\
2245 \\
2496 \\
2740\end{array}$ & $\begin{array}{r}20.297 \\
18.450 \\
17.740 \\
17.538 \\
17.348 \\
17.198 \\
16.059 \\
15.634 \\
15.548 \\
14.899 \\
14.101 \\
12.902 \\
11.752 \\
10.816 \\
9.484 \\
8.308 \\
6.971 \\
5.374 \\
4.412 \\
3.826 \\
3.410 \\
3.164 \\
3.030 \\
2.853\end{array}$ & $\begin{array}{l}36.502 \\
36.414 \\
36.397 \\
36.375 \\
36.373 \\
36.303 \\
36.176 \\
36.115 \\
36.102 \\
36.021 \\
35.904 \\
35.740 \\
35.593 \\
35.510 \\
35.463 \\
35.475 \\
35.402 \\
35.211 \\
35\end{array}$ & $\begin{array}{l}5.32 \\
5.59 \\
5.54 \\
5.29 \\
5.23 \\
5.03 \\
4.92 \\
4.98 \\
4.83 \\
4.80 \\
4.77\end{array}$ & $\begin{array}{l}0.072 \\
0.018\end{array}$ & 1.118 & $\begin{array}{r}20.296 \\
18.445 \\
17.732 \\
17.525 \\
17.331 \\
17.179 \\
16.034 \\
15.607 \\
15.516 \\
14.860 \\
14.057 \\
12.846 \\
11.686 \\
10.741 \\
9.391 \\
8.199 \\
6.845 \\
5.238 \\
4.263 \\
3.661 \\
3.228 \\
2.962 \\
2.804 \\
2.605\end{array}$ & $\begin{array}{l}25.830 \\
26.245 \\
26.410 \\
26.444 \\
26.490 \\
26.473 \\
26.646 \\
26.697 \\
26.707 \\
26.791 \\
26.875 \\
26.998 \\
27.110 \\
27.220 \\
27.417 \\
27.617 \\
27.757 \\
27.815 \\
27.823 \\
27.838 \\
27.855 \\
27.864 \\
27.874 \\
27.879\end{array}$ & $\begin{array}{l}32.182 \\
32.641 \\
32.823 \\
32.863 \\
32.913 \\
32.901 \\
33.104 \\
33.167 \\
33.180 \\
33.283 \\
33.389 \\
33.549 \\
33.697 \\
33.837 \\
34.077 \\
34.316 \\
34.504 \\
34.622 \\
34.668 \\
34.707 \\
34.742 \\
34.762 \\
34.778 \\
34.792\end{array}$ & $\begin{array}{l}38.327 \\
38.828 \\
39.027 \\
39.072 \\
39.127 \\
39.119 \\
39.351 \\
39.425 \\
39.441 \\
39.561 \\
39.689 \\
39.883 \\
40.066 \\
40.234 \\
40.515 \\
40.792 \\
41.026 \\
41.201 \\
41.284 \\
41.347 \\
41.398 \\
41.428 \\
41.451 \\
41.472\end{array}$ & $\begin{array}{r}3 \\
26 \\
50 \\
76 \\
102 \\
113 \\
149 \\
176 \\
202 \\
249 \\
297 \\
395 \\
495 \\
595 \\
793 \\
989 \\
1233 \\
1480 \\
1726 \\
1971 \\
2215 \\
2461 \\
2709 \\
2956\end{array}$ \\
\hline
\end{tabular}




\begin{tabular}{|c|c|c|c|c|c|c|c|c|c|c|}
\hline \multicolumn{3}{|c|}{$\begin{array}{l}\text { ENDEAVOR } 143 \text { STA- } 94 \\
\text { DATE } 17 / 5 / 86\end{array}$} & \multirow{2}{*}{$\begin{array}{l}\text { LAT }= \\
02 \\
\mathrm{ml} / \mathrm{l}\end{array}$} & \multicolumn{2}{|c|}{$3152.1 \mathrm{~N}$} & \multicolumn{2}{|c|}{$\mathrm{ON}=2847.1 \mathrm{~W}$} & \multicolumn{3}{|c|}{ SONIC DEPTH $=3660 \mathrm{~m}$} \\
\hline $\begin{array}{l}\text { PR } \\
\text { dbar }\end{array}$ & $\stackrel{T}{T} \mathrm{C}$ & $\begin{array}{l}S \\
0 / 00\end{array}$ & & $\stackrel{\theta}{\operatorname{Deg}} \mathrm{C}$ & $\begin{array}{l}\mathrm{SIG}-\theta \\
\mathrm{kg} / \mathrm{m} 3\end{array}$ & $\begin{array}{l}\mathrm{SIG}-1.5 \\
\mathrm{~kg} / \mathrm{m} 3\end{array}$ & $\begin{array}{l}\text { SIG-3 } \\
\mathrm{kg} / \mathrm{m} 3\end{array}$ & $\underset{m}{H G T H}$ & $\underset{c p h}{N}$ & $\underset{m}{\mathrm{DE}}$ \\
\hline $\begin{array}{r}1 \\
25 \\
50 \\
75 \\
100 \\
150 \\
200 \\
250 \\
300 \\
350 \\
400 \\
450 \\
500 \\
600 \\
700 \\
800 \\
900 \\
1000 \\
1200 \\
1400 \\
1600 \\
1800 \\
2000 \\
2200 \\
2400 \\
2600 \\
2800 \\
2999\end{array}$ & $\begin{array}{r}20.146 \\
19.507 \\
18.202 \\
17.736 \\
17.482 \\
16.899 \\
16.305 \\
15.516 \\
14.813 \\
14.224 \\
13.452 \\
12.989 \\
12.427 \\
11.327 \\
10.147 \\
9.463 \\
8.971 \\
8.412 \\
6.923 \\
5.813 \\
4.992 \\
4.380 \\
4.041 \\
3.639 \\
3.306 \\
3.107 \\
2.944 \\
2.823\end{array}$ & $\begin{array}{l}36.584 \\
36.515 \\
36.485 \\
36.404 \\
36.370 \\
36.301 \\
36.228 \\
36.096 \\
35.994 \\
35.913 \\
35.799 \\
35.738 \\
35.666 \\
35.548 \\
35.448 \\
35.459 \\
35.473 \\
35.476 \\
35.379 \\
35.256 \\
35.156 \\
35.084 \\
35.060 \\
35.013 \\
34.982 \\
34.967 \\
34.955 \\
34.945\end{array}$ & $\begin{array}{l}5.46 \\
5.33 \\
5.40 \\
5.28 \\
5.21 \\
4.87 \\
4.75 \\
4.76 \\
4.78 \\
4.79 \\
4.70 \\
4.65 \\
4.60 \\
4.47 \\
4.23 \\
4.25 \\
4.28 \\
4.35 \\
4.94 \\
5.34 \\
5.61 \\
5.79 \\
5.78 \\
5.86 \\
5.90 \\
5.87 \\
5.84 \\
5.80\end{array}$ & $\begin{array}{l}20.145 \\
19.502 \\
18.193 \\
17.723 \\
17.465 \\
16.874 \\
16.272 \\
15.477 \\
14.768 \\
14.172 \\
13.395 \\
12.926 \\
12.359 \\
11.250 \\
10.062 \\
9.370 \\
8.869 \\
8.302 \\
6.803 \\
5.682 \\
4.850 \\
4.226 \\
3.872 \\
3.457 \\
3.111 \\
2.895 \\
2.715 \\
2.576\end{array}$ & $\begin{array}{l}25.933 \\
26.050 \\
26.363 \\
26.418 \\
26.455 \\
26.545 \\
26.631 \\
26.712 \\
26.790 \\
26.857 \\
26.932 \\
26.981 \\
27.037 \\
27.157 \\
27.291 \\
27.417 \\
27.510 \\
27.602 \\
27.745 \\
27.796 \\
27.817 \\
27.829 \\
27.847 \\
27.852 \\
27.860 \\
27.869 \\
27.875 \\
27.880\end{array}$ & $\begin{array}{l}32.288 \\
32.420 \\
32.764 \\
32.832 \\
32.875 \\
32.981 \\
33.083 \\
33.186 \\
33.285 \\
33.368 \\
33.467 \\
33.529 \\
33.603 \\
33.757 \\
33.930 \\
34.078 \\
34.187 \\
34.298 \\
34.493 \\
34.586 \\
34.639 \\
34.676 \\
34.708 \\
34.730 \\
34.752 \\
34.769 \\
34.783 \\
34.793\end{array}$ & $\begin{array}{l}38.436 \\
38.583 \\
38.956 \\
39.036 \\
39.085 \\
39.206 \\
39.323 \\
39.447 \\
39.565 \\
39.665 \\
39.786 \\
39.862 \\
39.952 \\
40.139 \\
40.348 \\
40.517 \\
40.642 \\
40.771 \\
41.016 \\
41.149 \\
41.233 \\
41.293 \\
41.339 \\
41.377 \\
41.413 \\
41.439 \\
41.459 \\
41.475\end{array}$ & $\begin{array}{l}0.002 \\
0.052 \\
0.095 \\
0.137 \\
0.177 \\
0.255 \\
0.331 \\
0.402 \\
0.471 \\
0.537 \\
0.600 \\
0.660 \\
0.719 \\
0.830 \\
0.930 \\
1.019 \\
1.099 \\
1.171 \\
1.292 \\
1.396 \\
1.493 \\
1.587 \\
1.679 \\
1.768 \\
1.856 \\
1.942 \\
2.028 \\
2.113\end{array}$ & $\begin{array}{r}-2.00 \\
8.39 \\
2.49 \\
2.99 \\
2.46 \\
1.86 \\
2.99 \\
1.76 \\
2.30 \\
2.21 \\
2.28 \\
1.54 \\
1.88 \\
2.14 \\
2.27 \\
1.97 \\
1.94 \\
1.80 \\
1.46 \\
0.96 \\
0.77 \\
0.83 \\
0.69 \\
0.68 \\
0.69 \\
0.51 \\
0.56 \\
0.51\end{array}$ & $\begin{array}{r}1 \\
25 \\
50 \\
74 \\
99 \\
149 \\
199 \\
248 \\
298 \\
347 \\
397 \\
446 \\
496 \\
595 \\
694 \\
793 \\
892 \\
991 \\
1188 \\
1386 \\
1583 \\
1780 \\
1977 \\
2173 \\
2370 \\
2566 \\
2762 \\
2957\end{array}$ \\
\hline $\begin{array}{l}\text { PR } \\
\text { dbar }\end{array}$ & $\stackrel{T}{\operatorname{Deg} C}$ & $\stackrel{S}{S}$ & $\begin{array}{l}02 \\
\mathrm{ml} / \mathrm{I}\end{array}$ & $\begin{array}{c}\mathrm{F}-11 \\
\mathrm{pmol} / \mathrm{kg}\end{array}$ & $\begin{array}{c}\mathrm{F}-12 \\
\mathrm{pmol} / \mathrm{kg}\end{array}$ & $\stackrel{\theta}{\operatorname{Deg} C}$ & $\mathrm{~kg} / \mathrm{m} 3$ & $\underset{\mathrm{kg} / \mathrm{m} 3}{\mathrm{SIG}-1.5}$ & $\begin{array}{l}\mathrm{SIG}-3 \\
\mathrm{~kg} / \mathrm{m3}\end{array}$ & $\underset{m}{\mathrm{DE}}$ \\
\hline $\begin{array}{r}3 \\
30 \\
55 \\
77 \\
103 \\
128 \\
153 \\
178 \\
204 \\
253 \\
306 \\
402 \\
502 \\
603 \\
801 \\
1001 \\
1252 \\
1500 \\
1746\end{array}$ & $\begin{array}{r}20.240 \\
19.640 \\
18.058 \\
17.728 \\
17.625 \\
17.236 \\
16.934 \\
16.441 \\
15.979 \\
15.441 \\
14.615 \\
13.437 \\
12.141 \\
11.148 \\
9.455 \\
8.347 \\
6.622 \\
5.242 \\
4.394 \\
3.983 \\
3.550 \\
3.167 \\
2.971 \\
2.820\end{array}$ & $\begin{array}{l}36.569 \\
36.527 \\
36.416 \\
36.418 \\
36.392 \\
36.341 \\
36.302 \\
36.230 \\
36.180 \\
36.089 \\
35.975 \\
35.801 \\
35.639 \\
35.531 \\
35.462 \\
35.478 \\
35.355 \\
35.185 \\
35.080 \\
35.051 \\
35.007 \\
34.972\end{array}$ & $\begin{array}{l}5 \\
4 \\
4 \\
4 \\
4 \\
4 \\
4 \\
4 \\
4 \\
4 \\
4 \\
5 \\
5\end{array}$ & $\begin{array}{l}0.048 \\
0.048 \\
0.041\end{array}$ & 1.068 & $\begin{array}{r}20.240 \\
19.634 \\
18.048 \\
17.714 \\
17.607 \\
17.215 \\
16.909 \\
16.413 \\
15.946 \\
15.402 \\
14.569 \\
13.380 \\
12.074 \\
11.071 \\
9.362 \\
8.238 \\
6.498 \\
5.108 \\
4.245 \\
3.816 \\
3.365 \\
2.964 \\
2.747 \\
2.574\end{array}$ & $\begin{array}{l}25.896 \\
26.025 \\
26.346 \\
26.430 \\
26.437 \\
26.493 \\
26.537 \\
26.599 \\
26.669 \\
26.723 \\
26.820 \\
26.937 \\
27.072 \\
27.177 \\
27.421 \\
27.613 \\
27.768 \\
27.809 \\
27.824 \\
27.846 \\
27.856 \\
27.866 \\
27.874 \\
27.885\end{array}$ & $\begin{array}{l}32.249 \\
32.392 \\
32.752 \\
32.844 \\
32.853 \\
32.920 \\
32.972 \\
33.047 \\
33.130 \\
33.199 \\
33.319 \\
33.471 \\
33.646 \\
33.783 \\
34.082 \\
34.311 \\
34.527 \\
34.622 \\
34.670 \\
34.709 \\
34.738 \\
34.764 \\
34.781 \\
34.799\end{array}$ & $\begin{array}{l}38.395 \\
38.551 \\
38.948 \\
39.048 \\
39.060 \\
39.137 \\
39.196 \\
39.284 \\
39.379 \\
39.463 \\
39.605 \\
39.791 \\
40.003 \\
40.170 \\
40.521 \\
40.786 \\
41.061 \\
41.206 \\
41.287 \\
41.342 \\
41.388 \\
41.430 \\
41.456 \\
41.481\end{array}$ & $\begin{array}{r}3 \\
29 \\
55 \\
76 \\
102 \\
127 \\
152 \\
176 \\
202 \\
251 \\
303 \\
398 \\
497 \\
597 \\
792 \\
991 \\
1238 \\
1482 \\
1724 \\
1972 \\
2218 \\
2461 \\
2707 \\
2953\end{array}$ \\
\hline
\end{tabular}




\begin{tabular}{|c|c|c|c|c|c|c|c|c|c|c|}
\hline \multicolumn{3}{|c|}{$\begin{array}{l}\text { ENDEAVOR } 143 \text { STA- } 95 \\
\text { DATE } 17 / 5 / 86\end{array}$} & LAT $=$ & $3210.2 \mathrm{~N}$ & LON $=$ & \multicolumn{2}{|c|}{$2838.1 W$} & SONIC D & EPTH= & $450 \mathrm{~m}$ \\
\hline $\begin{array}{l}\text { PR } \\
\text { dbar }\end{array}$ & $\stackrel{T}{\operatorname{Teg}} \mathrm{C}$ & $\stackrel{5}{0 / 00}$ & $\begin{array}{l}02 \\
\mathrm{ml} / 1\end{array}$ & $\stackrel{\theta}{\operatorname{Deg} C}$ & $\begin{array}{l}\mathrm{SIG}-0 \\
\mathrm{~kg} / \mathrm{m} 3\end{array}$ & $\begin{array}{l}\mathrm{SIG}-1.5 \\
\mathrm{~kg} / \mathrm{m} 3\end{array}$ & & $\underset{\mathbf{m}}{\mathrm{HGTH}}$ & $\underset{c p h}{N}$ & \\
\hline $\begin{array}{r}3 \\
25 \\
50 \\
75 \\
100 \\
150 \\
200 \\
250 \\
300 \\
350 \\
400 \\
450 \\
500 \\
600 \\
700 \\
800 \\
900 \\
1000 \\
1200 \\
1400 \\
1600 \\
1800 \\
2000 \\
2200 \\
2400 \\
2600 \\
2800 \\
2999\end{array}$ & $\begin{array}{r}19.989 \\
20.013 \\
18.478 \\
18.152 \\
17.758 \\
17.331 \\
16.506 \\
15.935 \\
15.335 \\
14.698 \\
13.985 \\
13.219 \\
12.629 \\
11.730 \\
10.992 \\
9.821 \\
8.973 \\
8.306 \\
7.143 \\
6.201 \\
5.127 \\
4.525 \\
4.047 \\
3.656 \\
3.314 \\
3.102 \\
2.904 \\
2.837\end{array}$ & $\begin{array}{l}36.615 \\
36.583 \\
36.507 \\
36.491 \\
36.427 \\
36.361 \\
36.229 \\
36.148 \\
36.071 \\
35.986 \\
35.881 \\
35.769 \\
35.690 \\
35.583 \\
35.528 \\
35.472 \\
35.471 \\
35.476 \\
35.413 \\
35.315 \\
35.183 \\
35.120 \\
35.058 \\
35.016 \\
34.983 \\
34.968 \\
34.952 \\
34.945\end{array}$ & $\begin{array}{l}5.42 \\
5.42 \\
5.58 \\
5.38 \\
5.27 \\
5.07 \\
4.89 \\
4.77 \\
4.69 \\
4.74 \\
4.80 \\
4.69 \\
4.56 \\
4.53 \\
4.37 \\
4.20 \\
4.29 \\
4.37 \\
4.73 \\
5.10 \\
5.51 \\
5.64 \\
5.81 \\
5.88 \\
5.90 \\
5.88 \\
5.84\end{array}$ & $\begin{array}{l}19.989 \\
20.008 \\
18.469 \\
18.139 \\
17.741 \\
17.306 \\
16.473 \\
15.895 \\
15.288 \\
14.645 \\
13.927 \\
13.156 \\
12.560 \\
11.651 \\
10.904 \\
9.726 \\
8.871 \\
8.197 \\
7.020 \\
6.066 \\
4.984 \\
4.369 \\
3.879 \\
3.474 \\
3.118 \\
2.890 \\
2.676 \\
2.590\end{array}$ & $\begin{array}{l}25.998 \\
25.969 \\
26.311 \\
26.381 \\
26.431 \\
26.486 \\
26.584 \\
26.656 \\
26.734 \\
26.811 \\
26.884 \\
26.958 \\
27.016 \\
27.110 \\
27.205 \\
27.368 \\
27.508 \\
27.618 \\
27.742 \\
27.794 \\
27.822 \\
27.842 \\
27.846 \\
27.853 \\
27.861 \\
27.870 \\
27.876 \\
27.878\end{array}$ & $\begin{array}{l}32.356 \\
32.327 \\
32.705 \\
32.784 \\
32.844 \\
32.911 \\
33.030 \\
33.119 \\
33.214 \\
33.309 \\
33.403 \\
33.499 \\
33.576 \\
33.697 \\
33.816 \\
34.017 \\
34.185 \\
34.317 \\
34.482 \\
34.569 \\
34.639 \\
34.683 \\
34.706 \\
34.730 \\
34.752 \\
34.771 \\
34.786 \\
34.792\end{array}$ & $\begin{array}{l}38.507 \\
38.477 \\
38.891 \\
38.977 \\
39.047 \\
39.125 \\
39.266 \\
39.370 \\
39.480 \\
39.593 \\
39.707 \\
39.825 \\
39.919 \\
40.067 \\
40.208 \\
40.445 \\
40.640 \\
40.793 \\
40.998 \\
41.118 \\
41.228 \\
41.295 \\
41.337 \\
41.376 \\
41.413 \\
41.440 \\
41.464 \\
41.473\end{array}$ & $\begin{array}{l}0.006 \\
0.051 \\
0.097 \\
0.140 \\
0.181 \\
0.261 \\
0.340 \\
0.414 \\
0.486 \\
0.554 \\
0.620 \\
0.682 \\
0.742 \\
0.856 \\
0.964 \\
1.058 \\
1.140 \\
1.212 \\
1.333 \\
1.440 \\
1.537 \\
1.630 \\
1.721 \\
1.810 \\
1.898 \\
1.984 \\
2.069 \\
2.154\end{array}$ & $\begin{array}{r}-2.81 \\
4.78 \\
4.54 \\
2.18 \\
1.82 \\
2.09 \\
2.80 \\
2.48 \\
2.62 \\
2.36 \\
1.91 \\
2.27 \\
1.83 \\
1.72 \\
2.36 \\
2.11 \\
2.07 \\
1.71 \\
1.42 \\
1.25 \\
0.85 \\
0.73 \\
0.64 \\
0.72 \\
0.69 \\
0.58 \\
0.47 \\
0.32\end{array}$ & $\begin{array}{r}3 \\
25 \\
50 \\
74 \\
99 \\
149 \\
199 \\
248 \\
298 \\
347 \\
397 \\
446 \\
496 \\
595 \\
694 \\
793 \\
892 \\
991 \\
1188 \\
1386 \\
1583 \\
1780 \\
1977 \\
2173 \\
2370 \\
2566 \\
2762 \\
2957\end{array}$ \\
\hline $\begin{array}{l}\text { PR } \\
\text { dbar }\end{array}$ & $\stackrel{T}{\operatorname{Deg}} \mathrm{C}$ & $\stackrel{5}{1 / 00}$ & $\begin{array}{l}02 \\
\mathrm{ml} / \mathrm{I}\end{array}$ & $\begin{array}{c}\mathrm{F}-11 \\
\mathrm{pmol} / \mathrm{kg}\end{array}$ & $\begin{array}{c}\mathrm{F}-12 \\
\mathrm{pmol} / \mathrm{kg}\end{array}$ & $\stackrel{\theta}{D e g} \cdot C$ & $\begin{array}{l}\text { SI } \\
\mathrm{kg}\end{array}$ & $\mathrm{kg} / \mathrm{m} 3^{\mathrm{IG}-1} \cdot 5$ & $\begin{array}{l}\mathrm{SIG}-3 \\
\mathrm{~kg} / \mathrm{m}^{3}\end{array}$ & \\
\hline $\begin{array}{r}3 \\
26 \\
51 \\
77 \\
102 \\
124 \\
151 \\
177 \\
203 \\
252 \\
305 \\
407 \\
504 \\
594 \\
806 \\
1002 \\
1249 \\
1502 \\
1752 \\
1999 \\
2249 \\
2496\end{array}$ & $\begin{array}{r}20.008 \\
19.061 \\
18.271 \\
18.037 \\
17.634 \\
17.611 \\
17.198 \\
16.540 \\
16.261 \\
15.667 \\
14.792 \\
13.588 \\
12.403 \\
11.699 \\
9.576 \\
8.333 \\
6.889 \\
5.429 \\
4.615 \\
4.030 \\
3.479 \\
3.139 \\
2.917 \\
2.833\end{array}$ & $\begin{array}{l}36 \\
36 \\
36 \\
36 \\
36 \\
36 \\
36 \\
36 \\
36 \\
36 \\
35 \\
35 \\
35 \\
35 \\
35 \\
35 \\
35 \\
35 \\
35\end{array}$ & $\begin{array}{l}5 \\
5 \\
5 \\
5\end{array}$ & & & $\begin{array}{l}19.6 \\
18.2 \\
18.6 \\
17.6 \\
17.5 \\
17.5 \\
16.5 \\
16.2 \\
15.6 \\
14.7 \\
13.5 \\
12.3 \\
11.6 \\
9.5 \\
8.2 \\
6.9 \\
5.2 \\
4.2 \\
3.8 \\
3.2 \\
2.9 \\
2.6 \\
2.5\end{array}$ & $\begin{array}{l}26.210 \\
26.365 \\
26.401 \\
26.448 \\
26.455 \\
26.504 \\
26.591 \\
26.619 \\
26.693 \\
26.808 \\
26.927 \\
27.047 \\
27.116 \\
27.407 \\
27.615 \\
27.765 \\
27.820 \\
27.849 \\
27.848 \\
27.858 \\
27.867 \\
27.875 \\
27.881\end{array}$ & $\begin{array}{l}3 \\
32 \\
32 \\
32 \\
3 \\
3 \\
3 \\
3 \\
3\end{array}$ & $\begin{array}{l}38.762 \\
38.955 \\
39.003 \\
39.070 \\
39.079 \\
39.149 \\
39.271 \\
39.314 \\
39.420 \\
39.584 \\
39.772 \\
39.963 \\
40.076 \\
40.499 \\
40.788 \\
41.039 \\
41.202 \\
41.294 \\
41.340 \\
41.396 \\
41.033 \\
41 . .461 \\
41.476\end{array}$ & $\begin{array}{r}3 \\
26 \\
51 \\
77 \\
101 \\
122 \\
149 \\
176 \\
201 \\
250 \\
303 \\
403 \\
500 \\
588 \\
798 \\
991 \\
1235 \\
1484 \\
1731 \\
1973 \\
2218 \\
2461 \\
2707 \\
2955\end{array}$ \\
\hline
\end{tabular}




\begin{tabular}{|c|c|c|c|c|c|c|c|c|c|c|}
\hline \multicolumn{3}{|c|}{$\begin{array}{l}\text { ENDEAVOR } 143 \text { STA- } 96 \\
\text { DATE } 17 / 5 / 86\end{array}$} & LAT $=$ & \multicolumn{2}{|c|}{$3228.2 \mathrm{~N}$} & \multicolumn{2}{|c|}{ LON $=$} & \multicolumn{3}{|c|}{ SONIC DEPTH $=3260 \mathrm{~m}$} \\
\hline $\begin{array}{l}\text { PR } \\
\text { dbar }\end{array}$ & $\stackrel{T}{T} C$ & $\begin{array}{c}S \\
0 / 00\end{array}$ & $\mathrm{ml} / \mathrm{l}$ & $\stackrel{\ominus}{\operatorname{Deg} C}$ & $\begin{array}{l}\mathrm{SIG}-0 \\
\mathrm{~kg} / \mathrm{m} 3\end{array}$ & $\begin{array}{l}\mathrm{SIG}-1.5 \\
\mathrm{~kg} / \mathrm{m} 3\end{array}$ & $\begin{array}{l}\mathrm{SIG}-3 \\
\mathrm{~kg} / \mathrm{m} 3\end{array}$ & $\underset{m}{\text { HGTH }}$ & $\underset{\mathrm{cph}}{N}$ & $D E$ \\
\hline $\begin{array}{r}3 \\
25 \\
50 \\
75 \\
100 \\
150 \\
200 \\
250 \\
300 \\
350 \\
400 \\
450 \\
500 \\
600 \\
700 \\
800 \\
900 \\
1000 \\
200\end{array}$ & $\begin{array}{r}20.118 \\
19.901 \\
19.201 \\
18.286 \\
17.721 \\
17.115 \\
16.410 \\
15.357 \\
14.794 \\
14.333 \\
13.603 \\
13.130 \\
12.553 \\
11.103 \\
10.433 \\
9.659 \\
8.854 \\
8.394 \\
7.471 \\
6.443 \\
5.270 \\
4.611 \\
3.848 \\
3.466 \\
3.220 \\
3.086 \\
2.947 \\
2.850\end{array}$ & $\begin{array}{l}36.571 \\
36.563 \\
36.552 \\
36.504 \\
36.438 \\
36.338 \\
36.232 \\
36.069 \\
35.992 \\
35.930 \\
35.827 \\
35.759 \\
35.681 \\
35.507 \\
35.476 \\
35.470 \\
35.467 \\
35.480 \\
35.441 \\
35.340 \\
35.195 \\
35.121 \\
35.017 \\
34.982 \\
34.968 \\
34.964 \\
34.954 \\
34.946\end{array}$ & $\begin{array}{l}5.35 \\
5.33 \\
5.41 \\
5.46 \\
5.13 \\
4.92 \\
4.74 \\
4.73 \\
4.77 \\
4.77 \\
4.82 \\
4.76 \\
4.71 \\
4.43 \\
4.32 \\
4.24 \\
4.25 \\
4.33 \\
4.66 \\
5.09 \\
5.51 \\
5.73 \\
5.96 \\
6.02 \\
6.00 \\
5.91\end{array}$ & $\begin{array}{c}20.117 \\
19.896 \\
19.192 \\
18.273 \\
17.704 \\
17.090 \\
16.377 \\
15.318 \\
14.749 \\
14.281 \\
13.545 \\
13.066 \\
12.485 \\
11.027 \\
10.348 \\
9.565 \\
8.752 \\
8.284 \\
7.345 \\
6.305 \\
5.125 \\
4.454 \\
3.682 \\
3.287 \\
3.026 \\
2.874 \\
2.718 \\
2.602\end{array}$ & $\begin{array}{l}25.931 \\
25.983 \\
26.160 \\
26.357 \\
26.449 \\
26.521 \\
26.609 \\
26.727 \\
26.794 \\
26.847 \\
26.923 \\
26.969 \\
27.025 \\
27.166 \\
27.264 \\
27.394 \\
27.525 \\
27.607 \\
27.718 \\
27.781 \\
27.815 \\
27.834 \\
27.832 \\
27.843 \\
27.857 \\
27.868 \\
27.874 \\
27.878\end{array}$ & $\begin{array}{l}32.286 \\
32.344 \\
32.536 \\
32.756 \\
32.862 \\
32.951 \\
33.058 \\
33.205 \\
33.288 \\
33.355 \\
33.452 \\
33.513 \\
33.586 \\
33.774 \\
33.893 \\
34.048 \\
34.205 \\
34.304 \\
34.446 \\
34.548 \\
34.627 \\
34.672 \\
34.701 \\
34.728 \\
34.753 \\
34.769 \\
34.782 \\
34.791\end{array}$ & $\begin{array}{l}38.705 \\
38.947 \\
39.066 \\
39.171 \\
39.296 \\
39.471 \\
39.569 \\
39.649 \\
39.767 \\
39.841 \\
39.932 \\
40.163 \\
40.302 \\
40.481 \\
40.664 \\
40.777 \\
40.951 \\
41.088 \\
41.210 \\
41.280 \\
41.340 \\
41.382 \\
41.417 \\
41.439 \\
41.458 \\
41.472\end{array}$ & $\begin{array}{l}0.006 \\
0.051 \\
0.101 \\
0.145 \\
0.186 \\
0.265 \\
0.341 \\
0.413 \\
0.481 \\
0.547 \\
0.610 \\
0.672 \\
0.731 \\
0.842 \\
0.943 \\
1.035 \\
1.115 \\
1.187 \\
1.312 \\
1.424 \\
1.526 \\
1.622 \\
1.714 \\
1.804 \\
1.891 \\
1.978 \\
2.063 \\
2.149\end{array}$ & $\begin{array}{l}2.76 \\
3.21 \\
5.68 \\
4.92 \\
2.43 \\
2.07 \\
2.41 \\
2.74 \\
2.17 \\
1.99 \\
2.12 \\
1.66 \\
2.17 \\
2.15 \\
1.82 \\
2.33 \\
1.80 \\
1.71 \\
1.23 \\
1.25 \\
1.03 \\
0.75 \\
0.77 \\
0.68 \\
0.63 \\
0.53 \\
0.53 \\
0.42\end{array}$ & $\begin{array}{r}3 \\
25 \\
50 \\
74 \\
99 \\
149 \\
199 \\
248 \\
298 \\
347 \\
397 \\
446 \\
496 \\
595 \\
694 \\
793 \\
892 \\
991 \\
1188 \\
1386 \\
1583 \\
1780 \\
1977 \\
2173 \\
2370 \\
2566 \\
2762 \\
2957\end{array}$ \\
\hline $\begin{array}{l}\text { PR } \\
\text { dbar }\end{array}$ & $\stackrel{T}{T} C$ & $\stackrel{5}{0 / 00}$ & $\begin{array}{l}02 \\
\mathrm{~m} / / 1\end{array}$ & $\begin{array}{c}\mathrm{F}-11 \\
\mathrm{pmol} / \mathrm{kg}\end{array}$ & $\begin{array}{c}\mathrm{F}-12 \\
\mathrm{pmol} / \mathrm{kg}\end{array}$ & $\stackrel{\theta}{\operatorname{Deg} C}$ & ڤ & $\underset{\mathrm{kg} / \mathrm{m} 3}{\mathrm{SIG}-1.5}$ & $\mathrm{~kg} / \mathrm{m}^{3}$ & $D E$ \\
\hline $\begin{array}{r}5 \\
26 \\
53 \\
77 \\
104 \\
124 \\
154 \\
178 \\
202 \\
249 \\
302 \\
400 \\
499 \\
589 \\
799 \\
996 \\
1247 \\
1493 \\
1746 \\
1992 \\
2244 \\
2104\end{array}$ & $\begin{array}{r}19.945 \\
18.996 \\
18.570 \\
17.913 \\
17.646 \\
17.229 \\
16.906 \\
16.440 \\
16.204 \\
15.703 \\
14.865 \\
13.798 \\
12.617 \\
11.375 \\
9.711 \\
8.366 \\
7.208 \\
5.891 \\
4.830 \\
3.898 \\
3.435 \\
3.163 \\
2.984\end{array}$ & $\begin{array}{l}36 \\
36 \\
36 \\
36 \\
36 \\
36 \\
36 \\
36 \\
36 \\
36 \\
36 \\
35 \\
35 \\
35 \\
35 \\
35\end{array}$ & $\begin{array}{l}4.83 \\
4.73 \\
4.72 \\
4.68 \\
4.72 \\
4.77 \\
4.64 \\
4.43 \\
4.21 \\
4.33 \\
4.79 \\
5.30 \\
5.61 \\
5.93 \\
5.98 \\
5.96\end{array}$ & $\begin{array}{l}1.901 \\
1.694 \\
1.684\end{array}$ & $\begin{array}{l}1.002 \\
0.875 \\
0.869\end{array}$ & $\begin{array}{r}19.944 \\
18.991 \\
18.560 \\
17.899 \\
17.628 \\
17.208 \\
16.881 \\
16.411 \\
16.171 \\
15.663 \\
14.819 \\
13.740 \\
12.549 \\
11.299 \\
9.617 \\
8.257 \\
7.079 \\
5.750 \\
4.675 \\
3.732 \\
3.252 \\
2.961 \\
2.760 \\
2.600\end{array}$ & $\begin{array}{l}25.974 \\
26.218 \\
26.315 \\
26.426 \\
26.462 \\
26.528 \\
26.555 \\
26.616 \\
26.647 \\
26.696 \\
26.792 \\
26.908 \\
27.022 \\
27.142 \\
27.380 \\
27.611 \\
27.742 \\
27.801 \\
27.836 \\
27.836 \\
27.849 \\
27.863\end{array}$ & $\begin{array}{l}32.3 \\
32.6 \\
32.7 \\
32.8 \\
32.8 \\
32.9 \\
32.9 \\
33.0 \\
33.1 \\
33.1 \\
33.2 \\
33.4 \\
33.5 \\
33.7 \\
34.0 \\
34.3\end{array}$ & $\begin{array}{l}39.215 \\
39.301 \\
39.345 \\
39.421 \\
39.564 \\
39.741 \\
39.925 \\
40.121 \\
40.464 \\
40.783 \\
40.994 \\
41.149 \\
41.265 \\
41.339 \\
41.391 \\
41.428 \\
41.453 \\
41.472\end{array}$ & $\begin{array}{r}5 \\
26 \\
53 \\
76 \\
103 \\
123 \\
152 \\
176 \\
200 \\
247 \\
300 \\
396 \\
494 \\
584 \\
790 \\
986 \\
1233 \\
1475 \\
1724 \\
1966 \\
2214 \\
2456 \\
2704 \\
2955\end{array}$ \\
\hline
\end{tabular}




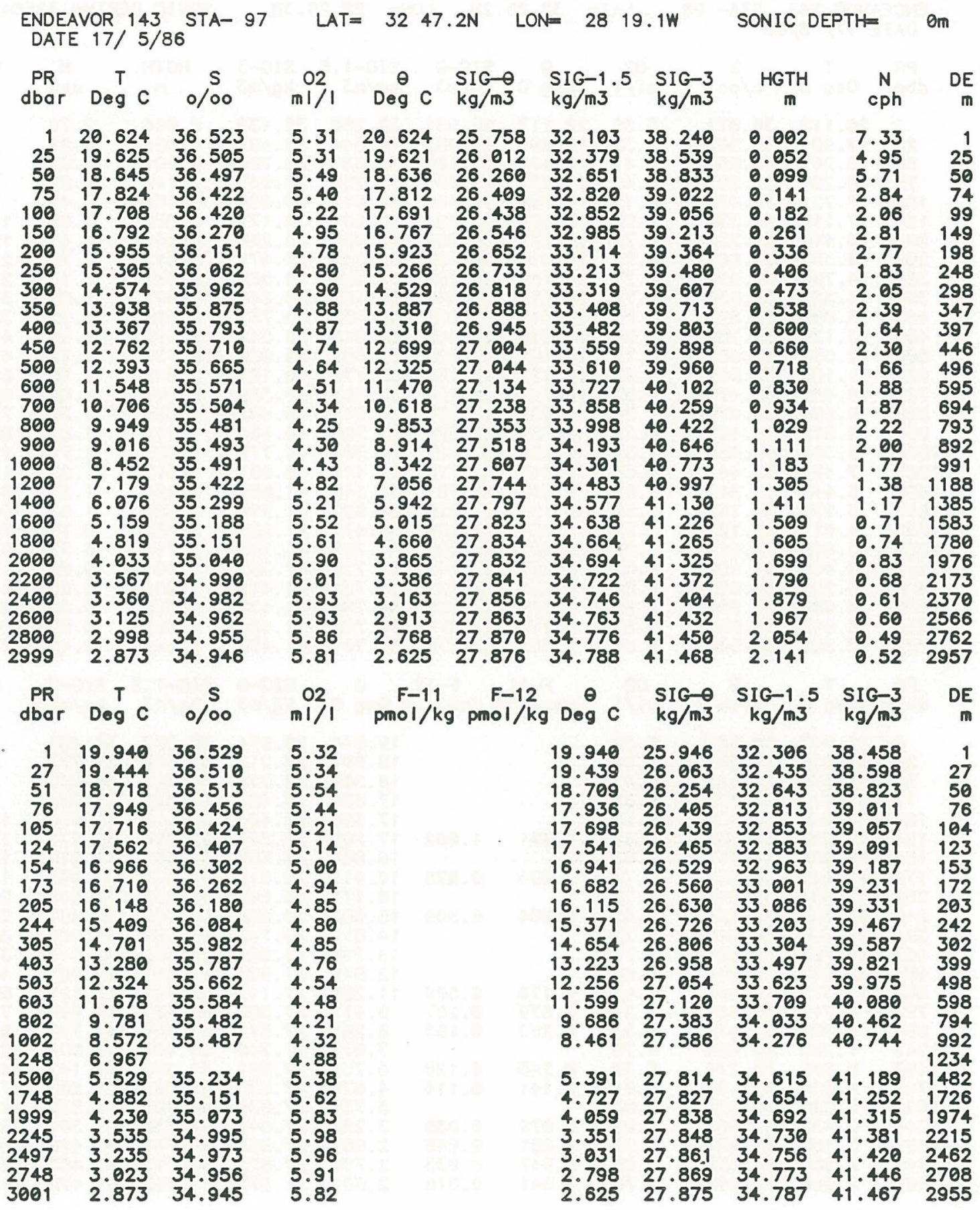




\begin{tabular}{|c|c|c|c|c|c|c|c|c|c|c|}
\hline \multicolumn{3}{|c|}{$\begin{array}{l}\text { ENDEAVOR } 143 \text { STA- } 98 \\
\text { DATE } 17 / 5 / 86\end{array}$} & LAT $=$ & 33 & LON $=$ & \multicolumn{2}{|r|}{$9.1 \mathrm{~W}$} & \multicolumn{3}{|c|}{ SONIC DEPTH $=2900 \mathrm{~m}$} \\
\hline $\begin{array}{l}\text { PR } \\
\text { dbar }\end{array}$ & $\stackrel{T}{\mathrm{D}} \mathrm{C}$ & $\stackrel{S}{0 / 00}$ & $\begin{array}{l}02 \\
\mathrm{ml} / \mathrm{l}\end{array}$ & $\stackrel{\theta}{\operatorname{Deg} C}$ & & $\underset{\mathrm{kg} / \mathrm{m} 3}{\mathrm{SIG}-1.5}$ & $\begin{array}{l}S I G-3 \\
\mathrm{~kg} / \mathrm{m} 3\end{array}$ & $\underset{\mathbf{m}}{\mathrm{HGTH}}$ & $\underset{c p h}{N}$ & $\begin{array}{l}\mathrm{DE} \\
\mathrm{m}\end{array}$ \\
\hline $\begin{array}{r}3 \\
25 \\
50 \\
75 \\
100 \\
150 \\
200 \\
250 \\
300 \\
350 \\
400 \\
450 \\
500 \\
600 \\
700 \\
800 \\
900 \\
1000 \\
200 \\
1400 \\
600 \\
800 \\
2000 \\
2200 \\
2400 \\
2600 \\
2625\end{array}$ & $\begin{array}{r}20.286 \\
19.634 \\
18.886 \\
18.771 \\
18.495 \\
18.297 \\
17.395 \\
16.147 \\
15.190 \\
14.593 \\
13.784 \\
13.278 \\
12.678 \\
11.475 \\
10.601 \\
9.819 \\
9.127 \\
8.590 \\
7.406 \\
6.163 \\
5.192 \\
4.621 \\
3.859 \\
3.514 \\
3.275 \\
3.112 \\
3.110\end{array}$ & $\begin{array}{l}36.625 \\
36.674 \\
36.730 \\
36.746 \\
36.695 \\
36.657 \\
36.477 \\
36.209 \\
36.064 \\
35.972 \\
35.854 \\
35.785 \\
35.703 \\
35.567 \\
35.505 \\
35.480 \\
35.490 \\
35.500 \\
35.447 \\
35.304 \\
35.174 \\
35.103 \\
35.019 \\
34.984 \\
34.970 \\
34.961 \\
34.961\end{array}$ & $\begin{array}{l}.56 \\
.44 \\
.30 \\
.14 \\
.86 \\
.77 \\
.76 \\
.78 \\
76 \\
.70 \\
.60\end{array}$ & $\begin{array}{ll}20.285 & 2 \\
19.630 & 2 \\
18.877 & 2 \\
18.758 & 2 \\
18.477 & 2 \\
18.270 & 2 \\
17.361 & 2 \\
16.107 & 2 \\
15.143 & 2 \\
14.540 & 2 \\
13.726 & 2 \\
13.214 & 2 \\
12.609 & 2 \\
11.397 & 2 \\
10.514 & 2 \\
9.723 & 2 \\
9.023 & 2 \\
8.479 & 2 \\
7.281 & 2 \\
6.029 & 2 \\
5.048 & 2 \\
4.464 & 2 \\
3.693 & 2 \\
3.334 & 2 \\
3.079 & 2 \\
2.900 & 2 \\
2.895 & 2\end{array}$ & $\begin{array}{ll}25.927 \\
26.138 \\
26.377 \\
26.420 \\
26.452 \\
26.475 \\
26.562 \\
26.654 \\
26.761 \\
26.824 \\
26.906 \\
26.958 \\
27.016 \\
27.144 \\
27.257 \\
27.375 \\
27.498 \\
27.593 \\
27.732 \\
27.789 \\
27.808 \\
27.819 \\
27.834 \\
27.841 \\
27.854 \\
27.863 \\
27.864\end{array}$ & $\begin{array}{l}32.278 \\
32.504 \\
32.760 \\
32.805 \\
32.844 \\
32.873 \\
32.984 \\
33.111 \\
33.245 \\
33.324 \\
33.430 \\
33.498 \\
33.574 \\
33.740 \\
33.880 \\
34.924 \\
34.170 \\
34.282 \\
34.462 \\
34.566 \\
34.623 \\
34.656 \\
34.702 \\
34.724 \\
34.747 \\
34.764 \\
34.764\end{array}$ & $\begin{array}{l}38.422 \\
38.662 \\
38.934 \\
38.982 \\
39.028 \\
39.062 \\
39.196 \\
39.355 \\
39.515 \\
39.611 \\
39.740 \\
39.822 \\
39.916 \\
40.117 \\
40.284 \\
40.452 \\
40.620 \\
40.749 \\
40.969 \\
41.117 \\
41.209 \\
41.265 \\
41.340 \\
41.376 \\
41.409 \\
41.433 \\
41.433\end{array}$ & $\begin{array}{l}0.006 \\
0.050 \\
0.093 \\
0.134 \\
0.175 \\
0.255 \\
0.334 \\
0.409 \\
0.480 \\
0.547 \\
0.612 \\
0.674 \\
0.734 \\
0.845 \\
0.947 \\
1.039 \\
1.122 \\
1.195 \\
1.320 \\
1.429 \\
1.530 \\
1.627 \\
1.721 \\
1.811 \\
1.900 \\
1.987 \\
1.998\end{array}$ & $\begin{array}{l}5.64 \\
5.99 \\
2.66 \\
1.87 \\
1.65 \\
1.50 \\
2.87 \\
2.79 \\
2.41 \\
2.15 \\
2.36 \\
2.16 \\
2.40 \\
2.02 \\
2.06 \\
2.07 \\
1.84 \\
1.80 \\
1.47 \\
1.18 \\
0.85 \\
0.84 \\
0.79 \\
0.64 \\
0.71 \\
0.33 \\
0.30\end{array}$ & $\begin{array}{r}3 \\
25 \\
50 \\
74 \\
99 \\
149 \\
198 \\
248 \\
298 \\
347 \\
397 \\
446 \\
496 \\
595 \\
694 \\
793 \\
892 \\
991 \\
1188 \\
1385 \\
1583 \\
1780 \\
1976 \\
2173 \\
2370 \\
2566 \\
2590\end{array}$ \\
\hline $\begin{array}{l}\text { PR } \\
\text { dbar }\end{array}$ & $\stackrel{T}{D} C$ & $\stackrel{5}{0 / 00}$ & $\begin{array}{l}02 \\
\mathrm{ml} / \mathrm{I}\end{array}$ & $\begin{array}{c}\mathrm{F}-11 \\
\mathrm{pmol} / \mathrm{kg}\end{array}$ & $\begin{array}{c}\mathrm{F}-12 \\
\mathrm{pmol} / \mathrm{kg}\end{array}$ & $\stackrel{\theta}{\operatorname{Deg} C}$ & $\begin{array}{l}\mathrm{SIG}-\theta \\
\mathrm{kg} / \mathrm{m} 3\end{array}$ & $\underset{\mathrm{kg} / \mathrm{m} 3}{\mathrm{SIG}-1.5}$ & $\begin{array}{l}S I G-3 \\
\mathrm{~kg} / \mathrm{m} 3\end{array}$ & DE \\
\hline $\begin{array}{r}1 \\
27 \\
54 \\
77 \\
104 \\
129 \\
155 \\
176 \\
202 \\
253 \\
303 \\
405 \\
504 \\
603 \\
802 \\
1003 \\
1250 \\
1499 \\
1739 \\
1995 \\
2247 \\
2496 \\
2625\end{array}$ & $\begin{array}{r}20.378 \\
19.496 \\
18.963 \\
18.914 \\
18.580 \\
18.456 \\
18.398 \\
18.056 \\
17.236 \\
15.974 \\
15.019 \\
13.674 \\
12.379 \\
11.568 \\
10.030 \\
8.579 \\
6.986 \\
5.487 \\
4.728 \\
4.001 \\
3.488 \\
3.156 \\
3.109 \\
3.108\end{array}$ & $\begin{array}{l}36 \\
36 \\
36 \\
36 \\
36 \\
36 \\
36 \\
36 \\
36 \\
36 \\
35 \\
35 \\
35 \\
35 \\
35 \\
35 \\
35 \\
35\end{array}$ & $\begin{array}{l}5.30 \\
5.43 \\
5.39 \\
5.32 \\
5.24 \\
5.21 \\
5.19 \\
5.28 \\
4.80 \\
4.71 \\
4.73 \\
4.70 \\
4.56 \\
4.34 \\
4.15 \\
4.32 \\
4.91 \\
5.45 \\
5.73 \\
5.95 \\
6.91\end{array}$ & & & $\begin{array}{r}20.378 \\
19.491 \\
18.953 \\
18.900 \\
18.561 \\
18.434 \\
18.371 \\
18.025 \\
17.202 \\
15.934 \\
14.973 \\
13.616 \\
12.310 \\
11.489 \\
9.933 \\
8.468 \\
6.860 \\
5.350 \\
4.576 \\
3.834 \\
3.304 \\
2.953 \\
2.894 \\
2.894\end{array}$ & $\begin{array}{l}25.919 \\
26.188 \\
26.399 \\
26.434 \\
26.468 \\
26.478 \\
26.477 \\
26.503 \\
26.568 \\
26.678 \\
26.783 \\
26.920 \\
27.047 \\
27.137 \\
27.341 \\
27.590 \\
27.753 \\
27.805 \\
27.817 \\
27.831 \\
27.841 \\
27.866 \\
27.862 \\
27.863\end{array}$ & $\begin{array}{l}32.267 \\
32.556 \\
32.780 \\
32.815 \\
32.858 \\
32.871 \\
32.872 \\
32.907 \\
32.994 \\
33.139 \\
33.271 \\
33.448 \\
33.614 \\
33.729 \\
33.983 \\
34.280 \\
34.499 \\
34.608 \\
34.650 \\
34.693 \\
34.725 \\
34.764 \\
34.763 \\
34.764\end{array}$ & $\begin{array}{l}39.5 \\
39.7 \\
39.9 \\
40.1 \\
40.4 \\
40.7 \\
41.0 \\
41.1 \\
41.2 \\
41.3 \\
41.3 \\
41.4 \\
41.4 \\
41.4\end{array}$ & $\begin{array}{r}0 \\
27 \\
53 \\
77 \\
103 \\
128 \\
154 \\
174 \\
200 \\
251 \\
300 \\
401 \\
500 \\
597 \\
794 \\
993 \\
1236 \\
1482 \\
1718 \\
1970 \\
2216 \\
2461 \\
2587 \\
2588\end{array}$ \\
\hline
\end{tabular}




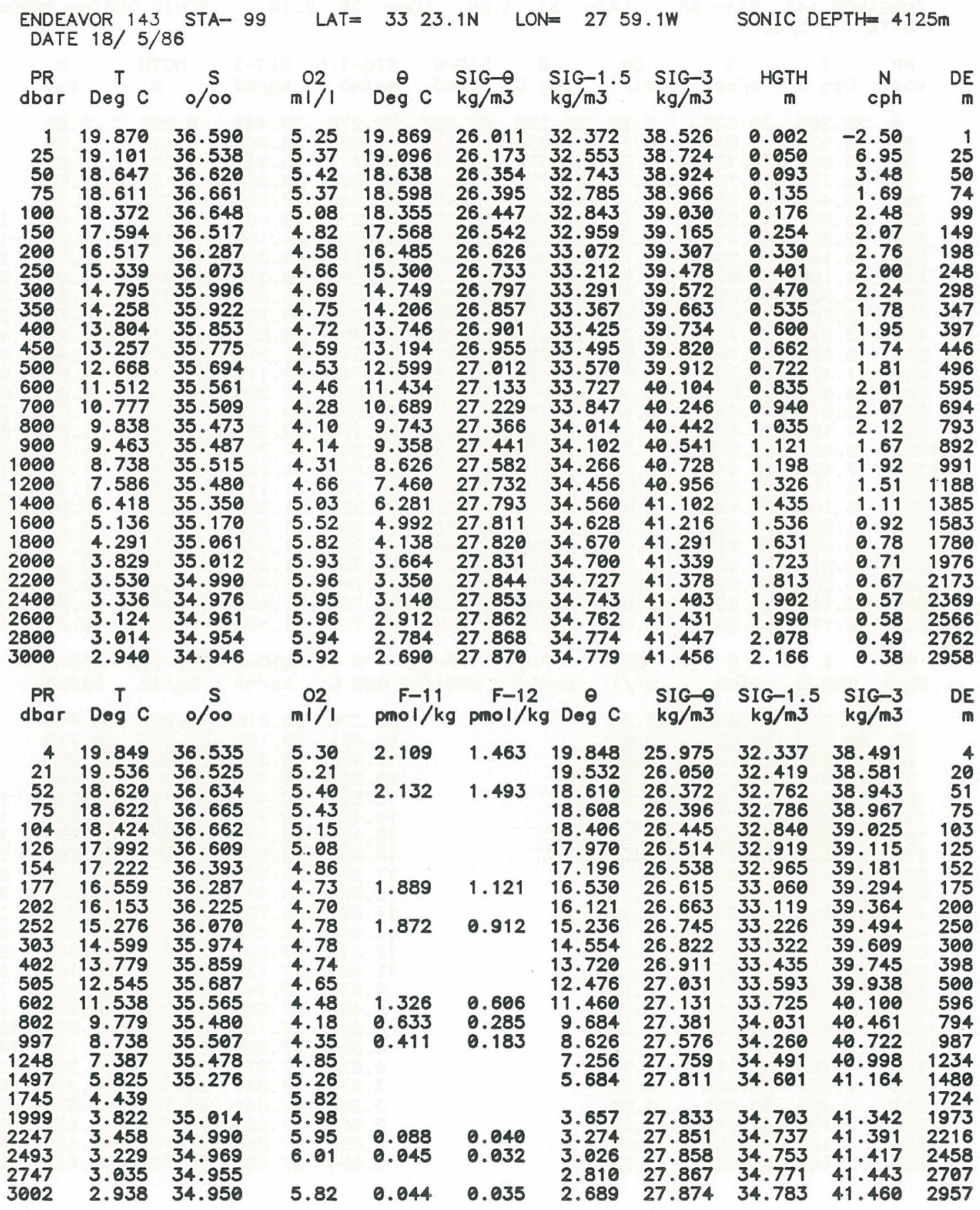




\begin{tabular}{|c|c|c|c|c|c|c|c|c|c|c|}
\hline \multicolumn{3}{|c|}{$\begin{array}{l}\text { ENDEAVOR } 143 \text { STA-100 } \\
\text { DATE } 18 / 5 / 86\end{array}$} & \multirow{2}{*}{$\begin{array}{l}\text { LAT }= \\
02 \\
\mathrm{ml} / \mathrm{l}\end{array}$} & \multicolumn{2}{|c|}{$3342.3 N$} & \multicolumn{2}{|c|}{$O N=2749.9 \mathrm{~W}$} & \multicolumn{3}{|c|}{ SONIC DEPTH $=4050 \mathrm{~m}$} \\
\hline $\begin{array}{l}\text { PR } \\
\text { dbar }\end{array}$ & $\stackrel{T}{\operatorname{Deg}} \mathrm{C}$ & $\stackrel{S}{0 / 00}$ & & $\stackrel{\theta}{\operatorname{Deg} C}$ & $\begin{array}{l}\mathrm{SIG}-\theta \\
\mathrm{kg} / \mathrm{m} 3\end{array}$ & $\begin{array}{l}\mathrm{SIG}-1.5 \\
\mathrm{~kg} / \mathrm{m} 3\end{array}$ & $\begin{array}{l}\mathrm{SIG}-3 \\
\mathrm{~kg} / \mathrm{m} 3\end{array}$ & $\underset{m}{\mathrm{HGTH}}$ & $\underset{c p h}{N}$ & DE \\
\hline $\begin{array}{r}3 \\
25 \\
50 \\
75 \\
100 \\
150 \\
200 \\
250 \\
300 \\
350 \\
400 \\
450 \\
500 \\
600 \\
700 \\
800 \\
900 \\
1000 \\
1200 \\
1400 \\
1600 \\
1800 \\
2000 \\
2200 \\
2400 \\
2600 \\
2800 \\
2999\end{array}$ & $\begin{array}{r}19.908 \\
19.915 \\
19.362 \\
18.657 \\
18.077 \\
17.308 \\
16.719 \\
15.799 \\
14.955 \\
14.565 \\
13.504 \\
13.132 \\
12.320 \\
11.249 \\
10.490 \\
9.783 \\
9.125 \\
8.688 \\
7.530 \\
6.246 \\
4.936 \\
4.325 \\
3.859 \\
3.574 \\
3.271 \\
3.104 \\
2.963 \\
2.862\end{array}$ & $\begin{array}{l}36.622 \\
36.621 \\
36.640 \\
36.593 \\
36.497 \\
36.345 \\
36.268 \\
36.130 \\
36.012 \\
35.959 \\
35.811 \\
35.756 \\
35.638 \\
35.522 \\
35.485 \\
35.497 \\
35.513 \\
35.544 \\
35.475 \\
35.322 \\
35.136 \\
35.064 \\
35.021 \\
35.002 \\
34.973 \\
34.962 \\
34.952 \\
34.946\end{array}$ & $\begin{array}{l}5.31 \\
5.31 \\
5.44 \\
5.36 \\
5.25 \\
5.15 \\
5.03 \\
5.09 \\
4.70 \\
4.73 \\
4.69 \\
4.73 \\
4.55 \\
4.39 \\
4.26 \\
4.09 \\
4.18 \\
4.33 \\
4.73 \\
5.16 \\
5.65 \\
5.86 \\
5.91 \\
5.94 \\
5.95 \\
5.93 \\
5.88 \\
5.83\end{array}$ & $\begin{array}{l}19.907 \\
19.911 \\
19.353 \\
18.644 \\
18.059 \\
17.283 \\
16.686 \\
15.759 \\
14.909 \\
14.512 \\
13.447 \\
13.069 \\
12.252 \\
11.172 \\
10.403 \\
9.688 \\
9.022 \\
8.576 \\
7.404 \\
6.111 \\
4.795 \\
4.172 \\
3.694 \\
3.393 \\
3.076 \\
2.892 \\
2.733 \\
2.615\end{array}$ & $\begin{array}{l}26.026 \\
26.024 \\
26.185 \\
26.332 \\
26.406 \\
26.480 \\
26.564 \\
26.674 \\
26.774 \\
26.819 \\
26.931 \\
26.966 \\
27.037 \\
27.151 \\
27.261 \\
27.394 \\
27.517 \\
27.612 \\
27.736 \\
27.793 \\
27.807 \\
27.819 \\
27.834 \\
27.849 \\
27.857 \\
27.865 \\
27.871 \\
27.877\end{array}$ & $\begin{array}{l}32.385 \\
32.384 \\
32.557 \\
32.722 \\
32.810 \\
32.905 \\
33.005 \\
33.140 \\
33.264 \\
33.321 \\
33.464 \\
33.510 \\
33.606 \\
33.754 \\
33.888 \\
34.043 \\
34.188 \\
34.298 \\
34.462 \\
34.567 \\
34.632 \\
34.668 \\
34.703 \\
34.730 \\
34.750 \\
34.766 \\
34.779 \\
34.789\end{array}$ & $\begin{array}{l}38.538 \\
38.536 \\
38.722 \\
38.902 \\
39.005 \\
39.120 \\
39.235 \\
39.394 \\
39.541 \\
39.608 \\
39.781 \\
39.838 \\
39.958 \\
40.139 \\
40.295 \\
40.473 \\
40.638 \\
40.761 \\
40.964 \\
41.114 \\
41.228 \\
41.288 \\
41.341 \\
41.379 \\
41.412 \\
41.435 \\
41.454 \\
41.469\end{array}$ & $\begin{array}{l}0.006 \\
0.050 \\
0.098 \\
0.142 \\
0.184 \\
0.265 \\
0.343 \\
0.417 \\
0.487 \\
0.554 \\
0.618 \\
0.679 \\
0.739 \\
0.849 \\
0.951 \\
1.042 \\
1.123 \\
1.195 \\
1.320 \\
1.429 \\
1.528 \\
1.623 \\
1.715 \\
1.805 \\
1.893 \\
1.980 \\
2.067 \\
2.153\end{array}$ & $\begin{array}{r}-1.11 \\
1.42 \\
5.90 \\
3.65 \\
2.81 \\
2.24 \\
2.57 \\
2.35 \\
2.55 \\
2.14 \\
2.23 \\
1.45 \\
2.19 \\
1.96 \\
2.07 \\
1.91 \\
1.98 \\
1.68 \\
1.44 \\
1.14 \\
0.89 \\
0.79 \\
0.76 \\
0.63 \\
0.61 \\
0.54 \\
0.52 \\
0.48\end{array}$ & $\begin{array}{r}3 \\
25 \\
50 \\
74 \\
99 \\
149 \\
198 \\
248 \\
298 \\
347 \\
397 \\
446 \\
496 \\
595 \\
694 \\
793 \\
892 \\
991 \\
1188 \\
1385 \\
1583 \\
1780 \\
1976 \\
2173 \\
2369 \\
2566 \\
2762 \\
2957\end{array}$ \\
\hline $\begin{array}{l}\text { PR } \\
\text { dbar }\end{array}$ & $\stackrel{T}{D e g} C$ & $\begin{array}{l}S \\
0 / 00\end{array}$ & $\begin{array}{l}02 \\
\mathrm{ml} / \mathrm{I}\end{array}$ & $\begin{array}{c}\mathrm{F}-11 \\
\mathrm{pmol} / \mathrm{kg}\end{array}$ & $\begin{array}{c}\mathrm{F}-12 \\
\mathrm{gmol} / \mathrm{kg}\end{array}$ & $\stackrel{\theta}{\operatorname{Deg} C}$ & $\underset{\mathrm{kg} / \mathrm{m} 3}{\mathrm{SIG}-0}$ & $\underset{\mathrm{kg} / \mathrm{m} 3}{\mathrm{SIG}-1.5}$ & $\begin{array}{l}\mathrm{SIG}-3 \\
\mathrm{~kg} / \mathrm{m} 3\end{array}$ & $D E$ \\
\hline $\begin{array}{r}3 \\
28 \\
51 \\
77 \\
105 \\
119 \\
137 \\
174 \\
204 \\
256 \\
305 \\
402 \\
503 \\
600 \\
800 \\
998 \\
1252 \\
1499 \\
1751 \\
1994 \\
2250 \\
2497 \\
2748 \\
3990\end{array}$ & $\begin{array}{r}20.028 \\
19.943 \\
19.070 \\
18.597 \\
17.765 \\
17.591 \\
17.369 \\
17.022 \\
16.716 \\
15.750 \\
15.040 \\
13.681 \\
12.284 \\
11.330 \\
9.742 \\
8.741 \\
7.341 \\
5.723 \\
4.502 \\
3.833 \\
3.492 \\
3.201 \\
2.996 \\
2.862\end{array}$ & $\begin{array}{l}36.640 \\
36.633 \\
36.647 \\
36.599 \\
36.452 \\
36.410 \\
36.368 \\
36.322 \\
36.277 \\
36.130 \\
36.027 \\
35.838 \\
35.634 \\
35.529 \\
35.485 \\
35.531 \\
35.460 \\
35.256 \\
35.090 \\
35.011 \\
34.991 \\
34.973 \\
34.954 \\
34.945\end{array}$ & $\begin{array}{l}5.27 \\
5.27 \\
5.36 \\
5.38 \\
5.14 \\
5.12 \\
5.12 \\
4.94 \\
5.07 \\
5.41 \\
4.70 \\
4.79 \\
4.42 \\
4.31 \\
4.76 \\
5.37 \\
5.88 \\
6.01 \\
5.97 \\
5.92\end{array}$ & & & $\begin{array}{r}20.027 \\
19.938 \\
19.060 \\
18.584 \\
17.747 \\
17.571 \\
17.346 \\
16.993 \\
16.683 \\
15.709 \\
14.994 \\
13.623 \\
12.216 \\
11.253 \\
9.647 \\
8.628 \\
7.211 \\
5.583 \\
4.351 \\
3.668 \\
3.308 \\
2.997 \\
2.772 \\
2.614\end{array}$ & $\begin{array}{l}26.007 \\
26.026 \\
26.266 \\
26.352 \\
26.448 \\
26.459 \\
26.482 \\
26.532 \\
26.571 \\
26.685 \\
26.766 \\
26.915 \\
27.040 \\
27.142 \\
27.391 \\
27.594 \\
27.752 \\
27.808 \\
27.820 \\
27.829 \\
27.849 \\
27.864 \\
27.869 \\
27.876\end{array}$ & $\begin{array}{l}32.364 \\
32.385 \\
32.645 \\
32.743 \\
32.861 \\
32.877 \\
32.905 \\
32.965 \\
33.012 \\
33.153 \\
33.254 \\
33.443 \\
33.611 \\
33.742 \\
34.043 \\
34.278 \\
34.485 \\
34.602 \\
34.662 \\
34.699 \\
34.733 \\
34.760 \\
34.775 \\
34.788\end{array}$ & $\begin{array}{l}38.514 \\
38.536 \\
38.816 \\
38.925 \\
39.064 \\
39.084 \\
39.119 \\
39.187 \\
39.242 \\
39.408 \\
39.529 \\
39.755 \\
39.964 \\
40.124 \\
40.473 \\
40.740 \\
40.993 \\
41.169 \\
41.275 \\
41.338 \\
41.386 \\
41.425 \\
41.449 \\
41.469\end{array}$ & $\begin{array}{r}28 \\
50 \\
76 \\
104 \\
118 \\
136 \\
173 \\
203 \\
254 \\
302 \\
398 \\
499 \\
594 \\
792 \\
987\end{array}$ \\
\hline
\end{tabular}




\begin{tabular}{|c|c|c|c|c|c|c|c|c|c|c|}
\hline \multicolumn{3}{|c|}{$\begin{array}{l}\text { ENDEAVOR } 143 \text { STA-101 } \\
\text { DATE } 18 / 5 / 86\end{array}$} & \multirow{2}{*}{$\begin{array}{l}\text { LAT }= \\
02 \\
\mathrm{ml} / \mathrm{l}\end{array}$} & \multicolumn{2}{|c|}{$3359.8 \mathrm{~N}$} & \multicolumn{2}{|c|}{$O N=2740.1 W$} & \multicolumn{3}{|c|}{ SONIC DEPTH= 4050m } \\
\hline $\begin{array}{l}\text { PR } \\
\text { dbar }\end{array}$ & $\stackrel{T}{\operatorname{Deg} C}$ & $\stackrel{S}{0 / 00}$ & & $\stackrel{\ominus}{\operatorname{Deg} C}$ & $\begin{array}{l}\text { IG-O } \\
g / \mathrm{m}^{3}\end{array}$ & $\begin{array}{l}\mathrm{SIG}-1.5 \\
\mathrm{~kg} / \mathrm{m} 3\end{array}$ & $\begin{array}{l}\text { SIG-3 } \\
\mathrm{kg} / \mathrm{m3}\end{array}$ & $\underset{m}{\text { HGTH }}$ & $\underset{c p h}{N}$ & $\begin{aligned} \mathrm{DE} \\
\mathbf{m}\end{aligned}$ \\
\hline $\begin{array}{r}1 \\
25 \\
50 \\
75 \\
100 \\
150 \\
200 \\
250 \\
300 \\
350 \\
400 \\
450 \\
500 \\
600 \\
700 \\
800 \\
900 \\
1000 \\
200 \\
400 \\
600 \\
800 \\
2000 \\
2200 \\
2400 \\
2600 \\
2800 \\
3000 \\
3059\end{array}$ & $\begin{array}{r}19.421 \\
19.221 \\
17.113 \\
16.511 \\
16.084 \\
15.680 \\
15.110 \\
14.549 \\
14.087 \\
13.535 \\
13.127 \\
12.711 \\
12.147 \\
11.053 \\
10.296 \\
9.708 \\
9.112 \\
8.728 \\
7.490 \\
6.069 \\
4.966 \\
4.305 \\
3.915 \\
3.525 \\
3.278 \\
3.141 \\
2.995 \\
2.872 \\
2.840\end{array}$ & $\begin{array}{l}36.277 \\
36.259 \\
36.229 \\
36.189 \\
36.134 \\
36.100 \\
36.029 \\
35.960 \\
35.897 \\
35.821 \\
35.761 \\
35.703 \\
35.629 \\
35.498 \\
35.479 \\
35.495 \\
35.509 \\
35.534 \\
35.477 \\
35.290 \\
35.136 \\
35.055 \\
35.019 \\
34.987 \\
34.971 \\
34.964 \\
34.956 \\
34.947 \\
34.945\end{array}$ & $\begin{array}{l}5.44 \\
5.41 \\
5.75 \\
5.44 \\
5.29 \\
5.20 \\
5.07 \\
4.89 \\
4.94 \\
4.94 \\
4.84 \\
4.85 \\
4.78 \\
4.36 \\
4.28 \\
4.11 \\
4.22 \\
4.31 \\
4.78 \\
5.24 \\
5.67 \\
5.92 \\
6.00 \\
6.04 \\
6.01 \\
5.96 \\
5.90 \\
5.84 \\
5.83\end{array}$ & $\begin{array}{r}19.421 \\
19.216 \\
17.105 \\
16.499 \\
16.068 \\
15.657 \\
15.079 \\
14.512 \\
14.043 \\
13.485 \\
13.070 \\
12.649 \\
12.080 \\
10.977 \\
10.211 \\
9.614 \\
9.009 \\
8.615 \\
7.365 \\
5.936 \\
4.824 \\
4.152 \\
3.748 \\
3.346 \\
3.083 \\
2.928 \\
2.765 \\
2.624 \\
2.586\end{array}$ & $\begin{array}{l}25.890 \\
25.929 \\
26.434 \\
26.547 \\
26.606 \\
26.674 \\
26.749 \\
26.820 \\
26.872 \\
26.930 \\
26.969 \\
27.008 \\
27.063 \\
27.168 \\
27.290 \\
27.405 \\
27.516 \\
27.598 \\
27.743 \\
27.791 \\
27.803 \\
27.814 \\
27.827 \\
27.842 \\
27.854 \\
27.863 \\
27.872 \\
27.877 \\
27.879\end{array}$ & $\begin{array}{l}32.264 \\
32.308 \\
32.865 \\
32.994 \\
33.064 \\
33.143 \\
33.234 \\
33.322 \\
33.387 \\
33.462 \\
33.513 \\
33.565 \\
33.637 \\
33.777 \\
33.923 \\
34.057 \\
34.187 \\
34.283 \\
34.470 \\
34.571 \\
34.627 \\
34.664 \\
34.693 \\
34.725 \\
34.747 \\
34.763 \\
34.778 \\
34.789 \\
34.792\end{array}$ & $\begin{array}{l}38.430 \\
38.479 \\
39.085 \\
39.229 \\
39.310 \\
39.400 \\
39.507 \\
39.609 \\
39.688 \\
39.778 \\
39.841 \\
39.906 \\
39.994 \\
40.168 \\
40.337 \\
40.488 \\
40.637 \\
40.745 \\
40.974 \\
41.125 \\
41.222 \\
41.285 \\
41.329 \\
41.376 \\
41.409 \\
41.430 \\
41.452 \\
41.469 \\
41.473\end{array}$ & $\begin{array}{l}0.002 \\
0.053 \\
0.098 \\
0.136 \\
0.173 \\
0.245 \\
0.314 \\
0.381 \\
0.444 \\
0.506 \\
0.567 \\
0.626 \\
0.683 \\
0.793 \\
0.892 \\
0.981 \\
1.060 \\
1.133 \\
1.258 \\
1.366 \\
1.465 \\
1.561 \\
1.654 \\
1.746 \\
1.834 \\
1.922 \\
2.009 \\
2.096 \\
2.121\end{array}$ & $\begin{array}{l}-0.75 \\
6.69 \\
5.94 \\
2.61 \\
2.20 \\
2.26 \\
1.89 \\
2.39 \\
1.89 \\
1.71 \\
1.87 \\
1.71 \\
1.69 \\
2.13 \\
2.03 \\
2.18 \\
1.64 \\
1.74 \\
1.48 \\
1.12 \\
0.81 \\
0.75 \\
0.73 \\
0.75 \\
0.59 \\
0.57 \\
0.54 \\
0.51 \\
0.48\end{array}$ & $\begin{array}{r}1 \\
25 \\
50 \\
74 \\
99 \\
149 \\
198 \\
248 \\
298 \\
347 \\
397 \\
446 \\
496 \\
595 \\
694 \\
793 \\
892 \\
990 \\
1188 \\
1385 \\
1583 \\
1779 \\
1976 \\
2173 \\
2369 \\
2566 \\
2762 \\
2958 \\
3015\end{array}$ \\
\hline $\begin{array}{l}\text { PR } \\
\text { dbar }\end{array}$ & $\stackrel{T}{\operatorname{Deg}} C$ & $\stackrel{S}{0 / 00}$ & $\begin{array}{ll}02 \\
\mathrm{n} / \mathrm{l}\end{array}$ & $\begin{array}{c}\mathrm{F}-11 \\
\mathrm{pmol} / \mathrm{kg}\end{array}$ & $\begin{array}{c}\mathrm{F}-12 \\
\mathrm{pmol} / \mathrm{kg}\end{array}$ & $\stackrel{\theta}{D e g} C$ & $\begin{array}{l}\text { SIG-O } \\
\mathrm{kg} / \mathrm{m3}\end{array}$ & $\underset{\mathrm{kg} / \mathrm{m} 3}{\mathrm{SIG}-1.5}$ & $\begin{array}{l}S I G-3 \\
\mathrm{~kg} / \mathrm{m} 3\end{array}$ & $\begin{array}{r}D E \\
m\end{array}$ \\
\hline $\begin{array}{r}5 \\
24 \\
50 \\
75 \\
100 \\
127 \\
151 \\
177 \\
176 \\
197 \\
299 \\
400 \\
500 \\
600 \\
800 \\
993 \\
1245 \\
1499 \\
1746 \\
1997 \\
2244 \\
2497\end{array}$ & $\begin{array}{r}19.496 \\
19.295 \\
17.510 \\
16.789 \\
16.476 \\
15.929 \\
15.573 \\
15.274 \\
15.267 \\
15.066 \\
13.947 \\
13.082 \\
12.079 \\
11.014 \\
9.581 \\
8.713 \\
7.349 \\
5.575 \\
4.452 \\
3.916 \\
3.465 \\
3.206 \\
3.018 \\
2.863\end{array}$ & $\begin{array}{l}36 . \\
36 \\
36 \\
36 \\
36 \\
36 . \\
36 \\
36 \\
36 . \\
35 \\
35 \\
35 \\
35 \\
35 \\
35 \\
35 \\
35 \\
35 \\
35 \\
34 \\
34 \\
34\end{array}$ & $\begin{array}{l}5.34 \\
5.40 \\
5.67 \\
5.70 \\
5.47 \\
5.34 \\
5.20 \\
5.16 \\
5.17 \\
5.05\end{array}$ & $\begin{array}{l}2.160 \\
2.176 \\
2.317 \\
2.303 \\
2.344 \\
2.327 \\
2.217 \\
2.260 \\
2.243 \\
2.145 \\
2.085 \\
2.010 \\
1.695 \\
1.105 \\
0.440 \\
0.334 \\
0.323 \\
0.301 \\
0.245 \\
0.127 \\
0.088 \\
0.061 \\
0.057 \\
0.041\end{array}$ & $\begin{array}{l}1.106 \\
1.023 \\
1.094 \\
1.097 \\
1.315 \\
1.163 \\
1.192 \\
1.190 \\
1.225 \\
1.000 \\
1.003 \\
0.916 \\
0.857 \\
0.513 \\
0.206 \\
0.205 \\
0.144 \\
0.118 \\
0.115 \\
0.098 \\
0.037 \\
0.025 \\
0.007 \\
0.002\end{array}$ & $\begin{array}{r}19.495 \\
19.291 \\
17.501 \\
16.777 \\
16.459 \\
15.908 \\
15.549 \\
15.247 \\
15.239 \\
15.036 \\
13.903 \\
13.026 \\
12.012 \\
10.938 \\
9.487 \\
8.602 \\
7.219 \\
5.436 \\
4.302 \\
3.750 \\
3.281 \\
3.003 \\
2.793 \\
2.615\end{array}$ & $\begin{array}{l}25.878 \\
25.918 \\
26.355 \\
26.495 \\
26.560 \\
26.635 \\
26.693 \\
26.726 \\
26.727 \\
26.756 \\
26.897 \\
26.981 \\
27.080 \\
27.175 \\
27.423 \\
27.602 \\
27.758 \\
27.801 \\
27.814 \\
27.830 \\
27.846 \\
27.859 \\
27.870 \\
27.879\end{array}$ & $\begin{array}{l}32.250 \\
32.296 \\
32.775 \\
32.934 \\
33.008 \\
33.097 \\
33.165 \\
33.207 \\
33.208 \\
33.243 \\
33.416 \\
33.526 \\
33.656 \\
33.785 \\
34.079 \\
34.287 \\
34.490 \\
34.600 \\
34.658 \\
34.696 \\
34.731 \\
34.756 \\
34.775 \\
34.791\end{array}$ & $\begin{array}{l}38.415 \\
38.465 \\
38.986 \\
39.162 \\
39.244 \\
39.347 \\
39.425 \\
39.475 \\
39.476 \\
39.516 \\
39.721 \\
39.856 \\
40.015 \\
40.176 \\
40.515 \\
40.750 \\
40.999 \\
41.173 \\
41.272 \\
41.332 \\
41.385 \\
41.421 \\
41.448 \\
41.472\end{array}$ & $\begin{array}{r}5 \\
24 \\
50 \\
74 \\
99 \\
125 \\
150 \\
176 \\
175 \\
195 \\
297 \\
397 \\
495 \\
594 \\
792 \\
982 \\
1231 \\
1481 \\
1724 \\
1972 \\
2214 \\
2462 \\
2708 \\
2954\end{array}$ \\
\hline
\end{tabular}


Attn: Stella Sanchez-Wade

Documents Section

Scripps Institution of Oceanography

Library, Mail Code C-075C

La Jolla, CA 92093

Hancock Library of Biology \& Oceanography

Alan Hancock Laboratory

University of Southern California

University Park

Los Angeles, CA 90089-0371

Gifts \& Exchanges

Library

Bedford Institute of Oceanography

P.O. Box 1006

Dartmouth, NS, B2Y 4A2, CANADA

Office of the International Ice Patrol

c/o Coast Guard R \& D Center

Avery Point

Groton, CT 06340

Library

Physical Oceanographic Laboratory

Nova University

8000 N. Ocean Drive

Dania, FL 33304

NOAA/EDIS Miami Library Center 4301 Rickenbacker Causeway

Miami, FL 33149

Library

Skidaway Institute of Oceanography

P.O. Box 13687

Savannah, GA 31416

Institute of Geophysics

University of Hawaii

Library Room 252

2525 Correa Road

Honolulu, HI 96822

Library

Chesapeake Bay Institute

4800 Atwell Road

Shady Side, MD 20876

MIT Libraries

Serial Journal Room 14E-210

Cambridge, MA 02139
Director, Ralph M. Parsons Laboratory

Room 48-31 1

MIT

Cambridge, MA 02139

Marine Resources Information Center

Building E38-320

MIT

Cambridge, MA 02139

Library

Lamont-Doherty Geological Observatory

Colombia University

Palisades, NY 10964

Library

Serials Department

Oregon State University

Corvallis, OR 97331

Pell Marine Science Library

University of Rhode Island

Narragansett Bay Campus

Narragansett, RI 02882

Working Collection

Texas A\&M University

Dept. of Oceanography

College Station, TX 77843

Library

Virginia Institute of Marine Science

Gloucester Point, VA 23062

Fisheries-Oceanography Library

151 Oceanography Teaching Bldg.

University of Washington

Seattle, WA 98195

Library

R.S.M.A.S.

University of Miami

4600 Rickenbacker Causeway

Miami, FL 33149

Maury Oceanographic Library

Naval Oceanographic Office

Bay St. Louis

NSTL, MS 39522-5001 



\begin{tabular}{|c|c|c|c|}
\hline $\begin{array}{l}\text { REPORT DOCUMENTATION } \\
\text { PAGE }\end{array}$ & $\begin{array}{l}\text { 1. REPORT NO. } \\
\text { WHOI-88-7 }\end{array}$ & 2. & 3. Recipient's Accession No. \\
\hline \multirow{2}{*}{\multicolumn{3}{|c|}{$\begin{array}{l}\text { 4. Title and Subtitle } \\
\text { Hydrographic Data from R/V Endeavor Cruise \#143 }\end{array}$}} & $\begin{array}{l}\text { 5. Report Date } \\
\text { March1988 }\end{array}$ \\
\hline & & & 6. \\
\hline \multicolumn{3}{|c|}{$\begin{array}{l}\text { 7. Author(s) } \\
\text { M.C. Stalcup, T.M. Joyce, J.L. Bullister, R.L. Barbour and J.A. Dunworth } \\
\end{array}$} & $\begin{array}{l}\text { 8. Performing Organization Rept. No. } \\
\text { WHOI-88-7 }\end{array}$ \\
\hline \multirow{2}{*}{\multicolumn{3}{|c|}{$\begin{array}{l}\text { 9. Performing Organization Name and Address } \\
\text { The Woods Hole Oceanographic Institution } \\
\text { Woods Hole, Massachusetts } 02543\end{array}$}} & 10. Project/Task/Work Unit No. \\
\hline & & & $\begin{array}{l}\text { 11. Contract(C) or Grant(G) No. } \\
\text { (C) } \\
\text { (G) OCE } 85-15642 \text {; OCE } 85-18372\end{array}$ \\
\hline \multirow{2}{*}{\multicolumn{3}{|c|}{$\begin{array}{l}\text { 12. Sponsoring Organization Name and Address } \\
\text { The National Science Foundation }\end{array}$}} & $\begin{array}{l}\text { 13. Type of Report \& Period Covered } \\
\text { Technical Report }\end{array}$ \\
\hline & & & 14. \\
\hline \multicolumn{4}{|c|}{$\begin{array}{l}\text { 15. Supplementary Notes } \\
\text { This report should be cited as: Woods Hole Oceanog. Inst. Tech. Rept., WHOI-88-7. }\end{array}$} \\
\hline \multicolumn{4}{|c|}{$\begin{array}{l}\text { 16. Abstract (Limit: } 200 \text { words) } \\
\text { Hydrographic data collected during R/V Endeavor cruise } 143 \text { is presented as a preliminary study of subduction in the northeast Atlantic } \\
\text { south of the Azores Front. The front is clearly defined at the northern end of CTD section \# } 1 \text { which also shows a layer of } 16-18^{\circ} \mathrm{C} \text { water } \\
\text { subducted to the south. Section } \# 2,280 \mathrm{~km} \text { to the east, is dominated by a large cyclonic ring with characteristics similar to 'eastern' rings } \\
\text { reported earlier. An anomalously salty parcel of Mediterranean water in this section is typical of highly saline lenses seen in the Canary } \\
\text { Basin. }\end{array}$} \\
\hline
\end{tabular}

17. Document Analysis a. Descriptors

1. Subduction

2. Cyclonic ring

3. Mediterranean water lenses

b. Identifiers/Open-Ended Terms

c. COSATI Field/Group

18. Availability Statement

Approved for publication; distribution unlimited.

\begin{tabular}{|l|l|}
\hline $\begin{array}{c}\text { 19. Security Class (This Report) } \\
\text { UNCLASSIFIED }\end{array}$ & $\begin{array}{c}\text { 21. No. of Pages } \\
118\end{array}$ \\
\hline 20. Security Class (This Page) & 22. Price \\
\hline
\end{tabular}

